\title{
1992 ANNUAL REPORT
}

\section{SANITARY LANDFILL GROUNDWATER \\ MONITORING REPORT (U)}

KEY WORDS

chloroethene (vinyl chloride)

LFW wells tetrachloroethylene trichloroethylene tritium

PUBLICATION DATE: FEBRUARY 1993

Authorized Derivative Classifier:
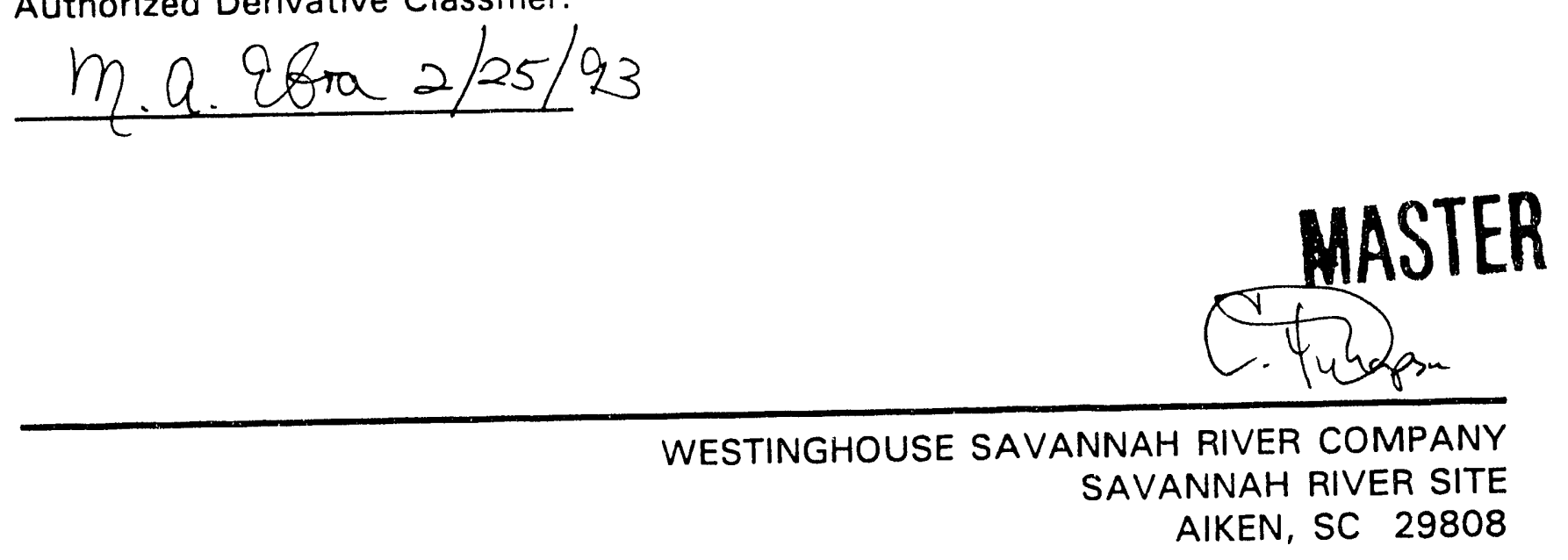

Prepared for the U.S. Department of Energy under Control Contract No. DE-ACO9-89SR18035 
-

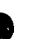

$c$

C $\begin{array}{lll}\text { Sanitary Landfill } & \text { ii } & \text { Fourth Quarter } 1992\end{array}$ 


\section{Abstract}

Fifty-seven wells of the LFW series monitor groundwater quality in Steed Pond Aquifer (formerly Aquifer Zone I/IIC ${ }_{2}$ ) (Water Table) beneath the Sanitary Landfill at the Savannah River Site (SRS). These wells are sampled quarterly as part of the SRS Groundwater Monitoring Program and to comply with the South Carolina Department of Health and Environmental Control Domestic Waste Permit 87A.

During first quarter 1992, 19 of the 57 LFW wells contained levels of trichloroethylene, tetrachloroethylene, 1,2-dichloroethane, tritium, chloroethene (vinyl chloride), 1,1dichloroethylene, cadmium, 1,1,1-trichloroethane, or benzene that exceeded the Safe Drinking Water Act final Primary Drinking Water Standards (PDWS) established by the U.S. Environmental Protection Agency. Trichloroethylene was detected most frequently, exceeding its final PDWS in 15 wells.

Elevated levels of trichloroethylene, benzene, chloroethene (vinyl chloride), 1,2dichloroethane, tritium, tetrachloroethylene, 1,2-dichloroethane, 1,1-dichloroethylene, or 1,2dichloropropane were detected above the final PDWS in one or more of 17 wells at the Sanitary Landfill during second quarter 1992. Trichloroethylene and benzene occurred most frequently: trichloroethylene exceeded its final PDWS in 13 wells and benzene in 4 wells.

During third quarter 1992, 17 of the 57 LFW wells contained levels of trichloroethylene, tetrachloroethylene, tritium, benzene, chloroethene (vinyl chloride), 1,2-dichloroethane, 1,1dichloroethylene, 1,2-dichloropropane, lead, or lindane that exceeded the final PDWS. Trichloroethylene and tetrachloroethylene were detected most frequently: trichloroethylene was elevated in 15 wells; tetrachloroethylene was elevated in 5 wells.

One or more of the following constituents exceeded the final PDWS in 21 wells at the Sanitary Landfill during fourth quarter 1992: trichloroethylene, tetrachloroethylene, chloroethene (vinyl chloride), benzene, 1,2-dichloroethane, tritium, 1,1-dichloroethylene, or mercury. Trichloroethylene, tetrachloroethylene, and chloroethene (vinyl chloride) occurred most frequently: trichloroethylene exceeded its final PDWS in 15 wells and tetrachloroethylene and chloroethene (vinyl chloride) in 5 wells each.

During 1992, no elevated constituents were detected in upgradient wells LFW 43B, 43C, and 43D.

The groundwater flow direction in the Steed Pond Aquifer (Water Table) beneath the Sanitary Landfill is to the south-southeast. Flow rates in this unit ranged between approximately $130 \mathrm{ft} / \mathrm{yr}$ and $140 \mathrm{ft} / \mathrm{yr}$ during first and fourth quarters 1992. 
WSRC-TR-93-068

0

0

○

.

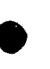

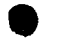


WSRC-TR-93-068

\section{Contents}

Page

Abstract $\ldots \ldots \ldots \ldots \ldots \ldots \ldots \ldots \ldots \ldots \ldots \ldots \ldots \ldots \ldots \ldots \ldots \ldots$

List of Figures $\ldots \ldots \ldots \ldots \ldots \ldots \ldots \ldots \ldots \ldots \ldots \ldots \ldots \ldots$

List of Tables $\ldots \ldots \ldots \ldots \ldots \ldots \ldots \ldots \ldots \ldots \ldots \ldots \ldots \ldots \ldots \ldots$

Executive $\operatorname{Summary} \ldots \ldots \ldots \ldots \ldots \ldots \ldots \ldots \ldots \ldots \ldots \ldots$

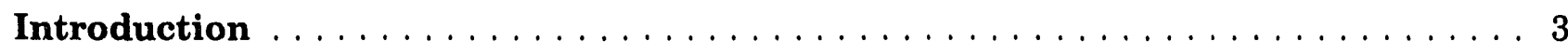

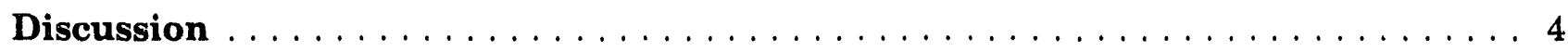

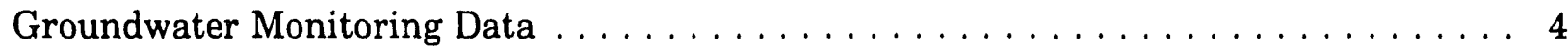

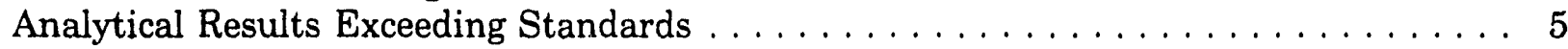

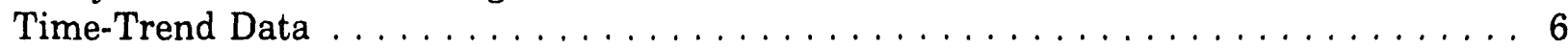

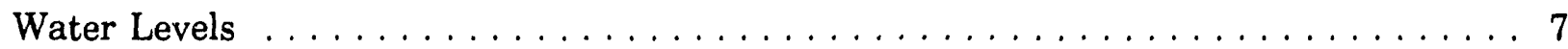

Groundwater Flow Rates and Directions $\ldots \ldots \ldots \ldots \ldots \ldots \ldots \ldots \ldots$

Upgradient Versus Downgradient Results $\ldots \ldots \ldots \ldots \ldots \ldots \ldots \ldots \ldots$

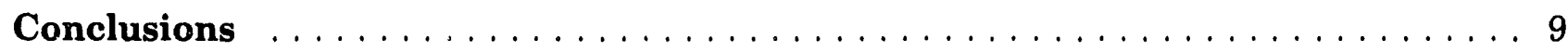

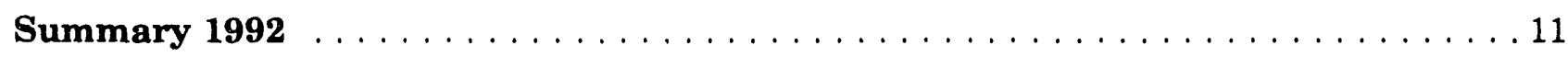

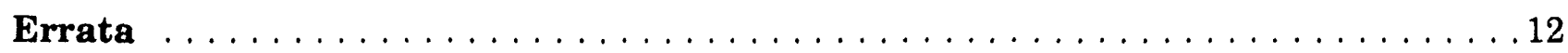

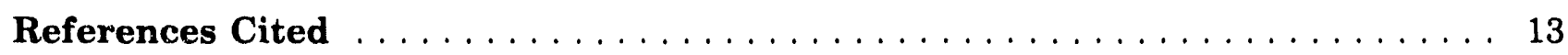

Appendix A-Primary Drinking Water Standards $\ldots \ldots \ldots \ldots \ldots \ldots$

Appendix B-Flagging Criteria $\ldots \ldots \ldots \ldots \ldots \ldots \ldots \ldots \ldots \ldots \ldots \ldots$

Appendix C-Figures $\ldots \ldots \ldots \ldots \ldots \ldots \ldots \ldots \ldots \ldots \ldots \ldots \ldots \ldots \ldots$

Appendix D-Groundwater Monitoring Results Tables $\ldots \ldots \ldots \ldots \ldots \ldots$

Appendix E-Data Quality/Useability Assessment $\ldots \ldots \ldots \ldots \ldots \ldots$

Appendix F-Time Series Plots $\ldots \ldots \ldots \ldots \ldots \ldots \ldots \ldots \ldots \ldots$

Appendix G-Hydrographs $\ldots \ldots \ldots \ldots \ldots \ldots \ldots \ldots \ldots \ldots \ldots \ldots$ 
WSRC-TR-93-068

0

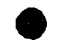

-

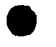




\section{List of Figures}

Page

1. Location of the Sanitary Landfill at the

Savannah River Site

2. Boundaries of Original Sanitary Landfill and

Expansions .............................

3. Location of Monitoring Wells at the Sanitary Landfill . . . . . . . . . . . . . C-4

4. Chloroethene (Vinyl chloride) Concentrations at the

Sanitary Landfill, First Quarter $1992 \ldots \ldots \ldots \ldots \ldots$. . . . . . . . . . . . C-5

5. Chloroethene (Vinyl chloride) Concentrations at the

Sanitary Landfill, Fourth Quarter $1992 \ldots \ldots \ldots \ldots \ldots$. . . . . . . . . . . C-6

6. Radium-226 Activities at the Sanitary Landfill, First

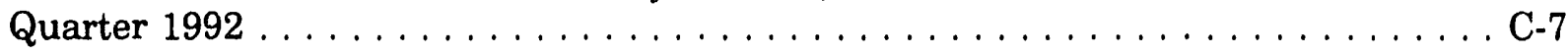

7. Radium-226 Activities at the Sanitary Landfill, Fourth

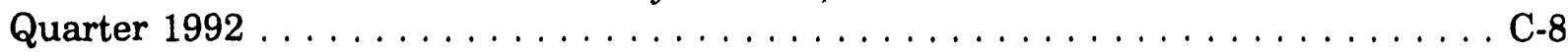

8. Trichloroethylene Concentrations at the Sanitary Landfill,

First Quarter 1992

9. Trichloroethylene Concentrations at the Sanitary Landfill,

Fourth Quarter $1992 \ldots \ldots \ldots \ldots \ldots \ldots \ldots \ldots \ldots \ldots$

10. Tritium Activities at the Sanitary Landfill, First Quarter 1992

11. Tritium Activities at the Sanitary Landfill, Fourth Quarter 1992

12. Water-Elevation Contour Map of Steed Pond Aquifer (Water

Table) at the Sanitary Landfill, First Quarter 1992 
13. Water-Elevation Contour Map of Steed Pond Aquifer (Water Table) at the Sanitary Landfill, Fourth Quarter 1992

14. Regional Correlation of Hydrostratigraphic and Lithostratographic Nomenclature C-15 


\section{List of Tables}

Page

1. Constituents Exceeding the Final Primary Drinking Water Standards . . . . . . D-5

2. Constituents Exceeding Half the Final Primary Drinking Water Standards

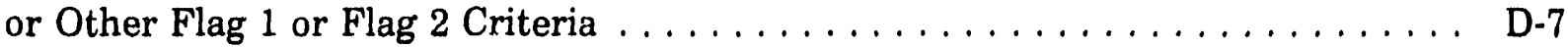

3. Groundwater Monitoring Results for Individual Wells . . . . . . . . . . . . . D-13 
WSRC-TR-93-068

-

$\bullet$

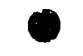

-

-

- 


\section{Executive Summary}

The Sanitary Landfill at the Savannah River Site (SRS) is composed of the original 32-acre landfill, plus expansion areas to the north and south that added 16 and 22 acres, respectively, to the facility. The landfill is subject to the requirements of the Resource Conservation and Recovery Act and currently operates under South Carolina Department of Health and Environmental Control (SCDHEC) Domestic Waste Permit 87A.

Fifty-seven wells of the LFW series monitor the groundwater quality in Steed Pond Aquifer (formerly Aquifer Zone I/IIC ${ }_{2}$ ) (Water Table) beneath the Sanitary Landfill. These wells are sampled quarterly for certain indicator parameters, inorganics, metals, radionuclides, volatile organics, and other constituents as part of the SRS Groundwater Monitoring Program and to comply with the SCDHEC domestic waste permit.

During first quarter 1992, 19 of the 57 LFW wells contained levels of trichloroethylene, tetrachloroethylene, 1,2-dichloroethane, tritium, chloroethene (vinyl chloride), 1,1dichloroethylene, cadmium, 1,1,1-trichloroethane, or benzene that exceeded the Safe Drinking Water Act final Primary Drinking Water Standards (PDWS) established by the U.S. Environmental Protection Agency. Trichloroethylene, tetrachloroethylene, and 1,2-dichloroethane occurred most frequently: trichloroethylene was detected in 15 wells, with the highest concentration in LFW $10 \mathrm{~A}$ at $49 \mu \mathrm{g} / \mathrm{L}$; tetrachloroethylene and 1,2-dichloroethane were detected in 3 wells each, with the maximum tetrachloroethylene concentration of $37 \mu \mathrm{g} / \mathrm{L}$ in LFW 10A and a maximum 1,2-dichloroethane concentration of $7.6 \mu \mathrm{g} / \mathrm{L}$ in $\mathrm{LFW} 36$.

Elevated levels of trichloroethylene, benzene, chloroethene (vinyl chloride), 1,2dichloroethane, tritium, tetrachloroethylene, 1,2-dichloroethane, 1,1-dichloroethylene, or 1,2dichloropropane were detected above the final PDWS in one or more of 17 wells at the Sanitary Landfill during second quarter 1992. Trichloroethylene and benzene occurred most frequently: trichloroethylene exceeded its final PDWS in 13 wells, with the highest concentration in LFW $10 \mathrm{~A}$ at $48 \mu \mathrm{g} / \mathrm{L}$; benzene was elevated in 4 wells, with the the highest concentration in LFW 21 at $21 \mu \mathrm{g} / \mathrm{L}$.

During third quarter 1992, 17 of the 57 LFW wells contained levels of trichloroethylene, tetrachloroethylene, tritium, benzene, chloroethene (vinyl chloride), 1,2-dichloroethane, 1,1dichloroethylene, 1,2-dichloropropane, lead, or lindane that exceeded the final PDWS in the groundwater beneath the Sanitary Landfill. Trichloroethylene and tetrachloroethylene were detected most frequently: trichloroethylene was elevated in 15 wells, with the highest concentration found in well LFW $10 \mathrm{~A}$ at $45 \mu \mathrm{g} / \mathrm{L}$; tetrachloroethylene was elevated in 5 wells, with the highest concentration in well LFW $10 \mathrm{~A}$ at $125 \mu \mathrm{g} / \mathrm{L}$.

Elevated levels of trichloroethylene, tetrachloroethylene, chloroethene (vinyl chloride), benzene, 1,2-dichloroethane, tritium, 1,1-dichloroethylene, or mercury were detected in 21 of the LFW wells at the Sanitary Landfill during fourth quarter 1992. Trichloroethylene, 
tetrachloroethylene, and chloroethene (vinyl chloride) occurred most frequently:

trichloroethylene exceeded its final PDWS in 15 wells, with the maximum concentration at 51 $\mu \mathrm{g} / \mathrm{L}$ in well LFW 10A; chloroethene (vinyl chloride) and tetrachloroethylene were elevated in 5 wells each, with a maximum chloroethene (vinyl chloride) concentration of $126 \mu \mathrm{g} / \mathrm{L}$ in well LFW 48D and a maximum tetrachloroethylene concentration of $27 \mu \mathrm{g} / \mathrm{L}$ in LFW 10A.

The groundwater flow direction in Steed Pond Aquifer (Water Table) beneath the Sanitary Landfill is to the south-southeast (SRS grid coordinates) toward Upper Three Runs Creek. Flow rates in this aquifer during the first and fourth quarters of 1992 were estimated to range between approximately $130 \mathrm{ft} / \mathrm{yr}$ and $140 \mathrm{ft} / \mathrm{yr}$.

No elevated constituents occurred in upgradient wells LFW 43B, 43C, and 43D during 1992. 


\section{Introduction}

The Sanitary Landfill (740-G) is located southwest of Road C, about midway down the slope from the Aiken Plateau to Upper Three Runs Creek. Since 1974, the site has been receiving sanitary waste, including paper and paper products, plastics, scrap metal, rubber, food waste, glass, packing material, miscellaneous construction debris, empty paint cans, and other nonradioactive waste materials. The bags of waste are placed in trenches and covered daily with soil (Heffner and Exploration Resources, 1991). The landfill is currently operating under South Carolina Domestic Waste Permit 87A (SCDHEC, 1991; WSRC, 1990).

In 1987, two expansion areas were permitted to the north and south of the original 32-acre landfill, adding 16 and 22 acres, respectively, to the site. The original landfill was filled to capacity in early 1987, and the southern expansion is more than three-quarters full at present. The northern expansion will be used beginning in early 1993 (WSRC, 1990).

In 1988, the Sanitary Landfill was placed on the Resource Conservation and Recovery Act (RCRA) Facility Investigation (RFI) list because elevated levels of hazardous constituents were detected in the groundwater beneath the facility (WSRC, 1990). The landfill was removed from the RFI list and a settlement agreement (\#91-51-SW) was executed as a result of disposal of wipes and rags used with RCRA-listed solvents. Fifty-seven wells of the LFW series presently monitor the groundwater quality in Steed Pond Aquifer (Water Table). Fourteen wells were installed between 1981 and 1984, prior to the practice of installing RCRA standard wells at the Savannah River Site (SRS). Seventeen RCRA wells were installed around the southern and northern expansions in late 1986. In accordance with the Groundwater Quality Assessment Plan (WSRC, 1990), 26 additional wells were installed in 1991 and first sampled third quarter 1991.

The monitoring wells at the Sanitary Landfill are sampled quarterly for certain indicator parameters, inorganics, metals, radionuclides, volatile organics, and other constituents as part of the SRS Groundwater Monitoring Program and to comply with the SCDHEC domestic waste permit. 


\section{Discussion}

\section{Groundwater Monitoring Data}

The groundwater sampling procedure was modified beginning fourth quarter 1992 in response to regulatory guidance and advances in sampling equipment design (WSRC, 1992b). The modified procedure requires evacuation of a minimum of two well volumes and stabilization of $\mathrm{pH}$, specific conductance, and turbidity prior to sample collection. Stability is established when a minimum of three successive measurements, taken within a given time period, are within a specified tolerance range. If a well pumps dry before two well volumes are purged or before stabilization is achieved, it must be revisited within 24 hours for the data to be considered a single sampling event. On the second visit within 24 hours, samples are taken without purging or measurements of stability; thus, these samples may not be representative of the groundwater quality.

A further modification in the procedure is that samples collected for metals analyses are not filtered. Thus, the analyses are for total metals rather than dissolved metals.

Throughout 1992, samples from wells at the Sanitary Landfill were analyzed for certain indicator parameters, heavy metals, radionuclides, and other constituents as part of the SRS Environmental Protection Department/Environmental Monitoring Section (EPD/EMS) Groundwater Monitoring Program. Monitoring results that exceeded the Safe Drinking Water Act (SDWA) final Primary Drinking Water Standards (PDWS) or screening levels established by the U.S. Environmental Protection Agency (EPA) (see Appendix A) or other SRS flagging criteria (see Appendix B) are discussed in this report. Constituent levels that equal or exceed the final PDWS, screening levels, or the Flag 2 criteria are described as elevated, and constituents that exceed Flag 1 criteria are described as slightly elevated.

The final PDWS for individual analytes provided in Appendix A may not always match the SRS flagging criteria provided in Appendix B. The final PDWS are used as guidelines in this compliance report to meet regulatory requirements; the flagging criteria are used by $\mathrm{EPD} / \mathrm{EMS}$ to identify relative levels of constituents in the groundwater and as guides for scheduling groundwater sampling.

Appendix $\mathrm{C}$ contains illustrations of the monitored waste management unit (Figures 1 and 2); the individual monitoring wells (Figure 3); the lateral distributions of chloroethene (vinyl chloride), radium-226, trichloroethylene, and tritium for first and fourth quarters 1992 (Figures 4 through 11); the water-elevation contours of the Steed Pond Aquifer (Water Table) hydrostratigraphic unit and the groundwater flow directions for third and fourth quarters 1992 (Figures 12 and 13); and the regional correlation of hydrostratigraphic and lithostratigraphic nomenclature (Figure 14). The monitoring results tables, as well as analyses that exceeded the holding times and the final PDWS or other flagging criteria, are presented in Appendix D; data quality/useability assessment information is in Appendix E; time series plots for benzene, chloroethene (vinyl chloride), 1,4-dichlorobenzene, dichloromethane, ethylbenzene, lead, tetrachloroethylene, 1,1,1-trichloroethane, 
trichloroethylene, trichlorofluoromethane, tritium, and xylenes for specified wells with elevated and slightly elevated concentrations of these constitutents are in Appendix F; and hydrographs are in Appendix G.

\section{Analytical Results Exceeding Standards}

Results for analytes that exceeded the final PDWS (see Appendix A) during each quarter of 1992 are summarized in Table 1 (Appendix D).

During first quarter 1992, 19 of the 57 LFW wells contained levels of one or more of the following constituents that exceeded the Safe Drinking Water Act final Primary Drinking Water Standards (PDWS) established by the U.S. Environmental Protection Agency: trichloroethylene, tetrachloroethylene, 1,2-dichloroethane, tritium, chloroethene (vinyl chloride), 1,1-dichloroethylene, cadmium, 1,1,1-trichloroethane, or benzene. Trichloroethylene, tetrachloroethylene, and 1,2-dichloroethane occurred most frequently: trichloroethylene was detected in 15 wells, with the highest concentration in LFW $10 \mathrm{~A}$ at 49 $\mu \mathrm{g} / \mathrm{L}$; tetrachloroethylene and 1,2-dichloroethane were detected in 3 wells each, with the maximum tetrachloroethylene concentration of $37 \mu \mathrm{g} / \mathrm{L}$ in LFW $10 \mathrm{~A}$ and a maximum 1,2dichloroethane concentration of $7.6 \mu \mathrm{g} / \mathrm{L}$ in LFW 36. Cadmium, chloroethene (vinyl chloride), 1,1-dichloroethylene, and tritium exceeded their final PDWS in 2 wells each; benzene and 1,1,1-trichloroethane were elevated in 1 well each.

Elevated levels of trichloroethylene, benzene, chloroethene (vinyl chloride), 1,2dichloroethane, tritium, tetrachloroethylene, 1,2-dichloroethane, 1,1-dichloroethylene, or 1,2dichloropropane were detected above the final PDWS in one or more of 17 wells at the Sanitary Landfill during second quarter 1992. Trichloroethylene and benzene occurred most frequently: trichloroethylene exceeded its final PDWS in 13 wells, with the highest concentration in LFW $10 \mathrm{~A}$ at $48 \mu \mathrm{g} / \mathrm{L}$; benzene was elevated in 4 wells, with the the highest concentration in LFW 21 at $21 \mu \mathrm{g} / \mathrm{L}$. Chloroethene (vinyl chloride) and tritium exceeded PDWS in 3 wells each; 1,2-dichloroethane and tetrachloroethylene exceeded standards in 2 wells each; and 1,1-dichloroethylene and 1,2-dichloropropane were elevated in 1 well each.

During third quarter 1992, one or more of the 17 wells at the Sanitary Landfill wells contained levels of trichloroethylene, tetrachloroethylene, tritium, benzene, chloroethene (vinyl chloride), 1,2-dichloroethane, 1,1-dichloroethylene, 1,2-dichloropropane, lead, or lindane that exceeded the final PDWS in the groundwater beneath the Sanitary Landfill.

Trichloroethylene and tetrachloroethylene occurred most frequently: trichloroethylene was elevated in 15 wells, with the highest concentration found in well LFW $10 \mathrm{~A}$ at $45 \mu \mathrm{g} / \mathrm{L}$; tetrachloroethylene was elevated in 5 wells, with the maximum concentration in well LFW $10 \mathrm{~A}$ at $125 \mu \mathrm{g} / \mathrm{L}$. Tritium exceeded its standard in 3 wells; benzene exceeded its standard in 2 wells; and chloroethene (vinyl chloride), 1,2-dichloroethane, 1,1-dichloroethylene, 1,2dichloropropane, lead, and lindane were elevated in 1 well each.

Elevated levels of trichloroethylene, chloroethene (vinyl chloride), tetrachloroethylene, benzene, 1,2-dichloroethane, tritium, 1,1-dichloroethylene, or mercury, were detected in one or more of the 21 of the LFW wells at the Sanitary Landfill during fourth quarter 1992. Trichloroethylene exceeded its final PDWS in 15 wells, with the maximum concentration at $51 \mu \mathrm{g} / \mathrm{L}$ in well LFW $10 \mathrm{~A}$; chloroethene (vinyl chloride) and tetrachloroethylene were elevated 
in 5 wells each, with a maximum chloroethene (vinyl chloride) concentration of $126 \mu \mathrm{g} / \mathrm{L}$ in well LFW 48D and a maximum tetrachloroethylene concentration of $27 \mu \mathrm{g} / \mathrm{L}$ in LFW 10A. Benzene exceeded its final PDWS in 4 wells; 1,2-dichloroethane and tritium exceeded their PWDS in 3 wells each; and 1,1-dichloroethylene and mercury exceeded their final PDWS in 1 well each.

Results for analytes that exceeded other SRS flagging criteria (see Appendix B) during the third and fourth quarters of 1992 are summarized in Table 2 (Appendix D) and include constituents with proposed drinking water standards. Table 3 (Appendix D) shows the results for all of the constituents and identifies the results that received modifiers or that exceeded the EPA-approved holding time, the final PDWS, or other standards during fourth quarter 1992.

Table 3 (Appendix D) also lists the number of well volumes purged from each of the LFW wells during fourth quarter 1992 . Wells LFW 28,60D, and 62D went dry during purging and were sampled after they recovered. Wells LFW 7 and 20 could not be sampled during fourth quarter 1992. Well LFW 7 is part of the Investigation Derived Waste Program because of high concentrations of 1,4-dichlorobenzene. The aboveground plumbing was inoperative on well LFW 20.

Some of the values for earlier quarters presented in the results tables of this report may differ from the values for those same quarters presented in previous reports, and reported values may not match reported sample dates. These differences result from the following: (1) the computer program that creates the analytical results tables was revised beginning second quarter 1992 to present the highest value for analytes with more than one result (previously, the program presented the first value encountered in the database); (2) a new computer program, which rounds numbers differently from the former program, was first used during third quarter 1992; and (3) some re-analyses may have been performed by the laboratory after the quarterly reports had gone to press. The sample dates in the tables reflect when the field data were collected. These dates may differ from the dates of the laboratory analyses if the highest results were obtained for samples collected on different dates.

The lateral distributions of chloroethene (vinyl chloride), radium-226, trichloroethylene, and tritium for first and fourth quarters 1992 are presented in Figures 4 through 11 (Appendix C).

\section{Time-Trend Data}

Appendix F presents time series plots for benzene in wells LFW 8, 10A, and 36; chloroethene (vinyl chloride) in wells LFW 8, 48C, and 48D; 1,4-dichlorobenzene in well LFW 7; dichloromethane in wells LFW 10A and 21; ethylbenzene in wells LFW 8, 10A, 22, 48C, and 48D; lead in well LFW 8; tetrachloroethylene in wells LFW 10A and 61D; 1,1,1-trichloroethane in wells LFW 39 and 61D; trichloroethylene in wells LFW 10A and 59D; trichlorofluoromethane in wells LFW 39, 58D, 61C, and 61D; tritium in well LFW 10A; and xylenes in wells LFW 21 and 22 in the Sanitary Landfill through fourth quarter 1992. Chloroethene (vinyl chloride), dichloromethane, tetrachloroethylene, trichloroethylene, and tritium have usually exceeded 
their final PDWS in these wells. Trichlorofluoromethane has consistently exceeded its SRS Flag 2 criterion.

The time series plots indicate that levels of benzene, dichloromethane, tetrachloroethylene, trichloroethylene, and tritium in well LFW 10A; ethylbenzene in well LFW 22; and 1,1,1trichloroethane and trichlorofluoromethane in well LFW 39 are increasing with time.

Benzene appears to be decreasing with time in well LFW 36. The data for the remaining wells show no detectable long-term trends or are insufficient to detect trends.

\section{Water Levels}

Hydrographs showing the water elevations through time for wells at the Sanitary Landfill are provided in Appendix F. The average water elevation in Steed Pond Aquifer (Water Table) during first quarter 1992 was $151.8 \mathrm{ft} \mathrm{msl}$ and was $151.2 \mathrm{ft}$ msl during second quarter 1992, a decrease of $0.6 \mathrm{ft} \mathrm{msl}$. The average water elevation in this zone during third quarter 1992 was $152.0 \mathrm{ft} \mathrm{msl}$, an increase of $0.8 \mathrm{ft}$ from second quarter 1992. The average water elevation in Steed Pond Aquifer (Water Table) during fourth quarter 1992 was $151.2 \mathrm{ft} \mathrm{msl}$, a decrease of $0.8 \mathrm{ft}$ from third quarter 1992.

\section{Groundwater Flow Rates and Directions}

The groundwater flow direction in the Steed Pond Aquifer (Water Table) hydrostratigraphic unit beneath the Sanitary Landfill is to the south-southeast (SRS grid coordinates) toward Upper Three Runs Creek (Figures 12 and 13). The change in hydraulic head from the extreme northern boundary of the landfill to the extreme southern boundary is approximately $20 \mathrm{ft}$.

The horizontal flow rate of groundwater for Steed Pond Aquifer (Water Table) is used to estimate the transport rate of any constituents originating from the Sanitary Landfill. Estimated horizontal flow rates in Steed Pond Aquifer (Water Table) are calculated along two paths (designated flow paths $A$ and $B$ ) that approximate the maximum and minimum groundwater flow rates beneath this unit. These calculations, based on the following onedimensional flow equation, employ inferred or estimated parameters and should be considered accurate to an order of magnitude only. Flow rates are calculated as follows: flow path length is calculated to the nearest $50 \mathrm{ft}$. Flow rate per day is calculated to two significant figures using the equation shown. This value is then multiplied by 365 and rounded to two significant figures for the flow rate per year.

$$
\text { Flow }(\mathrm{ft} / \text { day })=\frac{\text { Hydraulic Conductivity }(\mathrm{ft} / \text { day })}{\text { Porosity (unitless) }} \times \frac{d h(\mathrm{ft})}{d l(\mathrm{ft})}
$$

where the hydraulic conductivity ( $\mathrm{Kh}$ ) constant is $10 \mathrm{ft} /$ day (Geraghty \& Miller, 1990), the effective porosity value is $20 \%$, the change in head is $d h$, and the horizontal distance along each flow direction arrow is $d l$. Flow rate estimates vary depending upon the vertical gradient between wells, the size of the area under consideration, and the number of data points. 
The approximate range of groundwater flow rates in Steed Pond Aquifer (Water Table) during first quarter 1992 is estimated as follows (Figure 12, Appendix C):

Flow path $\mathrm{A}$

$$
\begin{aligned}
& \frac{10}{0.20} \times \frac{22}{3050} \cong 0.36 \mathrm{ft} / \text { day } \\
& 0.36 \mathrm{ft} / \text { day } \times 365 \text { days } \cong 130 \mathrm{ft} / \mathrm{yr}
\end{aligned}
$$

Flow path B

$$
\begin{aligned}
& \frac{10}{0.20} \times \frac{14}{1850} \cong 0.39 \mathrm{ft} / \text { day } \\
& 0.39 \mathrm{ft} / \text { day } \times 365 \text { days } \cong 140 \mathrm{ft} / \mathrm{yr}
\end{aligned}
$$

The approximate range of groundwater flow rates in Steed Pond Aquifer (Water Table) during fourth quarter 1992 is estimated as follows (Figure 13, Appendix C):

Flow path A

$$
\begin{aligned}
& \frac{10}{0.20} \times \frac{22}{3050} \cong 0.36 \mathrm{ft} / \text { day } \\
& 0.36 \mathrm{ft} / \text { day } \times 365 \text { days } \cong 130 \mathrm{ft} / \mathrm{yr}
\end{aligned}
$$

Flow path B

$$
\begin{aligned}
& \frac{10}{0.20} \times \frac{12}{1550} \cong 0.39 \mathrm{ft} / \text { day } \\
& 0.39 \mathrm{ft} / \text { day } \times 365 \text { days } \cong 140 \mathrm{ft} / \mathrm{yr}
\end{aligned}
$$

The flow direction and rate were very consistent throughout 1992.

\section{Upgradient Versus Downgradient Results}

Groundwater flow in the Steed Pond Aquifer (Water Table) hydrostratigraphic unit is to the south-southeast (SRS grid coordinates) toward Upper Three Runs Creek (Figures 12 and 13). Wells LFW 43B, 43C, and 43D, at the northern end of the Sanitary Landfill are designated upgradient wells for Steed Pond Aquifer (Water Table). All remaining Steed Pond Aquifer (Water Table) wells monitor downgradient or sidegradient water quality (Figures 3, 12, and 13, Appendix C). During 1992, no elevated constituents occurred in the upgradient wells. The designated background wells of the Sanitary Landfill are wells LFW 29, 30, 31, and cluster LFW 43. 


\section{Conclusions}

During first quarter 1992, 19 of the 57 LFW wells contained levels of trichloroethylene, tetrachloroethylene, 1,2-dichloroethane, tritium, chloroethene (vinyl chloride), 1,1dichloroethylene, cadmium, 1,1,1-trichloroethane, or benzene that exceeded the Safe Drinking Water Act final Primary Drinking Water Standards (PDWS) established by the U.S. Environmental Protection Agency. Trichloroethylene, tetrachloroethylene, and 1,2-dichloroethane occurred most frequently: trichloroethylene was detected in 15 wells, with the highest concentration in LFW 10A at $49 \mu \mathrm{g} / \mathrm{L}$. Tetrachloroethylene and 1,2-dichioroethane were detected in 3 wells ea $h$, with the maximum tetrachloroethylene concentration of $37 \mu \mathrm{g} / \mathrm{L}$ in LFW 10A and a maximum 1,2-dichloroethane concentration of $7.6 \mu \mathrm{g} / \mathrm{L}$ in LFW 36. All of the wells containing elevated trichlorocthylene, tetrachloroethylene, and 1,2-dichloroethane are located in or near either the original Sanitary Landfill or the southern expansion. Elevated levels of the remaining constituents also were detected mostly in the lower portion of the original facility and the southern expansion.

Elevated levels of trichloroethylene, benzene, chloroethene (vinyl chloride), 1,2dichloroethane, tritium, tetrachloroethylene, 1,2-dichloroethane, 1,1-dichloroethylene, or 1,2dichloropropane were detected above the final PDWS in one or more of 17 wells at the Sanitary Landfill during second quarter 1992. Trichloroethylene and benzene occurred most frequently: trichloroethylene exceeded its final PDWS in 13 wells, with the highest concentration in LFW $10 \mathrm{~A}$ at $48 \mu \mathrm{g} / \mathrm{L}$. Benzene was elevated in 4 wells, with the the highest concentration in LFW 21 at $21 \mu \mathrm{g} / \mathrm{L}$. All of the wells containing elevated trichloroethylene and benzene are located in or near either the original Sanitary Landfill or the southern expansion. Elevated levels of the remaining constituents also were detected mostly in the lower portion of the original facility and the southern expansion.

During third quarter 1992, 17 of the 57 LFW wells contained levels of trichloroethylene, tetrachloroethylene, tritium, benzene, chloroethene (vinyl chloride), 1,2-dichloroethane, 1,1dichloroethylene, 1,2-dichloropropane, lead, or lindane that exceeded the final PDWS in the groundwater beneath the Sanitary Landfill. Trichloroethylene and tetrachloroethylene occurred most frequently: trichloroethylene was elevated in 15 wells, with the highest concentration found in well LFW $10 \mathrm{~A}$ at $45 \mu \mathrm{g} / \mathrm{L}$; tetrachloroethylene was elevated in 5 wells, with the maximum concentration in well LFW $10 \mathrm{~A}$ at $125 \mu \mathrm{g} / \mathrm{L}$. All of the wells containing elevated trichloroethylene and tetrachloroethylene are located in or near either the original Sanitary Landfill or the southern expansion. Elevated levels of the remaining constituents were observed mostly in the lower portion of the original facility and the southern expansion.

Elevated levels of trichloroethylene, chloroethene (vinyl chloride), benzene, 1,2-dichloroethane, tetrachloroethylene, tritium, 1,1-dichloroethylene, or mercury were detected in 21 of the LFW wells at the Sanitary Landfill during fourth quarter 1992. Trichloroethylene and chloroethene (vinyl chloride) occurred most frequently: trichloroethylene exceeded its final PDWS in 15 wells, with the maximum concentration at $51 \mu \mathrm{g} / \mathrm{L}$ in well LFW $10 \mathrm{~A}$. 
Chloroethene (vinyl chloride) was elevated in 5 wells, with the maximum concentration at 126 $\mu \mathrm{g} / \mathrm{L}$ in well LFW 48D. All of the wells containing elevated trichloroethylene and chloroethene (vinyl chloride) are located in or near either the original Sanitary Landfill or the southern expansion. Elevated levels of the remaining constituents also were detected mostly in the lower portion of the original facility and the southern expansion.

The groundwater flow direction in Steed Pond Aquifer (Water Table) beneath the Sanitary Landfill is to the south-southeast (SRS grid coordinates) toward Lpper Three Runs Creek. Flow rates in this aquifer during first and fourth quarters 1992 were estimated to range between approximately $130 \mathrm{ft} / \mathrm{yr}$ and $140 \mathrm{ft} / \mathrm{yr}$ in Steed Pond Aquifer (Water Table).

No elevated constituents occurred in upgradient wells LFW 43B, 43C, and 43D during 1992. Generally, elevated levels of constituents found in downgradient wells but not in upgradient wells at a waste management unit are considered products of the waste management unit. 
WSRC-TR-93-068

\section{Summary 1992}

Fifty-seven wells of the LFW series monitor groundwater quality in Steed Pond Aquifer (Water Table) beneath the Sanitary Landfill at the Savannah River Site (SRS). These wells are sampled quarterly as part of the SRS Groundwater Monitoring Program and to comply with the South Carolina Department of Health and Environmental Control Domestic Waste Permit 87A. During the year, no new wells were added to the LFW groundwater monitoring network, and no wells were abandoned.

The groundwater flow direction in Steed Pond Aquifer (Water Table) beneath the Sanitary Landfill is to the south-southeast (SRS grid coordinates) toward Upper Three Runs Creek. Estimated flow rates in this aquifer during first and fourth quarters 1992 ranged between $130 \mathrm{ft} / \mathrm{yr}$ and $140 \mathrm{ft} / \mathrm{yr}$.

During 1992, samples analyzed from 25 of the 57 monitoring wells (44\%) exhibited levels of benzene, cadmium, chloroethere (vinyl chloride), 1,2-dichloroethane, 1,1-dichloroethylene, 1,2-dichloropropane, lead, lindane, mercury, 1,1,1-trichloroethane, tetrachloroethylene, trichloroethylene, or tritium that exceeded their final PDWS (Table 1, Appendix D).

Trichloroethylene occurred in concentrations that exceeded its final PDWS in 15 of the LFW wells during 1992 . The 15 wells are located in the lower portion of the original landfill and in the southern expansion. Trichloroethylene exceeded the final PDWS every quarter during 1992 in wells LFW 10A, 16, 21, 22, 37, 38, 39, 58D, 59D, 61C, 61D, 62C, and 62D; trichloroethylene exceeded its standard during three quarters of the year in wells LFW 40 and 59C. The highest concentration of trichloroethylene for the year occurred during fourth quarter 1992 in well LFW $10 \mathrm{~A}$ at $51 \mu \mathrm{g} / \mathrm{L}$.

Tetrachloroethylene exceeded its final PDWS in 9 wells, 1,2-dichloroethane in 6 wells, and benzene and chloroethene (vinyl chloride) in 5 wells each during 1992. These constituents occurred in wells located in the lower portion of the original landfill and the southern expansion. Usually, elevated levels of these constituents were not consistent throughout the year. The remaining volatile organic compounds (i.e., 1,2-dichloropropane, 1,1dichloroethylene, 1,1,1,-trichloroethane) that exhibited elevated levels in Sanitary Landfill wells during 1992 occurred with less frequency.

In addition to the volatile organic compounds found in elevated levels at the Sanitary Landfill during 1992, tritium was the only radioactive constituent that exceeded its final PDWS in wells LFW 10A, 16,38, and 61D. These wells are located in the lower portion of landfill and southern expansion. Elevated tritium appeared in well LFW 10A during all quarters of the year. 


\section{Errata}

Third Quarter 1992:

- Prior to third quarter 1992 , the results of certain analyses for nitrate-nitrite as nitrogen were reported incorrectly by the General Engineering laboratory as nitrate as nitrogen results. The analyses in the results tables for reports beginning third quarter 1992 are reported correctly (i.e., nitrate-nitrite results have been distinguished from true nitrate results).

- Page 29, Well LFW 25: The correct result for gross alpha is $<2.0 \mathrm{E}+00 \mathrm{pCi} / \mathrm{L}$; the correct result for nonvolatile beta is $8.2 \mathrm{E}+00 \mathrm{pCi} / \mathrm{L}$.

- Page 66, Well LFW 44D: The "B" modifier for the barium result should be deleted.

- Page 79, Well LFW 55C: The correct result for gross alpha is $<2.0 \mathrm{E}+00 \mathrm{pCi} / \mathrm{L}$; the correct result for nonvolatile beta is $<2.0 \mathrm{E}+00 \mathrm{pCi} / \mathrm{L}$.

- Page 84, Well LFW 57B: The correct result for gross alpha is $5.6 \mathrm{E}+00 \mathrm{pCi} / \mathrm{L}$; the correct result for nonvolatile beta is $5.3 \mathrm{E}+00 \mathrm{pCi} / \mathrm{L}$.

- Page 95, Well LFW 59D: The correct Weston laboratory results for nitrate as nitrogen are $126 \mu \mathrm{g} / \mathrm{L}$ and $166 \mu \mathrm{g} / \mathrm{L}$.

- Page 101, Well LFW 61D: The "B" modifier for the barium result should be deleted. 
WSRC-TR-93-068

\section{References Cited}

Geraghty \& Miller, Inc., 1990. Evaluation of Integrated Waste Facility Closure Capping on Ground-Water Flow and Solute Transport in General Separations Area, Savannah River Site: Flow Model and Particle-Tracking Analysis, Final Report.

Prepared by Geraghty \& Miller Modeling Group for Westinghouse Savannah River Company, Waste Management Technology, Savannah River Site, Aiken, SC.

Heffner, J. D., and Exploration Resources, Inc., 1991. Technical Summary of Groundwater Quality Protection Program at the Savannah River Site (1952-1986), Volume I-Site Geohydrology and Waste Sites, DPSP-88-1002. Westinghouse Savannah River Company, Aiken, SC.

SCDHEC (South Carolina Department of Health and Environmental Control), 1991.

Sanitary Landfill Domestic Waste Permit 87A, April 1991. Columbia, SC.

WSRC (Westinghouse Savannah River Company), 1990. Sanitary Landfill Groundwater Quality Assessment Plan, WSRC-TR-90-167. Savannah River Site, Aiken, SC.

WSRC (Westinghouse Savannah River Company), 1992. Hydrogeologic Data Collection Procedures and Specifications: Sampling Groundwater Monitoring Well, Manual 3Q5, Chapter 14, Revision 0. Environmental Protection Department, Environmental Monitoring Section, Savannah River Site, Aiken, SC. 
WSRC.TR-93-068

-

-

-

c

c

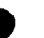


WSRC-TR-93-068

Appendix A - Final Primary Drinking Water Standards 
Final Primary Drinking Water Standards

\begin{tabular}{|c|c|c|c|c|}
\hline Analyte & Unit & Level & $\underline{\text { Status }}$ & Reference \\
\hline $\begin{array}{l}\text { Arsenic } \\
\text { Barium }\end{array}$ & $\mu g / L$ & 50 & Final & CFR, 1991 \\
\hline Barium & $\mu g / L$ & 2,000 & Final & CFR, 1991 \\
\hline Benzene & $\mu g / L$ & 5 & Final & CFR, 1991 \\
\hline Bromodichloromethane & $\mu g / L$ & $100^{\mathrm{a}}$ & Final & CFR, 1991 \\
\hline Bromoform & $\mu g / L$ & $100^{\circ}$ & Final & CFR, 1991 \\
\hline Cadmium & $\mu g / L$ & 5 & Final & CFR, 1991 \\
\hline Carbon tetrachloride & $\mu g / L$ & 5 & Final & CFR, 1991 \\
\hline Chlordane & $\mu g / L$ & 2 & Final & CFR, 1991 \\
\hline Chloroethene (Vinyl chloride) & $\mu g / L$ & 2 & Final & CFR, 1991 \\
\hline Chloroform & $\mu g / L$ & $100^{\mathrm{a}}$ & Final & CFR, 1991 \\
\hline Chromium & $\mu g / L$ & 100 & Final & CFR, 1991 \\
\hline Copper & $\mu g / L$ & 1,300 & Final & CFR, 1991 \\
\hline Dibromochloromethane & $\mu g / L$ & $100^{\circ}$ & Final & CFR, 1991 \\
\hline Dibromochloropropane & $\mu g / L$ & 0.2 & Final & CFR, 1991 \\
\hline 1,2-Dichlorobenzene & $\mu g / L$ & 600 & Final & CFR, 1991 \\
\hline 1,4-Dichlorobenzene & $\mu g / L$ & 75 & Final & CFR, 1991 \\
\hline 1,2-Dichloroethane & $\mu g / L$ & 5 & Final & CFR, 1991 \\
\hline 1,1-Dichloroethylene & $\mu \mathrm{g} / \mathrm{L}$ & 7 & Final & CFR, 1991 \\
\hline cis-1,2-Dichloroethylene & $\mu g / L$ & 70 & Final & CFR, 1991 \\
\hline trans-1,2-Dichloroethylene & $\mu g / L$ & 100 & Final & CFR, 1991 \\
\hline 2,4-Dichlorophenoxyacet:c acid & $\mu \mathrm{g} / \mathrm{L}$ & 70 & Final & CFR, 1991 \\
\hline 1,2-Dichloropropane & $\mu g / L$ & 5 & Final & CFR, 1991 \\
\hline Endrin & $\mu g / L$ & 0.2 & Final & CFR, 1991 \\
\hline Ethylbenzene & $\mu g / L$ & 700 & Final & CFR, 1991 \\
\hline Fluoride & $\mu g / L$ & 4,000 & Final & CFR, 1991 \\
\hline Gross alphab & $\mathrm{pCi} / \mathrm{L}$ & $1.5 E+01$ & Final & CFR, 1991 \\
\hline Heptachlor & $\mu g / L$ & 0.4 & Final & CFR, 1991 \\
\hline Heptachlor epoxide & $\mu g / L$ & 0.2 & Final & CFR, 1991 \\
\hline Lead & $\mu \mathrm{g} / \mathrm{L}$ & 15 & Final & CFR, 1991 \\
\hline Lindane & $\mu g / L$ & 0.2 & Final & CFR, 1991 \\
\hline Mercury & $\mu g / L$ & 2 & Final & CFR, 1991 \\
\hline Methoxychlor & $\mu g / L$ & 40 & Final & CFR, 1991 \\
\hline Nitrate as nitrogen & $\mu g / L$ & 10,000 & Final & CFR, 1991 \\
\hline Nitrate-nitrite as nitrogen & $\mu \mathrm{g} / \mathrm{L}$ & 10,000 & Final & CFR, 1991 \\
\hline Nitrite as nitrogen & $\mu g / L$ & 1,000 & Final & CFR, 1991 \\
\hline Nonvolatile betac & $\mathrm{pCi} / \mathrm{L}$ & $5 E+01$ & Final & EPA, 1977 \\
\hline $\mathrm{PCBs}^{\mathrm{d}}$ & $\mu g / L$ & 0.5 & Final & CFR, 1991 \\
\hline Pentachlorophenol & $\mu g / L$ & 1 & Final & CFR, 1991 \\
\hline Selenium & $\mu g / L$ & 50 & Final & CFR, 1991 \\
\hline Strontium-89/90 & $\mathrm{pCi} / \mathrm{L}$ & $8 E+00$ & Final & CFR, 1991 \\
\hline Strontium-90 & $\mathrm{pCi} / \mathrm{L}$ & $8 E+00$ & Final & CFR, 1991 \\
\hline Styrene & $\mu \mathrm{g} / \mathrm{L}$ & 100 & Final & CFR, 1991 \\
\hline Tetrachloroethylene & $\mu \mathrm{g} / \mathrm{L}$ & 5 & Final & CFR, 1991 \\
\hline Toluene & $\mu g / L$ & 1,000 & Final & CFR, 1991 \\
\hline Total radium (Radium-226 and -228) & $\mathrm{pCi} / \mathrm{L}$ & $5 E+00$ & Final & CFR, 1991 \\
\hline Total trihalomethanes & $\mu g / L$ & 100 & Final & CFR, 1991 \\
\hline Toxaphene & $\mu \mathrm{g} / \mathrm{L}$ & 3 & Final & CFR, 1991 \\
\hline 2,4,5-TP (Silvex) & $\mu g / L$ & 50 & Final & CFR, 1991 \\
\hline 1,1,1-Trichloroethane & $\mu g / L$ & 200 & Final & CFR, 1991 \\
\hline
\end{tabular}


Analyte

Trichloroethylene

Tritium

Xylenes

\section{Unit}

$\mu g / L$

$\mathrm{pCi} / \mathrm{mL}$

$\mu \mathrm{g} / \mathrm{L}$
Level

5

$2 \mathrm{E}+01$

10,000
Status

Final

Final

Final
Reference

CFR, 1991

CFR, 1991

CFR, 1991

a This value is the drinking water standard for total trihalomethanes (the sum of bromoform, bromodichloromethane, chloroform, and dibromochloromethane).

b The standard given is for gross alpha including radium-226 tut excluding radon and uranium.

c This is the screening level above which providers of public drinking water should perform analyses for specific man-made radionuclides. The standard for the total dose equivalent from all such radionuclides is 4 mrem per year.

d Analyses were conducted in 1992 for the following: PCB 1016, PCB 1221, PCB 1232, PCB 1242, PCB 1248, PCB 1254, and PCB 1260.

- For double radionuclide analyses where each separate radionuclide has its own standard, the more stringent standard is used.

\section{References}

CFR (Code of Federal Regulations), 1991. National Primary Drinking Water Regulations, 40 CFR, Part 141, pp. 578-715. Washington, DC.

EPA (U.S. Environmental Protection Agency), 1977. National Interim Primary Drinking Water Regulations, EPA-570/9-76-003. Washington, DC. 
WSRC-TR-93-068

-

-

C

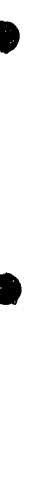

c 
WSRC-TR-93-068

\section{Appendix B - Flagging Criteria}




\section{Flagging Criteria}

Beginning in 1991, the Savannah River Site Environmental Protection Department/ Environmental Monitoring Section modified its guidelines for flagging constituents in the Groundwater Monitoring Program. These flagging criteria are as follows:

- Flag 2 criteria for constituents equal the U.S. Environmental Protection Agency (EPA) Primary Drinking Water Standard (PDWS), the EPA proposed PDWS, or the EPA Secondary Drinking Water Standard (SDWS). If a constituent does not have a drinking water standard, the Flag 2 criterion equals 10 times the method detection limit (MDL) calculated as the 90 th percentile detection limit obtained recently by one of the primary analytical laboratories.

- Flag 1 criteria for constituents equal one-half of the EPA PDWS, one-half the EPA proposed PDWS, or one-half the EPA SDWS. If a constituent does not have an EPA drinking water standard, the Flag 1 criterion equals 5 times the MDL calculated as the 90th percentile detection limit obtained recently by one of the primary analytical laboratories.

- Flag 0 criteria are assigned to constituent levels below Flag 1 criteria, constituent levels below the sample detection limits, or constituents having no flagging criteria.

The following parameters are not assigned flagging criteria: alkalinity, calcium, carbonate, color, corrosivity, magnesium, odor, potassium, Eh, silica, sodium, total dissolved solids, total phosphorus, total phosphates (as P), and turbidity. In addition, common laboratory contaminants and cleaners including phthalates, methylene chloride, ketones, and toluene are not assigned flagging criteria.

Analyte

Acenaphthene

Acenaphthylene

Acetone

Acetonitrile (Methyl cyanide)

Acetophenone

2-Acetylaminofluorene

Acrolein

Acrylonitrile

Aldrin

Alkalinity (as $\mathrm{CaCO}_{3}$ )

Allyl chloride

Aluminum

Americium-241

Americium-243

4-Aminobiphenyl

Ammonia

Ammonia nitrogen

Aniline

Anthracene

Antimony

Antimony-125

Aramite

\begin{tabular}{|c|c|c|}
\hline Unit & Flag 1 & Flag 2 \\
\hline$\mu g / L$ & 50 & 100 \\
\hline$\mu g / L$ & 50 & 100 \\
\hline$\mu g / L$ & 50 & 100 \\
\hline$\mu g / L$ & 500 & 1,000 \\
\hline$\mu \mathrm{g} / \mathrm{L}$ & 50 & 100 \\
\hline$\mu g / L$ & 50 & 100 \\
\hline$\mu g / L$ & 100 & 200 \\
\hline$\mu g / L$ & 100 & 200 \\
\hline$\mu g / L$ & 2.5 & 5 \\
\hline & No flag & No flag \\
\hline$\mu g / L$ & 250 & 500 \\
\hline$\mu g / L$ & 25 & 50 \\
\hline $\mathrm{pCi} / \mathrm{L}$ & $3.17 E+00$ & $6.34 E+00$ \\
\hline $\mathrm{pCi} / \mathrm{L}$ & $3.19 E+00$ & $6.37 E+00$ \\
\hline$\mu g / L$ & 50 & 100 \\
\hline$\mu g / L$ & 500 & 1,000 \\
\hline$\mu g / L$ & 50 & 100 \\
\hline$\mu \mathrm{g} / \mathrm{L}$ & 50 & 100 \\
\hline$\mu g / L$ & 50 & 100 \\
\hline$\mu \mathrm{g} / \mathrm{L}$ & 2.5 & 5 \\
\hline $\mathrm{pCi} / \mathrm{L}$ & $1.5 E+02$ & $3 E+02$ \\
\hline$\mu g / L$ & 50 & 100 \\
\hline
\end{tabular}

B-2

\section{Source}

EPA Method 8270

EPA Method 8270

EPA Method 8240

EPA Method 8240

EPA Method 8270

EPA Method 8270

EPA Method 8240

EPA Method 8240

EPA Method 8080

Set by EPD/EMS

EPA Method 8240

Secondary DWS (CFR, 1991b)

Proposed DWS (EPA, 1991)

Proposed DWS (EPA, 1991)

EPA Method 8270

APHA Method 417B

EPA Method 350.1

EPA Method 8270

EPA Method 8270

Proposed DWS (EPA, 1990)

Final DWS (EPA, 1977)

EPA Method 8270 


\begin{tabular}{|c|c|c|c|c|}
\hline Analyte & Unit & Flag 1 & Flag 2 & Source \\
\hline Arsenic & $\mu g / L$ & 25 & 50 & Final DWS (CFR, 1991a) \\
\hline Barium & $\mu g / L$ & 1,000 & 2,000 & Final DWS (CFR, 1991a) \\
\hline Barium-140 & $\mathrm{pCi} / \mathrm{L}$ & $4.5 E+01$ & $9 E+01$ & Final DWS (EPA, 1977) \\
\hline Benzene & $\mu g / L$ & 2.5 & 5 & Final DWS (CFR, 1991a) \\
\hline alpha-Benzene hexachloride & $\mu g / L$ & 2.5 & 5 & EPA Method 8080 \\
\hline beta-Benzene hexachloride & $\mu g / L$ & 2.5 & 5 & EPA Method 8080 \\
\hline delta-Benzene hexachloride & $\mu g / L$ & 2.5 & 5 & EPA Method 8080 \\
\hline Benzidine & $\mu g / L$ & 250 & 500 & EPA Method 8270 \\
\hline Benzola]anthracene & $\mu g / L$ & 0.05 & 0.1 & Proposed DWS (EPA, 1990) \\
\hline Benzolb]fluoranthene & $\mu g / L$ & 0.1 & 0.2 & Proposed DWS (EPA, 1990) \\
\hline Benzolk]fluoranthene & $\mu g / L$ & 0.1 & 0.2 & Proposed DWS (EPA, 1990) \\
\hline Benzolg,h,i]perylene & $\mu g / L$ & 50 & 100 & EPA Method 8270 \\
\hline Benzolalpyrene & $\mu g / L$ & 0.1 & 0.2 & Proposed DWS (EPA, 1990) \\
\hline Benzoic acid & $\mu g / L$ & 250 & 500 & EPA Method 8270 \\
\hline 1,4-Benzoquinone & $\mu g / L$ & 50 & 100 & EPA Method 8270 \\
\hline Benzyl alcohol & $\mu g / L$ & 100 & 200 & EPA Method 8270 \\
\hline Beryllium & $\mu g / L$ & 0.5 & 1 & Proposed DWS (EPA, 1990) \\
\hline Beryllium-7 & $\mathrm{pCi} / \mathrm{L}$ & $3 E+03$ & $6 E+03$ & Final DWS (EPA, 1977) \\
\hline Bis (2-chloroethoxy) methane & $\mu g / L$ & 50 & 100 & EPA Method 8270 \\
\hline Bis(2-chloroethyl) ether & $\mu g / L$ & 50 & 100 & EPA Method 8270 \\
\hline Bis(2-chloroisopropyl) ether & $\mu g / L$ & 50 & 100 & EPA Method 8270 \\
\hline Bis(chloromethyl) ether & $\mu g / L$ & 50 & 100 & EPA Method 8270 \\
\hline Bis (chloromethyl-ethyl) ether & $\mu g / L$ & 50 & 100 & EPA Method 8270 \\
\hline Bis(2-ethylhexyl) phthalate & & No flag & No flag & Set by EPD/EMS \\
\hline Bromide & $\mu g / L$ & 5,000 & 10,000 & EPA Method 300.0 \\
\hline Bromodichloromethane & $\mu g / L$ & 50 & 100 & Final DWS (CFR, 1991a) \\
\hline Bromoform & $\mu g / L$ & 50 & 100 & Final DWS (CFR, 1991a) \\
\hline Bromomethane (Methyl bromide) & $\mu g / L$ & 5 & 10 & EPA Method 8240 \\
\hline 4-Bromophenyl phenyl ether & $\mu g / L$ & 50 & 100 & EPA Method 8270 \\
\hline 2-sec-Butyl-4,6-dinitrophenol & $\mu g / L$ & 3.5 & 7 & Proposed DWS (EPA, 1990) \\
\hline Butylbenzyl phthalate & & No flag & No flag & Set by EPD/EMS \\
\hline Cadmium & $\mu g / L$ & 2.5 & 5 & Final DWS (CFR, 1991a) \\
\hline Calcium & & No flag & No flag & Set by EPD/EMS \\
\hline Carbon disulfide & $\mu \mathrm{g} / \mathrm{L}$ & 5 & 10 & EPA Method 8240 \\
\hline Carbon tetrachloride & $\mu g / L$ & 2.5 & 5 & Final DWS (CFR, 1991a) \\
\hline Carbon-14 & $\mathrm{pCi} / \mathrm{L}$ & $1 E+03$ & $2 E+03$ & Final DWS (EPA, 1977) \\
\hline Carbonate & $\mu g / L$ & 500 & 1,000 & EPA Method 310.1 \\
\hline Cerium-141 & $\mathrm{pCi} / \mathrm{L}$ & $1.5 E+02$ & $3 E+02$ & Final DWS (EPA, 1977) \\
\hline Cerium-144 & $\mathrm{pCi} / \mathrm{L}$ & $1.31 E+02$ & $2.61 E+02$ & Proposed DWS (EPA, 1991) \\
\hline Cesium-134 & $\mathrm{pCi} / \mathrm{L}$ & 4.07E+01 & $8.13 E+01$ & Proposed DWS (EPA, 1991) \\
\hline Cesium-137 & $\mathrm{pCi} / \mathrm{L}$ & $1 E+02$ & $2 E+02$ & Final DWS (EPA, 1977) \\
\hline Chlordane & $\mu g / L$ & 1 & 2 & Final DWS (CFR, 1991a) \\
\hline Chloride & $\mu \mathrm{g} / \mathrm{L}$ & 125,000 & 250,000 & Secondary DWS (CFR, 1991b) \\
\hline 4-Chloroaniline & $\mu g / L$ & 50 & 100 & EPA Method 8270 \\
\hline Chlorobenzene & $\mu g / L$ & 5 & 10 & EPA Method 8240 \\
\hline Chlorobenzilate & $\mu g / L$ & 50 & 100 & EPA Method 8270 \\
\hline Chloroethane & $\mu g / L$ & 5 & 10 & EPA Method 8240 \\
\hline Chloroethene (Vinyl chloride) & $\mu \mathrm{g} / \mathrm{L}$ & 1 & 2 & Final DWS (CFR, 1991a) \\
\hline Chloroethyl vinyl ether & $\mu \mathrm{g} / \mathrm{L}$ & 5 & 10 & EPA Method 8240 \\
\hline 2-Chloroethyl vinyl ether & $\mu g / L$ & 5 & 10 & EPA Method 8240 \\
\hline Chloroform & $\mu \mathrm{g} / \mathrm{L}$ & 50 & 100 & Final DWS (CFR, 1991a) \\
\hline para-Chloro-meta-cresol & $\mu g / L$ & 50 & 100 & EPA Method 8270 \\
\hline Chloromethane (Methyl chloride) & $\mu \mathrm{g} / \mathrm{L}$ & 5 & 10 & EPA Method 8240 \\
\hline 2-Chloronaphthalene & $\mu \mathrm{g} / \mathrm{L}$ & 50 & 100 & EPA Method 8240 \\
\hline 2-Chlorophenol & $\mu g / L$ & 50 & 100 & EPA Method 8270 \\
\hline
\end{tabular}




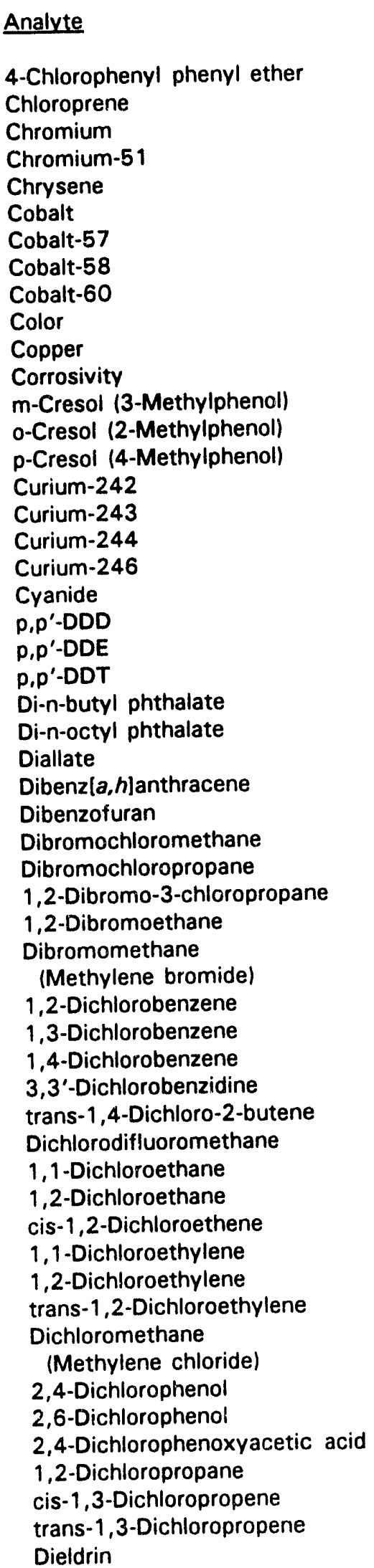

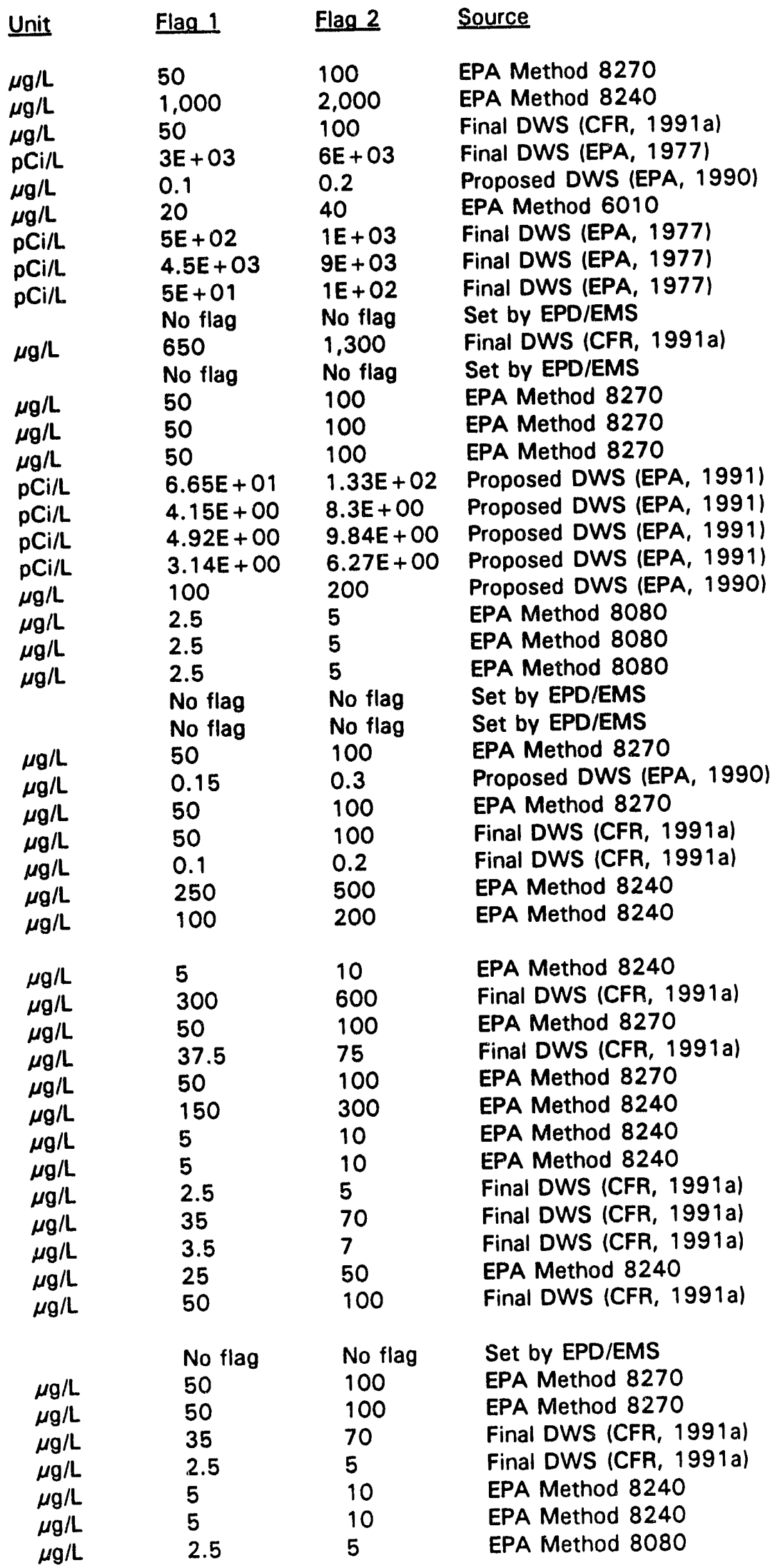




\begin{tabular}{|c|c|c|c|c|}
\hline Analyte & Unit & Flag 1 & Flag 2 & Source \\
\hline $\begin{array}{l}\text { Dimethoate } \\
\text { p-Dimethylaminoazobenzene }\end{array}$ & $\mu g / L$ & 50 & 100 & EPA Method 8270 \\
\hline $\begin{array}{l}\text { p-Dimethylaminoazobenzene } \\
\text { p-(Dimethylaminolethylbenzene }\end{array}$ & $\mu g / L$ & 50 & 100 & EPA Method 8270 \\
\hline & $\mu g / L$ & 50 & 100 & EPA Method 8270 \\
\hline $\begin{array}{l}\text { 7,12-Dimethylbenzla]anthracene } \\
\text { 3,3'-Dimethylbenzidine }\end{array}$ & $\mu g / L$ & 50 & 100 & EPA Method 8270 \\
\hline $\begin{array}{l}\text { 3,3'-Dimethylbenzidine } \\
\text { a,a-Dimethylphenethylamine }\end{array}$ & $\mu g / L$ & 50 & 100 & EPA Method 8270 \\
\hline & $\mu g / L$ & 50 & 100 & EPA Method 8270 \\
\hline $\begin{array}{l}\text { 2,4-Dimethyl phenol } \\
\text { Dimethyl phthalate }\end{array}$ & $\mu g / L$ & 50 & 100 & EPA Method 8270 \\
\hline & & No flag & No flag & Set by EPD/EMS \\
\hline $\begin{array}{l}\text { 1,3-Dinitrobenzene } \\
\text { 4,6-Dinitro-ortho-cresol }\end{array}$ & $\mu g / L$ & 50 & 100 & EPA Method 8270 \\
\hline $\begin{array}{l}\text { 4,6-Dinitro-orno-cresol } \\
\text { 2,4-Dinitrophenol }\end{array}$ & $\begin{array}{l}\mu g / L \\
\mu g / L\end{array}$ & 250 & 500 & EPA Method 8270 \\
\hline 2,4-Dinitrotoluene & $\begin{array}{l}\mu g / L \\
\mu g / L\end{array}$ & 250 & 500 & EPA Method 8270 \\
\hline 2,6-Dinitrotoluene & $\begin{array}{l}\mu g / L \\
\mu g / L\end{array}$ & 50 & 100 & EPA Method 8270 \\
\hline 1,4-Dioxane & $\begin{array}{l}\mu g / L \\
\mu g / L\end{array}$ & 50 & 100 & EPA Method 8270 \\
\hline Diphenylamine & $\begin{array}{l}\mu g / L \\
\mu g / L\end{array}$ & 50 & 100 & EPA Method 8270 \\
\hline 1,2-Diphenylhydrazine & $\begin{array}{l}\mu g / L \\
\mu g / L\end{array}$ & 50 & 100 & EPA Method 8270 \\
\hline Dissolved organic carbon & $\begin{array}{l}\mu g / L \\
\mu g / L\end{array}$ & 50 & 100 & EPA Method 8270 \\
\hline Disulfoton & $\mu g / L$ & $\begin{array}{l}5,000 \\
50\end{array}$ & $\begin{array}{l}10,000 \\
100\end{array}$ & \\
\hline Eh & & No flag & $\begin{array}{l}100 \\
\text { No flag }\end{array}$ & $\begin{array}{l}\text { EPA Method } 8270 \\
\text { Set by EPD/EMS }\end{array}$ \\
\hline alpha-Endosulfan & $\mu g / L$ & 50 & 100 & $\begin{array}{l}\text { Set by EPD/EMS } \\
\text { EPA Method } 8270\end{array}$ \\
\hline beta-Endosulfan & $\mu g / L$ & 50 & 100 & EPA Method 8270 \\
\hline Endosulfan 1 & $\mu g / L$ & 2.5 & 5 & EPA Method 8080 \\
\hline Endosulfan II & $\mu g / L$ & 2.5 & 5 & EPA Method 8080 \\
\hline Endosulfan sulfate & $\mu g / L$ & 2.5 & 5 & EPA Method 8080 \\
\hline Endrin & $\mu g / L$ & 0.1 & 0.2 & Final DWS (CFR, 1991a) \\
\hline Endrin aldehyde & $\mu \mathrm{g} / \mathrm{L}$ & 2.5 & 5 & EPA Method 8080 \\
\hline Endrin ketone & & No flag & No flag & Set by EPD/EMS \\
\hline Ethyl methacrylate & $\mu \mathrm{g} / \mathrm{L}$ & 50 & 100 & EPA Method 8270 \\
\hline Ethyl methanesulfonate & $\mu g / L$ & 50 & 100 & EPA Method 8270 \\
\hline Ethylbenzene & $\mu g / L$ & 350 & 700 & Final DWS (CFR, 1991a) \\
\hline Europium-154 & $\mathrm{pCi} / \mathrm{L}$ & $1 E+02$ & $2 E+02$ & Final DWS (EPA, 1977) \\
\hline Europium-155 & $\mathrm{pCi} / \mathrm{L}$ & $3 E+02$ & $6 E+02$ & Final DWS (EPA, 1977) \\
\hline Famphur & $\mu g / L$ & 50 & 100 & EPA Method 8270 \\
\hline Fluoranthene & $\mu g / L$ & 50 & 100 & EPA Method 8270 \\
\hline Fluorene & $\mu g / L$ & 50 & 100 & EPA Method 8270 \\
\hline Fluoride & $\mu g / L$ & 2,000 & 4,000 & Final DWS (CFR, 1991a) \\
\hline Gross alpha & $\mathrm{pCi} / \mathrm{L}$ & $7.5 E+00$ & $1.5 E+01$ & Final DWS (CFR, 1991a) \\
\hline Heptachlor & $\mu g / L$ & 0.2 & 0.4 & Final DWS (CFR, 1991a) \\
\hline Heptachlor epoxide & $\mu g / L$ & 0.1 & 0.2 & Final DWS (CFR, 1991a) \\
\hline $\begin{array}{l}\text { Heptachlorodibenzo-p-dioxin } \\
\text { isomers }\end{array}$ & & & & \\
\hline $\begin{array}{l}\text { isomers } \\
1,2,3,4,6,7,8 \text {-Heptachlorodibenzo- }\end{array}$ & $\mu g / L$ & 0.00325 & 0.0065 & EPA Method 8280 \\
\hline p-dioxin & $\mu g / L$ & 0.00325 & 0.0065 & EPA Method 8280 \\
\hline Heptachlorodibenzo-p-furan & & & & \\
\hline isomers & $\mu g / L$ & 0.00225 & 0.0045 & EPA Method 8280 \\
\hline $\begin{array}{l}1,2,3,4,6,7,8 \text {-Heptachlorodibenzo- } \\
\text { p-furan }\end{array}$ & & & & FPA Method 8280 \\
\hline Hexachlorobenzene & $\begin{array}{l}\mu g / L \\
\mu g / L\end{array}$ & $\begin{array}{l}0.00225 \\
0.5\end{array}$ & $\begin{array}{l}0.0045 \\
1\end{array}$ & $\begin{array}{l}\text { EPA Method } 8280 \\
\text { Proposed DWS (EPA, 1990) }\end{array}$ \\
\hline Hexachlorobutadiene & $\begin{array}{l}\mu g / L \\
\mu g / L\end{array}$ & $\begin{array}{l}0.5 \\
50\end{array}$ & & $\begin{array}{l}\text { Proposed DWS (EPA, 1990) } \\
\text { EPA Method } 8270\end{array}$ \\
\hline Hexachlorocyclopentadiene & $\mu g / L$ & $\begin{array}{l}50 \\
25\end{array}$ & $\begin{array}{l}100 \\
50\end{array}$ & Proposed DWS (EPA, 1990 ) \\
\hline Hexachlorodibenzo-p-dioxin isomers & $\mu g / L$ & 0.00225 & 0.0045 & EPA Method 8280 \\
\hline $\begin{array}{l}1,2,3,4,7,8-\text { Hexachlorodiben } \\
\text { p-dioxin }\end{array}$ & & & & \\
\hline $\begin{array}{l}\text { p-dioxin } \\
\text { Hexachlorodibenzo-p-furan iso }\end{array}$ & $\begin{array}{l}\mu g / L \\
\mu g / L\end{array}$ & 0.00225 & $\begin{array}{l}0.0045 \\
0.004\end{array}$ & EPA Method 8280 \\
\hline Hexachlorodibenzo-p-furan isom & $\mu g / L$ & 0.002 & 0.004 & EPA Method 8280 \\
\hline
\end{tabular}


Analyte

\section{$1,2,3,4,7,8$-Hexachlorodibenzo-} p-furan

Hexachloroethane

Hexachlorophene

Hexachloropropene

2-Hexanone

Indeno[1,2,3-c, 0]pyrene

lodine

lodine-129

lodine-131

lodomethane (Methyl iodide)

Iron

Iron-55

Iron-59

Isobutyl alcohol

Isodrin

Isophorune

Isosafrole

Kepone

Lanthanum-140

Lead

Lindane

Lithium

Magnesium

Manganese

Manganese-54

Mercury

Methacrylonitrile

Methapyrilene

Methoxychlor

3-Methylcholanthrene

2-Methyl-4,6-dinitrophenol

Methyl ethyl ketone

Methyl isobutyl ketone

Methyl methacrylate

Methyl methanesulfonate

2-Methylnaphthalene

Molybdenum

Naphthalene

1,4-Naphthoquinone

1-Naphthylamine

2-Naphthylamine

Neptunium-237

Nickel

Nickel-59

Nickel-63

Niobium-95

Nitrate as nitrogen

Nitrate-nitrite as nitrogen

Nitrite as nitrogen

2-Nitroaniline

3-Nitroaniline

4-Nitroaniline

Nitrobenzene

Nitrogen by Kjeldahl method
Unit

Flag 1

0.002

$\mu \mathrm{g} / \mathrm{L}$

$\mu \mathrm{g} / \mathrm{L}$

$\mu g / L$

$\mu g / L$

$\mu g / L$

$\mu g / L$

$\mu g / L$

$\mathrm{pCi} / \mathrm{L}$

pCi/L

$\mu g / L$

$\mu g / L$

$\mathrm{pCi} / \mathrm{L}$

pCi/L

$\mu g / L$

$\mu g / L$

$\mu g / L$

$\mu g / L$

$\mu g / L$

pCi/L

$\mu g / L$

$\mu \mathrm{g} / \mathrm{L}$

$\mu g / L$

$\mu g / L$

$\mathrm{pCi} / \mathrm{L}$

$\mu g / L$

$\mu g / L$

$\mu \mathrm{g} / \mathrm{L}$

$\mu g / L$

$\mu g / L$

$\mu g / L$

$\mu g / L$

$\mu g / L$

$\mu g / L$

$\mu g / L$

$\mu g / L$

$\mu g / L$

$\mu g / L$

$\mu g / L$

pCi/L

$\mu g / L$

pCi/L

pCi/L

pCi/L

$\mu g / L$

$\mu g / L$

$\mu g / L$

$\mu \mathrm{g} / \mathrm{L}$

$\mu g / L$

$\mu \mathrm{g} / \mathrm{L}$

$\mu \mathrm{g} / \mathrm{L}$

$\mu \mathrm{g} / \mathrm{L}$

50

5,000

5,000

500

50

50

50

50

500
$50 \quad 100$

$250 \quad 500$

$50 \quad 100$

$100 \quad 200$

$50 \quad 100$

$500 \quad 1,000$

$5 E-01 \quad 1 E+00$

$1.5 E+00 \quad 3 E+00$

$75 \quad 150$

$150 \quad 300$

$1 E+03 \quad 2 E+03$

$1 E+02 \quad 2 E+02$

$500 \quad 1,000$

$50 \quad 100$

$50 \quad 100$

$50 \quad 100$

$50 \quad 100$

$3 E+01 \quad 6 E+01$

$7.5 \quad 15$

$0.1 \quad 0.2$

$25 \quad 50$

No flag No flag

$25 \quad 50$

$1.5 E+02 \quad 3 E+02$

12

$250 \quad 500$

$50 \quad 100$

$20 \quad 40$

$50 \quad 100$

$250 \quad 500$

No flag No flag

No flag No flag

$50 \quad 100$

$50 \quad 100$

$50 \quad 100$

$250 \quad 500$

$50 \quad 100$

$50 \quad 100$

$50 \quad 100$

$50 \quad 100$

$3.53 E+00 \quad 7.06 E+00$

$1.5 \mathrm{E}+02$

$2.5 E+01$

$1.5 \mathrm{E}+02$

$3 E+02$

$5 E+01$

$3 E+02$

10,000

10,000

1,000

100

100

100

100

1,000
Source

EPA Method 8280

EPA Method 8270

EPA Method 8270

EPA Method 8270

EPA Method 8240

EPA Method 8270

EPA Method 415

Final DWS (EPA, 1977)

Final DWS (EPA, 1977)

EPA Method 8240

Secondary DWS (CFR, 1991b)

Final DWS (EPA, 1977)

Final DWS (EPA, 1977)

EPA Method 8240

EPA Method 8270

EPA Method 8270

EPA Method 8270

EPA Method 8270

Final DWS (EPA, 1977)

Final DWS (CFR, 1991a)

Final DWS (CFR, 1991a)

EPA Method 6010

Set by EPD/EMS

Secondary DWS (CFR, 1991b)

Final DWS (EPA, 1977)

Final DWS (CFR, 1991a)

EPA Method 8240

EPA Method 8270

Final DWS (CFR, 1991a)

EPA Method 8270

EPA Method 8270

Set by EPD/EMS

Set by EPD/EMS

EPA Method 8270

EPA Method 8270

EPA Method 8270

EPA Method 6010

EPA Method 8270

EPA Method 8270

EPA Method 8270

EPA Method 8270

Proposed DWS (EPA, 1991)

Proposed DWS (EPA, 1990)

Final DWS (EPA, 1977)

Final DWS (EPA, 1977)

Final DWS (EPA, 1977)

Final DWS (CFR, 1991a)

Final DWS (CFR, 1991a)

Final DWS (CFR, 1991a)

EPA Method 8270

EPA Method 8270

EPA Method 8270

EPA Method 8270

EPA Method 351.2 


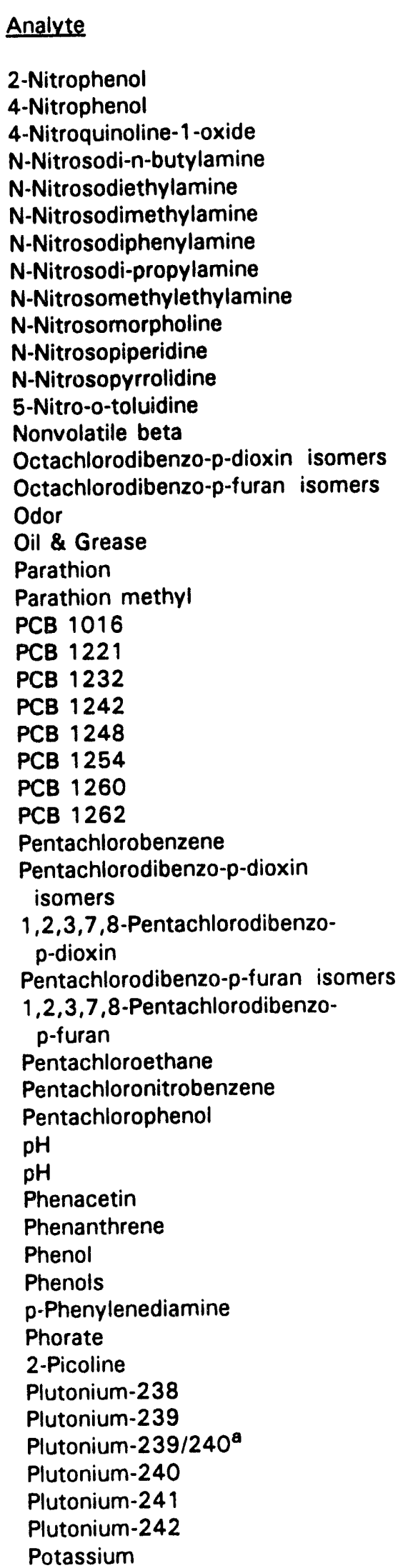

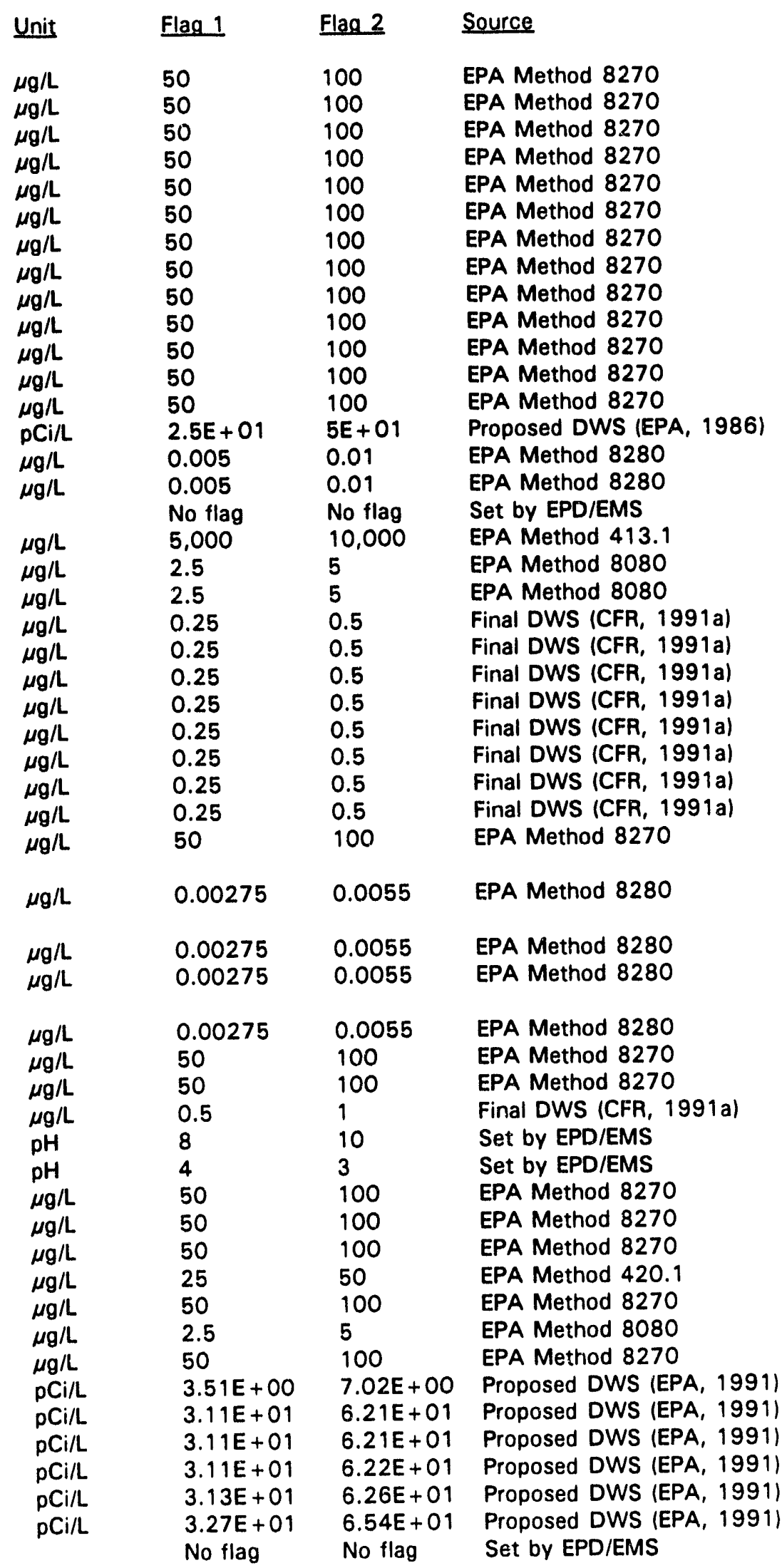




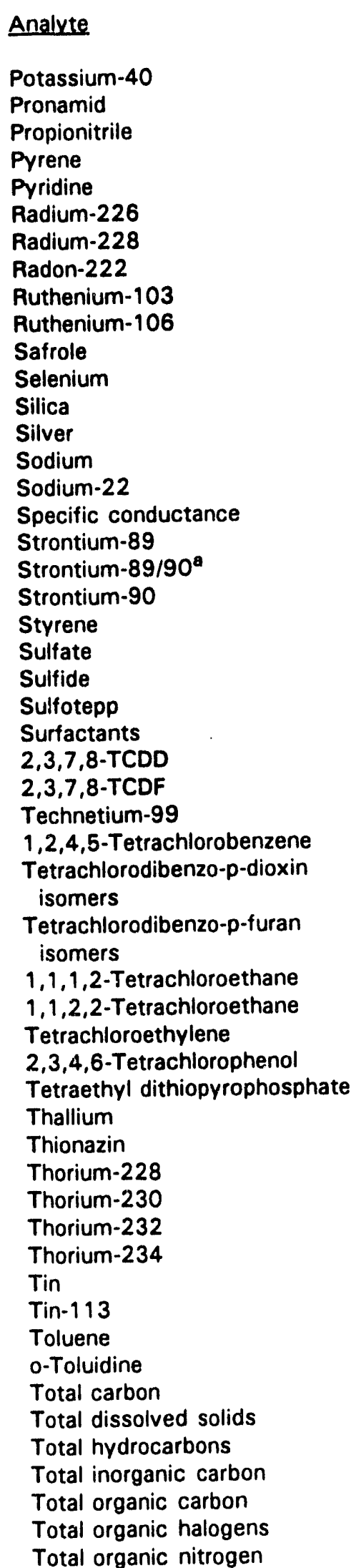

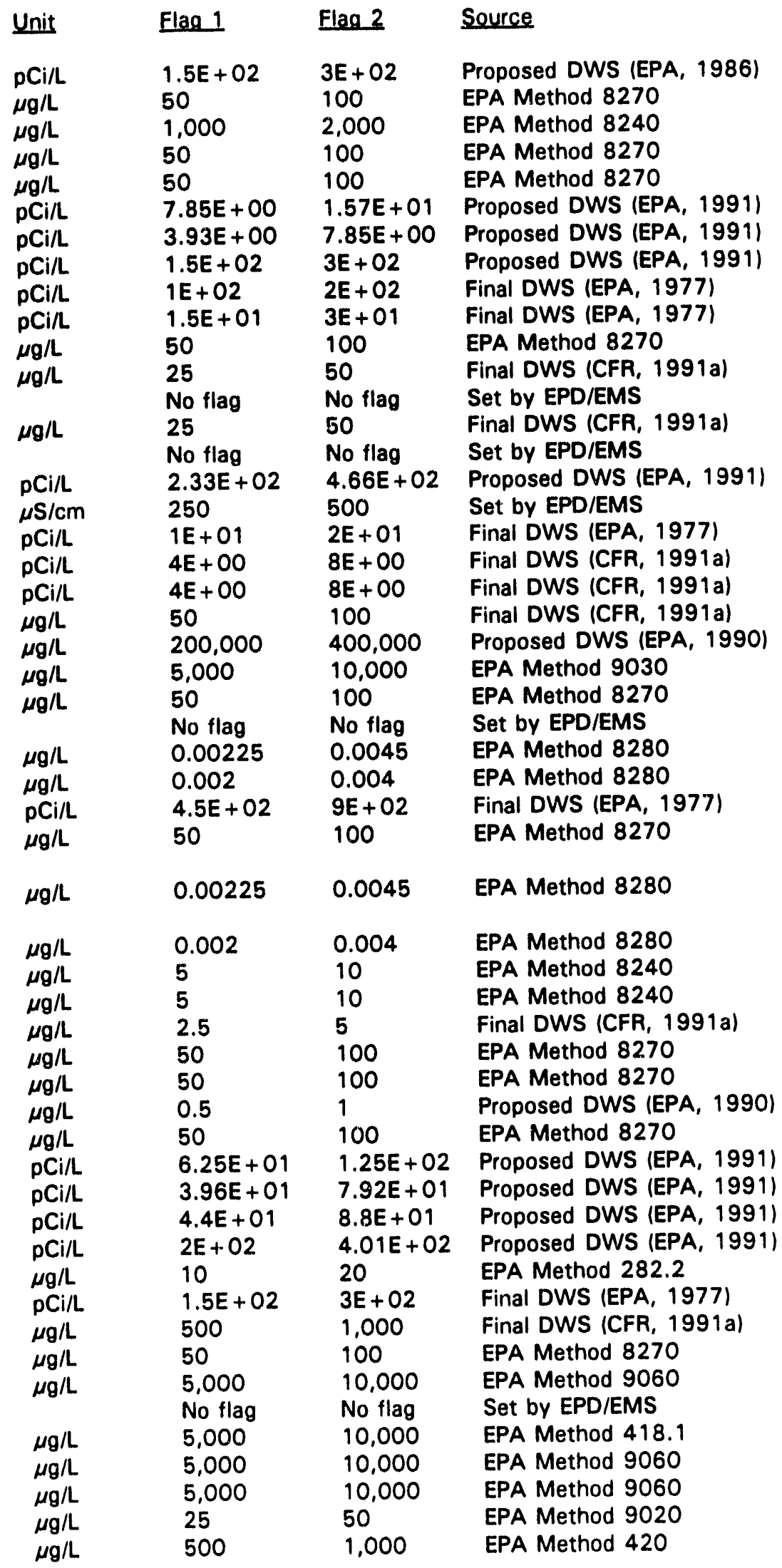




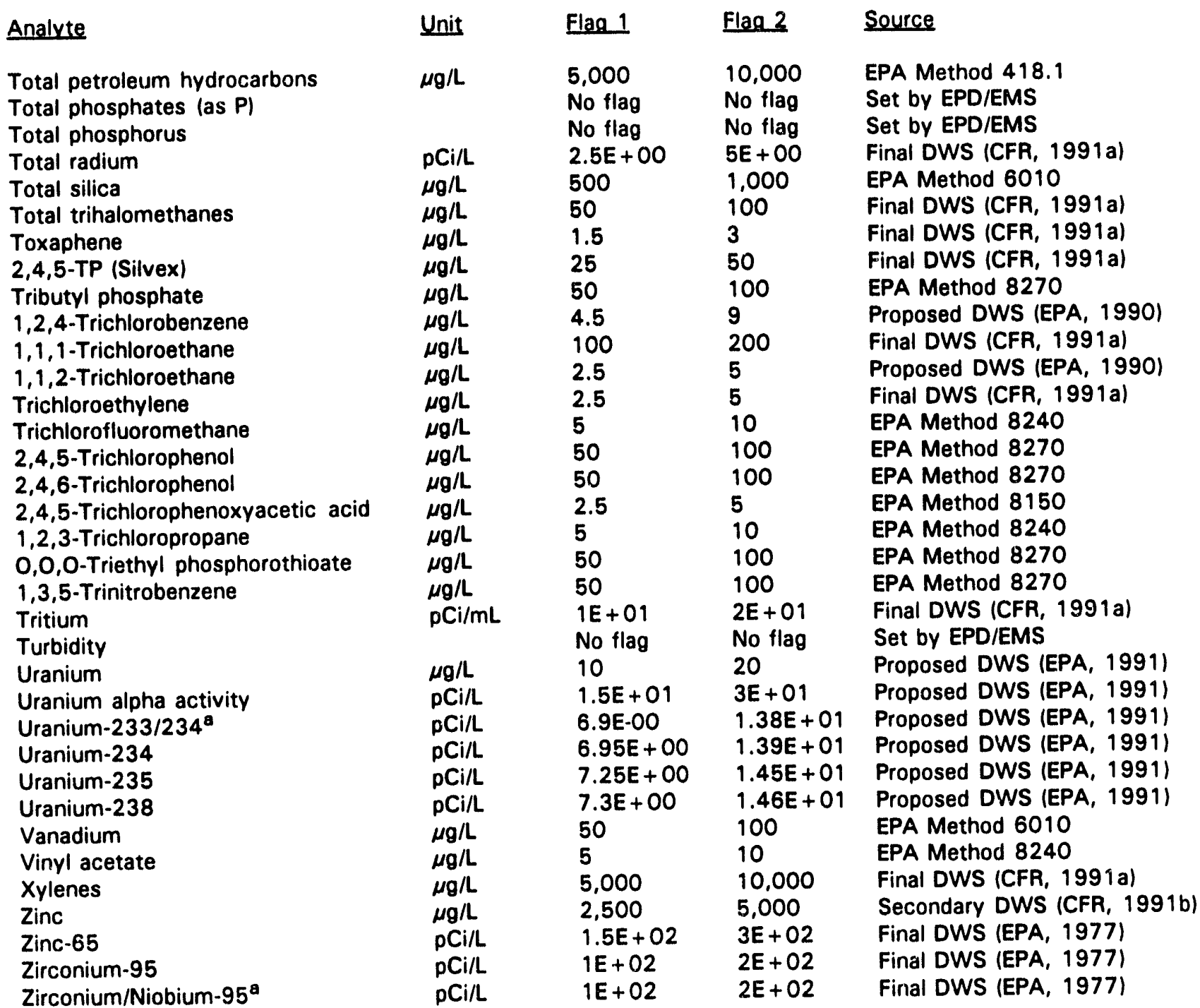

a For double radionuclide analyses where each separate radionuclide has its own standard, the more stringent standard is used.

\section{References}

APHA (American Public Health Association, American Water Works Association, and Water Pollution Control Federation), 1985. Standard Methods for the Examination of Water and Wastewater, 16th edition. Washington, DC.

CFR (Code of Federal Regulations), 1991a. National Primary Drinking Water Regulations, 40 CFR, Part 141, pp. 578-715. Washington, DC.

CFR (Code of Federal Regulations), 1991b. National Secondary Drinking Water Regulations, 40 CFR, Part 143, pp. 758-762. Washington, DC. 
EPA (U.S. Environmental Protection Agencyl, 1977. National Interim Primary Drinking Water Regulations, EPA-570/9-76-003. Washington, DC.

EPA (U.S. Environmental Protection Agencyl, 1986. Water Pollution Control; National Primary Drinking Water Regulations, Radionuclides (Proposed). Federal Register, September 30, 1986, pp. 34836-34862. Washington, DC.

EPA (U.S. Environmental Protection Agency), 1990. National Primary and Secondary Drinking Water Regulations; Synthetic Organic Chemicals and Inorganic Chemicals (Proposed Rule). Federal Register, July 25, 1990, pp. 30369-30448. Washington, DC.

EPA (U.S. Environmental Protection Agency), 1991. National Primary Drinking Water Regulations; Radionuclides; Proposed Rule. Federal Register, July 18, 1991, pp. 33052-33127. Washington, DC. 
WSRC.TR-93-068

\section{Appendix C - Figures}




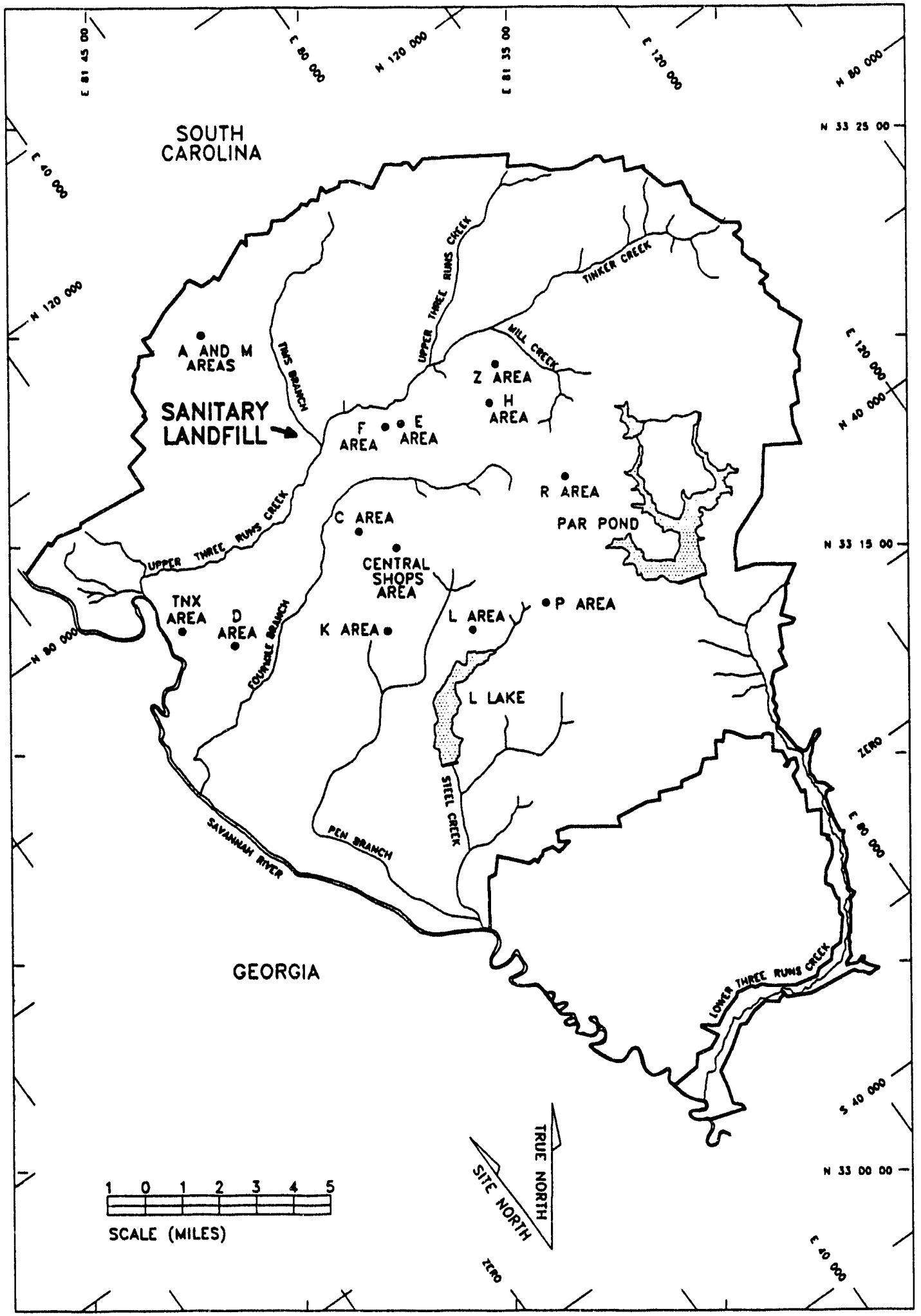

Figure 1. Location of the Sanitary Landfill at the Savannah River Site 


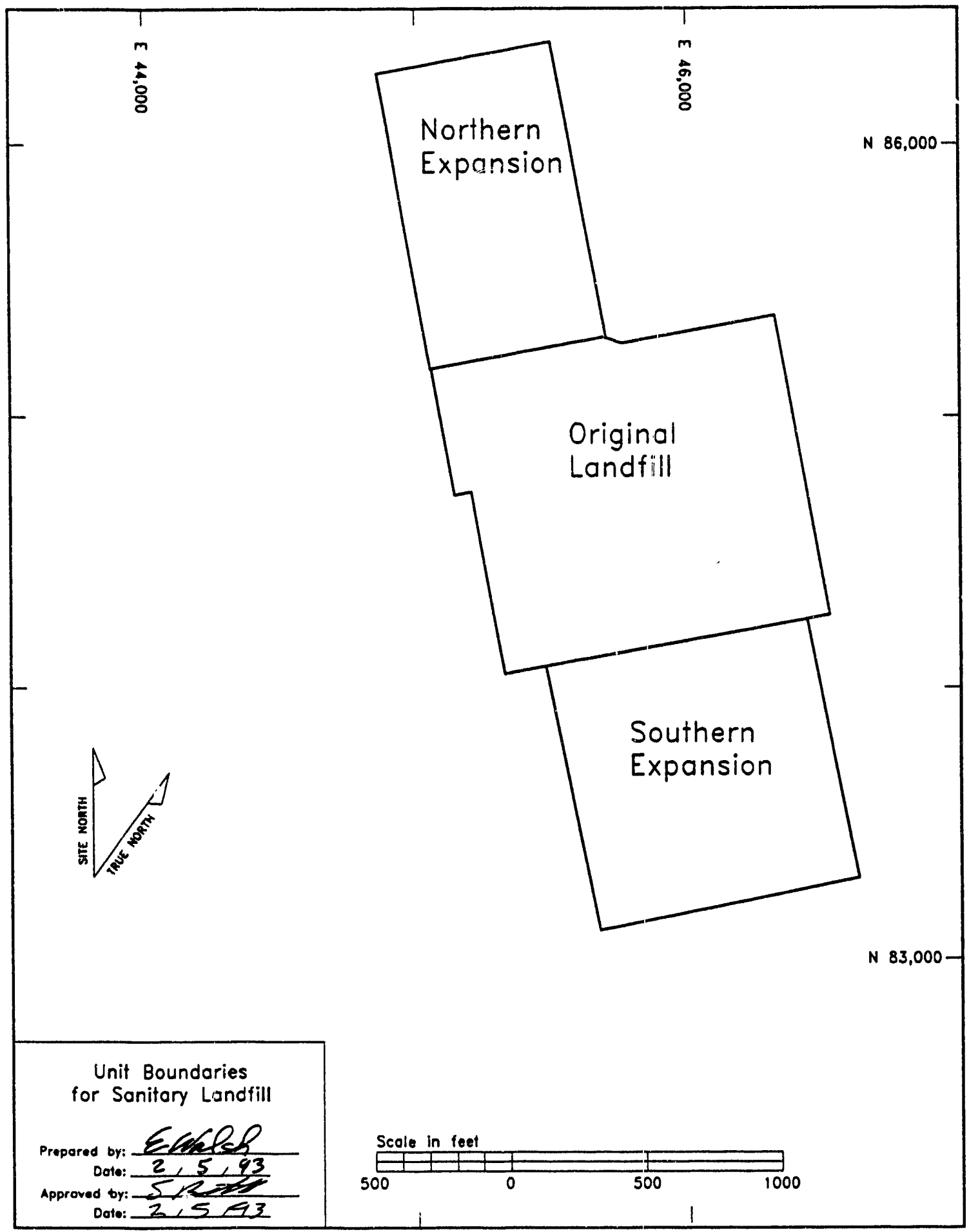

Figure 2. Boundaries of Original Sanitary Landfill and Expansions 


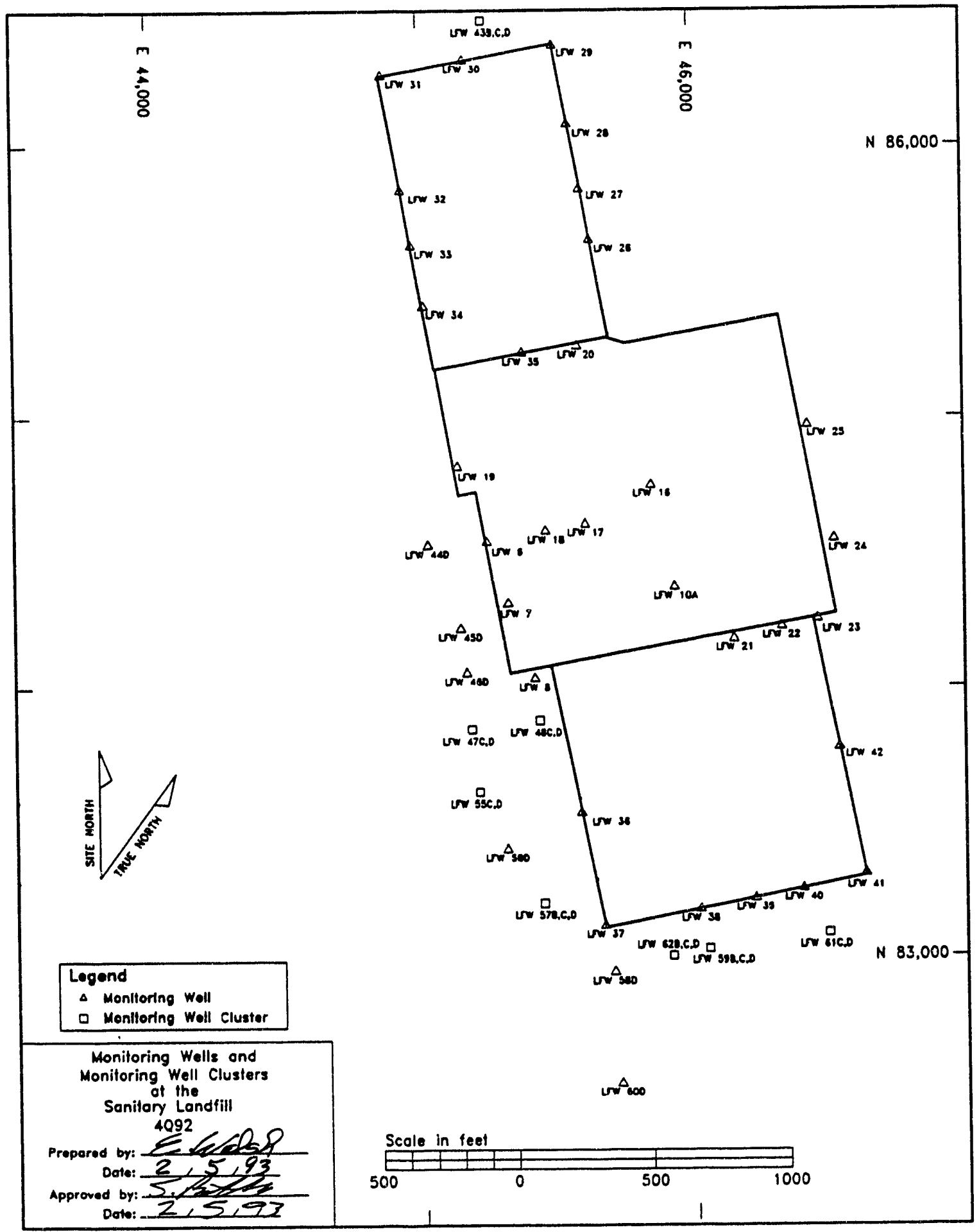

Figure 3. Location of Monitoring Wells at the Sanitary Landfill 


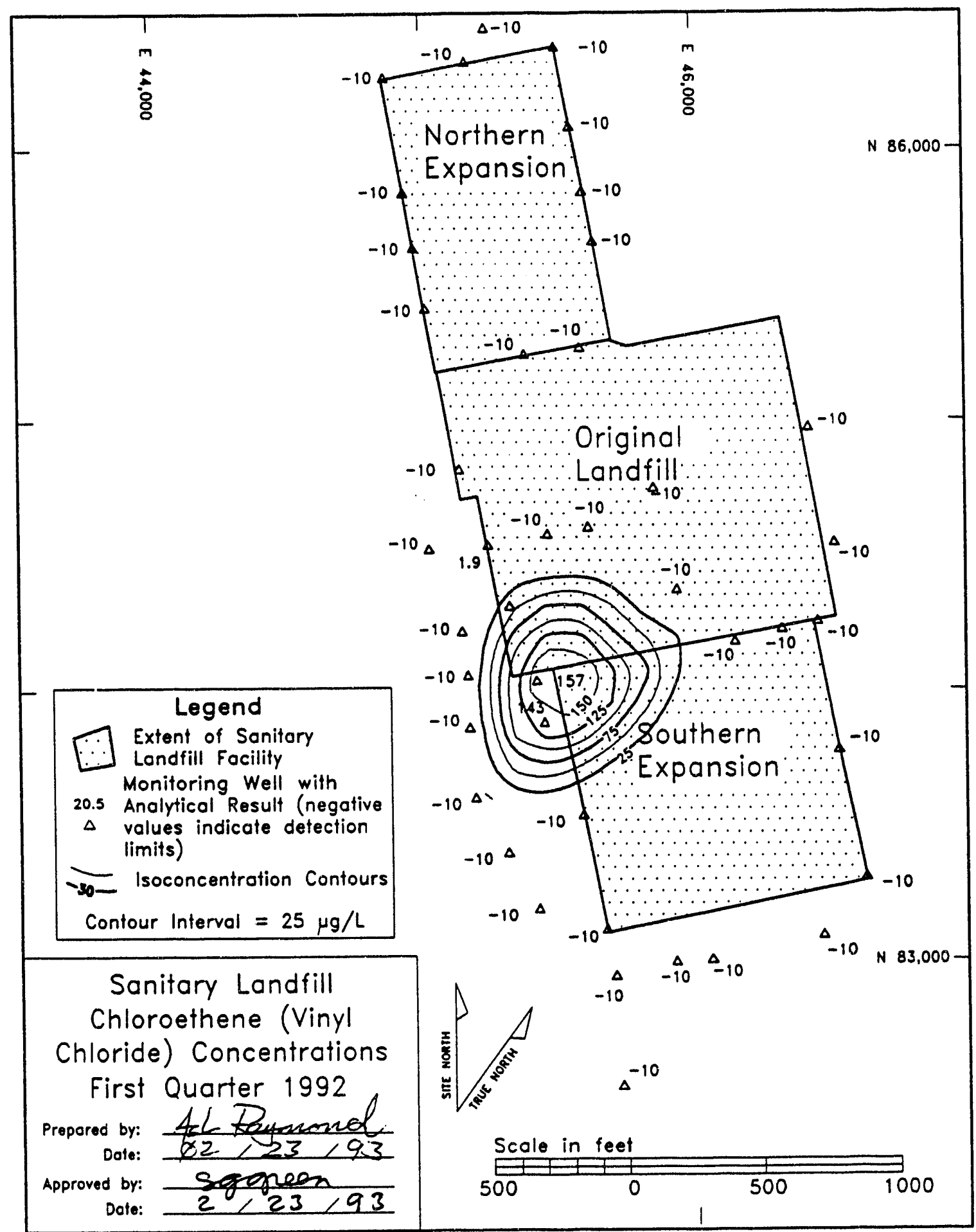

Figure 4. Chloroethene (Vinyl chloride) Concentrations at the Sanitary Landfill, First Quarter 1992 


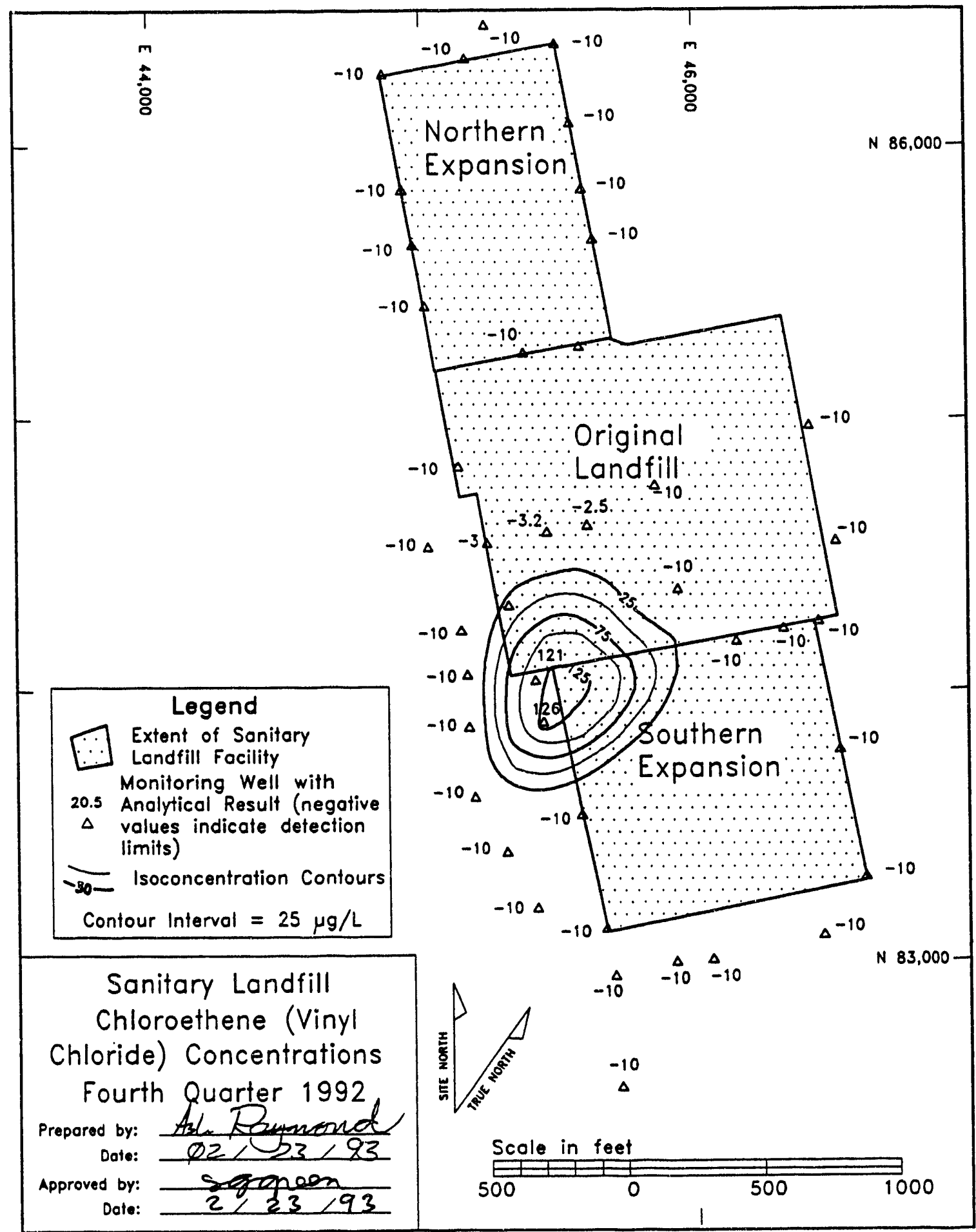

Figure 5. Chloroethene (Vinyl chloride) Concentrations at the Sanitary Landfill, Fourth Quarter 1992 


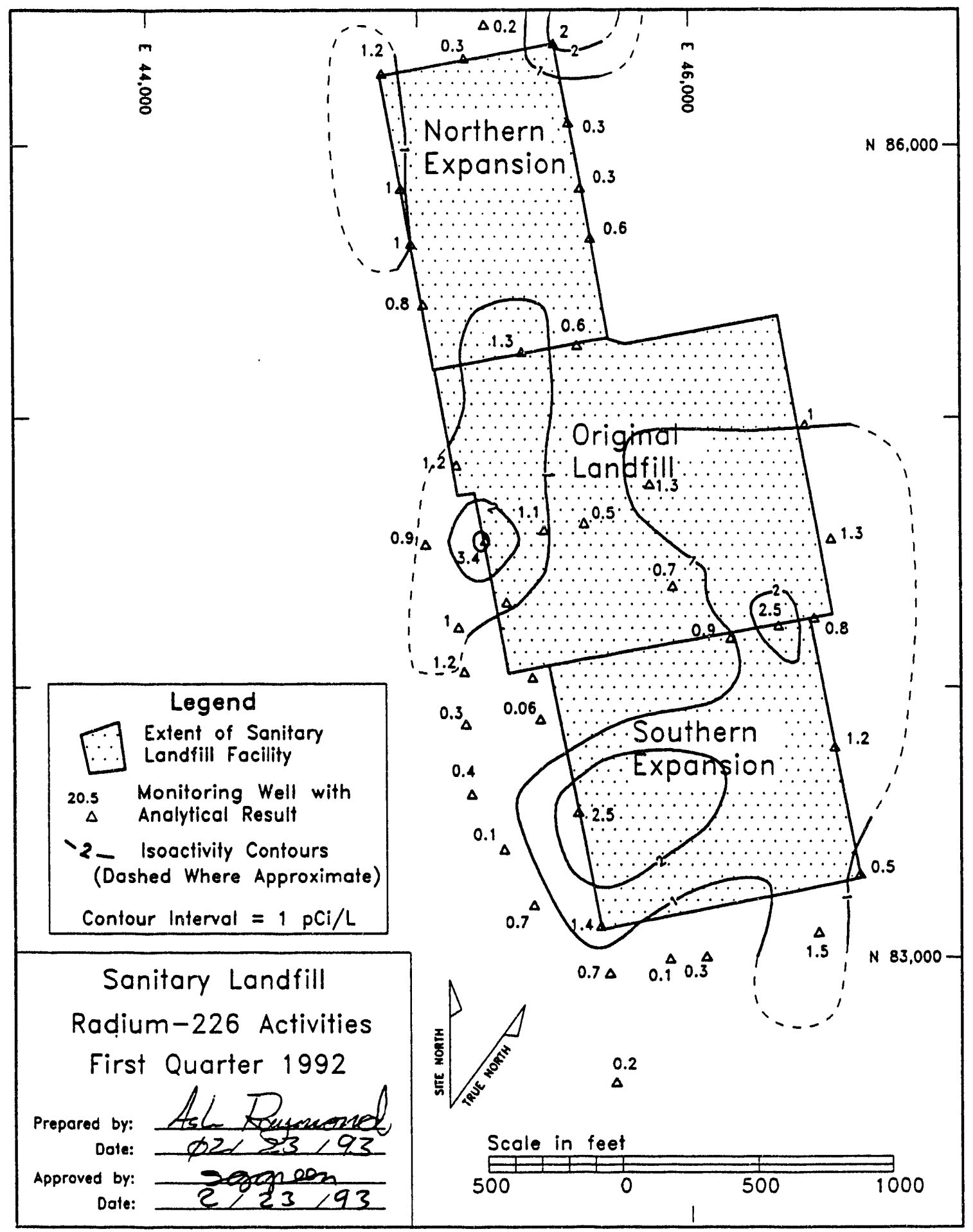

Figure 6. Radium-226 Activities at the Sanitary Landfill, First Quarter 1992 


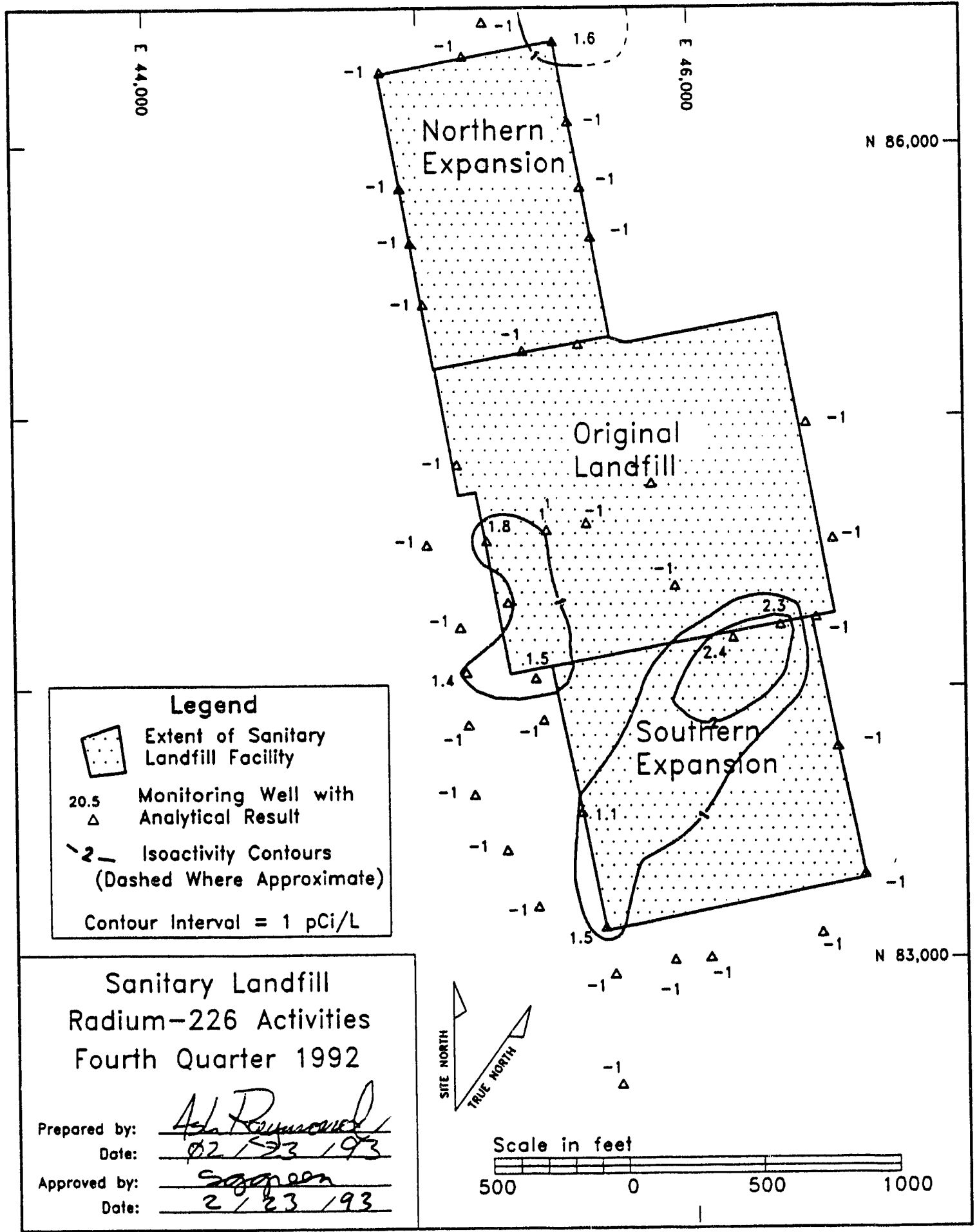

Figure 7. Radium-226 Activities at the Sanitary Landfill, Fourth Quarter 1992 


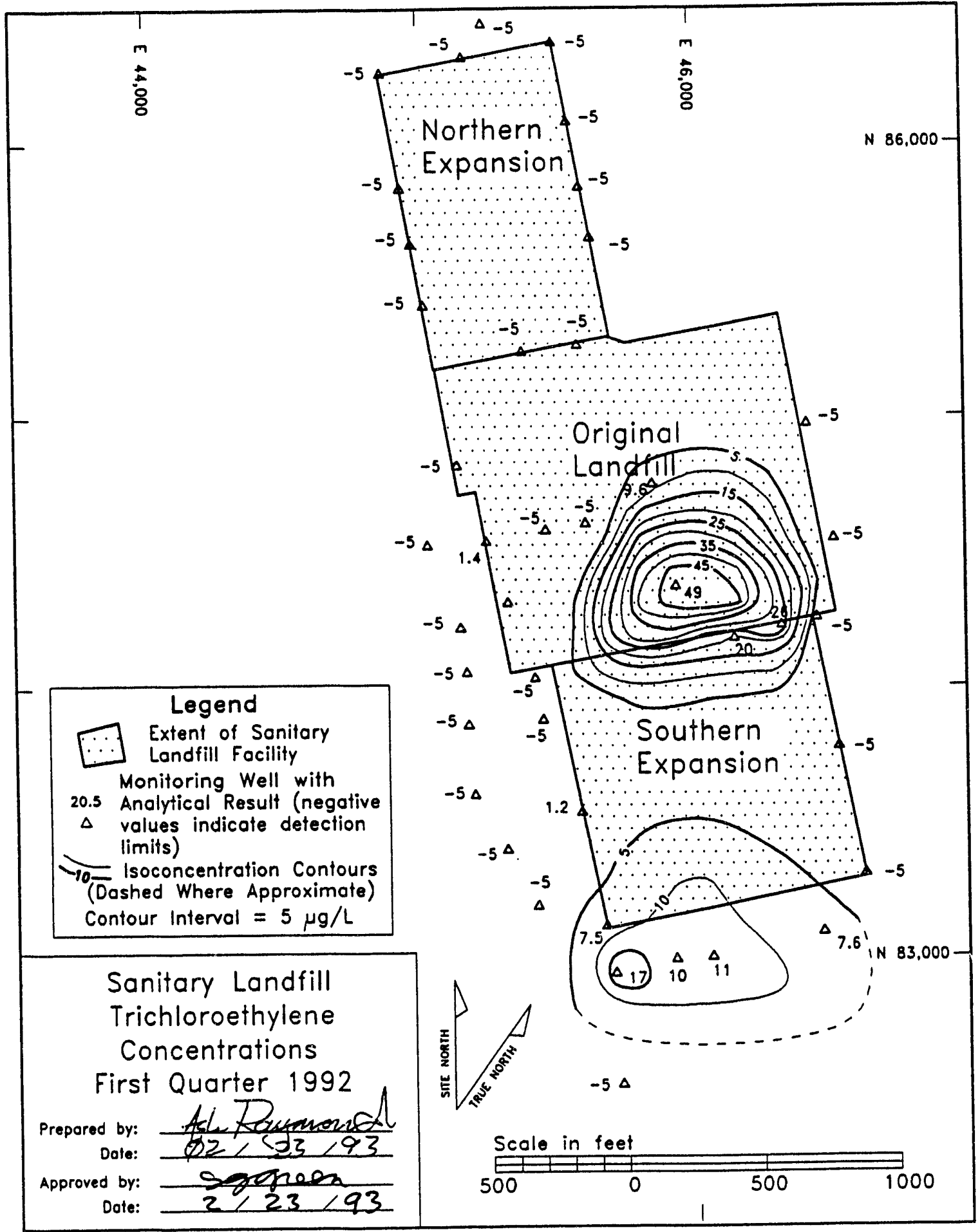

Figure 8. Trichloroethylene Concentrations at the Sanitary Landfill, First Quarter 1992 


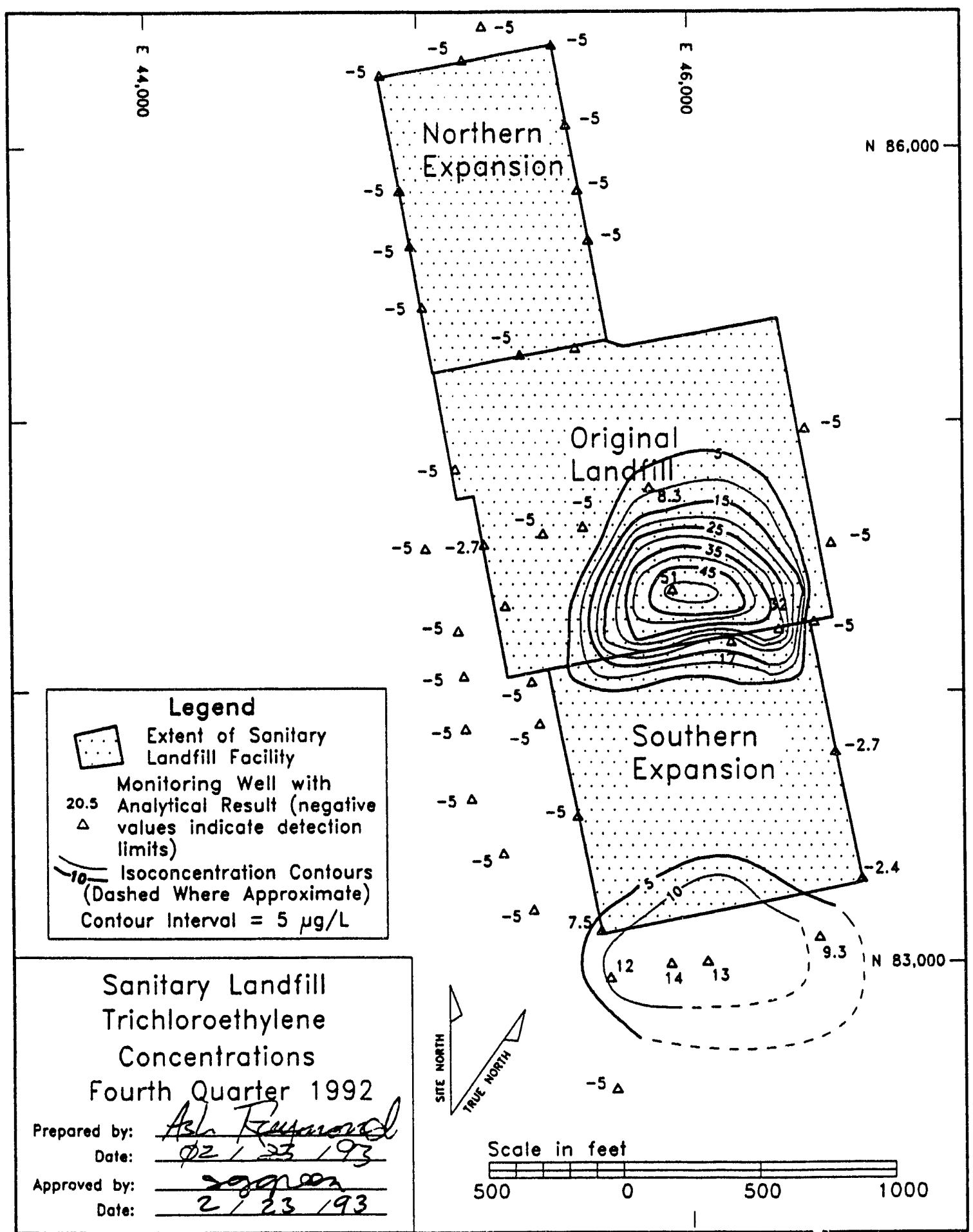

Figure 9. Trichloroethylene Concentrations at the Sanitary Landfill, Fourth Quarter 1992 


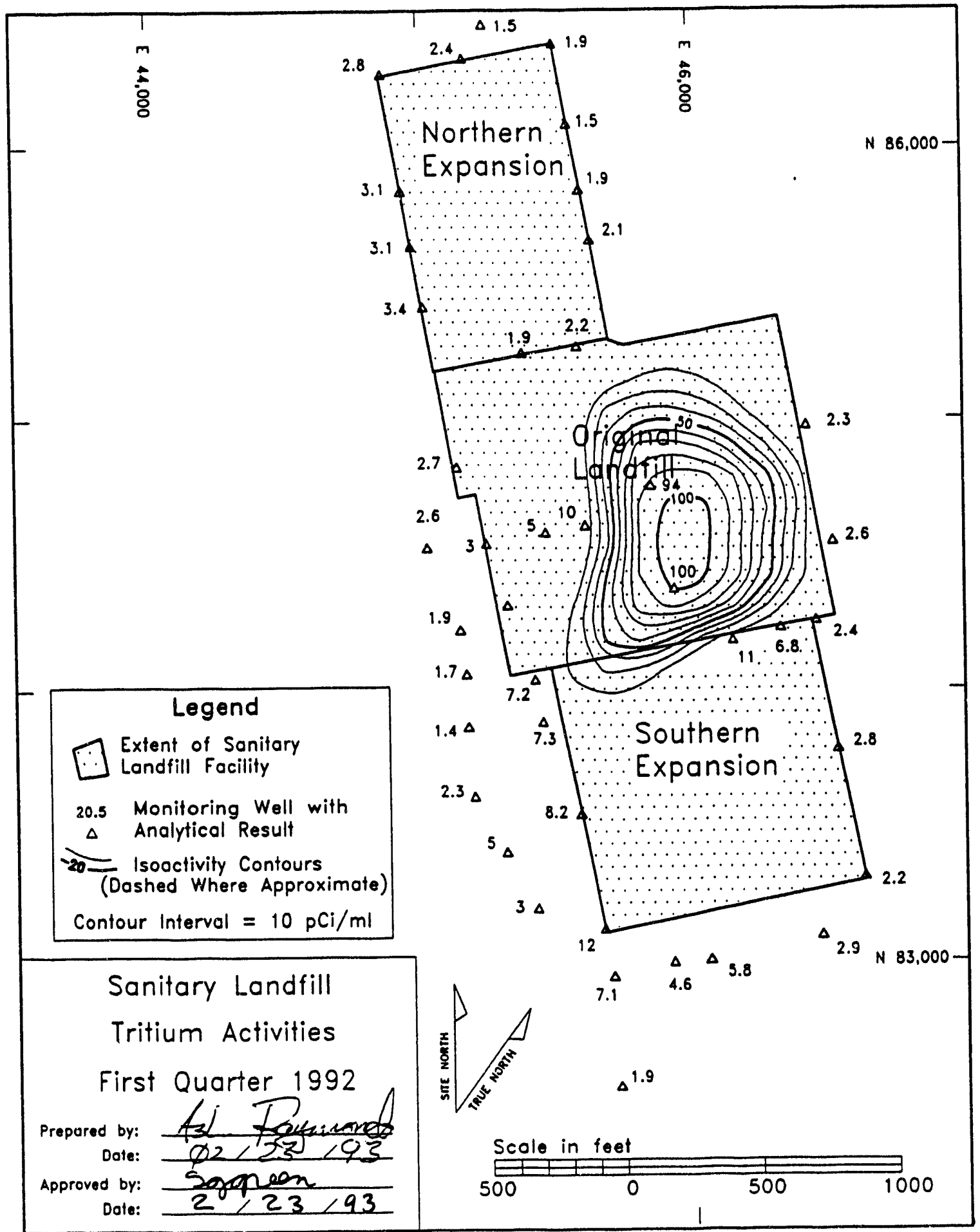

Figure 10. Tritium Activities at the Sanitary Landfill, First Quarter 1992 


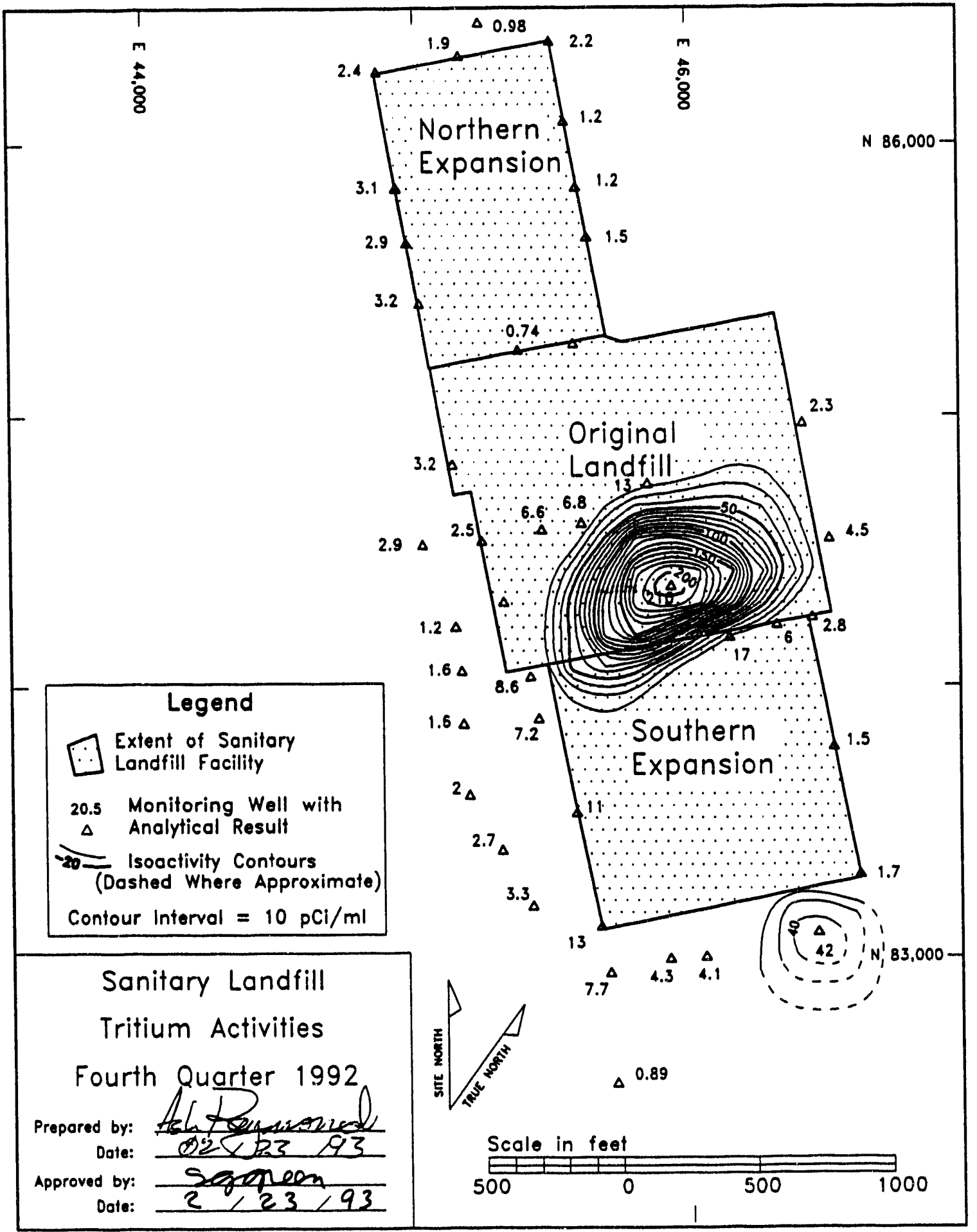

Figure 11. Tritium Activities at the Sanitary Landfill, Fourth Quarter 1992 


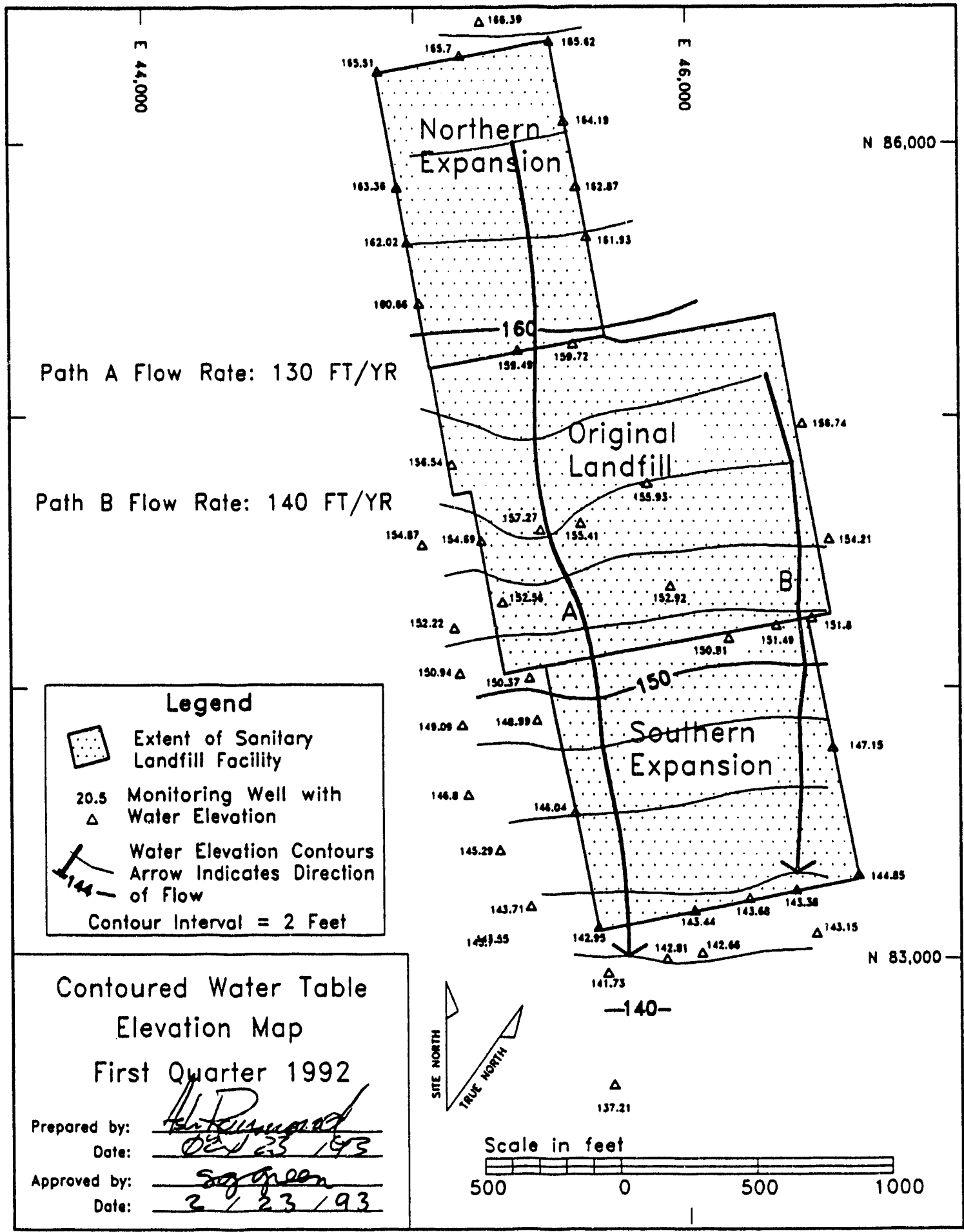

Figure 12. Water-Elevation Contour Map of Steed Pond Aquifer (Water Table) at the Sanitary Landfill, First Quarter 1992 


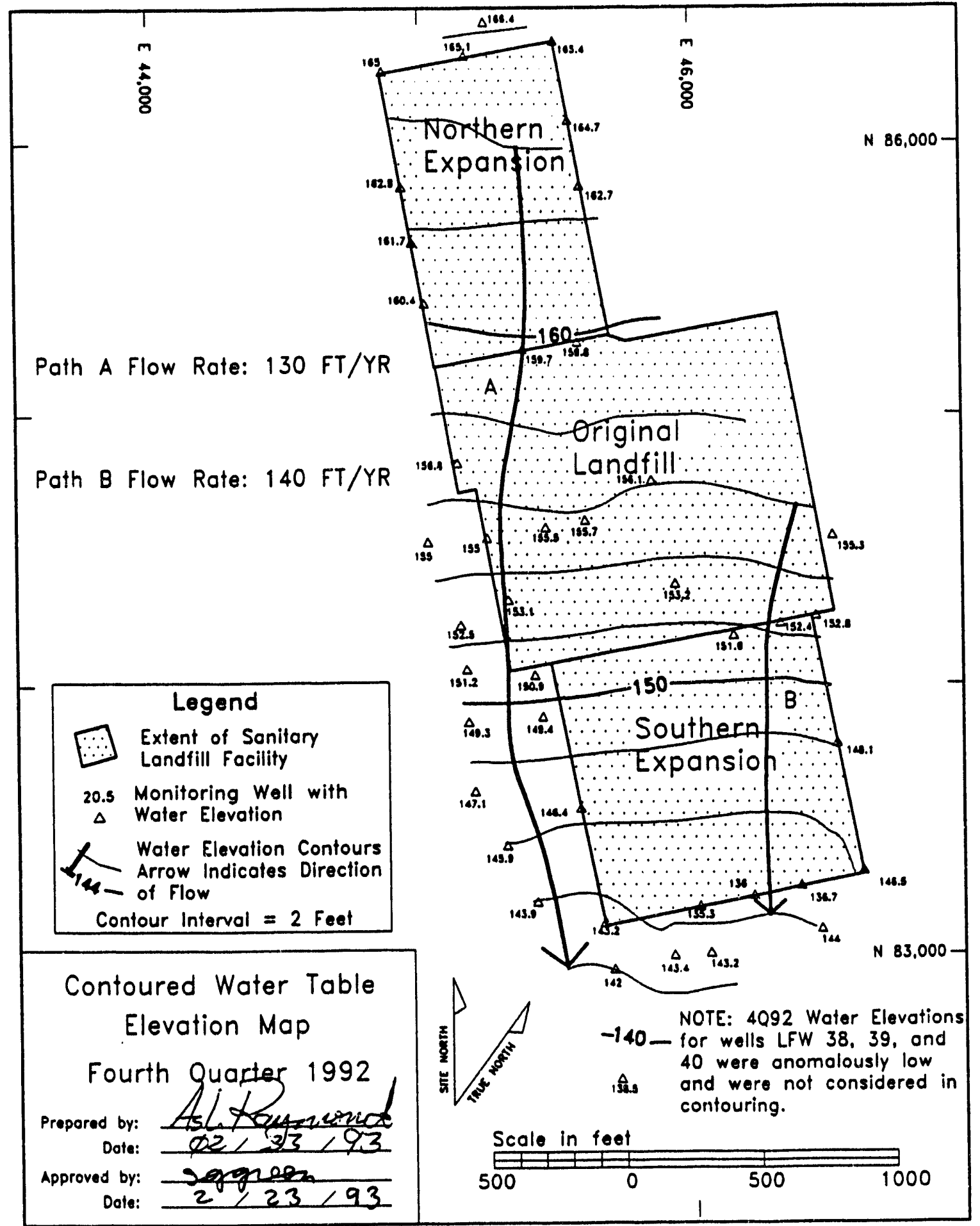

Figure 13. Water-Elevation Contour Map of Steed Pond Aquifer (Water Table) at the Sanitary Landfill, Fourth Quarter 1992 


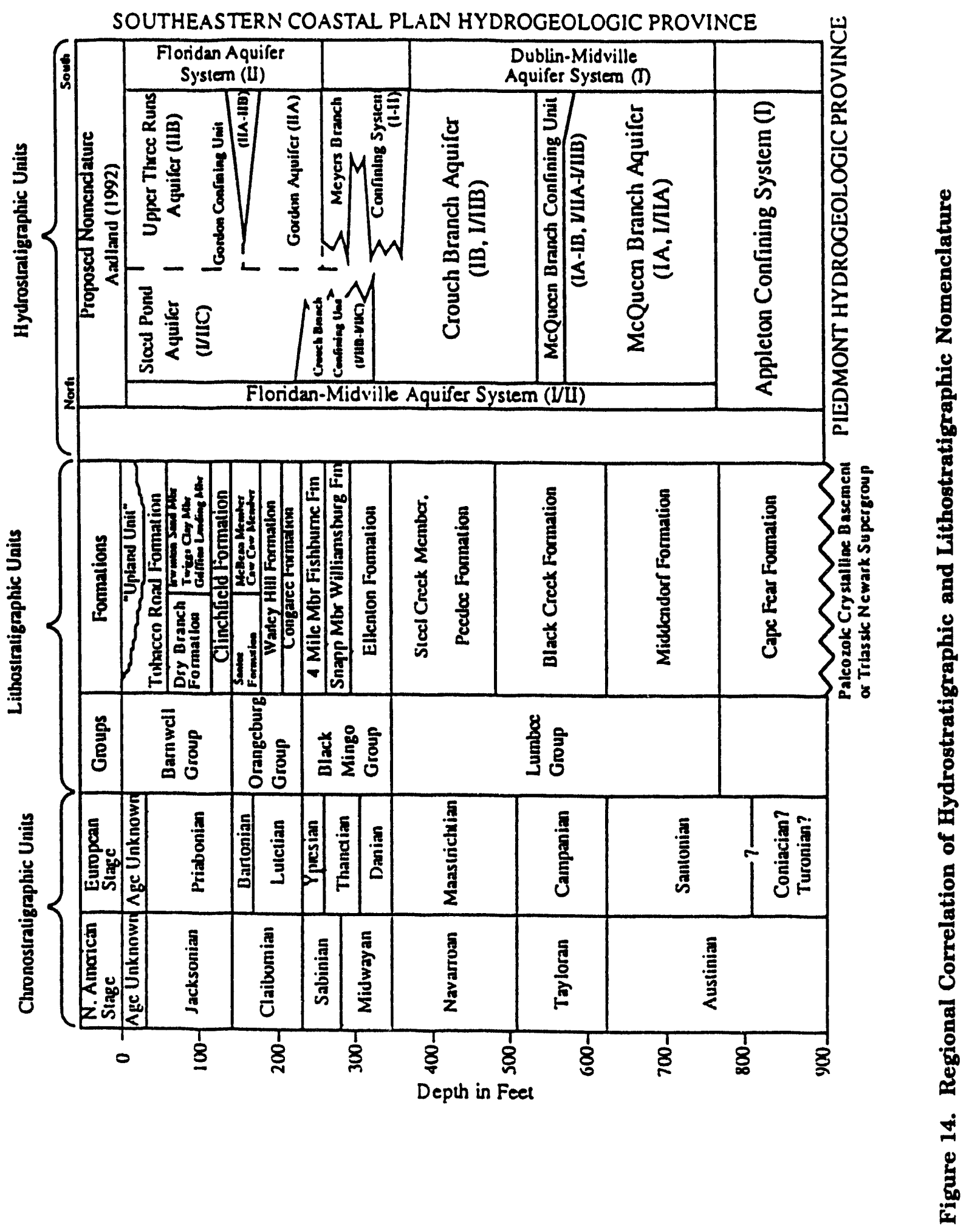




\section{References Cited}

Aadland, R. K., P. A. Thayer, and A. D. Smits, 1992. Hydrogeologic Atlas of WestCentral South Carolina (U), WSRC-RP-92-632. Westinghouse Savannah River Company, Savannah River Site, Aiken, SC.

Lewis, S. E., and R. K. Aadland, 1992. Hydrogeologic Setting of A/M Area: Framework for Groundwater Transport (U), WSRC-RC-92-355. Westinghouse Savannah River Company, Savannah River Site, Aiken, SC. 
WSRC-TR-93-068

Appendix D - Groundwater Monitoring Results Tables 


\section{Key to Reading the Tables}

The following abbreviations may appear in the tabular data:

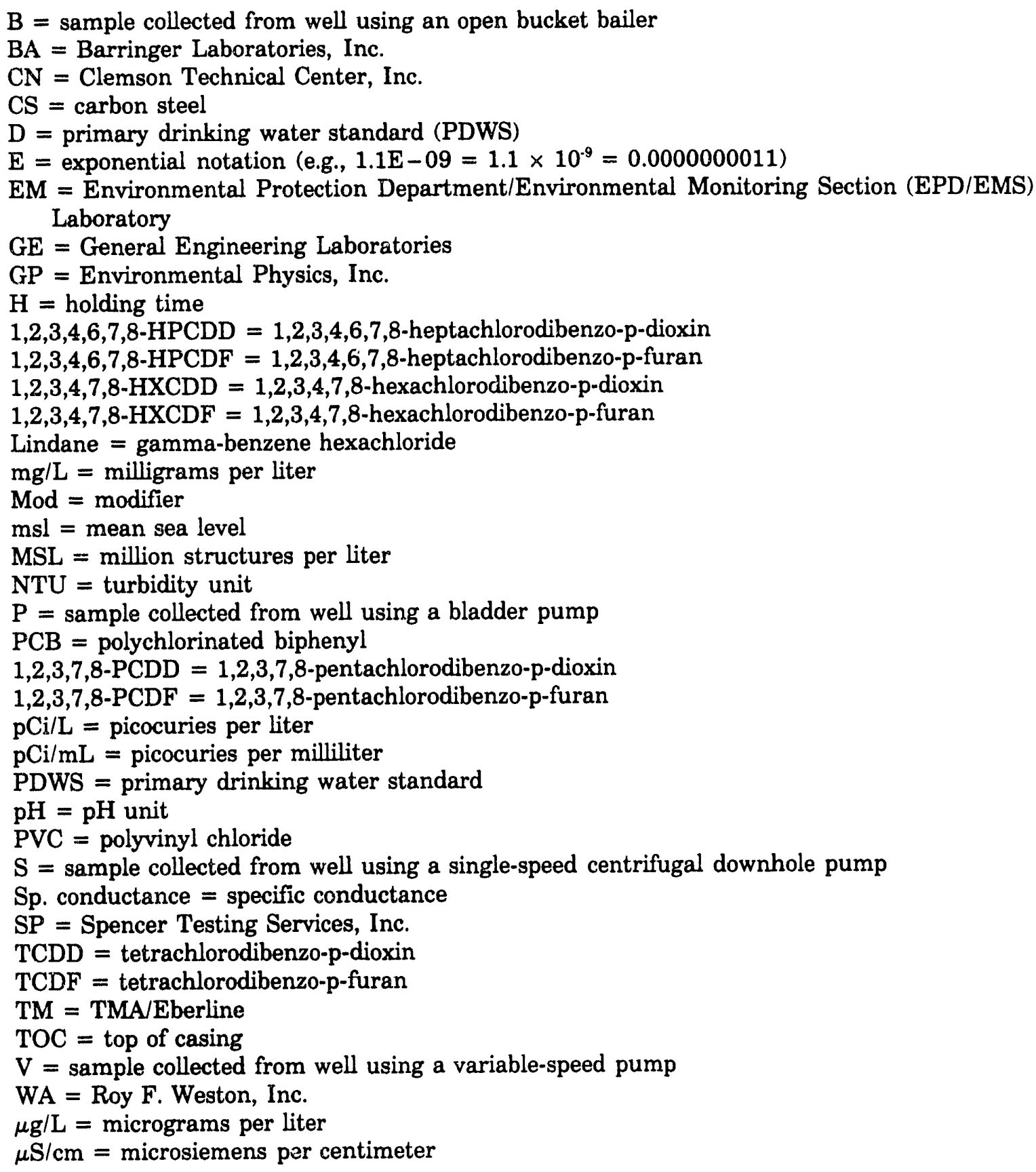




\section{Holding Times}

Standard analytical methods include a limit, called holding time, on the maximum elapsed time between sample collection and extraction or analysis by the laboratory. In the data tables, a large dot $(\bullet)$ in the $\mathrm{H}$ (holding time) column indicates that holding time was exceeded. Analyses performed beyond holding time may not yield valid results.

The South Carolina Department of Health and Environmental Control allows only 15 minutes to elapse between sampling and analysis for $\mathrm{pH}$. Thus, only field $\mathrm{pH}$ measurements can meet the holding time criterion; laboratory $\mathrm{pH}$ analyses will always exceed it.

Laboratory-initiated procedures for reducing the number of other analyses performed out of holding time include subcontracting analyses when difficulties with equipment, personnel, or work load would prevent timely analyses. Beginning fourth quarter 1991, SRS reduced the compensation to laboratories for analyses performed out of holding time.

\section{Data Qualification}

The contract laboratories continually assess their own accuracy and precision according to U.S. Environmental Protection Agency (EPA) guidelines. They submit sample- or batchspecific quality assurance/quality control information either at the same time as analytical results or in a quarterly summary. Properly defined and used result modifiers (also referred to as qualifiers) can be a key component in assessing data useability. Result modifiers designed by EPD/EMS and provided to the primary laboratories are defined below. These modifiers appear in the data tables under the column "Mod."

Result modifier

(Blank)

$A^{a}$

$\jmath^{\mathbf{a}}$

$L^{a}$

$M^{a}$

$R^{a}$

$T^{\mathbf{a}}$

$\mathrm{V}^{\mathbf{a}}$

1

2
Definition

Data are not qualified. Number should be interpreted exactly as reported.

Value reported is the mean of two or more determinations.

Value is estimated because quantitation in the sample or in associated quality control samples did not meet specifications.

Value is off-scale high. The actual value is not known but is known to be greater than the value shown.

Presence of the analyte is verified but not quantified.

Result was rejected because performance requirements in the sample analysis or associated quality control analyses were not met.

Analyte was not detected; if present, it was below the criteria for detection.

Analyte was detected in the associated method blank.

Result may be an underestimation of the true value due to analytical bias.

Result may be an overestimation of the true value due to analytical bias. 
Result modifier

3

6

a These codes are based on the STORET codes from EPA.
Definition

The associated result may be of poor precision (high variability) due to analytical bias.

The associated result is from a reanalysis performed out of holding time due to problems with an earlier analysis. 
Table 1. Constituents Exceeding the Final Primary Drinking Water Standards

\begin{tabular}{|c|c|c|c|c|c|c|c|}
\hline Well & Constituent & Unit & 1092 & $\underline{2092}$ & 3092 & 4092 & Mod \\
\hline LFW 6 & Chloroethene (Vinyl chloride) & $\mu g / L$ & - & - & - & 3.0 & $J$ \\
\hline LFW 8 & $\begin{array}{l}\text { Chloroethene (Vinyl chloride) } \\
\text { Chloroethene (Vinyl chloride) } \\
\text { Chloroethene (Vinyl chloride) } \\
\text { Chloroethene (Vinyl chloride) } \\
\text { Chloroethene (Vinyl chloride) }\end{array}$ & $\begin{array}{l}\mu \mathrm{g} / \mathrm{L} \\
\mu \mathrm{g} / \mathrm{L} \\
\mu \mathrm{g} / \mathrm{L} \\
\mu \mathrm{g} / \mathrm{L} \\
\mu \mathrm{g} / \mathrm{L}\end{array}$ & $\begin{array}{l}157 \\
\text { NA } \\
\text { NA } \\
\text { NA } \\
\text { NA }\end{array}$ & $\begin{array}{l}128^{c} \\
113^{c} \\
106^{c} \\
97^{c} \\
123^{c}\end{array}$ & $\begin{array}{l}\text { NA } \\
\text { NA } \\
\text { NA } \\
\text { NA }\end{array}$ & $\begin{array}{l}121 \\
\text { NA } \\
\text { NA } \\
\text { NA } \\
\text { NA }\end{array}$ & \\
\hline LFW 10A & $\begin{array}{l}\text { Benzene } \\
\text { Tetrachloroethylene } \\
\text { Trichloroethylene } \\
\text { Tritium } \\
\text { Tritium }\end{array}$ & $\begin{array}{l}\mu g / L \\
\mu g / L \\
\mu g / L \\
p C i / m L \\
p C i / m L\end{array}$ & $\begin{array}{l}9.5 \\
37 \\
49 \\
1.0 E+02^{d} \\
1.0 E+02^{d}\end{array}$ & $\begin{array}{l}8.6 \\
36 \\
48 \\
1.6 E+02 \\
\text { NA }\end{array}$ & $\begin{array}{l}- \\
125 \\
45 \\
1.4 E+02 \\
1.4 E+02\end{array}$ & $\begin{array}{l}9.9 \\
27 \\
51 \\
2.1 E+02 \\
\text { NA }\end{array}$ & $\begin{array}{l}\sqrt{ } 2 \\
\mathrm{~J} 2 \\
\mathrm{~J} 2\end{array}$ \\
\hline LFW 16 & $\begin{array}{l}\text { Tetrachloroethylene } \\
\text { Trichloroethylene } \\
\text { Tritium } \\
\text { Tritium }\end{array}$ & $\begin{array}{l}\mu g / L \\
\mu g / L \\
\mathrm{pCi} / \mathrm{mL} \\
\mathrm{pCi} / \mathrm{mL}\end{array}$ & $\begin{array}{l}- \\
9.6 \\
9.4 E+01^{d} \\
9.4 E+01^{d}\end{array}$ & $\begin{array}{l}- \\
7.8 \\
2.1 E+01 \\
\text { NA }\end{array}$ & $\begin{array}{l}15 \\
14 \\
- \\
\text { NA }\end{array}$ & $\begin{array}{l}5.0 \\
8.3 \\
- \\
\text { NA }\end{array}$ & $J$ \\
\hline LFW 17 & 1.2-Dichloroethane & $\mu g / L$ & 5.8 & - & - & - & \\
\hline LFW 18 & Chloroethene (Vinyl chloride) & $\mu_{g} / L$ & - & - & - & 3.2 & $J$ \\
\hline LFW 21 & $\begin{array}{l}\text { Benzene } \\
\text { Cadmium } \\
\text { 1,2-Dichloropropane } \\
\text { Lindane } \\
\text { Tetrachloroethylene } \\
\text { Trichloroethylene }\end{array}$ & $\begin{array}{l}\mu g / L \\
\mu g / L \\
\mu g / L \\
\mu g / L \\
\mu g / L \\
\mu g / L\end{array}$ & $\begin{array}{l}- \\
22 \\
- \\
- \\
- \\
20\end{array}$ & $\begin{array}{l}21 \\
- \\
7.7 \\
- \\
- \\
27\end{array}$ & $\begin{array}{l}16 \\
- \\
6.4 \\
0.48 \\
10 \\
24\end{array}$ & $\begin{array}{l}17 \\
- \\
- \\
- \\
- \\
17\end{array}$ & \\
\hline LFW 22 & $\begin{array}{l}\text { Cadmium } \\
\text { Tetrachloroethylene } \\
\text { Trichloroethylene }\end{array}$ & $\begin{array}{l}\mu g / L \\
\mu g / L \\
\mu g / L\end{array}$ & $\begin{array}{l}31 \\
- \\
26\end{array}$ & $\begin{array}{l}- \\
- \\
25\end{array}$ & $\begin{array}{l}- \\
9.6 \\
27\end{array}$ & $\begin{array}{l}- \\
- \\
32\end{array}$ & \\
\hline LFW 36 & $\begin{array}{l}\text { Benzene } \\
1,2 \text {-Dichloroethane }\end{array}$ & $\begin{array}{l}\mu \mathrm{g} / \mathrm{L} \\
\mu \mathrm{g} / \mathrm{L}\end{array}$ & - & $\begin{array}{l}5.8 \\
6.8\end{array}$ & - & - & \\
\hline LFW 37 & $\begin{array}{l}\text { Benzene } \\
\text { Benzene } \\
\text { 1,2-Dichloroethane } \\
\text { 1,2-Dichloroethane } \\
\text { 1,2-Dichloroethane } \\
\text { Trichloroethylene } \\
\text { Trichloroethylene }\end{array}$ & $\begin{array}{l}\mu \mathrm{g} / \mathrm{L} \\
\mu \mathrm{g} / \mathrm{L} \\
\mu \mathrm{g} / \mathrm{L} \\
\mu \mathrm{g} / \mathrm{L} \\
\mu \mathrm{g} / \mathrm{L} \\
\mu \mathrm{g} / \mathrm{L} \\
\mu \mathrm{g} / \mathrm{L}\end{array}$ & $\begin{array}{l}- \\
\text { NA } \\
7.0 \\
\text { NA } \\
\text { NA } \\
7.5 \\
\text { NA }\end{array}$ & $\begin{array}{l}5.6^{\mathrm{e}} \\
5.2^{\mathrm{e}} \\
7.8^{f} \\
7.1^{f} \\
6.6^{f} \\
7.2^{\mathrm{g}} \\
7.1^{\mathrm{g}}\end{array}$ & $\begin{array}{l}- \\
\text { NA } \\
- \\
\text { NA } \\
\text { NA } \\
9.3 \\
\text { NA }\end{array}$ & $\begin{array}{l}6.2 \\
\text { NA } \\
5.1 \\
\text { NA } \\
\text { NA } \\
7.5 \\
\text { NA }\end{array}$ & \\
\hline LFW 38 & $\begin{array}{l}\text { Benzene } \\
\text { 1,2-Dichloroethane } \\
\text { Trichloroethylene } \\
\text { Tritium }\end{array}$ & $\begin{array}{l}\mu g / L \\
\mu g / L \\
\mu g / L \\
p C i / m L\end{array}$ & $\begin{array}{l}- \\
- \\
17 \\
-\end{array}$ & $\begin{array}{l}- \\
- \\
18 \\
3.8 E+01\end{array}$ & $\begin{array}{l}5.1 \\
5.5 \\
21 \\
3.9 E+01\end{array}$ & $\begin{array}{l}5.3 \\
- \\
21 \\
2.1 E+01\end{array}$ & \\
\hline LFW 39 & $\begin{array}{l}\text { 1,1-Dichloroethylene } \\
\text { Tetrachloroethylene } \\
1,1,1 \text {-Trichloroethane } \\
\text { Trichloroethylene }\end{array}$ & $\begin{array}{l}\mu \mathrm{g} / \mathrm{L} \\
\mu \mathrm{g} / \mathrm{L} \\
\mu \mathrm{g} / \mathrm{L} \\
\mu \mathrm{g} / \mathrm{L}\end{array}$ & $\begin{array}{l}13 \\
7.0 \\
207 \\
11\end{array}$ & $\begin{array}{l}8.5 \\
- \\
- \\
9.8\end{array}$ & $\begin{array}{l}9.0 \\
- \\
- \\
14\end{array}$ & $\begin{array}{l}8.6 \\
5.6 \\
- \\
15\end{array}$ & \\
\hline
\end{tabular}


Steed Pond Aquifer (Water Table)

\begin{tabular}{|c|c|c|c|c|c|c|}
\hline Well & Constituent & $\underline{\text { Unit }}$ & 1092 & $\underline{2092}$ & $\underline{3092}$ & $\underline{4092}$ \\
\hline \multirow[t]{6}{*}{ LFW 40} & Tetrachloroethylene & $\mu g / L$ & - & - & 6.5 & - \\
\hline & Trichloroethylene & $\mu \mathrm{g} / \mathrm{L}$ & $7.1^{\circ}$ & - & 14 & 12 \\
\hline & Trichloroethylene & $\mu \mathrm{g} / \mathrm{L}$ & $5.3^{\circ}$ & NA & NA & NA \\
\hline & Trichloroethylene & $\mu g / L$ & $5.5^{\mathrm{g}}$ & NA & NA & NA \\
\hline & Trichloroethylene & $\mu \mathrm{g} / \mathrm{L}$ & $5.1^{0}$ & NA & NA & NA \\
\hline & Trichloroethylene & $\mu g / L$ & $6.5^{\mathfrak{g}}$ & NA & NA & NA \\
\hline LFW 42 & Tetrachloroethylene & $\mu g / L$ & - & - & - & 5.2 \\
\hline LFW 47C & Chloroethene (Vinyl chloride) & $\mu \mathrm{g} / \mathrm{L}$ & - & 7.7 & - & - \\
\hline LFW 47D & Lead & $\mu g / L$ & - & - & 21 & - \\
\hline LFW 48C & Mercury & $\mu \mathrm{g} / \mathrm{L}$ & - & - & - & 2.9 \\
\hline LFW 48D & Chloroethene (Vinyl chloride) & $\mu g / L$ & 143 & 104 & 85 & 126 \\
\hline \multirow[t]{2}{*}{ LFW 58D } & 1,2-Dichloroethane & $\mu g / L$ & - & - & - & 6.4 \\
\hline & Trichloroethylene & $\mu \mathrm{g} / \mathrm{L}$ & 17 & 15 & 16 & 12 \\
\hline \multirow[t]{2}{*}{ LFW 59C } & 1,2-Dichloroethane & $\mu \mathrm{g} / \mathrm{L}$ & - & - & - & 5.5 \\
\hline & Trichloroethylene & $\mu g / L$ & 10 & - & 11 & 11 \\
\hline \multirow[t]{3}{*}{ LFW 59D } & Trichloroethylene & $\mu \mathrm{g} / \mathrm{L}$ & 11 & 14 & $22^{9}$ & 13 \\
\hline & Trichloroethylene & $\mu g / L$ & NA & NA & $18^{0}$ & NA \\
\hline & Trichloroethylene & $\mu g / L$ & NA & NA & $20^{\circ}$ & NA \\
\hline \multirow[t]{2}{*}{ LFW 61C } & 1,1-Dichloroethylene & $\mu \mathrm{g} / \mathrm{L}$ & 24 & - & - & - \\
\hline & Trichloroethylene & $\mu \mathrm{g} / \mathrm{L}$ & 9.0 & 14 & 5.0 & 8.1 \\
\hline \multirow[t]{3}{*}{ LFW 61D } & Tetrachloroethylene & $\mu g / L$ & 5.3 & 8.1 & - & - \\
\hline & Trichloroethylene & $\mu g / L$ & 7.6 & 5.3 & 8.1 & 9.3 \\
\hline & Tritium & $\mathrm{pCi} / \mathrm{mL}$ & - & - & $8.3 E+01$ & $4.2 E+01$ \\
\hline LFW $62 \mathrm{C}$ & Trichloroethylene & $\mu g / L$ & 5.5 & 7.5 & 7.5 & 11 \\
\hline \multirow[t]{2}{*}{ LFW 620} & Tetrachloroethylene & $\mu \mathrm{g} / \mathrm{L}$ & - & - & - & 7.6 \\
\hline & Trichloroethylene & $\mu g / L$ & 9.9 & 11 & 11 & 14 \\
\hline
\end{tabular}

Note: The modifier column applies to 4092 data.

a $-=$ analyzed but not above final PDWS.

b $N A=$ not analyzed.

c Duplicate/replicate samples of chloroethene (vinyl chloride).

d Duplicate/replicate samples of tritium.

- Duplicate/replicate samples of benzene.

f Duplicate/replicate samples of 1,2-dichloroethane.

g Duplicate/replicate samples of trichloroethylene. 
Table 2. Constituents Exceeding Half the Final Primary Drinking Water Standards or Other Flag 1 or Flag 2 Criteria

Steed Pond Aquifer (Water Table)

\begin{tabular}{|c|c|c|c|c|c|c|}
\hline Well & Constituent & Unit & $\underline{3092}$ & 4092 & Mod & Flag \\
\hline LFW 6 & $\begin{array}{l}\text { Chloroethane } \\
\text { 1,1-Dichloroethane } \\
\text { 1,1-Dichloroethane } \\
\text { 1,1-Dichloroethane } \\
\text { 1,1-Dichloroethane } \\
\text { 1,1-Dichloroethane } \\
\text { Gross alpha } \\
\text { Iron } \\
\text { Iron } \\
\text { Iron } \\
\text { Iron } \\
\text { Manganese } \\
\text { Manganese } \\
\text { Manganese } \\
\text { Manganese } \\
\text { Tetrachloroethylene } \\
\text { Total organic halogens } \\
\text { Total organic halogens } \\
\text { Total organic halogens } \\
\text { Total organic halogens } \\
\text { Trichloroethylene }\end{array}$ & $\begin{array}{l}\mu \mathrm{g} / \mathrm{L} \\
\mu \mathrm{g} / \mathrm{L} \\
\mu \mathrm{g} / \mathrm{L} \\
\mu \mathrm{g} / \mathrm{L} \\
\mu \mathrm{g} / \mathrm{L} \\
\mu \mathrm{g} / \mathrm{L} \\
\mathrm{pCi} / \mathrm{L} \\
\mu \mathrm{g} / \mathrm{L} \\
\mu \mathrm{g} / \mathrm{L} \\
\mu \mathrm{g} / \mathrm{L} \\
\mu \mathrm{g} / \mathrm{L} \\
\mu \mathrm{g} / \mathrm{L} \\
\mu \mathrm{g} / \mathrm{L} \\
\mu \mathrm{g} / \mathrm{L} \\
\mu \mathrm{g} / \mathrm{L} \\
\mu \mathrm{g} / \mathrm{L} \\
\mu \mathrm{g} / \mathrm{L} \\
\mu \mathrm{g} / \mathrm{L} \\
\mu \mathrm{g} / \mathrm{L} \\
\mu \mathrm{g} / \mathrm{L} \\
\mu \mathrm{g} / \mathrm{L}\end{array}$ & $\begin{array}{l}<10 \\
7.9^{\mathrm{a}} \\
9.9^{\mathrm{a}} \\
7.5^{\mathrm{a}} \\
12^{\mathrm{a}} \\
7.1^{\mathrm{a}} \\
8.5 \mathrm{E}+00 \\
11,300^{\mathrm{d}} \\
9,660^{\mathrm{d}} \\
10,300^{\mathrm{d}} \\
13,100^{\mathrm{d}} \\
30^{\mathrm{e}} \\
33^{\mathrm{e}} \\
34^{\mathrm{e}} \\
38^{\mathrm{e}} \\
3.5^{\mathrm{f}} \\
58^{\mathrm{f}} \\
65^{\mathrm{f}} \\
118^{\mathrm{f}} \\
63^{\mathrm{f}} \\
-\end{array}$ & $\begin{array}{l}12 \\
20 \\
\text { NAb } \\
\text { NA } \\
\text { NA } \\
\text { NA } \\
-c \\
\text { NA } \\
\text { NA } \\
\text { NA } \\
- \\
118 \\
\text { NA } \\
\text { NA } \\
\text { NA } \\
2.6 \\
152 \\
\text { NA } \\
\text { NA } \\
\text { NA } \\
2.7\end{array}$ & J & $\begin{array}{l}2 \\
2 \\
\text { NA } \\
\text { NA } \\
\text { NA } \\
\text { NA } \\
- \\
\text { NA } \\
\text { NA } \\
\text { NA } \\
- \\
2 \\
\text { NA } \\
\text { NA } \\
\text { NA } \\
1 \\
2 \\
\text { NA } \\
\text { NA } \\
\text { NA } \\
1\end{array}$ \\
\hline LFW 8 & $\begin{array}{l}\text { Benzene } \\
\text { Chlorobenzene } \\
\text { Chloroethane } \\
\text { 1,1-Dichloroethane } \\
\text { Gross alpha } \\
\text { Iron } \\
\text { Radium-228 } \\
\text { Total organic carbon } \\
\text { Total organic halogens }\end{array}$ & $\begin{array}{l}\mu \mathrm{g} / \mathrm{L} \\
\mu \mathrm{g} / \mathrm{L} \\
\mu \mathrm{g} / \mathrm{L} \\
\mu \mathrm{g} / \mathrm{L} \\
\mathrm{pCi} / \mathrm{L} \\
\mu \mathrm{g} / \mathrm{L} \\
\mathrm{pCi} / \mathrm{L} \\
\mu \mathrm{g} / \mathrm{L} \\
\mu \mathrm{g} / \mathrm{L}\end{array}$ & $\begin{array}{l}3.3 \\
17 \\
<10 \\
32 \\
6.6 E+00 \\
47,800 \\
5.3 E+00 \\
4,740 \\
245\end{array}$ & $\begin{array}{l}4.0 \\
31 \\
13 \\
32 \\
1.1 E+01 \\
- \\
- \\
6,130 \\
219\end{array}$ & $\mathrm{~J}$ & $\begin{array}{l}1 \\
2 \\
2 \\
2 \\
1 \\
- \\
- \\
1 \\
2\end{array}$ \\
\hline LFW 10A & $\begin{array}{l}\text { Aluminum } \\
\text { Benzene } \\
\text { Chloroethane } \\
\text { 1,1-Dichloroethane } \\
\text { 1,1-Dichloroethylene } \\
\text { Gross alpha } \\
\text { Iron } \\
\text { Phenols } \\
\text { Specific conductance } \\
\text { Specific conductance } \\
\text { Total organic carbon } \\
\text { Total organic halogens }\end{array}$ & $\begin{array}{l}\mu g / L \\
\mu g / L \\
\mu g / L \\
\mu g / L \\
\mu g / L \\
p C i / L \\
\mu g / L \\
\mu g / L \\
\mu S / c m \\
\mu S / c m \\
\mu g / L \\
\mu g / L\end{array}$ & $\begin{array}{l}<15 \\
4.1 \\
<10 \\
153 \\
5.2 \\
7.3 \mathrm{E}+00 \\
90,000 \\
27 \\
331^{9} \\
331^{9} \\
13,000 \\
376\end{array}$ & $\begin{array}{l}71 \\
D^{i} \\
9.6 \\
148 \\
5.4 \\
8.2 \mathrm{E}+00 \\
- \\
143 \\
502 \\
\mathrm{NA} \\
94,600 \\
1,420\end{array}$ & $\begin{array}{l}\mathrm{J} 2 \\
\mathrm{~J} 2\end{array}$ & $\begin{array}{l}2 \\
D \\
1 \\
2 \\
1 \\
1 \\
- \\
2 \\
2 \\
N A \\
2 \\
2\end{array}$ \\
\hline LFW 16 & $\begin{array}{l}\text { Aluminum } \\
1,1 \text {-Dichloroethane } \\
\text { Total organic halogens }\end{array}$ & $\begin{array}{l}\mu g / L \\
\mu g / L \\
\mu g / L\end{array}$ & $\begin{array}{l}<15 \\
32 \\
<50\end{array}$ & $\begin{array}{l}40 \\
32 \\
116\end{array}$ & J3 & $\begin{array}{l}1 \\
2 \\
2\end{array}$ \\
\hline
\end{tabular}


Steed Pond Aquifer (Water Table)

\begin{tabular}{|c|c|c|c|c|c|c|}
\hline \multirow[t]{2}{*}{ Well } & Constituent & $\underline{\text { Unit }}$ & 3092 & 4092 & Mod & Flag \\
\hline & Tritium & $\mathrm{pCi} / \mathrm{mL}$ & $1.4 E+01$ & $1.3 E+01$ & & 1 \\
\hline \multirow[t]{9}{*}{ LFW 17} & Benzene & $\mu g / L$ & $<5.0$ & 4.1 & $\mathrm{~J}$ & 1 \\
\hline & Chlorobenzene & $\mu g / L$ & 41 & 84 & & 2 \\
\hline & Chloroethane & $\mu \mathrm{g} / \mathrm{L}$ & 7.2 & 11 & $\mathrm{~J}$ & 2 \\
\hline & Chloroethene (Vinyl chloride) & $\mu g / L$ & $<10$ & 1.9 & $\mathrm{~J}$ & 1 \\
\hline & 1,1-Dichloroethane & $\mu g / L$ & 25 & 25 & & 2 \\
\hline & 1,2-Dichloroethane & $\mu \mathrm{g} / \mathrm{L}$ & 4.5 & 3.9 & $\mathrm{~J}$ & 1 \\
\hline & Iron & $\mu \mathrm{g} / \mathrm{L}$ & 21,700 & - & & - \\
\hline & Specific conductance & $\mu \mathrm{S} / \mathrm{cm}$ & 250 & 316 & $J$ & 1 \\
\hline & Total organic halogens & $\mu g / L$ & 367 & 245 & & 2 \\
\hline \multirow[t]{8}{*}{ LFW 18} & Antimony & $\mu g / L$ & $<2.6$ & 4.0 & J3 & 1 \\
\hline & Arsenic & $\mu \mathrm{g} / \mathrm{L}$ & 27 & 35 & & 1 \\
\hline & Chlorobenzene & $\mu \mathrm{g} / \mathrm{L}$ & 2.7 & 5.2 & & 1 \\
\hline & Chloroethane & $\mu \mathrm{g} / \mathrm{L}$ & $<10$ & 17 & & 2 \\
\hline & 1,1-Dichloroethane & $\mu \mathrm{g} / \mathrm{L}$ & 15 & 15 & & 2 \\
\hline & Iron & $\mu \mathrm{g} / \mathrm{L}$ & 58,200 & - & & - \\
\hline & Total organic halogens & $\mu \mathrm{g} / \mathrm{L}$ & 260 & 298 & & 2 \\
\hline & Tritium & $\mathrm{pCi} / \mathrm{mL}$ & $1.4 E+01$ & - & & - \\
\hline LFW 19 & Iron & $\mu \mathrm{g} / \mathrm{L}$ & 172 & - & & - \\
\hline \multirow[t]{14}{*}{ LFW 21} & Aluminum & $\mu \mathrm{g} / \mathrm{L}$ & $<15$ & 50 & J3 & 1 \\
\hline & Antimony & $\mu \mathrm{g} / \mathrm{L}$ & 3.3 & - & & - \\
\hline & Arsenic & $\mu \mathrm{g} / \mathrm{L}$ & 27 & 34 & & 1 \\
\hline & 1,1-Dichloroethane & $\mu g / L$ & 361 & 184 & & 2 \\
\hline & 1,2-Dichloroethane & $\mu \mathrm{g} / \mathrm{L}$ & 4.6 & 3.9 & $J$ & 1 \\
\hline & 1,1-Dichloroethylene & $\mu g / L$ & 4.3 & - & & - \\
\hline & Iron & $\mu g / L$ & $1.8 E+08$ & - & & - \\
\hline & Manganese & $\mu g / L$ & 308 & 231 & & 2 \\
\hline & Phenols & $\mu \mathrm{g} / \mathrm{L}$ & 630 & 646 & & 2 \\
\hline & Specific conductance & $\mu \mathrm{S} / \mathrm{cm}$ & 1,250 & 1,120 & $\mathrm{~J}$ & 2 \\
\hline & Tetrachloroethylene & $\mu \mathrm{g} / \mathrm{L}$ & $D$ & 3.1 & J & 1 \\
\hline & Total organic carbon & $\mu \mathrm{g} / \mathrm{L}$ & 516,000 & 378,000 & & 2 \\
\hline & Total organic halogens & $\mu g / L$ & 504 & 1,900 & & 2 \\
\hline & Tritium & $\mathrm{pCi} / \mathrm{mL}$ & $1.7 E+01$ & $1.7 E+01$ & & 1 \\
\hline \multirow[t]{10}{*}{ LFW 22} & Benzene & $\mu g / L$ & 3.3 & 3.9 & $\mathrm{~J}$ & 1 \\
\hline & 1,1-Dichloroethane & $\mu \mathrm{g} / \mathrm{L}$ & 98 & 100 & & 2 \\
\hline & Iron & $\mu \mathrm{g} / \mathrm{L}$ & $1.1 E+08$ & - & & - \\
\hline & Manganese & $\mu \mathrm{g} / \mathrm{L}$ & 72 & 112 & & 2 \\
\hline & Phenols & $\mu g / L$ & 249 & 242 & & 2 \\
\hline & Specific conductance & $\mu \mathrm{S} / \mathrm{cm}$ & 598 & 661 & $J$ & 2 \\
\hline & retrachloroethylene & $\mu \mathrm{g} / \mathrm{L}$ & 9.6 & 4.8 & & 1 \\
\hline & Total organic carbon & $\mu \mathrm{g} / \mathrm{L}$ & 100,000 & 196,000 & & 2 \\
\hline & Total organic halogens & $\mu \mathrm{g} / \mathrm{L}$ & 593 & 1,250 & & 2 \\
\hline & Trichlorofluoromethane & $\mu \mathrm{g} / \mathrm{L}$ & $<5.0$ & 90 & & 2 \\
\hline
\end{tabular}


Steed Pond Aquifer (Water Table)

\begin{tabular}{|c|c|c|c|c|c|c|}
\hline Well & Constituent & Unit & $\underline{3092}$ & 40.92 & Mod & Flag \\
\hline LFW 23 & $\begin{array}{l}\text { Aluminum } \\
1,1 \text {-Dichloroethane } \\
\text { Lead } \\
\text { Total organic halogens }\end{array}$ & $\begin{array}{l}\mu g / L \\
\mu g / L \\
\mu g / L \\
\mu g / L\end{array}$ & $\begin{array}{l}<15 \\
<5.0 \\
7.1 \\
<50\end{array}$ & $\begin{array}{l}43 \\
6.8 \\
9.1 \\
134\end{array}$ & & $\begin{array}{l}1 \\
1 \\
1 \\
2\end{array}$ \\
\hline LFW 24 & Total organic halogens & $\mu g / L$ & 60 & - & & - \\
\hline LFW 25 & $\begin{array}{l}\text { Antimony } \\
\text { Radium-228 }\end{array}$ & $\begin{array}{l}\mu g / L \\
\mathrm{pCi} / L\end{array}$ & $\begin{array}{l}7.3 \\
4.5 E+00\end{array}$ & - & & - \\
\hline LFW 26 & $\begin{array}{l}\text { Antimony } \\
\text { Gross alpha } \\
\text { Nonvolatile beta }\end{array}$ & $\begin{array}{l}\mu g / L \\
p C i / L \\
p C i / L\end{array}$ & $\begin{array}{l}5.0 \\
9.6 \mathrm{E}+00 \\
2.6 \mathrm{E}+01\end{array}$ & $\begin{array}{l}- \\
- \\
-\end{array}$ & & $\begin{array}{l}- \\
- \\
-\end{array}$ \\
\hline LFW 28 & $\begin{array}{l}\text { Aluminum } \\
\text { Antimony }\end{array}$ & $\begin{array}{l}\mu \mathrm{g} / \mathrm{L} \\
\mu \mathrm{g} / \mathrm{L}\end{array}$ & $\begin{array}{l}<15 \\
4.2\end{array}$ & $\begin{array}{l}74 \\
-\end{array}$ & & $\begin{array}{l}2 \\
-\end{array}$ \\
\hline LFW 29 & Aluminum & $\mu g / L$ & $<15$ & 26 & $J 3$ & 1 \\
\hline LFW 30 & $\begin{array}{l}\text { Aluminum } \\
\text { Antimony }\end{array}$ & $\begin{array}{l}\mu g / L \\
\mu g / L\end{array}$ & $\begin{array}{l}26 \\
12\end{array}$ & $\begin{array}{l}- \\
-\end{array}$ & & $\begin{array}{l}- \\
-\end{array}$ \\
\hline LFW 31 & Antimony & $\mu g / L$ & 4.7 & - & & - \\
\hline LFW 32 & Antimony & $\mu g / L$ & 11 & - & & - \\
\hline LFW 33 & $\begin{array}{l}\text { Antimony } \\
\text { Radium-228 }\end{array}$ & $\begin{array}{l}\mu \mathrm{g} / \mathrm{L} \\
\mathrm{pCi} / \mathrm{L}\end{array}$ & $\begin{array}{l}8.4 \\
4.6 E+00\end{array}$ & - & & - \\
\hline LFW 34 & Antimony & $\mu g / L$ & 5.2 & - & & - \\
\hline LFW 35 & Total organic carbon & $\mu g / L$ & 5,210 & - & & - \\
\hline LFW 36 & $\begin{array}{l}\text { Benzene } \\
\text { Chlorobenzene } \\
\text { 1,1-Dichloroethane } \\
\text { 1,2-Dichloroethane } \\
\text { Iron } \\
\text { Total organic carbon } \\
\text { Total organic halogens } \\
\text { Trichlorofluoromethane } \\
\text { Tritium }\end{array}$ & $\begin{array}{l}\mu g / L \\
\mu g / L \\
\mu g / L \\
\mu g / L \\
\mu g / L \\
\mu g / L \\
\mu g / L \\
\mu g / L \\
\mathrm{pCi} / \mathrm{mL}\end{array}$ & $\begin{array}{l}3.3 \\
6.2 \\
45 \\
2.9 \\
10,800 \\
12,900 \\
197 \\
6.1 \\
1.2 E+01\end{array}$ & $\begin{array}{l}3.6 \\
10 \\
46 \\
- \\
- \\
- \\
402 \\
- \\
1.1 E+01\end{array}$ & J & $\begin{array}{l}1 \\
2 \\
2 \\
- \\
- \\
- \\
2 \\
- \\
1\end{array}$ \\
\hline LFW 37 & $\begin{array}{l}\text { Antimony } \\
\text { Benzene } \\
\text { 1,1-Dichloroethane } \\
\text { 1,2-Dichloroethane } \\
\text { Radium-228 } \\
\text { Total organic carbon } \\
\text { Total organic halogens } \\
\text { Trichlorofluoromethane } \\
\text { Tritium }\end{array}$ & $\begin{array}{l}\mu \mathrm{g} / \mathrm{L} \\
\mu \mathrm{g} / \mathrm{L} \\
\mu \mathrm{g} / \mathrm{L} \\
\mu \mathrm{g} / \mathrm{L} \\
\mathrm{pCi} / \mathrm{L} \\
\mu \mathrm{g} / \mathrm{L} \\
\mu \mathrm{g} / \mathrm{L} \\
\mu \mathrm{g} / \mathrm{L} \\
\mathrm{pCi} / \mathrm{mL}\end{array}$ & $\begin{array}{l}6.0 \\
4.2 \\
78 \\
4.8 \\
4.8 E+00 \\
15,900 \\
514 \\
46 \\
1.2 E+01\end{array}$ & $\begin{array}{l}- \\
D \\
86 \\
D \\
- \\
- \\
541 \\
36 \\
1.3 E+01\end{array}$ & & $\begin{array}{l}- \\
D \\
2 \\
D \\
- \\
- \\
2 \\
2 \\
1\end{array}$ \\
\hline
\end{tabular}


Steed Pond Aquifer (Water Table)

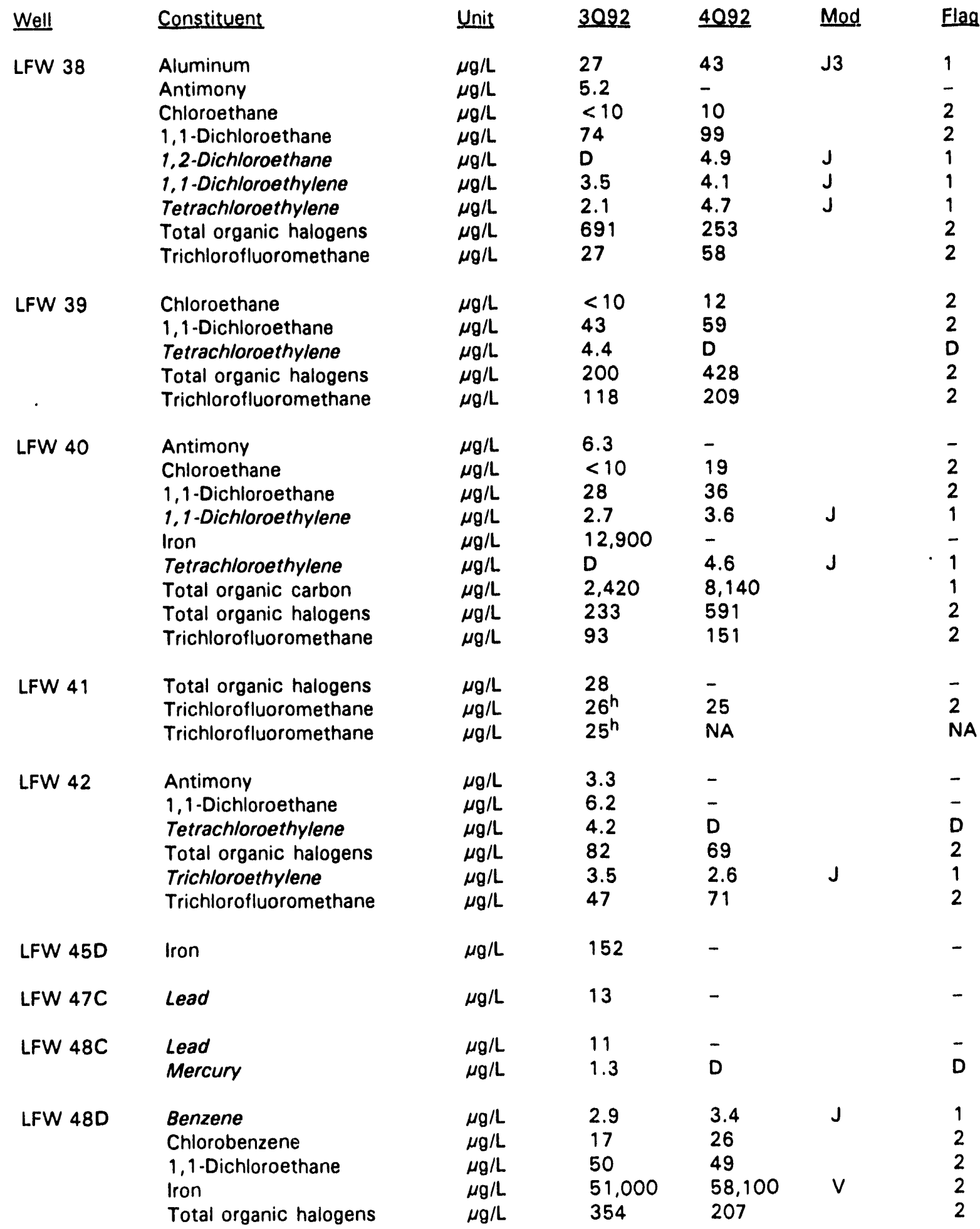


Steed Pond Aquifer (Water Table)

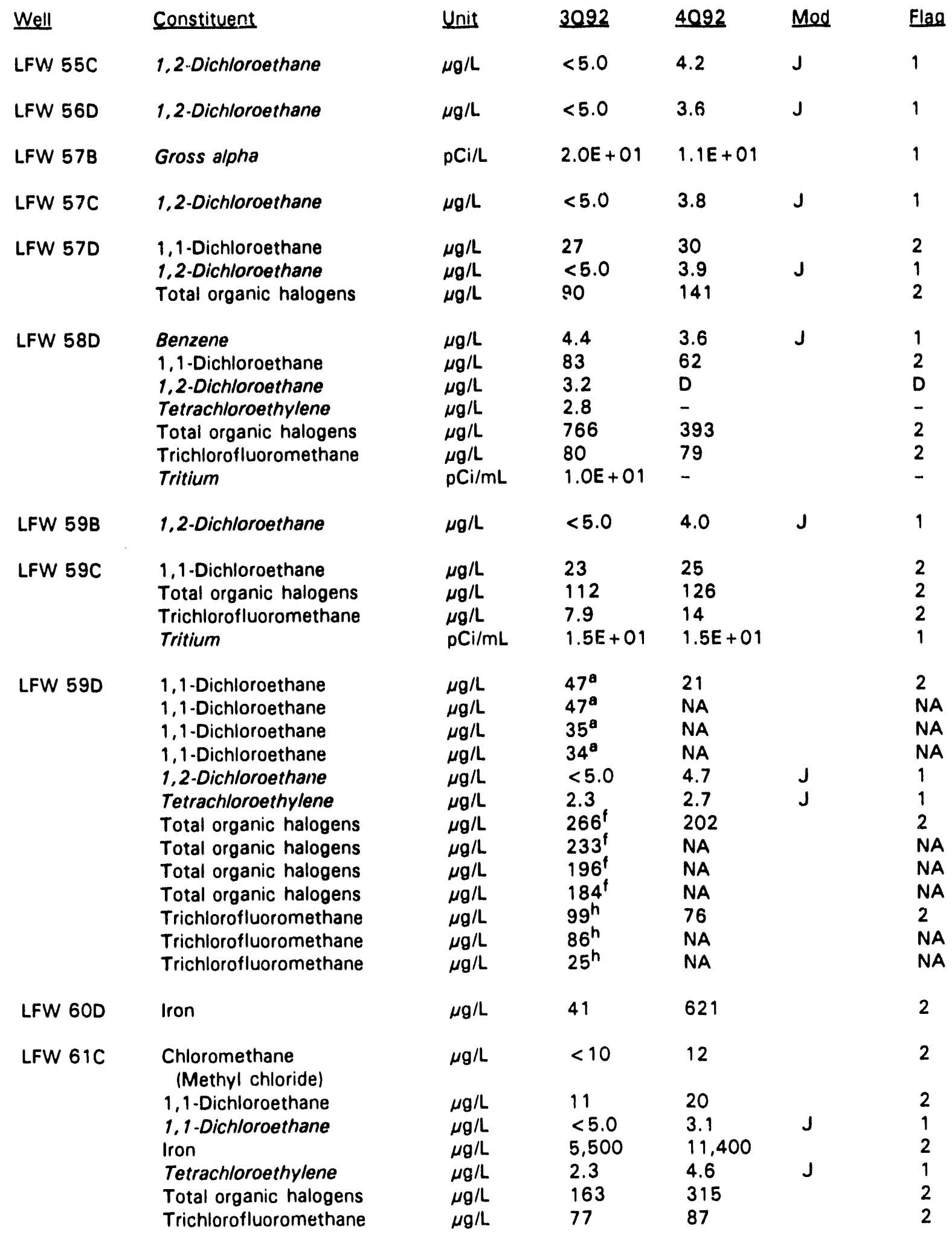


Steed Pond Aquifer (Water Table)

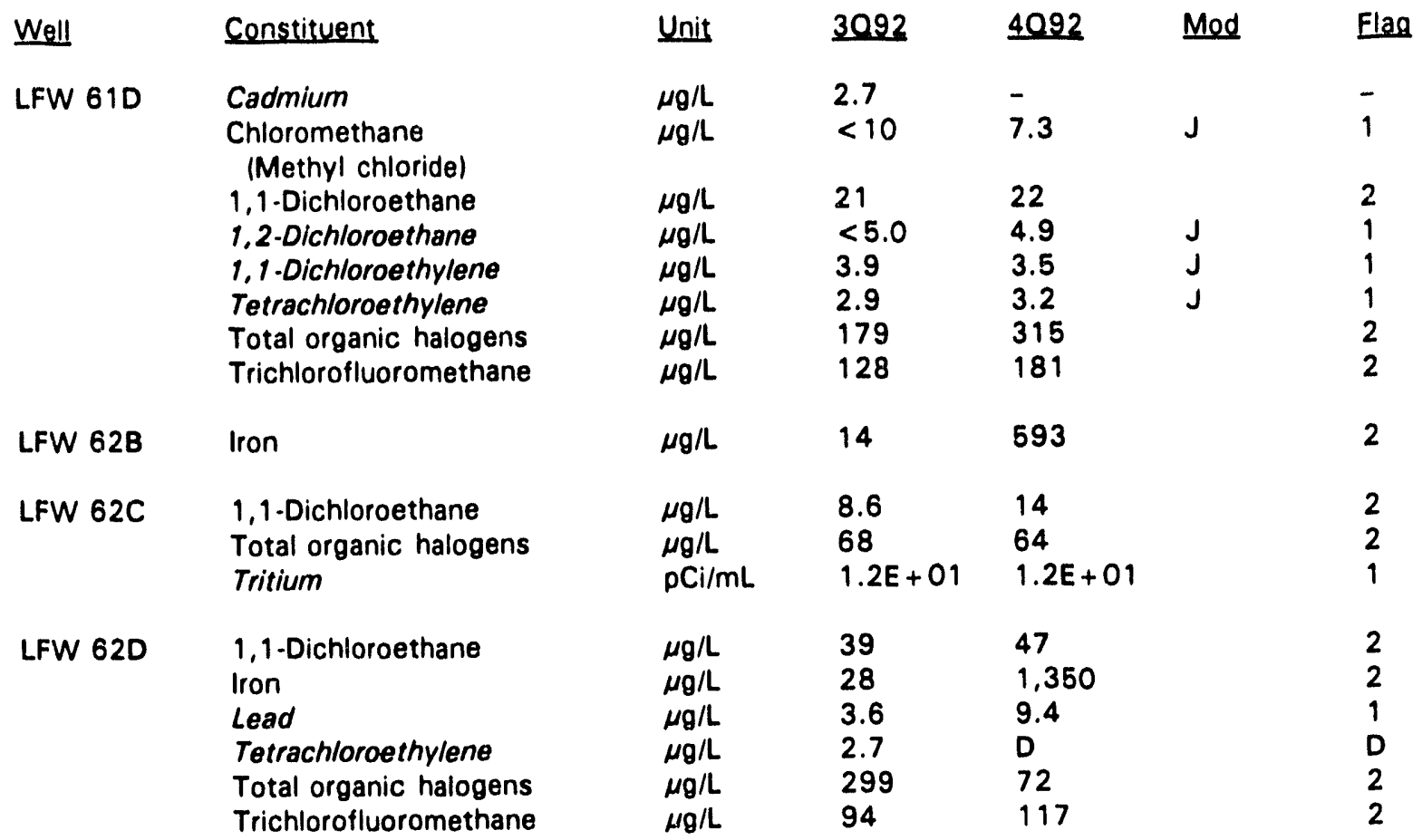

Note: Constituents exceeding half the final PDWS appear italicized. The modifier and flag columns apply to 4092 data.

a Duplicate/replicate samples of 1,1-dichloroethane.

b $\mathrm{NA}=$ not analyzed.

c - = not above Flag 1 or 2.

d Duplicate/replicate samples of iron.

- Duplicate/replicate samples of manganese.

f Duplicate/replicate samples of total organic halogens.

- Duplicate/replicate samples of specific conductance.

$\mathrm{h}$ Duplicate/replicate samples of trichlorofluoromethane.

$i \quad D=$ equaled or exceeded the final PDWS; refer to Table 1. 
WSRC-TR-93-068

Table 3. Groundwater Monitoring Results for Individual Wells

\section{WELL LFW 6}

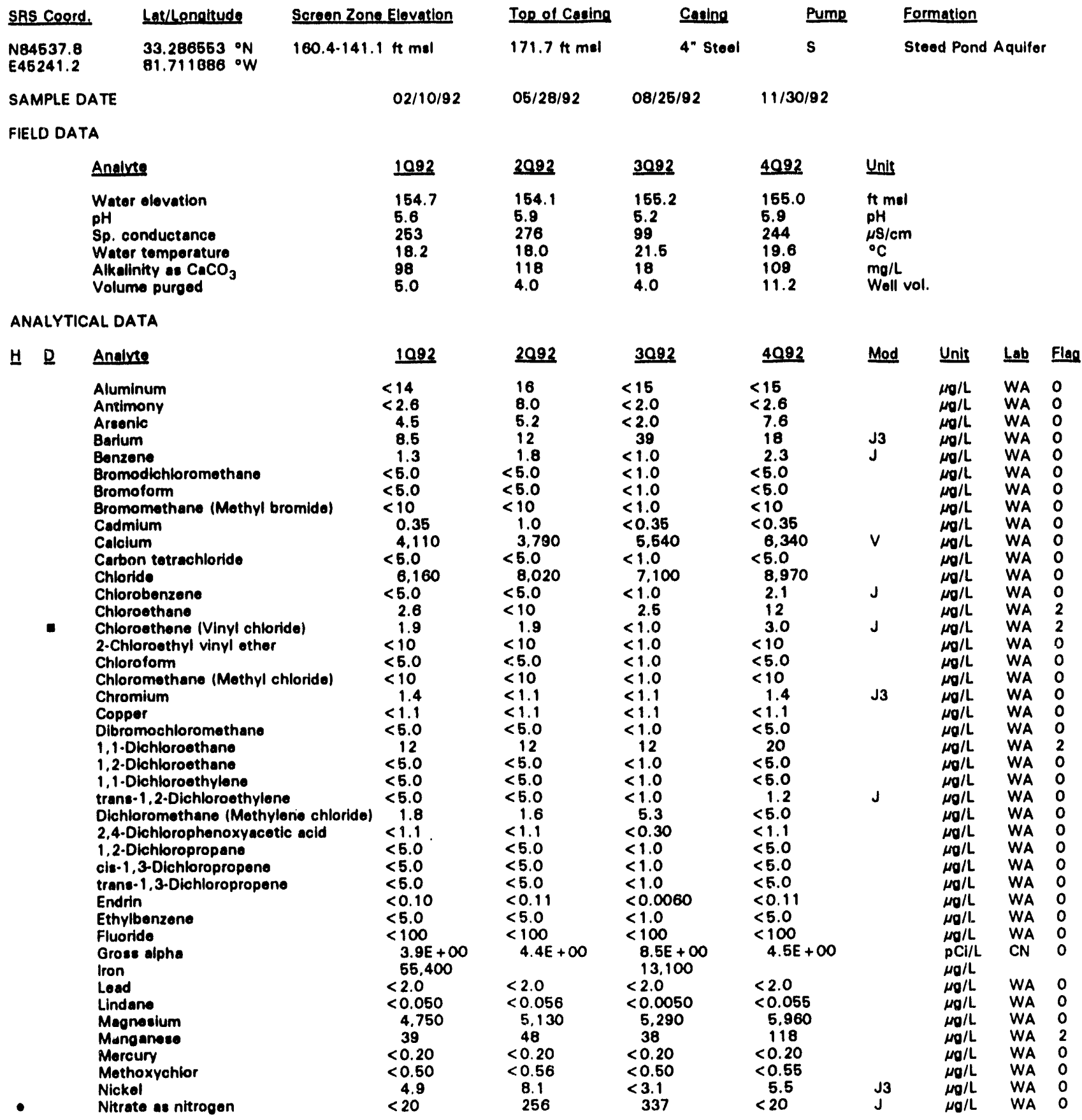

Note: Flagging levels, modifiers, and laboratory are for 4th querter 1992 data only. See Appendix B for flagging criteria.

- = exceeded holding time for 4th quarter 1992.

- =xceeded final primary drinking water standard for 4th quarter 1992. 
WSRC-TR-93-068

Well LFW 6 continued

ANALYTICAL DATA

H D Analute

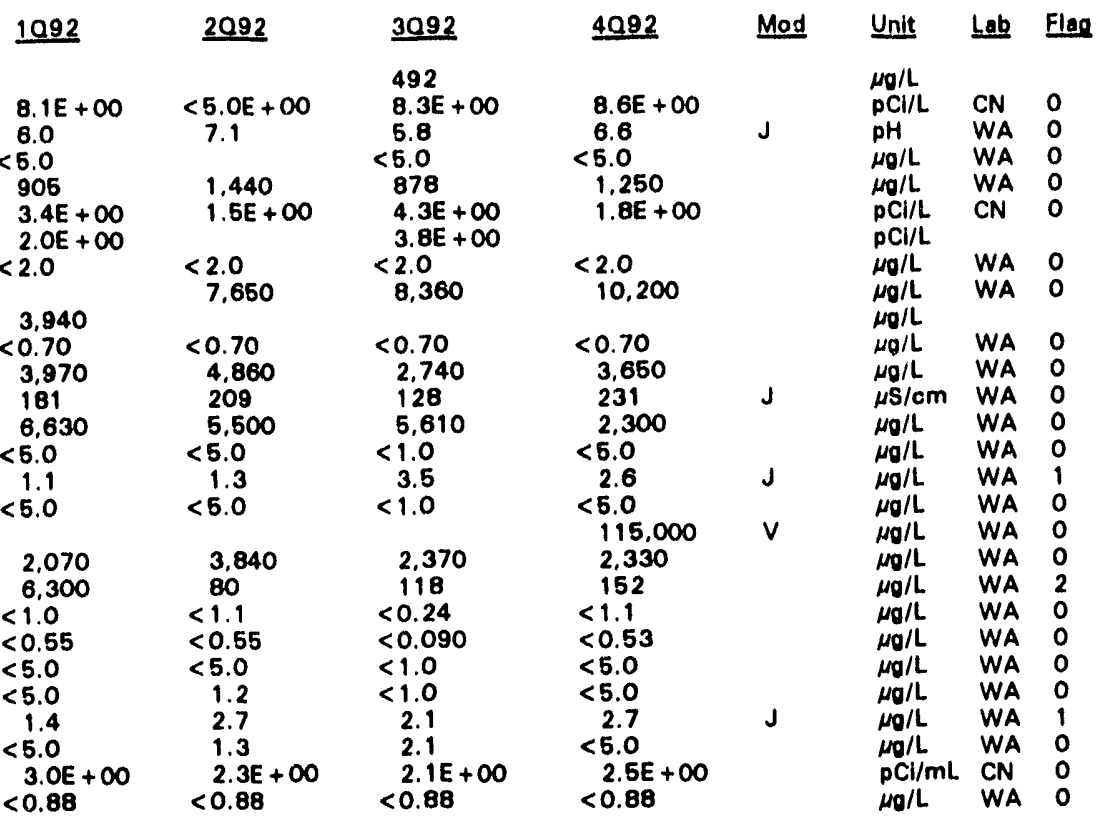

\section{WELL LFW 7}

\begin{tabular}{|c|c|c|c|c|c|c|}
\hline SRS Coord. & Lat/Lonoitude & Screen Zone Elevation & Top of Casing & Casing & Pump & Formation \\
\hline $\begin{array}{l}\text { N84310.3 } \\
E 45318.9\end{array}$ & $\begin{array}{l}33.286177{ }^{\circ} \mathrm{N} \\
81.711239{ }^{\circ} \mathrm{W}\end{array}$ & $159.8-140.5 \mathrm{ft} \mathrm{mal}$ & $171.2 \mathrm{ft} \mathrm{mol}$ & 4" Steol & $\mathbf{s}$ & Steed Pond Aquifer \\
\hline SAMPLE D & & $02 / 21 / 92$ & $06 / 04 / 92$ & $08 / 25 / 92$ & $11 / 30 / 92$ & \\
\hline FIELD DA & & & & & & \\
\hline & & 1092 & 2092 & 3092 & 4092 & Unit \\
\hline & $\begin{array}{l}\text { elevation } \\
\text { onductance } \\
\text { r temperature } \\
\text { inity as } \mathrm{CaCO}_{3} \\
\text { ne purged }\end{array}$ & 152.6 & 152.1 & 153.2 & 153.1 & $\begin{array}{l}\text { ft msl } \\
\mathrm{pH} \\
\mu \mathrm{S} / \mathrm{cm} \\
{ }^{\circ} \mathrm{C} \\
\mathrm{mg} / \mathrm{L} \\
\text { Woll vol. }\end{array}$ \\
\hline
\end{tabular}

Note: Flagging levels, modifiers, and laboratory are for 4th quarter 1992 data only. See Appendix B for flagging criteria.

- = exceeded holding time for 4th quarter 1992.

- exceeded final primary drinking water standard for 4th quarter 1992. 
WSRC-TR-93-068

WELL LFW 8

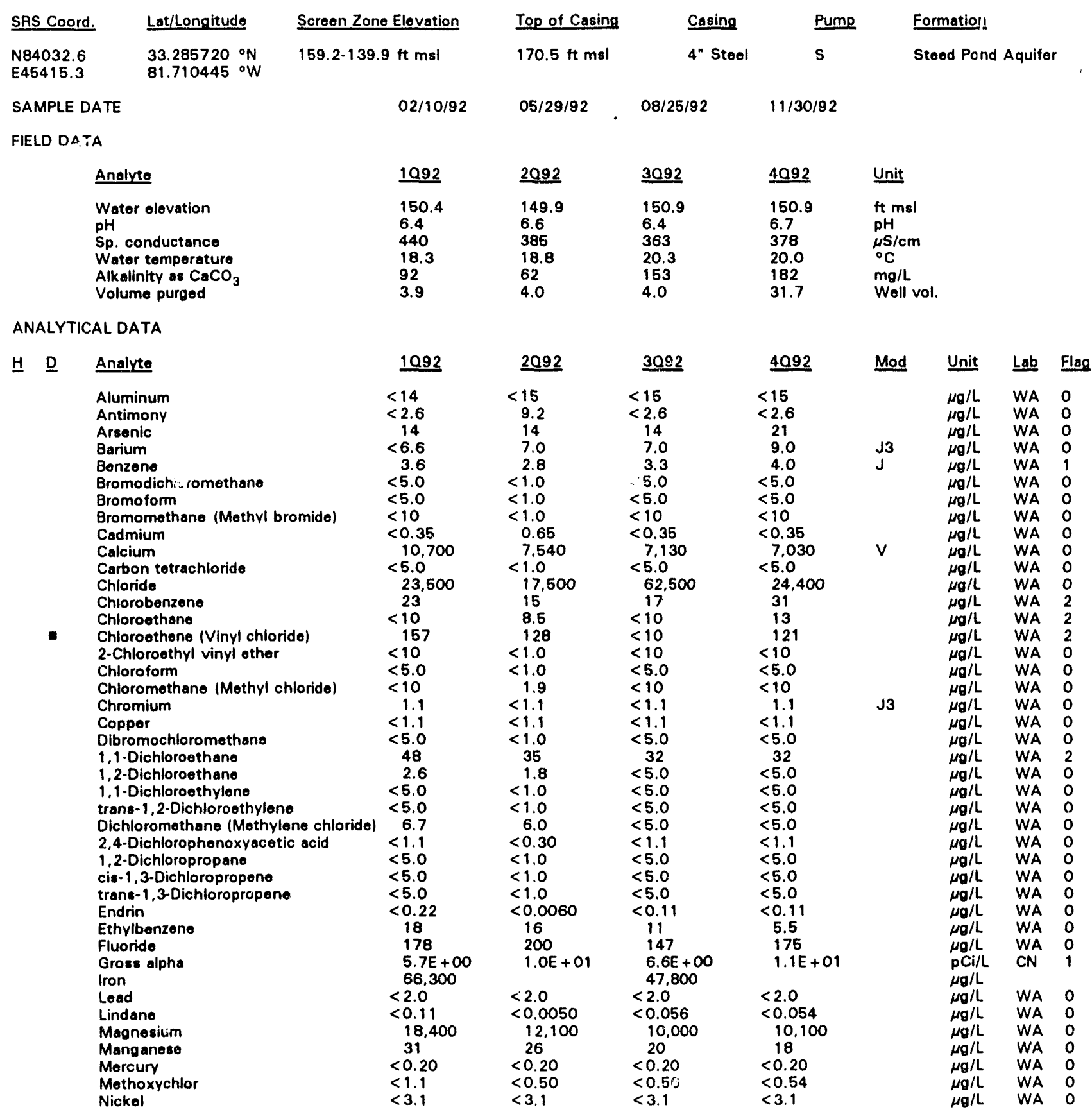

Note: Flagging levels, modifiers, and laboratory are for 4th quarter 1992 data only. See Appendix B for flagging criteria.

- = exceeded holding time for 4th quarter 1992.

- exceejsd final primary drinking water standard for 4th quarter "1992. 


D. Analute
Nitrate as nitrogen
Nitrate-nitrite as nitrogen
Nonvolatile beta
pH
Phenols
Potassium
Radium-226
Radium-228
Solenium
Silica
Silicon
Silver
Sodium
Specific conductance
Sulfate
1,1,2,2-Tetrachloroethane
Totrachloroethylene
Toluene
Total dissolved solids
Total organic carbon
Total organic halogens
Toxaphene
2,4,5-TP (Silvex)
$1,1,1-$ Trichloroethane
$1,1,2-$ Trichloroethane
Trichloroethylene
Trichlorofluoromethane
Tritium
Vanadium

\begin{tabular}{|c|c|c|c|c|c|c|c|}
\hline 1092 & 2092 & 3092 & 4092 & Mod & Unit & Lab & Flag \\
\hline$<20$ & $\begin{array}{l}210 \\
80\end{array}$ & 163 & $<20$ & $\mathrm{~J}$ & $\begin{array}{l}\mu \mathrm{g} / \mathrm{L} \\
\mu \mathrm{g} / \mathrm{L}\end{array}$ & WA & 0 \\
\hline $\begin{array}{l}1.2 E+01 \\
6.1 \\
11 \\
1.430 \\
2.4 E+00 \\
6.9 E+00\end{array}$ & $\begin{array}{l}1.2 E+01 \\
6.7 \\
1.720 \\
2.5 E+00\end{array}$ & $\begin{aligned} & 1.1 E+01 \\
& 6.1 \\
< & 5.0 \\
& 1.290 \\
< & 1.0 E+00 \\
& 5.3 E+00\end{aligned}$ & $\begin{array}{l}1.4 E+01 \\
6.3 \\
<5.0 \\
1.770 \\
1.5 E+00\end{array}$ & $J$ & $\begin{array}{l}\mathrm{pCi} / \mathrm{L} \\
\mathrm{pH} \\
\mu \mathrm{g} / \mathrm{L} \\
\mu \mathrm{g} / \mathrm{L} \\
\mathrm{pCi} / \mathrm{L} \\
\mathrm{pCi} / \mathrm{L}\end{array}$ & $\begin{array}{l}\text { CN } \\
\text { WA } \\
\text { WA } \\
\text { W'A } \\
\text { CN }\end{array}$ & $\begin{array}{l}0 \\
0 \\
0 \\
0 \\
0\end{array}$ \\
\hline$<2.0$ & $\begin{aligned}<2.0 \\
5.720\end{aligned}$ & $\begin{aligned}<2.0 \\
5.870\end{aligned}$ & $\begin{array}{r}<2.0 \\
6,600\end{array}$ & & $\begin{array}{l}\mu_{g} / \mathrm{L} \\
\mu g / L\end{array}$ & $\begin{array}{l}\text { WA } \\
\text { WA }\end{array}$ & $\begin{array}{l}0 \\
0\end{array}$ \\
\hline $\begin{aligned} & 2.930 \\
&< 0.70 \\
& 15.700 \\
& 228 \\
& 339 \\
&<5.0 \\
&<5.0 \\
& 5.7\end{aligned}$ & $\begin{aligned} &< 0.70 \\
& 16.200 \\
& 224 \\
& 535 \\
&<1.0 \\
&<1.0 \\
& 6.6\end{aligned}$ & $\begin{array}{l}<0.70 \\
19.200 \\
231 \\
594 \\
<5.0 \\
<5.0 \\
5.8\end{array}$ & $\begin{array}{l}<0.70 \\
20.900 \\
240 \\
356 \\
<5.0 \\
<5.0 \\
<5.0 \\
117,000\end{array}$ & V & $\begin{array}{l}\mu \mathrm{g} / \mathrm{L} \\
\mu \mathrm{g} / \mathrm{L} \\
\mu \mathrm{g} / \mathrm{L} \\
\mu \mathrm{S} / \mathrm{cm} \\
\mu \mathrm{g} / \mathrm{L} \\
\mu \mathrm{g} / \mathrm{L} \\
\mu \mathrm{g} / \mathrm{L} \\
\mu \mathrm{g} / \mathrm{L} \\
\mu \mathrm{g} / \mathrm{L}\end{array}$ & $\begin{array}{l}\text { WA } \\
\text { WA } \\
\text { WA } \\
\text { WA } \\
\text { WA } \\
\text { WA } \\
\text { WA } \\
\text { WA }\end{array}$ & $\begin{array}{l}0 \\
0 \\
0 \\
0 \\
0 \\
0 \\
0 \\
0\end{array}$ \\
\hline $\begin{aligned} & 5.310 \\
& 14.600 \\
< & 2.2 \\
< & 0.56 \\
< & 5.0 \\
< & 5.0 \\
< & 5.0 \\
< & 5.0 \\
& 7.2 E+00 \\
< & 0.88\end{aligned}$ & $\begin{aligned} & 7.660 \\
& 245 \\
< & 0.24 \\
< & 0.090 \\
< & 1.0 \\
& 1.1 \\
< & 1.0 \\
< & 1.0 \\
& 7.3 E+00 \\
< & 0.88\end{aligned}$ & $\begin{aligned} & 4.740 \\
& 245 \\
< & 1.1 \\
< & 0.56 \\
< & 5.0 \\
< & 5.0 \\
< & 5.0 \\
< & 5.0 \\
& 7.4 E+00 \\
< & 0.88\end{aligned}$ & $\begin{aligned} & 6.130 \\
& 219 \\
< & 1.1 \\
< & 0.53 \\
< & 5.0 \\
< & 5.0 \\
< & 5.0 \\
< & 5.0 \\
& 8.6 E+\infty \\
< & 0.88\end{aligned}$ & & $\begin{array}{l}\mu \mathrm{g} / \mathrm{L} \\
\mu \mathrm{g} / \mathrm{L} \\
\mu \mathrm{g} / \mathrm{L} \\
\mu \mathrm{g} / \mathrm{L} \\
\mu \mathrm{g} / \mathrm{L} \\
\mu \mathrm{g} / \mathrm{L} \\
\mu \mathrm{g} / \mathrm{L} \\
\mu \mathrm{g} / \mathrm{L} \\
\mathrm{pCi} / \mathrm{mL} \\
\mu \mathrm{g} / \mathrm{L}\end{array}$ & $\begin{array}{l}\text { WA } \\
\text { WA } \\
\text { WA } \\
\text { WA } \\
\text { WA } \\
\text { WA } \\
\text { WA } \\
\text { WA } \\
\text { CN } \\
\text { WA }\end{array}$ & $\begin{array}{l}1 \\
2 \\
0 \\
0 \\
0 \\
0 \\
0 \\
0 \\
0 \\
0\end{array}$ \\
\hline
\end{tabular}

\section{WELL LFW 10A}

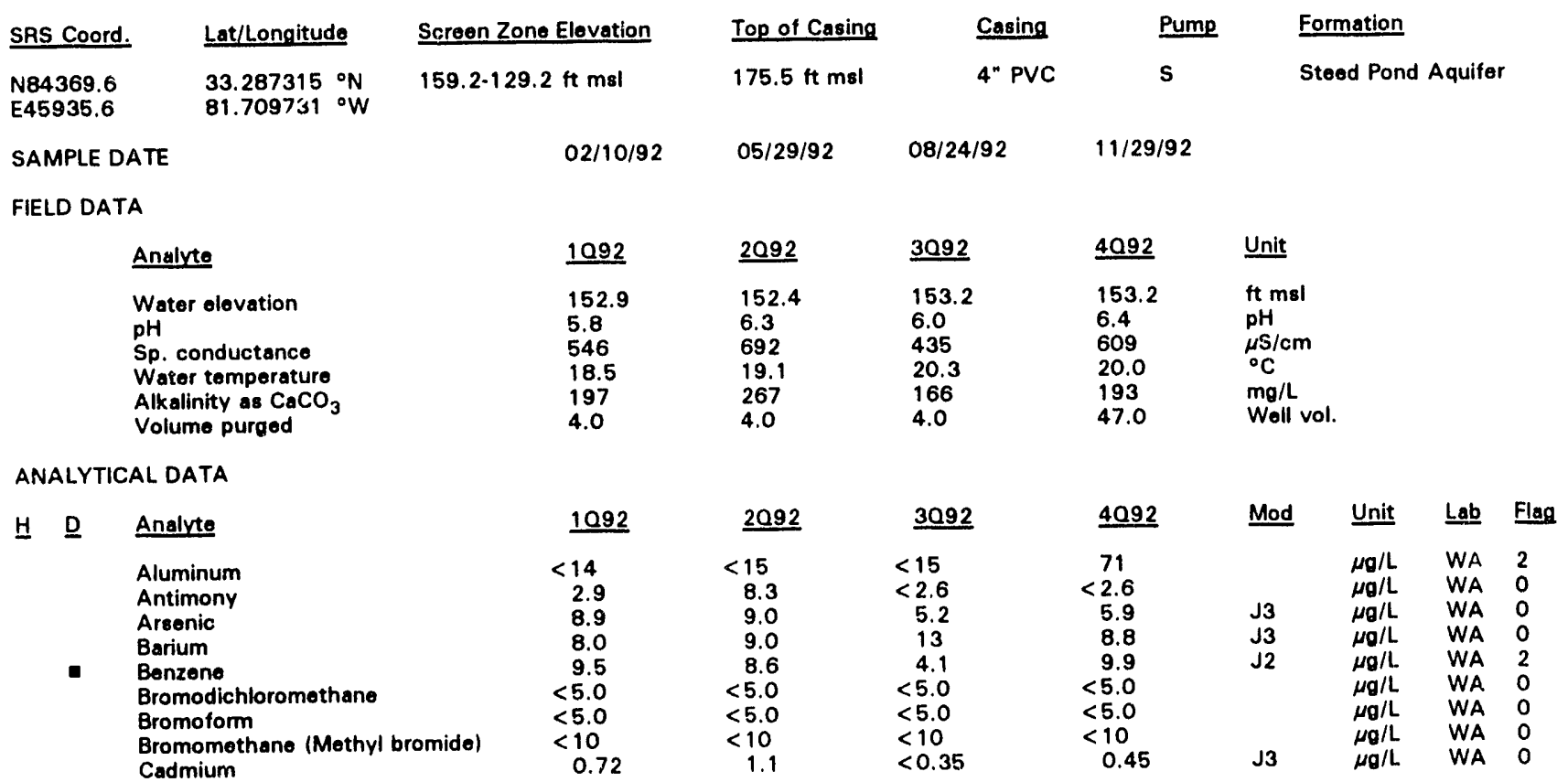

Note: Flagging levels, modifiers, and laboratory are for 4th quarter 1992 data only. See Appendix B for flagging criteria.

- exceeded holding time for 4th quarter 1992.

- exceeded final primary drinking water standard for 4th quarter 1992. 
Well LFW 10A continued

ANALYTICAL DATA.

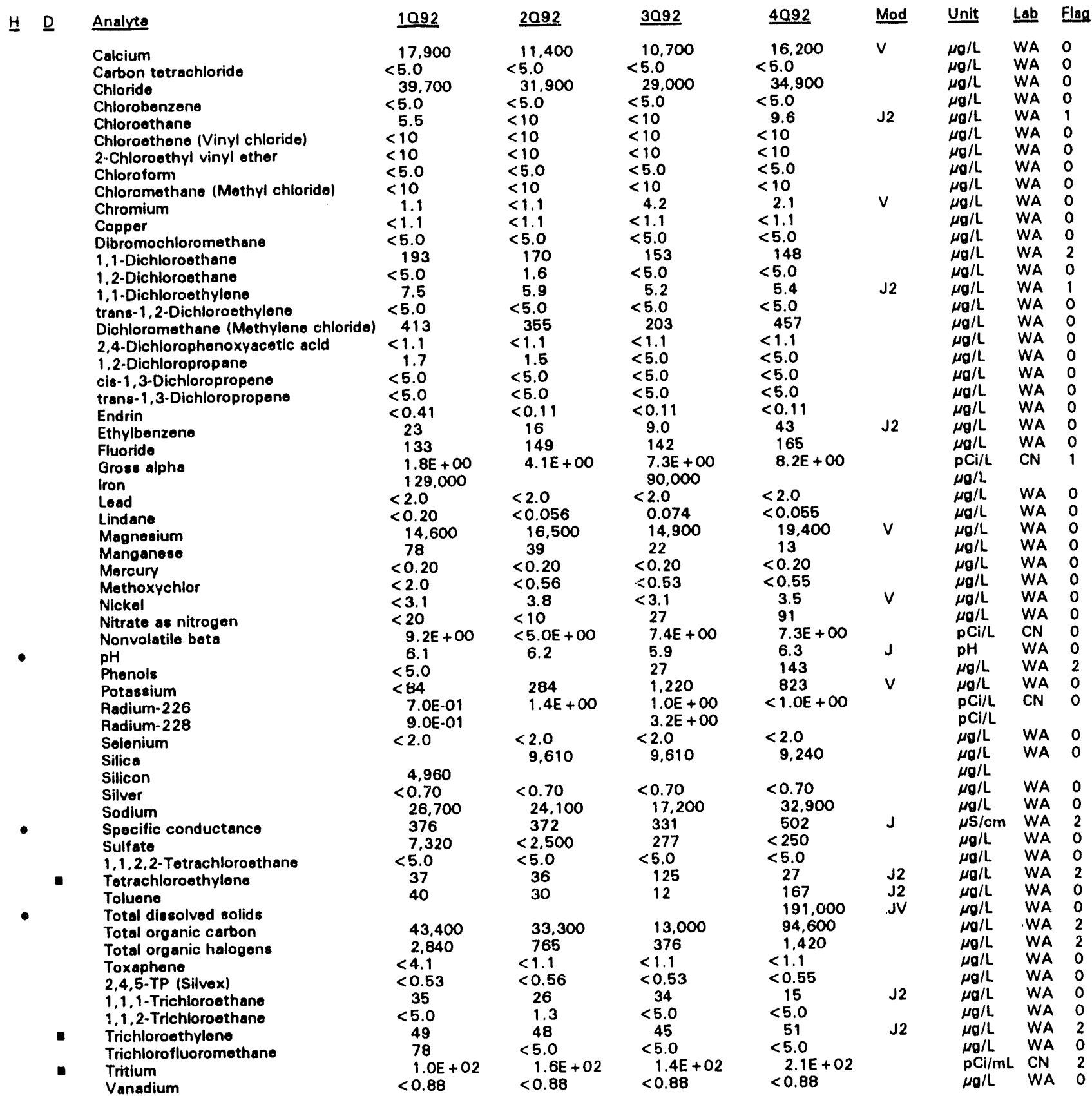

Note: Flagging levels, modifiers, and laboratory are for 4th quarter 1992 data only. See Appendix B for flagging criteria. - = exceeded holding time for 4th quarter 1992.

- exceeded final primary drinking water standard for 4th quarter 1992. 
WELL LFW 16

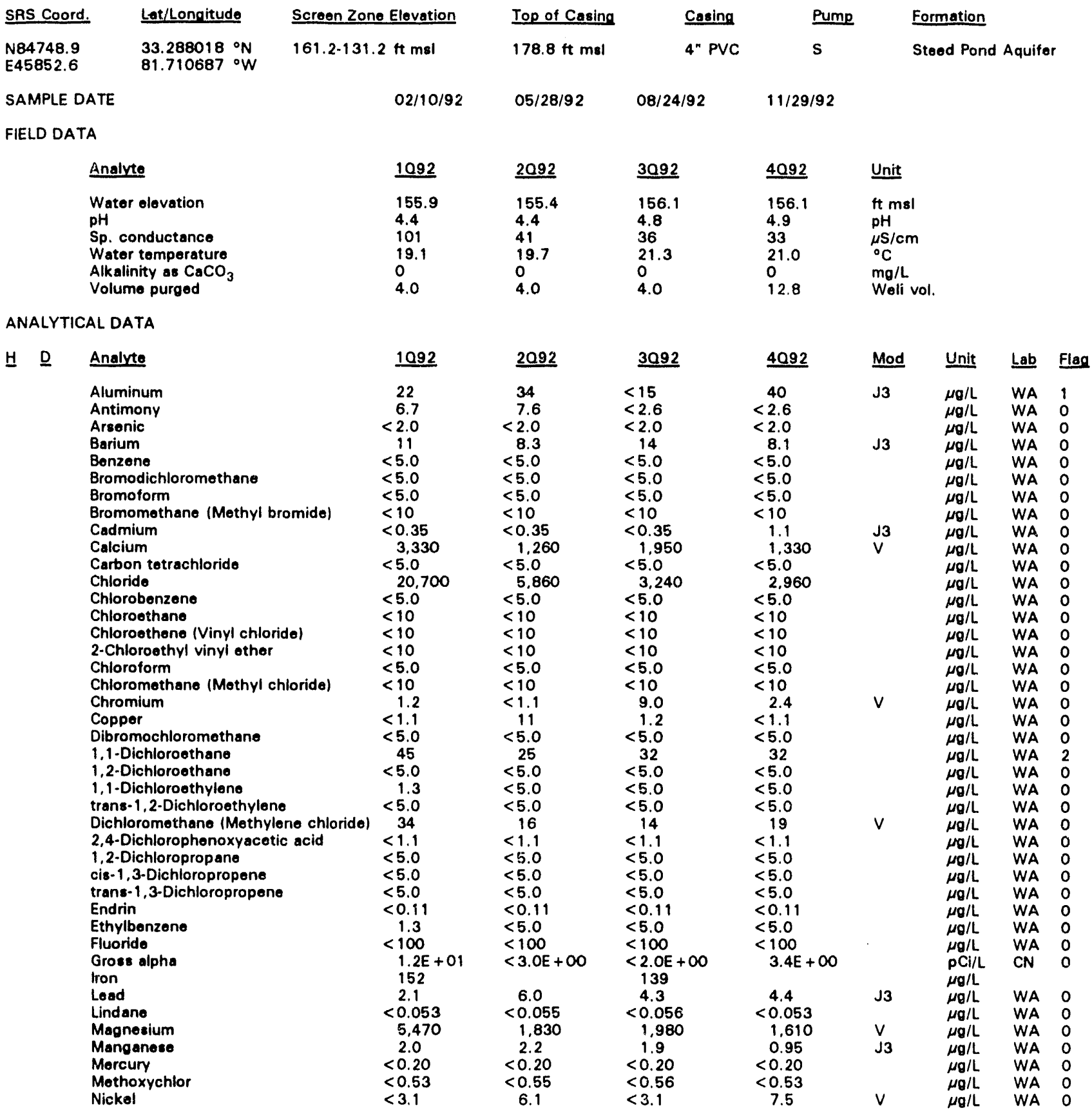

Note: Flagging levels, modifiers, and laboratory are for 4 th quarter 1992 data only. See Appendix B for flagging criteria.

- = exceeded holding time for 4th quarter 1992.

- exceeded final primary drinking water standard for 4th quarter 1992. 
Well LFW 16 continued

ANALYTICAL DATA

H. $\quad$ Analyte
Nitrate as nitrogen
Nonvolatile beta
pH
Phenols
Potassium
Radium-226
Radium-228
Selenium
Silica
Silicon
Silver
Sodium
Specific conductance
Sulfate
1,1,2,2-Tetrachloroethane
Totrachloroethylene
Toluene
Total dissolved solids
Total organic carbon
Total organic halogens
Toxaphene
2,4,5-TP (Silvex)
$1,1,1-$ Trichloroethane
$1,1,2-$ Trichloroethane
Trichloroethylene
Trichlorofluoromethane
Tritium
Vanadium

$\begin{array}{lcc}1092 & \underline{2092} & 3092 \\ 841 & 675 & 677 \\ 9.3 \mathrm{E}+00 & <5.0 \mathrm{E}+00 & 2.8 \mathrm{E}+00 \\ 5.2 & 6.6 & 5.1 \\ 7.7 & & <5.0 \\ 203 & 424 & 656 \\ 1.3 \mathrm{E}+00 & 5.3 \mathrm{E}-01 & 1.2 \mathrm{E}+00 \\ 5.0 \mathrm{E}+00 & & 1.8 \mathrm{E}+00 \\ <2.0 & <2.0 & <2.0 \\ & 8.820 & 11.100 \\ 5.820 & & \\ <0.70 & <0.70 & <0.70 \\ 5.800 & 1.910 & 1.590 \\ 79 & 42 & 41 \\ <2.500 & <2.500 & 318 \\ <5.0 & <5.0 & <5.0 \\ 3.2 & 2.7 & 15 \\ <5.0 & <5.0 & <5.0 \\ & & \\ 2.780 & 1.440 & 602 \\ 6.470 & 89 & <50 \\ <1.1 & <1.1 & <1.1 \\ <0.53 & <0.57 & <0.56 \\ 4.7 & 3.9 & 6.0 \\ <5.0 & 1.1 & <5.0 \\ 9.6 & 7.8 & 14 \\ 13 & <5.0 & <5.0 \\ 9.4 \mathrm{E}+01 & 2.1 \mathrm{E}+01 & 1.4 \mathrm{E}+01 \\ <0.88 & <0.88 & 1.2 \\ & & \end{array}$

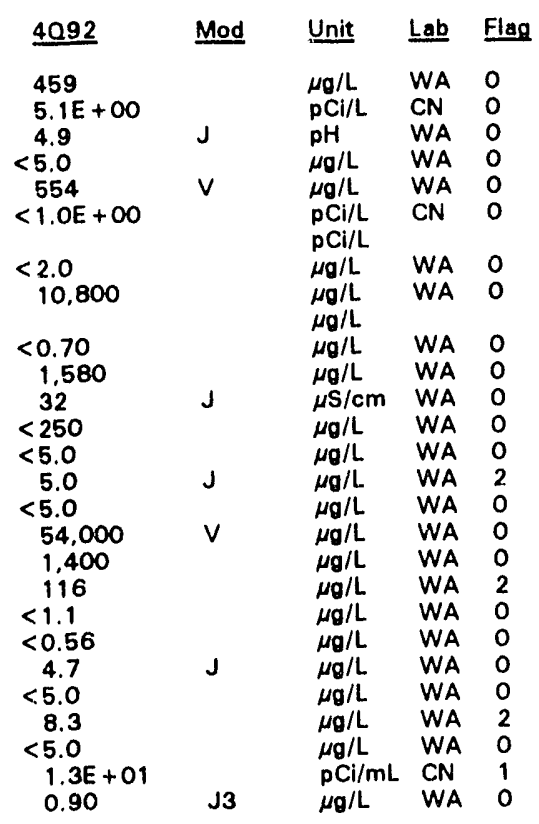

\section{WELL LFW 17}

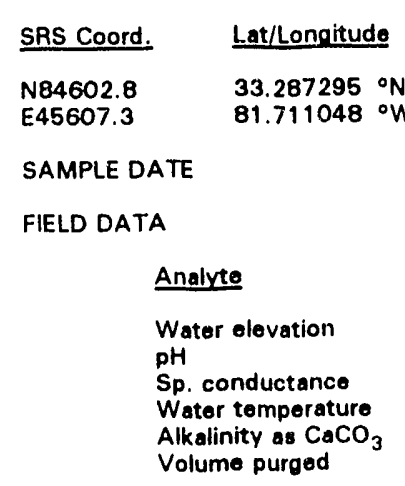

ANALYTICAL DATA

H D Analyte

Aluminum

Antimony

Arsenic

Barium

Benzene

Bromodichloromethane

Bromoform

Bromomethane (Mothyl bromide)

Cadmium

Calcium

\section{Screen Zone Elevation \\ 158.5-128.5 ft msl}

1092

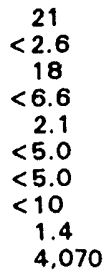

2092

$<15$
9.6
14
$<4.0$
2.6
$<5.0$
$<5.0$
$<10$
0.45
4.050

$02 / 11 / 92$

2092
154.7
6.0
239
19.1
70
4.0

Top of Casing

$177.8 \mathrm{ft} \mathrm{msl}$

1092
155.4
6.6
252
19.5
74
4.0

Casing

4" PVC

$08 / 25 / 92$

3092
155.6
6.3
306
19.7
86
4.0

3092

$<15$

$<2.6$

13
8.4

$<5.0$

$<5.0$

$<5.0$

$<10$

1.3
4.770
Pump

S

$11 / 30 / 92$

\section{Formation}

Steed Pond Aquifer

$\begin{array}{ll}155.7 & \mathrm{ft} \mathrm{msl} \\ 6.5 & \mathrm{pH} \\ 352 & \mu \mathrm{S} / \mathrm{cm} \\ 19.8 & { }^{\circ} \mathrm{C} \\ 144 & \mathrm{mg} / \mathrm{L} \\ 6.4 & \text { Well vol }\end{array}$

\begin{tabular}{|c|c|c|c|}
\hline 4Q992 & Mod & Unit & $\underline{\text { Lab }}$ \\
\hline$<15$ & & $\mu \boldsymbol{g} / \mathrm{L}$. & WA \\
\hline 2.6 & & $\mu g / L$ & WA \\
\hline 17 & & $\mu \mathrm{g} / \mathrm{L}$ & WA \\
\hline 4.9 & J3 & $\mu \mathrm{g} / \mathrm{L}$ & WA \\
\hline 4.1 & $\mathbf{J}$ & $\mu \mathrm{g} / \mathrm{L}$ & WA \\
\hline$<5.0$ & & $\mu g / L$ & WA \\
\hline$<5.0$ & & $\mu \mathrm{g} / \mathrm{L}$ & WA \\
\hline$<1$ & & $\mu \mathrm{g} / \mathrm{L}$ & WA \\
\hline 35 & & $\mu \mathrm{g} / \mathrm{L}$ & WA \\
\hline $44 C$ & V & $/ L$ & WA \\
\hline
\end{tabular}

Note: Flagging levels, modifiers, and laboratory are for 4 th quarter 1992 data only. See Appendix B for flagging criteria.

- = exceeded holding time for 4th quarter 1992.

- exceeded final primary drinking water standard for 4 th quarter 1992. 
Well LFW 17 continued

ANALYTICAL DATA

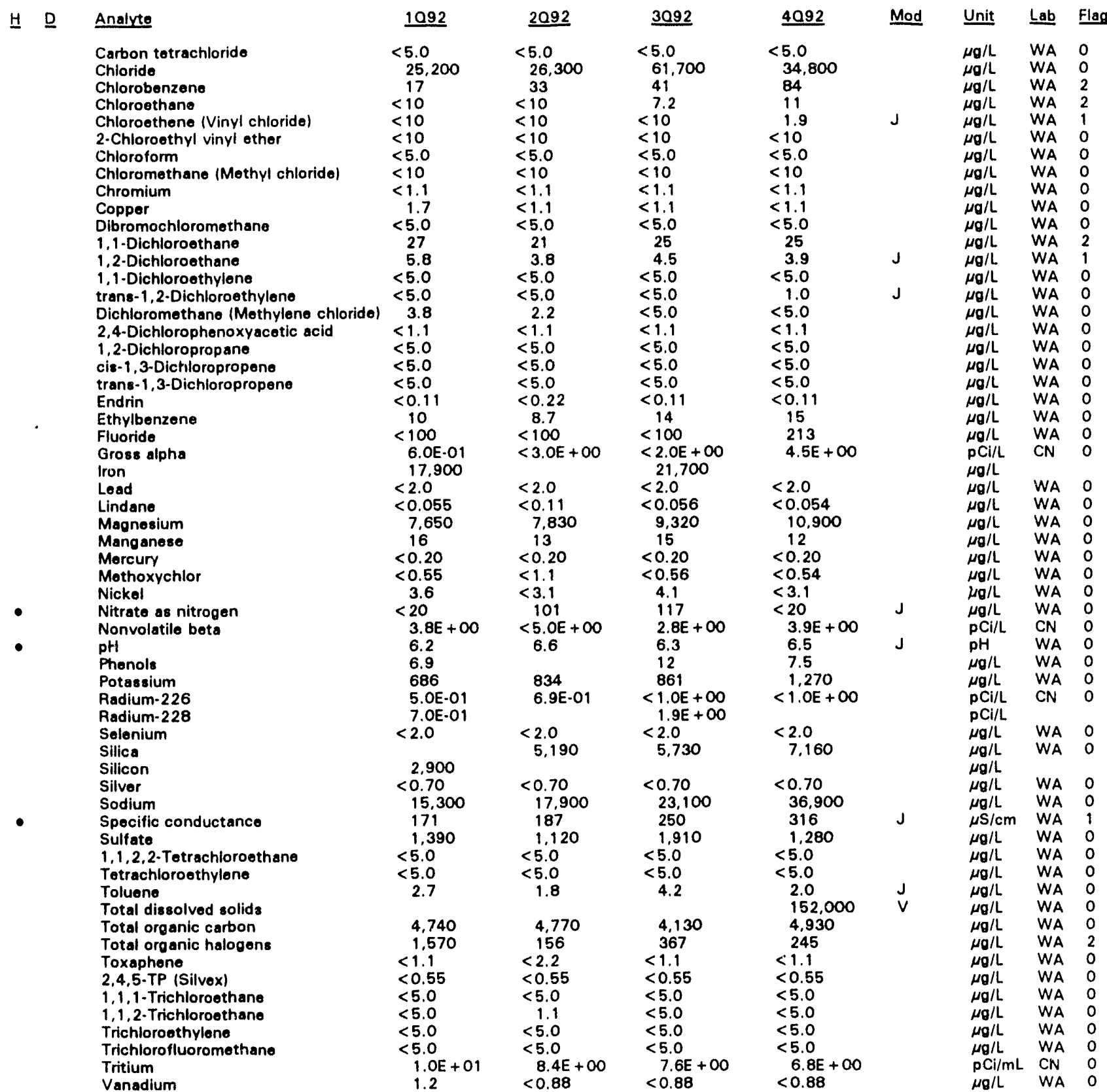

Note: Flagging levels, modifiers, and laboratory are for 4 th quarter 1992 data only. See Appendix B for flagging criteria.

- =xceeded holding time for 4th quarter 1992.

- = exceeded final primary drinking water standard for 4th quarter 1992. 
WSRC-TR-93-068

WELL LFW 18

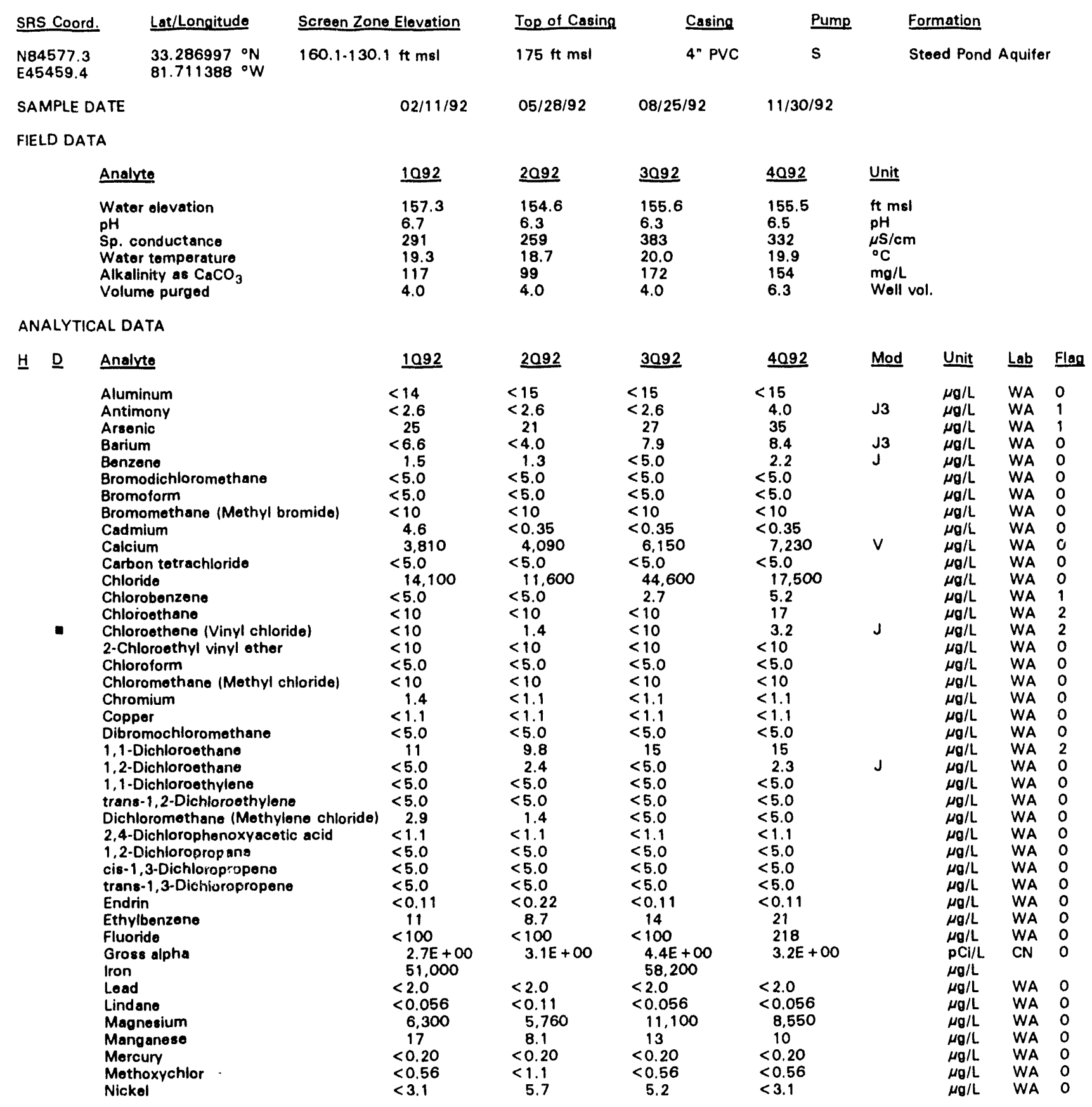

Note: Flagging levels, modifiers, and laboratory are for 4th quarter 1992 data only. See Appendix B for flagging criteria. - = exceeded holding time for 4th quarter 1992.

- =xceeded final primary drinking water standard for 4th quarter 1992. 
Woll LFW 18 continued

ANALYTICAL DATA

H D Analyte

- Nitrate as nitrogen

Nonvolatile beta

$\mathrm{pH}$

Phenols

Potassium

Radium-226

Radium-228

Solenium

Silica

Silicon

Silver

Sodium

- Specific conductance

Sulfate

1,1,2,2-Totrachloroethane

Tetrachloroethylene

Toluene

Total dissolved solids

Total organic carbon

Total organic halogens

Toxaphene

2,4,5-TP (Silvex)

1,1,1-Trichloroethane

1,1,2-Trichloroethane

Trichloroethylene

Trichlorofluoromethane

Tritium

Vanadium

\begin{tabular}{lll}
1092 & $\underline{2092}$ & $\underline{3092}$ \\
\hline 20 & 172 & 79 \\
$7.9 \mathrm{E}+00$ & $5.2 \mathrm{E}+00$ & $5.1 \mathrm{E}+00$ \\
6.1 & 6.7 & 6.2 \\
7.4 & & 9.0 \\
1.260 & 1.290 & 2.050 \\
$1.1 \mathrm{E}+00$ & $<5.0 \mathrm{E}-\mathrm{O} 1$ & $<1.0 \mathrm{E}+00$ \\
$3.9 \mathrm{E}+00$ & & $3.8 \mathrm{E}+00$ \\
$<2.0$ & $<2.0$ & $<2.0$ \\
& 6.210 & 8.250 \\
3.590 & & \\
$<0.70$ & $<0.70$ & $<0.70$ \\
11.200 & 9.930 & 16.300 \\
149 & 137 & 236 \\
518 & 511 & 958 \\
$<5.0$ & $<5.0$ & $<5.0$ \\
$<5.0$ & $<5.0$ & $<5.0$ \\
3.6 & 2.6 & 6.0 \\
& & \\
3.120 & 4.230 & 4.440 \\
840 & 150 & 260 \\
$<1.1$ & $<2.2$ & $<1.1$ \\
$<0.56$ & $<0.55$ & $<0.56$ \\
$<5.0$ & $<5.0$ & $<5.0$ \\
$<5.0$ & 1.2 & $<5.0$ \\
$<5.0$ & $<5.0$ & $<5.0$ \\
$<5.0$ & 1.1 & $<5.0$ \\
$5.0 \mathrm{E}+00$ & $5.0 \mathrm{E}+00$ & $1.4 \mathrm{E}+01$ \\
1.0 & $<0.88$ & $<0.88$ \\
& &
\end{tabular}

\begin{tabular}{|c|c|c|c|}
\hline 4092 & Mod & Unit & Lab \\
\hline $\begin{array}{l}<20 \\
9.3 E+00 \\
6.3 \\
5.1 \\
2.310 \\
1.0 E+\infty 0\end{array}$ & $\begin{array}{l}J \\
J\end{array}$ & $\begin{array}{l}\mu \mathrm{g} / \mathrm{L} \\
\mathrm{pCi} / \mathrm{L} \\
\mathrm{pH} \\
\mu \mathrm{g} / \mathrm{L} \\
\mu \mathrm{g} / \mathrm{L} \\
\mathrm{pCi} / \mathrm{L} \\
\mathrm{pCi} / \mathrm{L}\end{array}$ & $\begin{array}{l}\text { WA } \\
\text { CN } \\
\text { WA } \\
\text { WA } \\
\text { WA } \\
\text { CN }\end{array}$ \\
\hline $\begin{array}{l}<2.0 \\
7.960\end{array}$ & & $\begin{array}{l}\mu_{\mathbf{g}} / \mathrm{L} \\
\mu_{\boldsymbol{g}} / \mathrm{L} \\
\mu_{\mathbf{g}} / \mathrm{L}\end{array}$ & $\begin{array}{l}\text { WA } \\
\text { WA }\end{array}$ \\
\hline $\begin{array}{l}<0.70 \\
11.700 \\
238 \\
315 \\
<5.0 \\
<5.0\end{array}$ & $J$ & $\begin{array}{l}\mu \mathrm{g} / \mathrm{L} \\
\mu \mathrm{g} / \mathrm{L} \\
\mu \mathrm{S} / \mathrm{cm} \\
\mu \mathrm{g} / \mathrm{L} \\
\mu \mathrm{g} / \mathrm{L} \\
\mu \mathrm{g} / \mathrm{L}\end{array}$ & $\begin{array}{l}\text { WA } \\
\text { WA } \\
\text { WA } \\
\text { WA } \\
\text { WA } \\
\text { WA }\end{array}$ \\
\hline $\begin{array}{l}4.6 \\
100,000\end{array}$ & $\begin{array}{l}J \\
V\end{array}$ & $\mu \sigma / L$ & $\begin{array}{l}\text { WA } \\
\text { WA }\end{array}$ \\
\hline $\begin{aligned} & 4.030 \\
& 298 \\
< & 1.1\end{aligned}$ & & $\begin{array}{l}\mu g / L \\
\mu g / L\end{array}$ & $\begin{array}{l}\text { WA } \\
\text { WA } \\
\text { WA }\end{array}$ \\
\hline $\begin{array}{l}<0.55 \\
<5.0\end{array}$ & & $\begin{array}{l}\mu \mathrm{g} / \mathrm{L} \\
\mu \mathrm{g} / \mathrm{L}\end{array}$ & $\begin{array}{l}\text { WA } \\
\text { WA }\end{array}$ \\
\hline $\begin{array}{l}<5.0 \\
<5.0 \\
<5.0\end{array}$ & & $\begin{array}{l}\mu \mathrm{g} / \mathrm{L} \\
\mu \mathrm{g} / \mathrm{L}\end{array}$ & $\begin{array}{l}\text { WA } \\
\text { WA }\end{array}$ \\
\hline $\begin{array}{l}6.6 E+00 \\
<0.88\end{array}$ & & $\begin{array}{l}\mu_{\mathrm{g}} / \mathrm{L} \\
\mathrm{pCi} / \mathrm{mL} \\
\mu_{\mathrm{g}} / \mathrm{L}\end{array}$ & $\begin{array}{l}\text { WA } \\
\text { WA }\end{array}$ \\
\hline
\end{tabular}

\section{WELL LFW 19}

\begin{tabular}{|c|c|c|c|c|c|c|c|c|c|}
\hline SRS Coord. & Lat/Longitude & Screen Zone Elevation & Top of Casing & Casing & Pump & \multicolumn{4}{|c|}{ Formation } \\
\hline $\begin{array}{l}\text { N84817.2 } \\
E 45135.4\end{array}$ & $\begin{array}{l}33.286998{ }^{\circ} \mathrm{N} \\
81.712707{ }^{\circ} \mathrm{W}\end{array}$ & $160.0-130.0 \mathrm{ft} \mathrm{msl}$ & $176.7 \mathrm{ft} \mathrm{msl}$ & 4" PVC & $\mathbf{S}$ & \multicolumn{4}{|c|}{ Steed Pond Aquifer } \\
\hline \multicolumn{10}{|l|}{ FIELD DATA } \\
\hline \multicolumn{2}{|r|}{ Analvte } & 1092 & $\underline{2092}$ & $\underline{3092}$ & 4092 & \multicolumn{4}{|l|}{$\underline{\text { Unit }}$} \\
\hline \multicolumn{2}{|r|}{$\begin{array}{l}\text { Water elevation } \\
\text { pH } \\
\text { Sp. conductance } \\
\text { Water temperature } \\
\text { Alkalinity as } \mathrm{CaCO}_{3} \\
\text { Volume purged }\end{array}$} & $\begin{array}{l}156.5 \\
5.0 \\
18 \\
18.3 \\
0 \\
4.0\end{array}$ & $\begin{array}{l}156.0 \\
4.3 \\
19 \\
18.5 \\
0 \\
4.0\end{array}$ & $\begin{array}{l}156.8 \\
4.8 \\
21 \\
20.4 \\
0 \\
4.0\end{array}$ & $\begin{array}{l}156.8 \\
4.9 \\
18 \\
19.3 \\
0 \\
11.3\end{array}$ & \multicolumn{2}{|c|}{$\begin{array}{l}\text { ft msl } \\
\mathrm{pH} \\
\mu \mathrm{S} / \mathrm{cm} \\
{ }^{\circ} \mathrm{C} \\
\mathrm{mg} / \mathrm{L} \\
\text { Woll vol. }\end{array}$} & & \\
\hline \multicolumn{10}{|c|}{ ANALYTICAL DATA } \\
\hline \multirow[t]{2}{*}{$\underline{H} \quad \underline{D}$} & Analyte & 1092 & 2092 & $\underline{3092}$ & 4092 & Mod & Unit & $\underline{L a b}$ & Flag \\
\hline & $\begin{array}{l}\text { Aluminum } \\
\text { Antimony } \\
\text { Arsenic } \\
\text { Barium } \\
\text { Benzene } \\
\text { Bromodichloromethane } \\
\text { Bromoform } \\
\text { Bromomothane (Methyl } \\
\text { Cadmium } \\
\text { Calcium }\end{array}$ & $\begin{aligned} & 20 \\
&< 2.6 \\
&< 2.0 \\
&< 6.6 \\
&<5.0 \\
&<5.0 \\
&<5.0 \\
&<10 \\
&<0.35 \\
& \quad 386\end{aligned}$ & $\begin{aligned} &< 15 \\
& 6.3 \\
&< 2.0 \\
& 4.0 \\
&<5.0 \\
&<5.0 \\
&<5.0 \\
&<10 \\
&<0.35 \\
& \quad 442\end{aligned}$ & $\begin{aligned} &< 15 \\
&<2.6 \\
&<2.0 \\
& \\
& \quad 4.6 \\
&<5.0 \\
&<5.0 \\
&<5.0 \\
&<10 \\
&<0.35 \\
& \quad 508\end{aligned}$ & $\begin{aligned} &< 15 \\
&<2.6 \\
&<2.0 \\
& \\
& \quad 4.5 \\
&<5.0 \\
&<5.0 \\
&<5.0 \\
&<10 \\
&<0.35 \\
& \quad 450\end{aligned}$ & J3 & $\begin{array}{l}\mu \mathrm{g} / \mathrm{L} \\
\mu \mathrm{g} / \mathrm{L} \\
\mu \mathrm{g} / \mathrm{L} \\
\mu \mathrm{g} / \mathrm{L} \\
\mu \mathrm{g} / \mathrm{L} \\
\mu \mathrm{g} / \mathrm{L} \\
\mu \mathrm{g} / \mathrm{L} \\
\mu \mathrm{g} / \mathrm{L} \\
\mu \mathrm{g} / \mathrm{L} \\
\mu \mathrm{g} / \mathrm{L}\end{array}$ & $\begin{array}{l}\text { WA } \\
\text { WA } \\
\text { WA } \\
\text { WA } \\
\text { WA } \\
\text { WA } \\
\text { WA } \\
\text { WA } \\
\text { WA } \\
\text { WA }\end{array}$ & $\begin{array}{l}0 \\
0 \\
0 \\
0 \\
0 \\
0 \\
0 \\
0 \\
0 \\
0\end{array}$ \\
\hline
\end{tabular}

Note: Flagging levels, modifiers, and laboratory are for 4 th quarter 1992 data only. See Appendix B for flagging criteria.

- exceeded holding time for 4th quarter 1992.

- = exceeded final primary drinking water standard for 4th quarter 1992. 
Woll LFW 19 continued

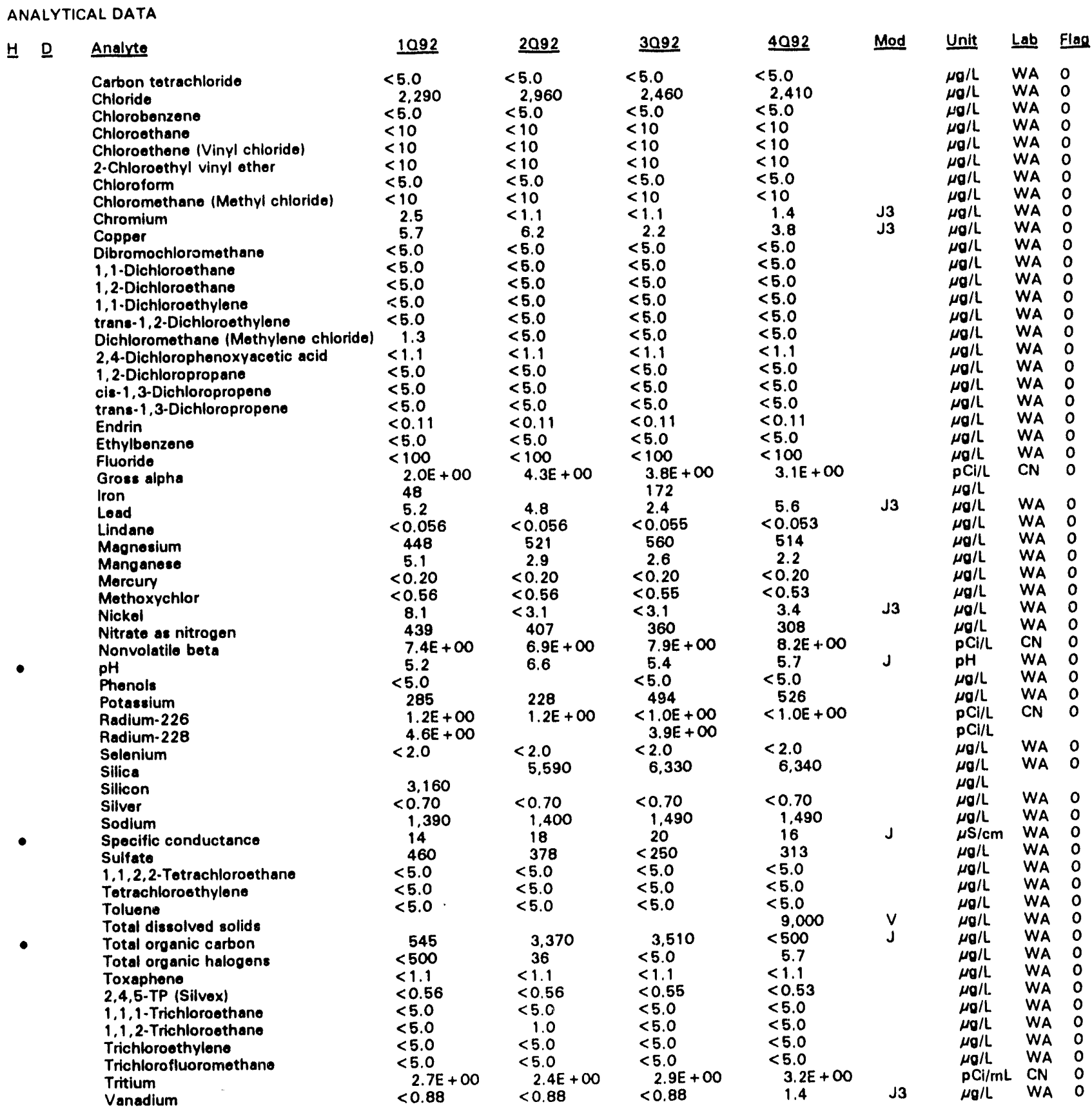

Note: Flagging levels, modifiers, and laboratory are for 4 th quarter 1992 data only. See Appendix B for flagging criteria. - = exceeded holding time for 4th quarter 1992.

- = exceeded final primary drinking water standara for 4th quarter 1992. 
WSRC-TR-93-068

\section{WELL LFW 20}

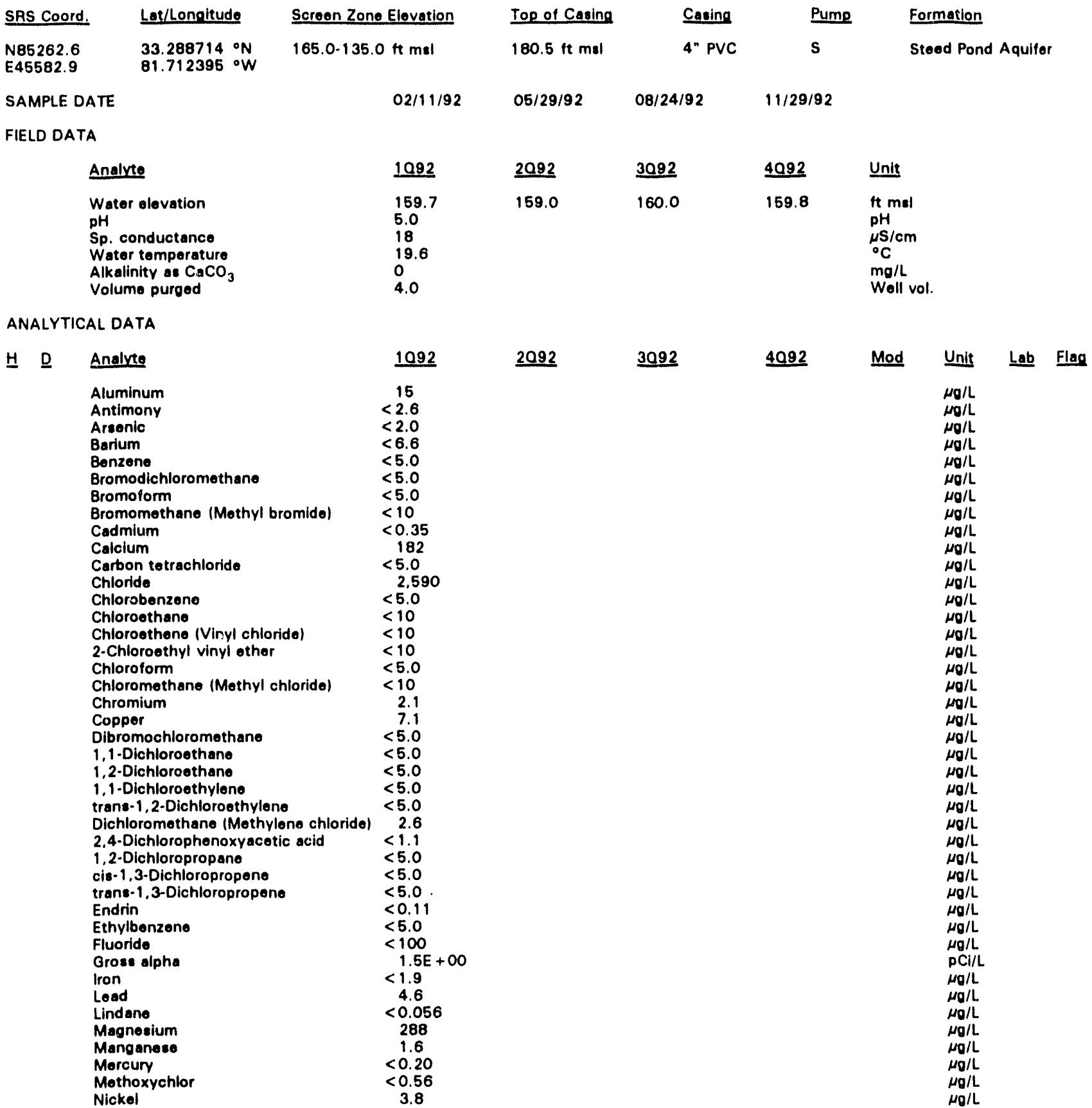

Note: Flagging lovels, modifiers, and laboratory are for 4th quarter 1992 data only. See Appendix B for flagging criteria.

- = exceeded holding time for 4th quarter 1992.

- exceeded final primary drinking water standard for 4th quarter 1992. 
WSRC-TR.93.068

Well LFW 20 continued

ANALYTICAL DATA

H D Analve

Nitrate as nitrogen

Nonvolatile beta

$\mathrm{pH}$

Phenols

Potassium

Radium-226

Radlum-228

Solenium

Silicon

Silver

Sodium

Specific conductance

Sulfate

1,1,2,2-Totrachloroethane

Tetrachloroethylene

Toluene

Total organic carbon

Total organic halogene

Toxaphene

2,4,5.TP (Silvex)

1,1,1-Trichloroethane

1.1.2.Trichloroothane

Trichloroethylene

Trichlorofluoromethane

Tritium

Vanadium
109

287

$5.3 E+00$

5.1

$<5.0$

380

6.0E.01

3. $2 E+\infty$

$<2.0$

2.870

$<0.70$

1,560

69

$<100$

$<5.0$

$<5.0$

$<5.0$

$<500$

$<500$

$<1.1$

$<0.67$

$<5.0$

$<5.0$

$<5.0$

$<5.0$

2. $2 \mathrm{E}+00$ $\underline{2092}$

$\underline{3092}$

1.1

\section{WELL LFW 21}

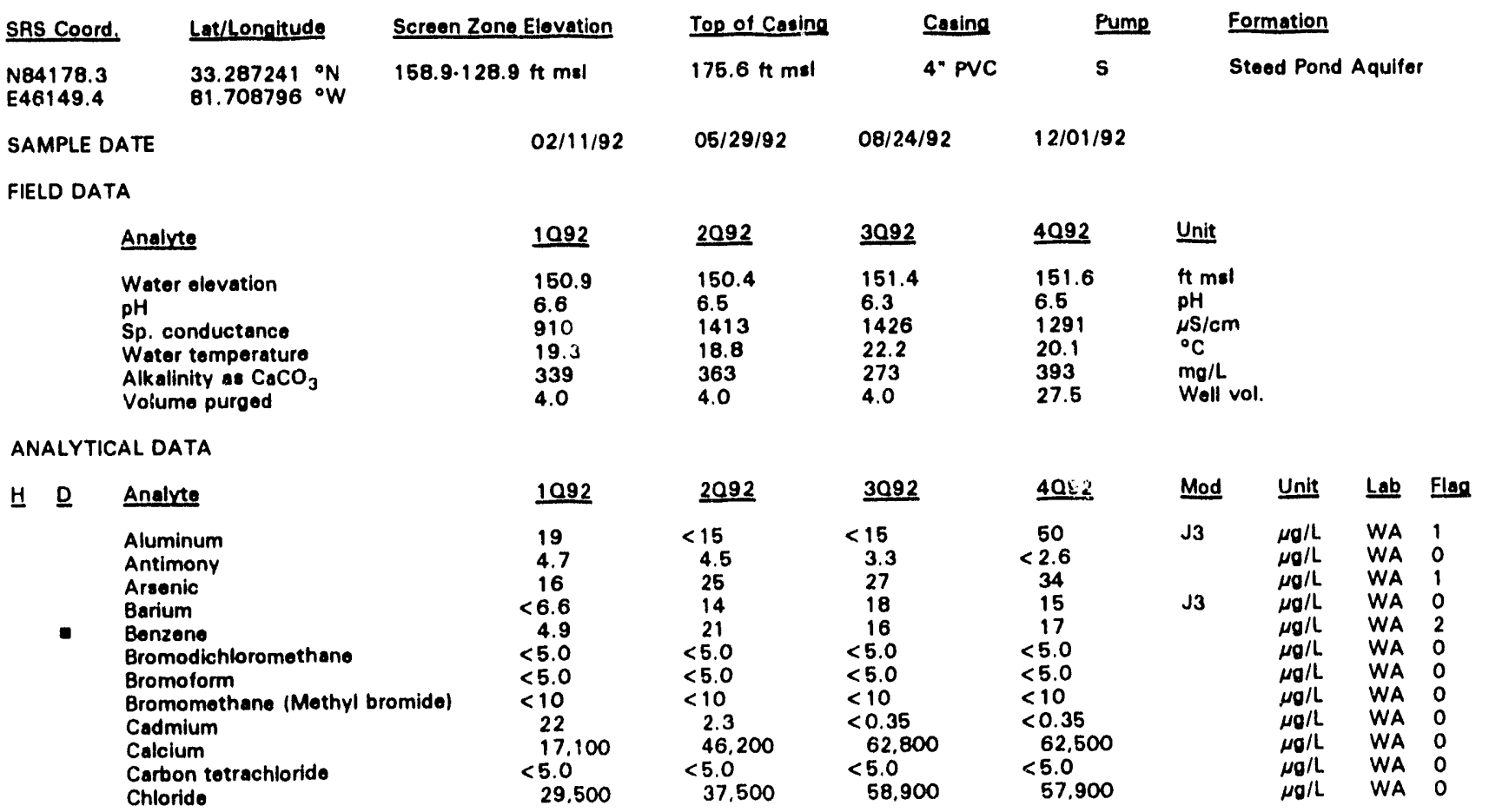

Note: Flagging levels, modifiers, and laboratory are for 4 th quarter 1992 data only. See Appendix B for flagging criteria.

- =xceeded holding tine for 4th quarter 1992.

- = exceeded final primary drinking water standard for 4th quarter 1992 . 


\begin{tabular}{|c|c|c|c|c|c|c|c|c|}
\hline D & Analis & 1092 & 2092 & 3092 & 4992 & Mod & Unit & $a b$ \\
\hline 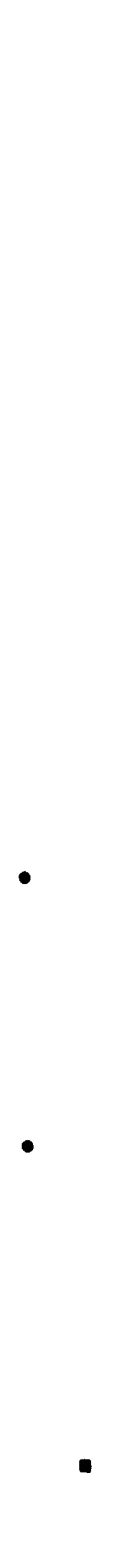 & 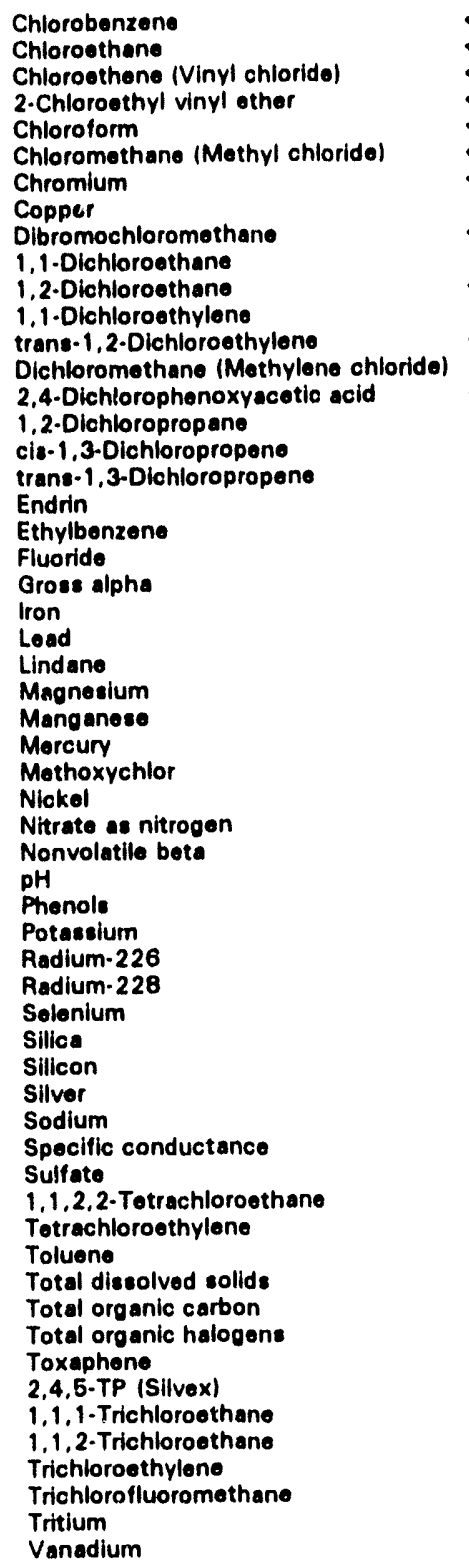 & 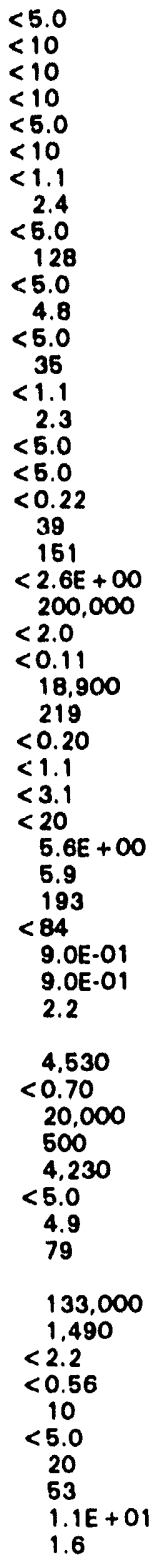 & 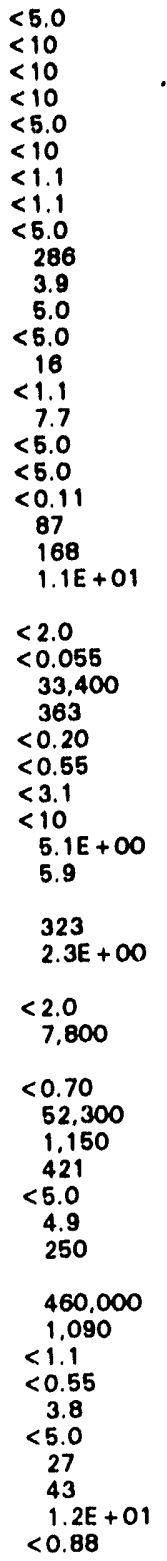 & 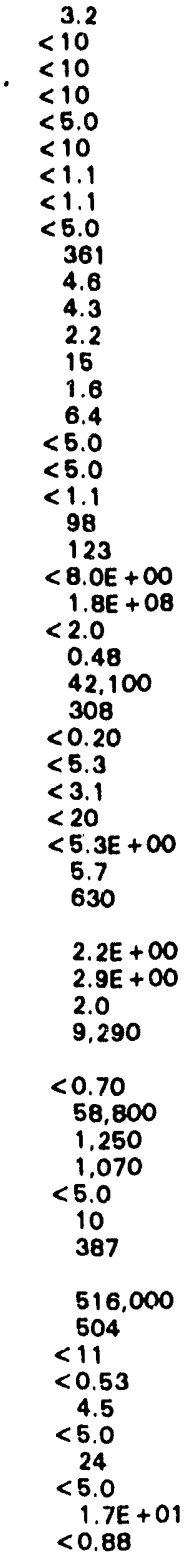 & 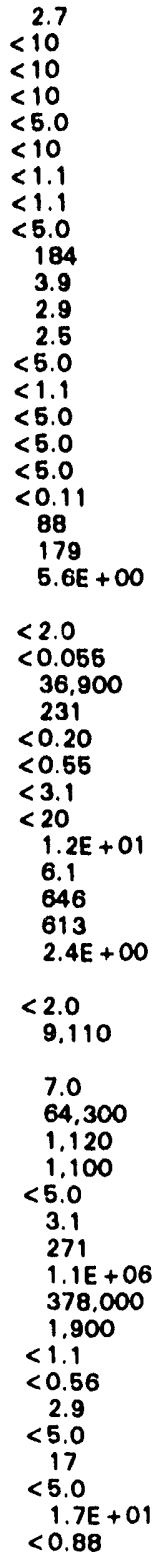 & $\begin{array}{l}\mathbf{J} \\
\mathbf{J} \\
\mathbf{J}\end{array}$ & 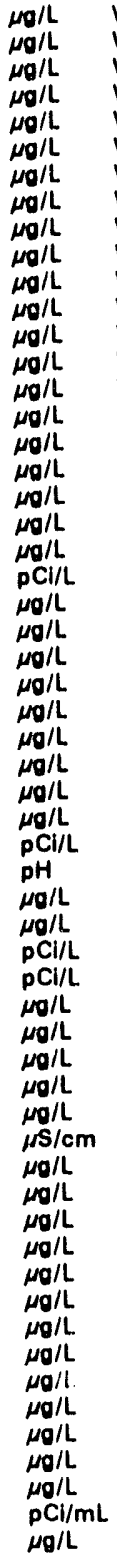 & $\begin{array}{l}\text { WA } \\
\text { WA } \\
\text { WA } \\
\text { WA } \\
\text { WA } \\
\text { WA } \\
\text { WA } \\
\text { WA } \\
\text { WA } \\
\text { WA } \\
\text { WA } \\
\text { WA } \\
\text { WA } \\
\text { WA } \\
\text { WA } \\
\text { WA } \\
\text { WA } \\
\text { WA } \\
\text { WA } \\
\text { WA } \\
\text { WA } \\
\text { CN } \\
\text { WA } \\
\text { WA } \\
\text { WA } \\
\text { WA } \\
\text { WA } \\
\text { WA } \\
\text { WA } \\
\text { WA } \\
\text { CN } \\
\text { WA } \\
\text { WA } \\
\text { WA } \\
\text { CN } \\
\text { WA } \\
\text { WA }\end{array}$ \\
\hline
\end{tabular}

Note: Flagging levels, modifiers, and laboratory are for 4 th quarter 1992 data only. Seo Appendix B for flagging criteria. - = exceeded holding time for 4th quarter 1992.

- =xceeded final primary drinking water standard for 4th quarter 1992. 
WSRC-TR-93.068

WELL LFW 22

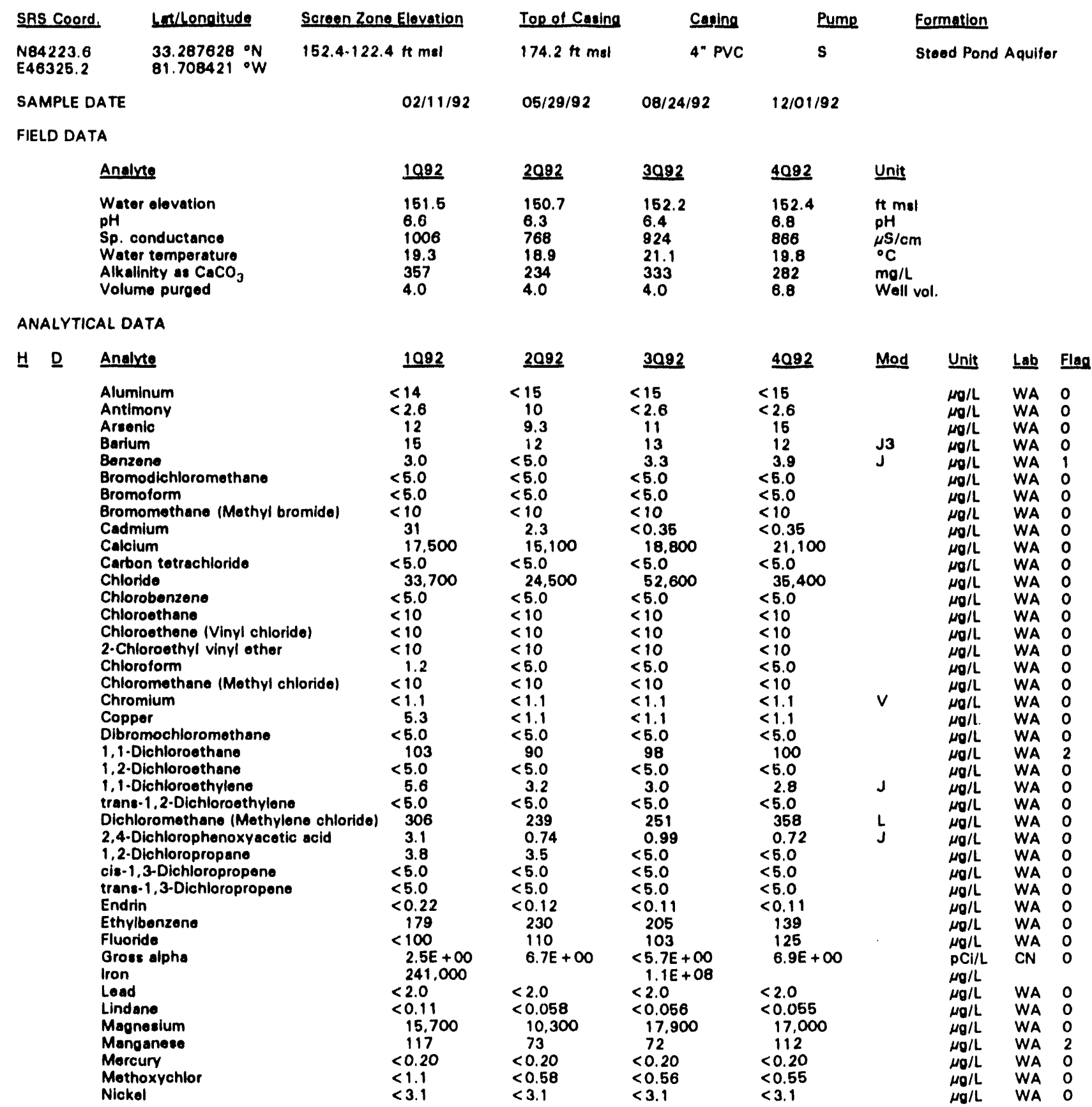

Note: Flagging levels, modifiers, and laboratory are for 4th quarter 1992 data only. See Appendix B for flagging criteria.

- = exceeded holding time for 4th quarter 1992.

- = exceeded final primary drinking water standard for 4th quarter 1992. 
Woll LFW 22 continued

ANALYTICAL DATA

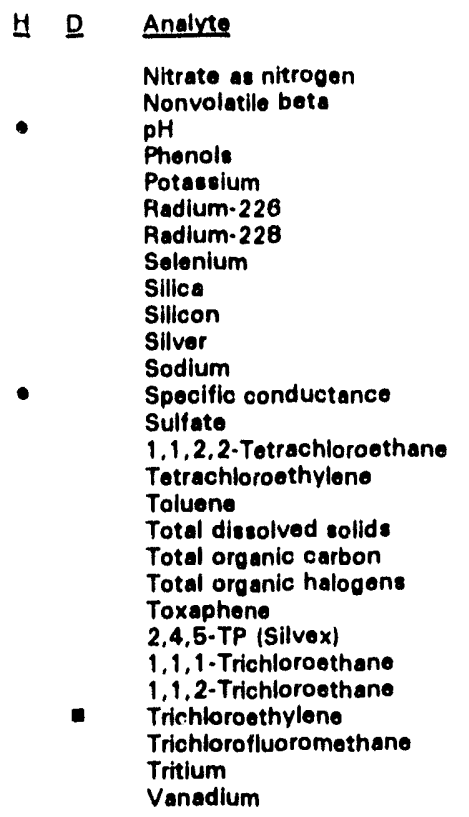

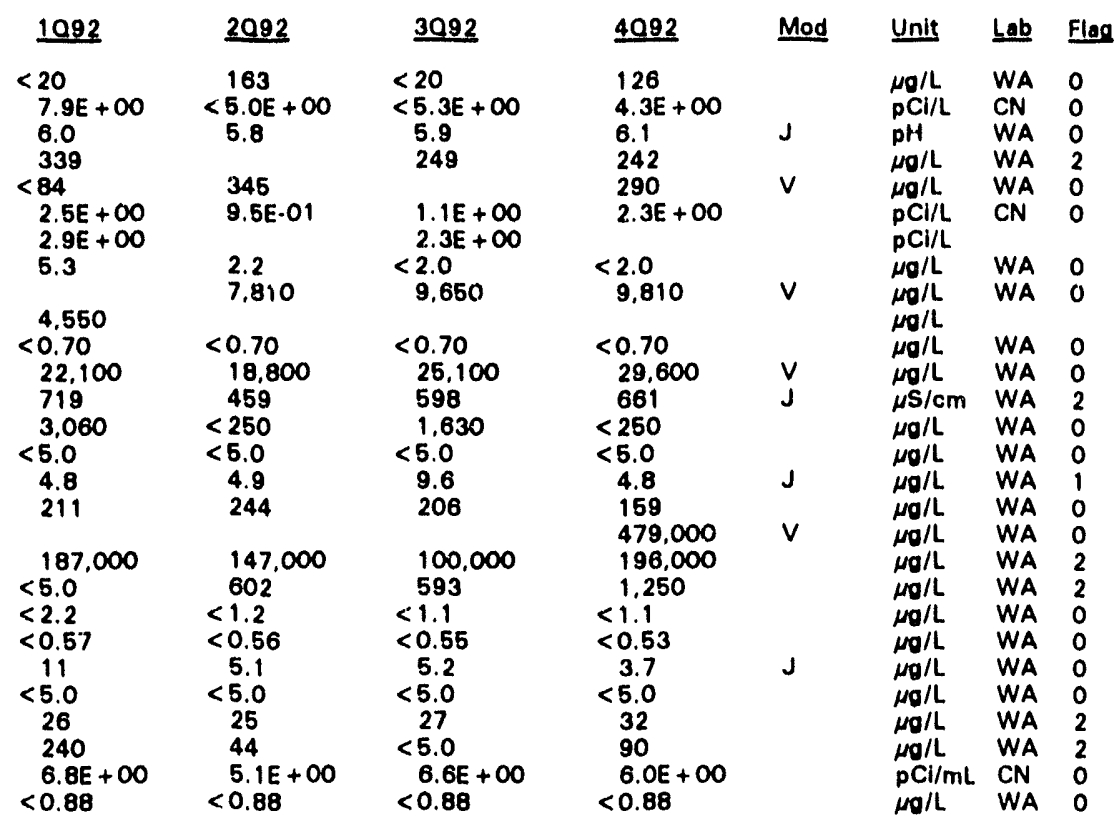

\section{WELL LFW 23}

\begin{tabular}{|c|c|}
\hline SAS Coord. & Lot/Longitude \\
\hline $\begin{array}{l}\text { N84251.3 } \\
\text { E46456.1 }\end{array}$ & $\begin{array}{l}33.287903{ }^{\circ} \mathrm{N} \\
81.708131^{\circ} \mathrm{W}\end{array}$ \\
\hline
\end{tabular}

\section{Screen Zone Elevation}

155.1-125.1 ft msl

SAMPLE DATE

$02 / 10 / 92$

$\frac{\text { Top of Casing }}{171.8 \mathrm{tt} \mathrm{msl}}$

$\begin{array}{ll}\text { Casing } & \text { Pump } \\ \text { 4" PVC } & \mathrm{S}\end{array}$

Formation

FIELD DATA

Analvte
Water elevation
pH
Sp. conductance
Water temperature
Alkalinity as $\mathrm{CaCO}_{3}$
Volume purged

1092
151.8
4.1
26
18.8
0
4.0

2092
151.0
4.2
25
18.4
0
4.0

\begin{tabular}{cc}
1092 & $\underline{2092}$ \\
\hline 18 & 21 \\
5.0 & $<2.6$ \\
$<2.0$ & $<2.0$ \\
$<6.6$ & $<4.0$ \\
$<5.0$ & $<5.0$ \\
$<5.0$ & $<5.0$ \\
$<5.0$ & $<5.0$ \\
$<10$ & $<10$ \\
0.50 & 0.45 \\
450 & 416
\end{tabular}

\begin{tabular}{cc}
1092 & $\underline{2092}$ \\
\hline 18 & 21 \\
5.0 & $<2.6$ \\
$<2.0$ & $<2.0$ \\
$<6.6$ & $<4.0$ \\
$<5.0$ & $<5.0$ \\
$<5.0$ & $<5.0$ \\
$<5.0$ & $<5.0$ \\
$<10$ & $<10$ \\
0.50 & 0.45 \\
450 & 416
\end{tabular}

$\begin{aligned} & 3092 \\ &< 15 \\ &<2.6 \\ &<2.0 \\ & 6.2 \\ &<5.0 \\ &<5.0 \\ &<5.0 \\ &<10 \\ & 0.97 \\ & 419\end{aligned}$

3092
152.8
4.6
27
21.1
0
4.0

$\begin{array}{ll}4092 & \text { Unit } \\ 152.8 & \mathrm{ft} \mathrm{msl} \\ 4.8 & \mathrm{pH} \\ 29 & \mu \mathrm{S} / \mathrm{cm} \\ 19.7 & { }^{\circ} \mathrm{C} \\ 0 & \mathrm{mg} / \mathrm{L} \\ 13.1 & \text { Well vol. }\end{array}$

ANALYTICAL DATA

H D Analve

Aluminum

Antimony

Arsenic

Benzene

Bromodichloromethane

Bromomethane (Mothyl bromide)

Cadmium

450

$\begin{aligned} & 4092 \\ & 43 \\ &< 2.0 \\ &< 2.0 \\ & 5.7 \\ &<1.0 \\ &<1.0 \\ &<1.0 \\ &<1.0 \\ & 0.38 \\ & 434\end{aligned}$

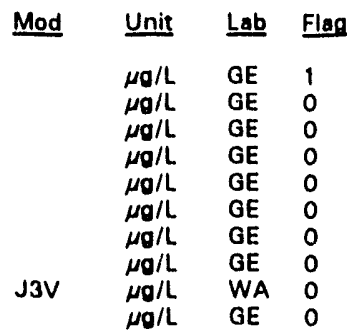

Note: Flagging levels, modifiers, and laboratory are for 4th quarter $19 y 2$ data only. See Appendix B for flagging criteria. - =xceeded holding time for 4th quarter 1992.

- =xceeded final primary drinking water standard for 4th quarter 1992. 
Well LFW 23 continued

\section{ANALYTICAL DATA}

H D Anaivte

Carbon tetrachioride

Chloride

Chlorobenzene

Chloroethane

Chloroethene (Vinyl chloride)

2-Chloroethyl vinyl ether

Chloroform

Chloromethane (Methyl chloride)

Chromium

Copper

Dibromochloromethane

1.1-Dichloroethane

1,2-Dichloroethane

1,1-Dichloroethylene

trans-1,2-Dichloroethylene

Dichloromethane (Methylene chloride)

2,4-Dichlorophenoxyacetic acid

1.2-Dichloropropane

cis-1,3-Dichloropropene

trans-1,3-Dichloropropene

Endrin

Ethylbenzene

Fluoride

Gross alpha

Iron

Lead

Lindano

Magnesium

Manganose

Mercury

Methoxychlor

Nickel

Nitrate as nitrogen

Nitrate-nitrite as nitre on

Phenols

Potassium

Radium-226

Radium-228

Selenium

Silica

Silicon

Silver

Sodium

- Specific conductance

Sulfote

1,1,2,2-Tetrachloroethane

Tetrachloroethylene

Tolueno

Total dissolved solids

Total organic carbon

Total organic halogens

Toxaphene

2,4,5-TP (Silvox)

1,1,1-Trichloroethane

1,1,2-Trichloroethane

Trichloroethylene

Trichlorofluoromethane

Tritium

Vanadium

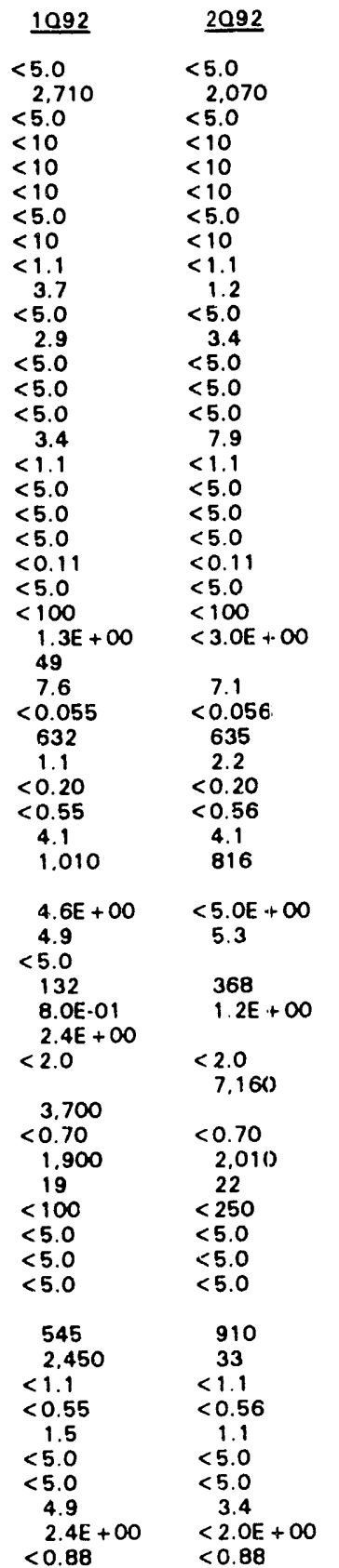

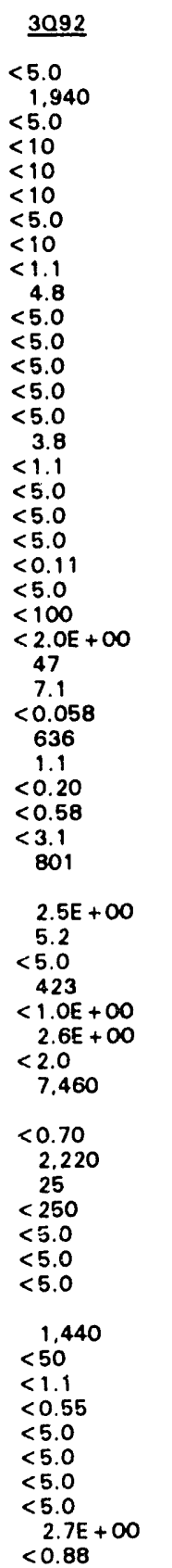

$\begin{aligned} & 4092 \\ &< 1.0 \\ & 1.820 \\ &<1.0 \\ & 3.2 \\ &<1.0 \\ &<1.0 \\ &<1.0 \\ &<1.0 \\ & 1.8 \\ & 4.7 \\ &<1.0 \\ & 6.8 \\ &<1.0 \\ &<1.0 \\ &<1.0 \\ & 6.9 \\ &<0.30 \\ &<1.0 \\ &<1.0 \\ &<1.0 \\ &<0.0060 \\ &<1.0 \\ &<100\end{aligned}$

$5.0 E+00$

9.1

$<0.0050$

714

1.3

$<0.20$

$<0.50$

4.9
1.200

1.200

4. $1 E+00$

5.2

$<5.0$

430

$1.4 E+\infty$

$<2.0$

$<.0$
8,020

0.88

2,270
27

$\begin{aligned} & 27 \\ < & 250\end{aligned}$

$<1.0$

$<1.0$

$<1.0$

$\begin{array}{lllll} & & \mu \mathrm{g} / \mathrm{L} & \mathrm{GE} & 0 \\ 84,000 & \mathrm{~J} & \mu_{\mathrm{g} / \mathrm{L}} & \mathrm{GE} & 0\end{array}$

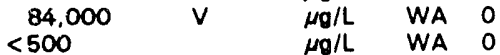

134

$<0.24$

$<0.090$

1.4
$<1.0$

$<1.0$

2.8E + 00

$\begin{array}{lll}\mu \mathrm{g} / \mathrm{L} & \mathrm{GE} & 0 \\ \mu \mathrm{g} / \mathrm{L} & \text { WA } & 0\end{array}$

$\mu g / L$ GE 0

$\mu \mathrm{g} / \mathrm{L} \quad \mathrm{GE}$

$\mu \mathrm{g} / \mathrm{L} \quad \mathrm{GE}$

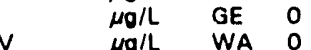

J3

$\mu g / L$ WA 0

$\begin{array}{lll}\mu \mathrm{g} / \mathrm{L} & \mathrm{GE} & 0 \\ \mu \mathrm{g} / \mathrm{L} & \mathrm{GE} & 1\end{array}$

$\mu \mathrm{g} / \mathrm{L}$ GE 0

$\mu g / L$ GE 0

$\mu g / L$ WA 0

$\mu_{g / L}$. GE 0

$\mu \mathrm{g} / \mathrm{L}$ GE 0

$\mu g / L$ GE 0

$\mu \mathrm{g} / \mathrm{L}$

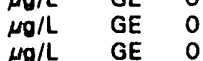

$\mu \mathrm{g} / \mathrm{L} \quad \mathrm{GE}$

$\mu \mathrm{g} / \mathrm{L}$

$\mu \sigma / L$ WA 1

$\mu \sigma / L$ GE 0

$\mu \sigma / L$ GE

$\mu \mathrm{g} / \mathrm{L}$ GE 0

$\begin{array}{lll}\mu \mathrm{g} / \mathrm{L} & \mathrm{GE} & 0 \\ \mu \mathrm{g} / \mathrm{L} & \text { WA } & 0\end{array}$

$\mu \sigma / L$ WA 0

$\mu g / L \quad G E \quad O$

$\mathrm{pCi} / \mathrm{L}$ GP 0

pH WA 0

$\mu g / L$ WA

$\mu \mathrm{g} / \mathrm{Ci} / \mathrm{L} \quad \mathrm{GP}$

$\mathrm{pCi} / \mathrm{L}$

$\begin{array}{lll}\mu \mathrm{g} / \mathrm{L} & \mathrm{GE} & 0 \\ \mu \mathrm{g} / \mathrm{L} & \mathrm{GE} & 0\end{array}$

$\mu \mathrm{g} / \mathrm{L}$

$\mu \mathrm{g} / \mathrm{L}$ WA

WA 0

J $\mu g / L$ WA 0

$<0.88$

1.3

$\sqrt{3}$

$\mu g / L$ WA

HQIL GE

$\mu \mathrm{g} / \mathrm{L}$ GE

$\mu \mathrm{g} / \mathrm{L} \quad \mathrm{GE} \quad 0$

$\mu \mathrm{g} / \mathrm{GE}$

PCi/mL CN

$\mu \mathrm{g} / \mathrm{L}$ WA

Note: Flagging levels, modifiers, and laboratory are for 4th quarter 1992 data only. See $/$ ppendix B for flagging criteria.

- = exceeded holding time for 4th quarter 1992.

a exceeded final primaiy drinking water etandard for 4th quarter 1992. 
WSRC-TR-93-068

\section{WELL LFW 24}

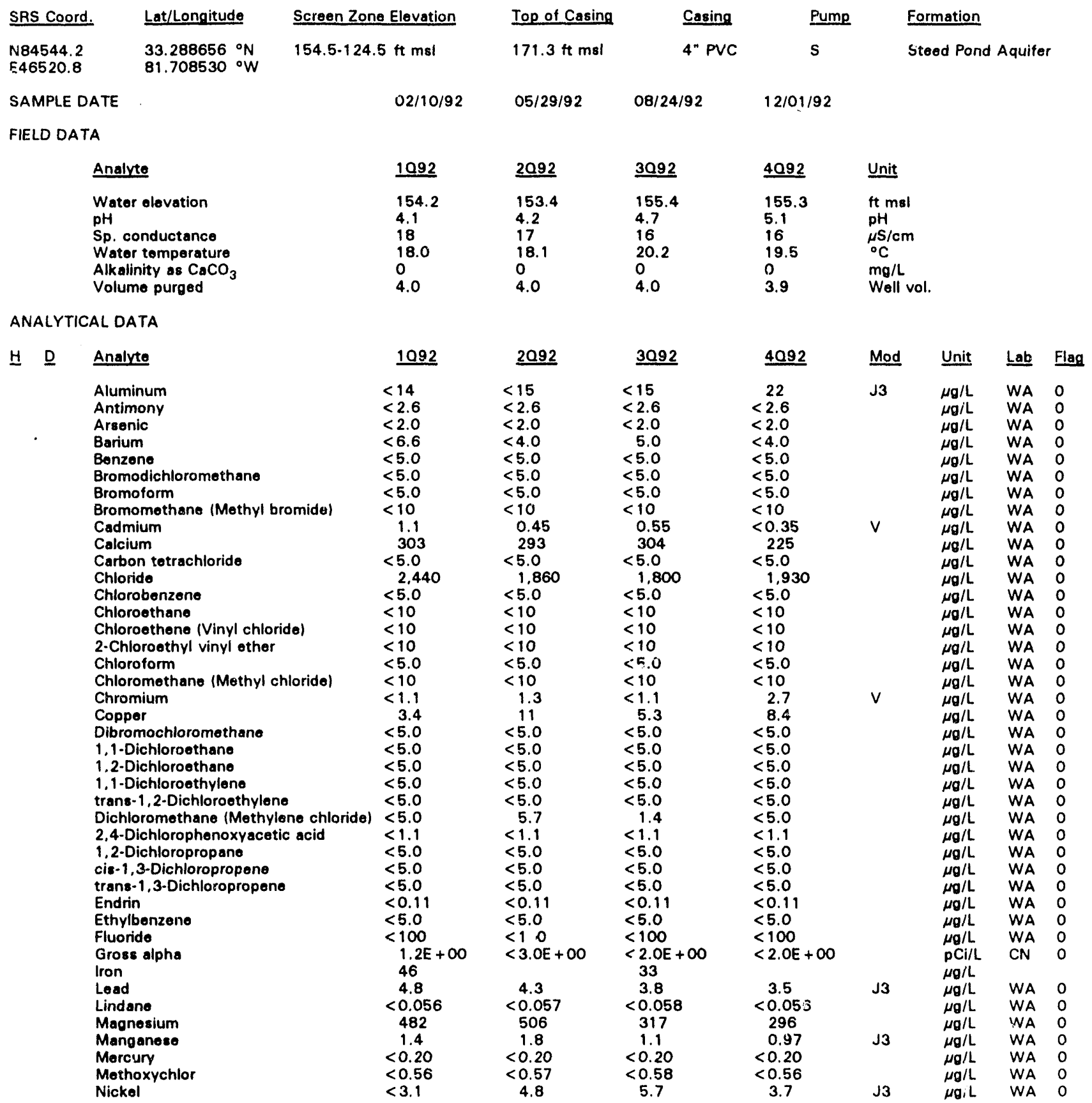

Note: Flagging levels, modifiers, and laboratory are for 4th quarter 1992 data only. See Appendix B for flagging criteria.

- =xceeded holding time for 4th quarter 1992.

- =xceeded final primary drinking water standard for 4th quarter 1992. 
Well LFW 24 continued

ANALYTICAL DATA

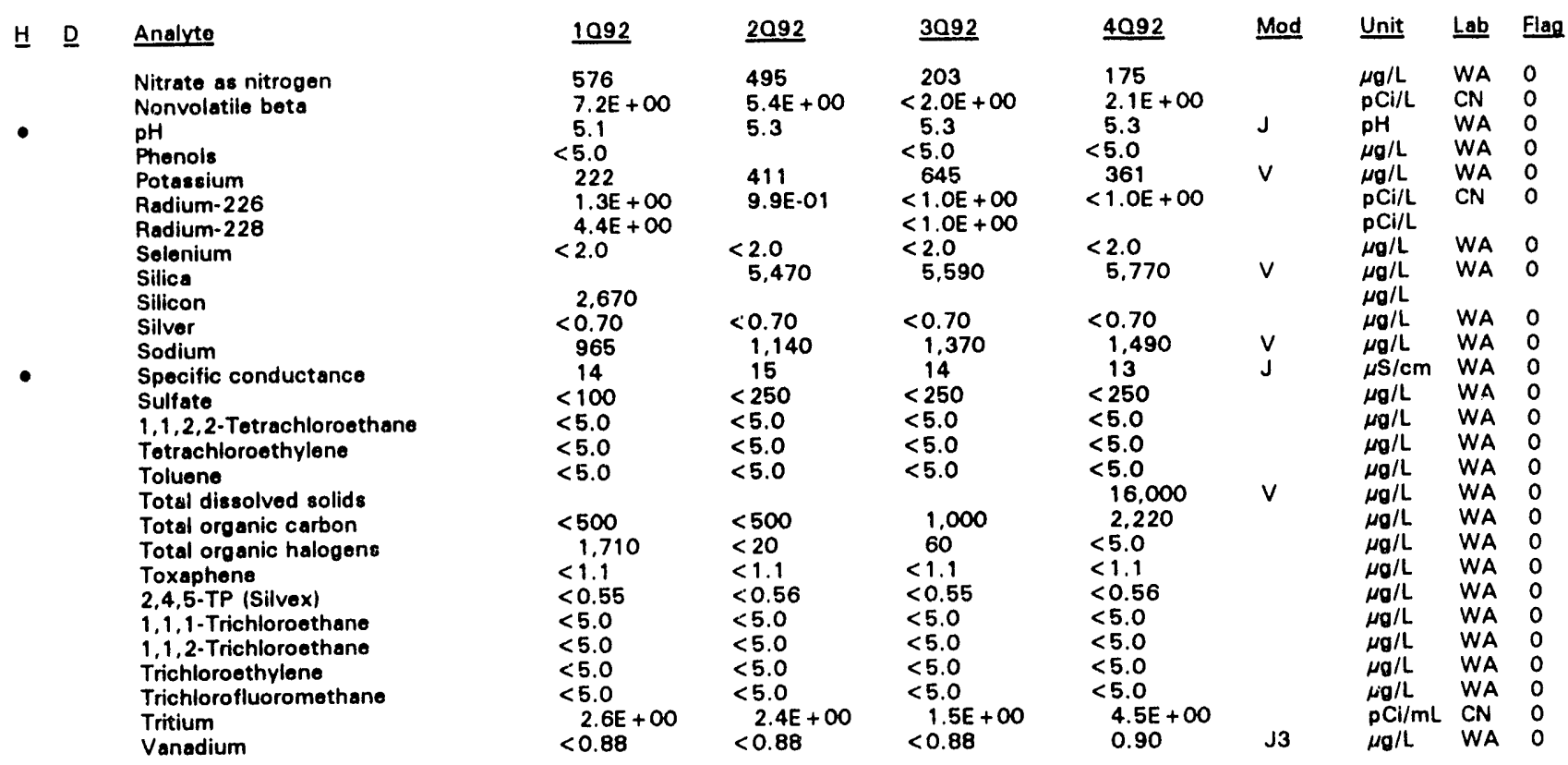

\section{WELL LFW 25}

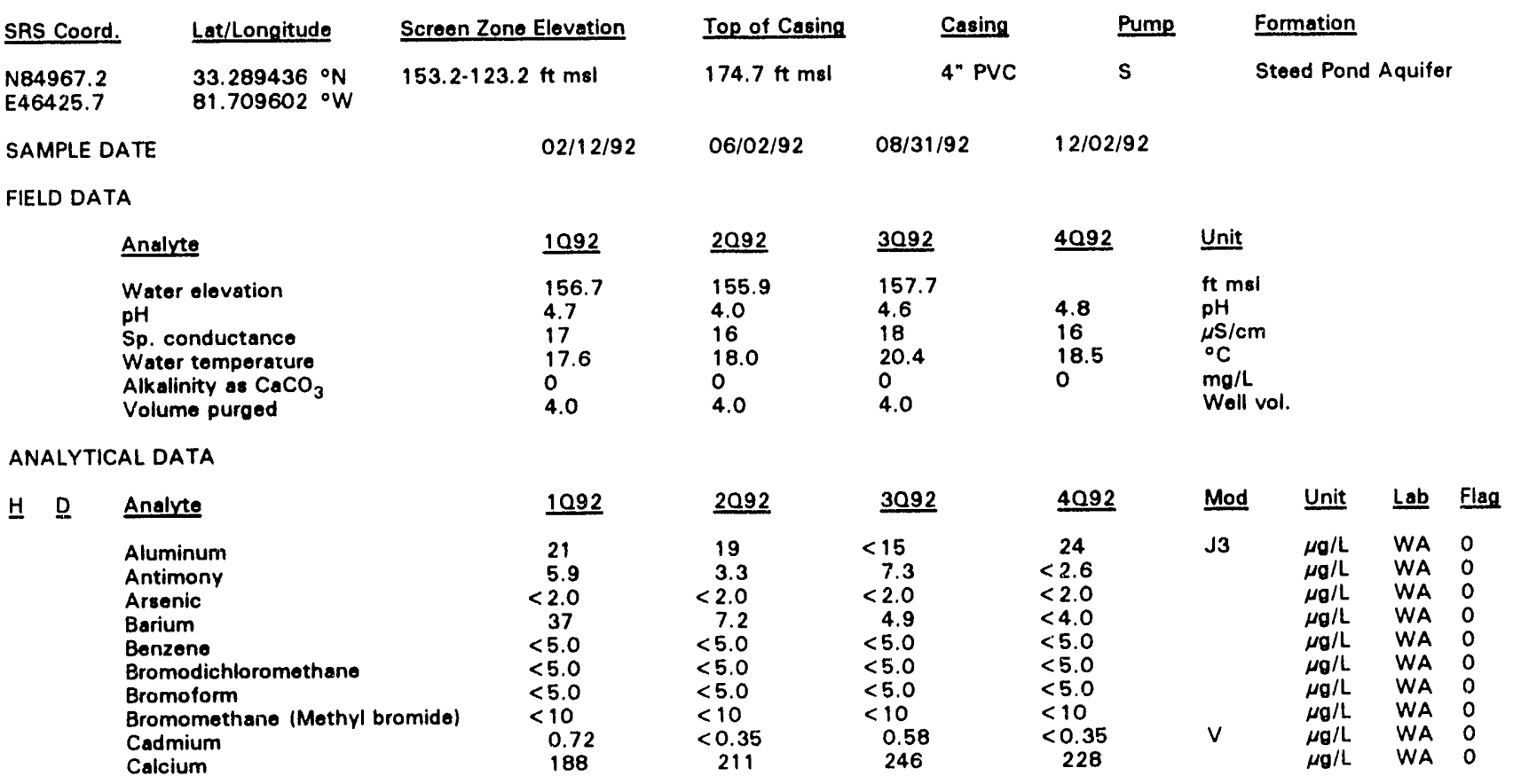

Note: Flagging levels, modifiers, and laboratory are for 4 th quarter 1992 data only. See Appendix B for flagging criteria.

- = exceeded holding time for 4th quarter 1992.

- =xceeded final primary drinking water standard for 4th quarter 1992. 
Well LFW 25 continued

ANALYTICAL DATA

H D Analyte

Carbon tetrachloride

Chloride

Chlorobenzene

Chloroethane

Chloroethene (Vinyl chloride)

2-Chloroethyl vinyl ether

Chloroform

Chloromethane (Methyl chloridel

Chromium

Copper

Dibromochloromethane

1.1.Dichloroethane

1,2-Dichloroethane

1,1-Dichloroethylene

trans-1,2-Dichloroethylene

Dichloromethane (Methylene chloride)

2,4-Dichlorophenoxyacetic acid

1,2-Dichloropropane

cis-1,3-Dichloropropene

trans-1,3-Dichloropropene

Endrin

Ethylbenzene

Fluorido

Gross alpha

Iron

Lead

Lindane

Magnesium

Manganese

Mercury

Methoxychlor

Nickol

Nitrate as nitrogen

Nonvolatilo beta

pH

Phenols

Potassium

Radium-226

Radium-228

Selenium

Silica

Silicon

Silver

Sodium

Specific conductance

Sulfate

1,1,2,2-Tetrachloroothane

Tetrachloroethylene

Toluene

Total dissolved solids

Total organic carbon

Total organic halogens

Toxaphene

2,4,5-TP (Silvex)

1,1,1-Trichloroethane

1,1,2-Trichloroethane

Trichloroethylene

Trichlorofluoromethane

Tritium

Vanadium

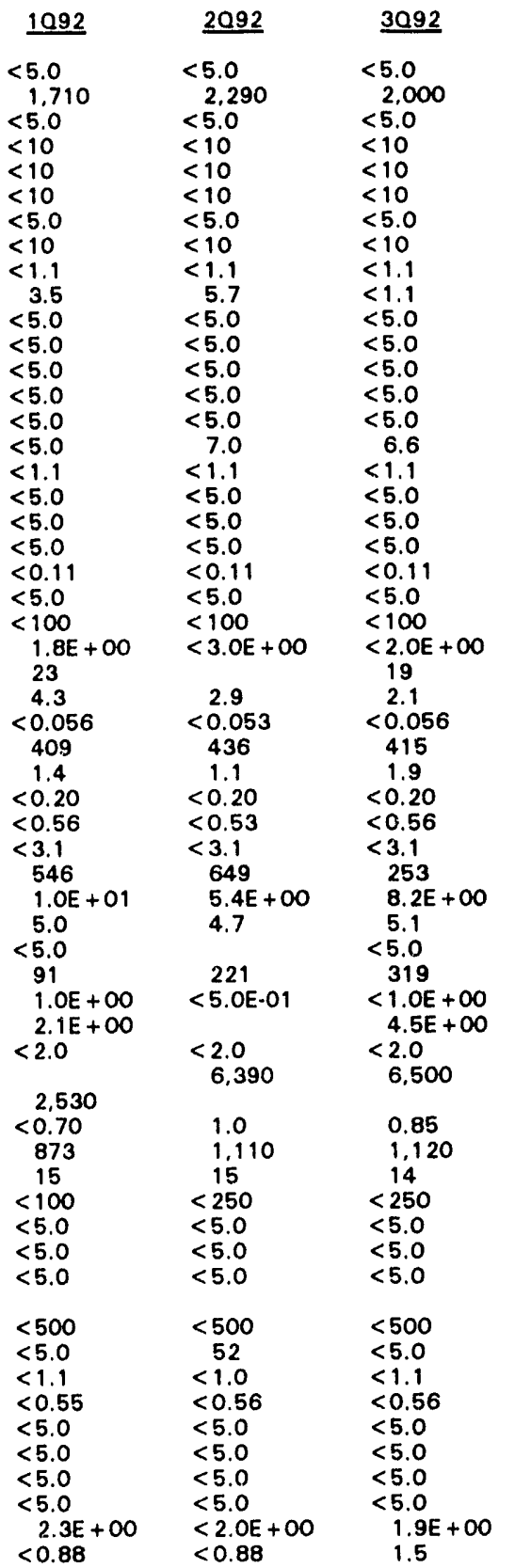

$\begin{aligned} & \underline{4092} \quad \text { Mod } \\ < & 5.0 \\ & 1,760 \\ < & 5.0 \\ < & 10 \\ < & 10 \\ < & 10\end{aligned}$

$\mu \sigma / L$ WA 0

$\mu g / L$ WA 0

$\mu g / L$ WA

$\mu g / L$ WA

$\mu g / L \quad W A$

$\mu \mathrm{g} / \mathrm{W}$ WA

$<5.0$

$<10$

2.2
$<1.1$

$<1.1$
$<5.0$

$<5.0$

$<5.0$

$<5.0$

$<5.0$

$<5.0$

$<5.0$

$<5.0$

$<5.0$

$<5.0$

$2.6 \mathrm{E}+00$

2.4
$<0.055$

433

1.6

$<0.20$
$<0.55$

5.0

311

$6.4 \mathrm{E}+00$

5.1

$<5.0$

343

$\mu \mathrm{g} / \mathrm{L}$

$\mu$ WA

$\mu g / L$ WA

$\mu g / L$ WA

$\mu g / L$ WA

$\mu g / L$ WA

$\mu g / L$ WA

$\mu g / L$ WA

$\mu \mathrm{g} / \mathrm{L}$ WA

$\mu g / L$ WA

$\mu g / L$ WA

$\mu g / L$ WA

$\mu g / L$ WA

$\mu g / L$ WA

$\mu g / L$ WA

$\mu \mathrm{g} / \mathrm{L}$ WA

$\mathrm{pCi} / \mathrm{L}$

$\mu \mathrm{g} / \mathrm{L}$

WA 0

$\mu \mathrm{g} / \mathrm{L}$ WA

$\mu / L$ WA

Ha/L WA

$\mu g$ WA

$\mu g / L$ WA 0

$\mu g / L$ WA

$\mu g / L$ WA

$\mu g / L$ WA

$\mathrm{pCi} / \mathrm{CN} \quad 0$

$\mathrm{pH}$

$\mu g / L$ WA 0

$\mu g / L$ WA 0

$6,580 \quad \vee \quad \mu g / L$ WA 0

$1.2 \quad J 3 \quad \mu / L$ WA

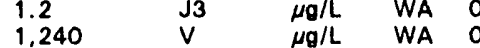

$\begin{array}{lllll}1,240 & V & \mu \mathrm{g} / \mathrm{L} & \text { WA } & 0 \\ 15 & \mathrm{~J} & \mu \mathrm{S} / \mathrm{cm} & \text { WA } & 0\end{array}$

$<250$

$<5.0$

$<5.0$

$<5.0$

20,000

$<500$

$<5.0$

$<1.1$

$<0.56$

$<5.0$

$<5.0$

$<5,0$

$<5.0$

2. $3 E+00$

$\mu g / L$

$\mu \mathrm{g} / \mathrm{L}$ WA

$\mu g / L$ WA 0

$\mu \mathrm{g} / \mathrm{L}$ WA 0

$\mu \mathrm{g} / \mathrm{L}$ WA 0

$\mu g / L$ WA 0

$\mu g / L$ WA 0

$\mu g, L$ WA 0

$\begin{array}{lll}\mu g i L & W A & 0 \\ \mu g / L & \text { WA } & 0\end{array}$

$\mu g / L$ WA 0

$\mu \mathrm{g} / \mathrm{L}$ WA

$\mu g / L$ WA 0

1.9

J3

0
0
0
0
0
0
0
0
0
0
0
0
0
0
0
0
0
0
0


WSRC-TR-93-068

\section{WELL LFW 26}

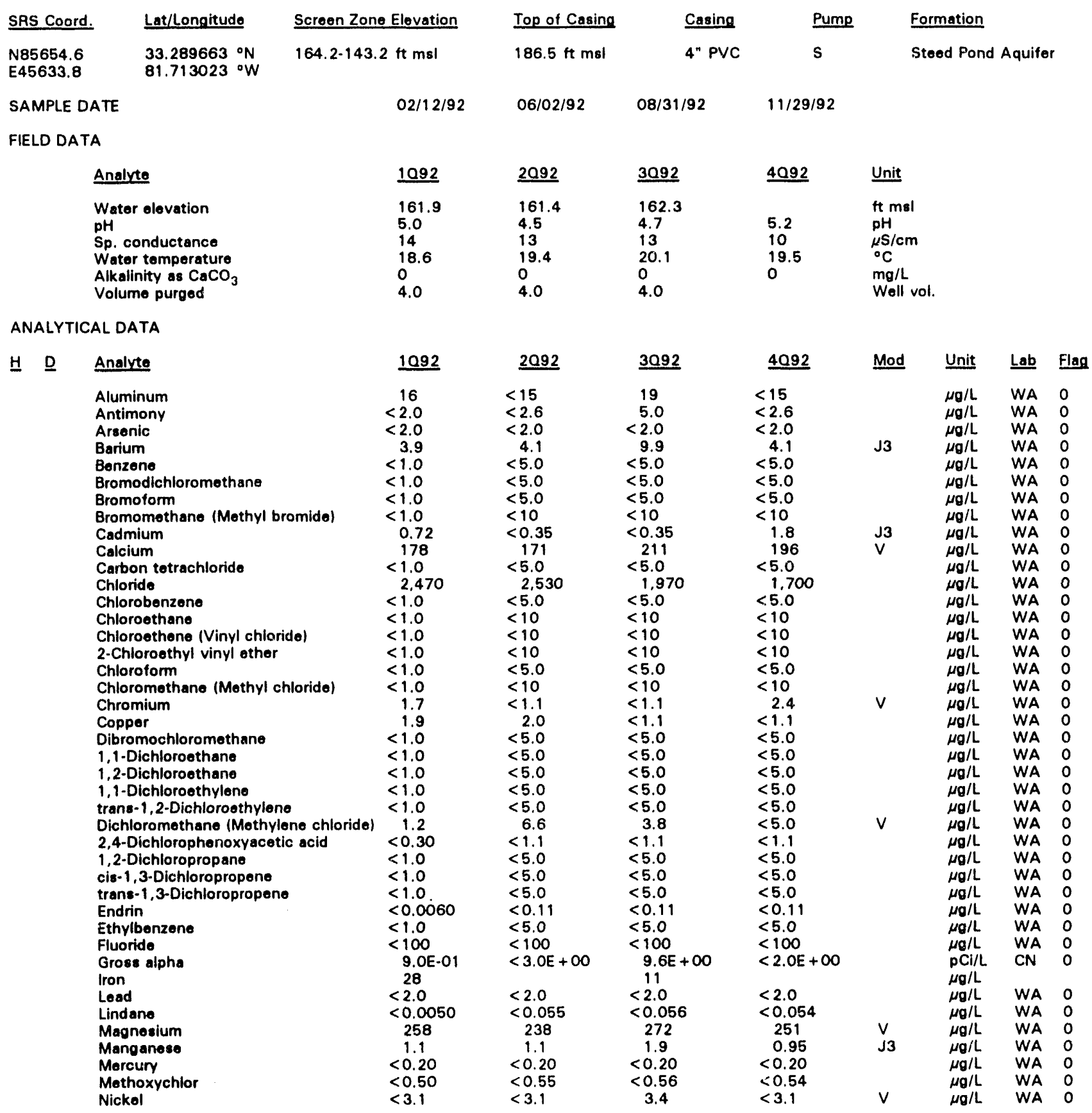

Note: Fegging levels, modifiers, and laboratory are for 4 th quarter 1992 data only. See Appendix B for flagging criteria.

- exceeded holding time for 4th quarter 1992.

a = exceeded final primary drinking water standard for 4th quarter 1992. 
Well LFW 26 continued

ANALYTICAL DATA

\begin{tabular}{|c|c|}
\hline$\underline{D}$ & Analyte \\
\hline & $\begin{array}{l}\text { Nitrate as nitrogen } \\
\text { Nonvolatile beta } \\
\text { pH } \\
\text { Phenols } \\
\text { Potassium } \\
\text { Radium-226 } \\
\text { Radium-228 } \\
\text { Solenium } \\
\text { Silica } \\
\text { Silicon } \\
\text { Silver } \\
\text { Sodium } \\
\text { Specific conductance } \\
\text { Sulfate } \\
1,1,2,2 \text {-Totrachloroethane } \\
\text { Totrachloroothylone } \\
\text { Toluene } \\
\text { Total alpha-emitting radium } \\
\text { Total dissolved solids } \\
\text { Total organic carbon } \\
\text { Total organic halogons } \\
\text { Toxapheno } \\
2,4,5-\text { TP (Silvex) } \\
1,1,1 \text {-Trichloroethane } \\
\text { 1,1,2-Trichloroethane } \\
\text { Trichloroethylene } \\
\text { Trichlorofluoromethane } \\
\text { Tritium } \\
\text { Vanadium }\end{array}$ \\
\hline
\end{tabular}

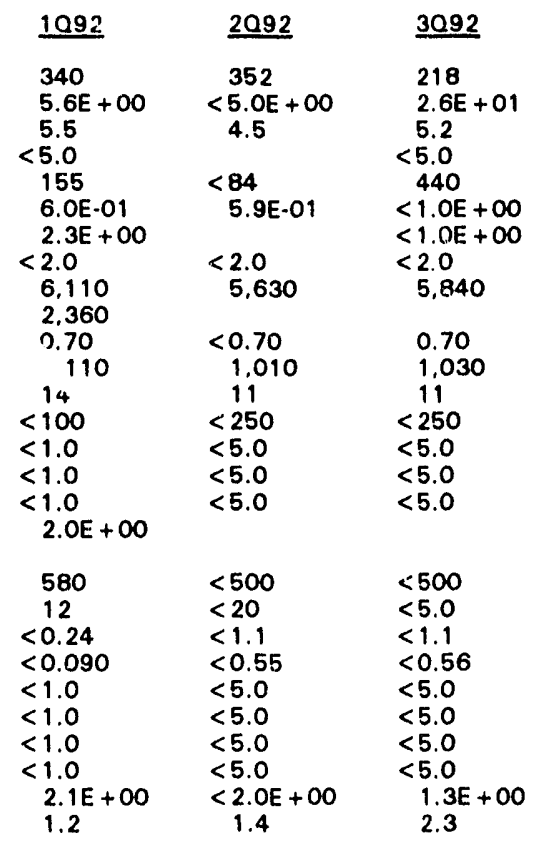

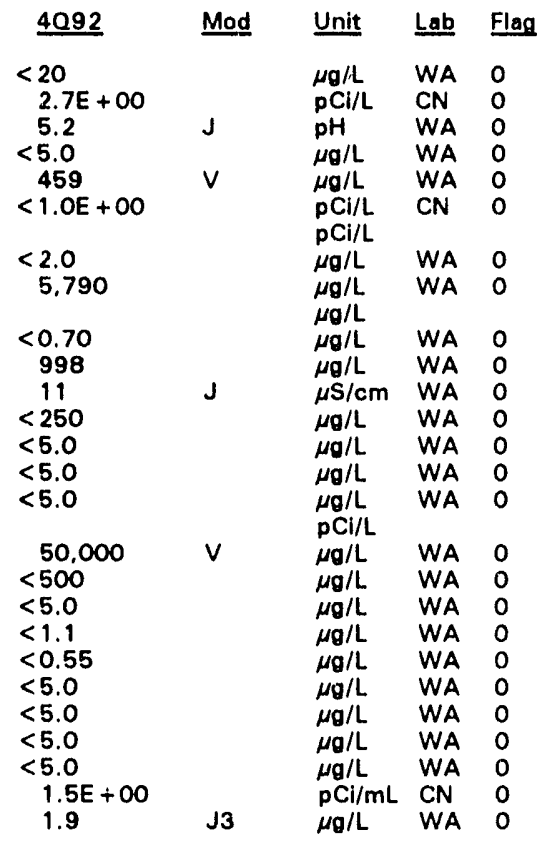

\section{WELL LFW 27}

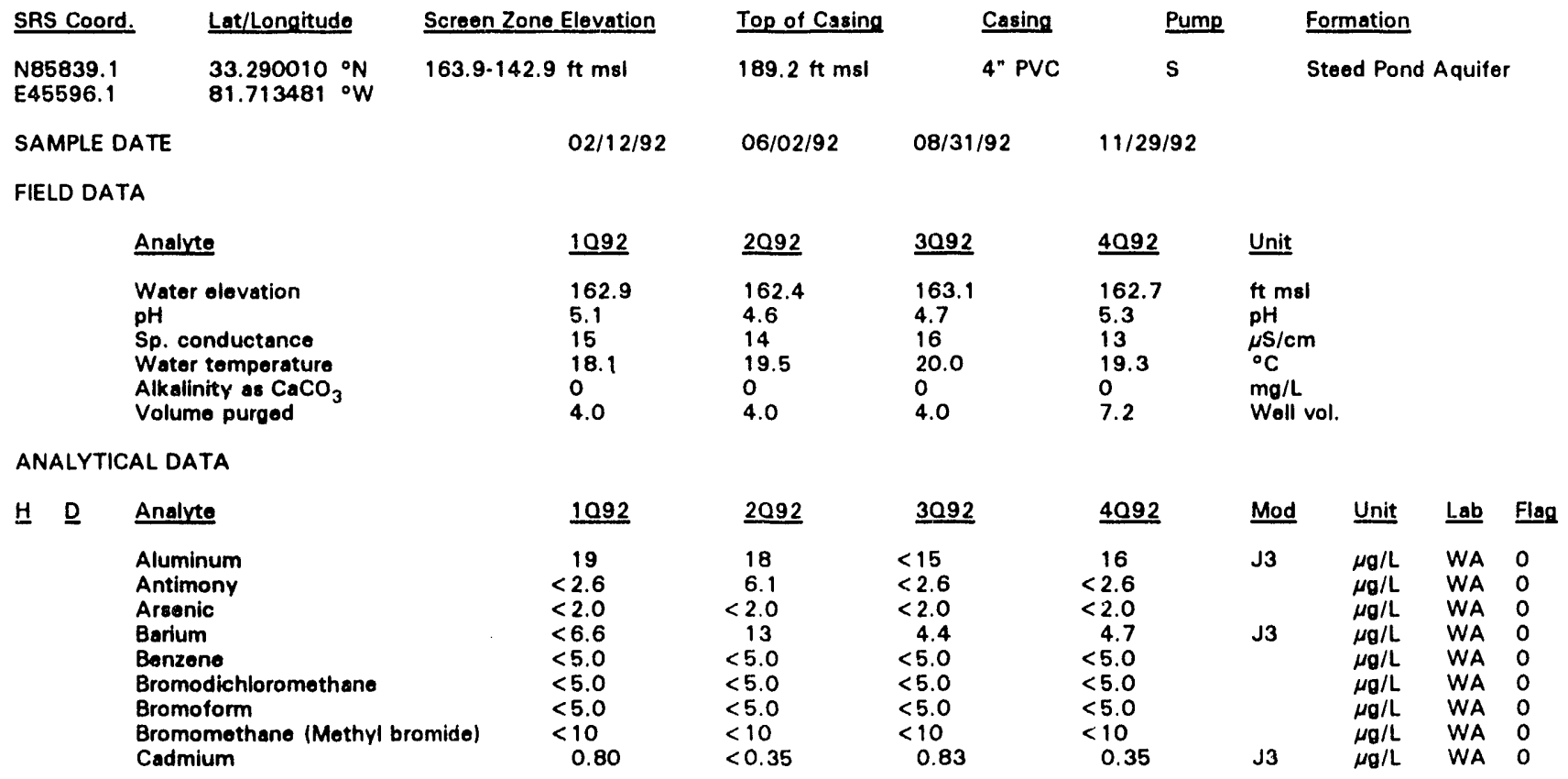

Note: Flagging levels, modifiers, and laboratory are for 4th quarter 1992 data only. See Appendix B for flagging criteria.

- = exceeded holding time for 4th quarter 1992.

- = exceeded final primary drinking water standard for 4th quarter 1992. 
WSRC-TR-93-068

Well LFW 27 continued

ANALYTICAL DATA

H D Analyte

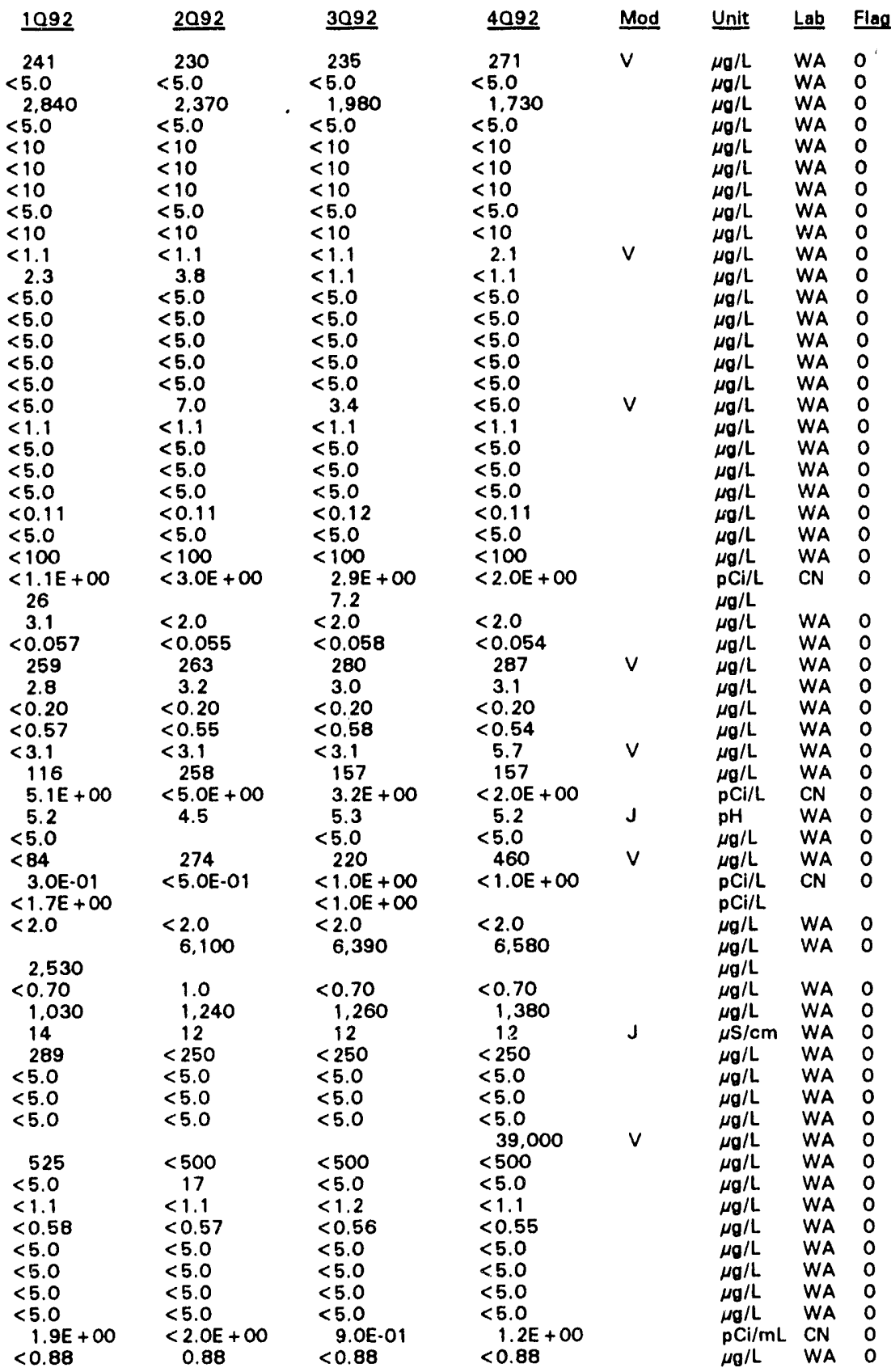

Note: Flagging levels, modifiers, and laboratory are for 4 th quarter 1992 data only. See Appendix 8 for flagging criteria.

- = exceeded holding time for 4th quarter 1992.

- = exceeded final primary drinking water standard for 4th quarter 1992. 
WSRC-TR-93-068

WELL LFW 28

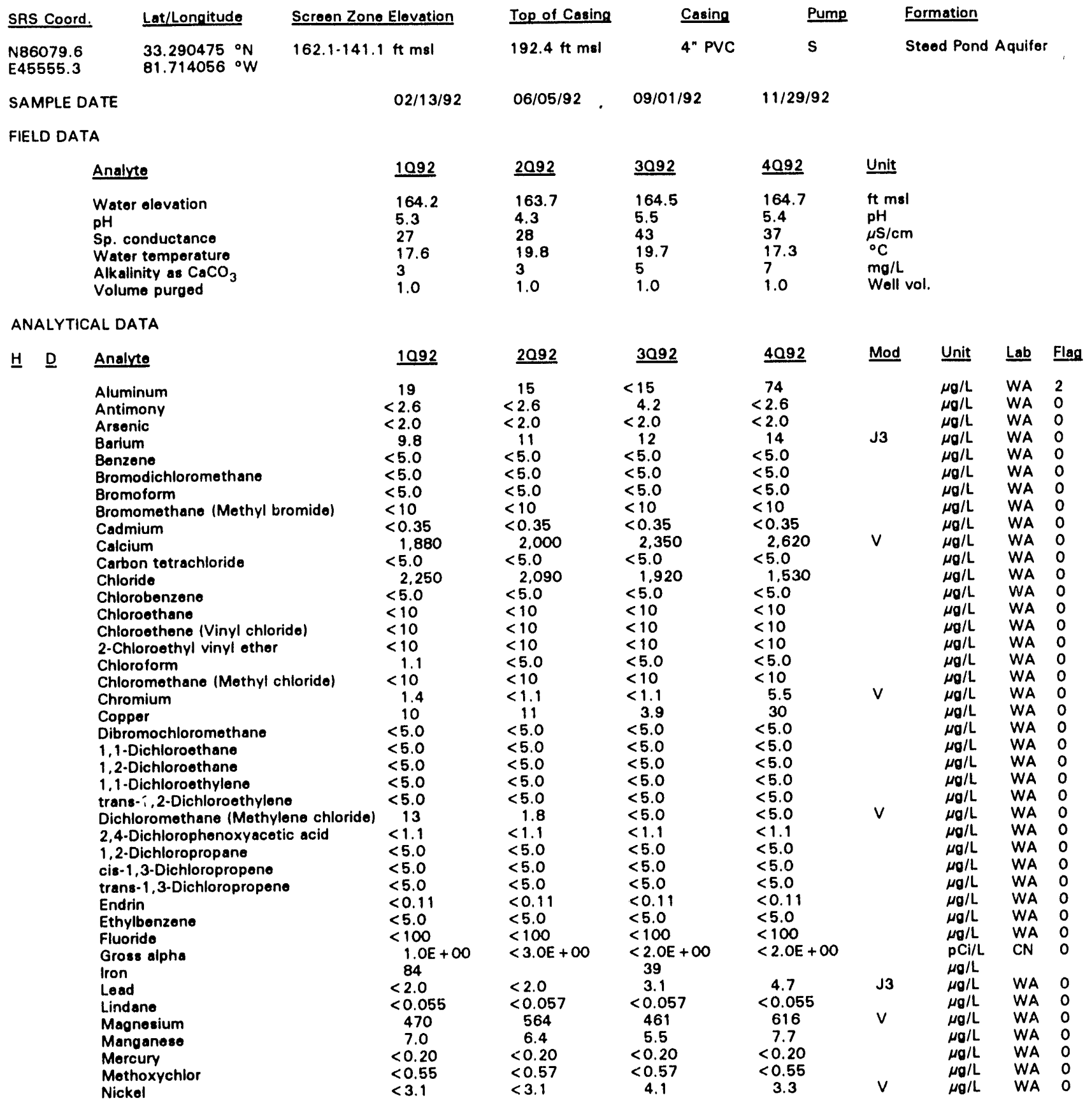

\footnotetext{
Note: Flagging levels, modifiers, and laboratory are for 4 th quarter 1992 data only. See Apperidix B for flagging criteria. - = exceeded holding time for 4th quarter 1992.

- =xceeded final primary drinking water standard for 4th quarter 1992.
} 
Well LFW 28 continued

ANALYTICAL DATA

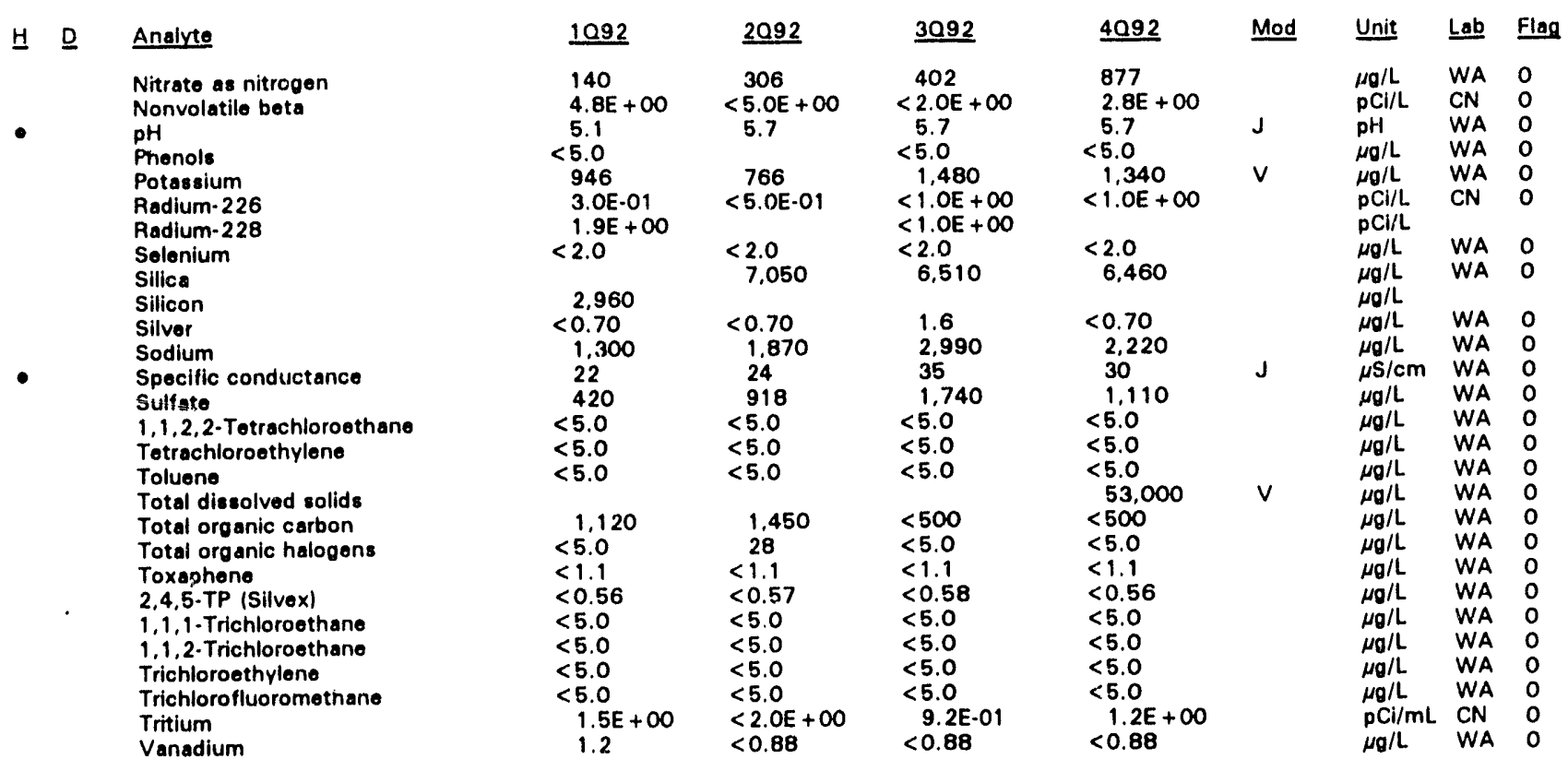

\section{WELL LFW 29}

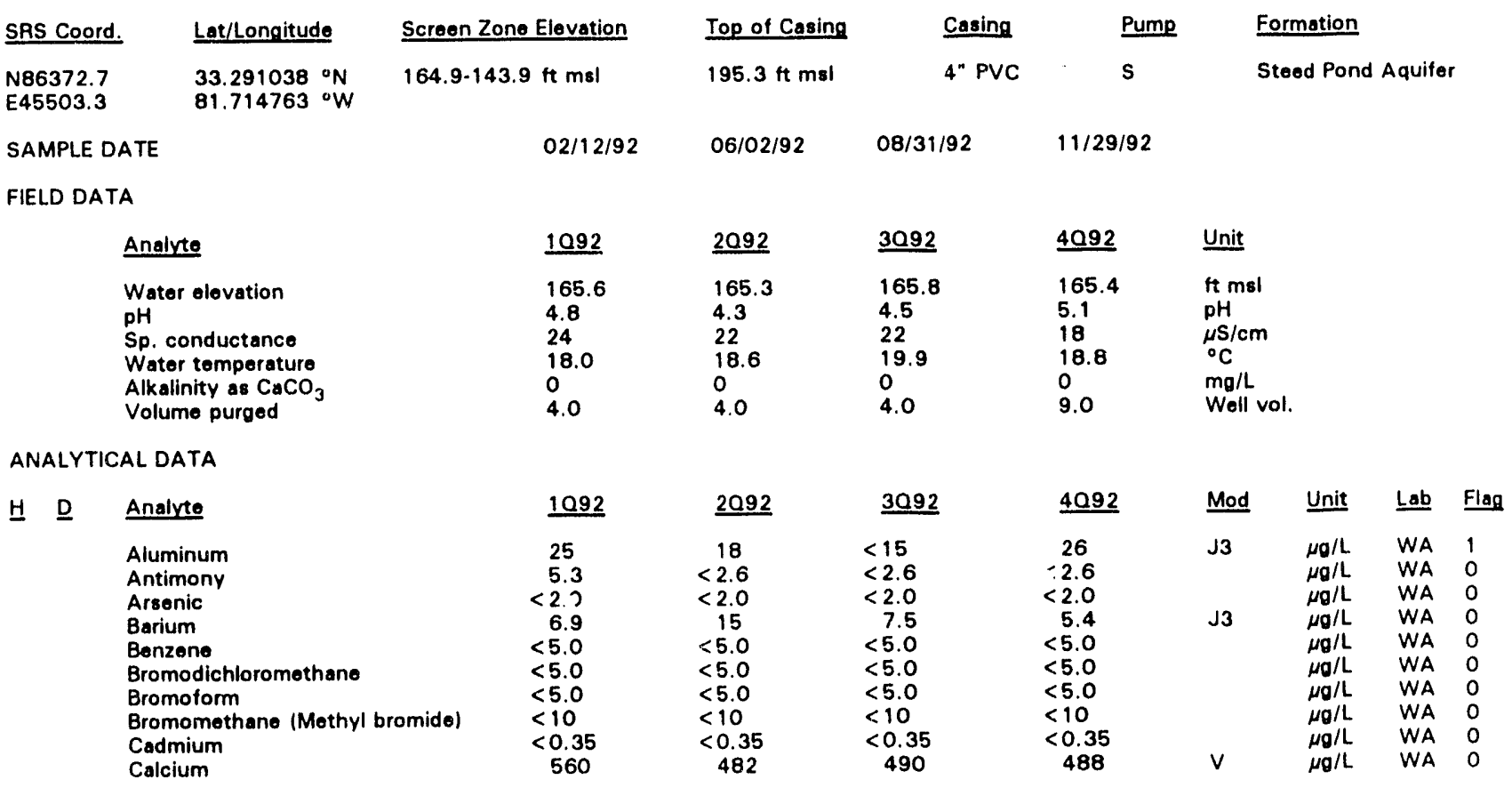

Note: Flagging levels, modifiers, and laboratory are for 4th quarter 1992 data only. See Appendix B for flagging criteria.

- = exceeded holding time for 4 th quarter 1992.

- =xceeded final primary drinking water standard for 4th quarter 1992. 


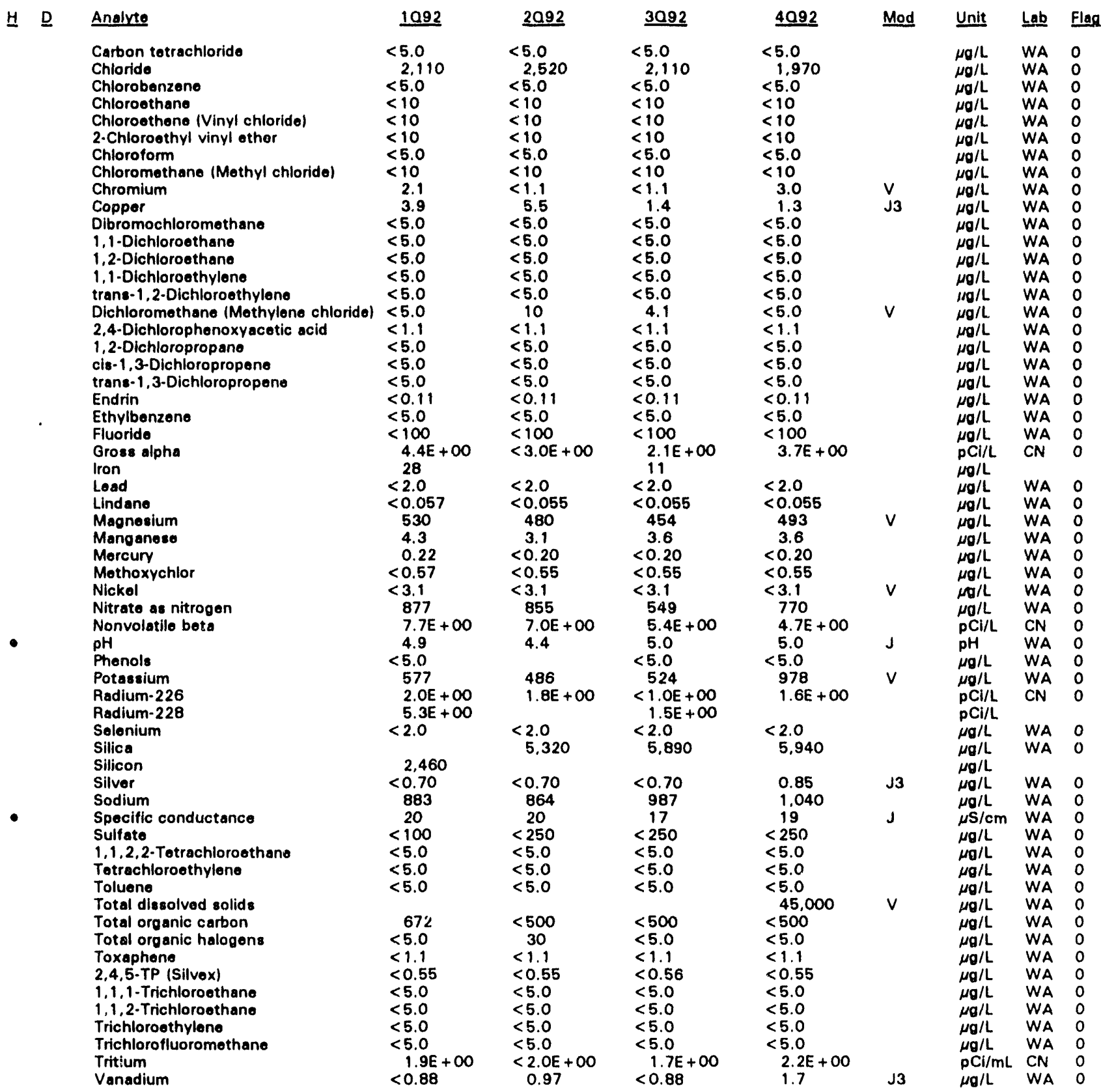

Note: Flagging levels, modifiers, and laboratory are for 4th quarter 1992 data only. See Appendix B for flagging criteria. - = exceeded holding time for 4th quarter 1992.

- = exceeded final primary drinking water standard for 4th quarter 1992. 
WSRC-TR-93.068

\section{WELL LFW 30}

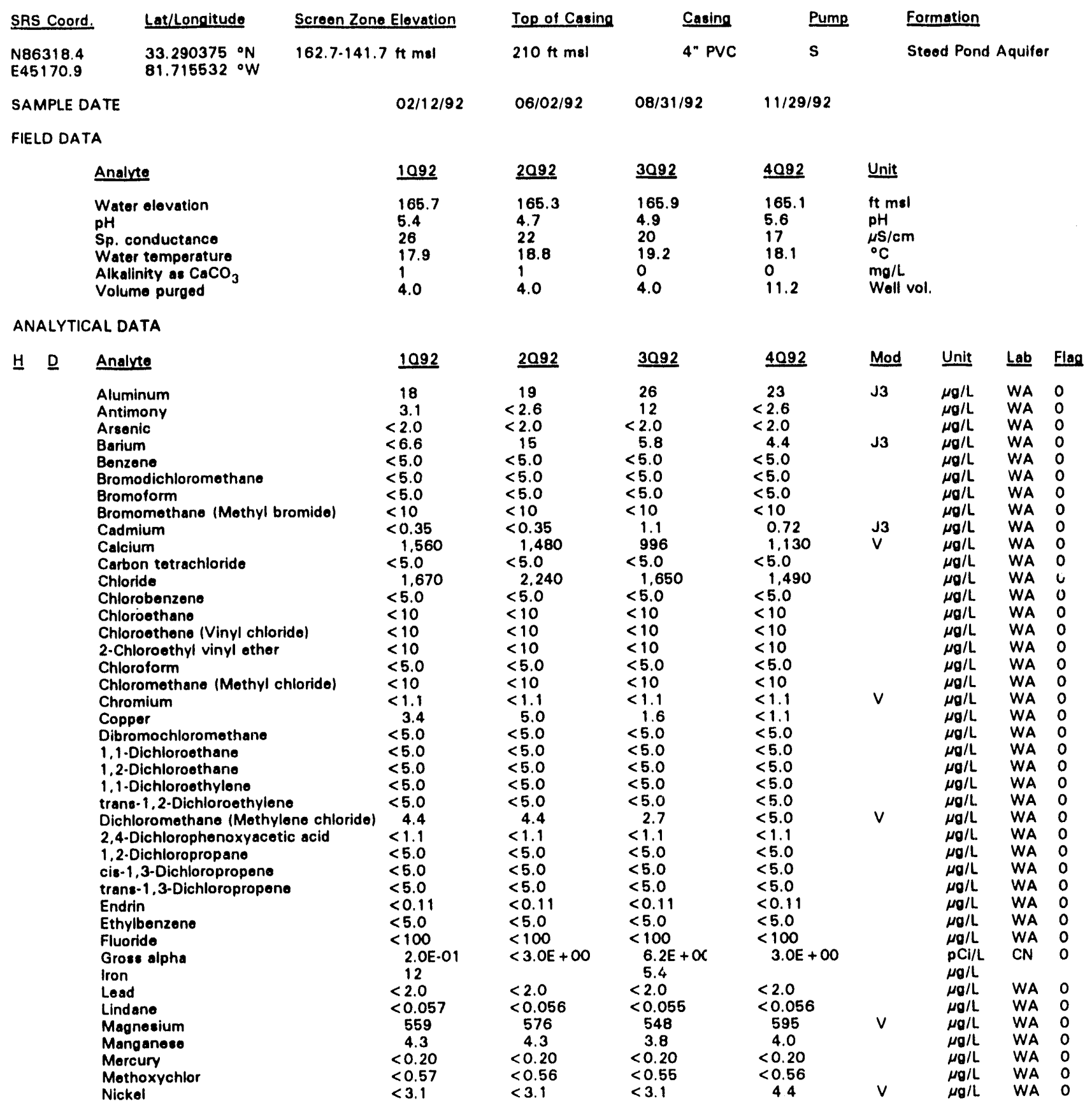

Note: Flagging levels, modifiers, and laboratory are for 4th quarter 1992 data only. See Appendix B for flagging criteria.

- =xceeded holding time for 4th quarter 1992.

- = exceeded final primary drinking water standard for 4 th quarter 1992. 
WSRC-TR-93-068

Well LFW 30 continued

ANALYTICAL DATA

H D Analyte

Nitrate as nitrogen

Nonvolatile beta

$\mathrm{pH}$

Phenols

Potaseium

Radium-226

Radium-228

Solenium

Silica

Silicon

Silver

Sodium

- Specific conductance

Sulfate

1,1,2,2-Tetrachloroethane

Tetrachloroothylene

Toluene

Total dissolved solids

Total organic carbon

Total organic halogens

Toxaphene

2,4,5-TP (Silvex)

1,1,1-Trichloroethane

1,1,2.Trichloroethane

Trichloroethylene

Trichlorofluoromethane

Tritium

Vanadium

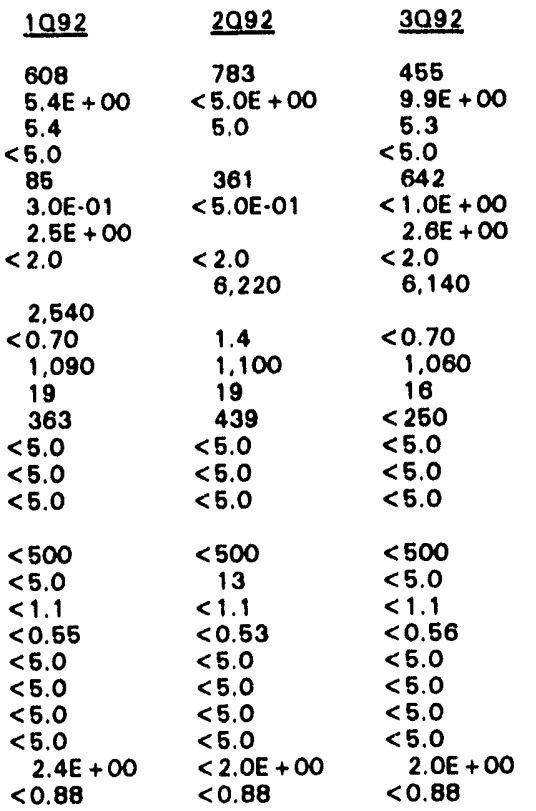

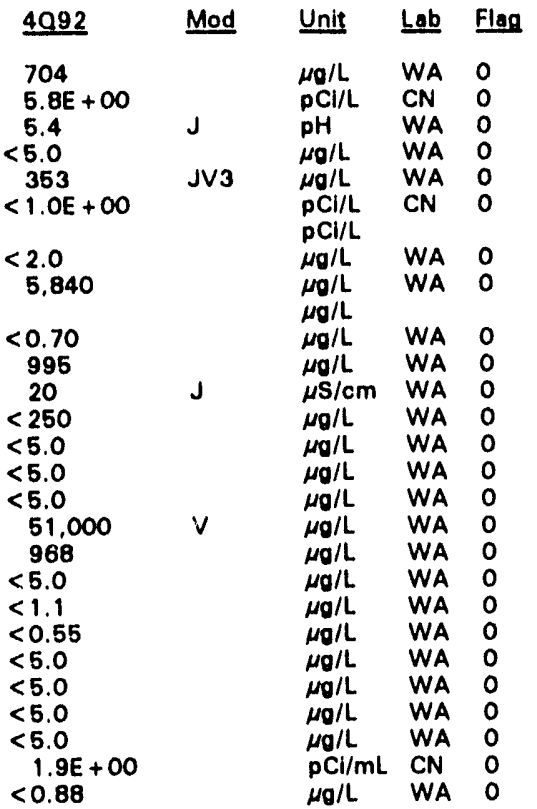

\section{WELL LFW 31}

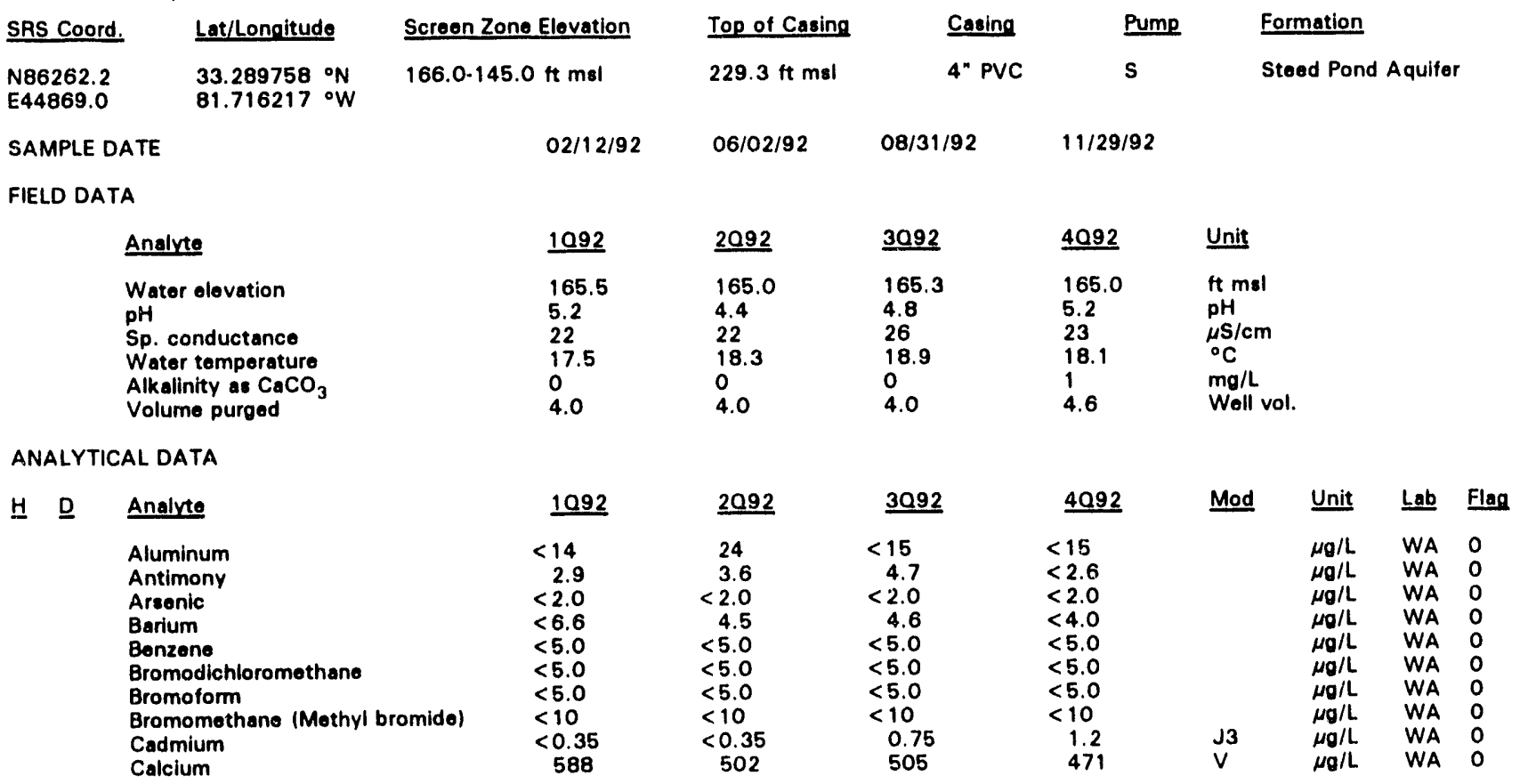

Note: Flagging levels, modifiers, and laboratory are fo 4 th quarter 1992 data only. See Appendix B for flagging criteria.

- = exceeded holding time for 4th quarter 1992.

- exceeded final primary di ing water standard for 4th quarter 1992. 
WSRC-TR-93-068

Well LFW 31 continued

ANALYTICAL DATA

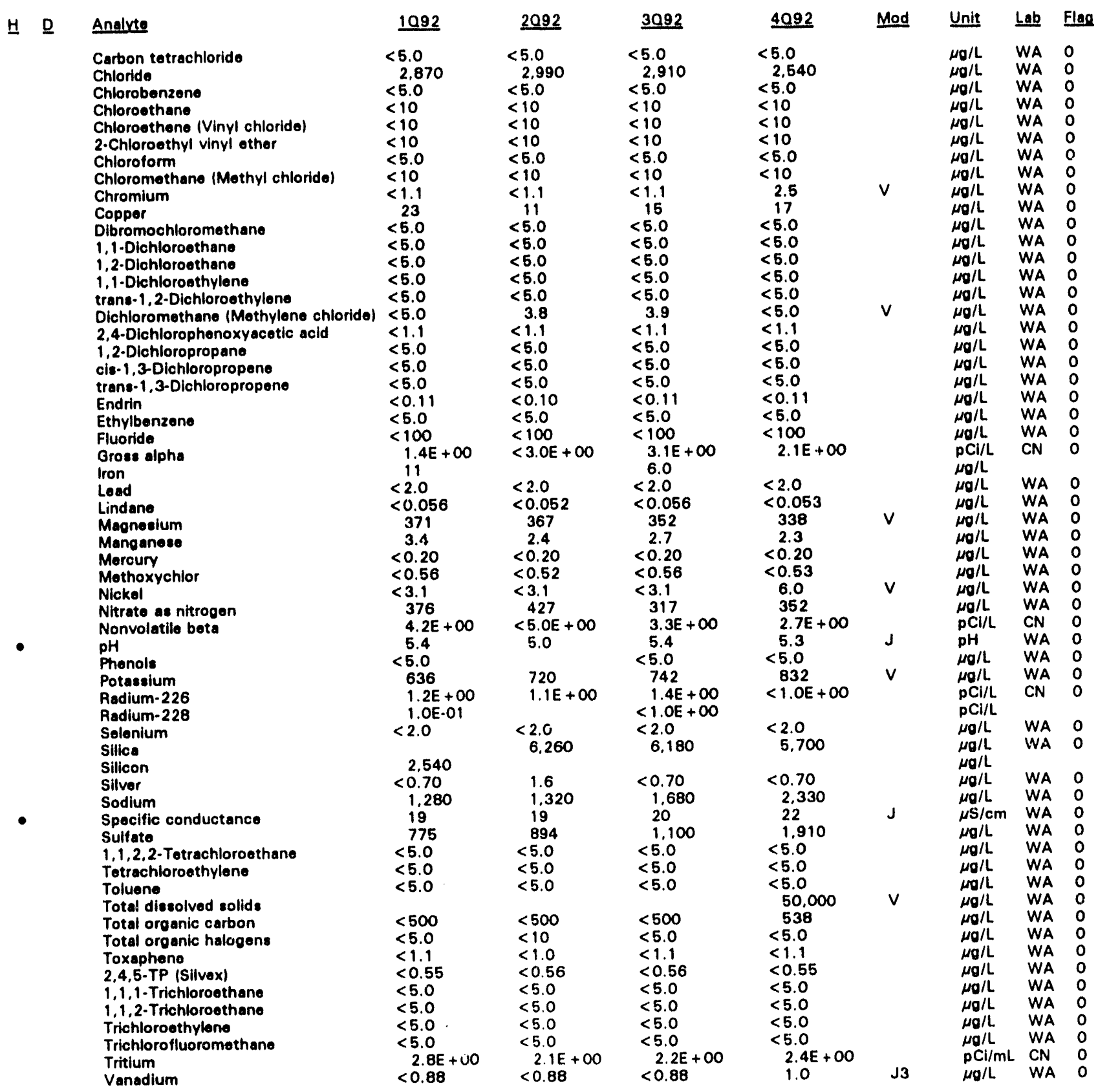

Note: Flagging lovels, modifiers, and laboratory are for 4th quarter 1992 data only. Seo Appendix B for flagging criteria. $-=$ exceeded holding time for 4 th quarter 1992.

- =xceeded final primary drinking water standard for 4th quarter 1992. 
WSRC-TR-93.068

WELL. LFW 32

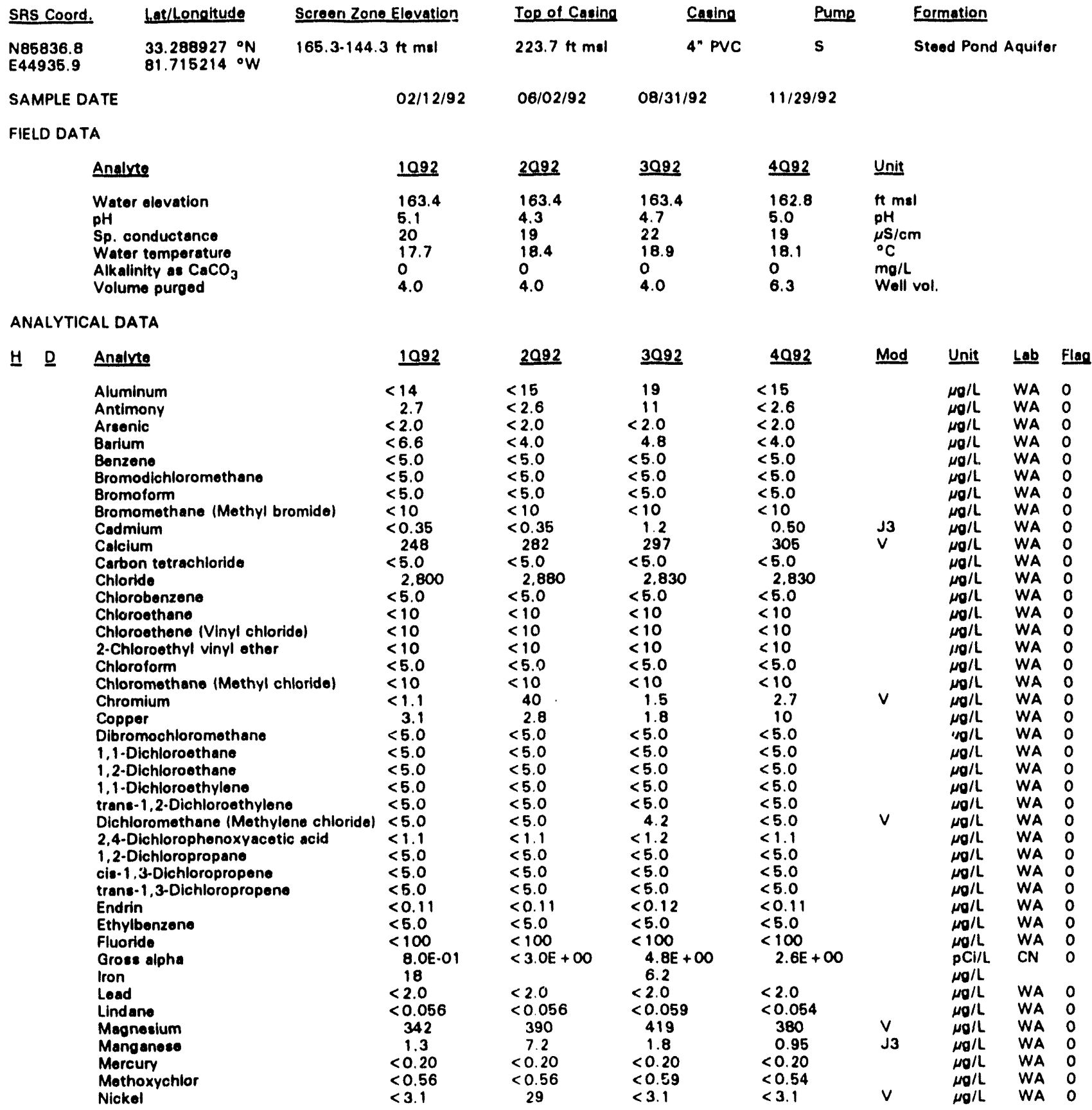

Note: Flagging levels, modifiers, and laboratory are for 4 th quarter 1992 deta only. See Appendix B for flagging criteria.

- = exceeded holding time for 4th quarter 1992.

- =xceeded final primary drinking water standard for 4th quarter 1992. 
Well LFW 32 continued

ANALYTICAL DATA

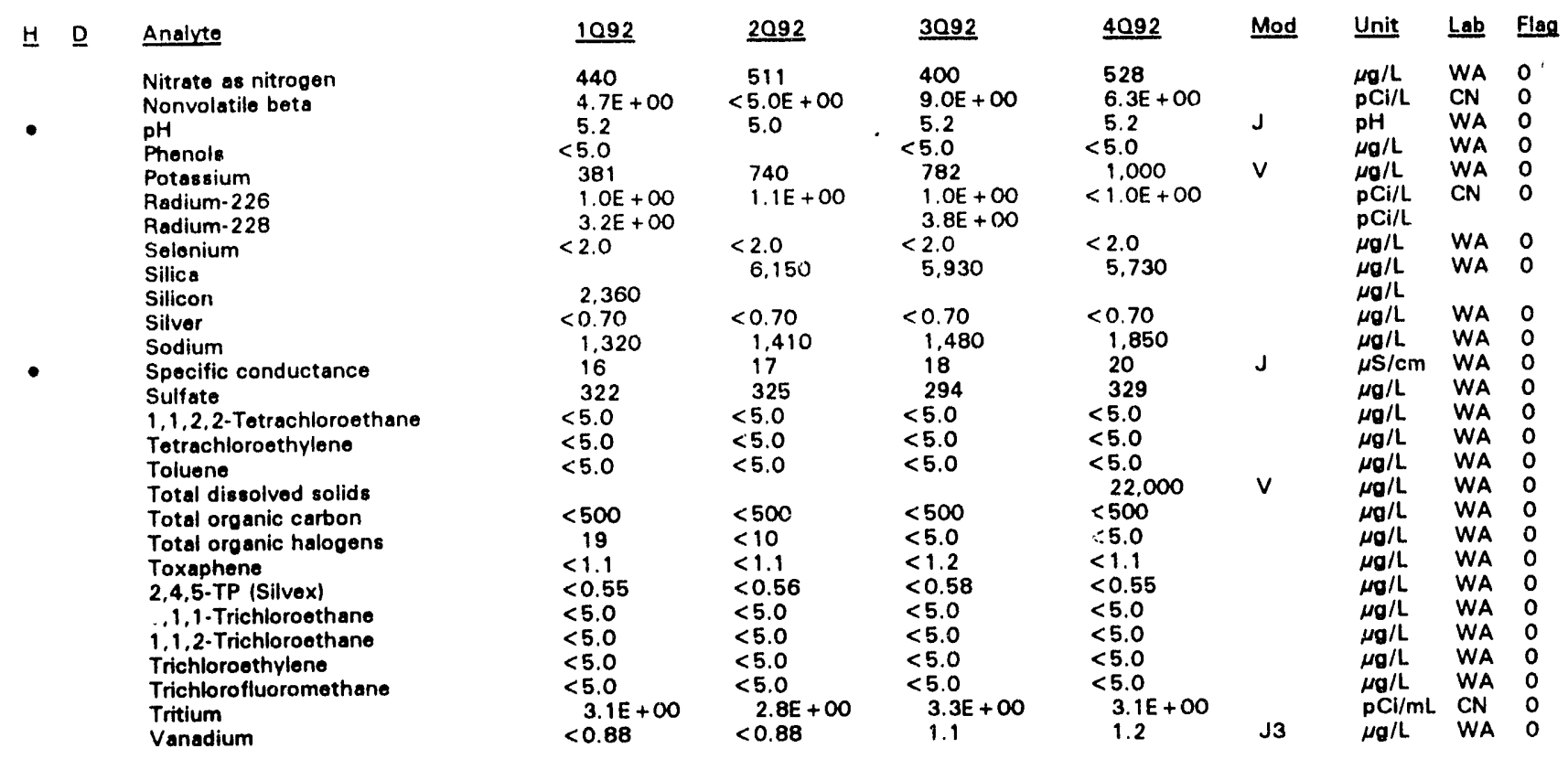

\section{WELL LFW 33}

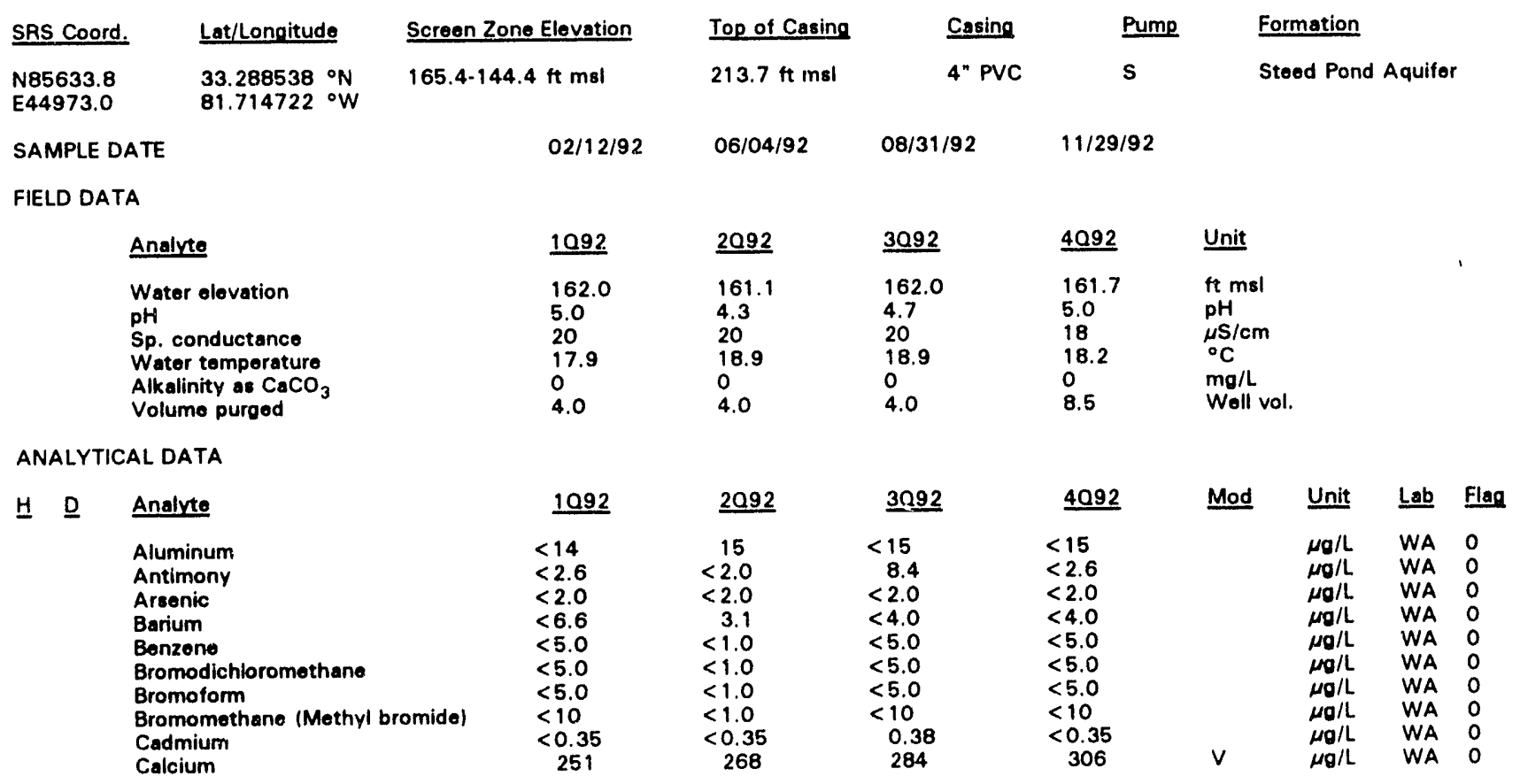

Note: Flagging levels, modifiers, and laboratory are for 4th quarter 1992 data only. See Appendix B for flagging criteria.

- = exceeded holding time for 4th quarter 1992.

- = exceeded final primary drinking water standard for 4th quarter 1992. 
Well LFW 33 continued

ANALYTICAL DATA

H D Analyte

Carbon tetrachloride

Chlorido

Chlorobenzene

Chloroethane

Chloroethene (Vinyl chloride)

2-Chloroethyl vinyi ether

Chloroform

Chloromethane (Methyl chloride)

Chromium

Coppor

Dibromochloromethane

1.1-Dichloroethane

1,2-Dichloroethane

1.1-Dichloroethylene

trans-1,2-Dichloroethylene

chloridel

1.2-Dichloropropane

cis-1,3-Dichloropropene

trans-1,3-Dichloropropene

Endrin

Ethylbenzene

Fluoride

Gross alpha

Iron

Lead

Lindane

Magnesium

Manganese

Mercury

Methoxychlor

Nickel

Nitrate as nitrogen

Nitrate-nitrite as nitrogen

Nonvolatile beta

pH

Phenols

Potassium

Radium-2?6

Radium-228

Solenium

Silica

Silicon

Silver
Sodium
Specific conductance

Sulfate

1,1,2,2-Tetrachloroethane

Totrachloroethylene

Tolueno

Total dissolved solids

Total organic carbon

Total organic halogens

Toxaphene

2,4,5-TP (Silvex)

1,1,1-Trichloroethane

1,1,2-Trichloroethane

Trichloroethyiene

Trichlorofluoromethane

Tritium

Vanadium

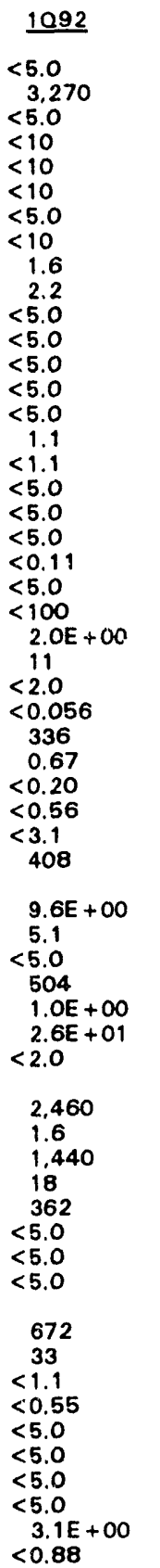

$\begin{aligned} & 2092 \\ &< 1.0 \\ & 2.850 \\ &<1.0 \\ &<1.0 \\ &<1.0 \\ &<1.0 \\ &<1.0 \\ &<1.0 \\ &<1.1 \\ & 2.0 \\ &<1.0 \\ &<1.0 \\ &<1.0 \\ &<1.0 \\ &<1.0 \\ & 4.1 \\ &<0.30 \\ &<1.0 \\ &<1.0 \\ &<1.0 \\ &<0.0060 \\ &<1.0 \\ &<100 \\ & 7.6 \mathrm{E}+00 \\ &<1\end{aligned}$

$\begin{array}{rl} & 3092 \\ <5.0 & 2.420 \\ <5.0 \\ <10 \\ <10 \\ <10 \\ <5.0 \\ <10 \\ <1.1 \\ 1.2 \\ <5.0 \\ <5.0 \\ <5.0 \\ <5.0 \\ <5.0 \\ 14 \\ <1.1 \\ <5.0 \\ <5.0 \\ <5.0 \\ <0.11 \\ <5.0 \\ <100 \\ 4.4 \mathrm{E}+00 \\ 7.6 \\ 2.0 \\ <0.056\end{array}$

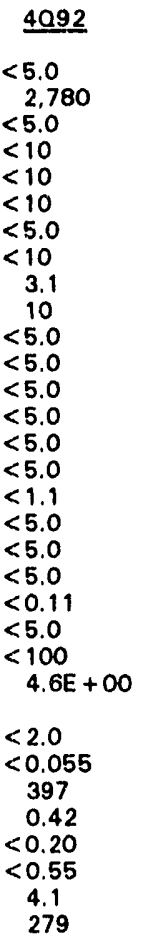

Mod

Unit Lab

$<0.0050$

$<0.056$

376

0.85

$<0.20$

$<0.50$

473

500
$2.2 E+01$

5.2
$<5.0$

642

$1.6 \mathrm{E}+00$

340
1.2

$<0.20$

$<0.56$

$<3.1$

279

1. $2 \mathrm{E}+01$

5.2
$<5.0$

$<5.0$

1,200

643
$1.9 E+00$

$<1.0 \mathrm{E}+00$

$<2.0$
6.450

$4.6 \mathrm{E}+00$

$<2.0$

6.210

5,940

1.0

1,390
20

402

$<1.0$

$<1.0$

$<1.0$

$<0.70$

669
182

$<0.24$

$<0.090$

$<1.0$

$<1.0$

$<1.0$

10
$9.1 E+00$

1.480
16
581

$<5.0$

$<5.0$
$<5.0$

$<500$

$<5.0$

$<1.1$

$<0.56$

$<5.0$

$<5.0$

$<5.0$

$<5.0$

3. $2 \mathrm{E}+00$

$<0.70$

1.620

19
440

440
$<5.0$

$<5.0$

$<5.0$

$38,000 \quad \mu g / L$ WA

$<\quad \sim \mathrm{g} / \mathrm{L}$ WA 0

$<1.1$

$<1.1$

$<5.0$

$<5.0$

$<5.0$

$<5.0$

$2.9 E+00$

$<0.88$

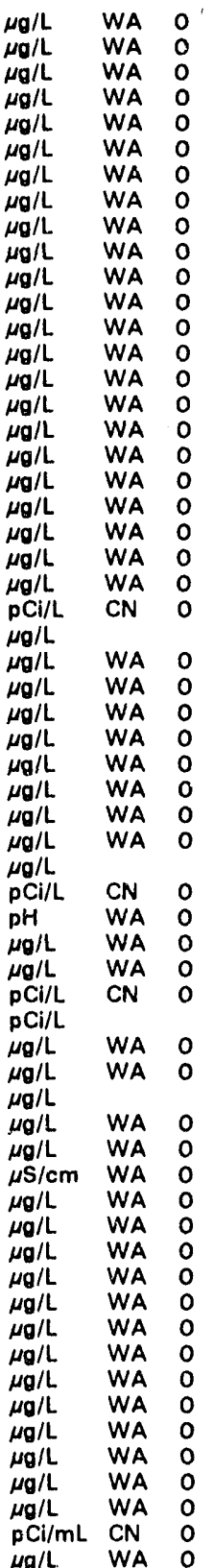

Note: Flagging levels, modifiers, and laboratory are for 4 th quarter 1992 data only. See Appendix B for flagging criteria.

- = exceeded holding time for 4th quarter 1992.

- exceeded final primary drinking water standard for 4 th quarter 1992. 
WSRC-TR-93-068

WELL LFW 34

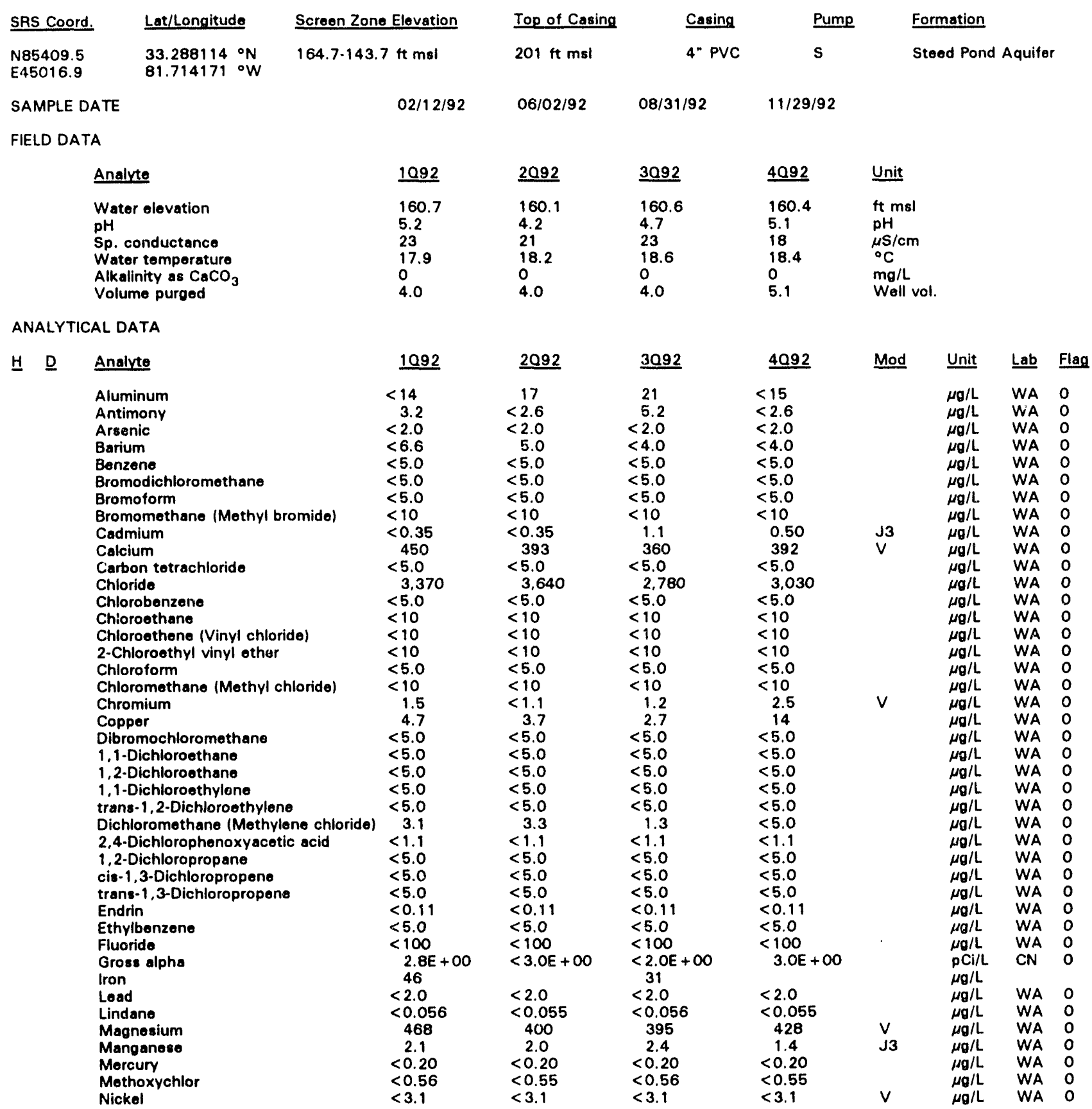

Note: Flagging levels, modifiers, and laboratory are for 4th quarter 1992 data only. See Appendix B for flagging criteria.

- = exceeded holding tine for 4 th quarter 1992.

- = exceeded final primary drinking water standard for 4th quarter 1992. 
Weil LFW 34 continued

ANALYTICAL DATA.

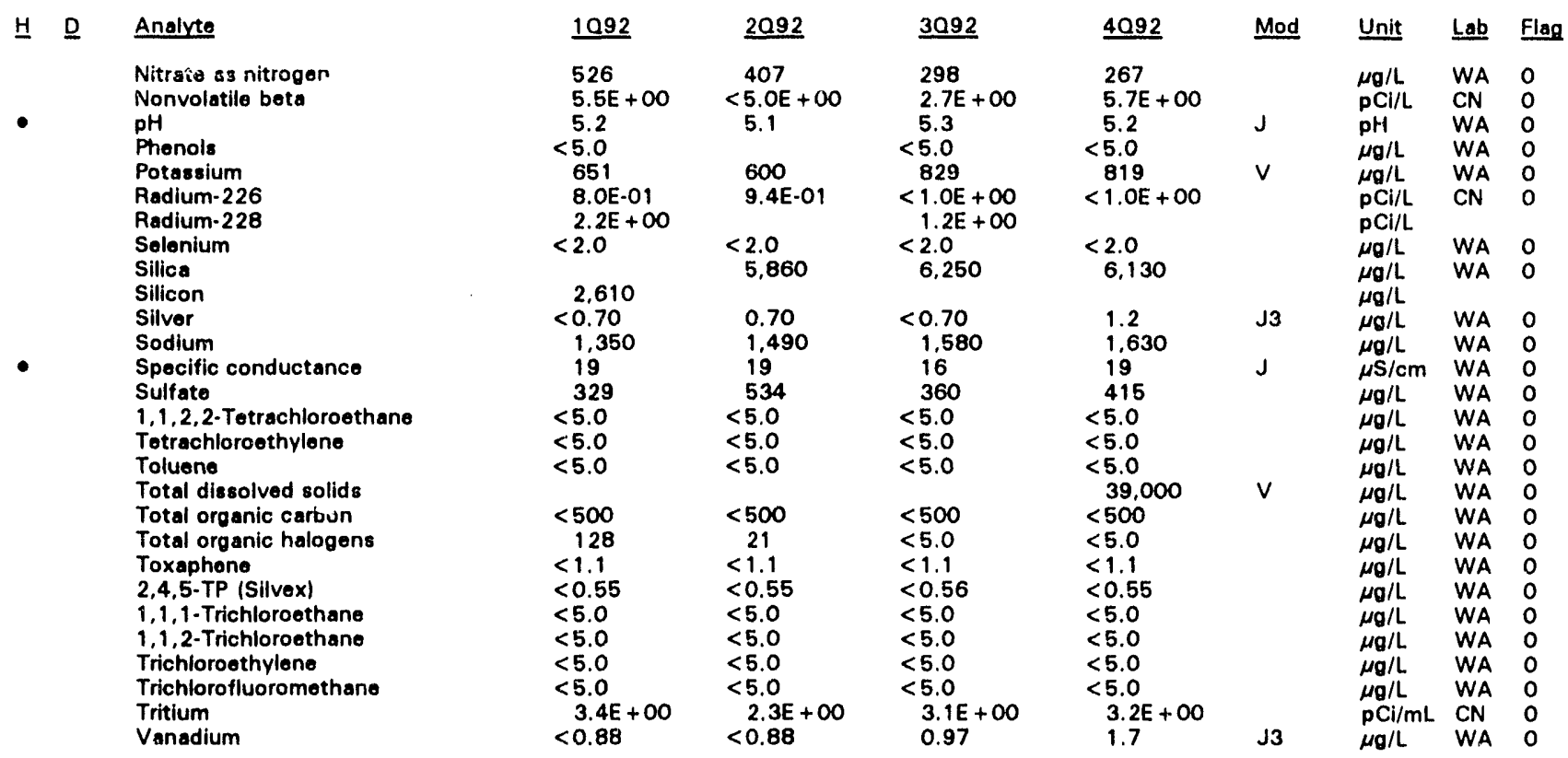

\section{WELL LFW 35}

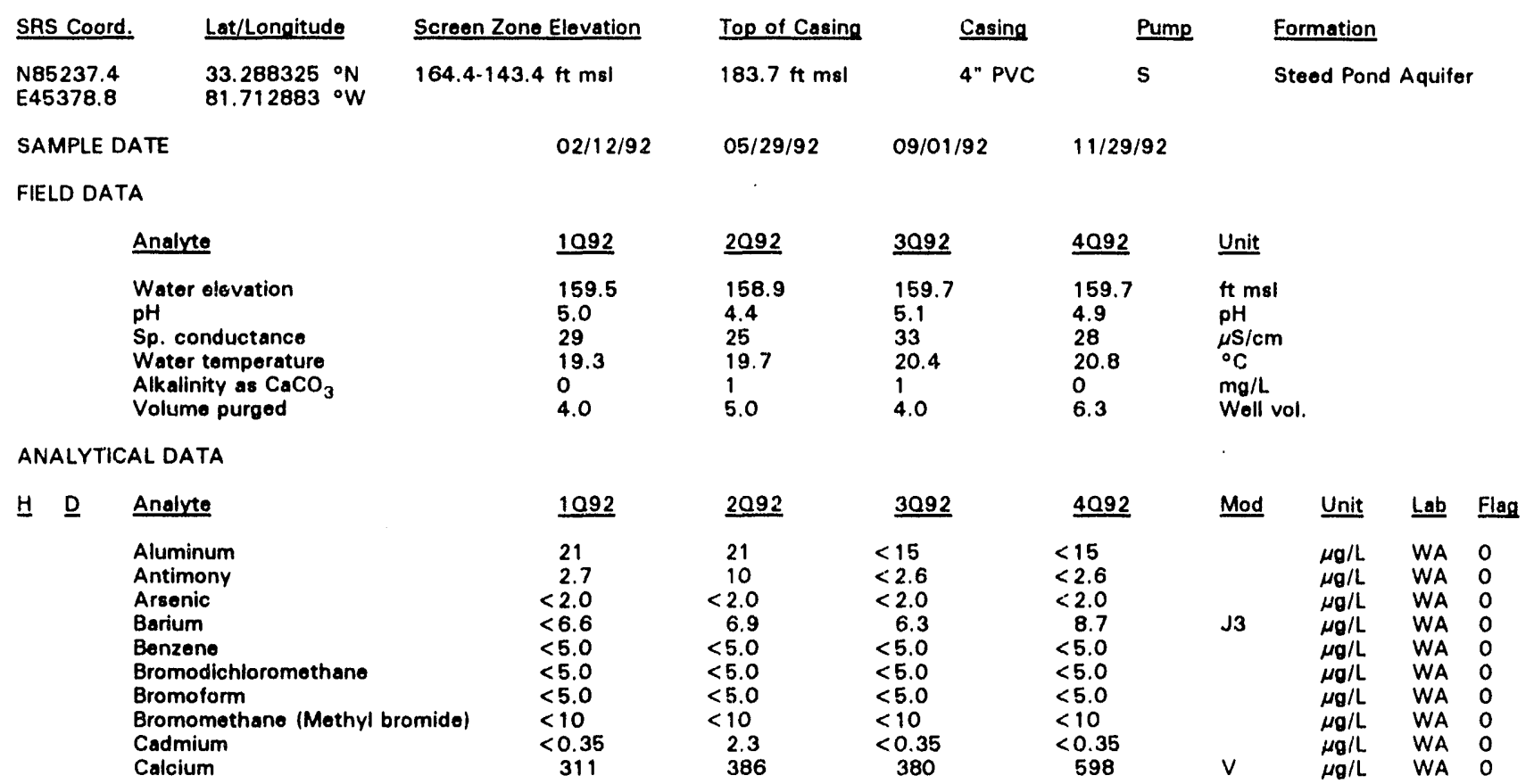

Note: Flagging levels, modifiers, and laboratory are for 4 th quarter 1992 data only. See Appendix B for flagging criteria.

- = exceeded holding time for 4th quarter 1992.

- = exceeded final primary drinking water standard for 4th quarter 1992 . 
Well LFW 35 continued

ANALYTICAL DATA

H D Analyte

Carbon tetrachloride

Chloride

Chlorobenzene

Chloroethane

Chloroethene (Vinyl chloride)

2-Chloroothyl vinyl other

Chloroform

Chloromethane (Methyl chloride)

Chromium

Copper

Dibromochloromethane

1,1-Dichloroethane

1,2-Dichloroethane

1,1-Dichloroethylene

trans-1,2-Dichloroethylene

Dichloromethane (Methylene chloride)

2,4-Dichlorophenoxyacetic acid

1.2-Dichloropropane

cis-1,3-Dichloropropene

trans-1,3-Dichloropropene

Endrin

Ethylbenzene

Fluoride

Gross alpha

Iron

Load

Lindane

Magnesium

Manganeso

Mercury

Mothoxychlor

Nickel

Nitrate as nitrogen

Nonvolatile bets

$\mathrm{pH}$

Phenols

Potassium

Radium-226

Radium-228

Selenium

Silica

Silicon

Silver

Sodium

Specific conductance

Sulfate

1,1,2,2-Tetrachloroethane

Tetrachloroethylene

Toluene

Total dissolved solids

Total organic carbon

Total organic halogens

Toxaphene

2.4,5-TP (Silvex)

1,1,1-Trichloroethane

1.1,2-Trichloroethano

Trichloroethylene

Trichlorofluoromethane

Tritium

Vanadium

\begin{tabular}{|c|c|c|}
\hline 1092 & $\underline{2092}$ & $\underline{3092}$ \\
\hline $\begin{aligned}< & 5.0 \\
& 2.860\end{aligned}$ & $\begin{aligned}<5.0 \\
3.060\end{aligned}$ & $\begin{aligned}<5.0 \\
\quad 2,720\end{aligned}$ \\
\hline$<5.0$ & $<5.0$ & $<5.0$ \\
\hline$<10$ & $<10$ & $<10$ \\
\hline$<10$ & $<10$ & $<10$ \\
\hline $\begin{array}{l}<10 \\
<5.0\end{array}$ & $<10$ & $<10$ \\
\hline $\begin{array}{l}<5.0 \\
<10\end{array}$ & $\begin{array}{l}<5.0 \\
<10\end{array}$ & $\begin{array}{l}<5.0 \\
<10\end{array}$ \\
\hline 1.6 & $<1.1$ & $<1.1$ \\
\hline 2.6 & 3.3 & $<1.1$ \\
\hline$<5.0$ & $<5.0$ & $<5.0$ \\
\hline$<5.0$ & $<5.0$ & $<5.0$ \\
\hline$<5.0$ & $<5.0$ & $<5.0$ \\
\hline$<5.0$ & $<5.0$ & $<5.0$ \\
\hline$<5.0$ & $<5.0$ & $<5.0$ \\
\hline$<5.0$ & $<5.0$ & $<5.0$ \\
\hline$<1.1$ & $<1.1$ & $<1.1$ \\
\hline$<5.0$ & $<5.0$ & $<5.0$ \\
\hline$<5.0$ & $<5.0$ & $<5.0$ \\
\hline$<5.0$ & $<5.0$ & $<5.0$ \\
\hline$<0.11$ & $<0.11$ & $<0.11$ \\
\hline $\begin{array}{l}<5.0 \\
<100\end{array}$ & $\begin{array}{l}<5.0 \\
<100\end{array}$ & $\begin{array}{l}<5.0 \\
<100\end{array}$ \\
\hline $\begin{array}{l}<100 \\
7.0 E-01 \\
7.9\end{array}$ & $\begin{array}{l}<100 \\
<3.0 E+00\end{array}$ & $\begin{array}{l}<100 \\
<2.0 E+\infty\end{array}$ \\
\hline $\begin{array}{r}7.9 \\
<2.0\end{array}$ & $<2.0$ & $\begin{array}{r}<1.9 \\
2.0\end{array}$ \\
\hline$<0.055$ & $<0.056$ & $<0.057$ \\
\hline 499 & 694 & 649 \\
\hline 2.9 & 4.0 & 3.0 \\
\hline$<0.20$ & $<0.20$ & $<0.20$ \\
\hline$<0.55$ & $<0.56$ & $<0.57$ \\
\hline$<3.1$ & $<3.1$ & $<3.1$ \\
\hline 1,580 & 1,120 & 1,050 \\
\hline $3.4 E+\infty 0$ & $<5.0 E+00$ & $3.7 E+\infty 0$ \\
\hline $\begin{array}{r}5.1 \\
<5.0\end{array}$ & 5.2 & $\begin{array}{r}5.2 \\
<5.0\end{array}$ \\
\hline 173 & 549 & $<84$ \\
\hline $\begin{array}{l}1.3 E+\infty \\
1.6 E+\infty\end{array}$ & $9.7 E-01$ & $\begin{array}{r}<1.0 E+00 \\
2.0 E+\infty\end{array}$ \\
\hline $\begin{array}{l}1.60+00 \\
<2.0\end{array}$ & $<2.0$ & $\begin{array}{l}2.0 E+\infty 0 \\
<2.0\end{array}$ \\
\hline & 5,800 & 6,460 \\
\hline $\begin{aligned} & 2,590 \\
&<0.70\end{aligned}$ & $<0.70$ & $<0.70$ \\
\hline 2,240 & $\begin{array}{l}2.350 \\
23\end{array}$ & 2,530 \\
\hline$<100$ & 251 & $<250$ \\
\hline$<5.0$ & $<5.0$ & $<5.0$ \\
\hline$<5.0$ & $<5.0$ & $<5.0$ \\
\hline$<5.0$ & $<5.0$ & $<5.0$ \\
\hline$<500$ & 1,960 & 5,210 \\
\hline$<5.0$ & $<20$ & $<5.0$ \\
\hline$<1.1$ & $<1.1$ & $<1.1$ \\
\hline$<0.55$ & $<0.56$ & $<0.56$ \\
\hline$<5.0$ & $<5.0$ & $<5.0$ \\
\hline$<5.0$ & $<5.0$ & $<5.0$ \\
\hline$<5.0$ & $<5.0$ & $<5.0$ \\
\hline$<5.0$ & $<5.0$ & $<5.0$ \\
\hline $\begin{aligned} & 1.9 E+00 \\
&<0.88\end{aligned}$ & $\begin{array}{l}<2.0 E+00 \\
<0.88\end{array}$ & $\begin{aligned} & 1.6 E+\infty \\
&<0.88\end{aligned}$ \\
\hline
\end{tabular}

\begin{tabular}{|c|c|c|c|c|}
\hline 4092 & Mod & Unit & $\underline{\text { Lab }}$ & \\
\hline$<5.0$ & & $\mu \mathrm{g} / \mathrm{L}$ & WA & 0 \\
\hline 2,680 & & $\mu g / L$ & WA & 0 \\
\hline$<5.0$ & & $\mu \mathrm{g} / \mathrm{L}$ & WA & 0 \\
\hline$<10$ & & $\mu \mathrm{g} / \mathrm{L}$ & WA & 0 \\
\hline $\begin{array}{l}<10 \\
<10\end{array}$ & & $\mu \mathrm{g} / \mathrm{L}$ & WA & 0 \\
\hline$<10$ & & $\mu_{g} / L$ & WA & 0 \\
\hline $\begin{array}{l}<5.0 \\
<10\end{array}$ & & $\mu_{\mathrm{o}} / \mathrm{L}$ & WA & 0 \\
\hline $\begin{array}{r}<10 \\
3.1\end{array}$ & & $\mu_{\theta} / L$ & WA & 0 \\
\hline $\begin{array}{l}3.1 \\
1.4\end{array}$ & v & $\mu \mathrm{g} / \mathrm{L}$ & WA & 0 \\
\hline 1.4 & J3 & $\mu \mathrm{g} / \mathrm{L}$ & WA & 0 \\
\hline$<5.0$ & & $\mu \mathrm{g} / \mathrm{L}$ & WA & 0 \\
\hline$<5.0$ & & $\mu \mathrm{g} / \mathrm{L}$ & WA & \\
\hline$<5.0$ & & $\mu \mathrm{g} / \mathrm{L}$ & WA & 0 \\
\hline$<5.0$ & & $\mu \mathrm{g} / \mathrm{L}$ & WA & \\
\hline$<5.0$ & & $\mu \mathrm{g} / \mathrm{L}$ & WA & 0 \\
\hline$<5.0$ & & $\mu \mathrm{g} / \mathrm{L}$ & WA & 0 \\
\hline$<1.1$ & & $\mu \mathrm{g} / \mathrm{L}$ & WA & 0 \\
\hline$<5.0$ & & $\mu \mathrm{g} / \mathrm{L}$ & WA & 0 \\
\hline$<5.0$ & & $\mu \mathrm{g} / \mathrm{L}$ & WA & 0 \\
\hline$<5.0$ & & $\mu \mathrm{g} / \mathrm{L}$ & WA & 0 \\
\hline$<0.11$ & & $\mu \mathrm{g} / \mathrm{L}$ & WA & 0 \\
\hline$<5.0$ & & $\mu \mathrm{g} / \mathrm{L}$ & WA & 0 \\
\hline$<100$ & & $\mu \mathrm{g} / \mathrm{L}$ & WA & \\
\hline $3.7 E+\infty 0$ & & ${ }_{\mu \mathrm{g} / \mathrm{L}}^{\mathrm{pCi}}$ & $\mathrm{CN}$ & 0 \\
\hline$<2.0$ & & $\mu \mathrm{g} / \mathrm{L}$ & WA & \\
\hline$<0.056$ & & $\mu \mathrm{g} / \mathrm{L}$ & WA & 0 \\
\hline 1.090 & v & $\mu_{\mathrm{g}} / \mathrm{L}$ & WA & 0 \\
\hline $\begin{array}{c}4.7 \\
<0.20\end{array}$ & & $\begin{array}{l}\mu_{0} / \mathrm{L} \\
\mu_{\mathrm{g}} / \mathrm{L}\end{array}$ & $\begin{array}{l}\text { WA } \\
\text { WA }\end{array}$ & 0 \\
\hline$<0.56$ & & $\mu_{\mathrm{g} / \mathrm{L}}$ & WA & 0 \\
\hline$<3.1$ & $v$ & $\mu \mathrm{g} / \mathrm{L}$ & WA & 0 \\
\hline $\begin{array}{l}2,150 \\
8,1 E+\infty\end{array}$ & & $\mu \mathrm{g} / \mathrm{L}$ & WA & 0 \\
\hline $\begin{array}{l}8.1 E+\infty 0 \\
4.8\end{array}$ & $J$ & $\begin{array}{l}\mathrm{pCI} / \mathrm{L} \\
\mathrm{pH}\end{array}$ & WA & 0 \\
\hline$<5.0$ & & $\mu \mathrm{g} / \mathrm{L}$ & WA & 0 \\
\hline 305 & JV3 & $\mu \mathrm{g} / \mathrm{L}$ & WA & 0 \\
\hline$<1.0 E+\infty 0$ & & $\begin{array}{l}\mathrm{PCi} / \mathrm{L} \\
\mathrm{pCi} / \mathrm{L}\end{array}$ & $\mathrm{CN}$ & 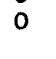 \\
\hline$<2.0$ & & $\mu_{g} / \mathrm{L}$ & WA & 0 \\
\hline 6,110 & & $\begin{array}{l}\mu \mathrm{g} / \mathrm{L} \\
\mu \mathrm{g} / \mathrm{L}\end{array}$ & WA & \\
\hline$<0.70$ & & $\mu \mathrm{g} / \mathrm{L}$ & WA & 0 \\
\hline $\begin{array}{l}1,550 \\
28\end{array}$ & $J$ & $\underset{\mu \mathrm{g} / \mathrm{cm}}{\mu \mathrm{cm}}$ & $\begin{array}{l}\text { WA } \\
\text { WA }\end{array}$ & 0 \\
\hline$<250$ & & $\mu_{\mathrm{g} / \mathrm{L}}$ & WA & 0 \\
\hline$<5.0$ & & $\mu \mathrm{g} / \mathrm{L}$ & WA & 0 \\
\hline$<5.0$ & & $\mu \mathrm{g} / \mathrm{L}$ & WA & 0 \\
\hline$<5.0$ & & $\mu \mathrm{g} / \mathrm{L}$ & WA & 0 \\
\hline 52.000 & v & $\mu \mathrm{g} / \mathrm{L}$ & WA & 0 \\
\hline$<500$ & & $\mu \mathrm{g} / \mathrm{L}$ & WA & 0 \\
\hline$<5.0$ & & $\mu \mathrm{g} / \mathrm{L}$ & WA & 0 \\
\hline$<1.1$ & & $\mu g / L$ & WA & 0 \\
\hline$<0,55$ & & $\mu \mathrm{g} / \mathrm{L}$ & WA & 0 \\
\hline$<5.0$ & & $\mu g / L$ & WA & 0 \\
\hline$<5.0$ & & $\mu \mathrm{g} / \mathrm{L}$ & WA & 0 \\
\hline$<5.0$ & & $\mu \mathrm{g} / \mathrm{L}$ & WA & 0 \\
\hline$<5.0$ & & $\mu \mathrm{g} / \mathrm{L}$ & WA & 0 \\
\hline 7.4E-01 & & $\mathrm{pCi} / \mathrm{mL}$ & $\mathrm{CN}$ & \\
\hline$<0.88$ & & $\mu \mathrm{g} / \mathrm{L}$ & WA & 0 \\
\hline
\end{tabular}

Note: Flagging levels, modifiers, and laboratory are for 4th quarter 1992 data only. See Appendix B for flagging criteria.

- = exceeded holding time for 4th quarter 1992.

- = exceeded final primary drinking water standard for 4th quarter 1992. 
WSRC-TR-93-068

WELL LFW 36

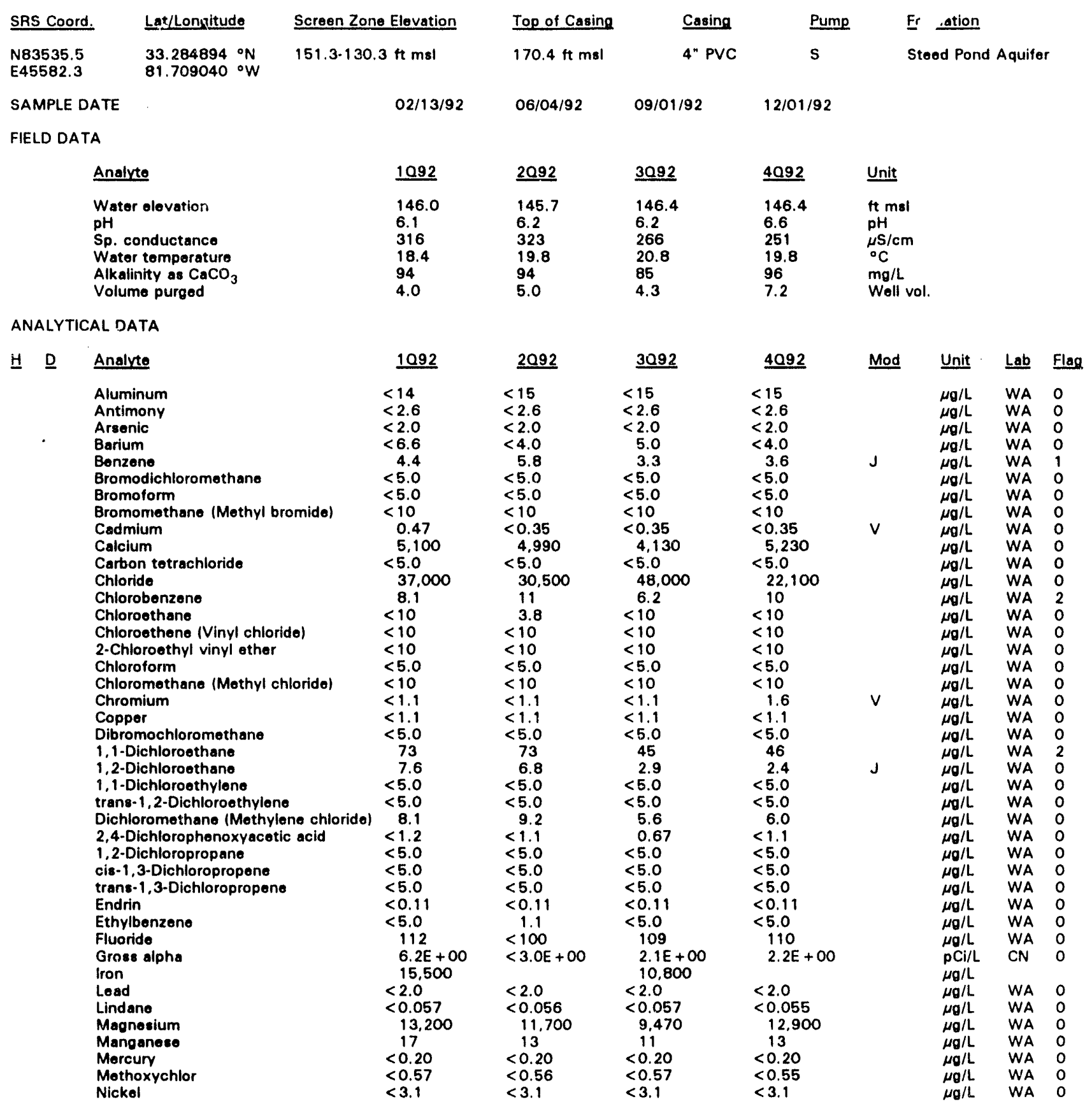

Note: Flagging levels, modifiers, and laboratory are for 4 th quarter 1992 data only. See Appendix B for flagging criteria.

- = exceeded holding time for 4th quarter 1992.

- =xceeded final primary drinking water standard for 4th quarter 1992. 
Well LFW 36 continued

ANALYTICAL DATA

H. $\quad$ Analyte
Nitrate as nitrogen
Nonvolatile beto
pH
Phenols
Potassium
Radium-226
Radium-228
Solenium
Silica
Silicon
Silvor
Sodium
Specific conductance
Sulfate
1,1,2,2-Totrachloroethane
Totrachloroethylene
Toluene
Total dissolved solids
Total organic carbon
Total organic halogens
Toxaphone
2,4.5-TP (Silvex)
1,1,1-Trichloroethane
1,1,2-Trichloroethane
Trichloroethylene
Trichlorofluoromethane
Tritium
Vanadium

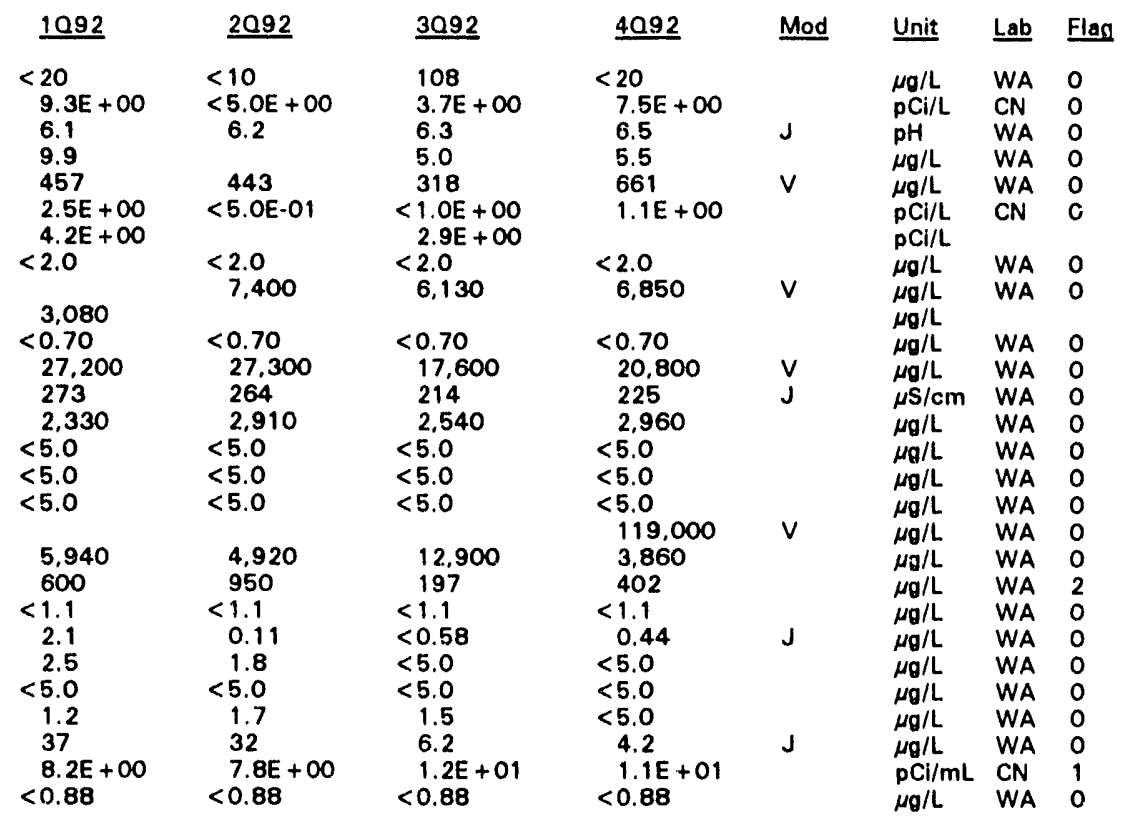

WELL LFW 37

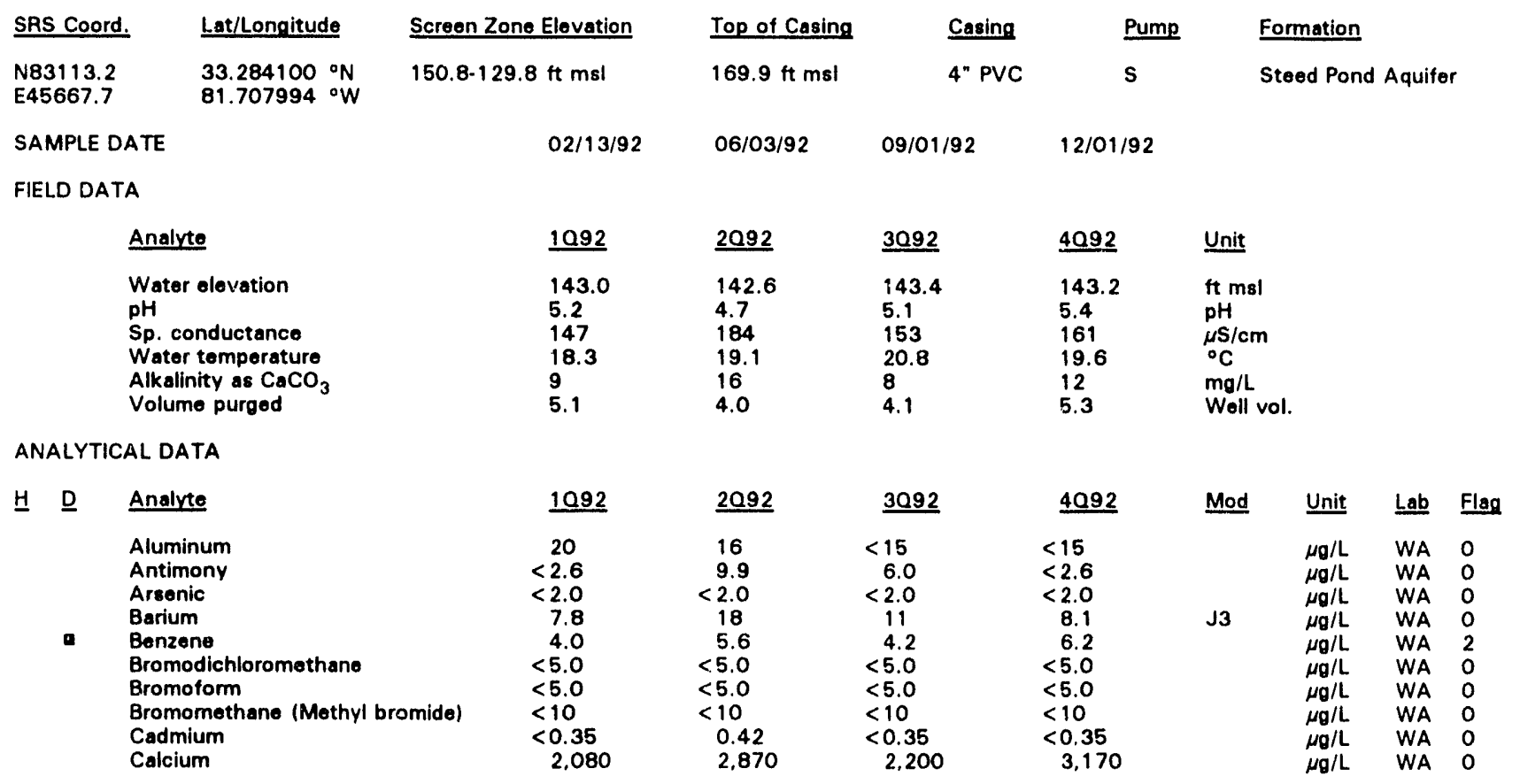

Note: Flagging levels, modifiers, and laboratory are for 4th quarter 1992 data only. See Appendix B for flagging criteria. - = exceeded holding time for 4th quarter 1992.

- = exceeded final primary drinking water standard for 4th quarter 1992. 
Well LFW 37 continued

ANALYTICAL DATA

H D Analute

Carbon tetrachloride

Chloride

Chlorobenzene

Chloroethane

Chloroethene (Vinyl chloride)

2-Chloroethyl vinyl ether

Chloroform

Chloromethane (Methyl chloride)

Chromium

Copper

Dibromochloromethane

1,1-Dichloroothane

- 1,2-Dichloroethano

1,1-Dichloroethylene

trans-1,2-Dichloroethylene

Dichloromethane (Methylene chloride)

2,4-Dichlorophenoxyacetic acid

1,2-Dichloropropane

cis-1,3-Dichloropropene

trans-1,3-Dichloropropene

Endrin

Ethylbenzene

Fluoride

Gross alpha

Iron

Lead

Lindane

Magnesium

Manganese

Mercury

Mothoxychior

Nickal

Nitrate as nitrogen

Nonvolatile beta

- $\quad$ pH

Phonols

Potassium

Radium-226

Radium-228

Solonium

Silica

Silicon

Silver

Sodium

- Specific conductance

Sulfate

1,1,2,2-Tetrachloroethane

Tetrachloroethylene

Toluene

Total dissolved solids

Total organic carbon

Total organic halogens

Toxaphene

2,4,5-TP (Silvex)

1,1,1-Trichloroethane

1,1,2-Trichloroethane

- Trichloroethylene

Trichlorofluoromethane

Tritium

Vanadium

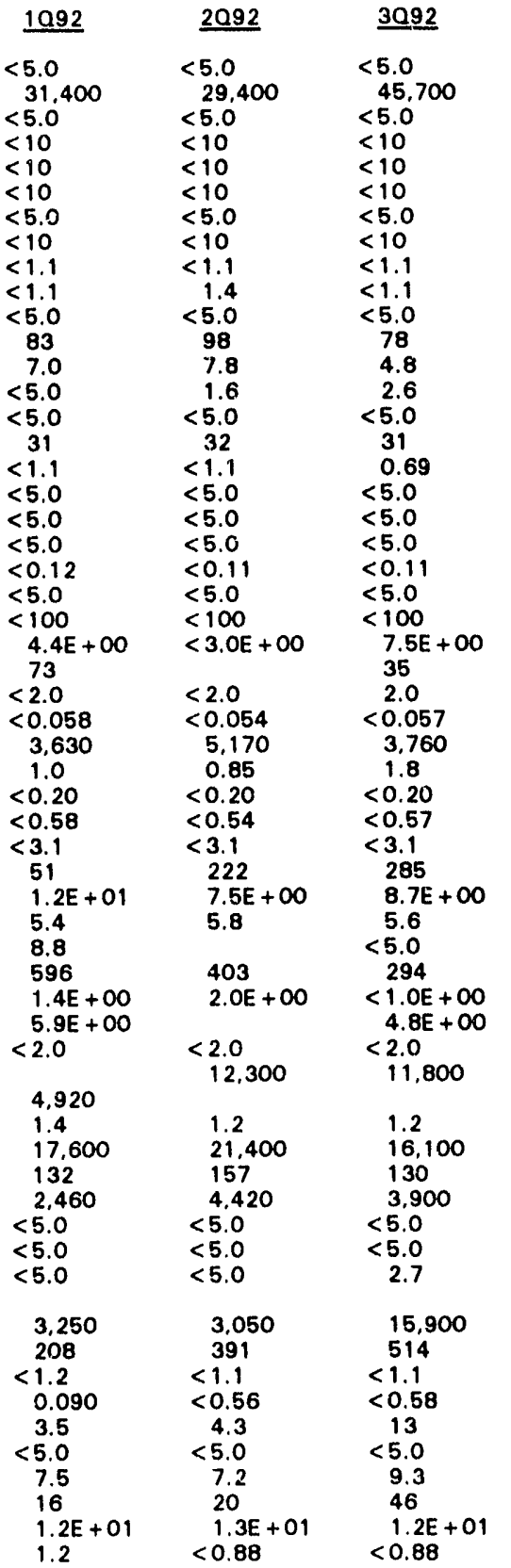

\begin{tabular}{|c|c|c|c|c|}
\hline 4092 & Mod & Unit & $\underline{\operatorname{Lab}}$ & Flag \\
\hline$<5.0$ & & $\mu \mathrm{g} / \mathrm{L}$ & WA & 0 \\
\hline 28,600 & & $\mu / \mathrm{g}$ & WA & 0 \\
\hline$<5.0$ & & $\mu \mathrm{g} / \mathrm{L}$ & WA & 0 \\
\hline$<10$ & & $\mu \sigma / L$ & WA & 0 \\
\hline $\begin{array}{l}<10 \\
<10\end{array}$ & & $\mu g / L$ & WA & 0 \\
\hline $\begin{array}{l}<10 \\
<5.0\end{array}$ & & $\begin{array}{l}\mu \mathrm{g} / \mathrm{L} \\
\mu \mathrm{g} / \mathrm{L}\end{array}$ & $\begin{array}{l}\text { WA } \\
\text { WA }\end{array}$ & $\begin{array}{l}0 \\
0\end{array}$ \\
\hline$<10$ & & $\mu_{g} / \mathrm{L}$ & WA & 0 \\
\hline 2.3 & $v$ & $\mu \mathrm{g} / \mathrm{L}$ & WA & 0 \\
\hline$<1.1$ & & $\mu \boldsymbol{g} / L$ & WA & 0 \\
\hline$<5.0$ & & $\mu \mathrm{g} / \mathrm{L}$ & WA & 0 \\
\hline $\begin{array}{l}86 \\
5.1\end{array}$ & & $\mu g / L$ & WA & 2 \\
\hline $\begin{array}{r}5.1 \\
<5.0\end{array}$ & & $\mu g / L$ & WA & 2 \\
\hline $\begin{array}{l}<5.0 \\
<5.0\end{array}$ & & $\mu g / L$ & WA & 0 \\
\hline $\begin{array}{c}<5.0 \\
29\end{array}$ & & $\mu g / L$ & WA & 0 \\
\hline $\begin{array}{r}29 \\
<1.1\end{array}$ & & $\mu \mathrm{g} / \mathrm{L}$ & WA & 0 \\
\hline $\begin{array}{l}<1.1 \\
<5.0\end{array}$ & & $\mu \mathrm{g} / \mathrm{L}$ & WA & 0 \\
\hline $\begin{array}{l}<5.0 \\
<5.0\end{array}$ & & $\mu \mathrm{g} / \mathrm{L}$ & WA & 0 \\
\hline $\begin{array}{l}<5.0 \\
<5.0\end{array}$ & & $\mu g / L$ & WA & 0 \\
\hline $\begin{array}{l}<5.0 \\
<0.11\end{array}$ & & $\mu \mathrm{g} / \mathrm{L}$ & $\begin{array}{l}\text { WA } \\
\text { WA }\end{array}$ & $\begin{array}{l}0 \\
0\end{array}$ \\
\hline$<5.0$ & & $\mu g / L$. & WA & 0 \\
\hline$<100$ & & $\mu \mathrm{g} / \mathrm{L}$ & WA & 0 \\
\hline $3.4 E+00$ & & $\begin{array}{l}\mathrm{pCi} / \mathrm{L} \\
\mu \mathrm{g} / \mathrm{L}\end{array}$ & $\mathrm{CN}$ & 0 \\
\hline$<2.0$ & & $\mu \mathrm{g} / \mathrm{L}$. & WA & 0 \\
\hline$<0.055$ & & $\mu g / L$ & WA & 0 \\
\hline 5,400 & & $\mu g / L$ & WA & 0 \\
\hline 0.97 & $\mathbf{J 3}$ & $\mu g / L$ & WA & 0 \\
\hline$<0.20$ & & $\mu g / L$ & WA & 0 \\
\hline $\begin{array}{l}<0.55 \\
<3.1\end{array}$ & & $\begin{array}{l}\mu \mathrm{g} / \mathrm{L} \\
\mu \mathrm{g} / \mathrm{L}\end{array}$ & $\begin{array}{l}\text { WA } \\
\text { WA }\end{array}$ & $\begin{array}{l}0 \\
0\end{array}$ \\
\hline$<20$ & & $\mu \mathrm{g} / \mathrm{L}$ & WA & 0 \\
\hline $7.9 E+00$ & & $\mathrm{pCi} / \mathrm{L}$ & CN & 0 \\
\hline 5.5 & $J$ & $\mathrm{pH}$ & WA & 0 \\
\hline$<5.0$ & & $\mu_{g} / \mathrm{L}$ & WA & 0 \\
\hline 604 & V & $\mu g / L$ & WA & 0 \\
\hline $1.5 E+00$ & & $\begin{array}{l}\mathrm{pCl} / \mathrm{L} \\
\mathrm{pCl} / \mathrm{L}\end{array}$ & $\mathrm{CN}$ & 0 \\
\hline$<2.0$ & & $\mu \mathrm{g} / \mathrm{L}$ & WA & 0 \\
\hline 11,900 & V & $\begin{array}{l}\mu \mathrm{g} / \mathrm{L} \\
\mu \mathrm{g} / \mathrm{L}\end{array}$ & WA & 0 \\
\hline$<0.70$ & & $\mu \mathrm{g} / \mathrm{L}$ & WA & 0 \\
\hline 19,100 & V & $\mu \mathrm{g} / \mathrm{L}$ & WA & 0 \\
\hline 148 & $J$ & $\mu \mathrm{S} / \mathrm{cm}$ & WA & 0 \\
\hline 6.290 & & $\mu g / L$ & WA & 0 \\
\hline$<5.0$ & & $\mu g / L$ & WA & 0 \\
\hline$<5.0$ & & $\mu \sigma / L$ & WA & 0 \\
\hline $\begin{array}{l}1.9 \\
105,000\end{array}$ & J & $\begin{array}{l}\mu \mathrm{g} / \mathrm{L} \\
\mu \mathrm{g} / \mathrm{L}\end{array}$ & $\begin{array}{l}\text { WA } \\
\text { WA }\end{array}$ & $\begin{array}{l}0 \\
0\end{array}$ \\
\hline $\begin{array}{l}105,000 \\
3,470\end{array}$ & & $\begin{array}{l}\mu \mathrm{g} / \mathrm{L} \\
\mu \mathrm{g} / \mathrm{L}\end{array}$ & WA & 0 \\
\hline 541 & & $\mu \mathrm{g} / \mathrm{L}$ & WA & 2 \\
\hline$<1.1$ & & $\mu g / L$ & WA & 0 \\
\hline 0.94 & & $\mu \mathrm{g} / \mathrm{L}$ & WA & 0 \\
\hline 6.9 & & $\mu g / L$ & WA & 0 \\
\hline$<5.0$ & & $\mu_{\boldsymbol{g}} / \mathrm{L}$ & WA & 0 \\
\hline 7.5 & & $\mu g / L$ & WA & 2 \\
\hline 36 & & $/ \mathrm{L}$ & WA & 2 \\
\hline $1.3 E+01$ & & $\mathrm{Cl} / \mathrm{mL}$ & $\mathrm{CN}$ & 1 \\
\hline 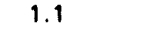 & J3 & /L & WA & 0 \\
\hline
\end{tabular}

Note: Flagging levels, modifiers, and laboratory are for 4th quarter 1992 data only. See Appendix B for flagging criteria.

- = exceeded holding time for 4th quarter 1992.

- exceeded final primary drinking water standard for 4th quarter 1992. 
WSRC-TR-93-068

WELL LFW 38

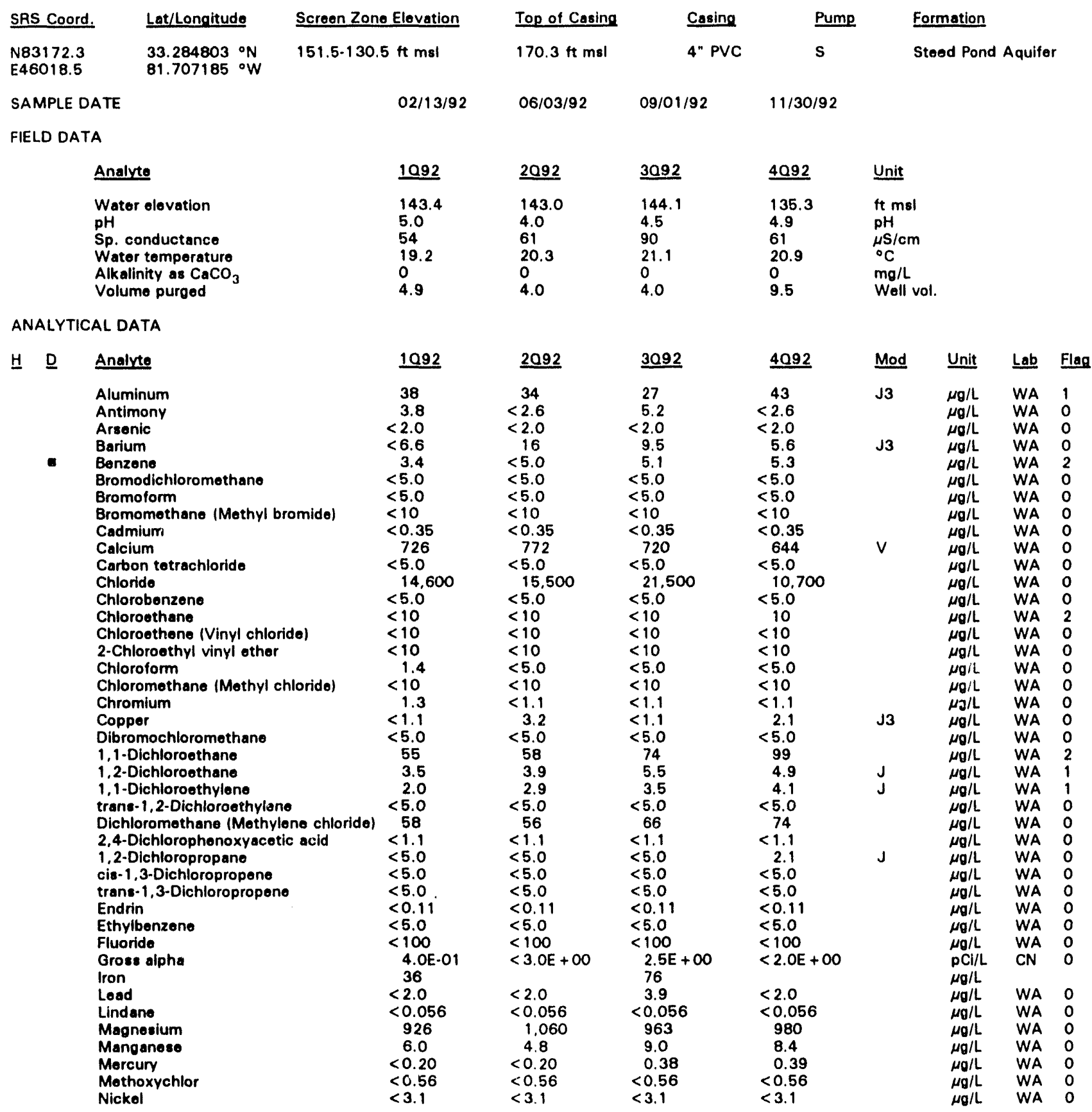

Note: Flagging levels, modifiers, and laboratory are for 4th quarter 1992 data only. See Appendix B for flagging criteria.

- = exceeded holding time for 4th quarter 1992.

- = exceeded final primary drinking water standard for 4th quarter 1992. 
Woll LFW 38 continued

ANALVTICAL DATA

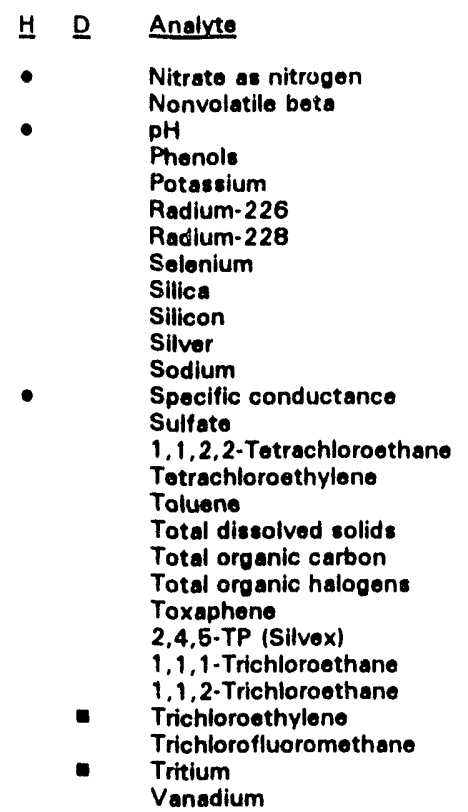

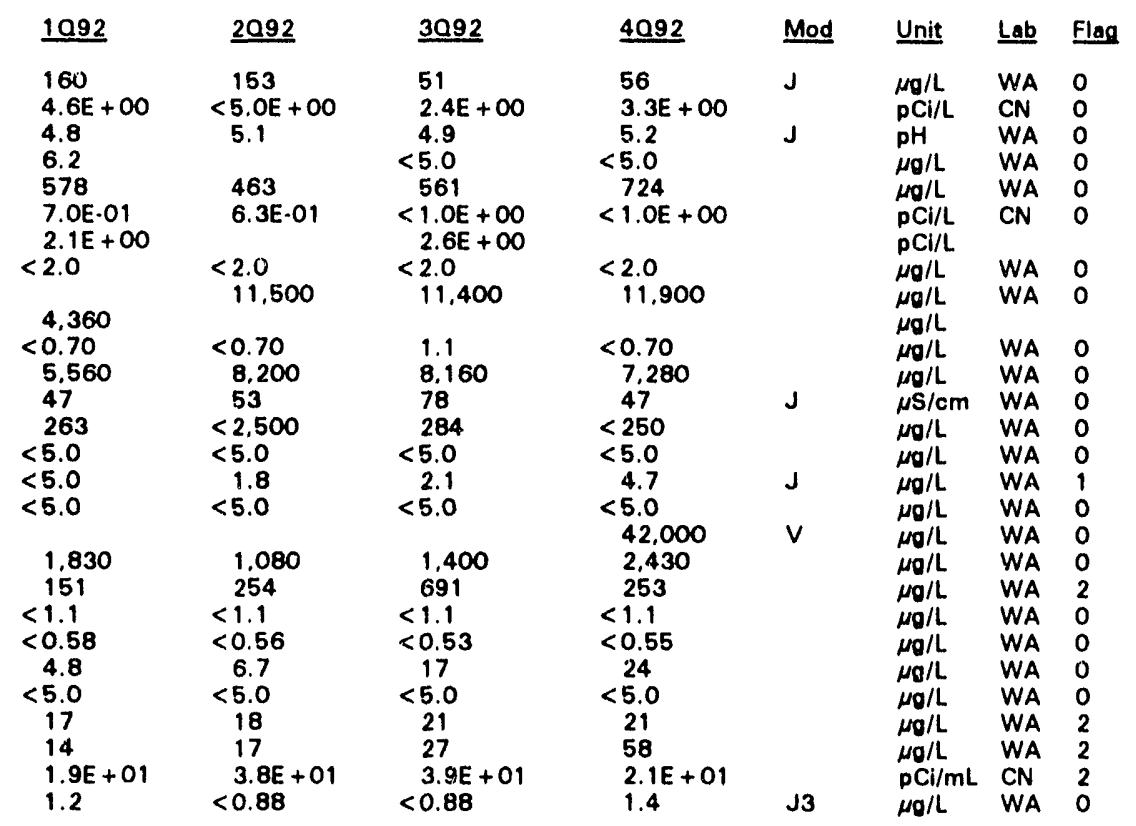

\section{WELL LFW 39}

\begin{tabular}{|c|c|c|c|c|c|c|c|c|c|}
\hline SAS Coord. & Lat/Lonqitude & Screen Zone Elevation & Top of Casing & Casing & Pump & \multicolumn{4}{|c|}{ Formation } \\
\hline $\begin{array}{l}\text { N83213.1 } \\
\text { E46218.5 }\end{array}$ & $\begin{array}{l}33.285220^{\circ} \mathrm{N} \\
81.706738^{\circ} \mathrm{W}\end{array}$ & $152.2-131.2 \mathrm{ft} \mathrm{msl}$ & $171.4 \mathrm{ft} \mathrm{msl}$ & $4^{n}$ PVC & $\mathbf{s}$ & \multicolumn{4}{|c|}{ Steod Pond Açuifer } \\
\hline \multicolumn{10}{|c|}{ FIELD DATA } \\
\hline \multicolumn{2}{|r|}{ Analyte } & 1092 & $\underline{2092}$ & 3092 & 4092 & \multicolumn{4}{|l|}{ Unit } \\
\hline \multicolumn{2}{|r|}{$\begin{array}{l}\text { Water elevation } \\
\text { pH } \\
\text { Sp. conductance } \\
\text { Water temperature } \\
\text { Alkalinity as } \mathrm{CaCO}_{3} \\
\text { Volume purged }\end{array}$} & $\begin{array}{l}143.7 \\
4.3 \\
38 \\
18.0 \\
0 \\
4.0\end{array}$ & $\begin{array}{l}143.2 \\
4.0 \\
31 \\
19.9 \\
0 \\
4.0\end{array}$ & $\begin{array}{l}144.2 \\
4.5 \\
32 \\
21.3 \\
0 \\
4.0\end{array}$ & $\begin{array}{l}136.0 \\
4.8 \\
35 \\
19.8 \\
0 \\
47.8\end{array}$ & \multicolumn{2}{|c|}{$\begin{array}{l}\text { ft mol } \\
\mathrm{pH} \\
\mu \mathrm{S} / \mathrm{cm} \\
{ }^{\circ} \mathrm{C} \\
\mathrm{mg} / \mathrm{L} \\
\text { Woll vol. }\end{array}$} & & \\
\hline \multicolumn{10}{|c|}{ ANALYTICAL DATA } \\
\hline \multirow[t]{2}{*}{$\underline{H} \quad \underline{D}$} & Analyte & 1092 & $\underline{2092}$ & 3092 & 4092 & Mod & Unit & $\underline{\text { Lab }}$ & Flag \\
\hline & $\begin{array}{l}\text { Aluminum } \\
\text { Antimony } \\
\text { Arcenic } \\
\text { Barium } \\
\text { Benzene } \\
\text { Bromodichloromethane } \\
\text { Bromoform } \\
\text { Bromomethane (Methyl } \\
\text { Cadmium } \\
\text { Calcium }\end{array}$ & $\begin{aligned} & 35 \\
< & 2.6 \\
< & 2.0 \\
< & 6.6 \\
< & 5.0 \\
< & 5.0 \\
< & 5.0 \\
< & 10 \\
< & 0.35 \\
& 497\end{aligned}$ & $\begin{aligned} & 25 \\
< & 2.6 \\
< & 2.0 \\
& 5.4 \\
< & 5.0 \\
< & 5.0 \\
< & 5.0 \\
< & 10 \\
< & 0.35 \\
& 358\end{aligned}$ & $\begin{aligned} & 17 \\
&<2.6 \\
&<2.0 \\
& \\
& 6.6 \\
&<5.0 \\
&<5.0 \\
&<5.0 \\
&<10 \\
&<0.35 \\
& \quad 308\end{aligned}$ & $\begin{aligned} & 19 \\
< & 2.6 \\
< & 2.0 \\
& 6.0 \\
< & 5.0 \\
< & 5.0 \\
< & 5.0 \\
< & 10 \\
< & 0.35 \\
& 433\end{aligned}$ & J3 & $\begin{array}{l}\mu g / L \\
\mu g / L \\
\mu g / L \\
\mu g / L \\
\mu g / L \\
\mu g / L \\
\mu g / L \\
\mu g / L \\
\mu g / L \\
\mu g / L\end{array}$ & $\begin{array}{l}\text { WA } \\
\text { WA } \\
\text { WA } \\
\text { WA } \\
\text { WA } \\
\text { WA } \\
\text { WA } \\
\text { WA } \\
\text { WA } \\
\text { WA }\end{array}$ & $\begin{array}{l}0 \\
0 \\
0 \\
0 \\
0 \\
0 \\
0 \\
0 \\
0 \\
0\end{array}$ \\
\hline
\end{tabular}

Note: Flagging levels, modifiers, and laboratory are for 4 th quarter 1992 data only. See Appendix B for flagging criteria.

- = exceeded holding time for 4th quarter 1992.

- = exceeded final primary drinking water standard for 4th quarter 1992. 
Well LFW 39 continued

ANALYTICAL DATA

H D Analyte

\begin{tabular}{|c|c|c|c|c|c|c|c|}
\hline 1092 & $\underline{2092}$ & 3092 & 4092 & Mod & Unit & Lab & Flag \\
\hline $\begin{array}{l}<5.0 \\
4.330\end{array}$ & $\begin{array}{l}<5.0 \\
3.650\end{array}$ & $\begin{array}{l}<5.0 \\
3.160\end{array}$ & $\begin{array}{l}<5.0 \\
3.860\end{array}$ & & $\underset{\mu \mathrm{g} / \mathrm{L}}{\mu \mathrm{g} / \mathrm{L}}$ & $\begin{array}{l}\text { WA } \\
\text { WA }\end{array}$ & $\begin{array}{l}0^{\prime} \\
0\end{array}$ \\
\hline$<5.0$ & $<5.0$ & $<<5.0$ & $<5.0$ & & $\mu \mathrm{g} / \mathrm{L}$ & WA & 0 \\
\hline$<10$ & $<10$ & $<10$ & 12 & & $\mu \mathrm{g} / \mathrm{L}$ & WA & 2 \\
\hline$<10$ & $<10$ & $<10$ & $<10$ & & $\mu \mathrm{g} / \mathrm{L}$ & WA & 0 \\
\hline$<10$ & $<10$ & $<10$ & $<10$ & & $\mu_{g} / \mathrm{L}$ & WA & 0 \\
\hline$<5.0$ & $<5.0$ & $<5.0$ & $<5.0$ & & $\mu \mathrm{q} / \mathrm{L}$ & WA & 0 \\
\hline$<10$ & $<10$ & $<10$ & $<10$ & & $\mu \mathrm{g} / \mathrm{L}$ & WA & 0 \\
\hline$<1.1$ & $<1.1$ & $<1.1$ & $<1.1$ & & $\mu \mathrm{g} / \mathrm{L}$ & WA & 0 \\
\hline 2.5 & 2.6 & 2.9 & 1.3 & J3 & $\mu \mathrm{g} / \mathrm{L}$ & WA & 0 \\
\hline$<5.0$ & $<5.0$ & $<5.0$ & $<5.0$ & & $\mu \mathrm{g} / \mathrm{L}$ & WA & 0 \\
\hline $\begin{array}{r}41 \\
<5.0\end{array}$ & $\begin{array}{r}38 \\
<5.0\end{array}$ & $\begin{array}{r}43 \\
<5.0\end{array}$ & $\begin{array}{r}59 \\
<5.0\end{array}$ & & $\begin{array}{l}\mu \mathrm{g} / \mathrm{L} \\
\mu \mathrm{g} / \mathrm{L}\end{array}$ & $\begin{array}{l}\text { WA } \\
\text { WA }\end{array}$ & $\begin{array}{l}2 \\
0\end{array}$ \\
\hline 13 & 8.6 & 9.0 & 8.6 & & $\mu \mathrm{g} / \mathrm{L}$ & WA & 2 \\
\hline$<5.0$ & $<5.0$ & $<5.0$ & $<5.0$ & & $\mu \mathrm{g} / \mathrm{L}$ & WA & 0 \\
\hline 13 & 13 & 17 & 44 & & $\mu \mathrm{g} / \mathrm{L}$ & WA & 0 \\
\hline$<1.1$ & $<1.1$ & $<1.1$ & $<1.1$ & & $\mu \mathrm{g} / \mathrm{L}$ & WA & 0 \\
\hline$<5.0$ & $<5.0$ & $<5.0$ & $<5.0$ & & $\mu \mathrm{g} / \mathrm{L}$ & WA & 0 \\
\hline$<5.0$ & $<5.0$ & $<5.0$ & $<5.0$ & & $\mu \mathrm{g} / \mathrm{L}$ & WA & 0 \\
\hline$<5.0$ & $<5.0$ & $<5.0$ & $<5.0$ & & $\mu \mathrm{g} / \mathrm{L}$ & WA & 0 \\
\hline$<0.11$ & $<0.11$ & $<0.10$ & $<0.12$ & & $\mu \mathrm{g} / \mathrm{L}$ & WA & 0 \\
\hline$<5.0$ & $<5.0$ & $<5.0$ & $<5.0$ & & $\mu \sigma / L$ & WA & 0 \\
\hline $\begin{array}{l}<100 \\
1.8 E+\infty \\
61\end{array}$ & $\begin{array}{l}<100 \\
<3.0 E+00\end{array}$ & $\begin{array}{l}<100 \\
<2.0 E+\infty 0 \\
34\end{array}$ & $\begin{array}{l}<100 \\
3.1 E+\infty\end{array}$ & & $\begin{array}{l}\mu \mathrm{g} / \mathrm{L} \\
\mathrm{pCi} / \mathrm{L} \\
\mu \mathrm{g} / \mathrm{L}\end{array}$ & $\begin{array}{l}\text { WA } \\
C N\end{array}$ & $\begin{array}{l}0 \\
0\end{array}$ \\
\hline$<2.0$ & $<2.0$ & 2.3 & $<2.0$ & & $\mu_{g} / \mathrm{L}$ & WA & 0 \\
\hline $\begin{array}{c}<0.056 \\
920\end{array}$ & $<0.056$ & $\begin{array}{c}<0.052 \\
641\end{array}$ & $<0.058$ & & $\mu_{\mathrm{g}} / \mathrm{L}$ & WA & 0 \\
\hline $\begin{array}{l}920 \\
1.6\end{array}$ & 0.67 & 1.3 & 1.5 & $\sqrt{13}$ & ${ }_{\mu \mathrm{g} / \mathrm{L}}^{\mu}$ & $\begin{array}{l}\text { WA } \\
\text { WA }\end{array}$ & 0 \\
\hline 0.24 & $<0.20$ & $<0.20$ & 0.35 & & $\mu_{\mathrm{g}} / \mathrm{L}$ & WA & 0 \\
\hline$<0.56$ & $<0.56$ & $<0.52$ & $<0.68$ & & $\mu \mathrm{g} / \mathrm{L}$ & WA & 0 \\
\hline$<3.1$ & $<3.1$ & $<3.1$ & $<3.1$ & & $\mu \mathrm{g} / \mathrm{L}$ & WA & 0 \\
\hline 1,250 & 1.110 & 843 & 414 & $J$ & $\mu \mathrm{g} / \mathrm{L}$ & WA & 0 \\
\hline $\begin{array}{l}4.9 \mathrm{E}+\infty \\
4.8\end{array}$ & $<5.0 \mathrm{OE}+00$ & $3.2 E+00$ & $4.5 E+\infty 0$ & & $\mathrm{pCi} / \mathrm{L}$ & $\mathrm{CN}$ & 0 \\
\hline$<5.0$ & & $\begin{array}{r}5.0 \\
<5.0\end{array}$ & $\begin{array}{r}4.9 \\
<5.0\end{array}$ & $\boldsymbol{J}$ & $\underset{\mu \mathrm{g} / \mathrm{L}}{\mathrm{pH}}$ & $\begin{array}{l}\text { WA } \\
\text { WA }\end{array}$ & $\begin{array}{l}0 \\
0\end{array}$ \\
\hline 459 & 253 & 96 & 510 & & $\mu \mathrm{g} / \mathrm{L}$ & WA & 0 \\
\hline $\begin{array}{l}1.0 E+\infty 0 \\
3.1 E+\infty 0\end{array}$ & $<5.0 E-01$ & $\begin{array}{r}<1.0 E+\infty 0 \\
2.7 E+\infty 0\end{array}$ & $<1.0 E+00$ & & $\begin{array}{l}\mathrm{pCl} / \mathrm{L} \\
\mathrm{pCi} / \mathrm{L}\end{array}$ & $\mathrm{CN}$ & 0 \\
\hline$<2.0$ & $\begin{aligned}<2.0 \\
\quad 8.630\end{aligned}$ & $\begin{array}{l}<2.0 \\
8.380\end{array}$ & $\begin{array}{l}<2.0 \\
11,300\end{array}$ & & $\begin{array}{l}\mu_{g} / \mathrm{L} \\
\mu_{g} / \mathrm{L}\end{array}$ & $\begin{array}{l}\text { WA } \\
\text { WA }\end{array}$ & $\begin{array}{l}0 \\
0\end{array}$ \\
\hline 3,920 & & & & & $\mu \mathrm{g} / \mathrm{L}$ & & \\
\hline $\begin{array}{l}0.85 \\
3.010\end{array}$ & $\begin{array}{l}<0.70 \\
2.650\end{array}$ & $\begin{array}{c}<0.70 \\
2390\end{array}$ & $\begin{array}{l}<0.70 \\
3.150\end{array}$ & & $\mu \mathrm{g} / \mathrm{L}$ & $\begin{array}{l}\text { WA } \\
\text { WA }\end{array}$ & $\begin{array}{l}0 \\
0\end{array}$ \\
\hline 30 & 26 & 26 & 31 & $J$ & $\mu \mathrm{S} / \mathrm{cm}$ & WA & 0 \\
\hline $\begin{array}{l}<100 \\
<5.0\end{array}$ & $\begin{array}{l}<250 \\
<5.0\end{array}$ & $\begin{array}{l}<250 \\
<5.0\end{array}$ & $\begin{array}{l}<250 \\
<5.0\end{array}$ & & $\mu_{\mu \sigma / L} / L$ & $\begin{array}{l}\text { WA } \\
\text { WA }\end{array}$ & $\begin{array}{l}0 \\
0\end{array}$ \\
\hline 7.0 & 4.5 & 4.4 & 5.6 & & $\mu \mathrm{g} / \mathrm{L}$ & WA & 2 \\
\hline$<5.0$ & $<5.0$ & $<5.0$ & $\begin{array}{l}<5.0 \\
38,000\end{array}$ & $V$ & $\begin{array}{l}\mu \mathrm{g} / \mathrm{L} \\
\mu \mathrm{g} / \mathrm{L}\end{array}$ & $\begin{array}{l}\text { WA } \\
\text { WA }\end{array}$ & $\begin{array}{l}0 \\
0\end{array}$ \\
\hline 808 & $<500$ & 1.740 & $<500$ & & $\mu \mathrm{g} / \mathrm{L}$ & WA & 0 \\
\hline $\begin{array}{r}243 \\
<1.1\end{array}$ & 288 & $\begin{array}{r}200 \\
<10\end{array}$ & 428 & & $\mu \sigma / L$ & WA & 2 \\
\hline $\begin{array}{l}<1.1 \\
<0.57\end{array}$ & $\begin{array}{l}<1.1 \\
<0.55\end{array}$ & $\begin{array}{l}<1.0 \\
<0.58\end{array}$ & $\begin{array}{l}<1.2 \\
<0.57\end{array}$ & & ${ }_{\mu \mathrm{g} / \mathrm{L}}$ & $\begin{array}{l}\text { WA } \\
\text { WA }\end{array}$ & $\begin{array}{l}0 \\
0\end{array}$ \\
\hline $\begin{array}{r}<07 \\
207\end{array}$ & 101 & 93 & $\begin{array}{l}<.68 \\
68\end{array}$ & & $\mu \mathrm{g} / \mathrm{L}$ & WA & 0 \\
\hline$<5.0$ & $<5.0$ & $<5.0$ & $<5.0$ & & $\mu \mathrm{g} / \mathrm{L}$ & WA & 0 \\
\hline 11 & 9.8 & 14 & 15 & & $\mu \mathrm{g} / \mathrm{L}$ & WA & 2. \\
\hline 285 & 134 & 118 & 209 & & $\mu \sigma / L$ & WA & 2 \\
\hline $\begin{array}{l}3.1 E+\infty 0 \\
1.3\end{array}$ & $\begin{array}{l}2.2 E+\infty 0 \\
<0.88\end{array}$ & $\begin{array}{l}3.2 E+00 \\
<0.88\end{array}$ & $\begin{array}{l}3.7 E+00 \\
1.2\end{array}$ & J3 & ${ }_{\mu \mathrm{g} / \mathrm{L}}^{\mathrm{pCI}}$ & $\begin{array}{l}\text { CN } \\
\text { WA }\end{array}$ & $\begin{array}{l}0 \\
0\end{array}$ \\
\hline
\end{tabular}

Note: Flagging levels, modifiers, and laboratory are for 4th quarter 1992 data only. See Appendix B for flagging criteria.

- =xceeded holding time for 4th quarter 1992.

- = exceeded final primary drinking water standard for 4th quarter 1992. 
WELL LFW 40

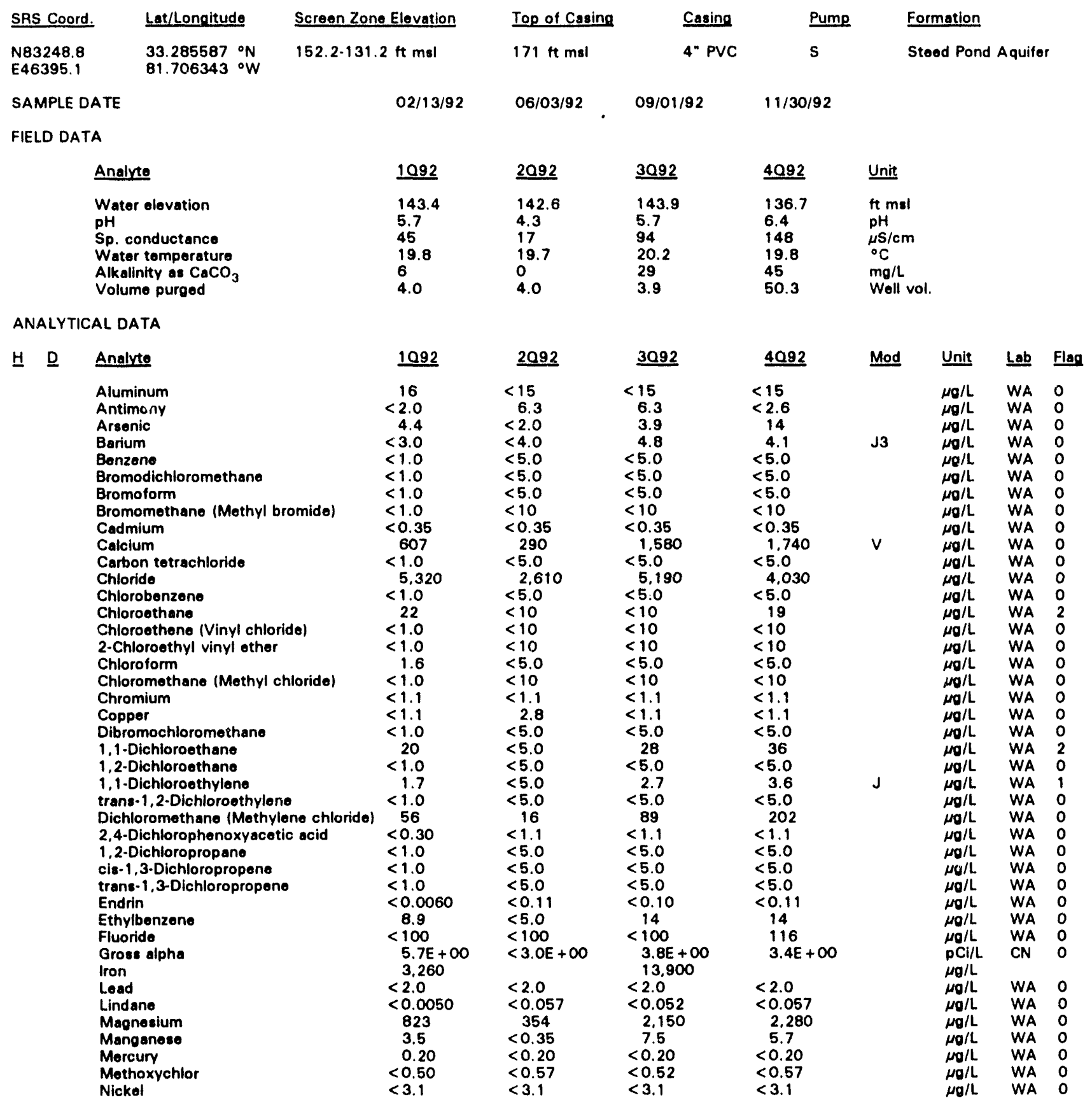

Note: Flagging levels, modifiers, and laboratory are for 4th quarter 1992 data only. See Appendix B for flagging criteria.

- = exceeded holding time for 4th quarter 1992.

- = exceeded final primary drinking water standard for 4th quarter 1992. 
WSRC-TR-93-068

Well LFW 40 continued

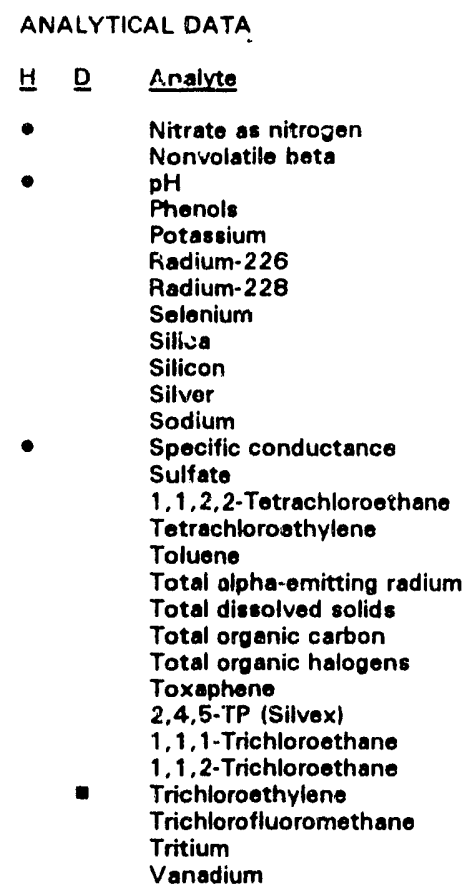

\begin{tabular}{|c|c|c|c|c|c|c|c|}
\hline 1092 & 2092 & 3092 & 4092 & Mod & Unit & Lab & Flag \\
\hline $\begin{array}{l}63 \\
3.4 E+00 \\
5.7 \\
16 \\
311 \\
7.0 E-01 \\
1.1 E+00\end{array}$ & $\begin{array}{l}\quad 202 \\
<5.0 \mathrm{E}+00 \\
5.3 \\
\\
350 \\
<5.0 \mathrm{E}-01\end{array}$ & $\begin{array}{l}50 \\
3.5 E+\infty \\
6.0 \\
6.0 \\
<84 \\
<1.0 E+\infty 0 \\
1.2 E+\infty 0\end{array}$ & $\begin{aligned}< & 20 \\
< & 2.0 \mathrm{E}+00 \\
& 6.2 \\
& 14 \\
& 181 \\
< & 1.0 \mathrm{E}+\infty\end{aligned}$ & $\begin{array}{l}\text { J3 } \\
\mathrm{J}\end{array}$ & $\begin{array}{l}\mu \mathrm{g} / \mathrm{L} \\
\mathrm{pCi} / \mathrm{L} \\
\mathrm{pH} \\
\mu \mathrm{g} / \mathrm{L} \\
\mu \mathrm{g} / \mathrm{L} \\
\mathrm{pCl} / \mathrm{L}\end{array}$ & $\begin{array}{l}\text { WA } \\
\text { CN } \\
\text { WA } \\
\text { WA } \\
\text { WA } \\
\text { CN }\end{array}$ & $\begin{array}{l}0 \\
0 \\
0 \\
0 \\
0 \\
0\end{array}$ \\
\hline $\begin{aligned}<2.0 \\
7.310 \\
2.930\end{aligned}$ & $\begin{array}{l}<2.0 \\
6.780\end{array}$ & $\begin{array}{l}<2.0 \\
\quad 6,740\end{array}$ & $\begin{array}{rl}<2 & 2.0 \\
7.590\end{array}$ & & $\begin{array}{l}\mu C / / L \\
\mu g / L \\
\mu g / L \\
\mu g / L\end{array}$ & $\begin{array}{l}\text { WA } \\
\text { WA }\end{array}$ & $\begin{array}{l}0 \\
0\end{array}$ \\
\hline $\begin{array}{l}<0.70 \\
3.620 \\
39 \\
508 \\
<1.0 \\
1.7 \\
6.3 \\
2.1 \mathrm{E}+00\end{array}$ & $\begin{array}{l}1.2 \\
2,000 \\
16 \\
407 \\
<5.0 \\
1.5 \\
<5.0\end{array}$ & $\begin{array}{c}<0.70 \\
3,300 \\
46 \\
680 \\
<5.0 \\
6.5 \\
11\end{array}$ & $\begin{array}{c}<0.70 \\
3.920 \\
114 \\
453 \\
<5.0 \\
4.6 \\
11\end{array}$ & $J$ & $\begin{array}{l}\mu \mathrm{g} / \mathrm{L} \\
\mu \mathrm{g} / \mathrm{L} \\
\mu \mathrm{S} / \mathrm{cm} \\
\mu \mathrm{g} / \mathrm{L} \\
\mu \mathrm{g} / \mathrm{L} \\
\mu \mathrm{g} / \mathrm{L} \\
\mu \mathrm{g} / \mathrm{L} \\
\mathrm{pCl} / \mathrm{L}\end{array}$ & $\begin{array}{l}\text { WA } \\
\text { WA } \\
\text { WA } \\
\text { WA } \\
\text { WA } \\
\text { WA } \\
\text { WA }\end{array}$ & $\begin{array}{l}0 \\
0 \\
0 \\
0 \\
0 \\
1 \\
0\end{array}$ \\
\hline $\begin{aligned} & 2,450 \\
& 192 \\
&< 0.24 \\
&< 0.090 \\
& 13< \\
&< 1.0 \\
& 7.1 \\
& 119 \\
& 3.2 \mathrm{E}+00 \\
& 1.3\end{aligned}$ & $\begin{array}{c}<500 \\
5.2 \\
<1.1 \\
<0.55 \\
6.8 \\
<5.0 \\
2.3 \\
21 \\
<2 . \text { J,E + } 00 \\
<\text { C.89 }\end{array}$ & $\begin{array}{rl} & 2.420 \\
233 & 233 \\
< & 1.0 \\
< & 0.58 \\
& 23 \\
< & 5.0 \\
14 \\
93 \\
1.5 E+00 \\
<0.88\end{array}$ & $\begin{array}{rl} & 53.000 \\
8.140 & 591 \\
& 591 \\
< & 0.1 \\
< & 0.57 \\
& 38 \\
<5.0 \\
12 \\
151 \\
2.1 E+00 \\
<0.88\end{array}$ & V & $\begin{array}{l}\mu \mathrm{g} / \mathrm{L} \\
\mu \mathrm{g} / \mathrm{L} \\
\mu_{\mathrm{g}} / \mathrm{L} \\
\mu_{\mathrm{g}} / \mathrm{L} \\
\mu_{\mathrm{g}} / \mathrm{L} \\
\mu_{\mathrm{g}} / \mathrm{L} \\
\mu_{\mathrm{g}} / \mathrm{L} \\
\mu_{\mathrm{g}} / \mathrm{L} \\
\mu_{\mathrm{g}} / \mathrm{L} \\
\mathrm{pCl} / \mathrm{mL} \\
\mu \mathrm{g} / \mathrm{L}\end{array}$ & $\begin{array}{l}\text { WA } \\
\text { WA } \\
\text { WA } \\
\text { WA } \\
\text { WA } \\
\text { WA } \\
\text { WA } \\
\text { WA } \\
\text { WA } \\
\text { CN } \\
\text { WA }\end{array}$ & $\begin{array}{l}0 \\
1 \\
2 \\
0 \\
0 \\
0 \\
0 \\
2 \\
2 \\
0 \\
0\end{array}$ \\
\hline
\end{tabular}

\section{WELL LFW 41}

$\begin{array}{ll}\text { SRS Coord. } & \text { Lat/Lonqitude } \\ \text { N83304.9 } & 35.286089{ }^{\circ} \mathrm{N} \\ \text { E46626.9 } & 81.705841^{\circ} \mathrm{W} \\ \text { SAMPLE DATE } & \\ \text { FIELD DATA } & \end{array}$

\section{Analyte}

Water olovation

pH

Sp. conductance

Water temperature

Alkalinity as $\mathrm{CaCO}_{3}$

Volume purged

Screen Zone Elovation

151.3.130.3 ft ms!

$02 / 13 / 92$
Top of Casing

$170.5 \mathrm{ft} \mathrm{msl}$

$06 / 03 / 92$

2092
144.1
4.2
15
19.1
0
4.0

\begin{tabular}{l}
$\underline{3092}$ \\
\hline 145.5 \\
4.8 \\
16 \\
20.5 \\
0 \\
4.0
\end{tabular}

2092

$<15$

Antimony

Arsenic

Barium

Benzene

Bromodichloromethane

Bromoform

Bromomothane (Methyl bromide)

Cadmium

1092

18
$<2.6$

1092
144.9
5.1
16
18.4
0
4.0

$<2.6$
$<2.0$

$<6.6$

$<5.0$

$<5.0$

$<5.0$

$<10$

$<0.35$

$\begin{array}{ll}\text { Casing } & \text { Pump } \\ \text { 4" PVC } & \mathrm{s}\end{array}$

Formation

Steed Pond Aquifer

\footnotetext{
(a)
} 


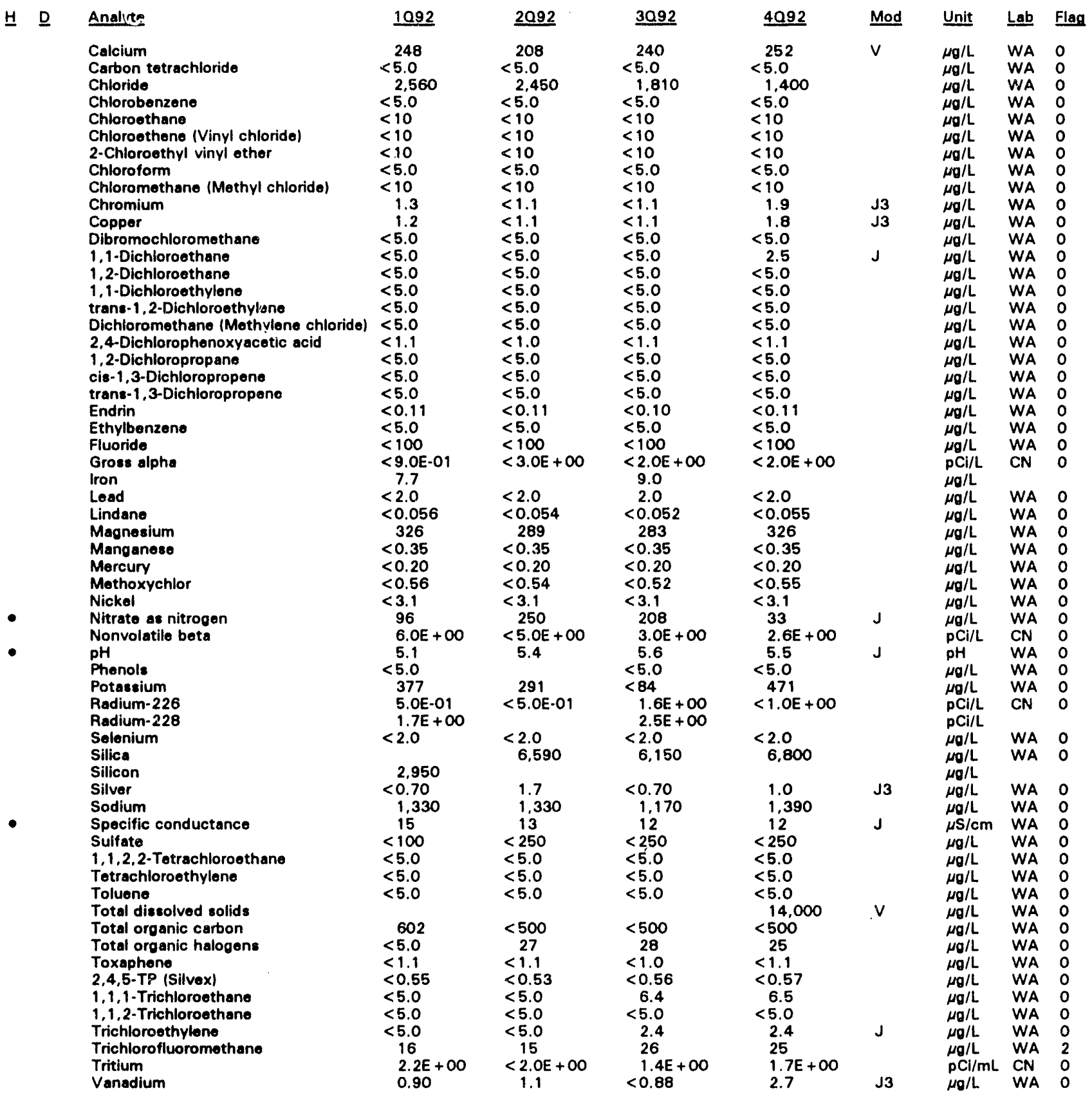

Note: Flagging levels, modifiers, and laboratory are for 4th quarter 1992 data only. See Appendix B for flagging criteria. - = exceeded holding time for 4th quarter 1992.

- = exceeded final primary drinking water standard for 4th quarter 1992. 
WSRC-TR-93-068

\section{WELL LFW 42}

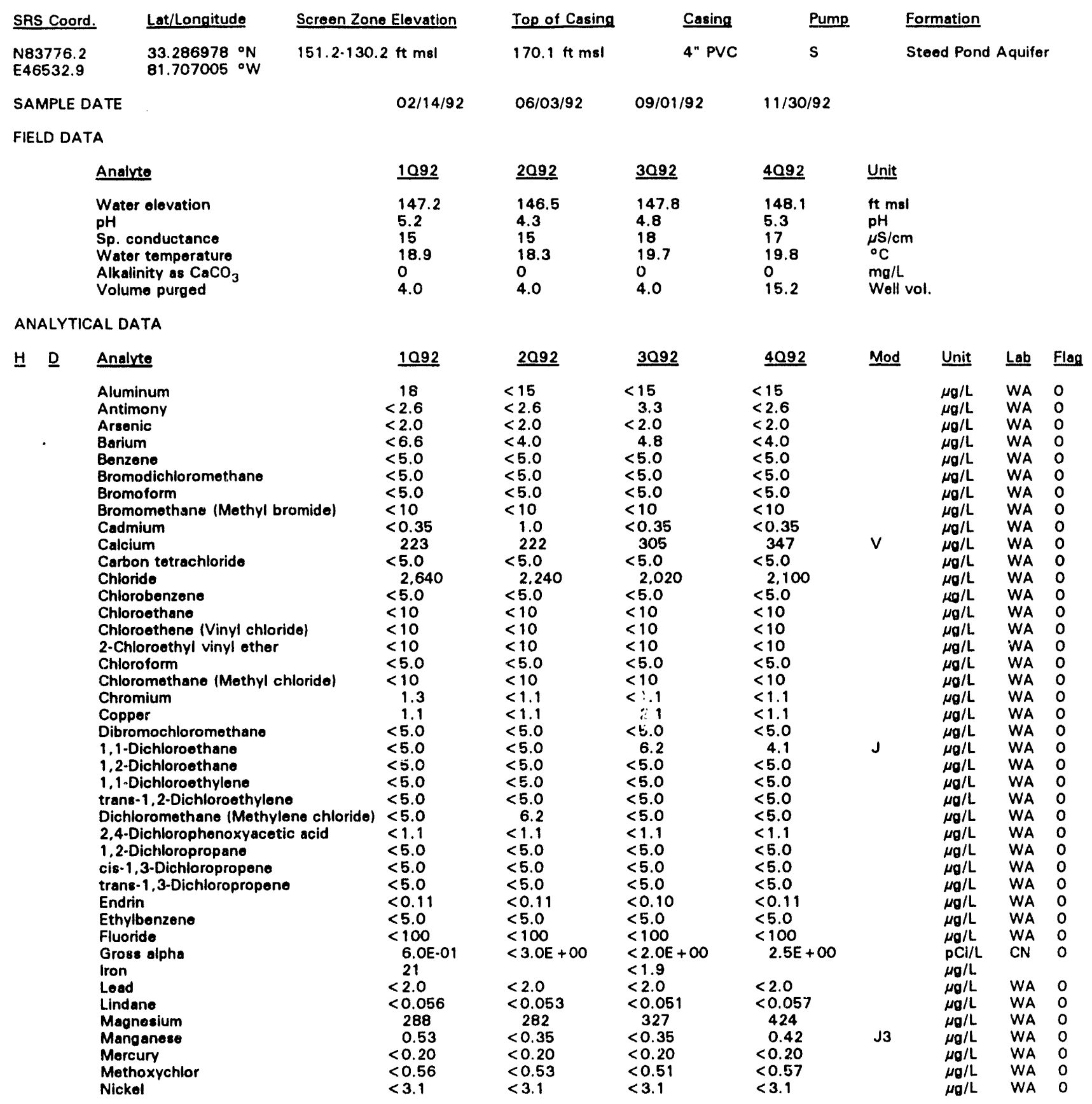

\footnotetext{
Note: Flagging levels, modifiers, and laboratory are for 4th quarter 1992 data only. See Appendix B for flagging criteria. - = exceeded holding time for 4th quarter 1992.

- exceeded finol primary drinking water standard for 4th quarter 1992.
} 
Woll LFW 42 continued

ANALYTICAL DATA
- D $\quad$ Analyte
- Nitrate as nitrogen
Nonvolatile beta
pH
Phenols
Potassium
Radium-226
Radium-228
Solenium
Silica
Silicon
Silver
Sodium
Specific conductance
Sulfate
1.1,2,2-Totrachloroethane
Totrachloroothylene
Toluene
Total diseolved solids
Total organic carbon
Total organic halogens
Toxaphene
2,4,5-TP (Silvex)
$1,1,1-$ Trichloroethane
1,1,2-Trichloroethane
Trichloroethylene
Trichlorofluoromethane
Tritium
Vanadium

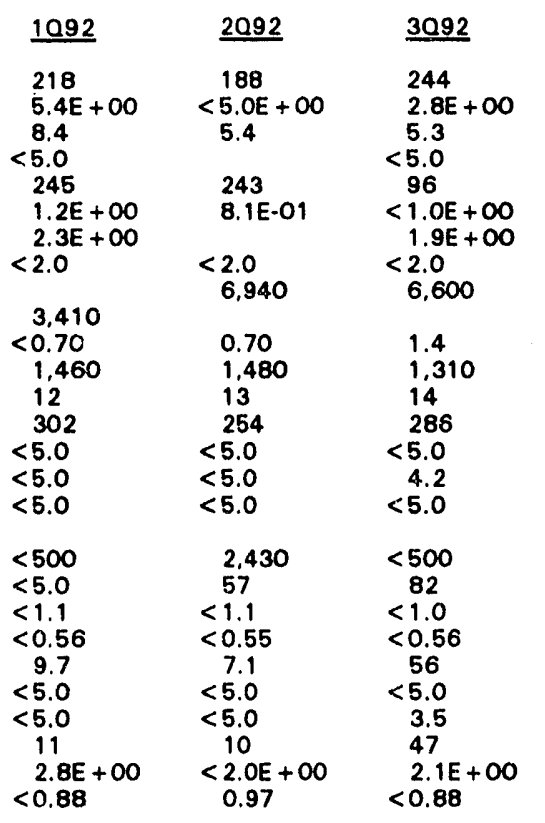

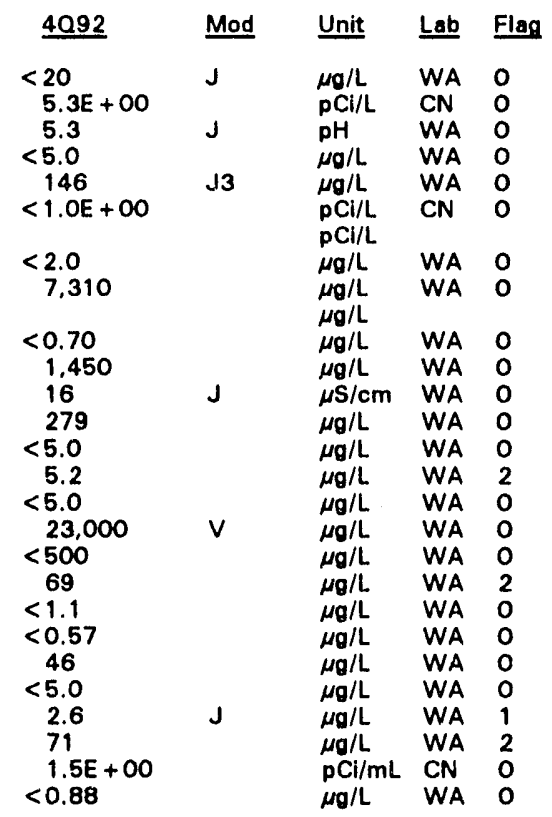

WELL LFW 43B

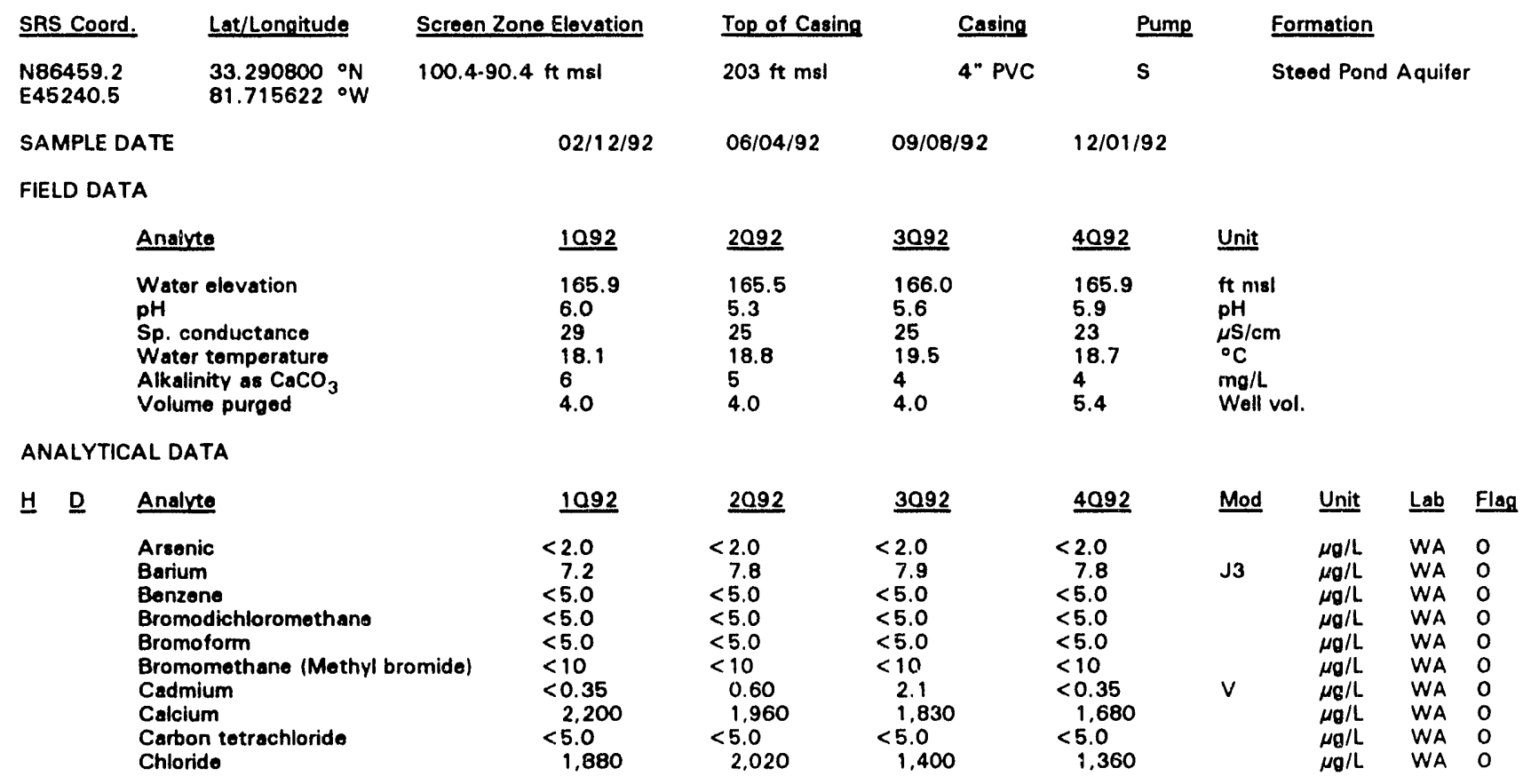

Note: Flagging levels, modifiers, and laboratory are for 4th quarter 1992 data only. See Appendix B for flagging criteria.

- = exceeded holding time for 4th quarter 1992.

- = exceeded final primary drinking water standard for 4th quarter 1992. 
WSRC-TR-93-068

Well LFW 43B continued

ANALYTICAL DATA

H D Analyte

Chlorobenzene

Chloroethane

Chloroethene (Vinyl chloride)

2-Chloroethyl vinyl ether

Chloroform

Chloromethane (Methyl chloride)

Chromium

Copper

Dibromochloromethane

1.1-Dichloroethane

1,2-Dichloroethane

1,1-Dichloroethylene

trans-1,2-Dichloroethylene

Dichloromethane (Methylene chloride)

2,4-Dichlorophenoxyacetic acid

1,2-Dichloropropane

cis-1.3-Dichloropropene

trans-1,3-Dichloropropene

Endrin

Ethylbenzene

Fluoride

Gross alpha

Iron

Lindane

Magnesium

Manganose

Mercury

Methoxychlor

Nickel

Nitrate as nitrogen

Nonvolatile beta

Nonvoletilo
PH
Phenols

Phonols

Potassium

Radium-228

Selenium

Silica

Silicon

Silver

Sodium

Specific conductance

Sulfate

1,1,2,2-Tetrachloroethane

Totrachloroethylene

Toluene

Total dissolved solids

Total organic carbon

Total organic halogens

Total phosphates las P

Toxaphene

2,4,5-TP (Silvex)

$1,1,1$-Trichloroethane

1,1,2-Trichloroethane

Trichloroethylene

rrichlorofluoromathane

Tritium

Vanadium

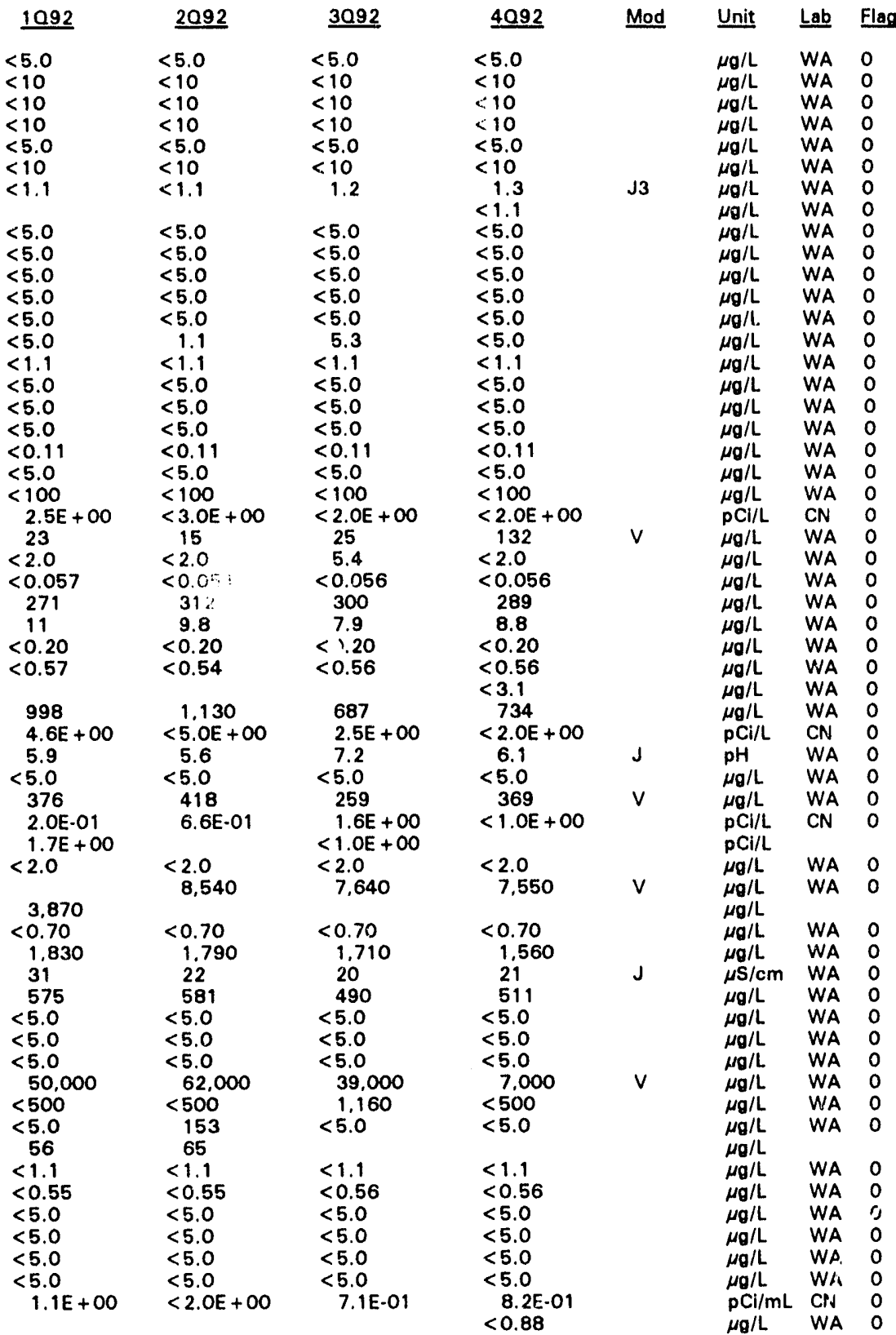

Note: Flagging levels, modifiers, and laboratory are for 4th quarter 1992 data only. See Appendix B for flagging criteria.

- = exceeded holding time for 4th quarter 1992.

- = exceeded final primary drinking water standard for 4th quarter 1992. 
WELL LFW 43C

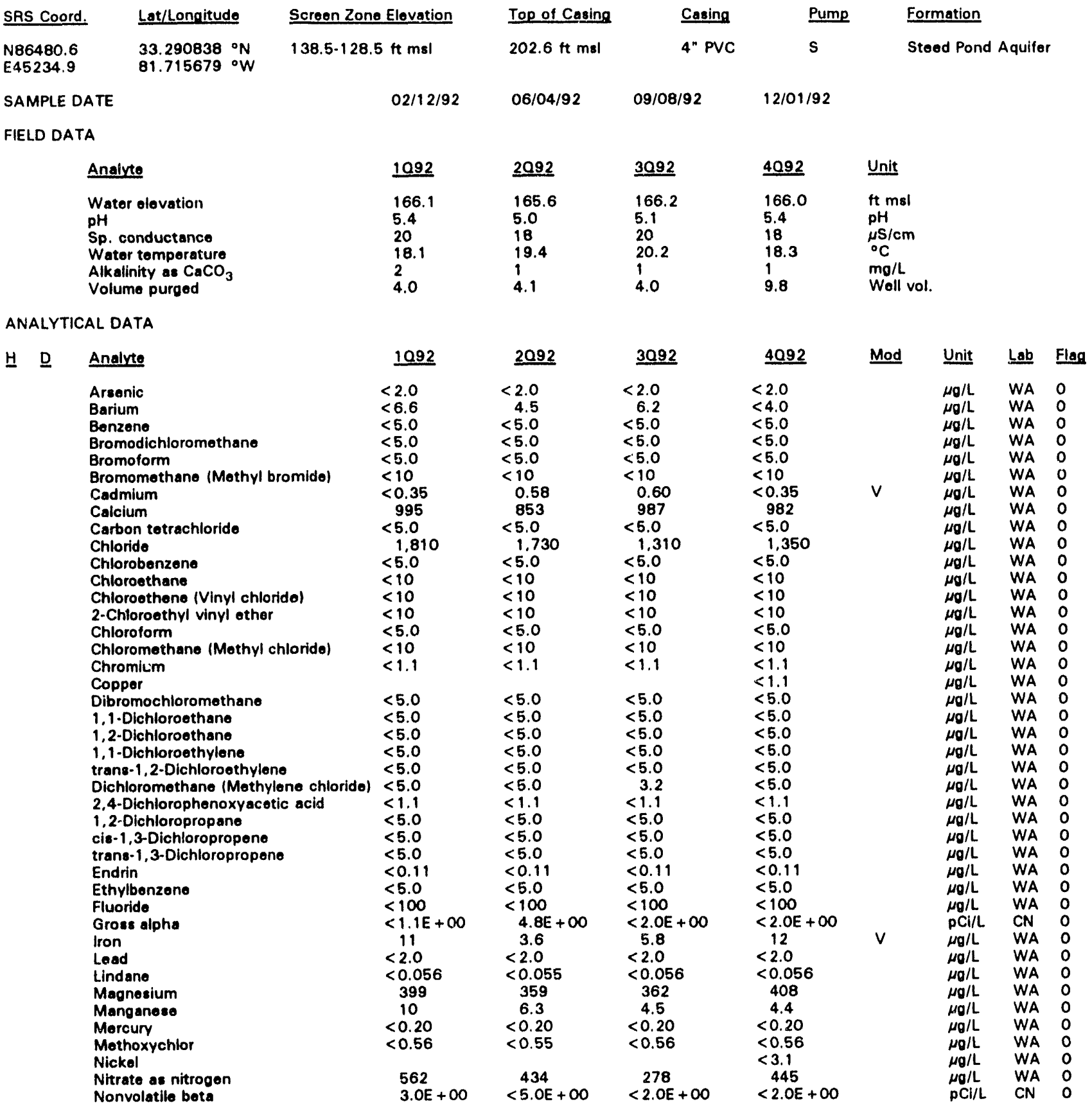

Note: Flagging levels, modifiers, and laboratory are for 4 th quarter 1992 data only. See Appendix B for flagging criteria.

- exceeded holding time for 4th quarter 1992.

- exceeded final primary drinking water standard for 4th quarter 1992. 
WSRC-TR-93-068

Well LFW 43 C continued

ANALYTICAL DATA

- D $\quad$ Analyte
pH
Phenols
Potassium
Radium-226
Radium-228
Selenium
Silica
Silicon
Silver
Sodium
Specific conductance
Sulfate
1,1,2,2-Totrachloroethane
Tetrachloroethylene
Toluene
Total dissolved solids
Total organic carbon
Total organic halogens
Total phosphates (as P)
Toxaphene
2,4,5-TP (Silvex)
1,1,1-Trichloroethane
1,1,2-Trichloroethane
Trichloroethylene
Trichlorofluoromethane
Tritium
Vanadium

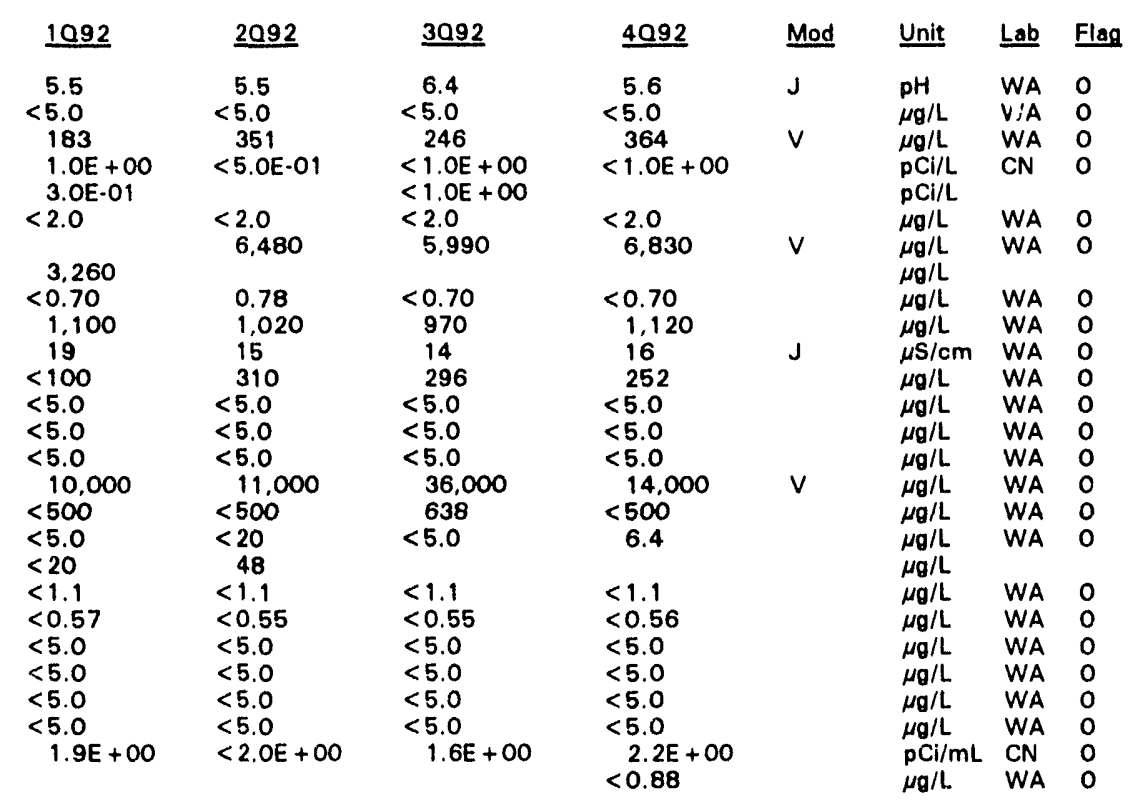

\section{WELL LFW 43D}

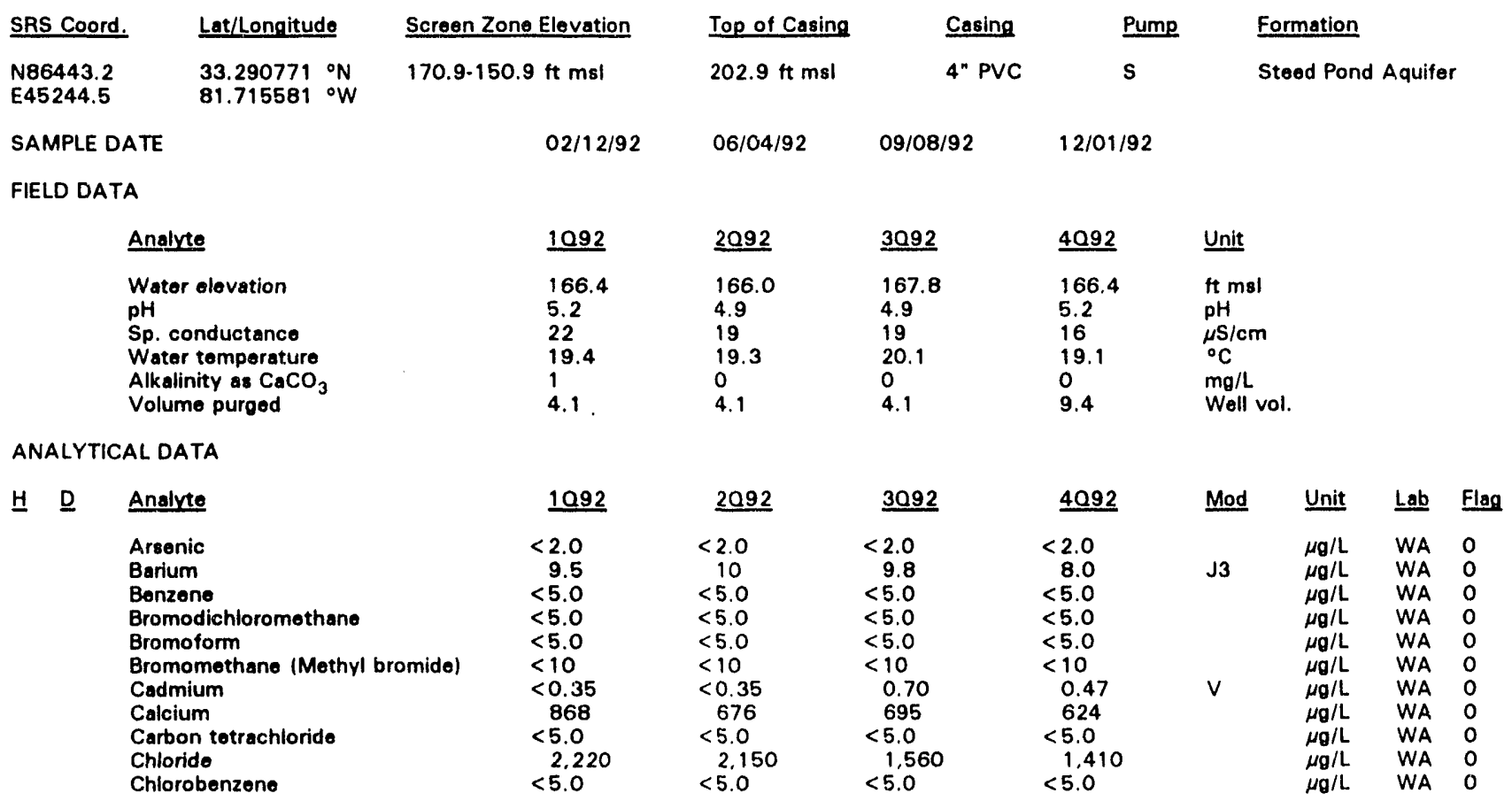

Note: Flagging levels, modifiers, and laboratory are for 4th quarter 1992 data only. See Appendix B for flagging criteria.

- = exceeded holding time for 4th quarter 1992.

- = exceeded final primary drinking water standard for 4th quarter 1992. 


\begin{tabular}{|c|c|c|c|c|c|c|c|c|}
\hline$\underline{H} \quad \underline{D}$ & Analvte & 1092 & 2092 & 3092 & 4092 & Mod & Unit & $\underline{\text { Lab }}$ \\
\hline & 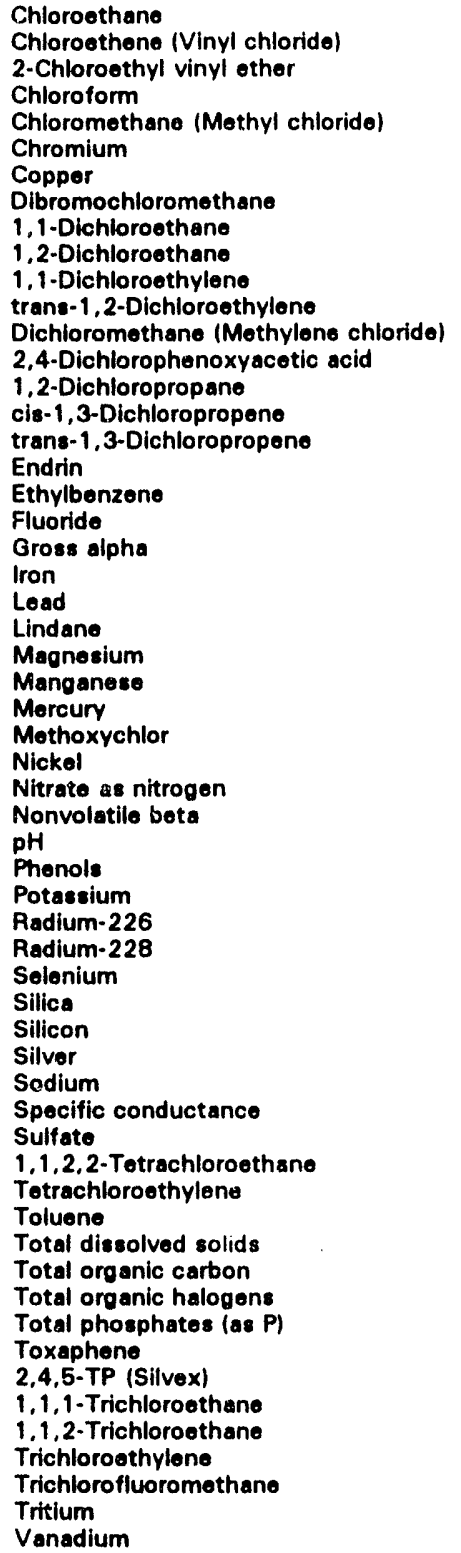 & 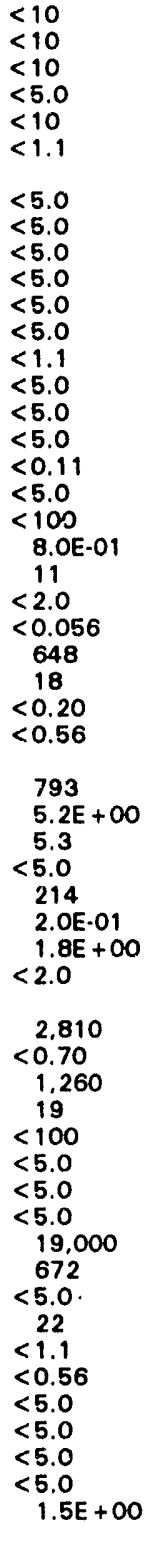 & $\begin{array}{l}<10 \\
<10 \\
<10 \\
<5.0 \\
<10 \\
<1.1 \\
<5.0 \\
<5.0 \\
<5.0 \\
<5.0 \\
<5.0 \\
11.0 \\
<1.1 \\
<5.0 \\
<5.0 \\
<5.0 \\
<0.11 \\
<5.0 \\
<100 \\
<3.0 E+00 \\
6.1 \\
<2.0 \\
<0.054 \\
599 \\
13 \\
<0.20 \\
<0.54 \\
\\
\quad 930 \\
<5.0 E+\infty \\
5.3 \\
<5.0 \\
201 \\
<5.0 E .01 \\
<2.0 \\
<2.0 \\
5.920 \\
<0.70 \\
1.060 \\
19 \\
<250 \\
<5.0 \\
<5.0 \\
<5.0 \\
15.000 \\
<500 \\
72 \\
48 \\
<1.1 \\
<0.55 \\
<5.0 \\
<5.0 \\
<5.0 \\
<5.0 \\
<2.0 E+00\end{array}$ & $\begin{array}{l}<10 \\
<10 \\
<10 \\
<5.0 \\
<10 \\
<1.1 \\
<5.0 \\
<5.0 \\
<5.0 \\
<5.0 \\
<5.0 \\
4.1 \\
<1.1 \\
<5.0 \\
<5.0 \\
<5.0 \\
<0.11 \\
<5.0 \\
<100 \\
<2.0 E+\infty \\
12 \\
<2.0 \\
<0.056 \\
546 \\
9.4 \\
<0.20 \\
<0.56 \\
\\
417 \\
4.0 E+00 \\
6.2 \\
<5.0 \\
<84 \\
<1.0 \mathrm{E}+\infty \\
1.3 \mathrm{E}+\infty \\
<2.0 \\
5.270 \\
<0.70 \\
840 \\
841 \\
15 \\
<250 \\
<5.0 \\
<5.0 \\
<5.0 \\
32.000 \\
1.480 \\
<5.0 \\
<1.1 \\
<0.57 \\
<5.0 \\
<5.0 \\
<5.0 \\
<5.0 \\
1.0 \mathrm{E}+00\end{array}$ & $\begin{array}{l}<10 \\
<10 \\
<10 \\
<5.0 \\
<10 \\
<1.1 \\
1.7 \\
<5.0 \\
<5.0 \\
<5.0 \\
<5.0 \\
<5.0 \\
<5.0 \\
<1.1 \\
<5.0 \\
<5.0 \\
<5.0 \\
<0.11 \\
<5.0 \\
<100 \\
<2.0 E+00 \\
32 \\
<2.0 \\
<0.054 \\
529 \\
9.3 \\
<0.20 \\
<0.54 \\
4.3 \\
519 \\
3.8 E+\infty \\
5.4 \\
<5.0 \\
375 \\
<1.0 E+\infty \\
<2.0 \\
<2.0 \\
5.360 \\
<0.70 \\
961 \\
16 \\
<250 \\
<5.0 \\
<5.0 \\
<5.0 \\
29.000 \\
<500 \\
<5.0 \\
<1.1 \\
<1.1 \\
<0.56 \\
<55.0 \\
<5.0 \\
<5.0 \\
<5.0 \\
9.8 E-01 \\
<0.88 \\
\end{array}$ & $\begin{array}{l}J \\
v\end{array}$ & $\begin{array}{l}\mu \mathrm{g} / \mathrm{L} \\
\mu \mathrm{g} / \mathrm{L} \\
\mu \mathrm{g} / \mathrm{L} \\
\mu \mathrm{g} / \mathrm{L} \\
\mu \mathrm{g} / \mathrm{L} \\
\mu \mathrm{g} / \mathrm{L} \\
\mu \mathrm{g} / \mathrm{L} \\
\mu \mathrm{g} / \mathrm{L} \\
\mu \mathrm{g} / \mathrm{L} \\
\mu \mathrm{g} / \mathrm{L} \\
\mu \mathrm{g} / \mathrm{L} \\
\mu \mathrm{g} / \mathrm{L} \\
\mu \mathrm{g} / \mathrm{L} \\
\mu \mathrm{g} / \mathrm{L} \\
\mu \mathrm{g} / \mathrm{L} \\
\mu \mathrm{g} / \mathrm{L} \\
\mu \mathrm{g} / \mathrm{L} \\
\mu \mathrm{g} / \mathrm{L} \\
\mu \mathrm{g} / \mathrm{L} \\
\mu \mathrm{g} / \mathrm{L} \\
\mathrm{pCC} / \mathrm{L} \\
\mu \mathrm{g} / \mathrm{L} \\
\mu \mathrm{g} / \mathrm{L} \\
\mu \mathrm{g} / \mathrm{L} \\
\mu \mathrm{g} / \mathrm{L} \\
\mu \mathrm{g} / \mathrm{L} \\
\mu \mathrm{g} / \mathrm{L} \\
\mu \mathrm{g} / \mathrm{L} \\
\mu \mathrm{g} / \mathrm{L} \\
\mu \mathrm{g} / \mathrm{L} \\
\mathrm{pC} \mathrm{Ci} / \mathrm{L} \\
\mathrm{pH} \\
\mu \mathrm{g} / \mathrm{L} \\
\mu \mathrm{g} / \mathrm{L} \\
\mathrm{pC \textrm {C }} / \mathrm{L} \\
\mathrm{pCC} / \mathrm{L} \\
\mu \mathrm{g} / \mathrm{L} \\
\mu \mathrm{g} / \mathrm{L} \\
\mu \mathrm{g} / \mathrm{L} \\
\mu \mathrm{g} / \mathrm{L} \\
\mu \mathrm{g} / \mathrm{L} \\
\mu \mathrm{S} / \mathrm{cm} \\
\mu \mathrm{g} / \mathrm{L} \\
\mu \mathrm{g} / \mathrm{L} \\
\mu \mathrm{g} / \mathrm{L} \\
\mu \mathrm{g} / \mathrm{L} \\
\mu \mathrm{g} / \mathrm{L} \\
\mu \mathrm{g} / \mathrm{L} \\
\mu \mathrm{g} / \mathrm{L} \\
\mu \mathrm{g} / \mathrm{L} \\
\mu \mathrm{g} / \mathrm{L} \\
\mu \mathrm{g} / \mathrm{L} \\
\mu \mathrm{g} / \mathrm{L} \\
\mu \mathrm{g} / \mathrm{L} \\
\mu \mathrm{g} / \mathrm{L} \\
\mu \mathrm{g} / \mathrm{L} \\
\mathrm{pCCl} / \mathrm{mL} \\
\mu \mathrm{g} / \mathrm{L}\end{array}$ & $\begin{array}{l}\text { WA } \\
\text { WA } \\
\text { WA } \\
\text { WA } \\
\text { WA } \\
\text { WA } \\
\text { WA } \\
\text { WA } \\
\text { WA } \\
\text { WA } \\
\text { WA } \\
\text { WA } \\
\text { WA } \\
\text { WA } \\
\text { WA } \\
\text { WA } \\
\text { WA } \\
\text { WA } \\
\text { WA } \\
\text { WA } \\
\text { CN } \\
\text { WA } \\
\text { WA } \\
\text { WA } \\
\text { WA } \\
\text { WA } \\
\text { WA } \\
\text { WA } \\
\text { WA } \\
\text { WA } \\
\text { CN } \\
\text { WA } \\
\text { WA } \\
\text { WA } \\
\text { CN } \\
\text { WA } \\
\text { WA } \\
\text { WA } \\
\text { WA } \\
\text { WA } \\
\text { WA } \\
\text { WA } \\
\text { WA } \\
\text { WA } \\
\text { WA } \\
\text { WA } \\
\text { WA } \\
\text { WA } \\
\text { WA } \\
\text { WA } \\
\text { WA } \\
\text { WA } \\
\text { WA } \\
\text { CN } \\
\text { WA }\end{array}$ \\
\hline
\end{tabular}

Note: Flagging levels, modifiers, and laboratory are for 4th quarter 1992 data only. See Appendix $B$ for flagging criteria. - = exceeded holding time for 4th quarter 1992.

- = excueded final primary drinking water standard for 4th quarter 1992. 
WSRC-TR-93-068

\section{WELL LFW 44D}

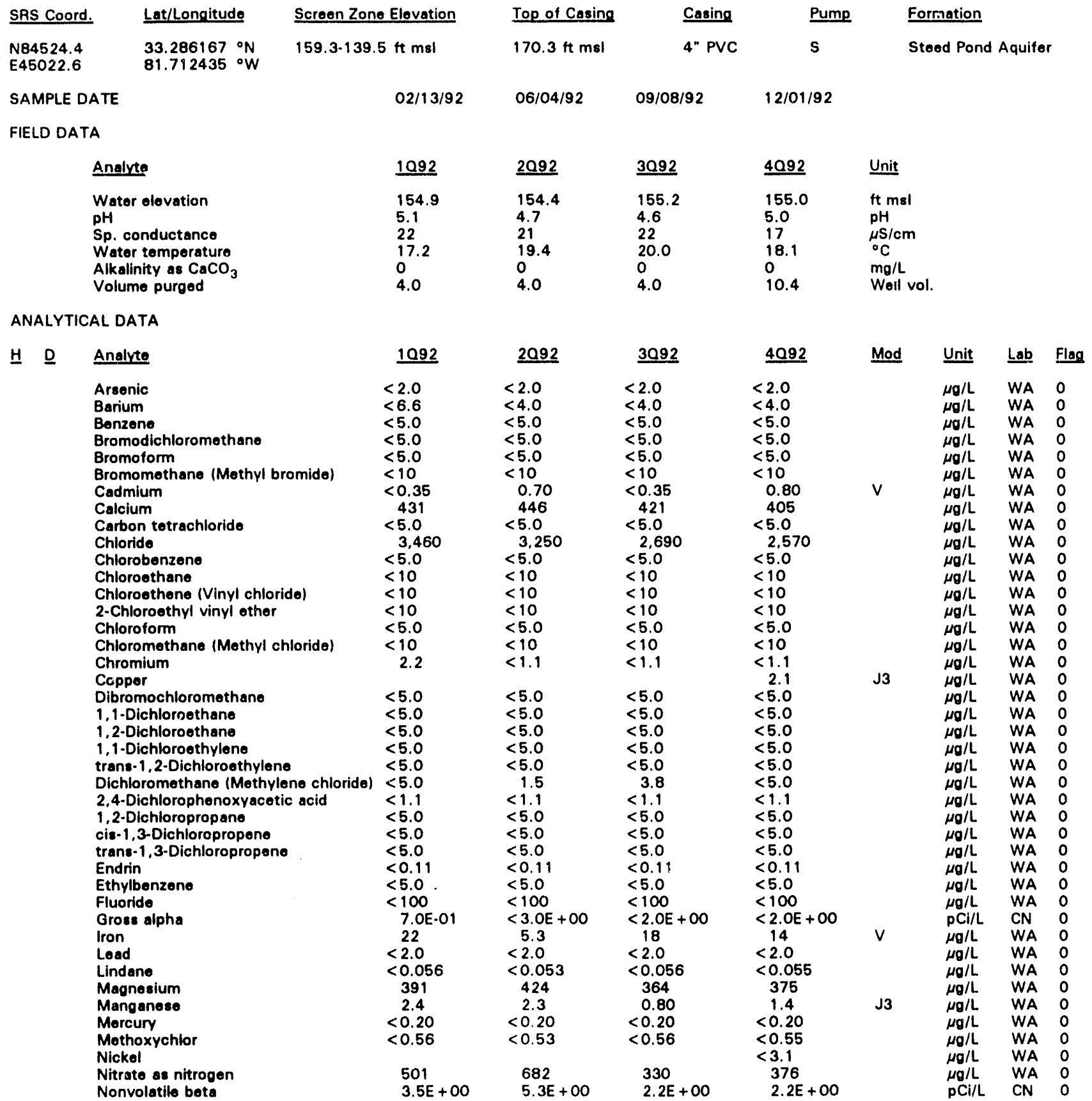

Note: Flagging levels, modifiers, and laboratory are for 4th quarter 1992 data only. See Appendix B for flagging criteria.

- =xceeded holding time for 4th quarter 1992.

- exceeded final primary drinking water standard for 4th quarter 1992 . 
Well LFW 440 continued

ANALYTICAL DATA

H. Analyte
pH
Phenols
Potassium
Radium-226
Radium-228
Selenium
Silica
Silicon
Silver
Sodium
Specific conductance
Sulfate
1,1,2,2-Tetrachloroethane
Totrachloroethylene
Toluene
Total dissolved solids
Total organic carbon
Total organic halogens
Total phosphates (as P)
Toxaphene
2,4,5-TP (Silvex)
$1,1,1-$ Trichloroethane
$1,1,2-$ Trichloroethane
Trichloroethylene
Trichlorofluoromethane
Tritium
Vanadium

\begin{tabular}{l}
1092 \\
\hline 4.5 \\
$<5.0$ \\
708 \\
$9.0 \mathrm{E} \cdot 01$ \\
$1.3 E+00$ \\
$<2.0$ \\
\\
2.650 \\
0.75 \\
1.270 \\
20 \\
$<100$ \\
$<5.0$ \\
$<5.0$ \\
$<5.0$ \\
39.000 \\
1.310 \\
8.4 \\
$<20$ \\
$<1.1$ \\
$<0.56$ \\
$<5.0$ \\
$<5.0$ \\
$<5.0$ \\
$<5.0$ \\
$2.6 E+00$ \\
\end{tabular}

\begin{tabular}{l}
2092 \\
\hline 5.2 \\
$<5.0$ \\
879 \\
$<5.0 \mathrm{E} \cdot 01$ \\
\\
$<2.0$ \\
6.420 \\
$<0.70$ \\
1.440 \\
19 \\
288 \\
$<5.0$ \\
$<5.0$ \\
$<5.0$ \\
13.000 \\
$<500$ \\
83 \\
34 \\
$<1.1$ \\
$<0.56$ \\
$<5.0$ \\
$<5.0$ \\
$<5.0$ \\
$<5.0$ \\
$2.8 E+00$ \\
\end{tabular}

$\begin{aligned} & 3092 \\ & 6.1 \\ &<5.0 \\ & 642 \\ &<1.0 \mathrm{E}+00 \\ &<1.0 \mathrm{E}+00 \\ &<2.0 \\ & 5.470 \\ &<0.70 \\ & 1.220 \\ & 17 \\ &<250 \\ &<5.0 \\ &<5.0 \\ &<5.0 \\ & 29.000 \\ &<552 \\ &<5.0 \\ &<1.1 \\ &<0.55 \\ &<5.0 \\ &<5.0 \\ &<5.0 \\ &<5.0 \\ & 3.2 \mathrm{E}+00 \\ &\end{aligned}$

$\begin{aligned} & 4092 \\ & 5.2 \\ &<5.0 \\ & 891 \\ &<1.0 E+00 \\ &< \\ &<2.0 \\ & 6.020 \\ &<0.70 \\ & 1.510 \\ & 16 \\ &<250 \\ &<5.0 \\ &<5.0 \\ &<5.0 \\ & 10.000 \\ &<500 \\ &<5.0 \\ &<1.1 \\ &<0.55 \\ &<5.0 \\ &<5.0 \\ &<5.0 \\ &<5.0 \\ &<0.88+00 \\ &<08 \\ &\end{aligned}$

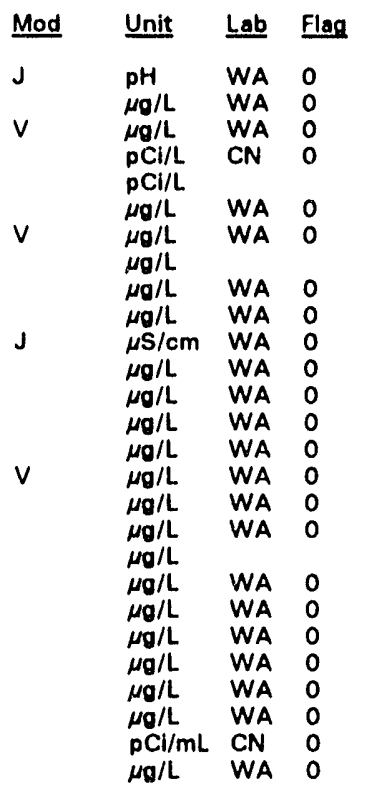

\section{WELL LFW 45D}

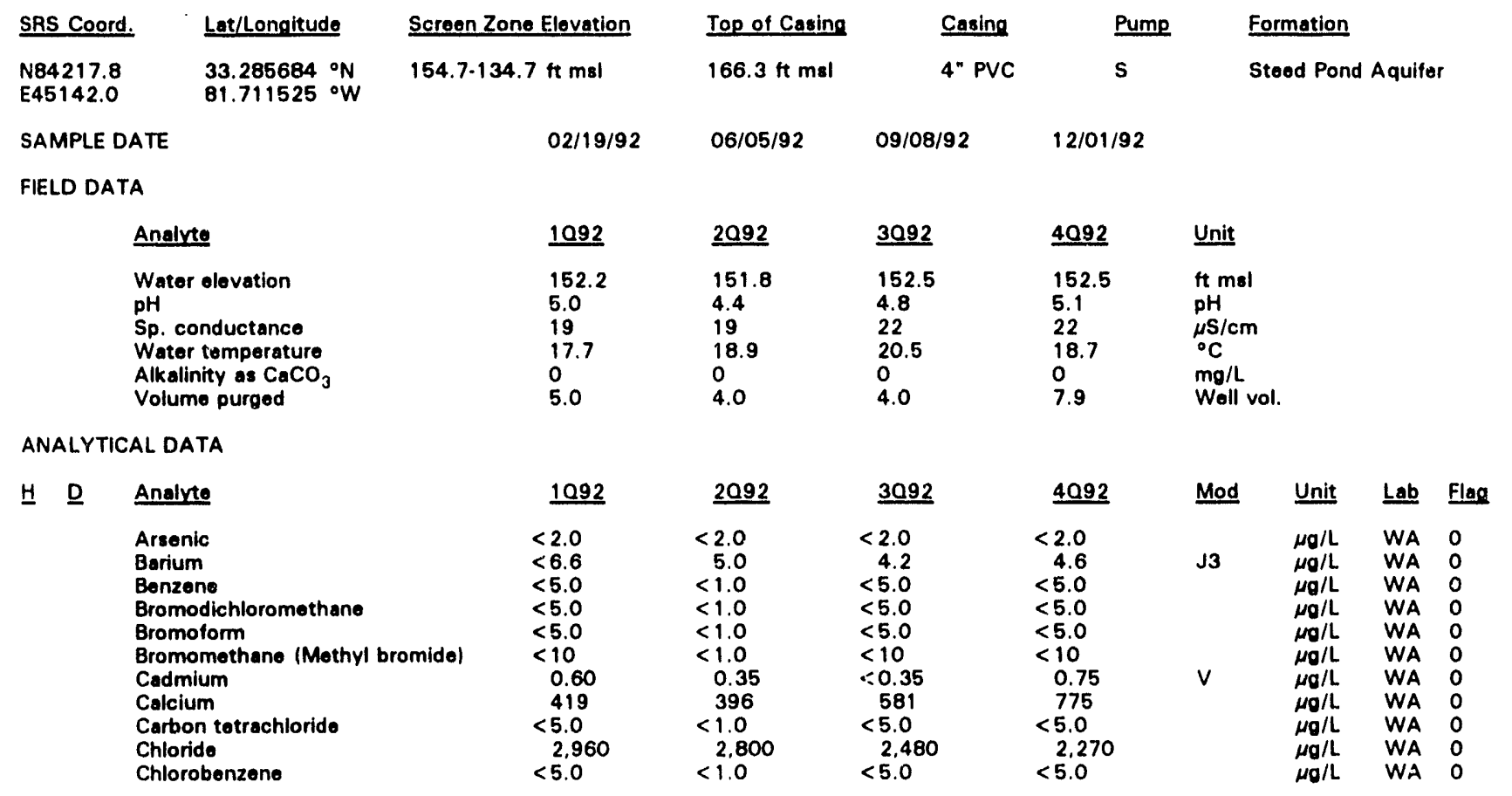

Note: Flagging levels, modifiers, and laboratory are for 4 th quarter 1992 data only. See Appendix B for flagging criteria.

- = exceeded holding time for 4th quarter 1992.

- exceeded final primary drinking water standard for 4th quarter 1992. 
WSRC-TR-93-068

Well LFW 450 continued

ANALYTICAL DATA

H D Analvte

Chloroethane

Chloroetherie (Vinyl chloride)

2. Chloroethyl vinyl other

Chloroform

Chloromethane (Methyl chloride)

Chromium

Copper

Dibromochloromethane

1.1-Dichloroethane

1.2-Dichloroethane

1.1-Dichloroethylene

trans-1,2-Dichloroethylene

Dichloromethane (Methylene chloride)

2,4-Dichlorophenoxyacetic acid

1,2-Dichloropropane

cis-1,3-Dichloropropene

trane-1,3-Dichloropropene

Endrin

Ethylbenzene

Fluoride

Gross alpha

Iron

Lead

Lindano

Magnesium

Manganese

Mercury

Mothoxychlor

Nickel

Nitrate as nitrogen

Nitrate-nitrite as nitrogen

Nonvolatils bets

pH

Phenols

Potassium

Radium-226

Radium-228

Selenium

Silica

Silicon

Silver

Sodlum

Specific conductance

Sulfate

$1,1,2,2$-Totrachloroothane

Tetrachloroethylene

Toluene

Total dissolved solids

Total organic carbon

Total organic halogens

Total phosphates (as P)

Toxaphene

2,4,5-TP (Silvex)

1,1,1-Trichloroethane

1,1,2-Trichloroethane

Trichloroethylene

Trichlorofluoromethane

Tritium

Uranium

Vanadium

$<10$

$<10$

$<10$

$<5.0$

$<10$

$<1.1$

$<5.0$

$<5.0$

$<5.0$

$<5.0$

$<5.0$

7.5

$<1.1$

$<5.0$

$<5.0$

$<0.11$

$<5.0$

$<100$

$1.3 E+00$

10

$<2.0$

$<0.055$
390
3.1

$<0.20$

$<0.55$

$$
224
$$

3. $1 E+00$

$$
5.7
$$

$<5.0$

553

$1.0 E+00$

$2.0 E+00$

$<2.0$

3,130

$<0.70$

1.260

14
467

467
$<5.0$

$<5.0$

$<5.0$

22.000

1.020

64
$<20$

$<20$

$<1.1$

$<0.55$

$<5.0$

$<5.0$

$<5.0$

$<5.0$

1. $9 E+00$

0.060

$\begin{aligned} & 2092 \\ &< 1.0 \\ &< 1.0 \\ &< 1.0 \\ &< 1.0 \\ &< 1.0 \\ &< 1.1 \\ &< 1.0 \\ &< 1.0 \\ &< 1.0 \\ &< 1.0 \\ &< 1.0 \\ & 11 \\ &<0.30 \\ &< 1.0 \\ &<1.0 \\ &<1.0 \\ &<0.0060 \\ &<1.0 \\ &<100 \\ & 2.4 \mathrm{E}+00 \\ & 26 \\ &< 2.0 \\ &< 0.0050 \\ & 429 \\ &<3.0 \\ &<0.20 \\ &<0.50 \\ &\end{aligned}$

3092

$<10$
$<10$

$<10$

$<5.0$

$<10$

$<1.1$

$<5.0$

$<5.0$

$<5.0$

$<5.0$

$<1.1$

$<5.0$

$<5.0$

$<5.0$

$<0.11$

$<5.0$

$<100$

$<2.0 E+00$

152

$<2.0$

$<0.056$

396
2.6

$<0.20$

$<0.56$

335

$<2.0 \mathrm{E}+00$

5.7

$<5.0$

597
$1.4 E+$

$1.4 E+00$

$<2.0$
6,660

$<0.70$

1,290
20

20
780

$<1.0$

2.9

$<1.0$

15,000

$<500$

37
62

$<0.24$

$<0.090$

$<1.0$

$<1.0$

1.5

$<1.0$

$1.4 E+00$

$<20$

$<2.0 E+00$

6.1

$<5.0$

565

$<1.0 E+00$

$<1 . \mathrm{OE}+00$

$<2.0$

6,080

$<0.70$

1,330

17

897

$<5.0$

$<5.0$

$<50$

29,000

638

$<5.0$

$<1.1$

$<0.56$

$<5.0$

$<5.0$

$<5.0$

$<5.0$

\begin{tabular}{|c|c|}
\hline 4092 & Mod \\
\hline $\begin{array}{l}<10 \\
<10 \\
<10 \\
<5.0 \\
<10 \\
<1.1\end{array}$ & \\
\hline 2.1 & J3 \\
\hline
\end{tabular}

1. $4 \mathrm{E}+00$
Unit Lab Fla

$\mu \mathrm{g} / \mathrm{L}$ WA 0

$\mu \sigma / L$ WA

$\mu \mathrm{g} / \mathrm{L}$ WA 0

$\mu g / L$ WA 0

$\mu_{\theta} / L$ WA 0

$\mu g / L$ WA 0

$\mu_{0} / \mathrm{L}$ WA 0

$\mu g / L$ WA 0

$\mu g / L$ WA 0

$\mu g / L$ WA 0

$\mu \mathrm{g} / \mathrm{L}$ WA 0

$\mu \mathrm{g} / \mathrm{L}$ WA 0

$\mu_{\mathrm{g} / \mathrm{L}}$ WA 0

$\mu \mathrm{g} / \mathrm{L}$ WA 0

$\mu g / L$ WA 0

$\mu g / L$ WA

$\mu g / L$ WA

$\mu \sigma / L$ WA 0

$\mu g / L$ WA 0

$\mathrm{PCI} / \mathrm{L}$ CN 0

$\mu g / L$ WA 0

$\mu \mathrm{g} / \mathrm{L}$ WA 0

$\mu g / L$ WA

$\begin{array}{lll}\mu g / L & \text { WA } & 0 \\ \mu g / L & \text { WA } & 0\end{array}$

$\mu \mathrm{g} / \mathrm{L}$ WA

$\mu g / L$ WA 0

$\mu g / L$ WA

$\begin{array}{lll}\mu g / L & W A & 0 \\ \mu g / L & W A & 0\end{array}$

$\mu \mathrm{g} / \mathrm{L}$

pCi/L CN $O$

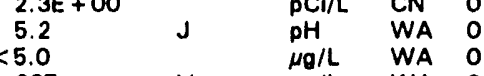

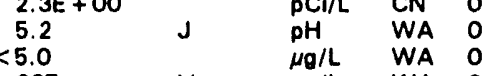

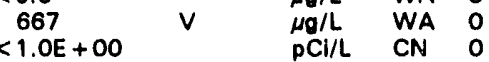

$<2.0 \quad \stackrel{\mathrm{pCi} / \mathrm{L}}{\mu \mathrm{g} / \mathrm{L}}$ WA 0

$5,990 \vee \quad \vee \mu g / L$ WA 0

$<0.70$

1.820

2000

2.400
$<5.0$

$<5.0$

$<5.0$

5.000

$<500$

$<5.0$

$<1.1$

$<0.55$

$<5.0$

$<5.0$

$<5.0$

$<5.0$

1. $2 \mathrm{E}+00$

$<0.88$

$\mu g / L$

$\mu g / L$ WA 0

HOL WA O

$\mu \mathrm{g} / \mathrm{L}$ WA 0

$\mu \sigma / L$ WA 0

$\mu \mathrm{g} / \mathrm{L}$ WA 0

$\mu g / L$ WA 0

$\mathrm{pCl} / \mathrm{mL}$

$\mu \mathrm{g} / \mathrm{L}$

$\mu g / L$ WA $O$

Note: Flagging levels, modifiers, and laboratory are for 4th quarter 1992 data only. See Appendix B for flagging criteria.

- = exceeded holding time for 4 th quarter 1992 .

- =xceeded final primary drinking water standard for 4th quarter 1992 . 
WSRC-TR-93-068

WELL LFW 46D

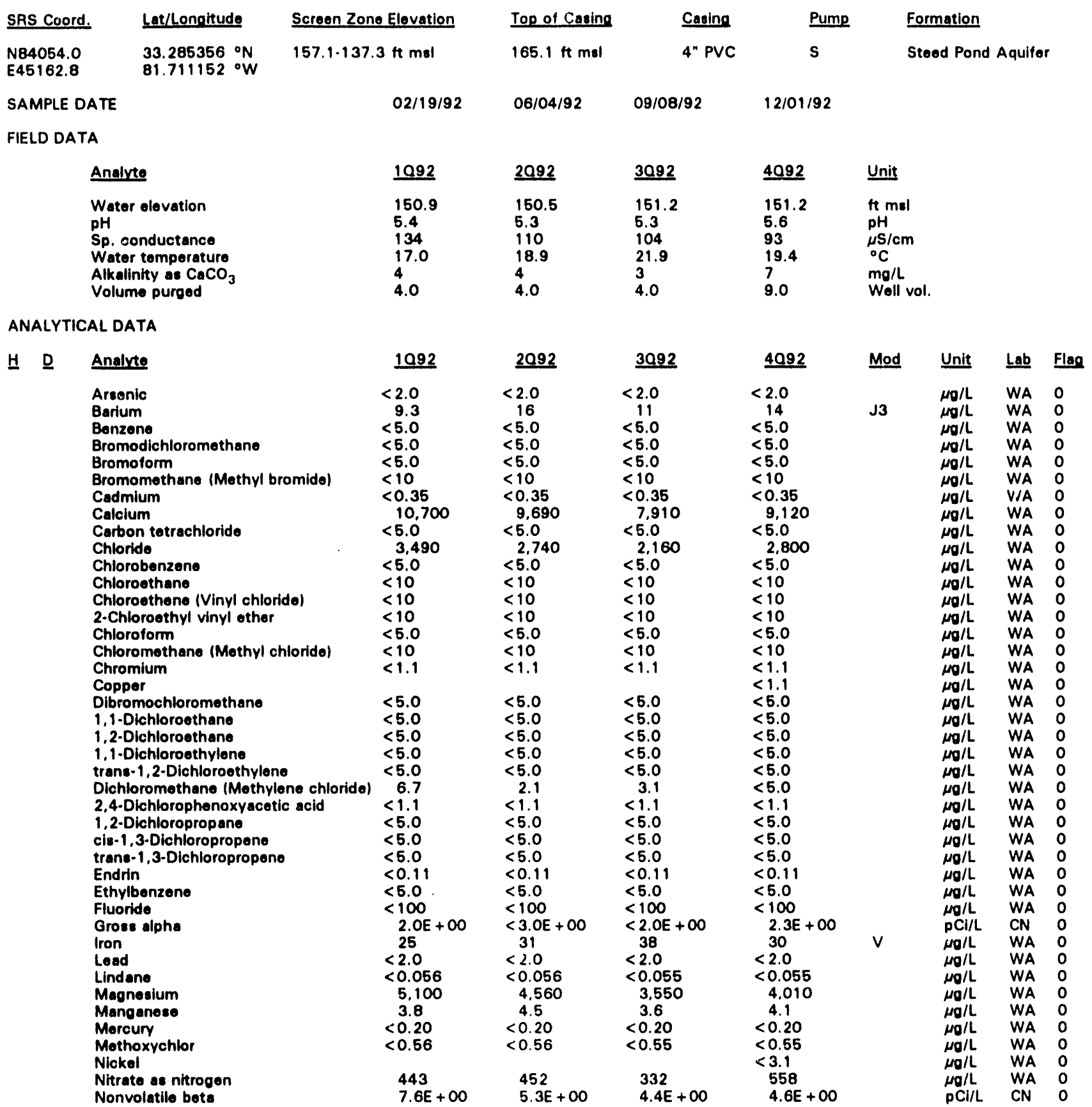

Note: Flagging levels, modifiers, and laboratory are for 4 th querter 1992 data only. See Appendix B for flagging criteria.

- =xceeded holding time for 4th quarter 1992.

- = exceeded final primary drinking watur standard for 4th quarter 1992. 
Well LFW 460 continued

ANALYTICAL DATA.

$\begin{array}{ll}\text { H. } & \text { Analyte } \\ \text { - } & \text { Ph } \\ \text { Phenols } \\ \text { Potassium } \\ \text { Radium-226 } \\ \text { Radium-228 } \\ \text { Selenium } \\ \text { Silica } \\ \text { Silicon } \\ \text { Silver } \\ \text { Sodium } \\ \text { Specific conductance } \\ \text { Sulfate } \\ \text { 1,1,2,2-Tetrachloroethane } \\ \text { Totrachloroethylene } \\ \text { Toluene } \\ \text { Total dissolved solids } \\ \text { Total organic carbon } \\ \text { Total organic halogens } \\ \text { Total phosphates (as P) } \\ \text { Toxaphene } \\ 2,4,5 \cdot \text { TP (Silvex) } \\ \text { 1,1,1-Trichloroethane } \\ \text { 1,1,2-Trichloroethane } \\ \text { Trichloroethylene } \\ \text { Trichlorofluoromethane } \\ \text { Tritium } \\ \text { Uranium } \\ \text { Vanadium }\end{array}$

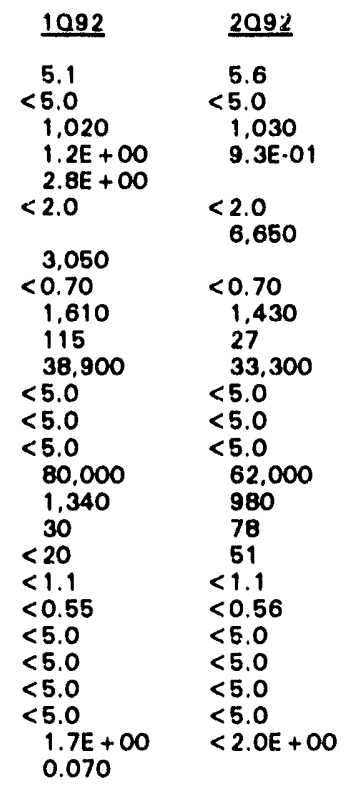

3092
5.3
$<5.0$
1.030
$<1.0 \mathrm{E}+00$
$2.1 \mathrm{E}+00$
$<2.0$
5.740
$<0.70$
1.150
82
28.400
$<5.0$
$<5.0$
$<5.0$
77.000
743
$<5.0$
$<1.1$
$<0.56$
$<5.0$
$<5.0$
$<5.0$
$<5.0$
$1.7 \mathrm{E}+00$

\begin{tabular}{|c|c|c|c|}
\hline 4092 & Mod & Unit & $\underline{L a b}$ \\
\hline $\begin{aligned} & 5.9 \\
&< 5.0 \\
& 763 \\
& 1.4 \mathrm{E}+00\end{aligned}$ & $J$ & $\begin{array}{l}\mathrm{pH} \\
\mu \mathrm{g} / \mathrm{L} \\
\mu \mathrm{g} / \mathrm{L} \\
\mathrm{pCi} / \mathrm{L} \\
\mathrm{pCi} / \mathrm{L}\end{array}$ & $\begin{array}{l}\text { WA } \\
\text { WA } \\
\text { WA } \\
\text { CN }\end{array}$ \\
\hline $\begin{aligned}<2.0 \\
\quad 6.240\end{aligned}$ & V & $\begin{array}{l}\mu \mathrm{g} / \mathrm{L} \\
\mu \mathrm{g} / \mathrm{L} \\
\mu \mathrm{g} / \mathrm{L}\end{array}$ & $\begin{array}{l}\text { WA } \\
\text { WA }\end{array}$ \\
\hline $\begin{array}{l}<0.70 \\
1.770 \\
99 \\
30,100 \\
<5.0 \\
<5.0 \\
<5.0 \\
65.000 \\
683 \\
5.2\end{array}$ & $J$ & $\begin{array}{l}\mu \mathrm{g} / \mathrm{L} \\
\mu \mathrm{g} / \mathrm{L} \\
\mu \mathrm{S} / \mathrm{cm} \\
\mu \mathrm{g} / \mathrm{L} \\
\mu \mathrm{g} / \mathrm{L} \\
\mu \mathrm{g} / \mathrm{L} \\
\mu \mathrm{g} / \mathrm{L} \\
\mu \mathrm{g} / \mathrm{L} \\
\mu \mathrm{g} / \mathrm{L} \\
\mu \mathrm{g} / \mathrm{L} \\
\mu \mathrm{g} / \mathrm{L}\end{array}$ & $\begin{array}{l}\text { WA } \\
\text { WA } \\
\text { WA } \\
\text { WA } \\
\text { WA } \\
\text { WA } \\
\text { WA } \\
\text { WA } \\
\text { WA } \\
\text { WA }\end{array}$ \\
\hline $\begin{aligned} &< 1.1 \\
&<0.55 \\
&<5.0 \\
&<5.0 \\
&<5.0 \\
&<5.0 \\
& \quad 1.6 E+00 \\
&<0.88\end{aligned}$ & & $\begin{array}{l}\mu \mathrm{g} / \mathrm{L} \\
\mu \mathrm{g} / \mathrm{L} \\
\mu \mathrm{g} / \mathrm{L} \\
\mu \mathrm{g} / \mathrm{L} \\
\mu \mathrm{g} / \mathrm{L} \\
\mu \mathrm{g} / \mathrm{L} \\
\mathrm{pCl} / \mathrm{mL} \\
\mu \mathrm{g} / \mathrm{L} \\
\mu \mathrm{g} / \mathrm{L}\end{array}$ & $\begin{array}{l}\text { WA } \\
\text { WA } \\
\text { WA } \\
\text { WA } \\
\text { WA } \\
\text { WA } \\
\text { CN } \\
\text { WA }\end{array}$ \\
\hline
\end{tabular}

\section{WELL LFW 47C}

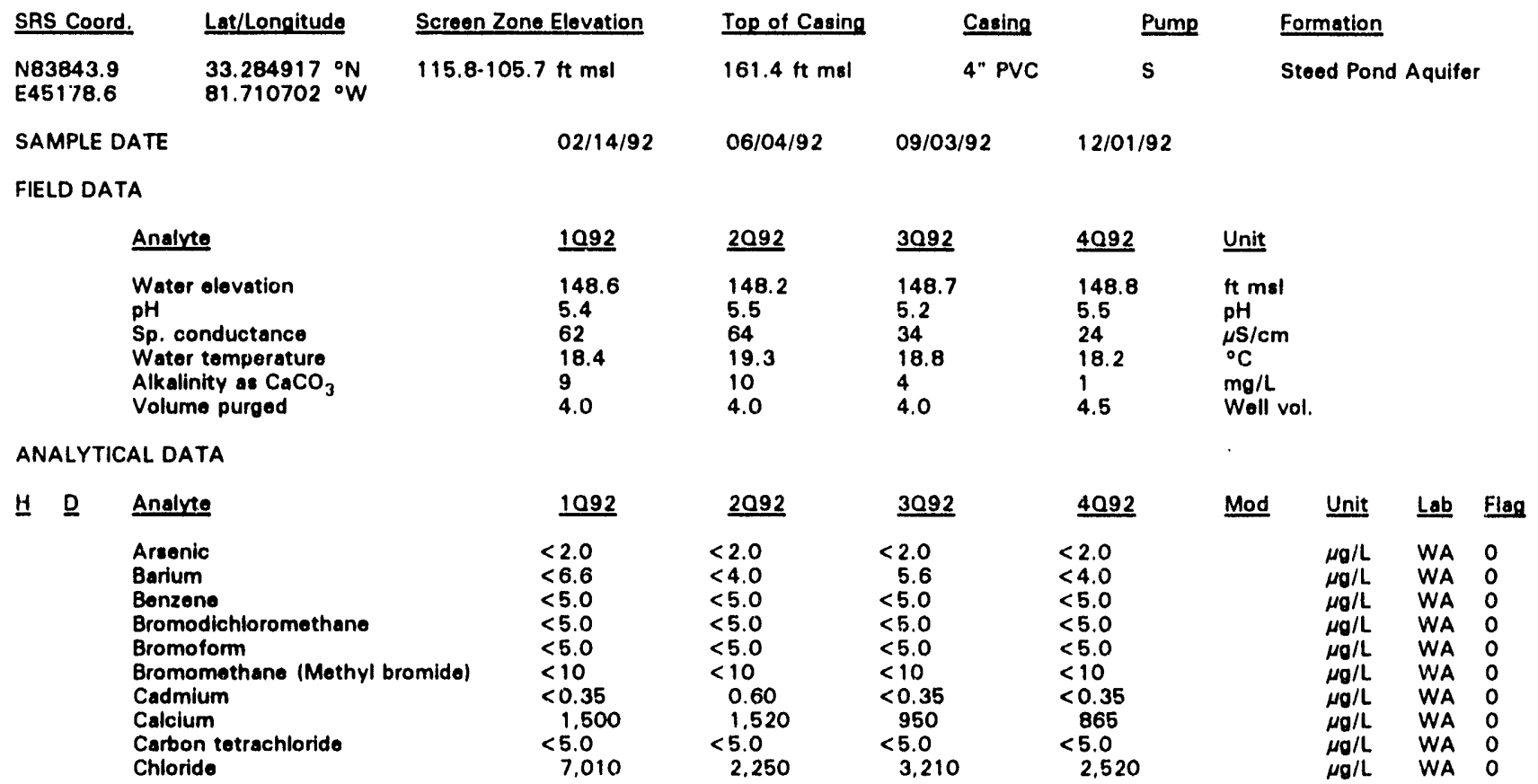

Note: Flagging levels, modifiers, and laboratory are for 4 th quarter 1992 data only. See Appendix B for flagging criteria.

- =xceeded holding time for 4 th quarter 1992.

- = exceeded final primary drinking water standard for 4th quarter 1992. 
Woll LFW 47C continued

ANALYTICAL DATA

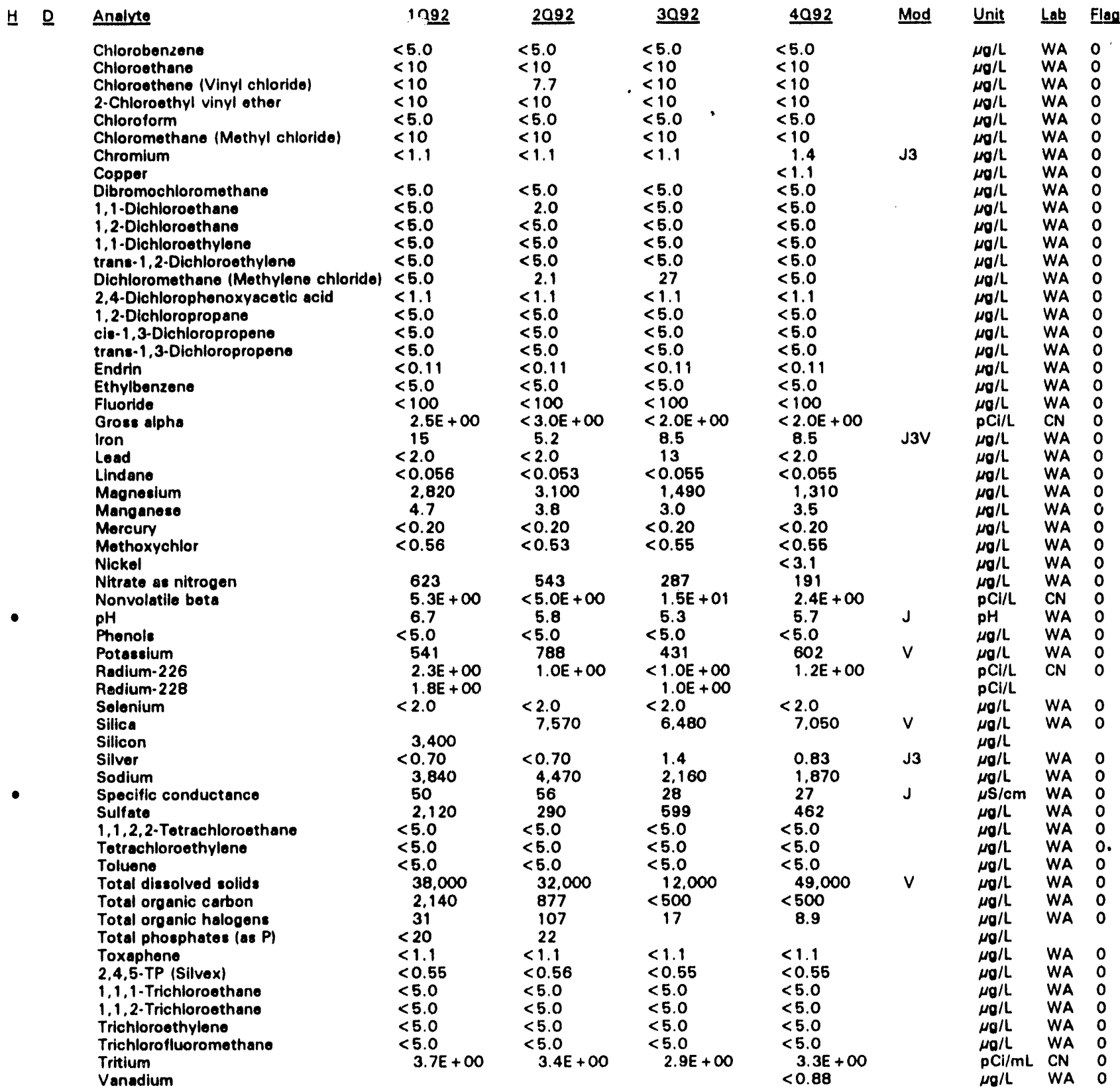

Note: Flagging levels, modifiers, and laboratory are for 4th quarter 1992 data only. See Appendix B for flagging criteria.

- = exceeded holding time for 4 th quarter 1992.

- = exceeded final primary drinking water standard for 4th quarter 1992. 
WSRC-TR-93-068

WELL LFW 47D

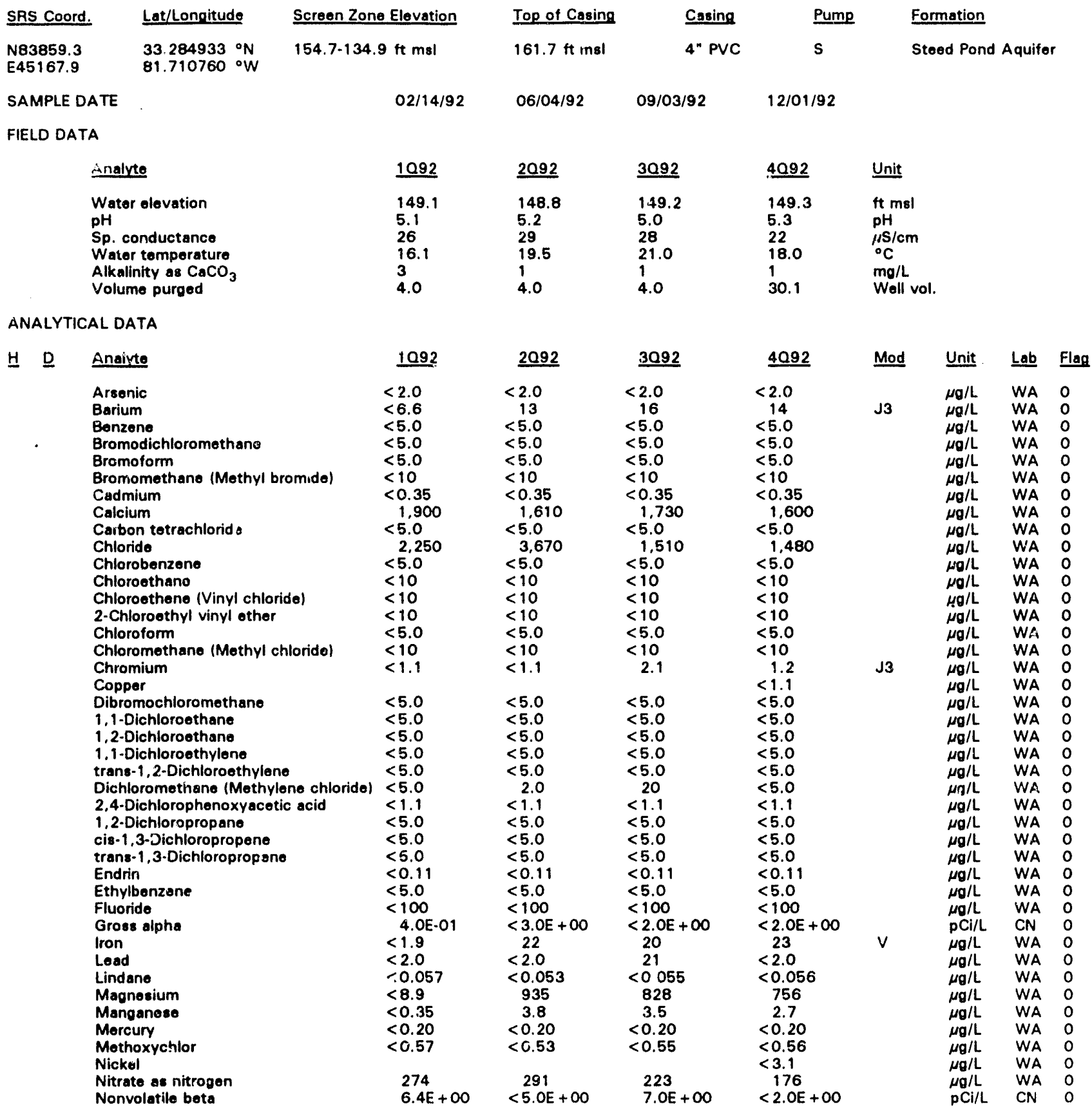

Note: Flagging levels, modifiers, and laboratory are for 4th quarter 1992 deta only. See Appendix B for flagging criteria. - =xceeded holding time for 4th quarter 1992.

- exceeded final primary drinking water standard for 4th quarter 1992. 
Well LFW 470 continued

ANALYTICAL DATA .

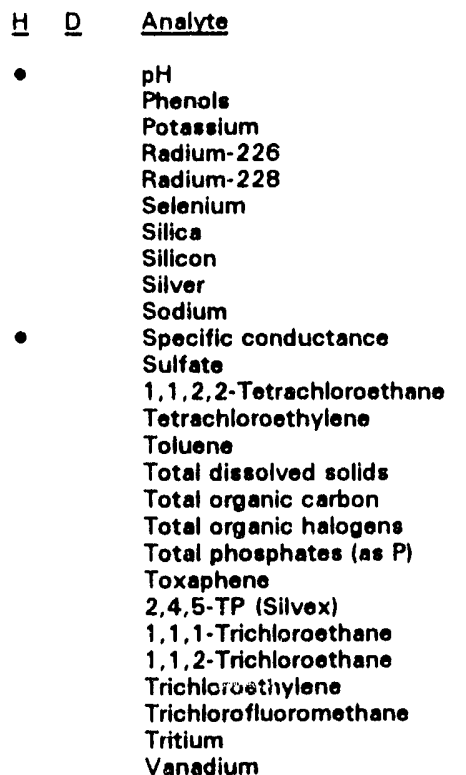

\begin{tabular}{|c|c|c|c|c|c|c|c|}
\hline 1092 & $\underline{2092}$ & $\underline{3092}$ & 4092 & Mod & Unit & Lab & Flag \\
\hline $\begin{aligned} & 6.7 \\
&< 5.0 \\
&< 84 \\
& 3.0 \mathrm{E}-01 \\
& 1.1 \mathrm{E}+\infty\end{aligned}$ & $\begin{aligned} & 5.6 \\
&< 5.0 \\
& 919 \\
&<5.0 E-01\end{aligned}$ & $\begin{aligned} & 5.2 \\
< & 5.0 \\
& 1.120 \\
< & 1.0 E+\infty 0 \\
< & 1.0 E+\infty 0\end{aligned}$ & $\begin{aligned} & 5.6 \\
< & 5.0 \\
& 853 \\
< & 1.0 \mathrm{E}+00\end{aligned}$ & v & $\begin{array}{l}\mathrm{pH} \\
\mu \mathrm{g} / \mathrm{L} \\
\mu \mathrm{g} / \mathrm{L} \\
\mathrm{pCl} / \mathrm{L} \\
\mathrm{pCi} / \mathrm{L}\end{array}$ & $\begin{array}{l}\text { WA } \\
\text { WA } \\
\text { WA } \\
\text { CN }\end{array}$ & $\begin{array}{l}0 \\
0 \\
0 \\
0\end{array}$ \\
\hline$<2.0$ & $\begin{array}{l}<2.0 \\
5.250\end{array}$ & $\begin{array}{r}<2.0 \\
4,880\end{array}$ & $\begin{array}{r}<2.0 \\
4.930\end{array}$ & v & $\begin{array}{l}\mu \sigma / L \\
\mu \sigma / L\end{array}$ & $\begin{array}{l}\text { WA } \\
\text { WA }\end{array}$ & $\begin{array}{l}0 \\
0\end{array}$ \\
\hline $\begin{array}{l}2,260 \\
<0.70 \\
<106 \\
22 \\
4,580 \\
<5.0 \\
<5.0 \\
<5.0 \\
28,000 \\
763 \\
6.7 \\
52\end{array}$ & $\begin{array}{l}<0.70 \\
930 \\
28 \\
9.880 \\
<5.0 \\
<5.0 \\
<5.0 \\
13,000 \\
566 \\
20 \\
166\end{array}$ & $\begin{array}{l}2.3 \\
1.010 \\
23 \\
4.600 \\
<5.0 \\
<5.0 \\
<5.0 \\
6.000 \\
<500 \\
5.5\end{array}$ & $\begin{array}{l}1.2 \\
1.080 \\
24 \\
3.860 \\
<5.0 \\
<5.0 \\
<5.0 \\
58,000 \\
<500 \\
<5.0\end{array}$ & $\begin{array}{l}\text { J3 } \\
\mathrm{J}\end{array}$ & $\begin{array}{l}\mu \mathrm{g} / \mathrm{L} \\
\mu \mathrm{g} / \mathrm{L} \\
\mu \mathrm{g} / \mathrm{L} \\
\mu \mathrm{S} / \mathrm{cm} \\
\mu \mathrm{g} / \mathrm{L} \\
\mu \mathrm{g} / \mathrm{L} \\
\mu \mathrm{g} / \mathrm{L} \\
\mu \mathrm{g} / \mathrm{L} \\
\mu \mathrm{g} / \mathrm{L} \\
\mu \mathrm{g} / \mathrm{L} \\
\mu \mathrm{g} / \mathrm{L} \\
\mu \mathrm{g} / \mathrm{L}\end{array}$ & $\begin{array}{l}\text { WA } \\
\text { WA } \\
\text { WA } \\
\text { WA } \\
\text { WA } \\
\text { WA } \\
\text { WA } \\
\text { WA } \\
\text { WA } \\
\text { WA } \\
\text { WA }\end{array}$ & $\begin{array}{l}0 \\
0 \\
0 \\
0 \\
0 \\
0 \\
0 \\
0 \\
0 \\
0\end{array}$ \\
\hline$<1.1$ & $<1.0$ & $<1.1$ & $<1.1$ & & $\mu \mathrm{g} / \mathrm{L}$ & WA & 0 \\
\hline$<0.57$ & $<0.55$ & $<0.57$ & $<0.56$ & & $\mu \mathrm{g} / \mathrm{L}$ & WA & 0 \\
\hline$<5.0$ & $<5.0$ & $<5.0$ & $<5.0$ & & $\mu \mathrm{g} / \mathrm{L}$ & WA & \\
\hline$<5.0$ & $<5.0$ & $<5.0$ & $<5.0$ & & $\mu \mathrm{g} / \mathrm{L}$ & WA & \\
\hline$<5.0$ & $<5.0$ & $<5.0$ & $<5.0$ & & $\mu \mathrm{g} / \mathrm{L}$ & WA & \\
\hline$<5.0$ & $<5.0$ & $<5.0$ & $<5.0$ & & $\mu \mathrm{g} / \mathrm{L}$ & WA & 0 \\
\hline $1.4 E+$ & $<2.0 E+00$ & $1.1 E+\infty$ & $\begin{aligned} & 1.6 E+00 \\
&<0.88\end{aligned}$ & & $\underset{\mu \mathrm{pCl} / \mathrm{mL}}{ }$ & $\begin{array}{l}\text { CN } \\
\text { WA }\end{array}$ & $\begin{array}{l}0 \\
0\end{array}$ \\
\hline
\end{tabular}

\section{WELL LFW 48C}

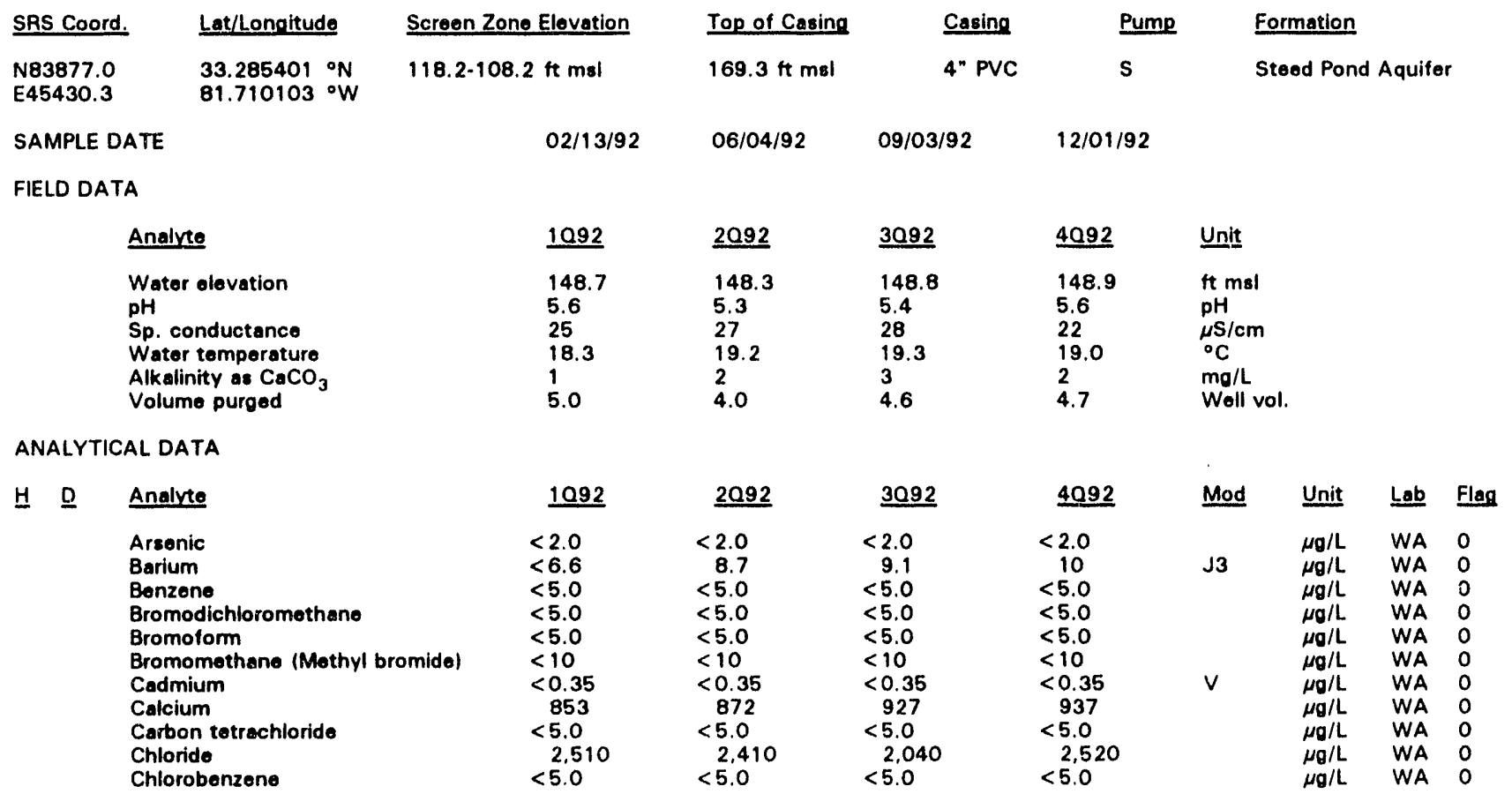

Note: Flagging levels, modifiers, and laboratory are for 4th quarter 1992 data only. See Appendix $B$ for flagging criteria.

- =xceeded holding time for 4th quarter 1992.

- exceeded final primary drinking water standard for 4th quarter 1992. 




Note: Flagging levels, modifiers, and laboratory are fur 4th quarter 1992 data only. See Appendix B for flagging criteria. - = exceeded holding time for 4th quarter 1992.

- = exceeded final primary drinking water standard for 4th quarter 1992. 
WSRC-TR-93-068

WELL LFW 48D

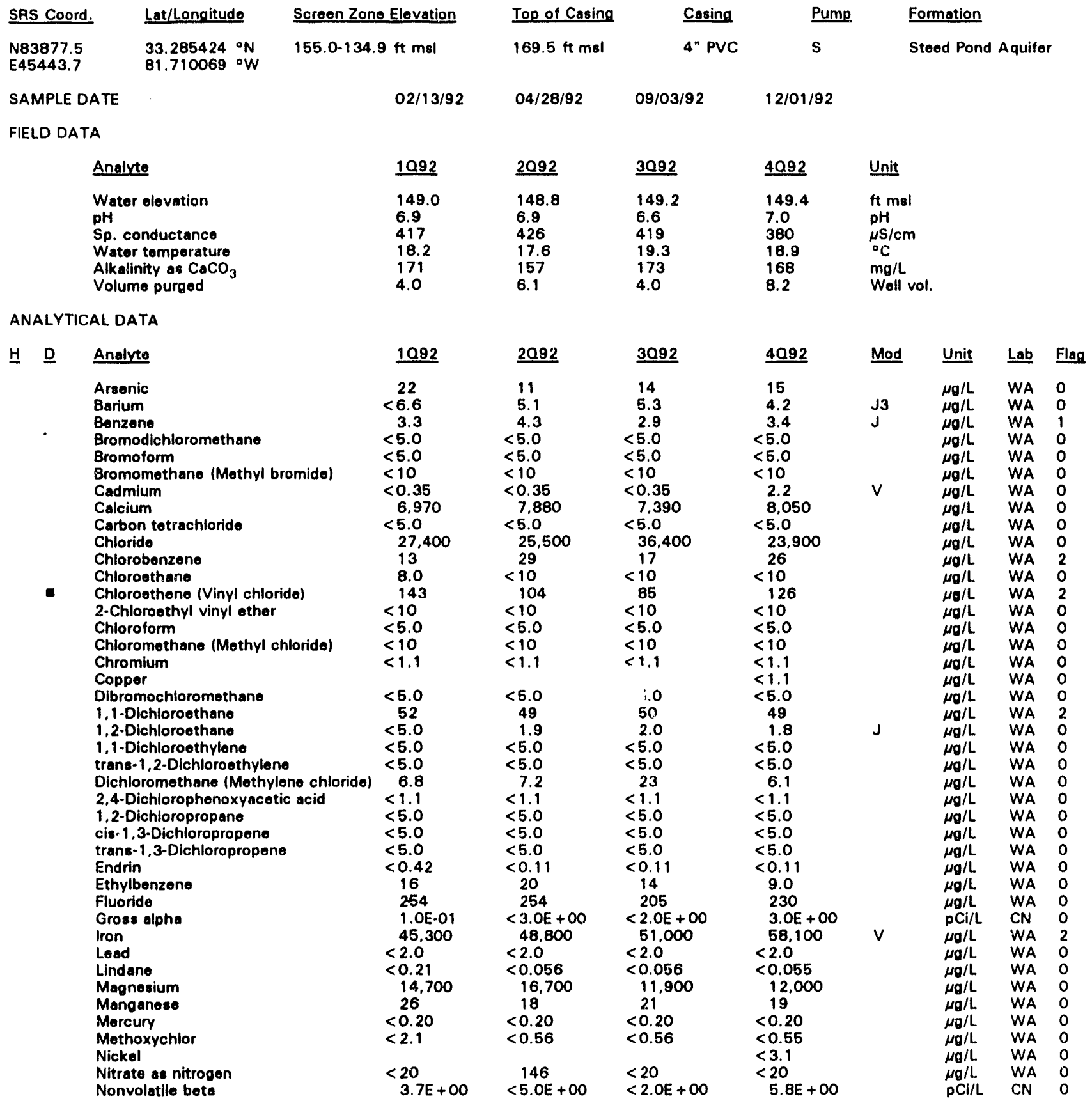

Note: Flagging levels, modiriers, and laboratory are for 4 th quarter 1992 data only. See Appendix B for flagging criteria.

- = exceeded holding time for 4th quarter 1992.

- =xceeded final primary drinking water standard for 4th quarter 1992. 
WSRC-TR-93-068

Well LFW 48D continued

ANALYTICAL DATA

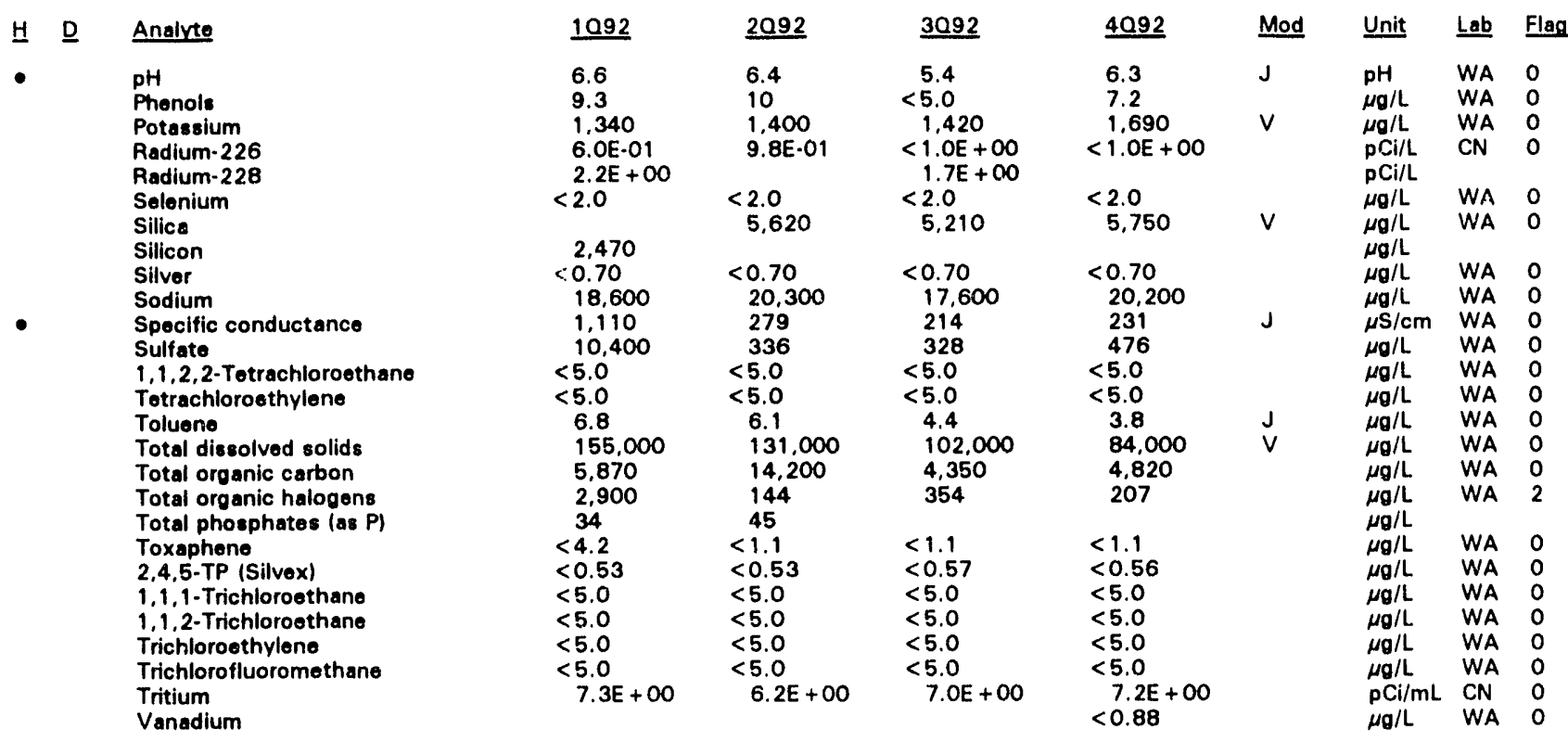

WELL LFW 55C

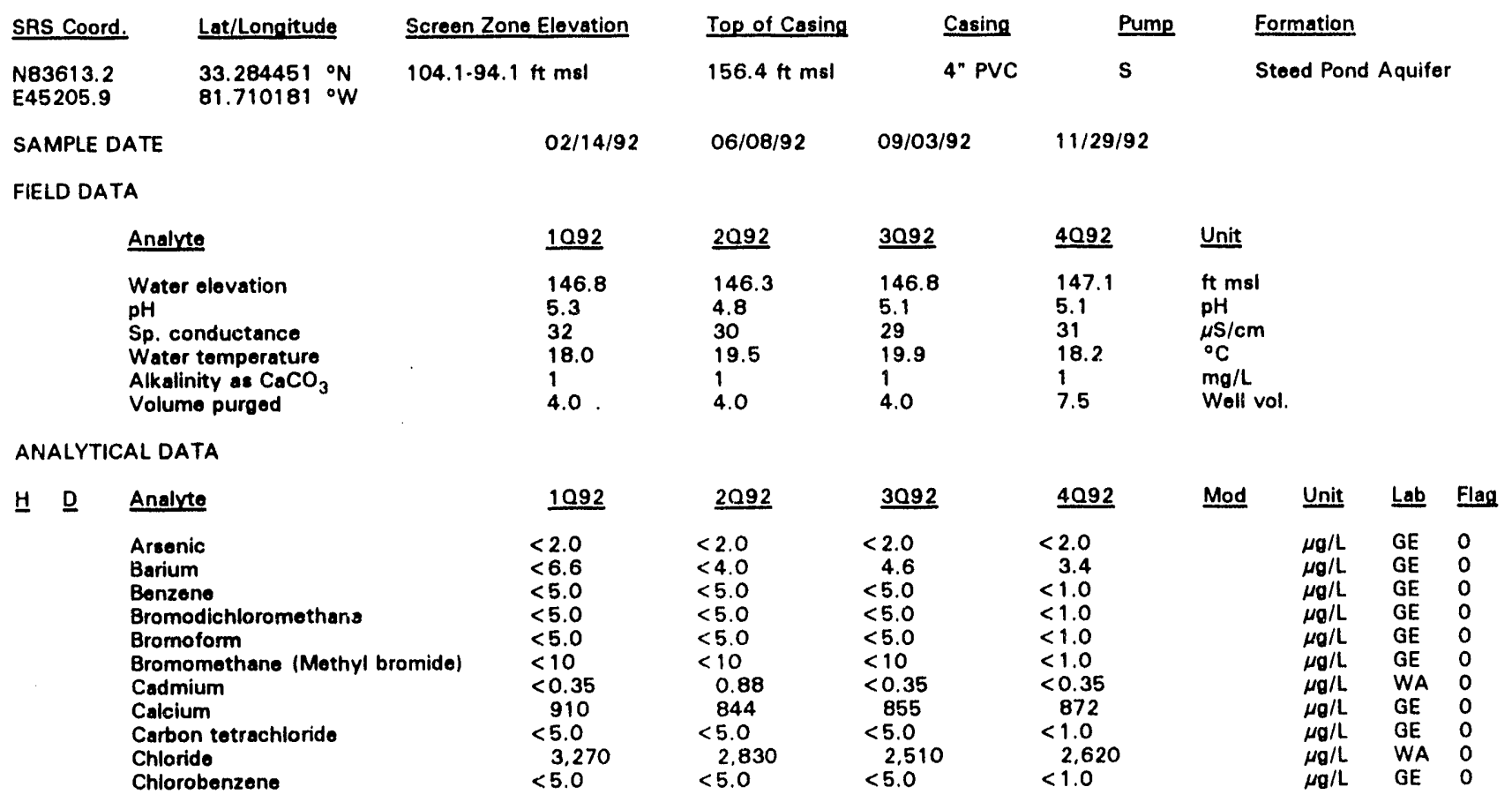

Note: Flagging levels, modifiers, and laboratory are for 4 th quarter 1992 data only. See Appendix B for flagging criteria.

- = exceeded holding time for 4th quarter 1992.

- = exceeded final primary drinking water standard for 4th quarter 1992. 
Woll LFW 55C continued

\section{ANALYTICAL DATA}

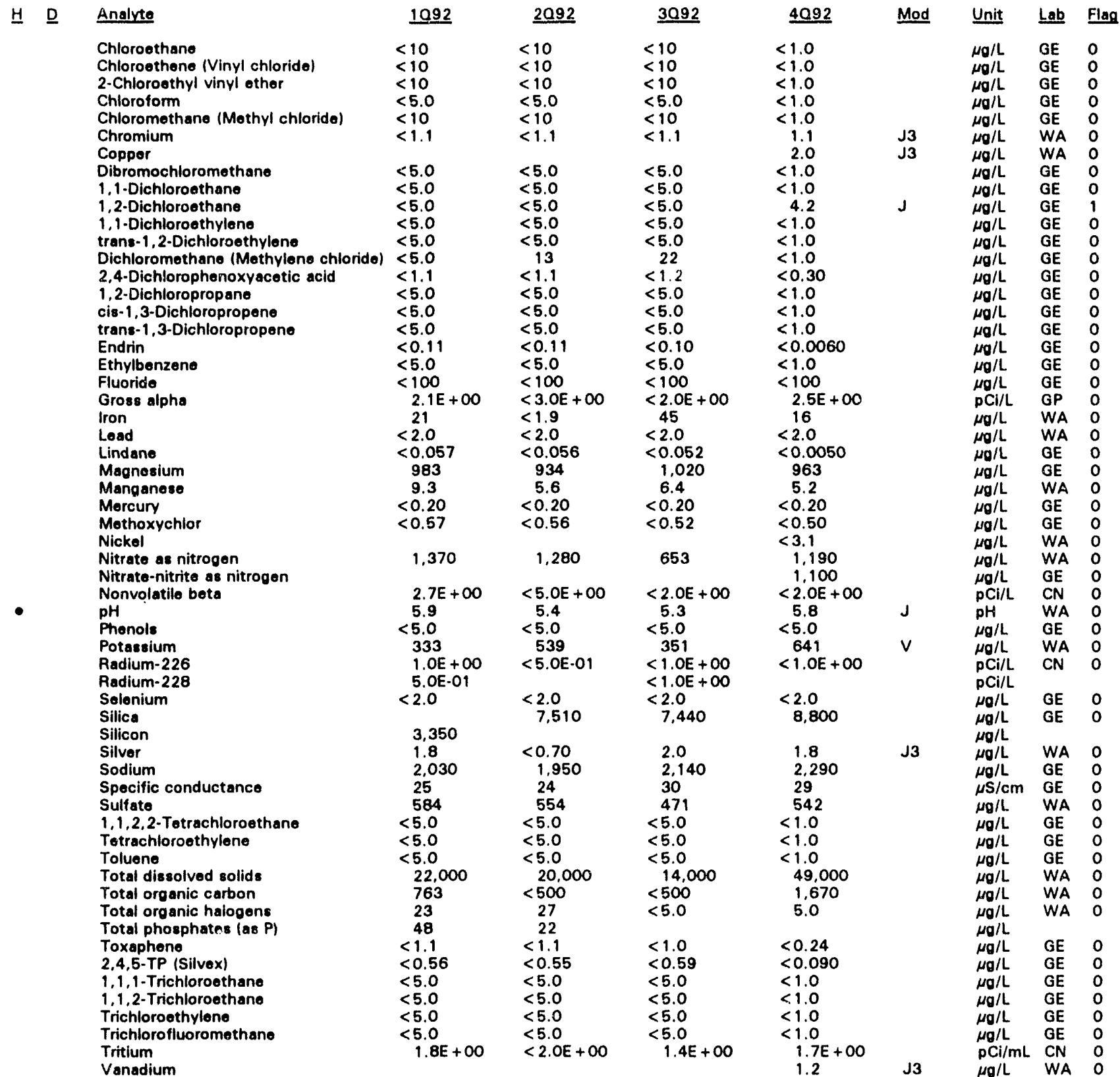

Note: Flagging levels, modifiers, and laboratory are for 4th quarter 1992 data only. See Appendix B for flagging criteria. - = exceeded holding time for 4th quarter 1992.

- =xceeded final primary drinking water standard for th quarter 1992. 
WSRC-TR-93-068

WELL LFW 55D

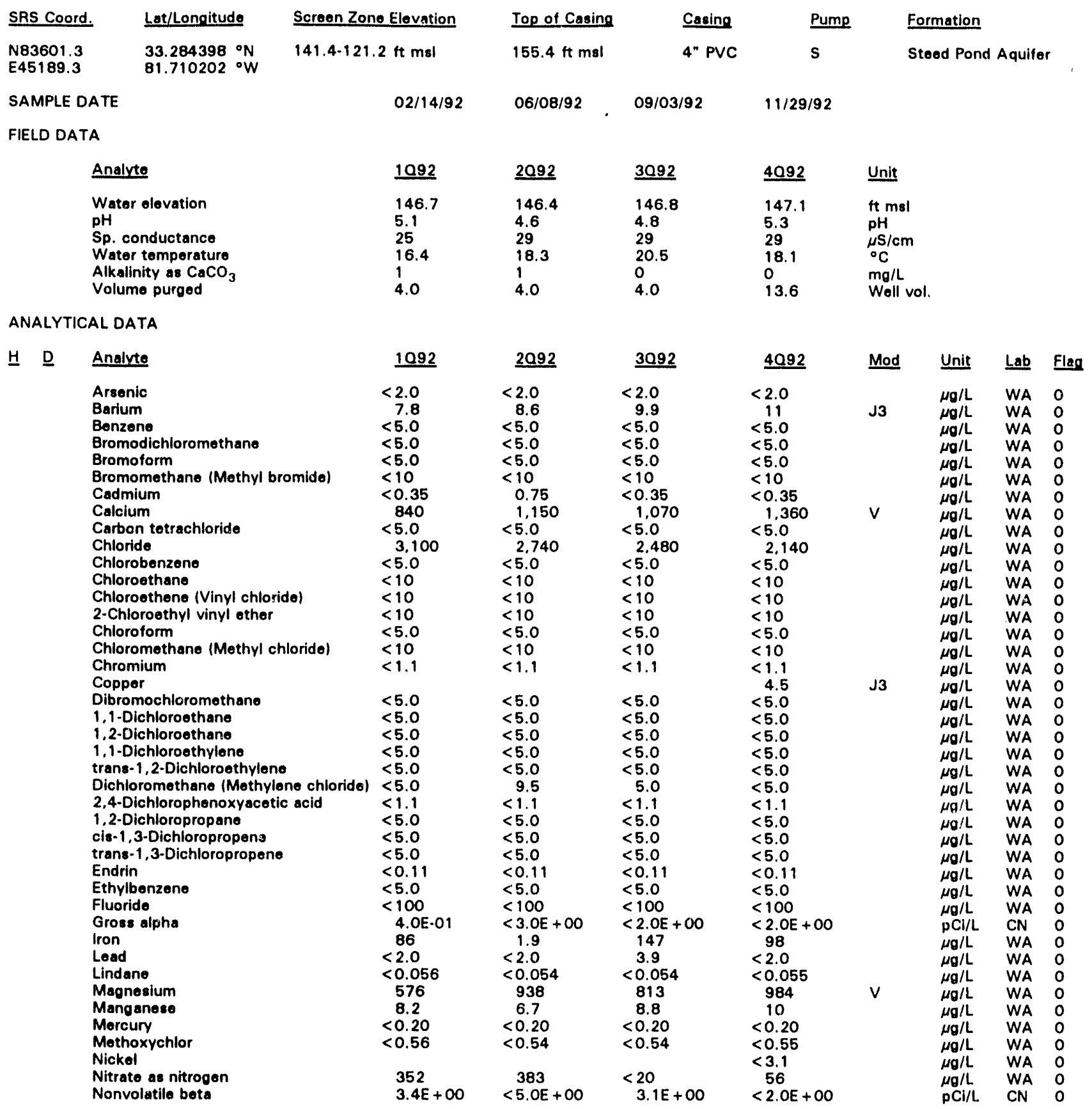

Note: Flagging levels, modifiers, and laboratory are for 4 th quarter 1992 data only. See Appendix B for flagging criteria.

- =xceeded holding time for 4th quarter 1992.

- =xceeded final primary drinking water standard for 4 th quarter 1992. 
Well LFW 55D continued

ANALYTICAL DATA
H D $\quad$ Analyte
pH
Phenols
Potassium
Radium-226
Radium-228
Selenium
Sillca
Silicon
Silver
Sodium
Specific conductance
Sulfate
1,1,2,2-Totrachloroethane
Totrachloroethylene
Toluene
Total dissolved solids
Total organic carbon
Total organic halogens
Total phosphates las P)
Toxaphene
2,4,5-TP (Silvex)
1.1,1-Trichloroethane
1,1,2-Trichloroethane
Trichloroethylene
Trichlorofluoromethane
Tritium
Vanadium

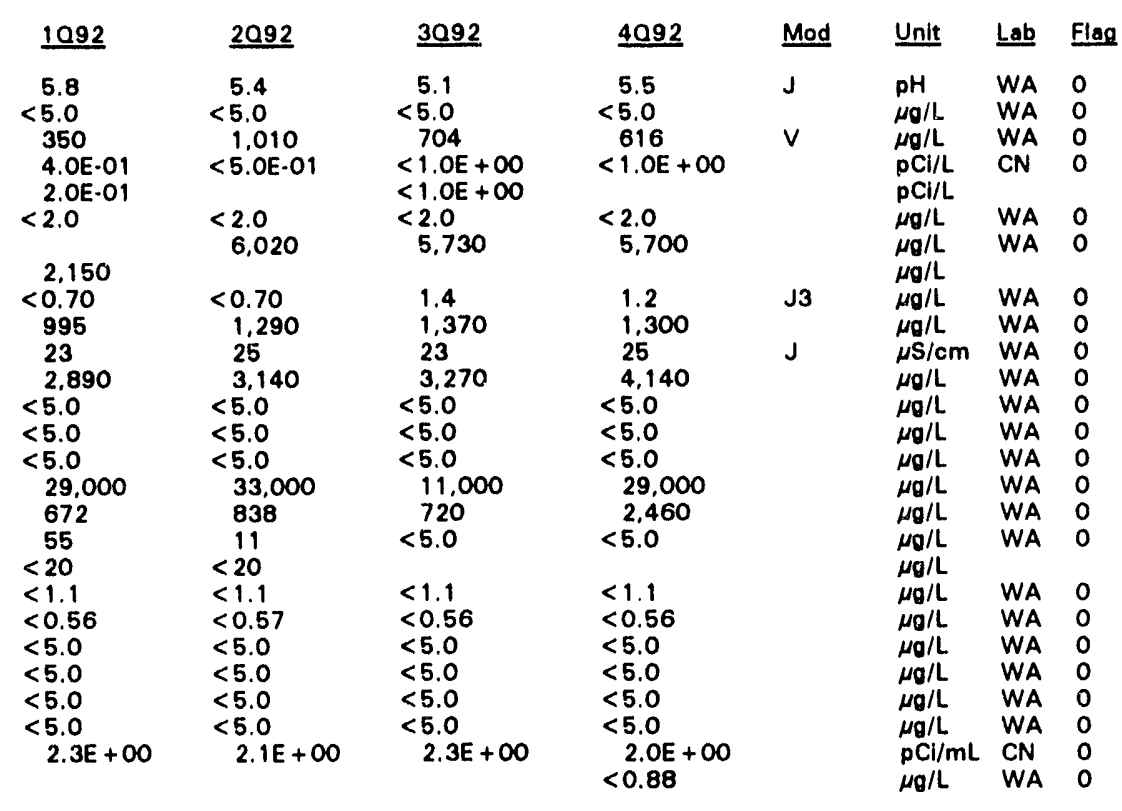

\section{WELL LFW 56D}

$\begin{array}{ll}\text { SRS Coord. } & \text { Lat/Longitude } \\ \text { N83398.0 } & 33.284140^{\circ} \mathrm{N} \\ \text { E45306.6 } & 81.709498{ }^{\circ} \mathrm{W}\end{array}$

\section{Screen Zone Elevation}

SAMPLE DATE

$02 / 14 / 92$

\begin{tabular}{|c|c|}
\hline Top of Casing & \\
\hline $158.1 \mathrm{ft} \mathrm{msl}$ & $4^{\prime \prime}$ \\
\hline $06 / 08 / 92$ & $09 / 03 / 92$ \\
\hline 2092 & 3092 \\
\hline $\begin{array}{l}144.9 \\
4.3 \\
23 \\
18.7 \\
0 \\
4.0\end{array}$ & $\begin{array}{l}145.3 \\
4.7 \\
17 \\
20.9 \\
0 \\
4.0\end{array}$ \\
\hline
\end{tabular}

1092
145.3
5.2
18
17.2
0
4.0

\begin{tabular}{lc}
$\quad 1092$ & $\underline{2092}$ \\
\hline$<2.0$ & $<2.0$ \\
$<6.6$ & 4.3 \\
$<5.0$ & $<5.0$ \\
$<5.0$ & $<5.0$ \\
$<5.0$ & $<5.0$ \\
$<10$ & $<10$ \\
$<0.35$ & $<0.35$ \\
4992 & 479 \\
$<5.0$ & $<5.0$ \\
2.820 & 3.570 \\
$<5.0$ & $<5.0$
\end{tabular}

\begin{tabular}{lc}
$\quad 1092$ & $\underline{2092}$ \\
\hline 2.0 & $<2.0$ \\
$<6.6$ & 4.3 \\
$<5.0$ & $<5.0$ \\
$<5.0$ & $<5.0$ \\
$<5.0$ & $<5.0$ \\
$<10$ & $<10$ \\
$<0.35$ & $<0.35$ \\
492 & 479 \\
$<5.0$ & $<5.0$ \\
2.820 & 3.570 \\
$<5.0$ & $<5.0$
\end{tabular}

$\begin{aligned} & 3092 \\ &< 2.0 \\ & 4.5 \\ &<5.0 \\ &<5.0 \\ &<5.0 \\ &<10 \\ &<0.35 \\ & 361 \\ &<5.0 \\ & 1.910 \\ &<5.0\end{aligned}$

$\begin{aligned} & 4092 \\ &< 2.0 \\ &<4.0 \\ &<5.0 \\ &<5.0 \\ &<5.0 \\ &<10 \\ &<0.35 \\ & 402 \\ &<5.0 \\ & 1.860 \\ &<5.0\end{aligned}$

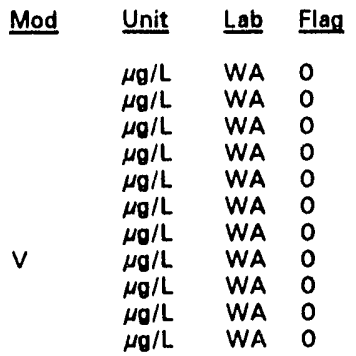

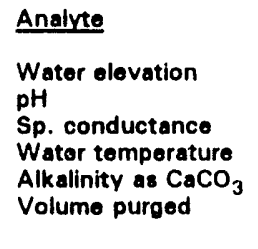

Analyte
Arsenic
Barium
Benzene
Bromodichloromethane
Bromoform
Bromomethane (Methyl bromide)
Cadmium
Calcium
Carbon tetrachloride
Chloride
Chlorobenzene

\begin{tabular}{|c|c|}
\hline 4092 & Unit \\
\hline $\begin{array}{l}145.9 \\
5.1 \\
16 \\
18.5 \\
0 \\
27.8\end{array}$ & $\begin{array}{l}\text { ft msl } \\
\mathrm{pH} \\
\mu \mathrm{S} / \mathrm{cm} \\
{ }^{\circ} \mathrm{C} \\
\mathrm{mg} / \mathrm{L} \\
\text { Woll vol. }\end{array}$ \\
\hline
\end{tabular}

Note: Flagging levels, modifiers, and laboratory are for 4th quarter 1992 data only. See Appendix B for flagging criteria. - = exceeded holding time for 4th quarter 1992.

- = exceeded final primary drinking water standard for 4th quarter 1992. 
Woll LFW 560 continued

ANALYTICAL DATA.

\begin{tabular}{|c|c|c|c|c|c|c|c|c|}
\hline$\underline{H} \quad \underline{D}$ & Analyte & 1092 & 2092 & 3092 & 4092 & Mod & Unit & $\underline{\text { Lab }}$ \\
\hline & 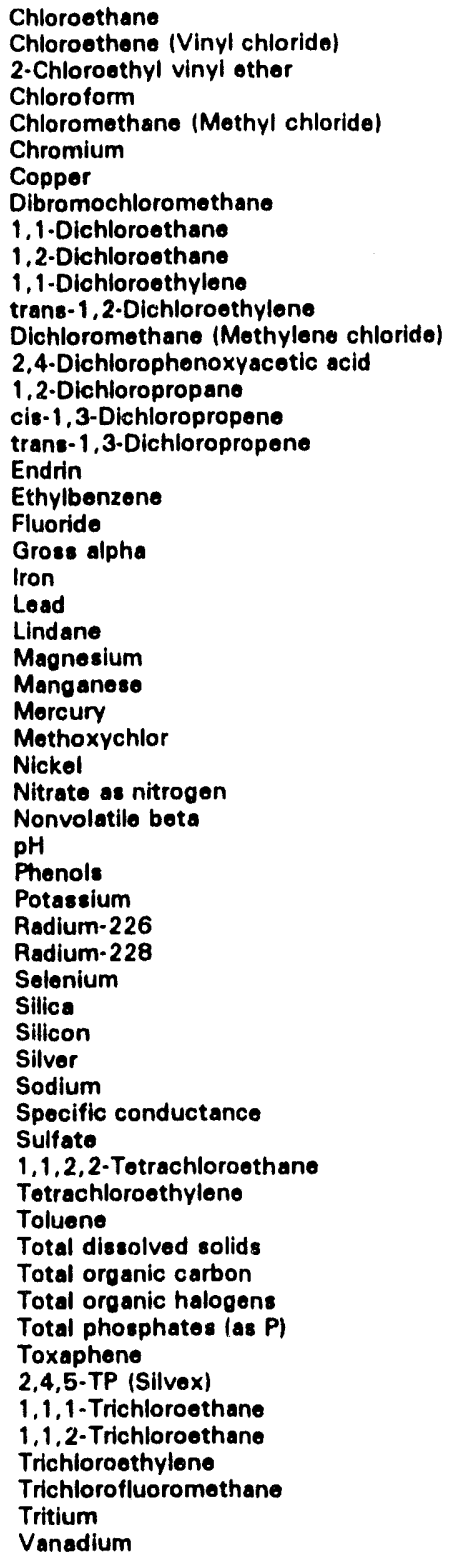 & 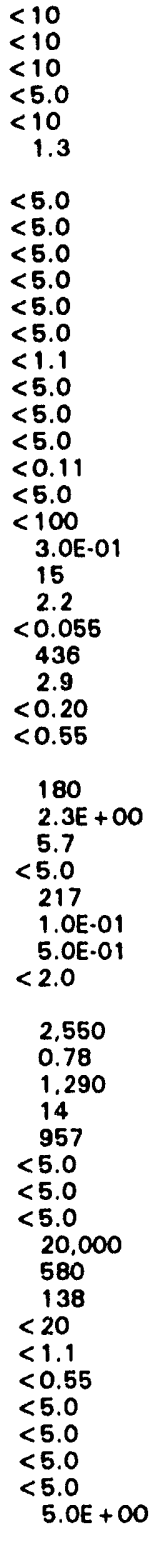 & 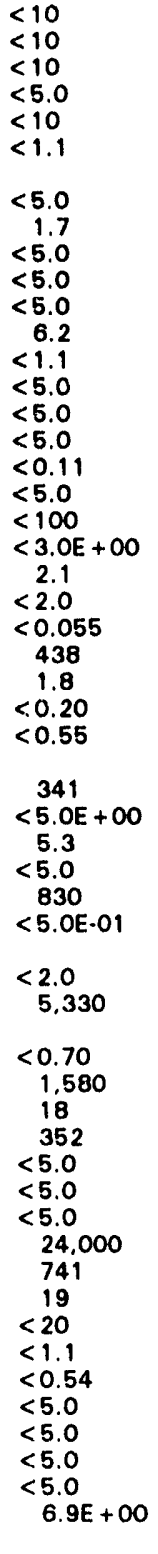 & 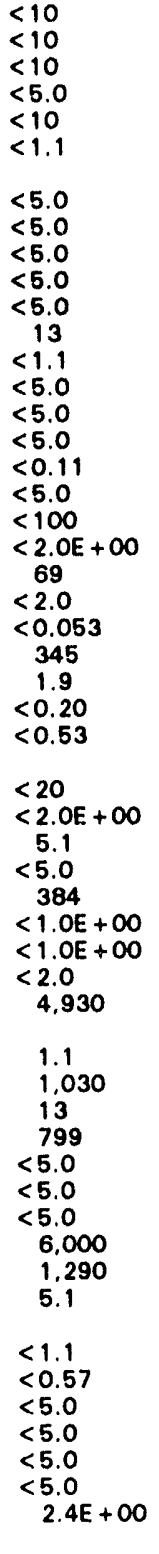 & 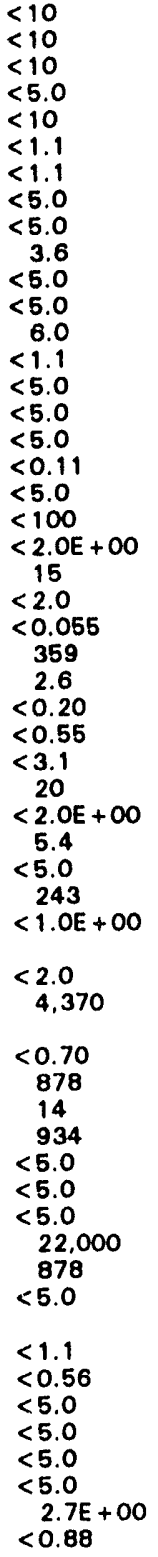 & $\begin{array}{l}\mathrm{J} \\
\mathrm{V}\end{array}$ & $\begin{array}{l}\mu g / L \\
\mu g / L \\
\mu g / L \\
\mu g / L \\
\mu g / L \\
\mu g / L \\
\mu g / L \\
\mu g / L \\
\mu g / L \\
\mu g / L \\
\mu g / L \\
\mu g / L \\
\mu g / L \\
\mu g / L \\
\mu g / L \\
\mu g / L \\
\mu g / L \\
\mu g / L \\
\mu g / L \\
\mu g / L \\
p C I / L \\
\mu g / L \\
\mu g / L \\
\mu g / L \\
\mu g / L \\
\mu g / L \\
\mu g / L \\
\mu g / L \\
\mu g / L \\
\mu g / L \\
p C I / L \\
p H \\
\mu g / L \\
\mu g / L \\
p C i / L \\
p C I / L \\
\mu g / L \\
\mu g / L \\
\mu g / L \\
\mu g / L \\
\mu g / L \\
\mu S / d \\
\mu g / L \\
\mu g / L \\
\mu g / L \\
\mu g / L \\
\mu g / L \\
\mu g / L \\
\mu g / L \\
\mu g / L \\
\mu g / L \\
\mu g / L \\
\mu g / L \\
\mu g / L \\
\mu g / L \\
\mu g / L \\
p C D \\
\mu g / L\end{array}$ & $\begin{array}{l}\text { WA } \\
\text { WA } \\
\text { WA } \\
\text { WA } \\
\text { WA } \\
\text { WA } \\
\text { WA } \\
\text { WA } \\
\text { WA } \\
\text { WA } \\
\text { WA } \\
\text { WA } \\
\text { WA } \\
\text { WA } \\
\text { WA } \\
\text { WA } \\
\text { WA } \\
\text { WA } \\
\text { WA } \\
\text { WA } \\
\text { CN } \\
\text { WA } \\
\text { WA } \\
\text { WA } \\
\text { WA } \\
\text { WA } \\
\text { WA } \\
\text { WA } \\
\text { WA } \\
\text { WA } \\
\text { CN } \\
\text { WA } \\
\text { WA } \\
\text { WA } \\
\text { CN } \\
\text { WA } \\
\text { WA } \\
\text { WA } \\
\text { WA } \\
\text { WA } \\
\text { WA } \\
\text { WA } \\
\text { WA } \\
\text { WA } \\
\text { WA } \\
\text { WA } \\
\text { WA } \\
\text { WA } \\
\text { WA } \\
\text { WA } \\
\text { WA } \\
\text { WA } \\
\text { WA } \\
\text { CN } \\
\text { WA } \\
\text { WA }\end{array}$ \\
\hline
\end{tabular}

Note: Flagging levels, modifiers, and laboratory are for 4th quarter 1992 data only. See Appendix B for flagging criteria.

- = exceeded holding time for 4th quarter 1992.

a = exceeded final primary drinking water standard for 4th quarter 1992. 
WSRC-TR-93-068

WELL LFW 57B

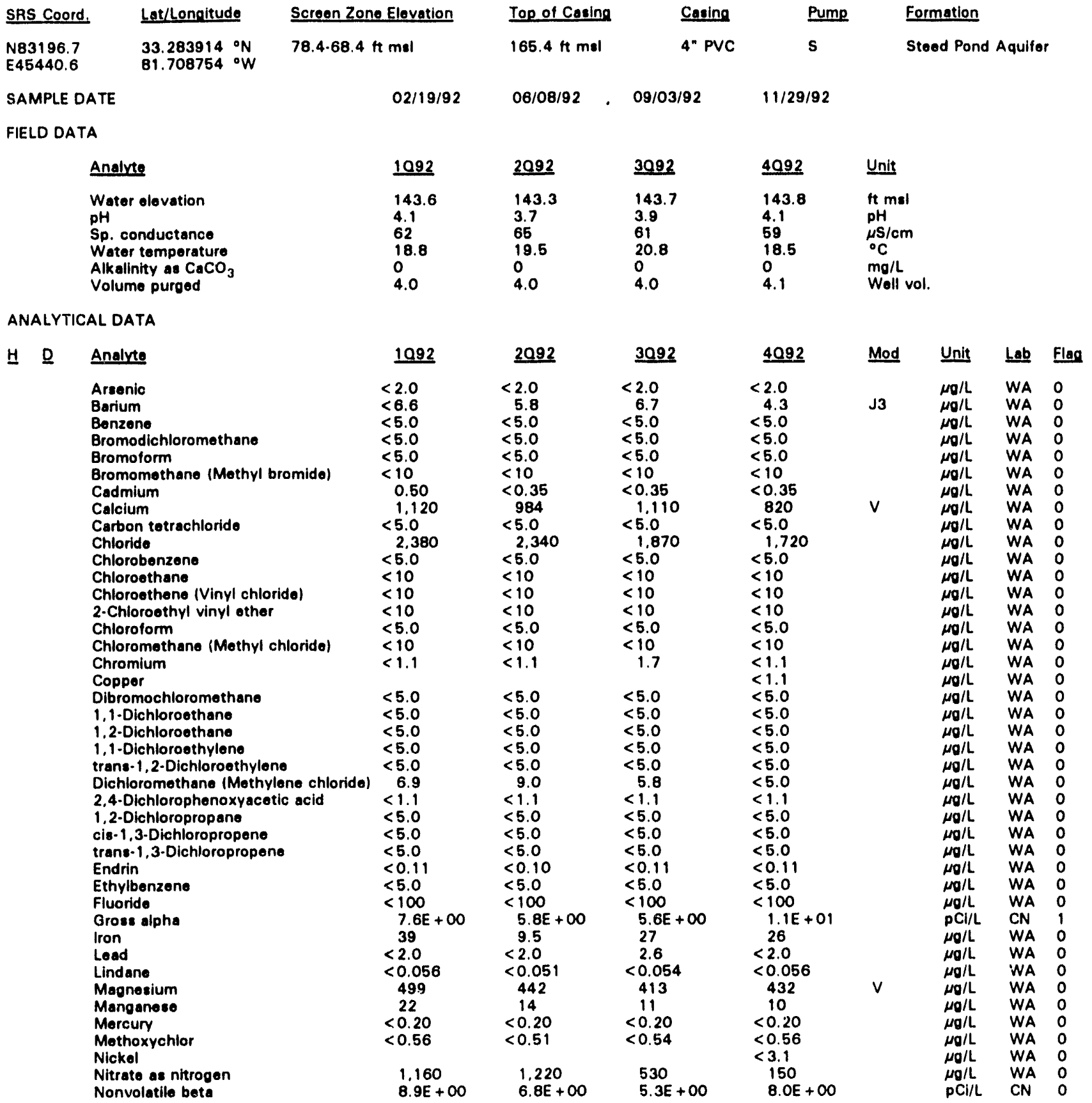

Note: Flagging levels, modifiers, and laboratory are for 4th quarter 1992 data only. See Appendix B for flagging criteria.

- = exceeded holding time for 4th quarter 1992.

- = exceeded final primary drinking water standard for 4th quarter 1992. 
Woll LFW 578 continued

ANALYTICAL DATA

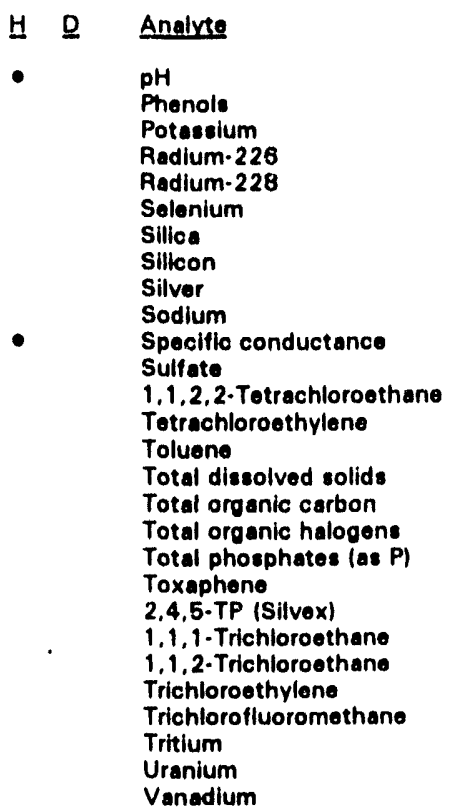

\begin{tabular}{|c|c|c|c|c|c|c|c|}
\hline 1092 & $\underline{2092}$ & $\underline{3092}$ & 4092 & Mod & Unit & Lab & Fleg \\
\hline $\begin{array}{r}4.8 \\
<5.0 \\
331 \\
2.2 E+\infty \\
3.9 E+\infty\end{array}$ & $\begin{array}{l}4.3 \\
<5.0 \\
864 \\
3.2 E+00\end{array}$ & $\begin{array}{r}4.7 \\
<5.0 \\
787 \\
1.2 E+\infty \\
2.7 E+\infty\end{array}$ & $\begin{array}{l}4.4 \\
<5.0 \\
443 \\
2.2 E+\infty\end{array}$ & J & $\begin{array}{l}\mathrm{pH} \\
\mu \mathrm{g} / \mathrm{L} \\
\mu \mathrm{g} / \mathrm{L} \\
\mathrm{pCi} / \mathrm{L} \\
\mathrm{pCl} / \mathrm{L}\end{array}$ & $\begin{array}{l}\text { WA } \\
\text { WA } \\
\text { WA } \\
C N\end{array}$ & $\begin{array}{l}0 \\
0 \\
0 \\
0\end{array}$ \\
\hline$<2.0$ & $\begin{array}{l}<2.0 \\
\quad 8,060\end{array}$ & $\begin{array}{l}<2.0 \\
\quad 8.560\end{array}$ & $\begin{array}{l}<2.0 \\
\quad 9,650\end{array}$ & & $\begin{array}{c}\mu \mathrm{g} / \mathrm{L} \\
\mu \mathrm{g} / \mathrm{L}\end{array}$ & $\begin{array}{l}\text { WA } \\
\text { WA }\end{array}$ & $\begin{array}{l}0 \\
0\end{array}$ \\
\hline $\begin{array}{l}4.810 \\
0.72 \\
1.700 \\
46 \\
8.540 \\
<5.0 \\
<5.0 \\
<5.0 \\
45.000 \\
<500 \\
12\end{array}$ & $\begin{array}{l}<0.70 \\
1.610 \\
52 \\
8.650 \\
<5.0 \\
<5.0 \\
<5.0 \\
40.000 \\
<500 \\
<10 \\
<50\end{array}$ & $\begin{array}{l}1.4 \\
1.640 \\
48 \\
10,400 \\
<5.0 \\
<5.0 \\
<5.0 \\
25,000 \\
<500 \\
<5.0\end{array}$ & $\begin{array}{l}1.3 \\
1.630 \\
50 \\
9.370 \\
<5.0 \\
<5.0 \\
<5.0 \\
31.000 \\
779 \\
<5.0\end{array}$ & $\begin{array}{l}\text { J3 } \\
\text { J }\end{array}$ & $\begin{array}{l}\mu \mathrm{g} / \mathrm{L} \\
\mu \mathrm{g} / \mathrm{L} \\
\mu \mathrm{g} / \mathrm{L} \\
\mu \mathrm{S} / \mathrm{cm} \\
\mu \mathrm{g} / \mathrm{L} \\
\mu \mathrm{g} / \mathrm{L} \\
\mu \mathrm{g} / \mathrm{L} \\
\mu \mathrm{g} / \mathrm{L} \\
\mu \mathrm{g} / \mathrm{L} \\
\mu \mathrm{g} / \mathrm{L} \\
\mu \mathrm{g} / \mathrm{L}\end{array}$ & $\begin{array}{l}\text { WA } \\
\text { WA } \\
\text { WA } \\
\text { WA } \\
\text { WA } \\
\text { WA } \\
\text { WA } \\
\text { WA } \\
\text { WA } \\
\text { WA }\end{array}$ & $\begin{array}{l}0 \\
0 \\
0 \\
0 \\
0 \\
0 \\
0 \\
0 \\
0 \\
0\end{array}$ \\
\hline $\begin{array}{l}<20 \\
<1.1 \\
<0.56 \\
<5.0 \\
<5.0 \\
<5.0 \\
<5.0 \\
\quad 1.0 \mathrm{E}+00\end{array}$ & $\begin{array}{l}<20 \\
<1.0 \\
<0.55 \\
<5.0 \\
<5.0 \\
<5.0 \\
<5.0 \\
<2.0 E+\infty\end{array}$ & $\begin{array}{l}<1.1 \\
<0.56 \\
<5.0 \\
<5.0 \\
<5.0 \\
<5.0 \\
<7.0 \mathrm{E} \cdot 01\end{array}$ & $\begin{array}{l}<1.1 \\
<0.56 \\
<5.0 \\
<5.0 \\
<5.0 \\
<5.0 \\
<7.0 \text { E. } 01\end{array}$ & & $\begin{array}{l}\mu \sigma / L \\
\mu g / L \\
\mu g / L \\
\mu g / L \\
\mu g / L \\
\mu g / L \\
\mu g / L \\
\mathrm{pCl} / \mathrm{mL}\end{array}$ & $\begin{array}{l}\text { WA } \\
\text { WA } \\
\text { WA } \\
\text { WA } \\
\text { WA } \\
\text { WA } \\
\text { CN }\end{array}$ & $\begin{array}{l}0 \\
0 \\
0 \\
0 \\
0 \\
0 \\
0\end{array}$ \\
\hline & & & $<0.88$ & & ${ }_{\mu \sigma / L}^{\mu / L}$ & WA & 0 \\
\hline
\end{tabular}

\section{WELL LFW 57C}

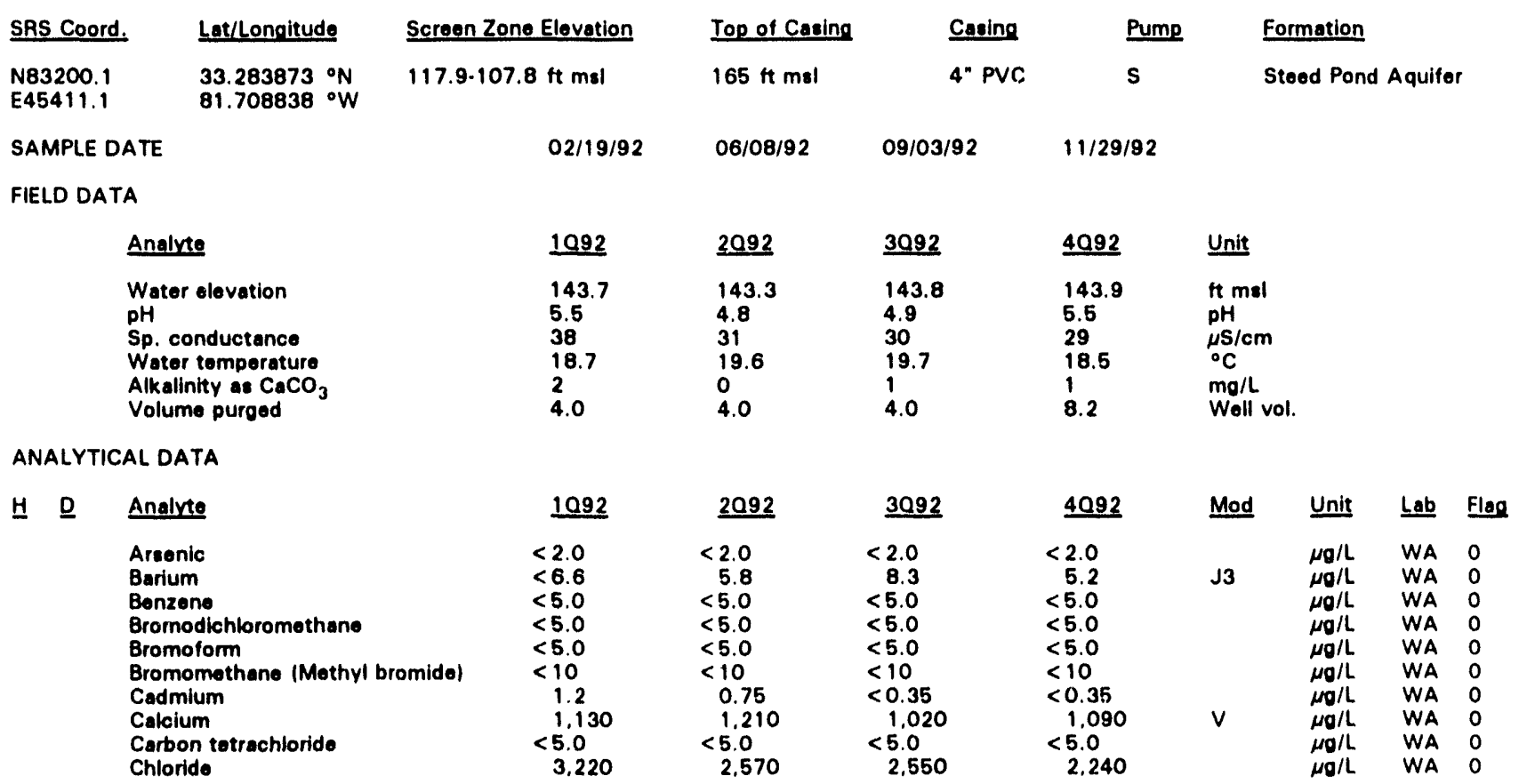

Note: Flagging levels, modifiers, and laboratory are for 4th quarter 1992 data only. See Appendix B for flagging criteria.

- =xceeded holding time for 4th quarter 1992.

- excesded final primary drinking water standard for 4th quarter 1992. 
WSRC-TR-93.068

Woll LFW $57 C$ continued

ANALYTICAL DATA

H D Analve

Chlorobenzene

Chloroethane

Chloroethene (Vinyl chloride)

2.Chloroethyl vinyl other

Chtoroform

Chloromethane (Methyl chloride)

Chromium

Copper

Dibromochloromethane

1,1-Dichloroethane

1.2-Oichloroethane

1,1-Dichloroethyleno

trans-1,2-Dichloroethylene

Dichloromethane (Methylene chloride)

2,4-Dichlorophenoxyacotic acld

1,2-Dichloropropane

cis-1,3-Dichloropropene

trane-1,3-Dichloropropene

Endrin

Ethylbenzene

Fluorido

Grose alphe

Iron

Lead

Lindane

Magnetium

Manganose

Mercury

Mothoxychlor

Nickel

Nitrate as nitrogen

Nonvolatile beta

- $\quad$ pH

Phenols

Potassium

Aadium-226

Radium -228

Selonium

Silica

Silicon

Silver

Sodium

- Specific conductance

Sulfate

1,1,2,2-Totrachloroethane

Tetrachloroethylene

Toluene

Total dissolved solids

Total organic carbon

Total organic halogens

Total phosphates (as P)

Toxaphene

2,4,5-TP (Silvax)

1,1,1-Trichloroethane

1,1,2-Trichloroethane

Trichloroothylene

Trichlorofluoromethane

Tritium

Uranium

1092

$<5$.

$<10$

$<10$

$<10$

$<5.0$

$<10$

$<1.1$

$<5.0$

$<5.0$

$<5.0$

$<5.0$
$<5.0$

2.2

$<1.1$

$<5.0$

$<5.0$

$<5.0$

$<0.11$

$<5.0$

$<100$

$1.1 \mathrm{E}+00$

6.2

$<2.0$

$<0.055$

912
9.6

9.6
$<0.20$

$<0.55$

1.480

$2.4 E+\infty 0$

5.2

$<5.0$
341

341
8.0E-01

$<1.9 E+\infty 0$

$<2.0$

4,000

$<0.70$

2,140

722

$<5.0$

$<5.0$

$<5.0$

48,000

$<500$

59
20

20
$<1.1$

$<0.56$

$<5.0$

$<5.0$

$<5.0$

$<5.0$

1. $6 \mathrm{E}+00$

0.060

\begin{aligned} & 2092 \\ &$< 5.0 \\ &<10 \\ &<10 \\ &<10 \\ &<5.0 \\ &<10 \\ &<1.1 \\ &<5.0 \\ &<5.0 \\ &<5.0 \\ &<5.0 \\ &<5.0 \\ & 112 \\ &<1.1 \\ &<5.0 \\ &<5.0 \\ &<5.0 \\ &<0.11 \\ &<5.0 \\ &<100 \\ &<3.0 E+00 \\ &<1.9 \\ &<2.0 \\ &<0.065 \\ & 915 \\ &<5.8 \\ &<0.20 \\ &<0.55 \\ &$\hline 0\end{aligned}

$<5.0$

$<10$

$<10$

$<10$

$<5.0$
$<10$

2.0

$<5.0$

$<5.0$

$<5.0$

$<5.0$

$<5.0$

7.6

$<1.1$

$<5.0$

$<5.0$

$<0.11$

$<5.0$

$<100$

$<2.0 E+00$

8.9

2.0

$<0.054$

767

5.3

$<0.20$

$<0.54$

1.420

$<5.0 E+00$

5.2

$<5.0$

619

7.OE-01

$<2.0$

7,800

$<0.70$

1.900

25

650

$<5.0$

$<5.0$

$<5.0$

35,000

$<500$

18

23

$<1.1$

$<5.0$

5.0

$<5.0$

$<5.0$

$<2.0 E+00$

707

3. $3 E+00$

5.0

$<5.0$

418

$<1$. OE +00
$<1$. OE +00

$<2.0$

7.420

3.3

1.870

24

$<5.0$

$<5.0$

$<5.0$

36,000

641

$<1.1$

$<0.56$

$<5.0$

$<5.0$

$<5.0$

$<5.0$

$1.9 E+00$

$\quad 4092 \quad$ Mod
$<5.0$
$<10$
$<10$

$\begin{array}{lll}\text { Unit } & \text { Lab } & \text { Flag } \\ \mu g / L & \text { WA } & 0 \\ \mu g / L & W A & 0\end{array}$

$<10$

$<6.0$

$<10$

$<1.1$

$<5.0$

$<5.0$

3.8

$<5.0$
$<5.0$

6.9

$<1.1$

$<5.0$

$<5.0$

$<5.0$

$<0.11$

$<5.0$

$<100$

2.7E +00

13

$<0.055$

1.000

4.5

$<0.20$

$<0.55$

$<3.1$

597

$3.4 E+\infty 0$

5.6

$<5.0$

412

$<1 . \mathrm{OE}+00$

$<2.0$

7,570

$<0.70$

2,000

25

759

$<5.0$

$<5.0$

$<5.0$

53,000

1,080

$<1.1$

$<0.55$

$<5.0$

$<5.0$

$<5.0$

$<5.0$

$2.0 E+O O$

$<0.88$ 
WSRC-TR-93-068

WELL LFW 57D

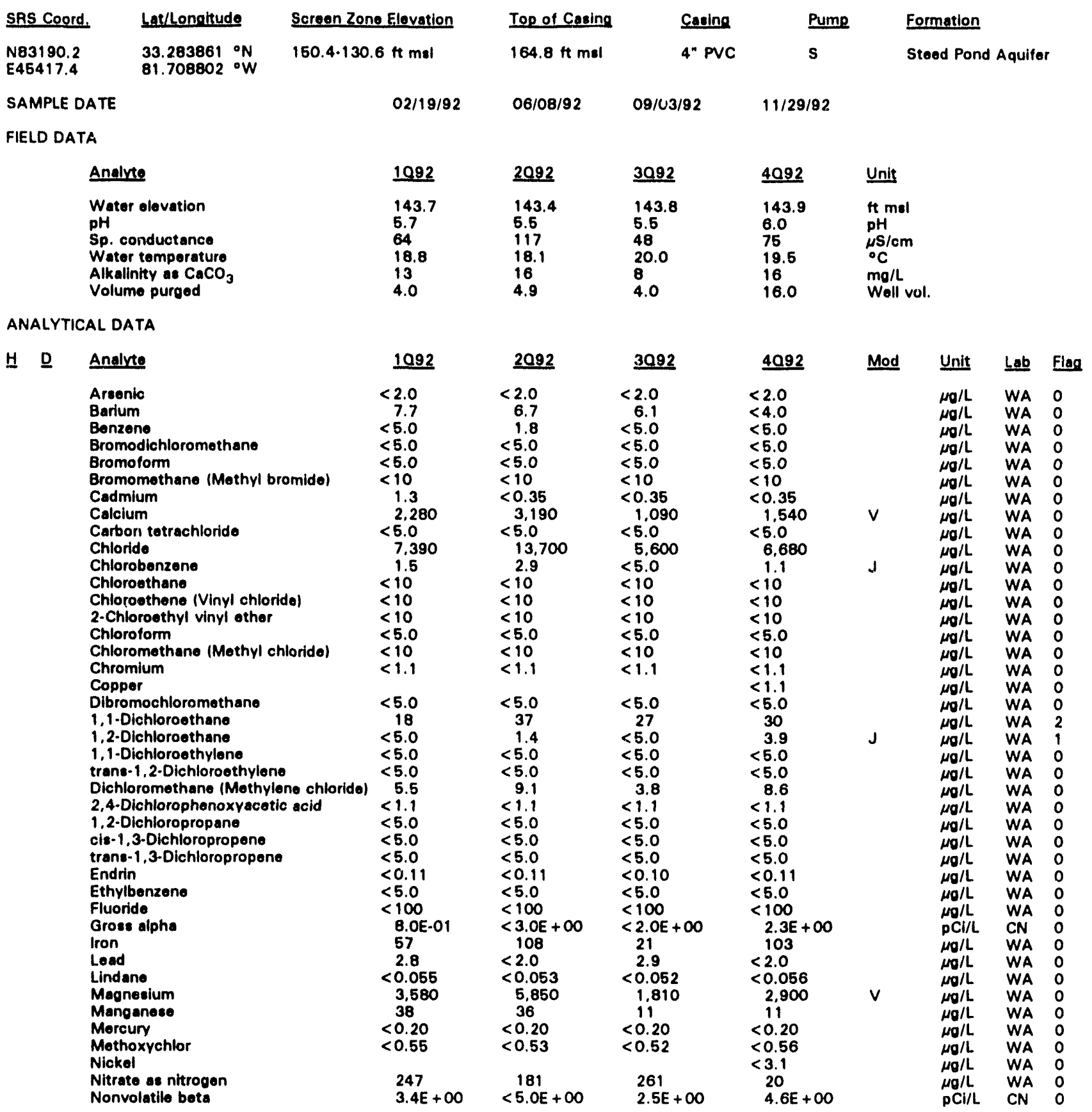

Note: Flagging levels, modifiers, and laboratory are for 4th quarter 1992 data only. See Appendix B for flagging criteria.

- = exceeded holding time for 4th quarter 1992

- = exceeded final primary drinking water standard for 4th quarter 1992. 
Woll LFW 570 continued

ANALYTICAL DATA

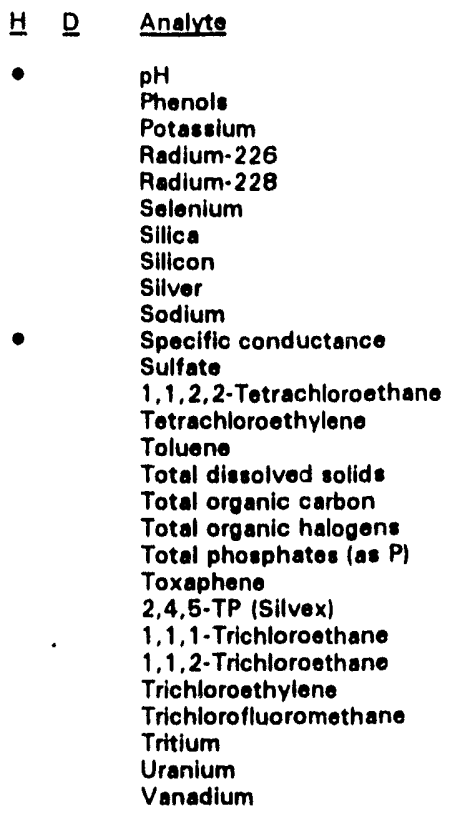

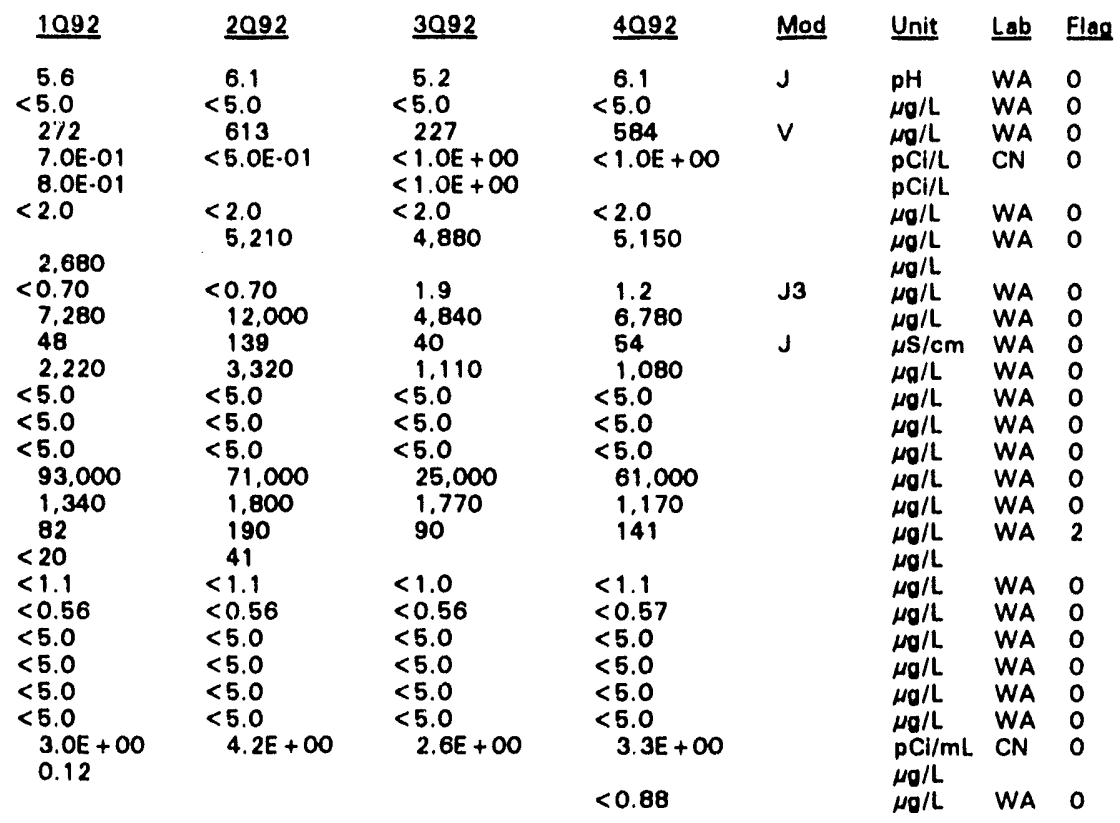

\section{WELL LFW 58D}

$\begin{array}{ll}\text { SRS Coord. } & \text { Lat/Longitude } \\ \text { N82940.6 } & 33.283771^{\circ} \mathrm{N} \\ \text { E45700.2 } & 81.707573^{\circ} \mathrm{W} \\ \text { SAMPLE DATE } & \\ \text { FIELD DATA } & \end{array}$

\section{Screen Zone Elovation}

47.6-127.5 $\mathrm{ft} \mathrm{msl}$

\section{Top of Casing}

$167.6 \mathrm{ft} \mathrm{mal}$

$\begin{array}{ll}\text { Casing } & \text { Pump } \\ \text { 4" PVC } & \mathrm{s}\end{array}$

Formation

$02 / 19 / 92$

$06 / 08 / 92$

$09 / 04 / 92$

$11 / 29 / 92$

\section{Analyte}

Water elovation

pH

Sp. conductance

Water temperature

Alkalinity as $\mathrm{CaCO}_{3}$

Volume purged

$\begin{array}{ll}1092 & \underline{2092} \\ 141.7 & 141.4 \\ 4.8 & 4.4 \\ 50 & 72 \\ 19.1 & 19.3 \\ 0 & 0 \\ 4.0 & 4.0\end{array}$

3092
142.0
4.7
79
19.6
0
4.0

40.92
142.0
4.9
80
19.6
0
6.9

Unit

ft msl

$\mathrm{pH}$

$\mu \mathrm{S} / \mathrm{cm}$

${ }^{\circ} \mathrm{C}$

Woll vol.

\section{ANALYTICAL DATA}

H $\underline{D}$

Analyte
Arsenic
Barium
Benzene
Bromodichloromethane
Bromoform
Bromomethane (Methyl bromide)
Cadmium
Calcium
Carbon tetrachloride
Chloride

1092
$<2.0$
7.6
2.3
$<5.0$
$<5.0$
$<10$
1.3
1.310
$<5.0$
9.340

$\begin{aligned} & 2092 \\ &< 2.0 \\ & 6.7 \\ & 2.9 \\ &<5.0 \\ &<5.0 \\ &<10 \\ &<0.35 \\ & 1.240 \\ &<5.0 \\ & 13.100\end{aligned}$

$\begin{aligned} & 3092 \\ &< 2.0 \\ & 6.6 \\ & 4.4 \\ &<5.0 \\ &<5.0 \\ &<10 \\ &< 0.35 \\ & 1.130 \\ &<5.0 \\ & 36.400\end{aligned}$

$\begin{aligned} & 4092 \\ &< 2.0 \\ & 7.2 \\ & 3.6 \\ &<5.0 \\ &<5.0 \\ &<10 \\ &<0.35 \\ & 1.280 \\ &<5.0 \\ & 17.900\end{aligned}$

Mod

\begin{tabular}{|c|c|c|}
\hline Unit & Lab & \\
\hline$\mu \mathrm{g} / \mathrm{L}$ & WA & 0 \\
\hline$\mu \mathrm{g} / \mathrm{L}$ & WA & \\
\hline$\mu g / L$ & WA & 1 \\
\hline$\mu \mathrm{g} / \mathrm{L}$ & WA & \\
\hline$\mu \mathrm{g} / \mathrm{L}$ & WA & \\
\hline$\mu_{\mathrm{g}} / \mathrm{L}$ & WA & \\
\hline$\mu \mathrm{g} / \mathrm{l}$ & WA & \\
\hline & WA & \\
\hline & WA & \\
\hline & WA & \\
\hline
\end{tabular}

\footnotetext{
Note: Flagging levels, modifiers, and laboratory are for 4 th quarter 1792 data only. See Appendix B for flagging criteria.

- = exceeded holding time for 4th quarter 1992.

- exceeded final primary drinking water standard for 4th quarter 1992.
} 


\begin{tabular}{|c|c|c|c|c|c|c|}
\hline 1092 & $\underline{2092}$ & $\underline{3092}$ & 4092 & Mod & Unit & Lab \\
\hline $\begin{aligned} &<5.0 \\
&<10 \\
&<10 \\
&<10 \\
&<5.0 \\
&<<10 \\
& 1.1\end{aligned}$ & $\begin{array}{l}<5.0 \\
1.3 \\
<10 \\
<10 \\
<5.0 \\
<10 \\
<1.1\end{array}$ & $\begin{array}{l}<5.0 \\
<10 \\
<10 \\
<10 \\
<5.0 \\
<10 \\
<1.1\end{array}$ & $\begin{array}{l}<5.0 \\
<10 \\
<10 \\
<10 \\
<5.0 \\
<10 \\
<1.1 \\
<1.1\end{array}$ & & $\begin{array}{l}\mu \mathrm{g} / \mathrm{L} \\
\mu \mathrm{g} / \mathrm{L} \\
\mu \mathrm{g} / \mathrm{L} \\
\mu \mathrm{g} / \mathrm{L} \\
\mu \mathrm{g} / \mathrm{L} \\
\mu \mathrm{g} / \mathrm{L} \\
\mu \mathrm{g} / \mathrm{L} \\
\mu \mathrm{g} / \mathrm{L}\end{array}$ & $\begin{array}{l}\text { WA } \\
\text { WA } \\
\text { WA } \\
\text { WA } \\
\text { WA } \\
\text { WA } \\
\text { WA } \\
\text { WA }\end{array}$ \\
\hline $\begin{array}{r}51 \\
<5.0\end{array}$ & $\begin{array}{l}56 \\
2.6\end{array}$ & $\begin{array}{l}83 \\
3.2\end{array}$ & $\begin{array}{l}62 \\
6.4\end{array}$ & & ${ }_{\mu \mathrm{g} / \mathrm{L}}$ & $\begin{array}{l}\text { WA } \\
\text { WA }\end{array}$ \\
\hline 3.5 & 1.8 & 2.7 & $<5.0$ & & $\mu \mathrm{g} / \mathrm{L}$ & WA \\
\hline $\begin{array}{c}<5.0 \\
47\end{array}$ & $\begin{array}{c}<5.0 \\
51\end{array}$ & $\begin{array}{c}<5.0 \\
60\end{array}$ & $\begin{array}{r}<5.0 \\
47\end{array}$ & & $\mu \mathrm{g} / \mathrm{L}$ & $\begin{array}{l}\text { WA } \\
\text { WA }\end{array}$ \\
\hline$<1.1$ & 0.35 & 0.56 & $<1.1$ & & $\mu \mathrm{g} / \mathrm{L}$ & WA \\
\hline$<5.0$ & $<5.0$ & $<5.0$ & $<5.0$ & & $\mu \mathrm{g} / \mathrm{L}$ & WA \\
\hline$<5.0$ & $<5.0$ & $<5.0$ & $<5.0$ & & $\mu \mathrm{g} / \mathrm{L}$ & WA \\
\hline $\begin{array}{l}<5.0 \\
<0.11\end{array}$ & $\begin{array}{l}<5.0 \\
<0.11\end{array}$ & $\begin{array}{l}<5.0 \\
<0.11\end{array}$ & $\begin{array}{l}<5.0 \\
<0.11\end{array}$ & & $\mu_{\mu / L} / L$ & $\begin{array}{l}\text { WA } \\
\text { WA }\end{array}$ \\
\hline$<5.0$ & $<5.0$ & $<5.0$ & $<5.0$ & & $\mu \mathrm{g} / \mathrm{L}$ & WA \\
\hline $\begin{array}{l}<100 \\
7.0 \mathrm{E}-01\end{array}$ & $\begin{array}{l}<100 \\
<3.0 E+00\end{array}$ & $\begin{array}{l}<100 \\
<2.0 E+\infty 0\end{array}$ & $\begin{array}{l}<100 \\
3.3 \mathrm{E}+\mathrm{OC}\end{array}$ & & $\begin{array}{l}\mu \mathrm{g} / \mathrm{L} \\
\mathrm{pCi} / \mathrm{L}\end{array}$ & $\begin{array}{l}\text { WA } \\
\mathrm{CN}\end{array}$ \\
\hline $\begin{array}{l}77 \\
4.7\end{array}$ & $\begin{array}{l}95 \\
<2.0 \\
\end{array}$ & $\begin{array}{l}115 \\
3.2\end{array}$ & $\begin{array}{r}59 \\
<2.0 \\
\end{array}$ & & $\mu \mathrm{g} / \mathrm{L}$ & $\begin{array}{l}\text { WA } \\
\text { WA }\end{array}$ \\
\hline $\begin{array}{c}<0.055 \\
926\end{array}$ & $\begin{array}{r}<0.055 \\
1.060\end{array}$ & $\begin{array}{r}<0.057 \\
1.060\end{array}$ & $\begin{array}{r}<0.057 \\
1.380\end{array}$ & & $\mu \mathrm{g} / \mathrm{L}$ & WA \\
\hline $\begin{array}{c}926 \\
43 \\
<0.20\end{array}$ & $\begin{array}{l}1,060 \\
28 \\
<0.20\end{array}$ & $\begin{array}{l}1,060 \\
19 \\
0.25\end{array}$ & $\begin{array}{l}1,380 \\
13 \\
0.33\end{array}$ & V & $\begin{array}{l}\mu \mathrm{g} / \mathrm{L} \\
\mu \mathrm{g} / \mathrm{L} \\
\mu \mathrm{g} / \mathrm{L}\end{array}$ & $\begin{array}{l}\text { WA } \\
\text { WA } \\
\text { WA }\end{array}$ \\
\hline$<0.55$ & $<0.55$ & $<0.57$ & $\begin{array}{l}<0.57 \\
<3.1\end{array}$ & & $\mu_{\mu \mathrm{g} / \mathrm{L}}$ & $\begin{array}{l}\text { WA } \\
\text { WA }\end{array}$ \\
\hline $\begin{array}{l}326 \\
2.4 E+\infty\end{array}$ & $\begin{aligned} & 503 \\
< & 5.0 E+\infty\end{aligned}$ & $\begin{array}{l}<20 \\
2.9 \mathrm{E}+\infty\end{array}$ & $\begin{array}{l}20 \\
3.9 E+O C)\end{array}$ & & $\begin{array}{l}\mu \mathrm{g} / \mathrm{L} \\
\mathrm{pCi} / \mathrm{L}\end{array}$ & $\begin{array}{l}\text { WA } \\
\text { CN }\end{array}$ \\
\hline 5.5 & 5.3 & 6.5 & 5.2 & $\mathrm{~J}$ & $\mathrm{pH}$ & WA \\
\hline $\begin{array}{c}<5.0 \\
433\end{array}$ & $\begin{array}{r}<5.0 \\
857\end{array}$ & $\begin{array}{r}<5.0 \\
300\end{array}$ & $\begin{array}{r}<5.0 \\
549\end{array}$ & v & $\underset{\mu \mathrm{g} / \mathrm{L}}{\mu \mathrm{g} / \mathrm{L}}$ & $\begin{array}{l}\text { WA } \\
\text { WA }\end{array}$ \\
\hline $\begin{array}{l}\text { 7.0E-01 } \\
\text { 8.0E-01 }\end{array}$ & $<5.0 E-01$ & $\begin{array}{l}<1.0 E+\infty 0 \\
<1.0 E+\infty 0\end{array}$ & $<1.0 E+\infty)$ & & $\begin{array}{l}\mathrm{pCi} / \mathrm{L} \\
\mathrm{pCi} / \mathrm{L}\end{array}$ & $\mathrm{CN}$ \\
\hline$<2.0$ & $\begin{aligned}<2.0 \\
\quad 11,700\end{aligned}$ & $\begin{array}{l}<2.0 \\
10.400\end{array}$ & $\begin{aligned}<2.0 \\
11,400\end{aligned}$ & & $\begin{array}{l}\mu \mathrm{g} / \mathrm{L} \\
\mu \mathrm{g} / \mathrm{L}\end{array}$ & $\begin{array}{l}\text { WA } \\
\text { WA }\end{array}$ \\
\hline $\begin{array}{r}5,650 \\
<0.70\end{array}$ & $<0.70$ & $<0.70$ & 0.83 & & $\mu \mathrm{g} / \mathrm{L}$ & WA \\
\hline $\begin{array}{r}<.70 \\
4,850\end{array}$ & 8,500 & 8.690 & 8,870 & $\sqrt{3}$ & $\underset{\mu \mathrm{g} / \mathrm{L}}{\mu \mathrm{g} / \mathrm{L}}$ & WA \\
\hline 48 & 60 & 67 & 63 & $\mathrm{~J}$ & $\mu \mathrm{S} / \mathrm{cm}$ & WA \\
\hline $\begin{array}{r}399 \\
<5.0\end{array}$ & $\begin{array}{r}406 \\
<5.0\end{array}$ & 272 & 272 & & $\mu \mathrm{g} / \mathrm{L}$ & WA \\
\hline $\begin{array}{r}<5.0 \\
3.7\end{array}$ & $<5.0$ & $<5.0$ & $<5.0$ & & $\mu \boldsymbol{g} / \mathrm{L}$ & WA \\
\hline $\begin{array}{r}3.7 \\
<5.0\end{array}$ & $\begin{array}{r}3.0 \\
<5.0\end{array}$ & $\begin{array}{r}2.8 \\
<5.0\end{array}$ & $\begin{array}{r}2.2 \\
<5.0\end{array}$ & $J$ & $\underset{\mu \mathrm{g} / \mathrm{L}}{\mu \mathrm{g}}$ & $\begin{array}{l}\text { WA } \\
\text { WA }\end{array}$ \\
\hline 60,000 & 50.000 & 43,000 & 82,000 & & $\mu \mathrm{g} / \mathrm{L}$ & WA \\
\hline $\begin{array}{l}1.230 \\
199\end{array}$ & $\begin{array}{l}1,610 \\
330\end{array}$ & $\begin{array}{l}1,870 \\
766\end{array}$ & $\begin{array}{l}4,830 \\
393\end{array}$ & & $\underset{\mu \mathrm{g} / \mathrm{L}}{\mu \mathrm{L}}$ & $\begin{array}{l}\text { WA } \\
\text { WA }\end{array}$ \\
\hline$<20$ & $<20$ & & & & g/L & WA \\
\hline$<1.1$ & $<1.1$ & $<1.1$ & $<1.1$ & & $\mu \mathrm{g} / \mathrm{L}$ & WA \\
\hline$<0.56$ & $<0.55$ & $<0.56$ & $<0.57$ & & $\mu \mathrm{g} / \mathrm{L}$ & WA \\
\hline 21 & 9.5 & 11 & 8.3 & & $\mu \mathrm{g} / \mathrm{L}$ & WA \\
\hline$<5.0$ & $<5.0$ & $<5.0$ & $<5.0$ & & $\mu_{\boldsymbol{g}} / \mathrm{L}$ & WA \\
\hline $\begin{array}{l}17 \\
98\end{array}$ & $\begin{array}{l}15 \\
65\end{array}$ & $\begin{array}{l}16 \\
80\end{array}$ & $\begin{array}{l}12 \\
79\end{array}$ & & $\mu \mathrm{g} / \mathrm{L}$ & $\begin{array}{l}\text { WA } \\
\text { WA }\end{array}$ \\
\hline $7.1 E+\infty 0$ & $6.6 E+00$ & $1.0 E+01$ & $7.7 E+00$ & & $\mathrm{pCi} / \mathrm{mL}$ & $\mathrm{CN}$ \\
\hline & & & $<0.88$ & & $\mu \mu^{\mu / L} / \mathrm{L}$ & WA \\
\hline
\end{tabular}

Note: Flagging levels, modifiers, and laboratory are for 4th quarter 1992 data only. See Appendix B for flagging criteria. - = exceeded holding time for 4th quarter 1992.

- =xceeded final primary drinking water standard for 4th quarter 1992. 
WELL LFW 59B

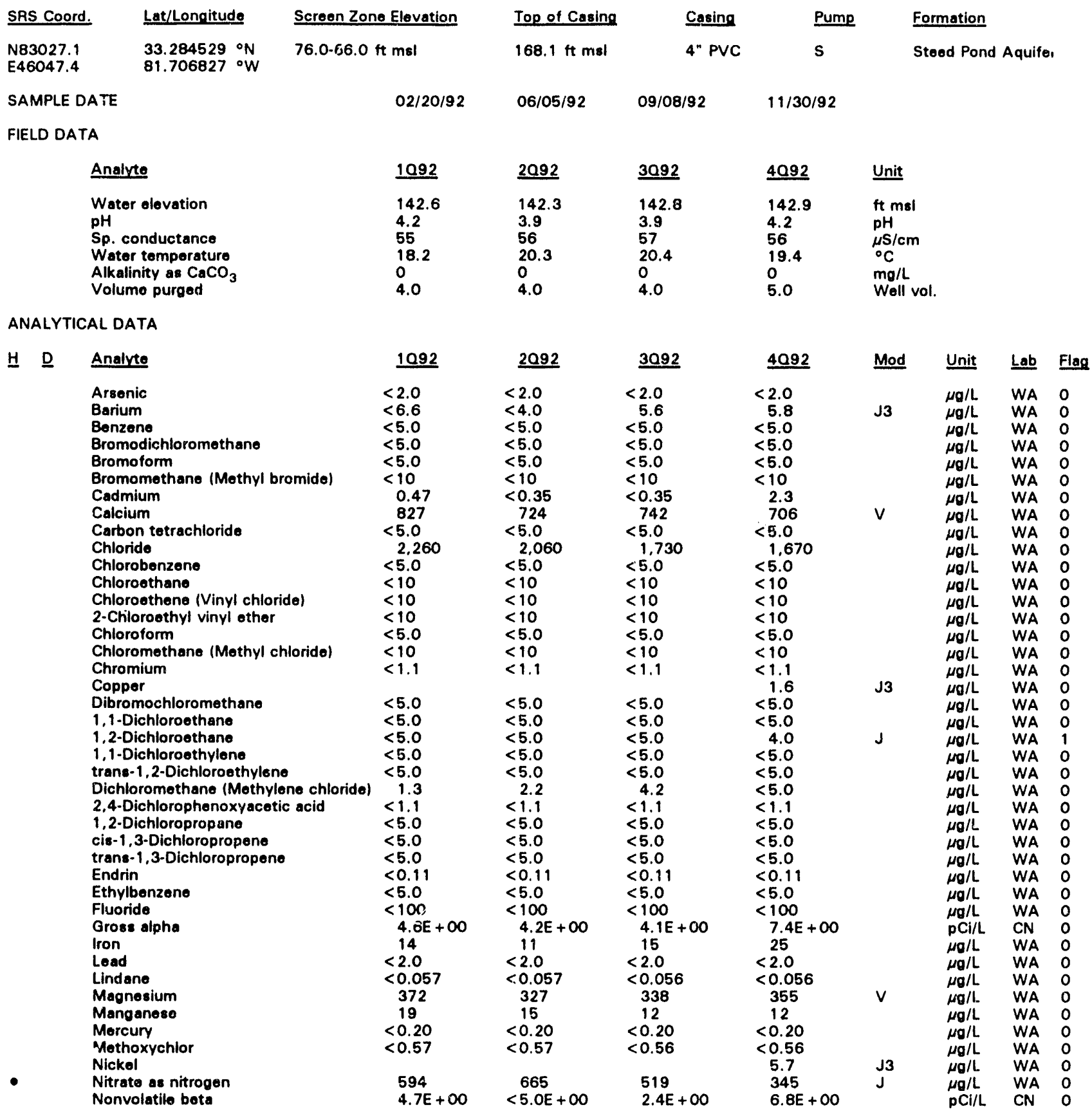

Note: Flagging levels, modifiers, and laboratory are for 4 th quarter 1992 data only. See Appendix B for flagging criteria. - = exceeded holding time for 4th quarter 1992.

- =xceeded final primary drinking water standard for 4th quarter 1992. 
Well LFW 598 continued

ANALYTICAL DATA

H D Analvte
pH
Phenols
Potassium
Radium-226
Radium-228
Solenium
Silica
Silicon
Silver
Sodium
Specific conductance
Sulfate
1,1,2,2-7 otrachloroethane
Totrachloroethylene
Toluene
Total dissolved solids
Total organic carbon
Total organic halogens
Total phosphates (as P)
Toxaphene
2,4,5-TP (Silvex)
1,1,1-Trichloroothane
1,1,2-Trichloroethane
Trichloroethylene
Trichlorofluoromethane
Tritium
Uranium
Vanadium

1092
4.5
$<5.0$
329
$2.7 \mathrm{E}+00$
$1.3 \mathrm{E}+00$
$<2.0$

4,900
0.72
1.400
42
8,720
$<5.0$
$<5.0$
$<5.0$
31.000
1,230
20
29
$<1.1$
$<0.56$
$<5.0$
$<5.0$
$<5.0$
$<5.0$
$3.5 \mathrm{E}-01$
0.060

$\begin{aligned} & 2092 \\ & 4.2 \\ &<5.0 \\ & 238 \\ & 2.2 \mathrm{E}+00 \\ &<2.0 \\ &<.800 \\ &<0.70 \\ & 1.410 \\ & 1.270 \\ & 8.280 \\ &<5.0 \\ &<5.0 \\ &<5.0 \\ & 21.000 \\ & 617 \\ &<20 \\ &<20 \\ &<1.1 \\ &<0.54 \\ &<5.0 \\ &<5.0 \\ &<5.0 \\ &<5.0 \\ &<2.0 \mathrm{E}+\infty 0 \\ &\end{aligned}$

\begin{tabular}{l}
3092 \\
\hline 5.6 \\
$<5.0$ \\
244 \\
$2.0 \mathrm{E}+00$ \\
$1.6 \mathrm{E}+00$ \\
$<2.0$ \\
9.720 \\
$<0.70$ \\
1.380 \\
45 \\
8.900 \\
$<5.0$ \\
$<5.0$ \\
$<5.0$ \\
35.000 \\
638 \\
$<5.0$ \\
$<1.1$ \\
$<0.57$ \\
$<5.0$ \\
$<5.0$ \\
$<5.0$ \\
$<5.0$ \\
$<7.0 \mathrm{E}-01$ \\
\end{tabular}

\begin{tabular}{|c|c|c|c|c|}
\hline 4092 & Mod & Unit & Lab & Flag \\
\hline $\begin{aligned} & 4.2 \\
&<5.0 \\
& 318 \\
& 1.5 E+00\end{aligned}$ & $\begin{array}{l}\mathrm{J} 3 \\
\mathrm{~J}\end{array}$ & $\begin{array}{l}\mathrm{pH} \\
\mu \mathrm{g} / \mathrm{L} \\
\mu \mathrm{g} / \mathrm{L} \\
\mathrm{pCl} / \mathrm{L} \\
\mathrm{pCi} / \mathrm{L}\end{array}$ & $\begin{array}{l}\text { WA } \\
\text { WA } \\
\text { WA } \\
\text { CN }\end{array}$ & $\begin{array}{l}0 \\
0 \\
0 \\
0\end{array}$ \\
\hline $\begin{array}{l}<2.0 \\
10,300\end{array}$ & $V$ & $\begin{array}{l}\mu_{v} / L \\
\mu_{0} / L \\
\mu_{g} / L\end{array}$ & $\begin{array}{l}\text { WA } \\
\text { WA }\end{array}$ & $\begin{array}{l}0 \\
0\end{array}$ \\
\hline $\begin{array}{l}<0.70 \\
1,500 \\
32 \\
8,520 \\
<5.0 \\
<5.0 \\
<5.0 \\
23,000 \\
582 \\
<5.0\end{array}$ & V & $\begin{array}{l}\mu g / L \\
\mu g / L \\
\mu S / c m \\
\mu g / L \\
\mu g / L \\
\mu g / L \\
\mu g / L \\
\mu g / L \\
\mu g / L \\
\mu g / L \\
\mu g / L\end{array}$ & $\begin{array}{l}\text { WA } \\
\text { WA } \\
\text { WA } \\
\text { WA } \\
\text { WA } \\
\text { WA } \\
\text { WA } \\
\text { WA } \\
\text { WA } \\
\text { WA }\end{array}$ & $\begin{array}{l}0 \\
0 \\
0 \\
0 \\
0 \\
0 \\
0 \\
0 \\
0 \\
0\end{array}$ \\
\hline $\begin{array}{l}<1.1 \\
<0.57 \\
<5.0 \\
<5.0 \\
<5.0 \\
<5.0 \\
<7.0 \mathrm{E}-01\end{array}$ & & $\begin{array}{l}\mu \mathrm{g} / \mathrm{L} \\
\mu \mathrm{g} / \mathrm{L} \\
\mu \mathrm{g} / \mathrm{L} \\
\mu \mathrm{g} / \mathrm{L} \\
\mu \mathrm{g} / \mathrm{L} \\
\mu \mathrm{g} / \mathrm{L} \\
\mathrm{pCi} / \mathrm{mL} \\
\mu \mathrm{g} / \mathrm{L}\end{array}$ & $\begin{array}{l}\text { WA } \\
\text { WA } \\
\text { WA } \\
\text { WA } \\
\text { WA } \\
\text { WA } \\
\text { CN }\end{array}$ & $\begin{array}{l}0 \\
0 \\
0 \\
0 \\
0 \\
0 \\
0\end{array}$ \\
\hline$<0.88$ & & $\mu \boldsymbol{g} / \mathrm{L}$ & WA & 0 \\
\hline
\end{tabular}

\section{WELL LFW 59C}

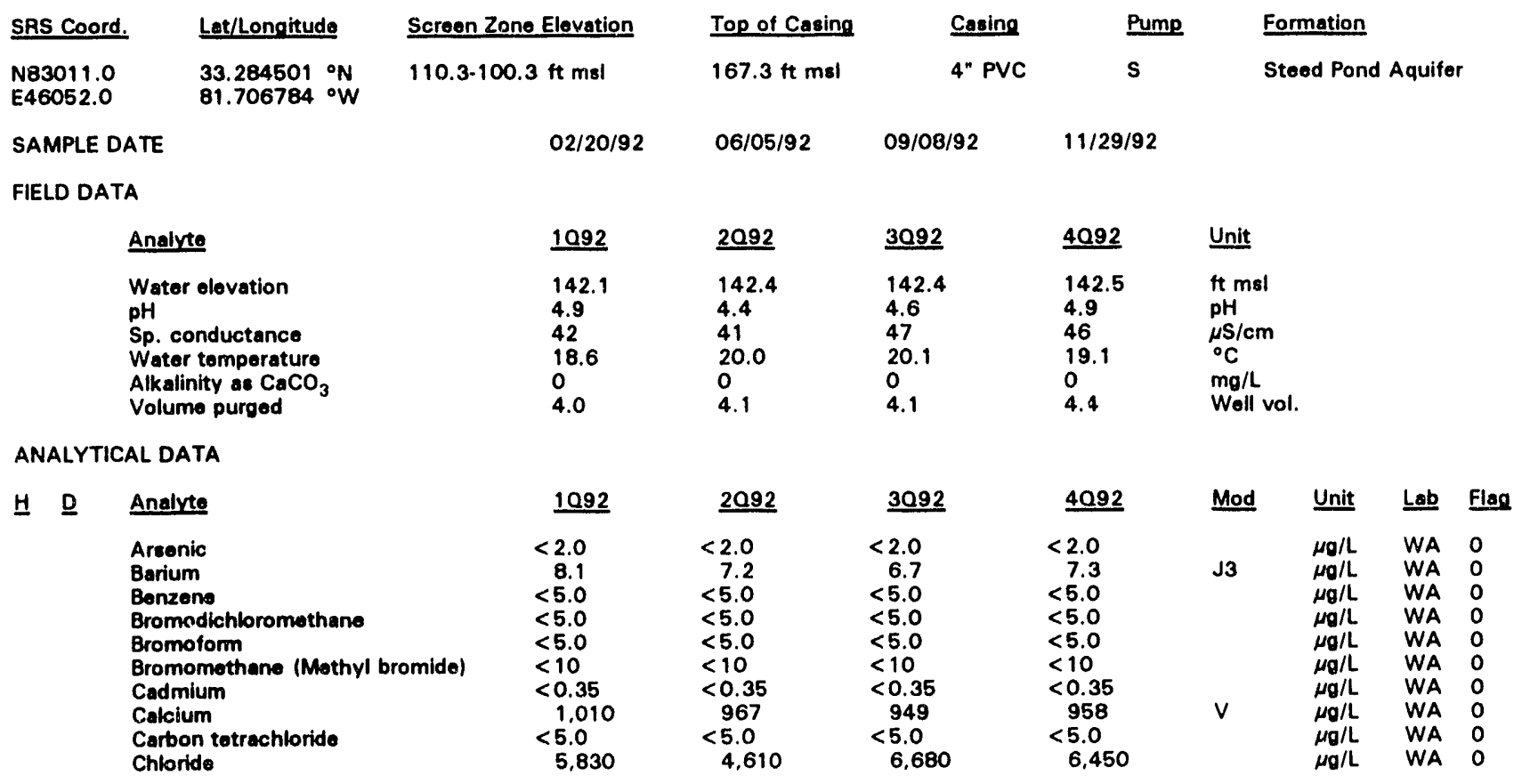

Note: Flagging levels, modifiers, and laboratory are for 4th quarter 1992 data only. Seo Appendix B for flagging criteria.

- =xceeded holding time for 4th quarter 1992.

w = exceeded final primary drinking water standard for 4th quarter 1992. 
Well LFW 59 C continued

ANALYTICAL DATA

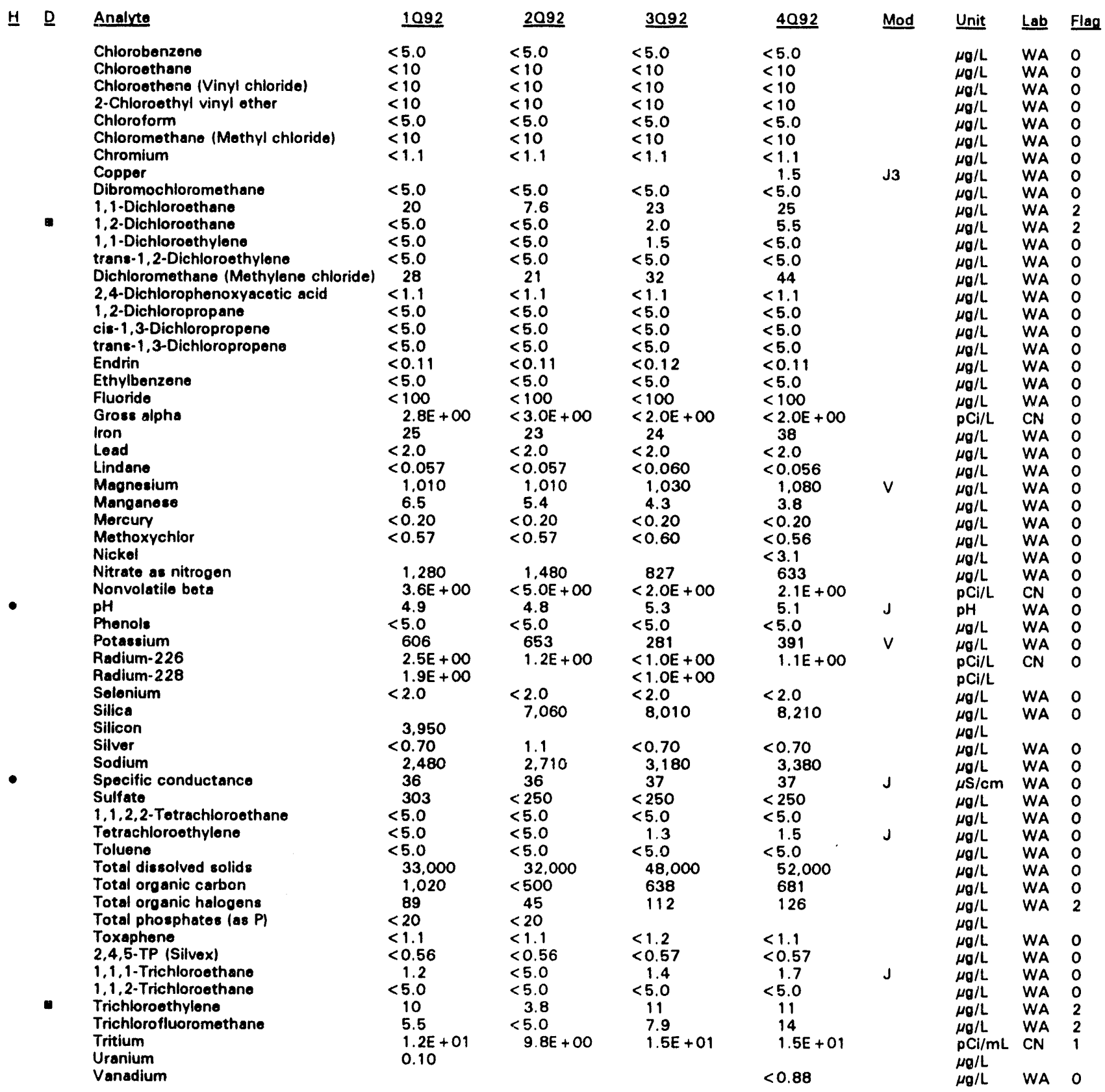

Note: Flagging levels, modifiers, and laboratory are for 4th quarter 1992 data only. See Appendix B for flagging criteria. - = exceeded holding time for 4th quarter 1992.

- = exceeded final primary drinking water standard for 4th quarter 1992. 
WSRC-TR-93-068

\section{WELL LFW 59D}

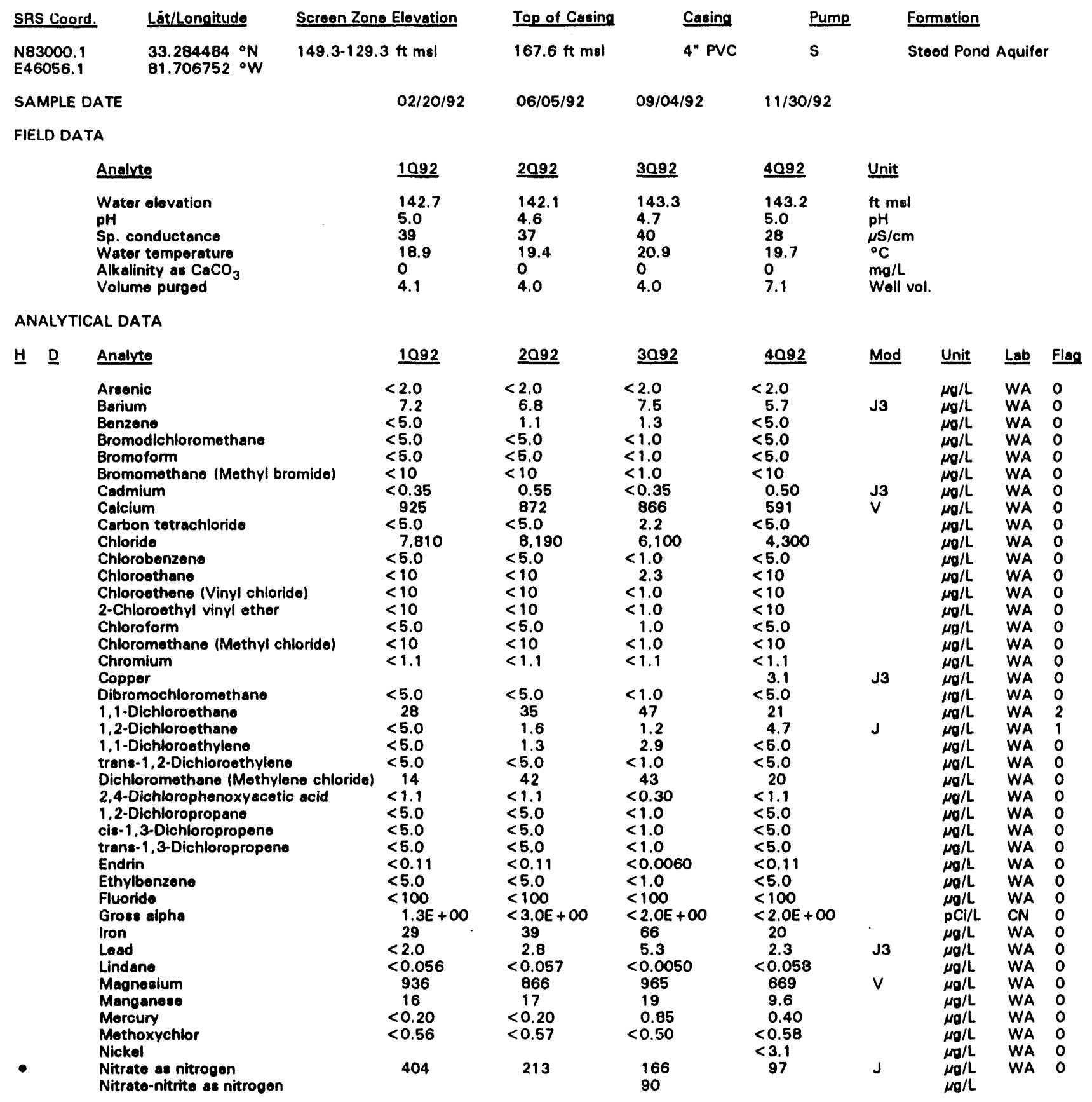

Note: Flagging levels, modifiers, and laboratory are for 4th quarter 1992 data only. See Appendix $B$ for flagging criteria.

- = exceeded holding time for 4th quarter 1992.

- exceeded final primary drinking water standard for 4th quarter 1992. 
WSRC-TR-93-068

Well LFW 590 continued

ANALYTICAL DATA

H D $\quad$ Analyte
Nonvolatile beta
pH
Phenols
Potassium
Radium-226
Radium-228
Solenium
Silica
Silicon
Silver
Sodium
Specific conductance
Sulfate
1.1,2,2-Totrachloroethane
Totrachloroethylene
Toluene
Total dissolved solids
Total organic carbon
Total organic halogens
Total phosphates (as P)
Toxaphene
$2,4,5-T P$ (Silvex)
1.1,1-Trichloroethane
1,1,2-Trichloroethane
Trichloroethylene
Trichlorofluoromothane
Tritium
Uranium
Vanadium

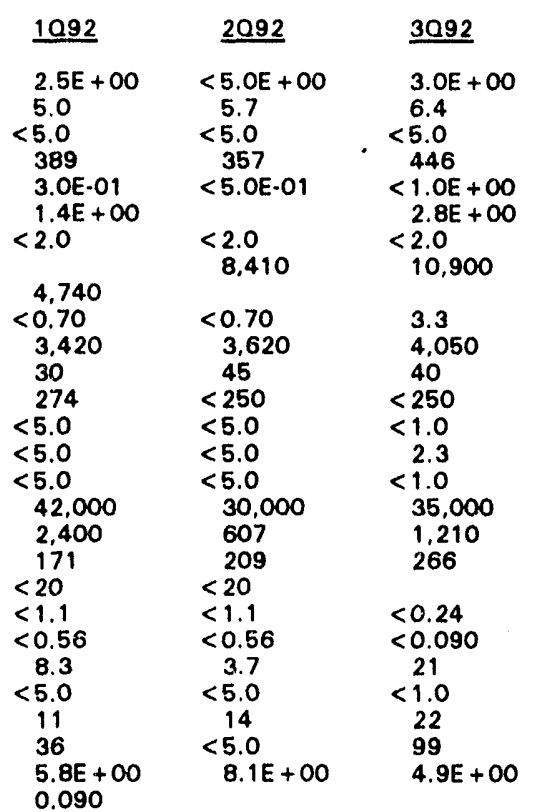

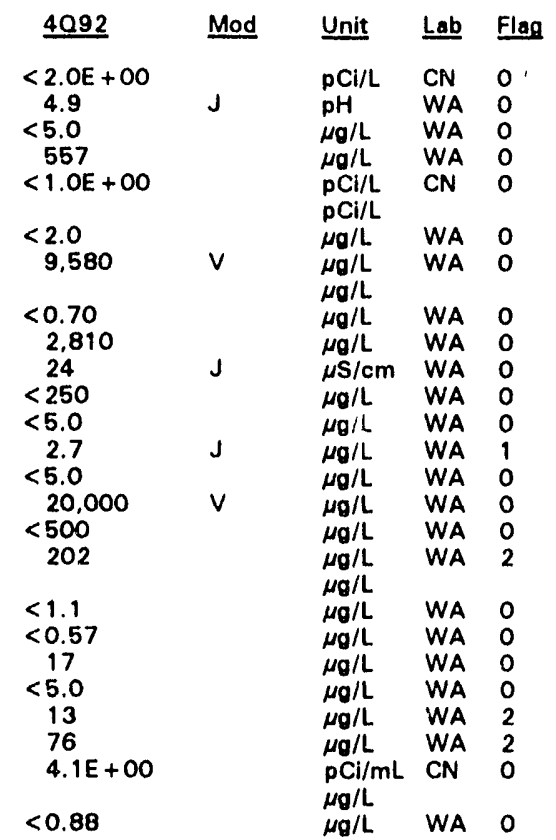

\section{WELL LFW 60D}

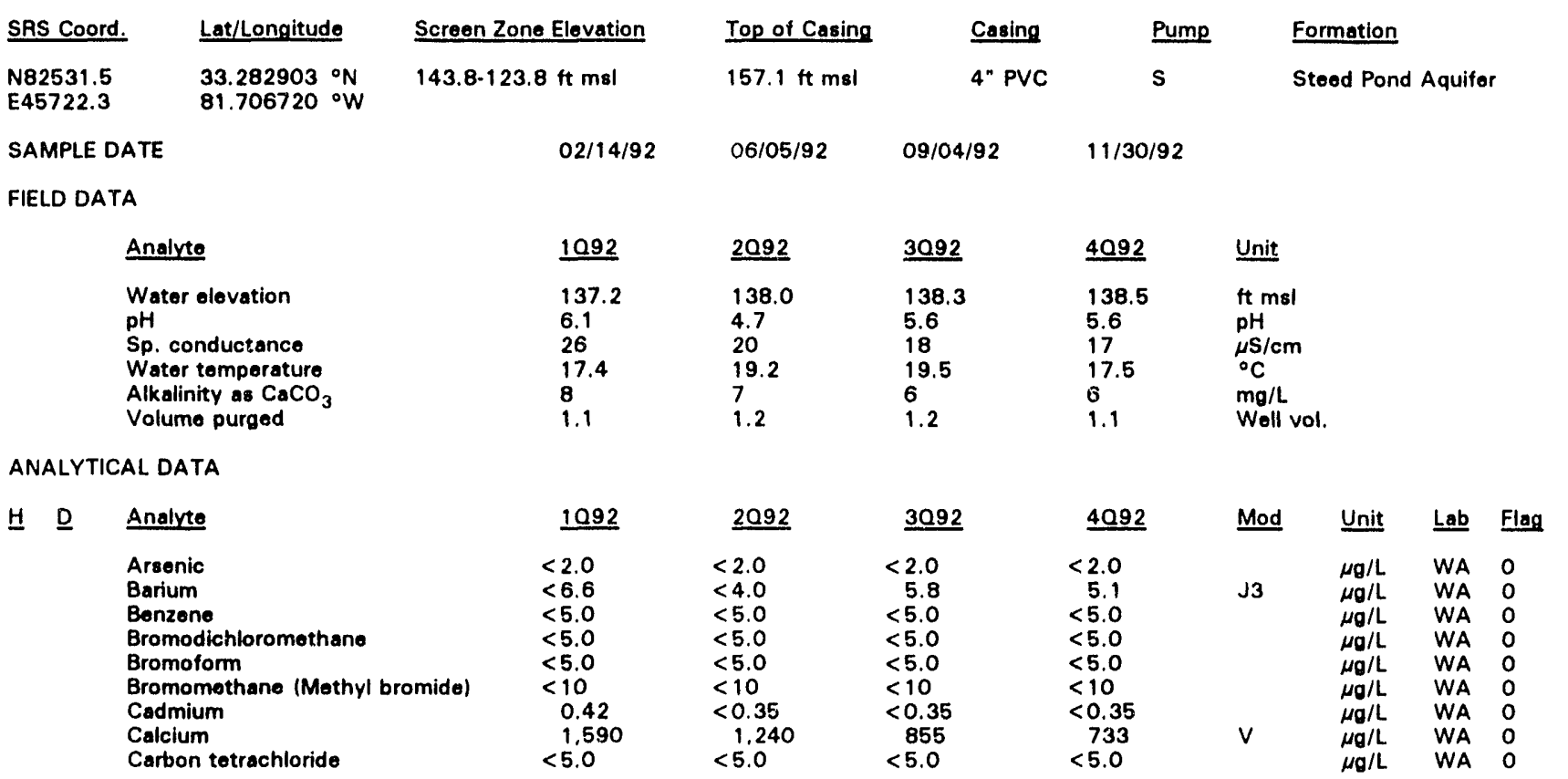

Note: Flagging levels, modifiers, and laboratory are for 4th quarter 1992 data only. See Appendix B for flagging criteria.

- =xceeded holding time for 4th quarter 1992.

- = exceeded final primary drinking water standard for 4th quarter 1992. 
WSRC-TR-93-068

Well LFW 600 continued

\section{ANALYTICAL DATA}

H. D Analyte

Chloride

Chlorobenzene

Chloroethane

Chloroethene (Vinyl chloride)

2-Chloroethyl vinyl other

Chloroform

Chloromethane (Methyl chloride)

Chromium

Copper

Dibromochloromethane

1.1-Dichloroethane

1,2-Dichloroethane

1,1-Dichloroothylene

trans-1,2-Dichloroethylene

Dichloromethane (Methylene chloride)

2,4-Dichlorophenoxyacetic acid

1,2-Dichloropropano

cis-1,3-Dichloropropene

trans-1,3-Dichloropropene

Endrin

Ethylbenzene

Fluoride

Grose alpha

Iron

Lead

Lindane

Magnesium

Manganese

Morcury

Mothoxychlor

Nickel

- Nitrate as nitrogen

Nonvolatile bota

pH

Phenols

Potassium

Radium-226

Radium-228

Solenium

Silica

Silicon

Silver

Sodium

Specific conductance

Sulfate

1,1,2,2-Totrachloroethane

Totrachloroethylene

Toluene

Total dissolved solids

Total organic carbon

Total organic halogene

Total phosphates (as P)

Toxaphene

2,4,5-TP (Silvex)

1,1,1-Trichloroethane

1,1,2-Trichloroethane

Trichloroethylene

Trichlorofluoromethane

Tritium

Vanadium

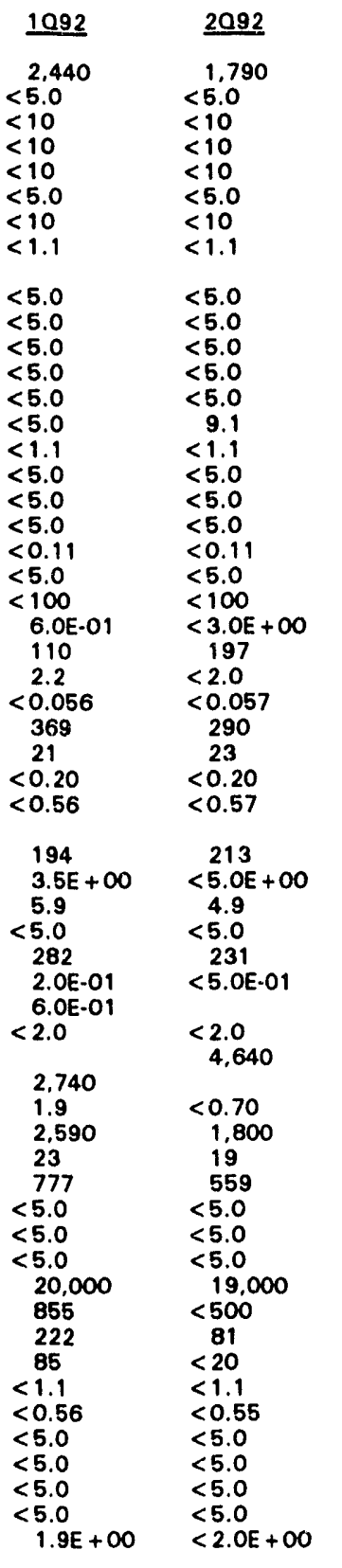

$\begin{aligned} & 3092 \\ & 1.490 \\ &<5.0<10 \\ &< 10 \\ &<10 \\ &<5.0 \\ &<10 \\ & 1.3 \\ &<5.0 \\ &<5.0 \\ &<5.0 \\ &<5.0 \\ &<5.0 \\ & 5.6 \\ &<1.1 \\ &<5.0 \\ &<5.0 \\ &<5.0 \\ &<0.11 \\ &<5.0 \\ &<100 \\ &<2.0 \mathrm{E}+00 \\ & 41 \\ & 2.3 \\ &<0.056 \\ & 215 \\ & 16 \\ &<0.20 \\ &<0.56\end{aligned}$

4092 Mod

1,380

$<5.0$

$<10$

$<10$
$<10$

$<5.0$

$<10$

$\begin{array}{lll}\mu \mathrm{g} / \mathrm{L} & \text { WA } & 0 \\ \mu \mathrm{g} / \mathrm{L} & \text { WA } & 0 \\ \mu \mathrm{g} / \mathrm{L} & \text { WA } & 0\end{array}$

$4.4 \quad \mathrm{~J} 3$

$<5.0$

$<5.0$

$<5.0$

$<5.0$

$<5.0$

$<5.0$

$<1.1$

$<5.0$

$<5.0$

$<0.11$

$<5.0$

$<100$

$<2.0 E+00$

621
5.7

$<0.056$

259
17

17
$<0.20$

$<0.56$

4.2

327

$<2.0 \mathrm{E}+00$

6.9

$<5.0$

239

$<1.0 E+00$

$<1$. OE + 00

$<2.0$

4.920

$<2.0 E+00$

$<2.0 \mathrm{E}+\mathrm{O}$
5.7

5.7
$<5.0$

126

$<1.0 E+00$

$<2.0$

$<0.70$

1,720

16

641

$<5.0$

$<5.0$

$<5.0$

$<1,000$

$\begin{aligned} & 573 \\ < & 5.0\end{aligned}$

5.440

$<0.70$

1.340

13
796

796
$<5.0$

$<5.0$

$<5.0$

25,000
3,250

9.4

$<1.1$
$<0.55$

$<0.55$
$<5.0$

$<5.0$

$<5.0$

$<5.0$

7.9E-01

$<1.1$

$<0.57$

$<5.0$

$<5.0$

$<5.0$

$8.9 E \cdot 01$
$<0.88$

$\mu \mathrm{g} / \mathrm{L}$ WA 0

$\mu \sigma / L$ WA 0

$\mu g / L$ WA 0

$\mu g / L$ WA 0

$\mu g / L$ WA

J3 $\mu g / L$ WA 0

$\mu g / L$ WA 0

$\mu \mathrm{g} / \mathrm{L}$ WA 0

$\mu g / L$ WA 0

$\mu g / L$ WA 0

$\mu \mathrm{g} / \mathrm{L}$ WA 0

$\mu g / L$ WA

$\mu \sigma / L$ WA 0

$\mu \sigma / L$ WA 0

$\mu g / L$ WA 0

$\mu g / L$ WA 0

CIIL CN O

$\mathrm{PCI} / \mathrm{LN} 0$

$\mu \mathrm{g} / \mathrm{L}$ WA 2

$\mu g / L$ WA 0

$\mu \mathrm{g} / \mathrm{L}$ WA 0

$\mu g / L$ WA 0

$\mu \mathrm{G} / \mathrm{L}$ WA 0

$\mu g / L$ WA 0

$\mu \mathrm{g} / \mathrm{L}$ WA 0

$\mathrm{pCi} / \mathrm{L}$ CN 0

$\begin{array}{llll}J & \mathrm{pH} & \text { WA } & 0 \\ & \mu \mathrm{g} / \mathrm{L} & \text { WA } & 0\end{array}$

J3 $\mu \mathrm{g} / \mathrm{L}$ WA 0

$\mathrm{pCi} / \mathrm{L}$ CN

$\mathrm{pCi} / \mathrm{L}$

$\begin{array}{lll}\mu \mathrm{g} / \mathrm{L} & \text { WA } & 0 \\ \mu \mathrm{g} / \mathrm{L} & \text { WA } & 0\end{array}$

$\mathrm{Hg} / \mathrm{L}$ WA 0

$\mu g / L$ WA 0

$\begin{array}{lll}\mu / \mathrm{s} / \mathrm{cm} & \text { WA } & 0 \\ \mathrm{~g} & \text { WA } & 0\end{array}$

$\mu g / L$ WA 0

$\mu \mathrm{g} / \mathrm{L}$ WA 0

WIL WA 0

$\mu g / L$ WA 0

$\mu g / L$ WA 0

$\mu g / L$ WA 0

$\mu \mathrm{g} / \mathrm{L}$

$\mu / L$ WA 0

$\mu g / L$ WA 0

$\mu g / L$ WA 0

$\mu g / L$ WA 0

$\mu g / L$ WA 0

$\mu g / L$ WA 0

$\mathrm{pCi} / \mathrm{mL}$ CN 0

$\mu \mathrm{g} / \mathrm{L}$ WA 0

Note: Flagging levels, modifiers, and laboratory are for 4th quarter 1992 data only. See Appendix B for flagging criteria.

- = exceeded holding time for 4th quarter 1992.

- exceeded final primary drinking water standard for 4th quarter 1992. 
WELL LFW 61C

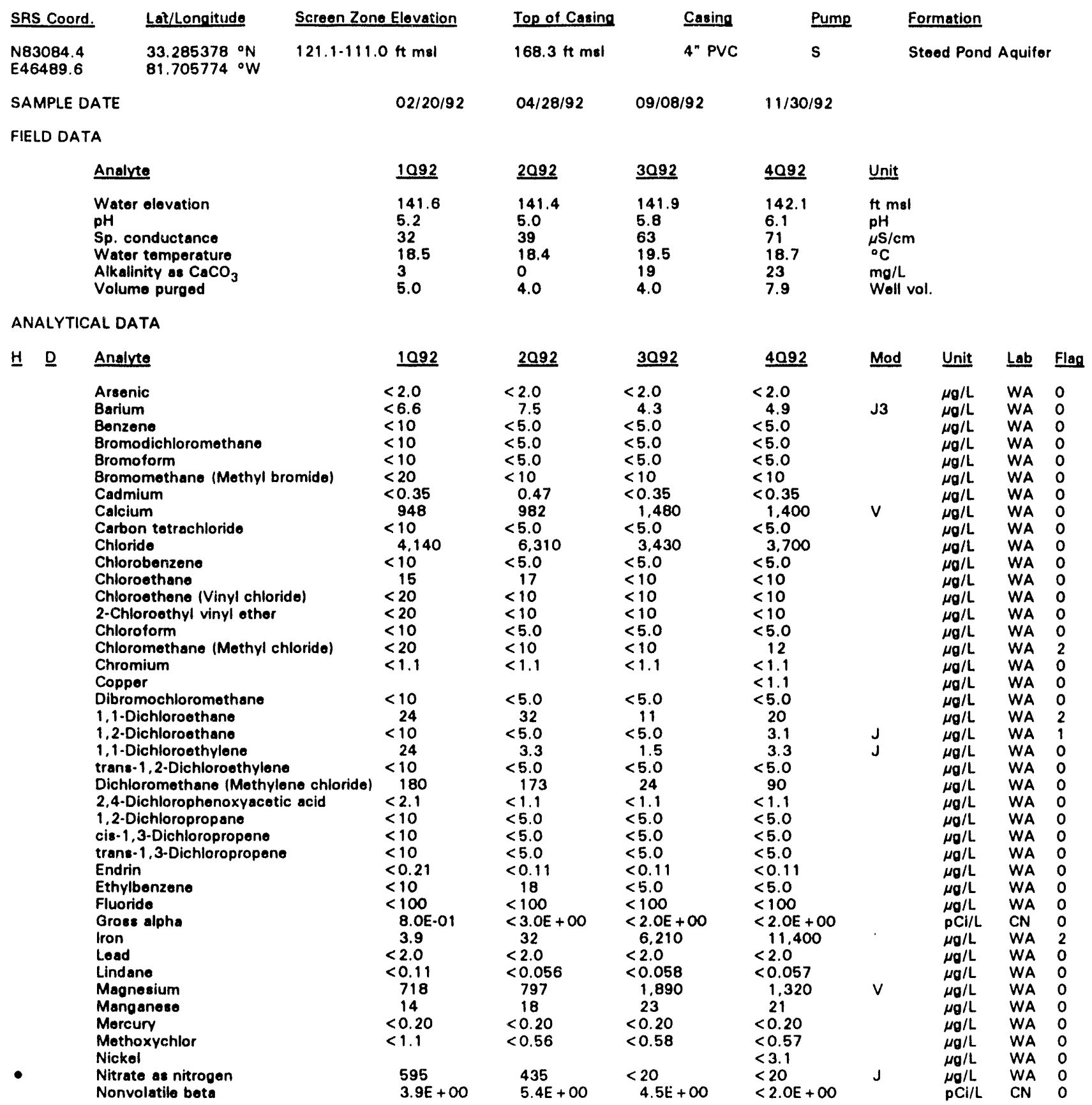

Note: Flagging levels, modifiers, and laboratory are for 4th quarter 1992 data only. See Appendix B for flagging criteria.

- = exceeded holding time for 4th quarter 1992.

- = exceeded final primary drinking water standard for 4th quarter 1992. 
Well LFW 61C continued

ANALYTICAL DATA

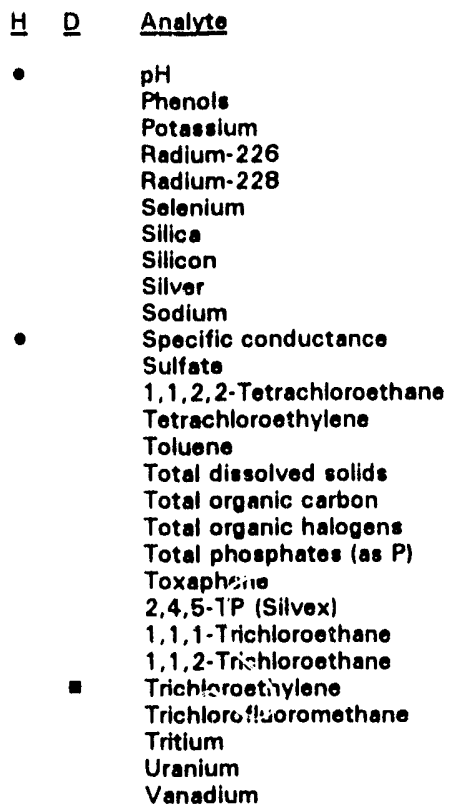

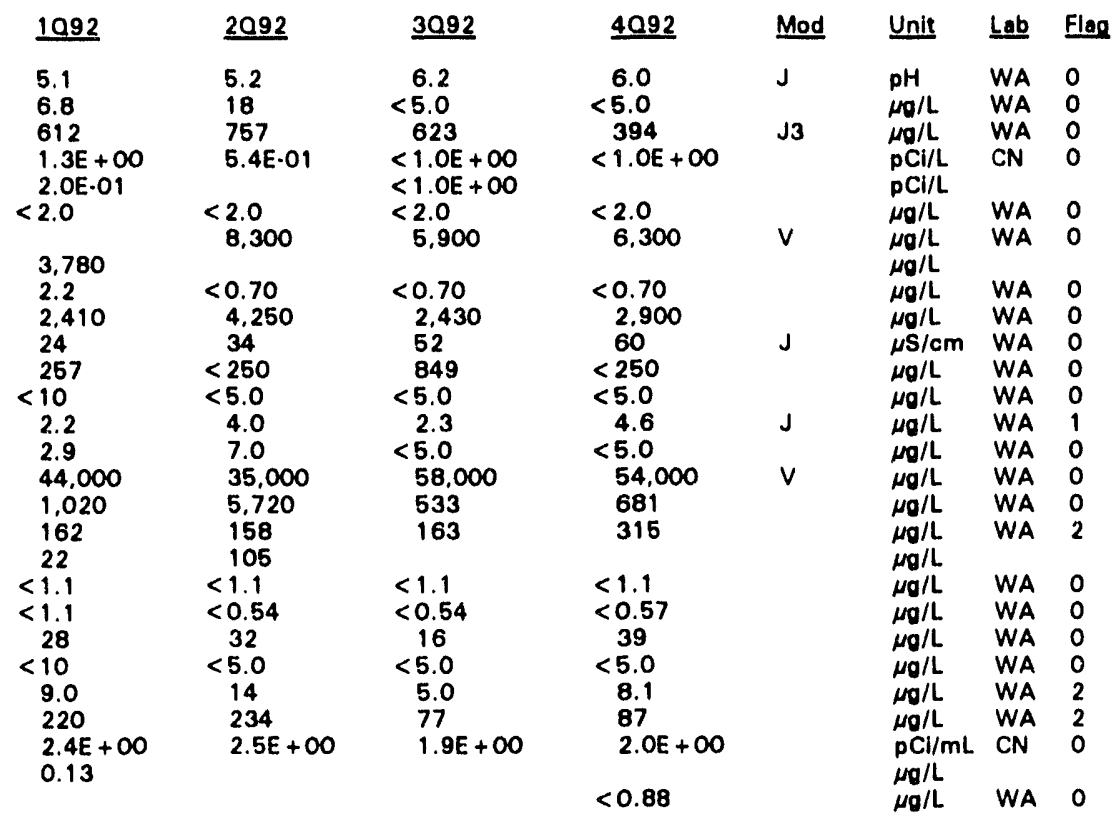

\section{WELL LFW 61D}

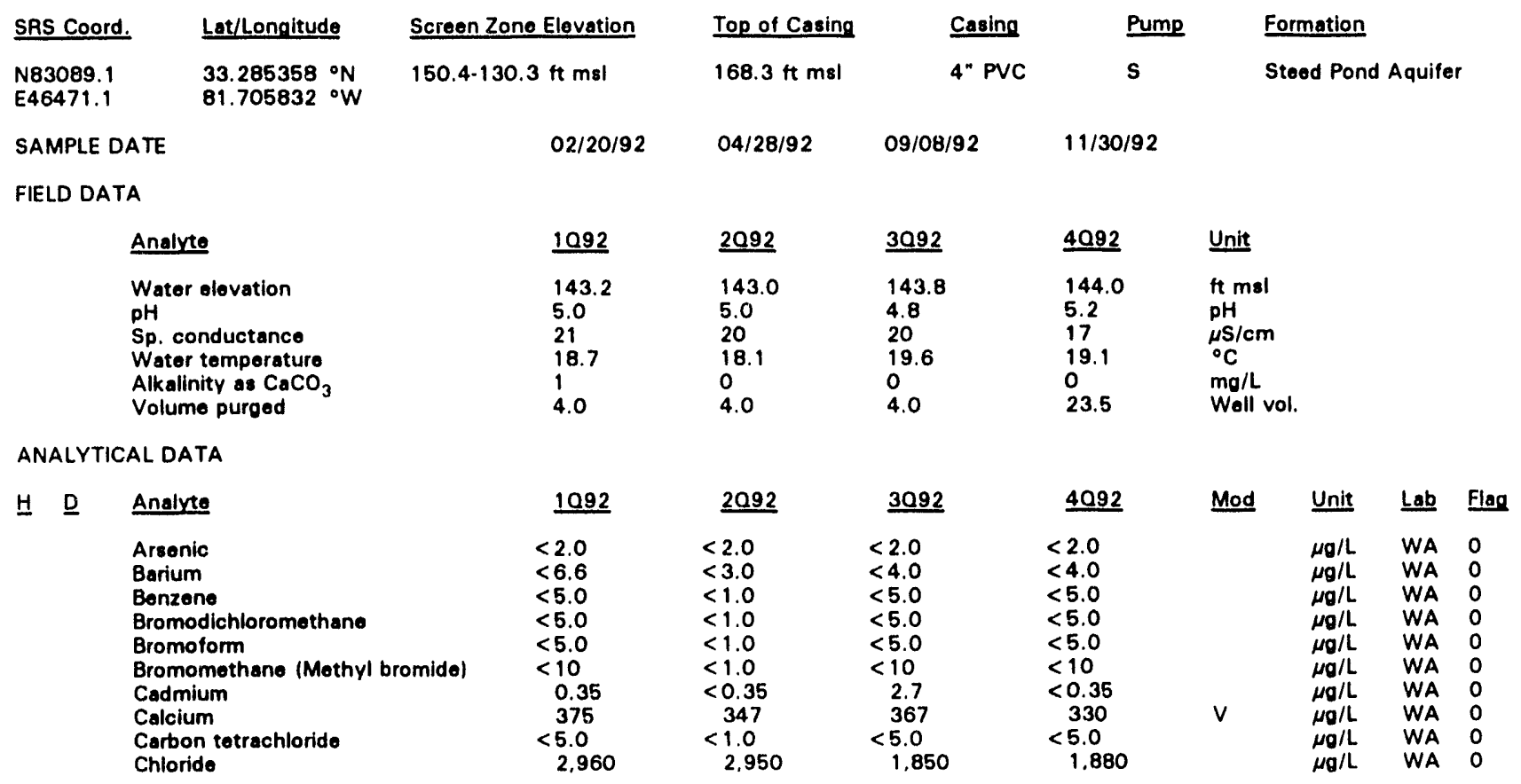

Note: Flagging lovels, modifiers, and laboratory are for 4 th quarter 1992 data only. See Appendix 8 for flagging criteria.

- =xceeded holding time for 4th quarter 1992.

- exceeded final primary drinking water standard for 4 th quarter 1992. 
Well LFW 610 continued

\section{ANALYTICAL DATA}

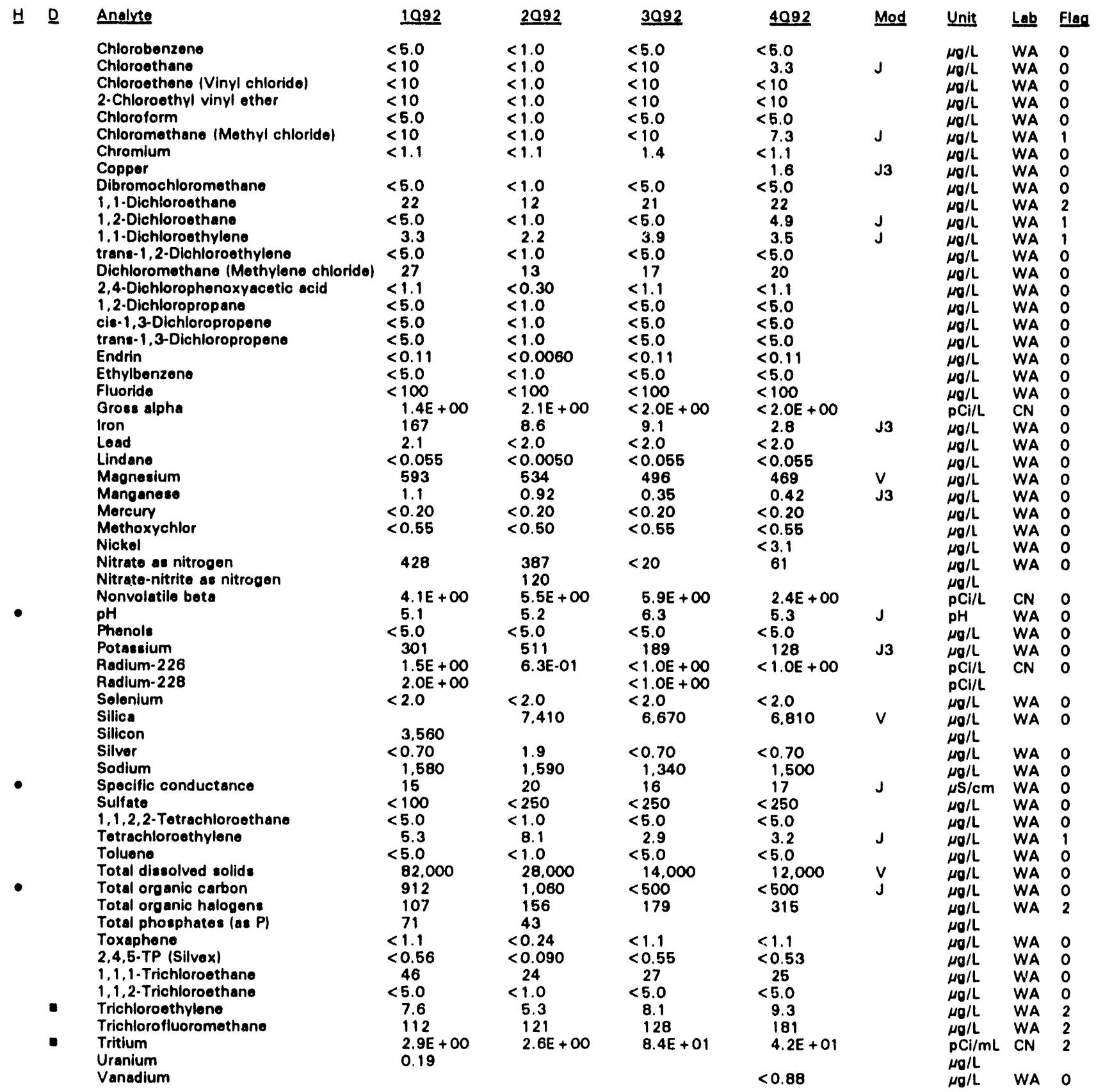

Note: Flagging levels, modifiers, and laboratory are for 4th quarte, 1992 data only. See Appendix B for flagging criteria. - = exceeded holding time for 4th quarter 1992.

- =xceeded final primary drinking water standard for 4th quarter 1992. 
WSRC-TR-93.068

WELL LFW 62B

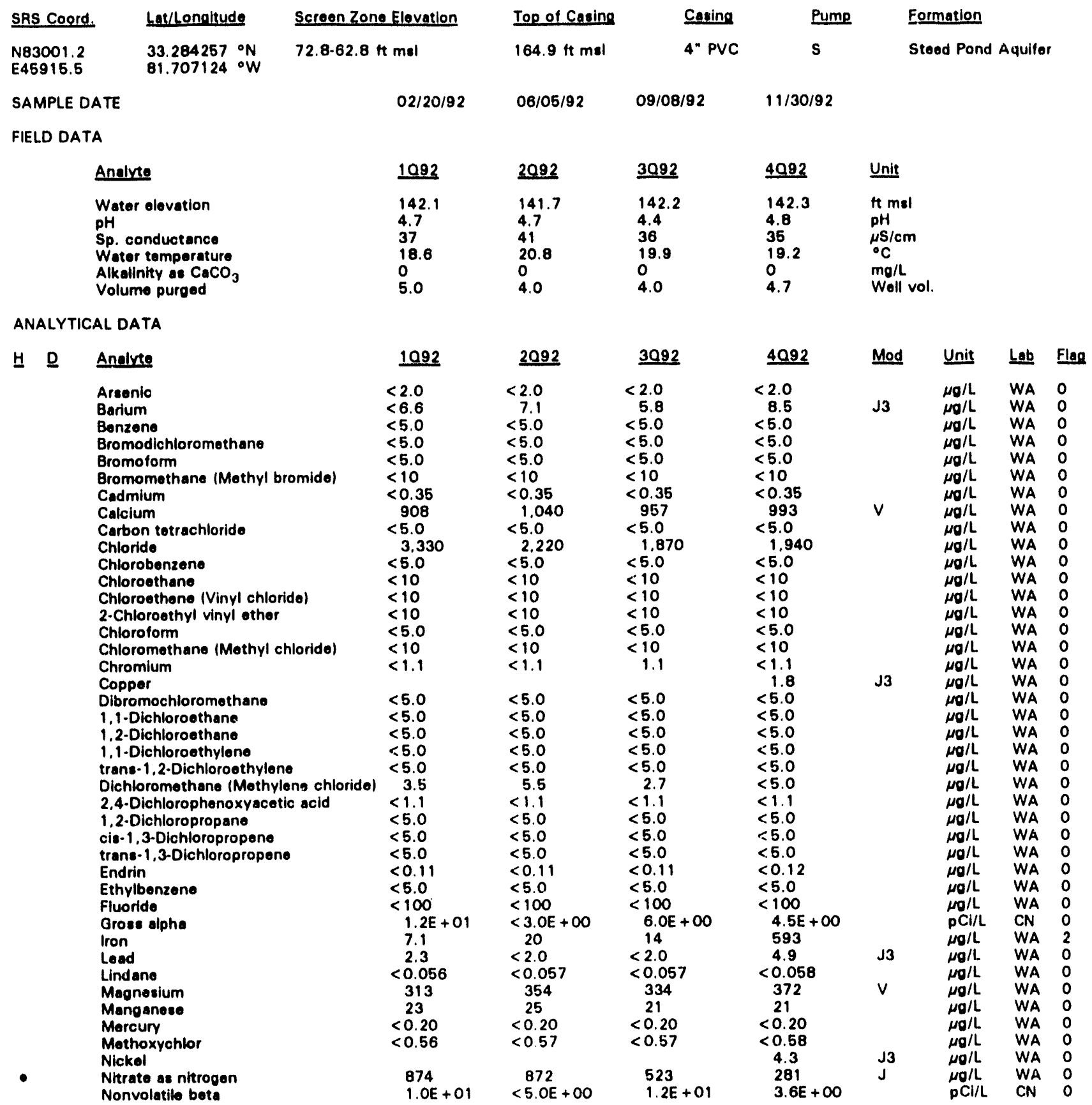

Note: Flagging levels, modifiers, and laboratory are for 4 th quarter 1992 data only. See Appendix B for flagging criteria.

- =xceeded holding time for 4th quarter 1992.

- exceeded final primary drinking water standard for 4th quarter 1992. 
Well LFW 628 continued

ANALYTICAL DATA

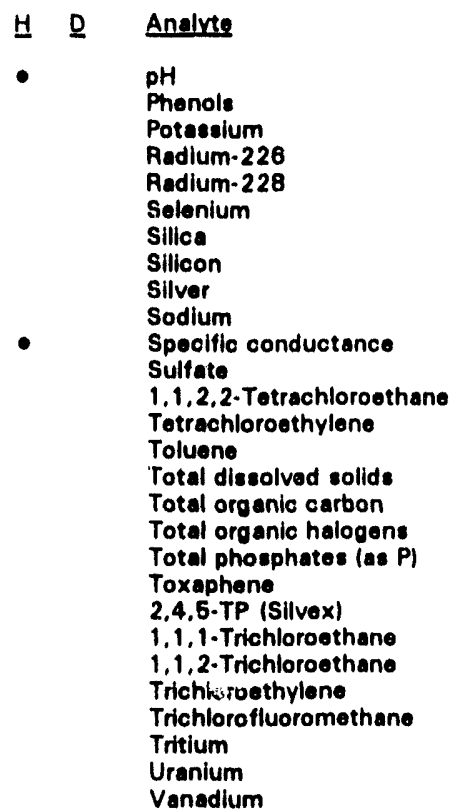

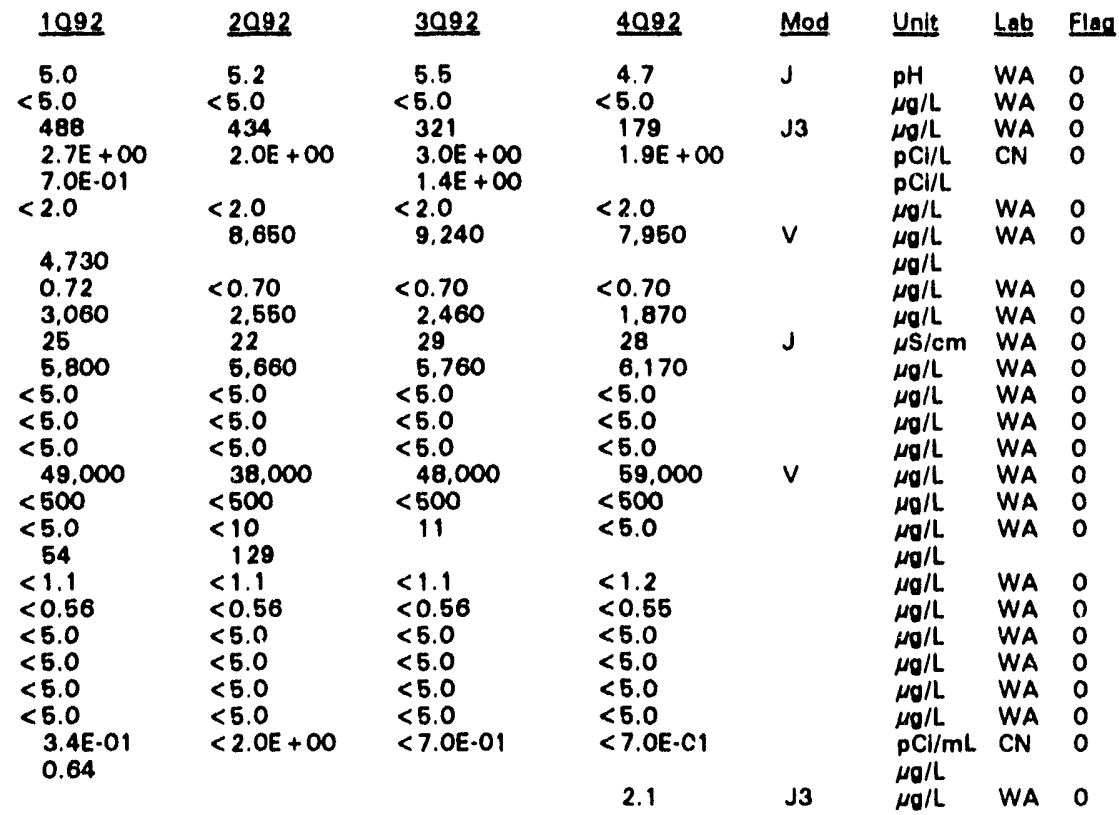

WELL LFW 62C

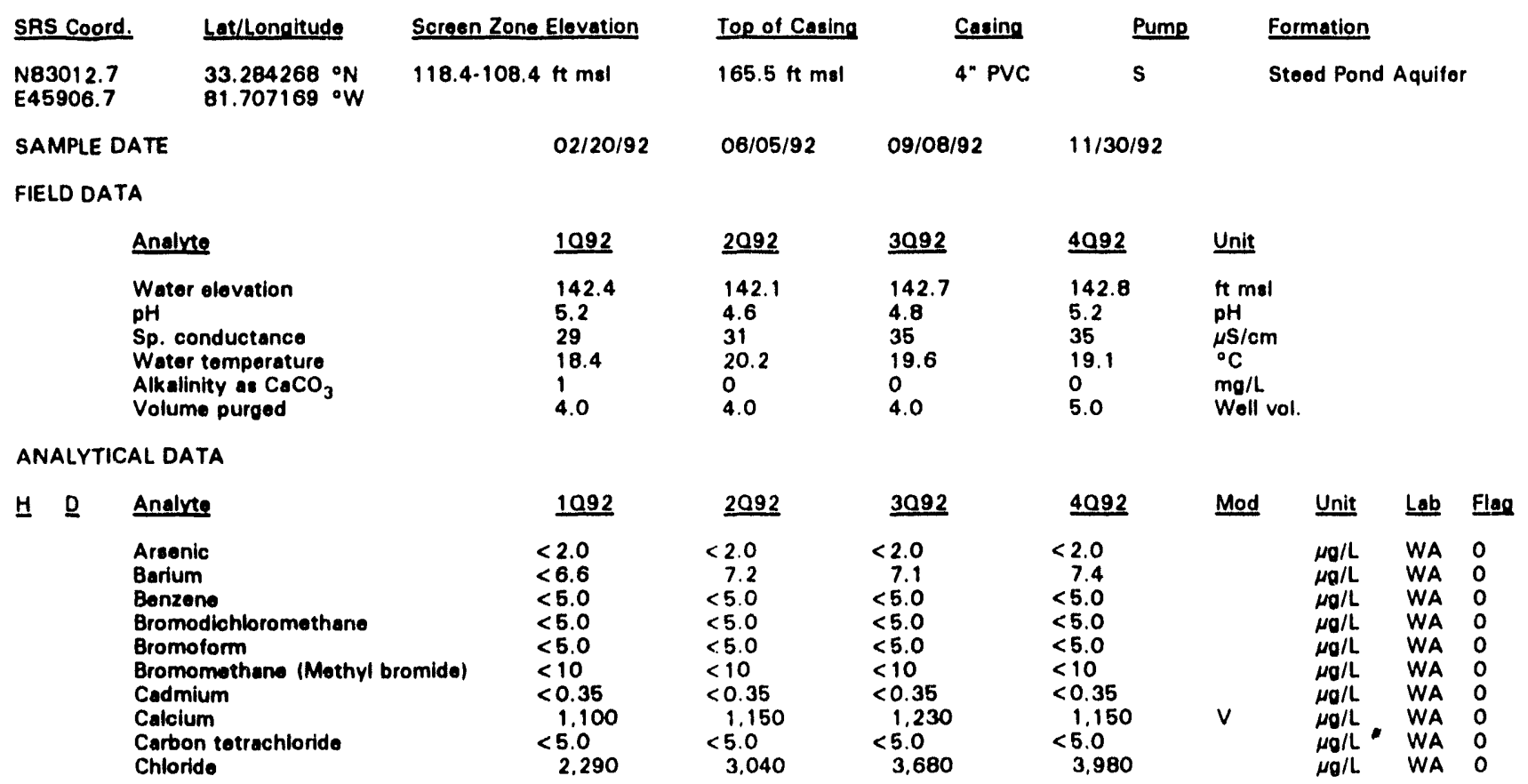

Note: Flagging levels, modifiers, and laboratory are for 4th quarter 1992 data only. See Appendix B for flagging criteria.

- = exceeded holding time for 4th quarter 1992.

- exceeded final primary drinking water standard for 4th quarter 1992. 
WSRC-TR-93-068

Woll LFW 62C continued

ANALYTICAL DATA

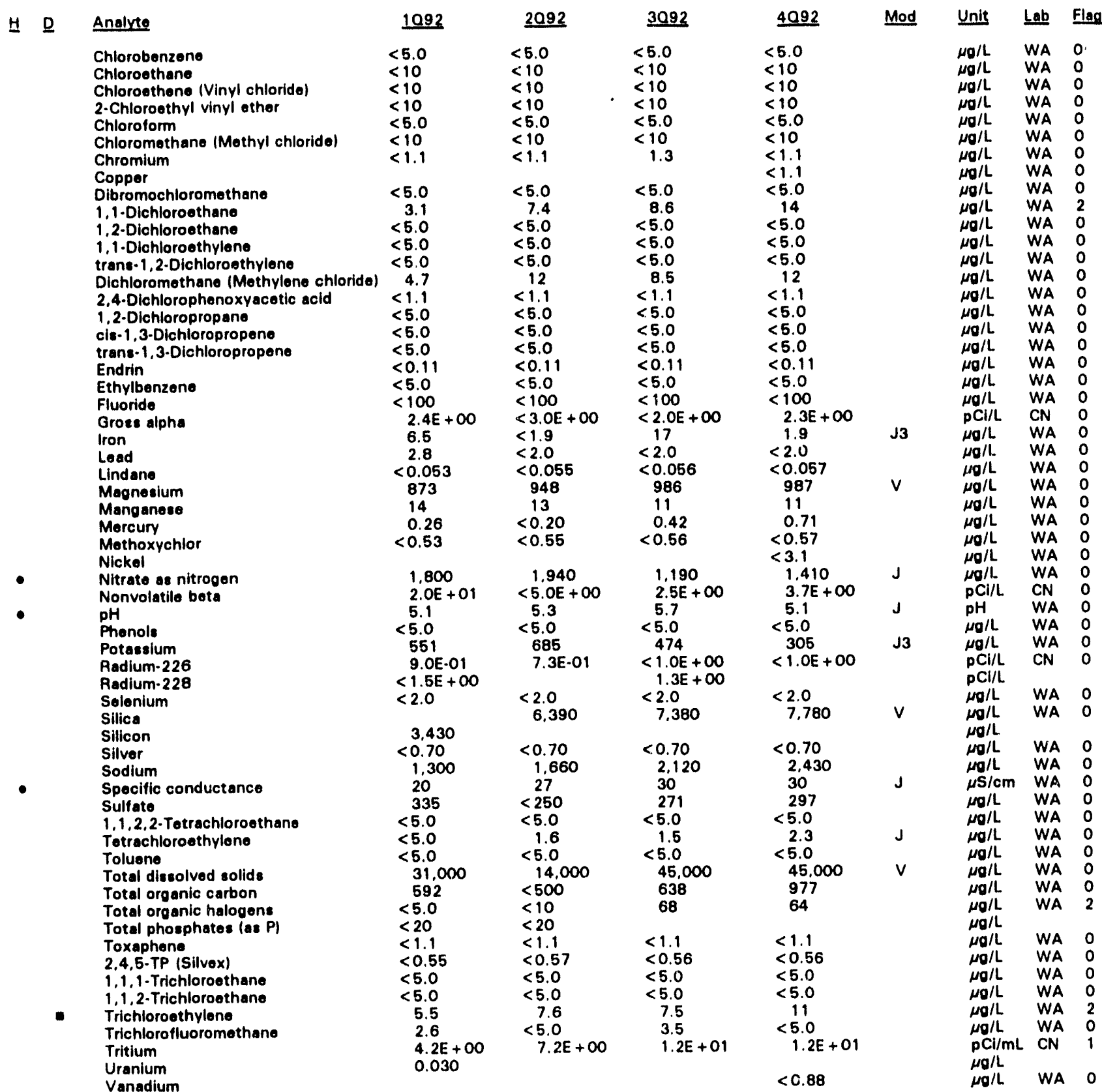

Note: Flagging levels, modifiers, and laboratory are for 4 th quarter 1992 data only. See Appendix B for flagging criteria. - = exceeded holding time for 4th quarter 1992.

- = exceeded final primary drinking water standard for 4th quarter 1992. 
WELL LFW 62D

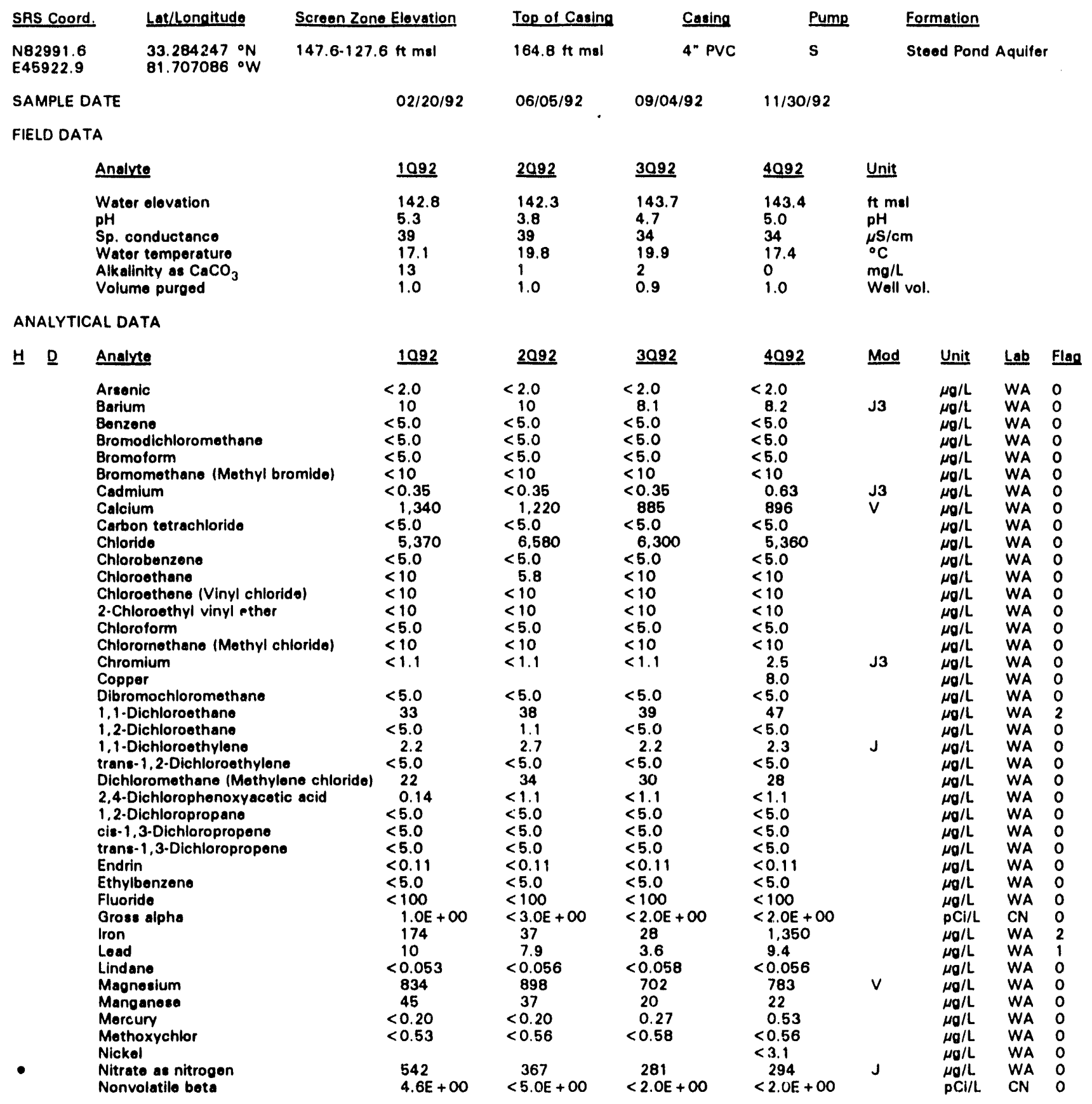

Note: Flagging levels, modifiers, and labnratory are for 4 th quarter 1992 data only. See Appendix B for flagging criteria.

- = exceeded holding time for 4th quarter 1992.

- =xceeded final primary drinking water standard for 4th quarter 1992. 
WSRC-TR-93-068

Well LFW 62D continued

ANALYTICAL DATA.

H D Analyte

- $\mathrm{pH}$

Phenols

Potassium

Radium-226

Radium-228

Selonium

Silica

Silicon

Silver

Sodium

- Specific conductance

Specifio

1,1,2,2-Totrachloroethane

- Totrachloroethylene

Toluene

Total dissolved solids

Total organic carbon

Total organic halogens

Total phosphates (as P)

Toxaphene

2,4,5-TP (Silvex)

1,1,1-Trichloroethane

1,1,2-Trichloroethane

- Trichloroethylene

Trichlorofluoromethane

Tritium

Uranium

Vanadium

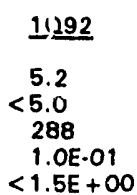

$\underline{2092}$

5.3
$<5.0$

603

$<1.5 E+00$

$<2.0$

4,800

1.0

3,030

32

684

$<5.0$
2.6

2.6
$<5.0$

34,000

1,230

177

50

$<1.1$

$<0.55$

31
$<5.0$

$<5.0$
10.0

95

$4.6 \mathrm{E}+00$

0.030

3092
6.3
$<5.0$
90
$<1.0 E+00$
$<1.0 \mathrm{E}+00$
$<2.0$
8.140
0.92
2.640
36
578
$<5.0$
2.7
$<5.0$
$<1.000$
897
299
$<1.1$
$<0.56$
24
$<5.0$
11
94
$4.3 E+00$

\begin{tabular}{ll} 
& Mog \\
\hline 5.1 & $\mathrm{~J}$ \\
$<5.0$ & $\mathrm{J3}$ \\
320 & \\
$<1.0 \mathrm{E}+00$ & \\
$<$ & \\
& 2.0 \\
8,460 & $\mathrm{~V}$ \\
$<0.70$ & \\
2.970 & \\
35 & $\mathrm{~J}$ \\
415 & \\
$<5.0$ & \\
7.6 & \\
$<5.0$ & \\
36.000 & $\mathrm{~V}$ \\
725 & \\
72 & \\
$<1.1$ & \\
$<0.53$ & \\
39 & \\
$<5.0$ & \\
14 & \\
117 & \\
$4.3 \mathrm{E}+00$ & \\
3.7 & $\mathrm{J3}$
\end{tabular}

Note: Flagging levels, modifiers, and laboratory are for 4th quarter 1992 data only. See Appendix B for flagging criteria.

- = exceeded holding time for 4th quarter 1992.

- =xceeded final primary drinking water standard for 4th quarter 1992. 
WSRC-TR-93-068

-

?

$\bullet$

b 


\section{Appendix E - Data Quality/Useability Assessment}




\section{Data Quality/Useability Assessment}

Quality assurance/quality control (QA/QC) procedures relating to accuracy and precision of analyses performed on groundwater samples are followed in the field and laboratory and are reviewed prior to publication of results. The Environmental Protection Department/ Environmental Monitoring Section's (EPD/EMS) review of the volume of analytical data acquired each quarter and presented in various reports is an ongoing process; its review of the QA/QC data cannot be completed in time to meet the deadlines for the reports required by the Resource Conservation and Recovery Act and associated regulations. Other site and regulatory personnel can obtain further information on the data quality and useability in a variety of ways.

\section{Data Qualification}

The contract laboratories continually assess their own accuracy and precision according to U.S. Environmental Protection Agency (EPA) guidelines. They submit sample- or batchspecific QA/QC information either at the same time as analytical results or in a quarterly summary. Properly defined and used result modifiers (also referred to as qualifiers) can be a key component in assessing data useability. Result modifiers designed by EPD/EMS and provided to the primary laboratories are presented in Appendix D.

\section{Assessment of Accuracy of the Data}

Accuracy, or the nearness of the reported result to the true concentration of a constituent in a sample, can be assessed in several ways.

A laboratory's general accuracy can be judged by analysis of results obtained from known samples. The non-radionuclide contract laboratories analyze commercial reference samples every quarter at EPD/EMS' request. The results of these analyses are presented in the EPD/EMS quarterly report, The Savannah River Site's Groundwater Monitoring Program. The primary laboratories also seek or maintain state certification by participating periodically in performance studies; reference samples and analysis of results are provided by EPA. Results of these studies also are published in the EPD/EMS quarterly reports.

Analysis of blanks provides a tool for assessing the accuracy of both sampling and laboratory analysis. Results for all field blanks for the quarter can be found in the EPD/EMS quarterly reports. Any field or laboratory blanks that exceeded established minimums are identified in the same reports, in tables associating them with groundwater samples analyzed in the same batches.

Surrogates, organic compounds similar in chemical behavior to the compounds of interest but not normally found in environmental samples, are used to monitor the effect of the matrix on the accuracy of analyses for organic parameters. For example, for analyses of volatile organics by EPA method 8240, three surrogate compounds are added to all samples and blanks in each analytical batch. In analyses of semivolatile organics, three to four acid compounds and three to four base/neutral compounds are used. Other surrogates are used 
in pesticides analyses. Percent recoveries for surrogate analyses are calculated by laboratory personnel, reported to EPD/EMS, reviewed, and entered into the database, but they are not published. If recoveries are not within specified limits, the laboratory is expected to re-run the samples or attach result qualifiers to the data identifying the anomalous results.

Sample-specific accuracy for both organic and inorganic parameters can be assessed by examination of matrix spike/matrix spike duplicate results. A sample is analyzed unspiked to determine a baseline set of values. A second portion of sample is spiked with known concentrations of compounds appropriate to the analyses being performed, typically 5 volatile organic compounds for volatile organic analyses, 11 semivolatile compounds for semivolatiles, 6 pesticide compounds for pesticides, all metals for metals analyses, and a known quantity of cyanide for cyanide analysis. The percentage of the spike compound which is recovered, i.e., measured in excess of the value obtained for the unspiked sample, is a direct measure of analytical accuracy. EPA requires matrix spike/matrix spike duplicates to be run at least once per 20 samples of similar matrix.

Matrix spike/matrix spike duplicate results are reported to EPD/EMS but are not published. For organic compounds, according to EPA guidelines, no action is taken on the basis of matrix spike/matrix spike duplicate data alone (i.e., no result modifiers are assigned solely on the basis of matrix spike results); however, the results can indicate if a lab is having a systematic problem in the analysis of one or more analytes.

In the case of inorganic compounds, such as metals, the matrix spike sample analysis provides information about the effect of each sample matrix on the digestion and measurement methodology. Data qualifiers can be assigned on the basis of the percentage of spike recovery and are reported in the published results tables.

\section{Assessment of Precision}

Precision of the analyses, or agreement of a set of replicate results among themselves, is assessed through the use of duplicates (laboratory-initiated) and blind replicates (provided by EPD/EMS). The results of duplicate and replicate analyses are presented in the results tables in these reports as multiple entries for an analyte under a single table heading.

The laboratories assess precision by calculating the relative percent difference, or RPD, for each pair of laboratory-initiated duplicate results. During 1992, at least one of the contract laboratories used a data qualifier (J3) to modify metals analyses when the RPD for laboratory duplicates was greater than $20 \%$.

Additional statistical comparisons of laboratory duplicate and blind replicate results, both intra- and interlaboratory, are presented in the EPD/EMS quarterly reports. The calculation used for these reports is the MRD, or mean relative difference, which is similar to EPA's RPD except that the MRD provides a single value for all of the analyses of a particular compound, either inter- or intralaboratory, during one quarter. Because detection limits may vary among samples, the MRD requires calculation of a reference detection limit, which is the detection limit at the 90 th percentile of the array of limits in the population of all replicate 
and duplicate analyses for a given analyte during a particular quarter. The MRD is not method-specific.

\section{Method-Specific Accuracy and Precision}

The contract laboratories' EPA-approved laboratory procedures include QA/QC requirements as an integral part of the methods. Thus, knowledge of the method used in obtaining data is an important component of determining data useability. EPA has conducted extensive research and development on the methods approved for the analysis of water and waste water; information on the accuracy and precision of the method is available from EPA publications, as is full information on required QA/QC procedures. A listing of the methods used by the primary laboratories during first quarter 1992 is given below along with the source for the method description. Many, if not all, of these sources include presentations of representative accuracy and precision results.

Method

EPA 120.1

EPA 150.1

EPA 160.1

EPA 160.2

EPA 180.1

EPA200.7

EPA206. 2

EPA208.2

EPA239.2

EPA245. 1

EPA270.2

EPA279.2

EPA300.0

EPA310.1

EPA325.2

EPA335.3

EPA340.2

EPA353.1

EPA353.2

EPA353.3

EPA354. 1

EPA365. 1

EPA365. 2

EPA375.4

EPA376.2

APHA403

EPA4 13.1

APHA415A

EPA4 15.1

EPA418.1

EPA420.1

EPA420.2

APHA705

ASTMD3869C

APHA5320

EPA6010
Used to Analyze

Specific conductance

$\mathrm{pH}$

Filterable residue (total dissolved solids)

Nonfilterable residue

Turbidity

Trace elements

Arsenic

Barium

Lead

Mercury

Selenium

Thallium

Inorganics, non-metallics

Alkalinity

Chloride

Cyanide

Fluoride

Nitrogen, nitrate-nitrite

Nitrogen, nitrate, nitrite, or combined

Nitrogen, nitrate-nitrite, or nitrite only

Nitrogen, nitrite

Phosphorus, all forms (reported as total phosphates)

Phosphorus, all forms (reported as total phosphates)

Sulfate, turbidimetric

Sulfide

Alkalinity

Oil \& grease

lodine

Total organic carbon

Petroleum hydrocarbons

Phenolics

Phenolics

Total alpha-emitting radium

lodide

Dissolved organic halogen

Metals
Source

EPA EMSL 1983

EPA EMSL 1983

EPA EMSL 1983

EPA EMSL 1983

EPA EMSL 1983

EPA EMSL 1983

EPA EMSL 1983

EPA EMSL 1983

EPA EMSL 1983

EPA EMSL 1983

EPA EMSL 1983

EPA EMSL 1983

EPA EMSL 1991

EPA EMSL 1983

EPA EMSL 1983

EPA EMSL 1983

EPA EMSL 1983

EPA EMSL 1983

EPA EMSL 1983

EPA EMSL 1983

EPA EMSL 1983

EPA EMSL 1983

EPA EMSL 1983

EPA EMSL 1983

EPA EMSL 1983

APHA 1985

EPA EMSL 1983

APHA 1985

EPA EMSL 1983

EPA EMSL 1983

EPA EMSL 1983

EPA EMSL 1983

APHA 1985

ASTM 1992

APHA 1989

EPA 1986 
Method

EPA7041

EPA7060

EPA7421

EPA7470

EPA7740

EPA7841

EPA8010

EPA8020

EPA8080

EPA8140

EPA8150

EPA8240

EPA8270

EPA8280

EPA9012

EPA9020

EPA9030
Used to Analyze

Antimony
Arsenic
Lead
Mercury
Selenium
Thallium
Halogenated volatile organics
Aromatic volatile organics
Organochlorine pesticides and PCBs
Organophosphorus pesticides
Chlorinated herbicides
GCMS VOA
GCMS semivolatiles
Dioxins and furans
Total cyanide
Total organic halides
Sulfides

Source

EPA 1986
EPA 1986
EPA 1986
EPA 1986
EPA 1986
EPA 1986
EPA 1986
EPA 1986
EPA 1986
EPA 1986
EPA 1986
EPA 1986
EPA 1986
EPA 1986
EPA 1986
EPA 1986
EPA 1986

An example of the available method-specific QA/QC information is that for the analysis of metals by EPA Method 6010/200.7 (EPA 1986/EPA EMSL 1983). The primary laboratories, General Engineering Laboratories (GE) and Roy F. Weston, Inc. (Weston), use this inductively coupled plasma (ICP) atomic emission spectrometric method.

The following precision and accuracy data are based on the experience of seven laboratories that applied the ICP technique to acid-distilled water matrices that had been dosed with various metal concentrates. (Note: not all seven laboratories analyzed all 14 elements.) The references give results for samples having three concentration ranges; the results here are for samples having the lowest values, similar to actual groundwater results for SRS.

ICP Precision and Accuracy Data

\begin{tabular}{llll} 
Element & True value $(\mu \mathrm{g} / \mathrm{L})$ & $\begin{array}{l}\text { Mean reported } \\
\text { value }(\mu \mathrm{g} / \mathrm{L})\end{array}$ & Mean \\
\cline { 2 - 4 } & & & RSD \\
Beryllium & 20 & 20 & 9.8 \\
Manganese & 15 & 15 & 6.7 \\
Vanadium & 70 & 69 & 2.9 \\
Arsenic & 22 & 19 & 23 \\
Chromium & 10 & 10 & 18 \\
Copper & 11 & 11 & 40 \\
Iron & 20 & 19 & 15 \\
Aluminum & 60 & 62 & 33 \\
Cadmium & 2.5 & 2.9 & 16 \\
Cobalt & 20 & 20 & 4.1 \\
Nickel & 30 & 28 & 11 \\
Lead & 24 & 30 & 32 \\
Zinc & 16 & 19 & 45 \\
Selenium & 6 & 8.5 & 42
\end{tabular}

a Relative standard deviation. Note: In EPA (1986), the column heading is Mean Standard Deviation (\%). 
As another example, EPA Method 601/8010 (EPA 1991a/EPA 1986) is used by both GE and Weston for analyses of halogenated volatile organics. In the presentation of the method in both references, the following table gives method-specific accuracy and precision as functions of concentration. Contract laboratories are expected to achieve or at least approach these limits.

\section{Accuracy and Precision as Functions of Concentration for EPA Method 601/8010}

Parameter
Bromodichloromethane
Bromoform
Bromomethane
Carbon tetrachloride
Chlorobenzene
Chloroethane
2-Chloroethyl vinyl ether
Chloroform
Chloromethane
Dibromochloromethane
1,2-Dichlorobenzene
1,3-Dichlorobenzene
1,4-Dichlorobenzene
1,1-Dichloroethane
1,2-Dichloroethane
1,1-Dichloroethene
trans-1,2-Dichloroethene
1,2-Dichloropropane
cis-1,3-Dichloropropene
trans-1,3-Dichloropropene
Methylene chloride
1,1,2,2-Tetrachlorethane
Tetrachloroethylene
1,1,1-Trichloroethane
1,1,2-Trichloroethane
Trichloroethylene
Trichlorofluoromethane
Vinyl chloride

\section{Parameter}

Bromoform

Bromomethane

Chlorobenzene

Chloroethane

2-Chloroethyl vinyl ether ${ }^{f}$

Dibromochloromethane

1,2-Dichlorobenzene

1,3-Dichlorobenzene

1,1-Dichloroethane

1,2-Dichloroethane

1.1-Dichloroethene trans-1,2-Dichloroethene 1,2-Dichloropropane ${ }^{f}$ cis-1,3-Dichloropropene ${ }^{f}$ trans-1,3-Dichloropropene

Methylene chloride

Tetrachloroethylene 1,1,1-Trichloroethane 1,1,2-Trichloroethane Trichlorofluoromethane inyl chloride

\begin{tabular}{l} 
Accuracy as \\
recovery, $\mathrm{X}^{\mathrm{a}}$ u \\
\hline $1.12 C-1.02^{d}$ \\
$0.96 C-2.05$ \\
$0.76 C-1.27$ \\
$0.98 C-1.04$ \\
$1.00 C-1.23$ \\
$0.99 C-1.53$ \\
$1.00 C$ \\
$0.93 C-0.39$ \\
$0.77 C+0.18$ \\
$0.94 C+2.72$ \\
$0.93 C+1.70$ \\
$0.95 C+0.43$ \\
$0.93 C-0.09$ \\
$0.95 C-1.08$ \\
$1.04 C-1.06$ \\
$0.98 C-0.87$ \\
$0.97 C-0.16$ \\
$1.00 C$ \\
$1.00 C$ \\
$1.00 C$ \\
$0.91 C-0.93$ \\
$0.95 C+0.19$ \\
$0.94 C+0.06$ \\
$0.90 C-0.16$ \\
$0.86 C+0.30$ \\
$0.87 C+0.48$ \\
$0.89 C-0.07$ \\
$0.97 C-0.36$
\end{tabular}

Single analyst

precision $(\mu \mathrm{g} / \mathrm{L})^{b}$

$0.11 \bar{x}+0.04^{\circ}$

$0.12 \bar{X}+0.58$

$0.28 \bar{x}+0.27$

$0.15 \bar{X}+0.38$

$0.15 \bar{x}-0.02$

$0.14 \bar{X}-0.13$

$0.20 \bar{X}$

$0.13 \bar{x}+0.15$

$0.28 \bar{x}-0.31$

$0.11 \bar{X}+1.10$

$0.20 \bar{X}+0.97$

$0.14 \bar{x}+2.33$

$0.15 \bar{x}+0.29$

$0.09 \bar{x}+0.17$

$0.11 \bar{x}+0.70$

$0.21 \bar{x}-0.23$

$0.11 \bar{x}+1.46$

$0.13 \bar{x}$

$0.18 \bar{x}$

$0.18 \bar{x}$

$0.11 \bar{X}+0.33$

$0.14 \bar{X}+2.41$

$0.14 \bar{X}+0.38$

$0.15 \bar{x}+0.04$

$0.13 \bar{x}-0.14$

$0.13 \bar{x}-0.03$

$0.15 \bar{x}+0.67$

$0.13 \bar{X}+0.65$
Overall precision $(\mu \mathrm{g} / \mathrm{L})^{\mathrm{c}}$

$0.20 \bar{x}+1.00$

$0.21 \bar{x}+2.41$

$0.36 \bar{x}+0.94$

$0.20 \bar{x}+0.39$

$0.18 \bar{x}+1.21$

$0.17 \bar{x}+0.63$

$0.35 \bar{X}$

$0.19 \bar{X}-0.02$

$0.52 \bar{X}+1.31$

$0.24 \bar{X}+1.68$

$0.13 \bar{X}+6.13$

$0.26 \bar{X}+2.34$

$0.20 \bar{X}+0.41$

$0.14 \bar{X}+0.94$

$0.15 \bar{X}+0.94$

$0.29 \bar{X}-0.40$

$0.17 \bar{x}+1.46$

$0.23 \bar{x}$

$0.32 \bar{x}$

$0.32 \bar{x}$

$0.21 \bar{x}+1.43$

$0.23 \bar{X}+2.79$

$0.18 \bar{X}+2.21$

$0.20 \bar{X}+0.37$

$0.19 \bar{X}+0.67$

$0.23 \bar{x}+0.30$

$0.26 \bar{x}+0.91$

$0.27 \bar{X}+0.40$

a $X^{\prime}=$ expected recovery for one or more measurements of a sample containing a concentration of $C$, in $\mu \mathrm{g} / \mathrm{L}$.

b Expected single analyst standard deviation of measurements.

c Expected interlaboratory standard deviation of measurements.

d $C=$ true value for the concentration, in $\mu \mathrm{g} / \mathrm{L}$.

e $\bar{X}=$ average recovery found for measurements of samples containing a concentration of $C$, in $\mu \mathrm{g} / \mathrm{L}$.

$f$ Estimates based on performance in a single laboratory. 


\section{References}

APHA (American Public Health Association), 1985. Standard Methods for the Examination of Water and Wastewater, 16th edition. Washington, DC.

APHA (American Public Health Association), 1989. Standard Methods for the Examination of Water and Wastewater, 17th edition. Washington, DC.

ASTM (American Society for Testing and Materials), 1992. 1992 Annual Book of ASTM Standards, Volume 11.02, Water (II). Philadelphia, PA.

CFR (Code of Federal Regulations), 1991. Analytical Methods, Title 40, Part 136, Appendix A. Washington, DC. Revised July 1, 1991.

EPA (U.S. Environmental Protection Agency), 1986. Test Methods for Evaluating Solid Waste (SW-846), Volumes IA-IC. Washington, DC.

EPA (U.S. Environmental Protection Agency), 1987. Data Quality Objectives for Remedial Response Activities. PB88-131870; EPA/540/G-87/003. Washington, DC.

EPA (U.S. Environmental Protection Agency), 1988a. Contract Laboratory Program Statement of Work for Inorganics Analysis, Multi-Media, Multi-Concentration. SOW No. 788. Washington, DC.

EPA (U.S. Environmental Protection Agency), 1988b. Contract Laboratory Program Statement of Work for Organics Analysis Multi-Media, Multi-Concentration. SOW No. 288. Washington, DC.

EPA (U.S. Environmental Protection Agency), 1990. Guidance for Data Useability in Risk Assessment. Interim Final. EPA/540/G-90/008. Washington, DC.

EPA EMSL (U.S. Environmental Protection Agency, Environmental Monitoring and Systems Laboratory), 1979. Handbook for Analytical Quality Control in Water Wastewater Laboratories. PB-297 451; EPA-600/4-79-019. Cincinnati, OH.

EPA EMSL (U.S. Environmental Protection Agency, Environmental Monitoring and Systems Laboratory), 1983. Methods for Chemical Analysis of Water and Wastes. Cincinnati, OH. Revised March 1983.

EPA EMSL (U.S. Environmental Protection Agency, Environmental Monitoring and Systems Laboratory), 1991. Test Method, The Determination of Inorganic Anions in Water by Ion Chromatography-Method 300.0. Cincinnati, OH. Revised August 1991. 
WSRC-TR-93-068 
WSRC-TR-93.068

Appendix F - Time Series Plots 
$\infty$

\section{3}

$\overline{1}$

ำ

g

ญ्d

$\infty$

to

흠

a

.

ॐ

E a
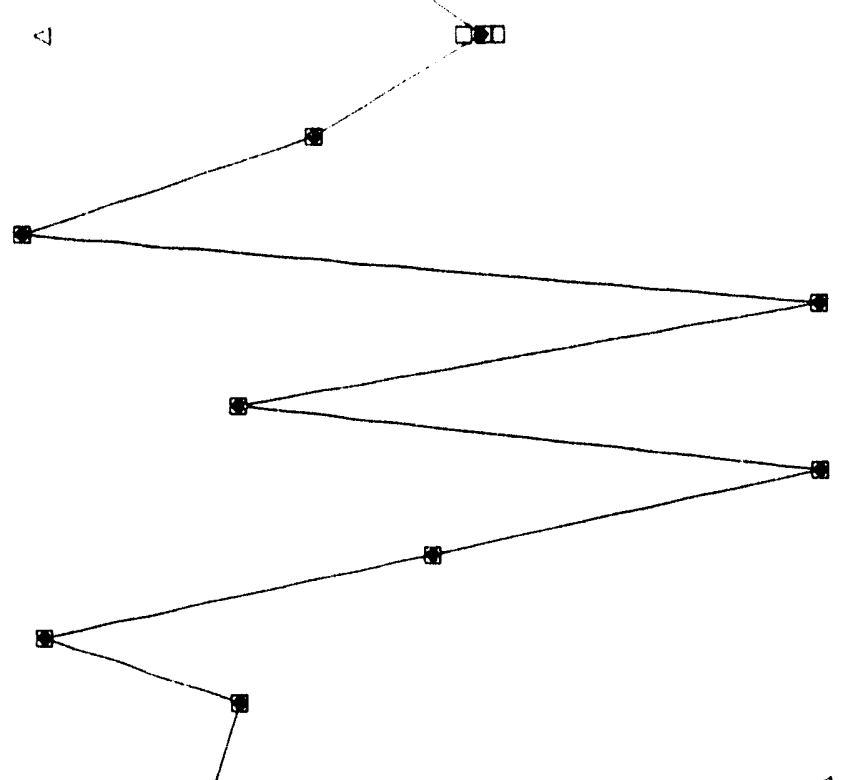

$\triangleleft$

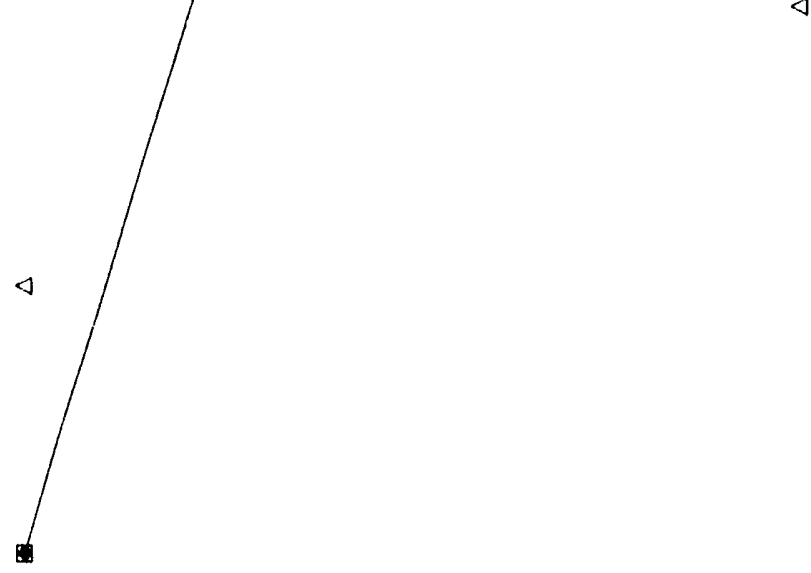

$\triangleleft$

$\triangleleft$

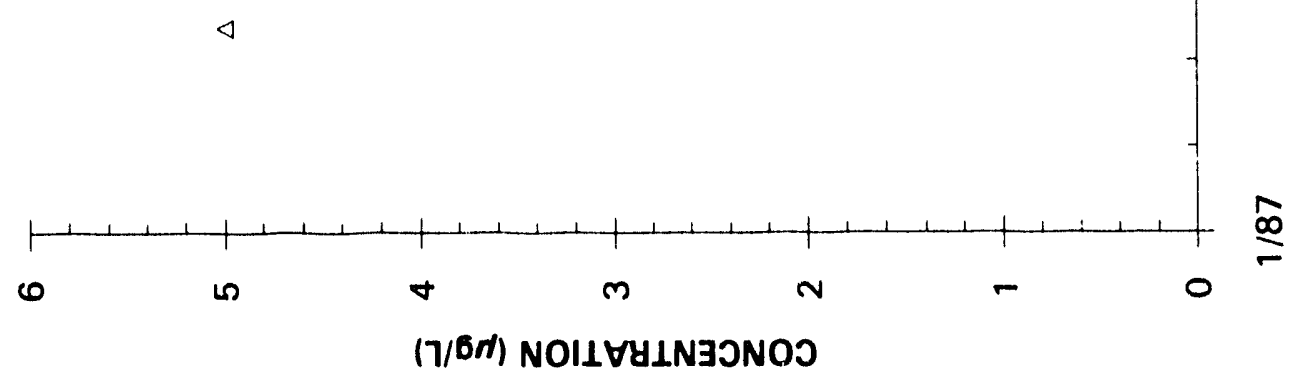

(7/6n) NOII $\forall Y \perp N 3 J N O J$ 


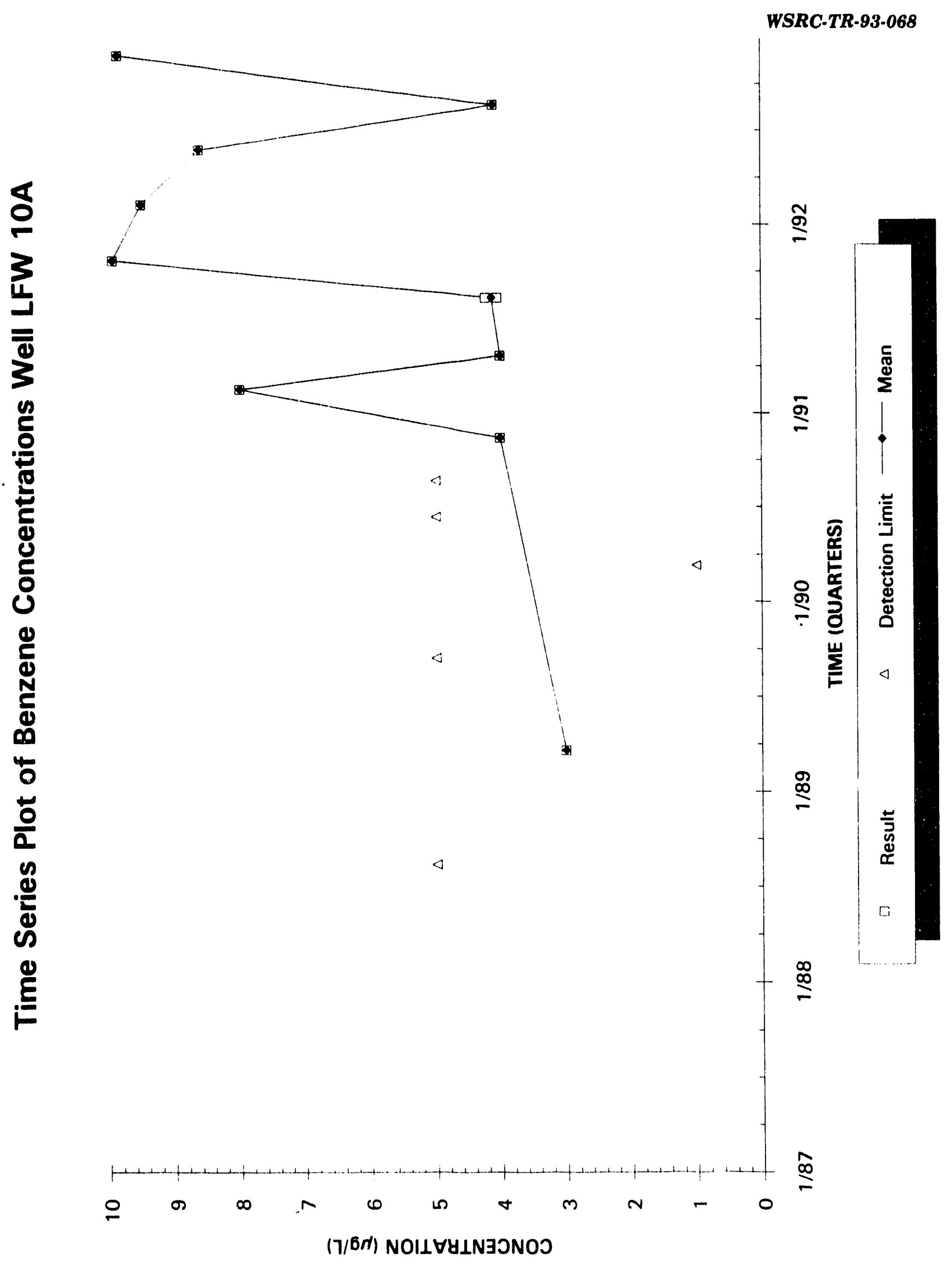


WSRC-TR.93-068

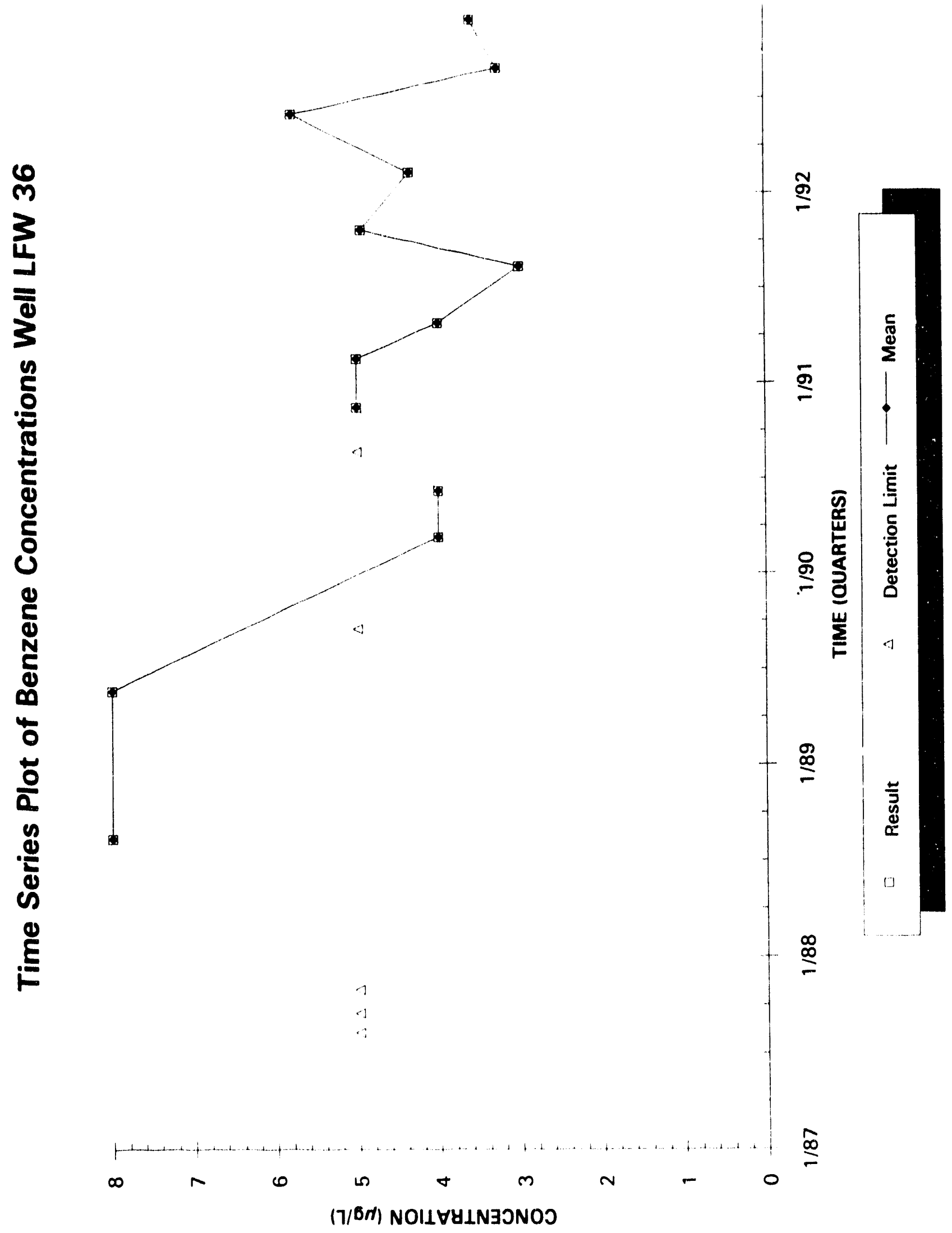


을

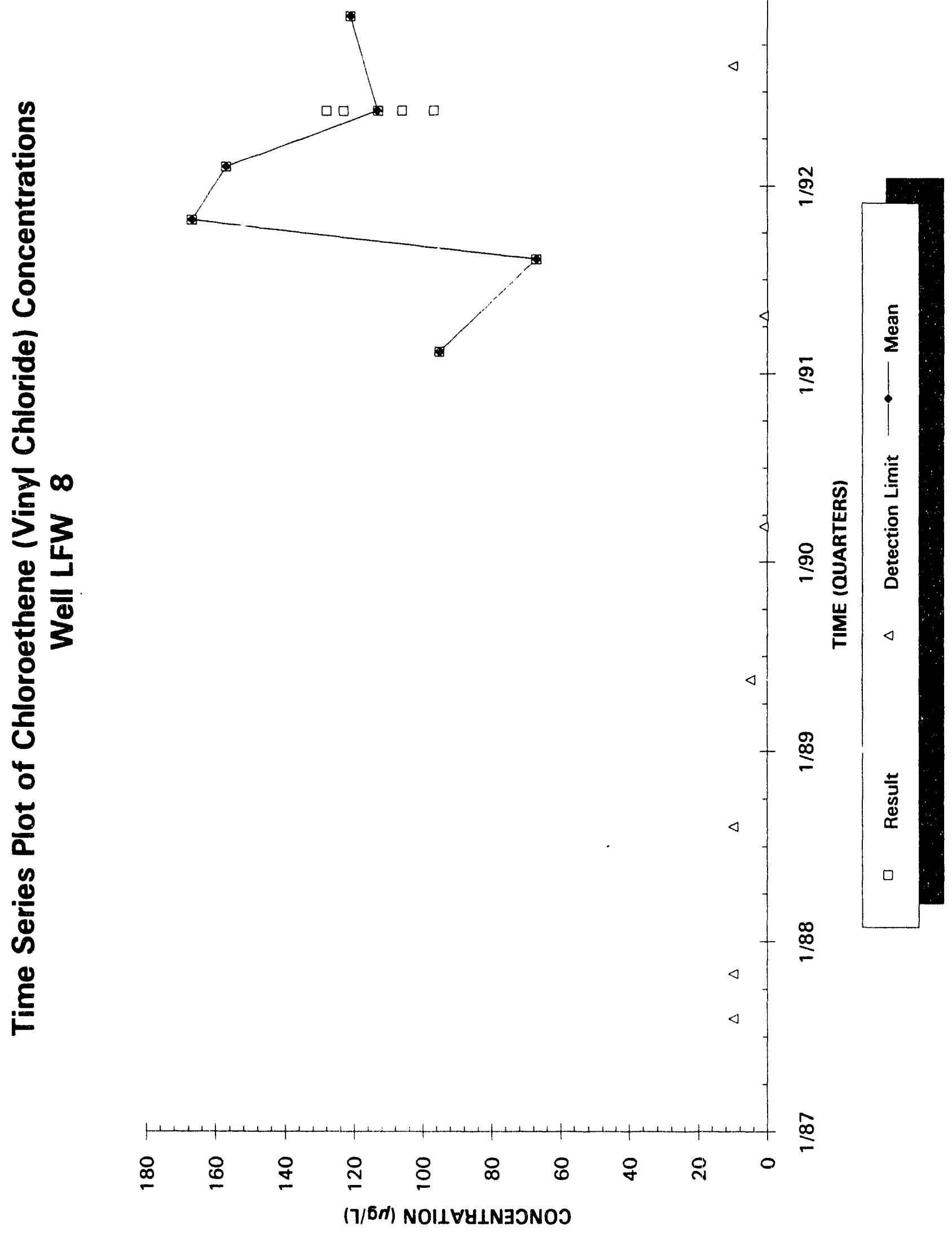

WSRC-TR-93-068

은

$\sum \infty$

$\sum_{0}^{5} \frac{5}{4}$

- 


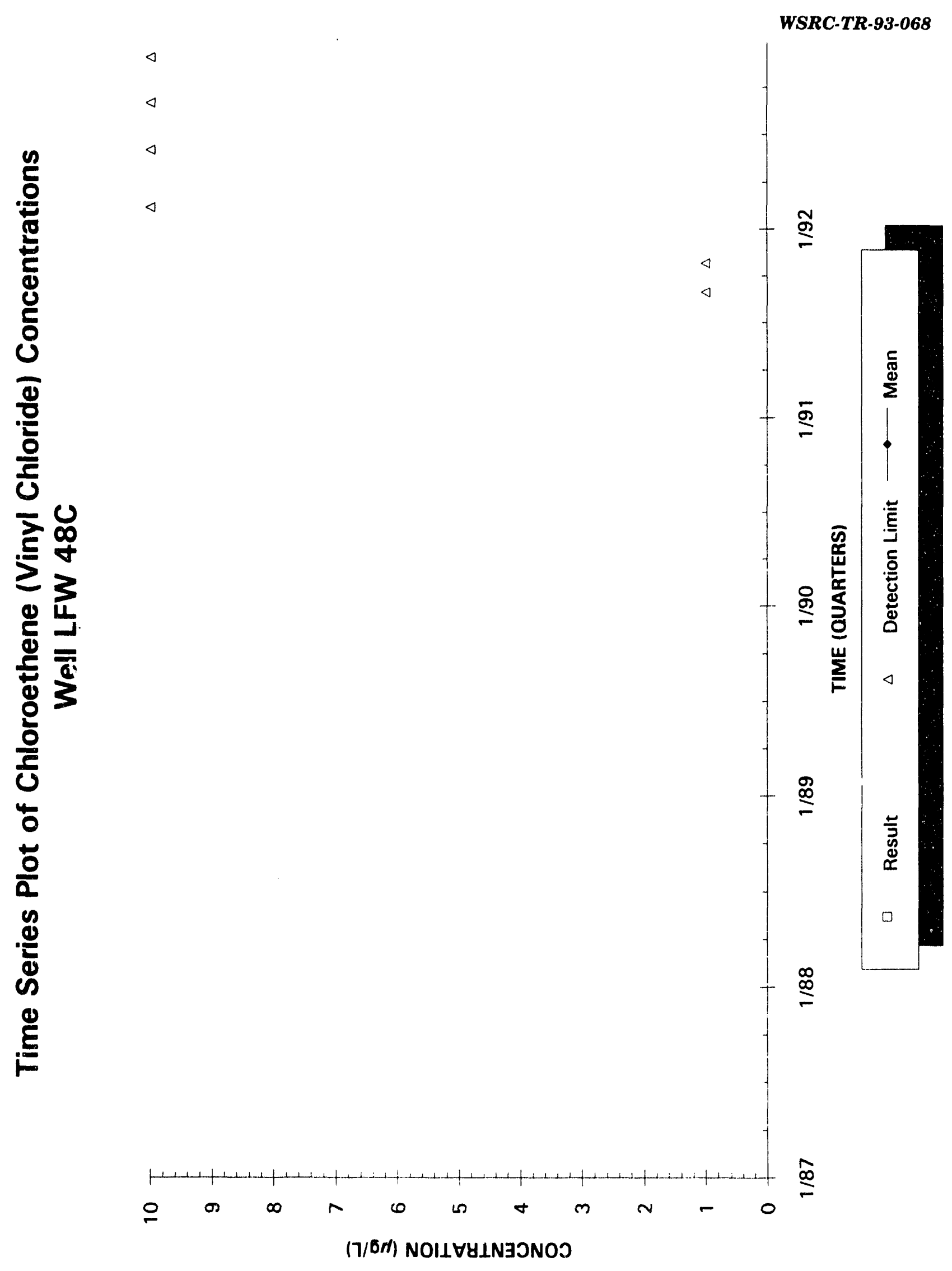




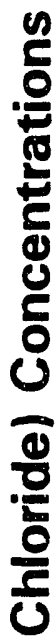

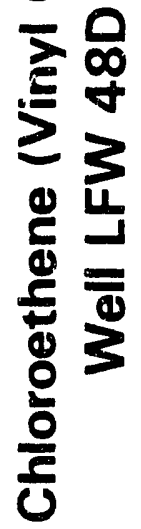

32

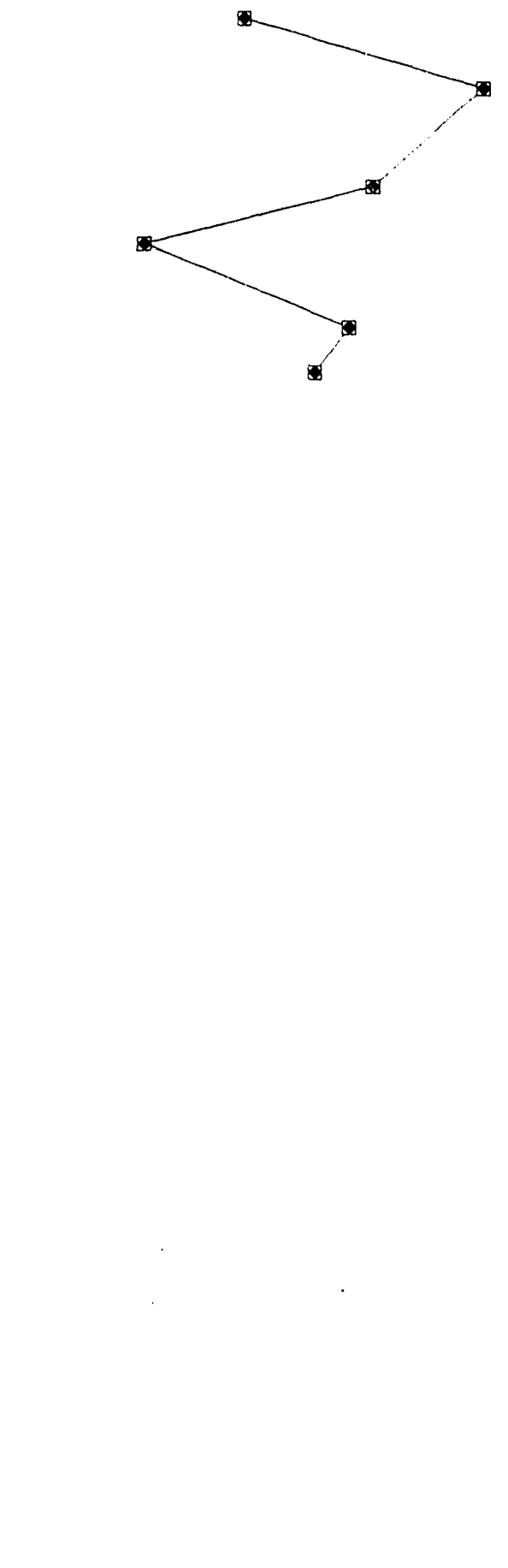

-

to

$\frac{\circ}{\alpha}$

恋

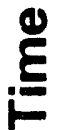

$\stackrel{\infty}{\infty}$

รั

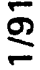

$\stackrel{\infty}{\mathscr{\infty}}$

우

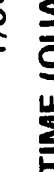



焉

ڤ్ర

$\underline{E}$

$\triangleleft$

$\mid$

$\square$

$\stackrel{5}{3}$

(1)

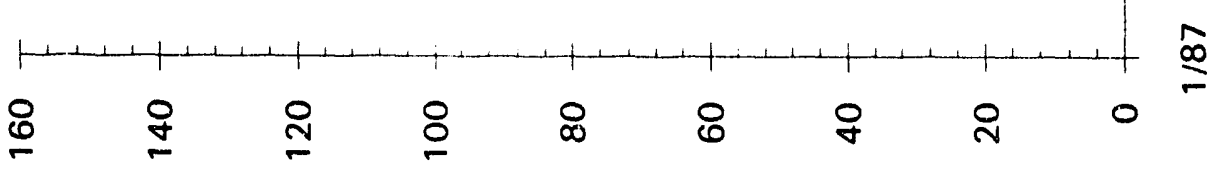

$(7 / 6 n)$ NOIL $\forall Y \perp N B O N O J$ 


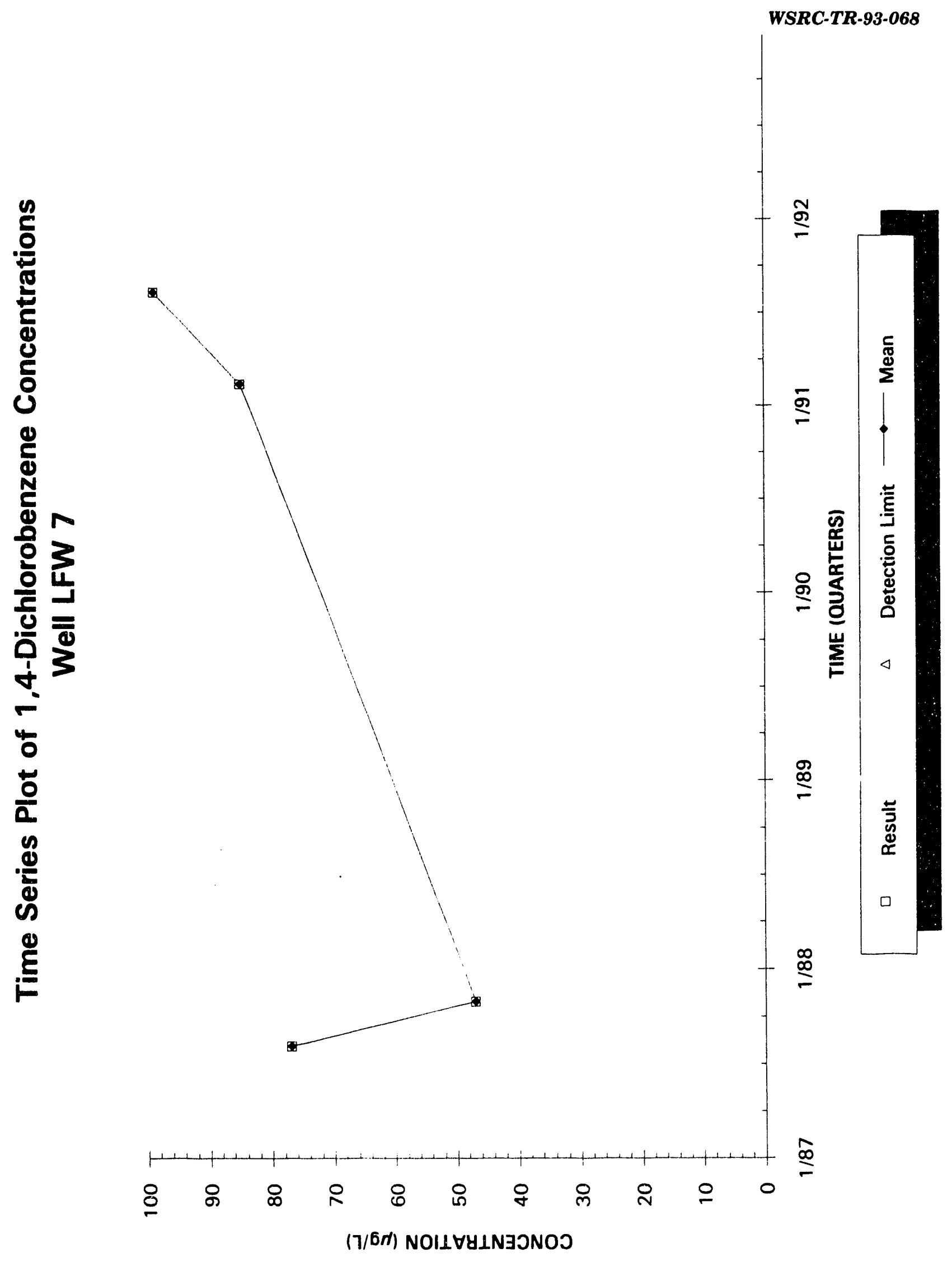




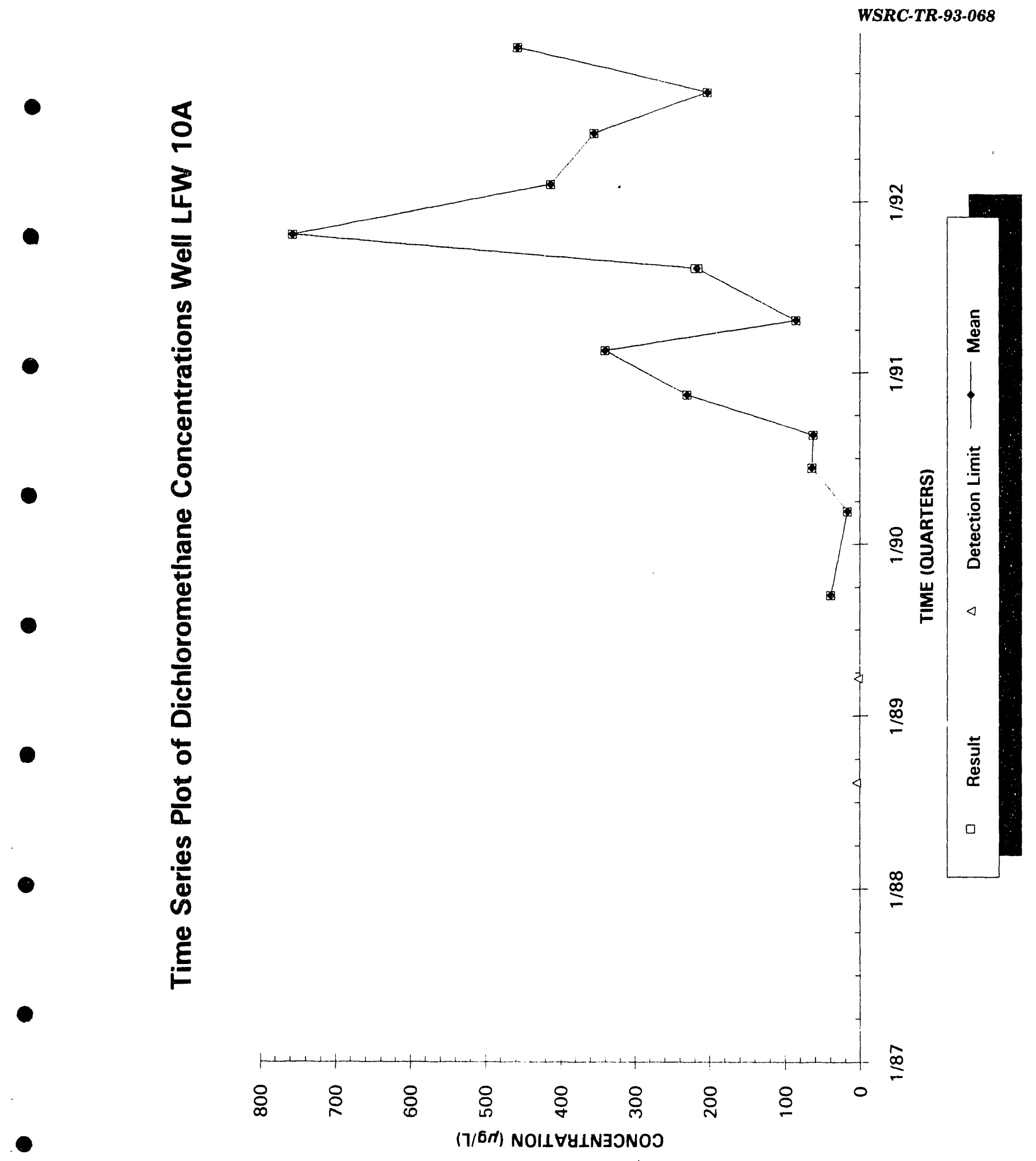



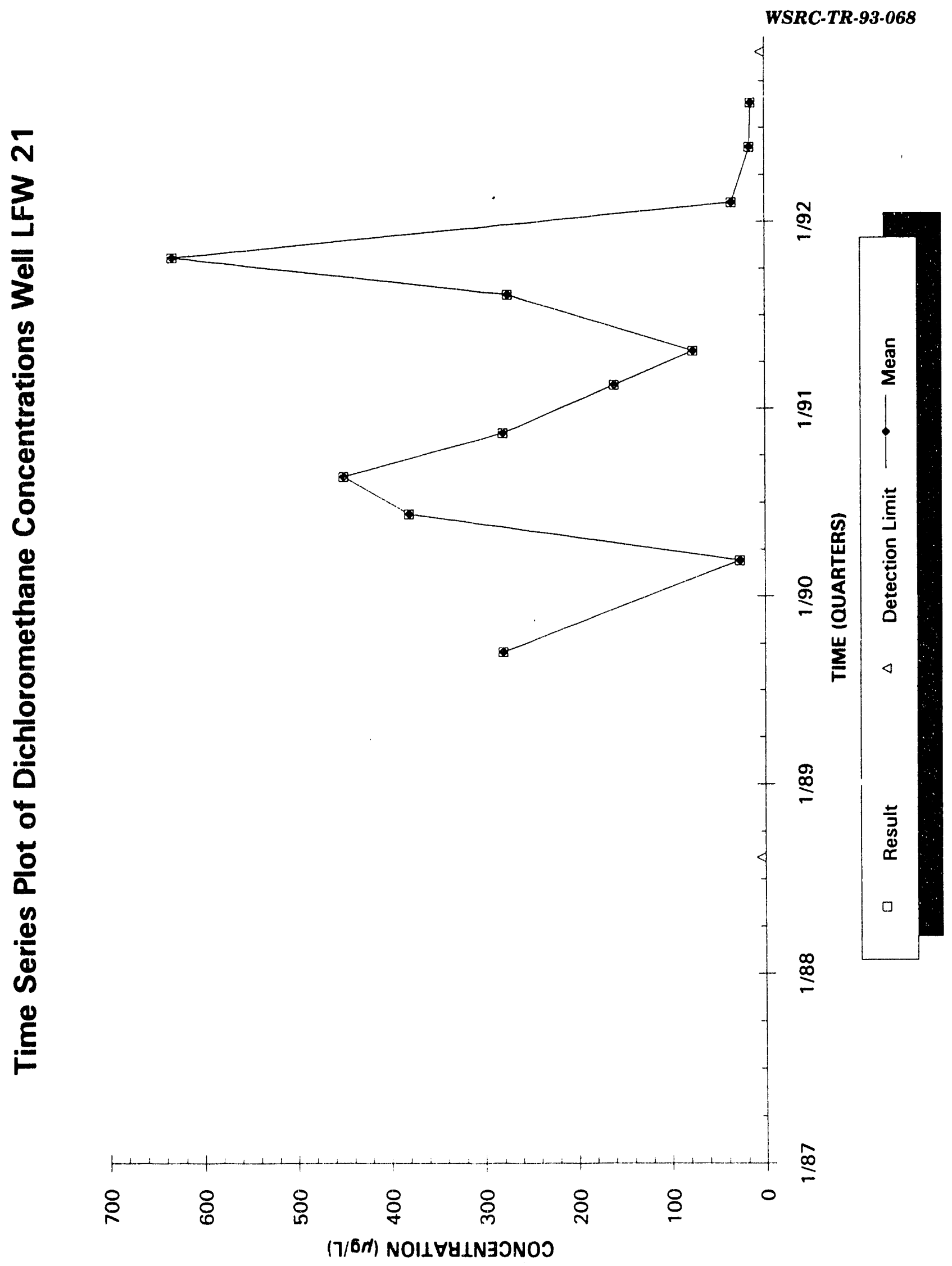


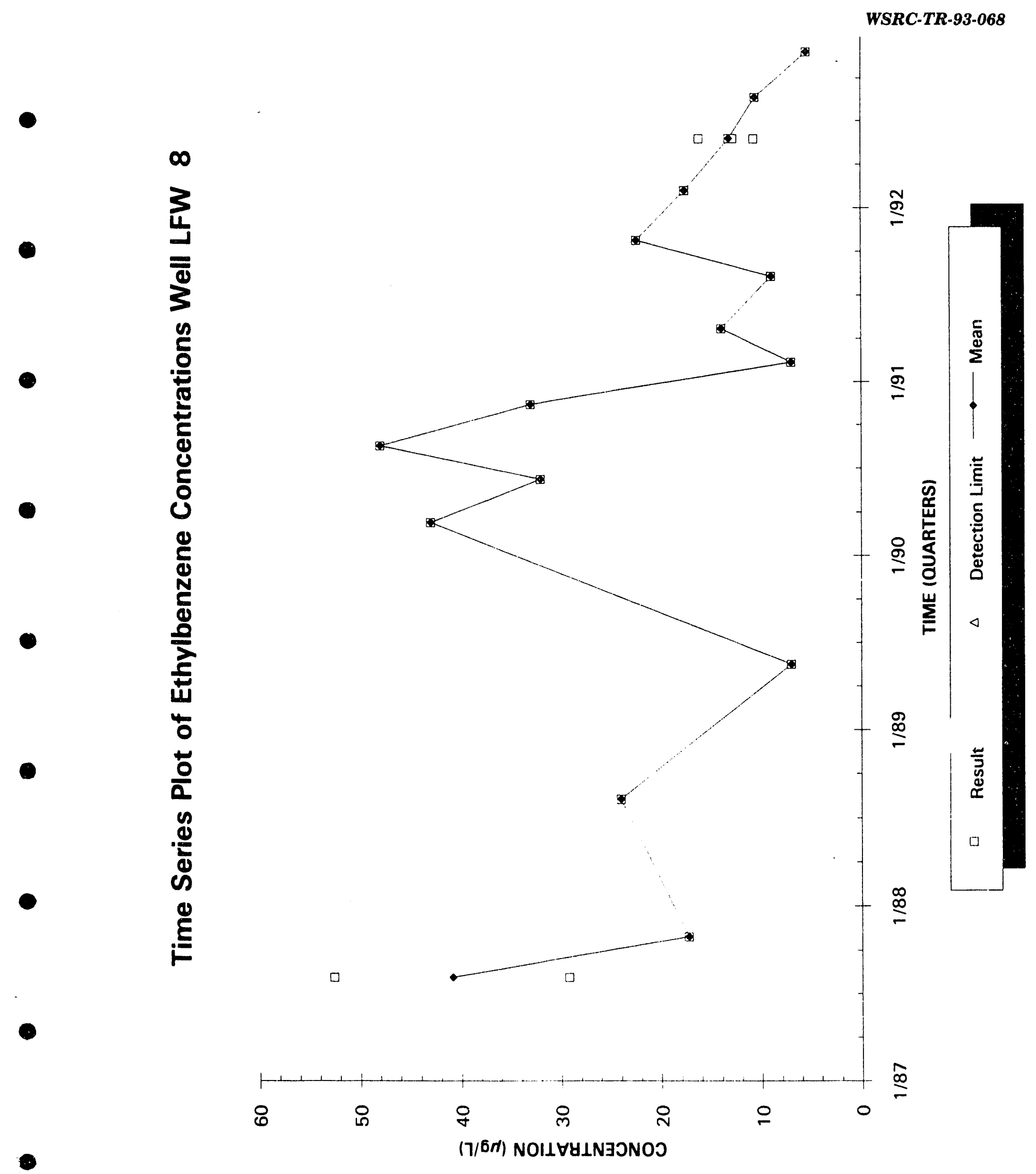




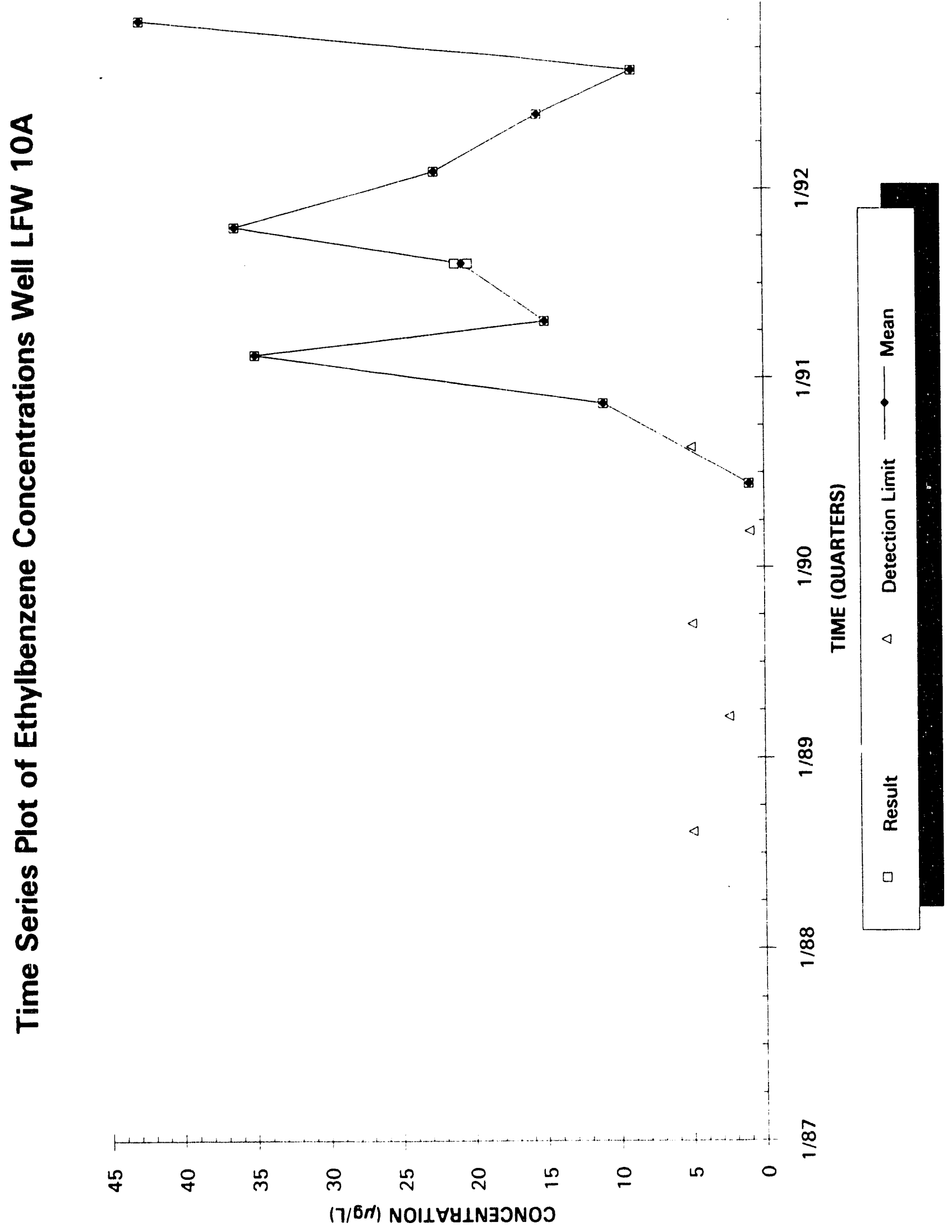


J

롤

to

흠

a

ڤั

E

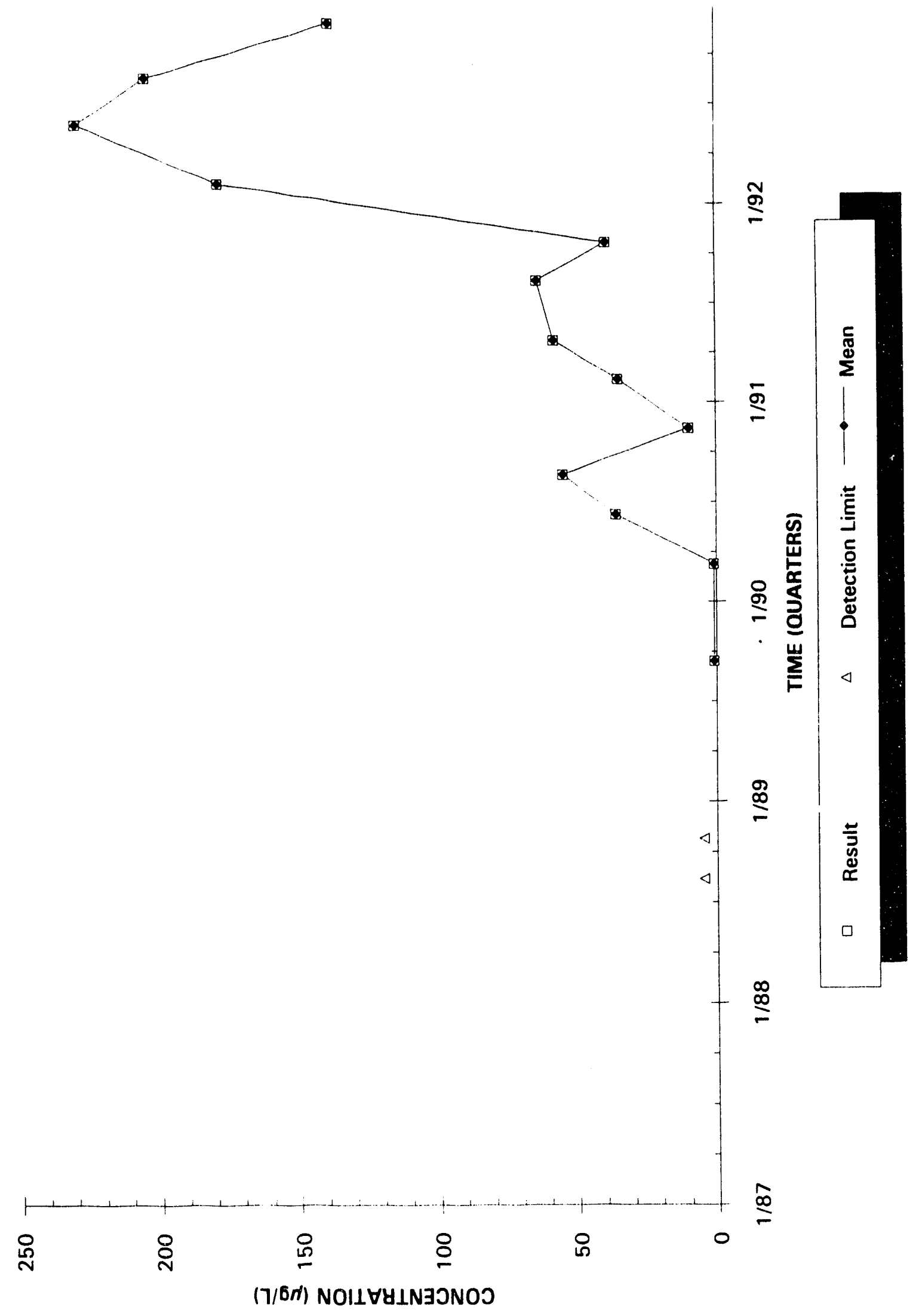




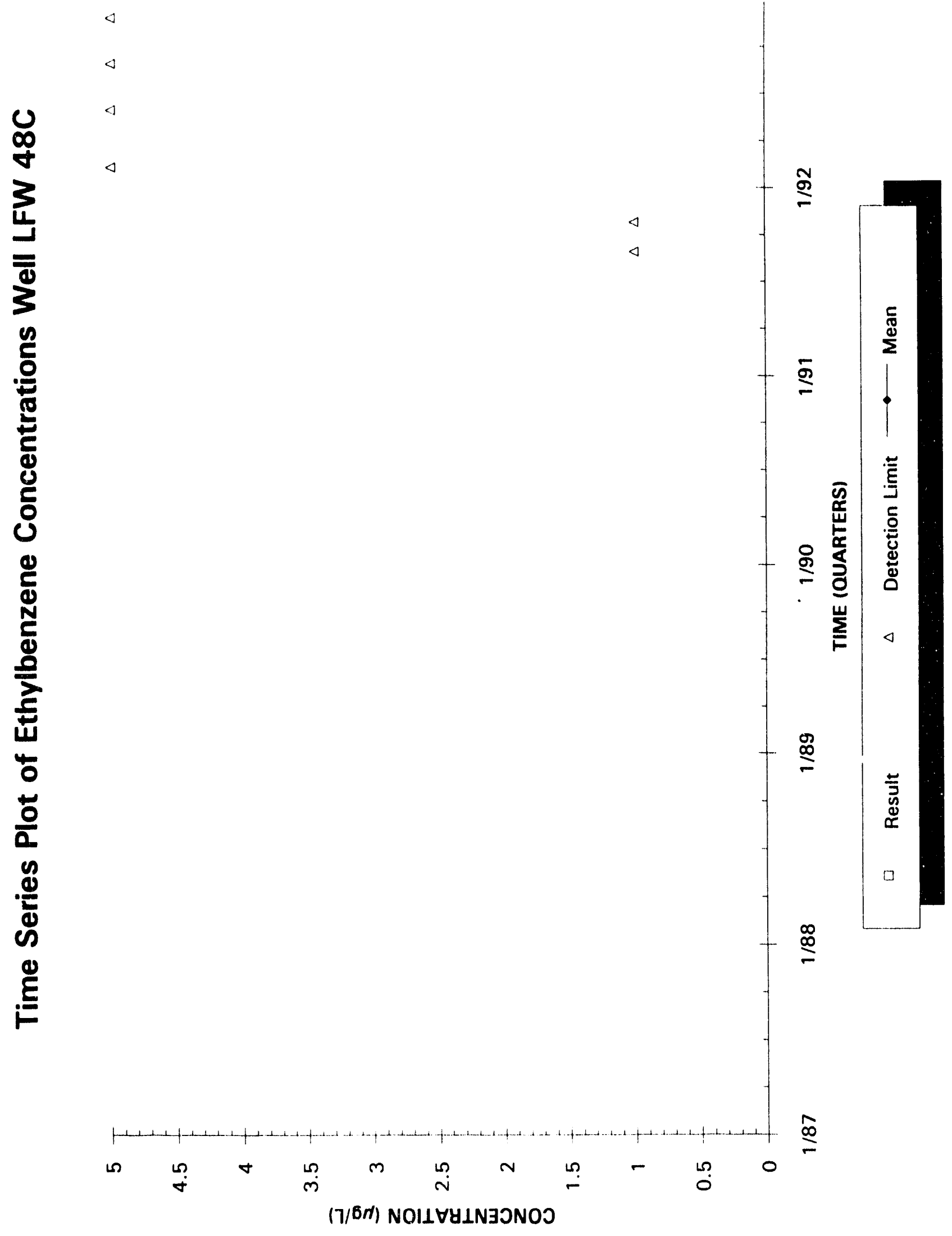




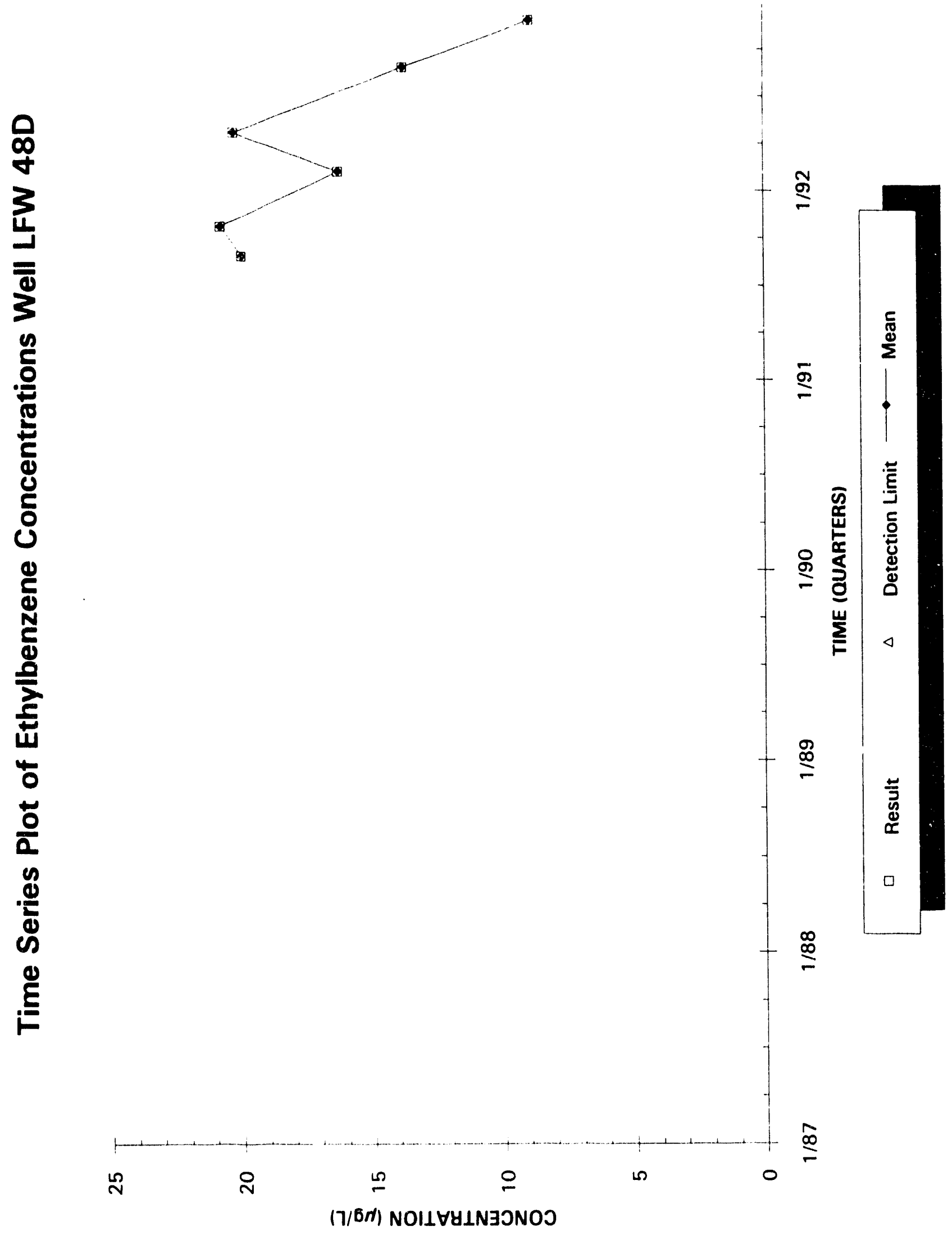




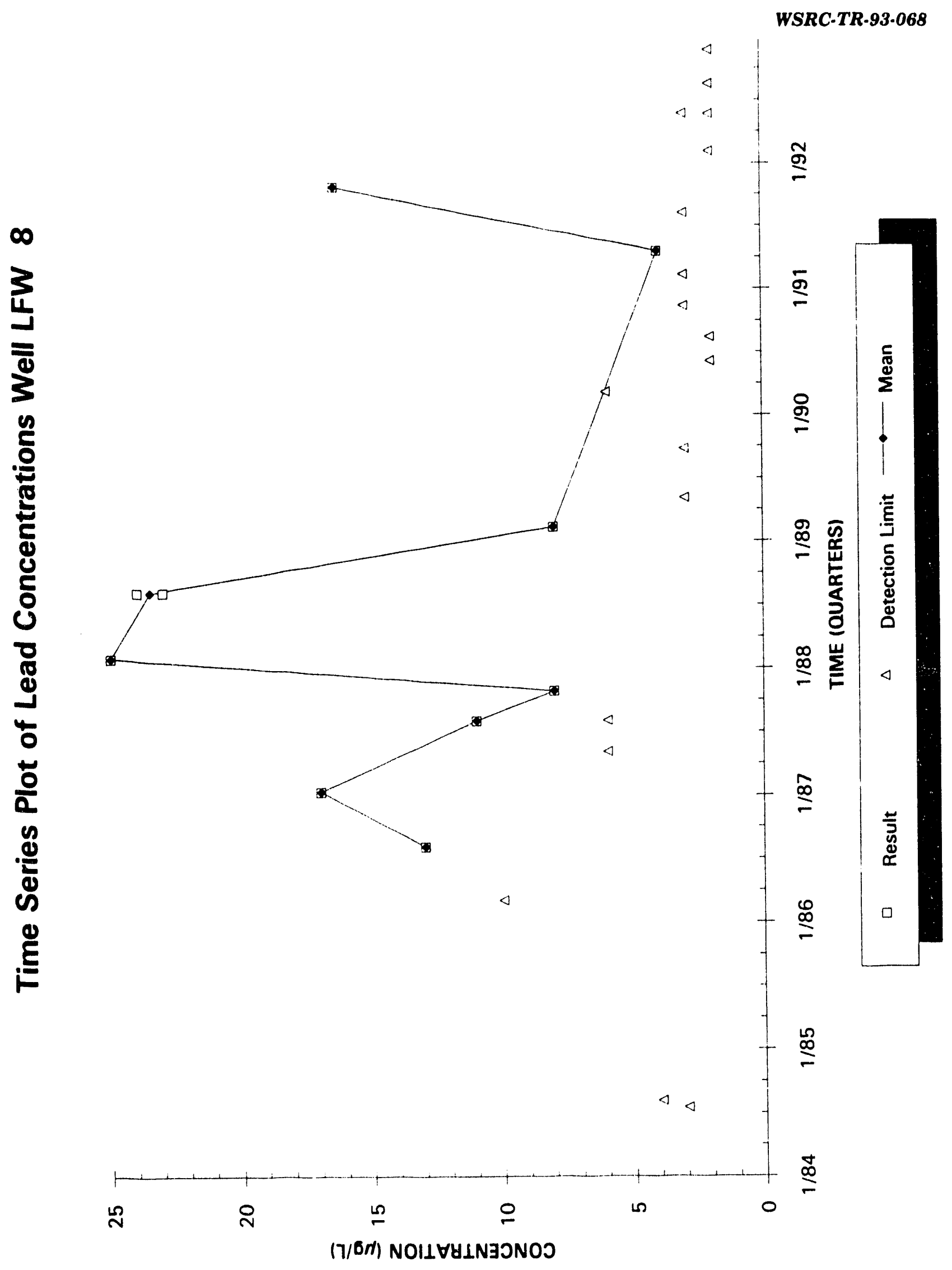




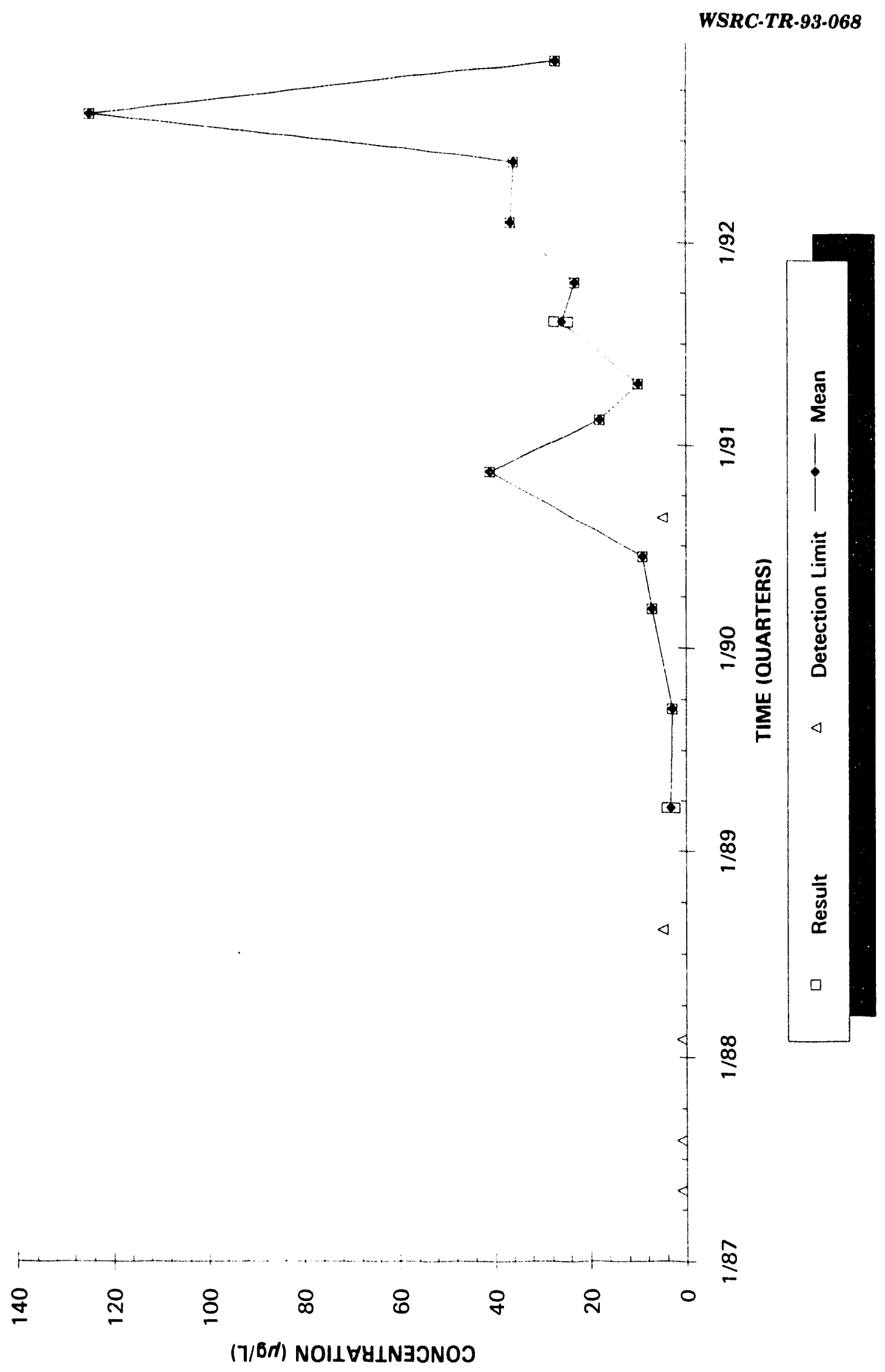




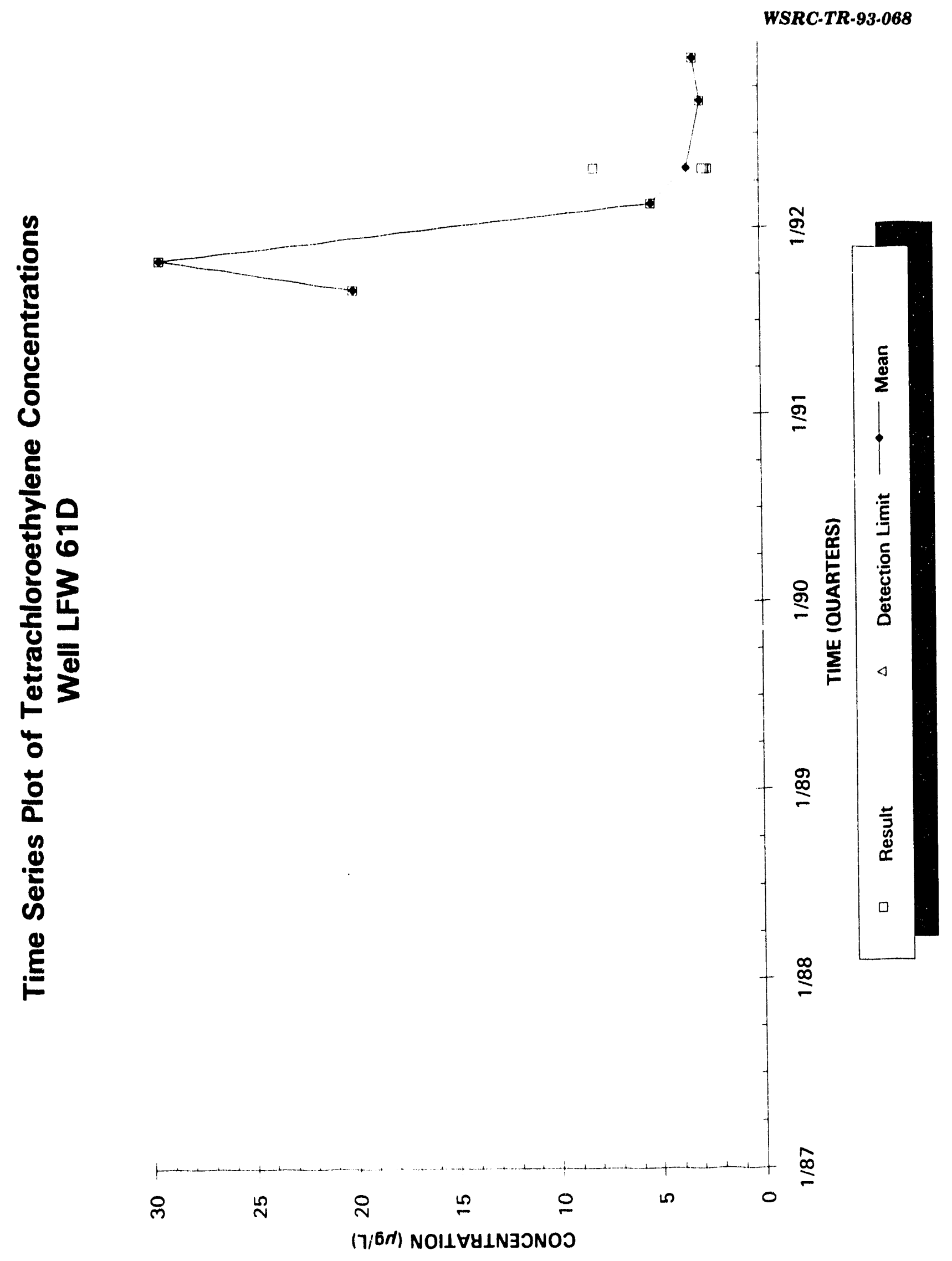




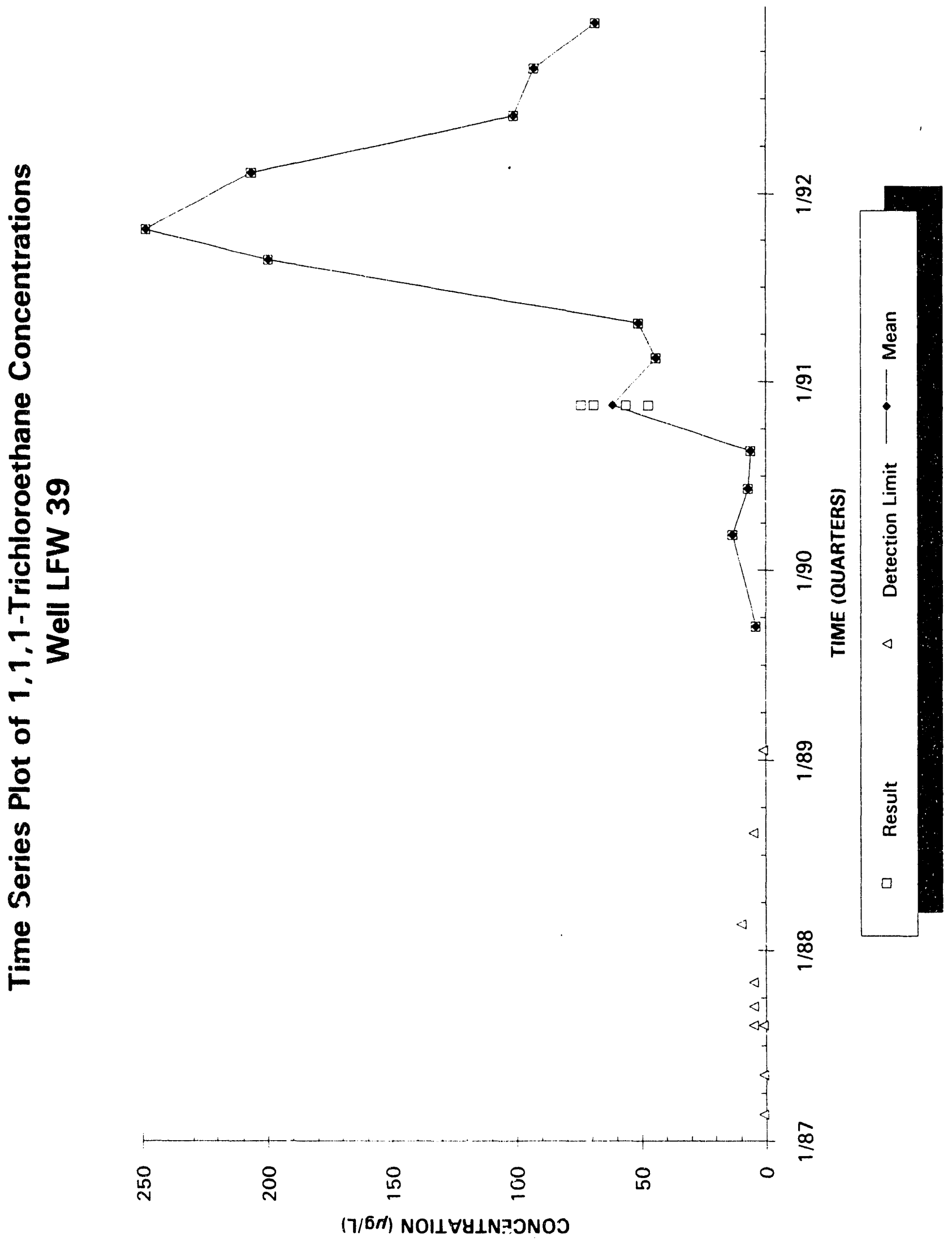



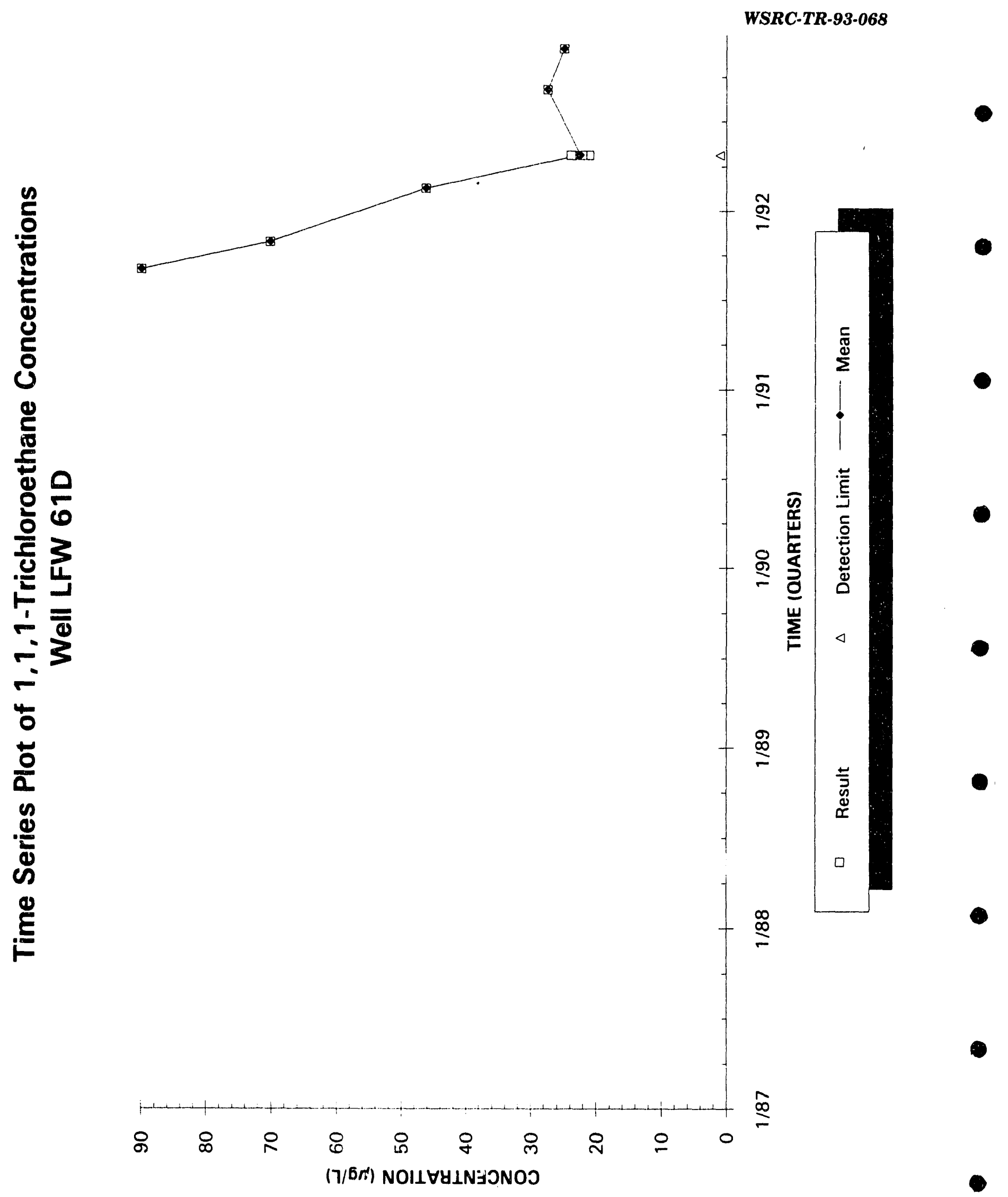
WSRC-TR-93-068

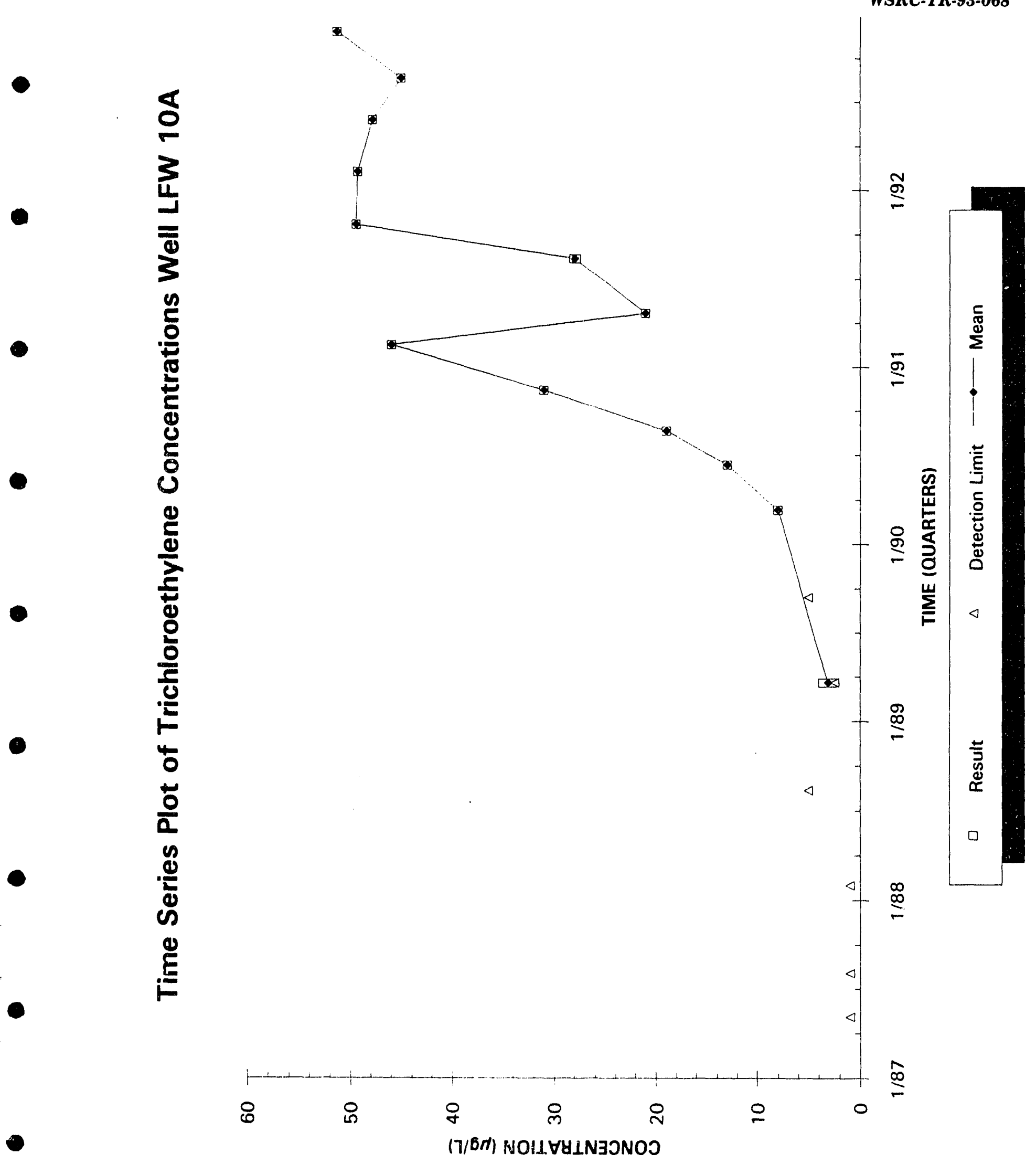



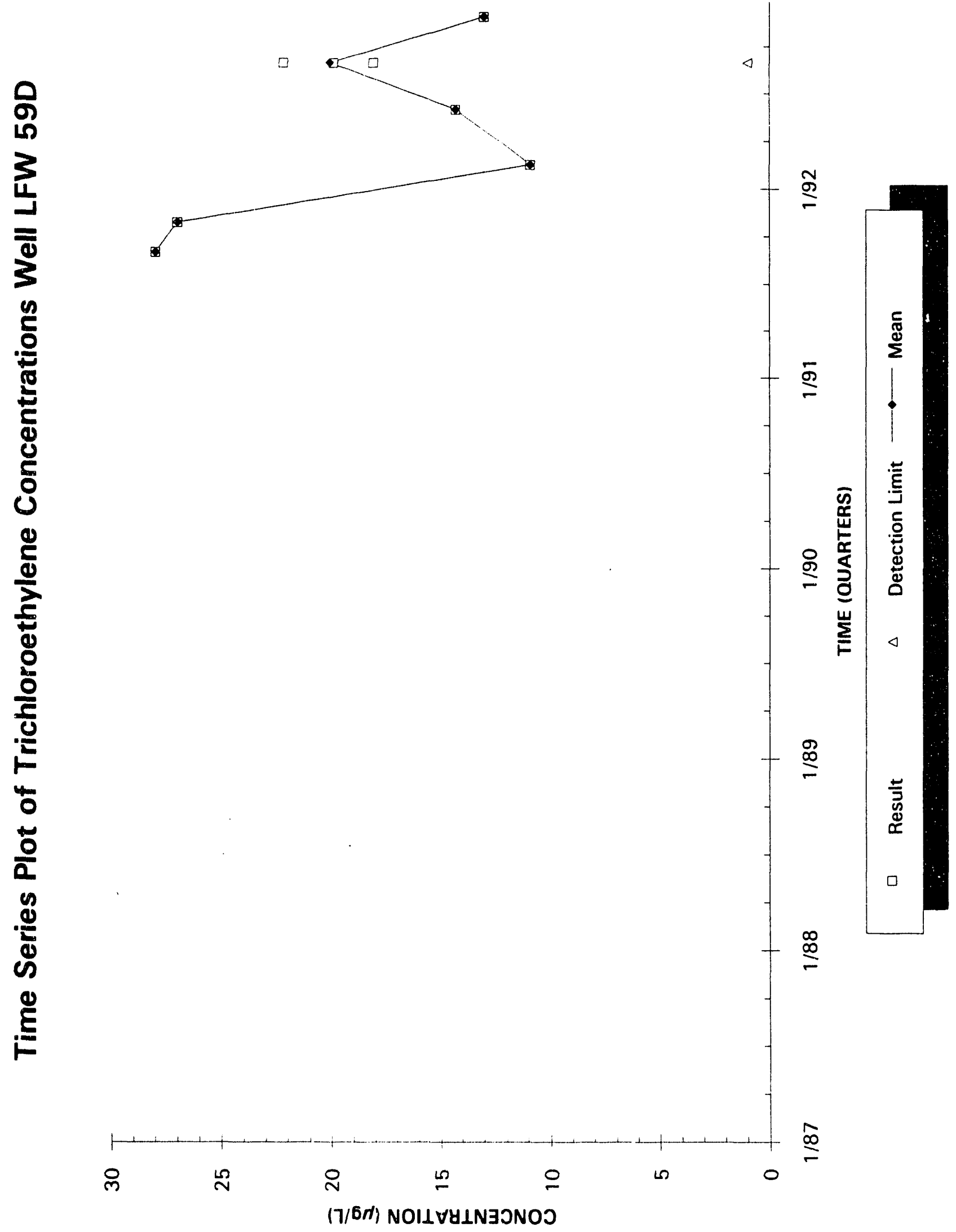

WSRC-TR-93-068

9 


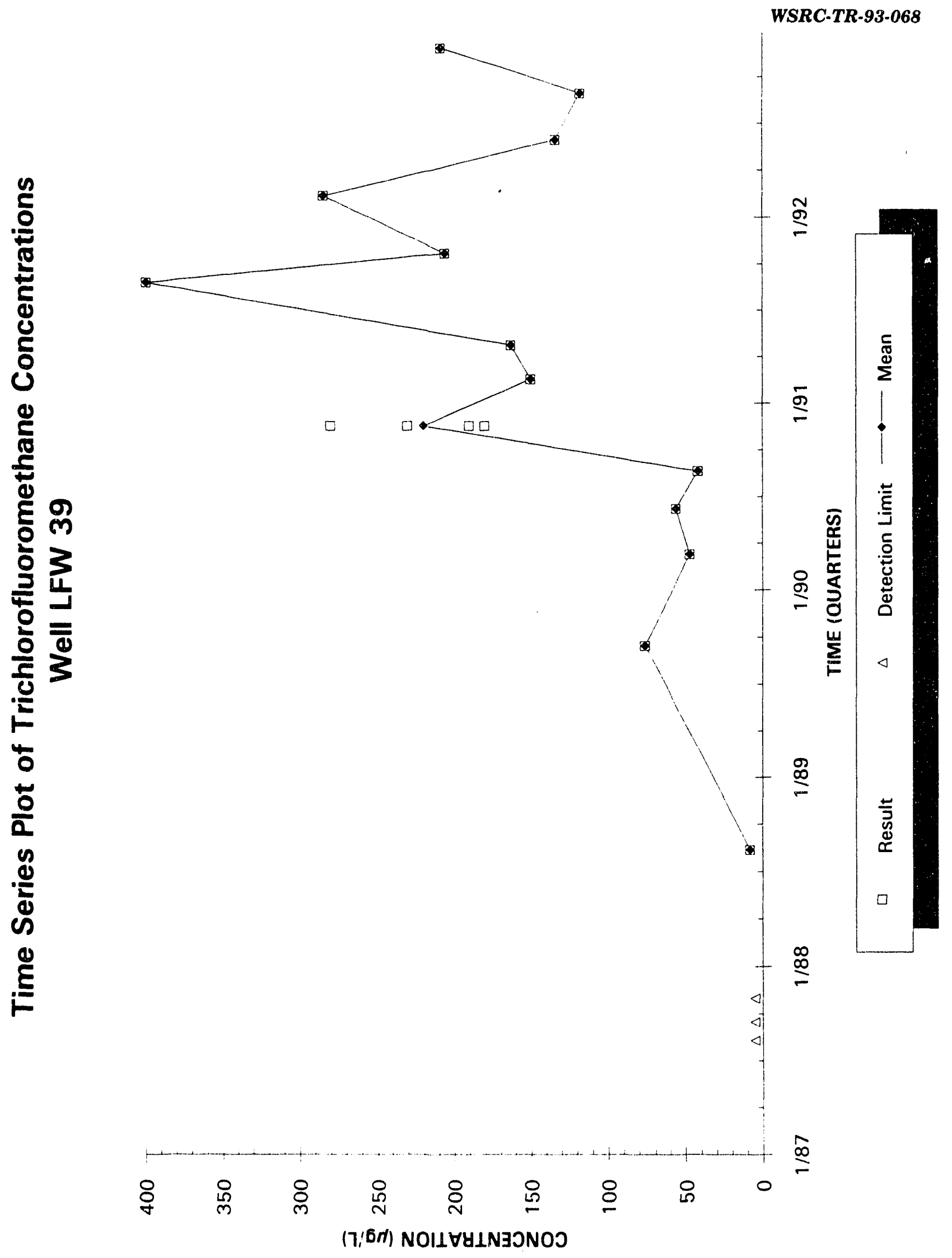



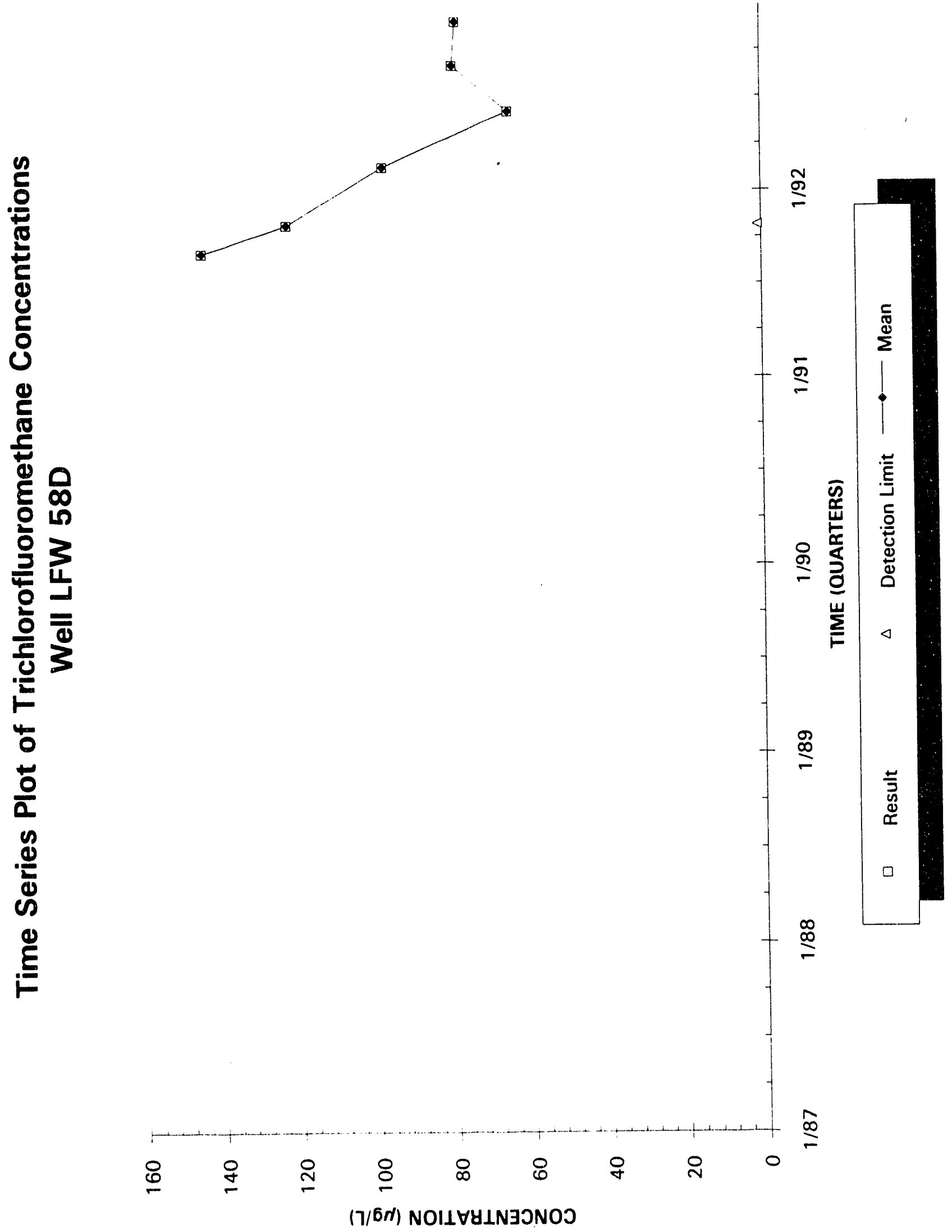


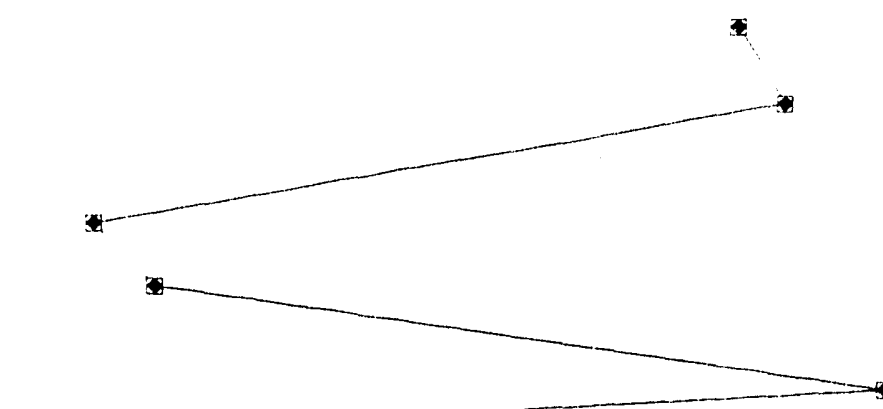

5

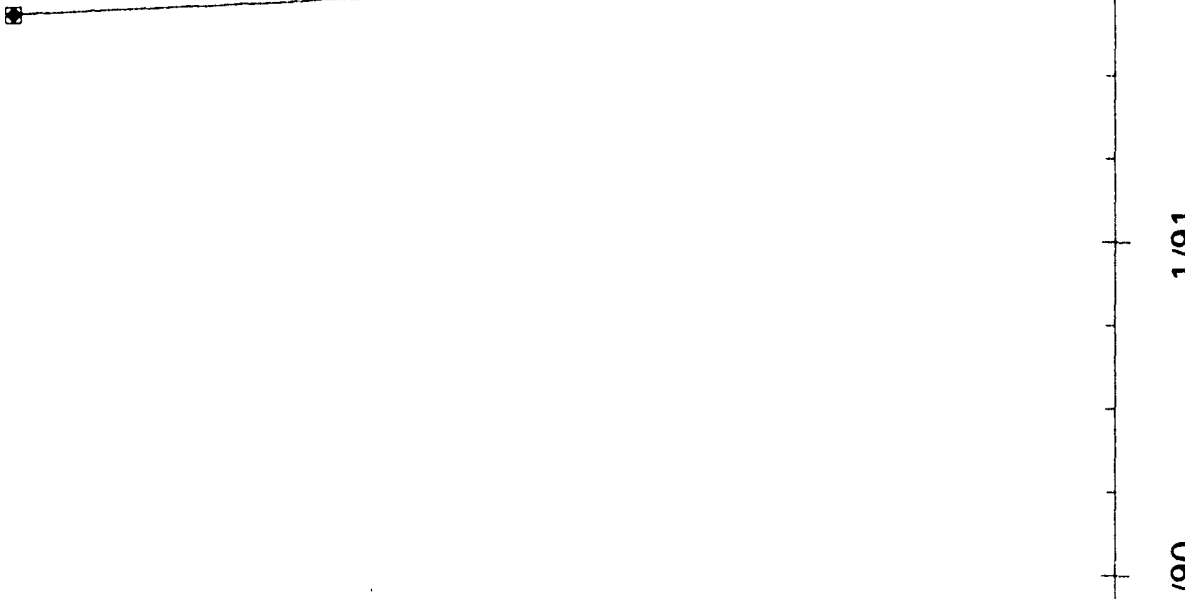

\%ั

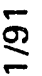

d)

$\frac{2}{2}=$

$\frac{0}{\frac{1}{0}} \sum^{\overline{0}}$

E

ธ

뭄

o

응

๘

$\stackrel{\Xi}{E}$

$\stackrel{\boldsymbol{\Xi}}{\underline{\Xi}}$

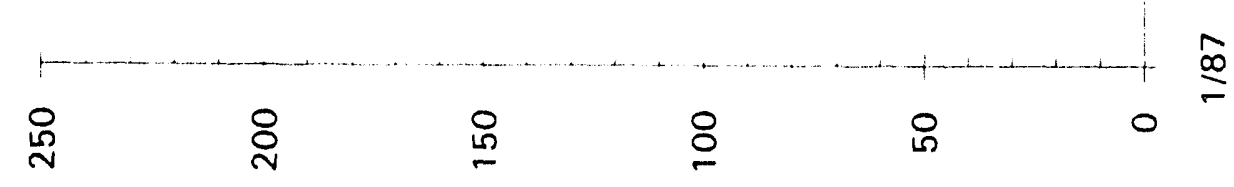

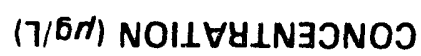


WSRC-TR-93-068
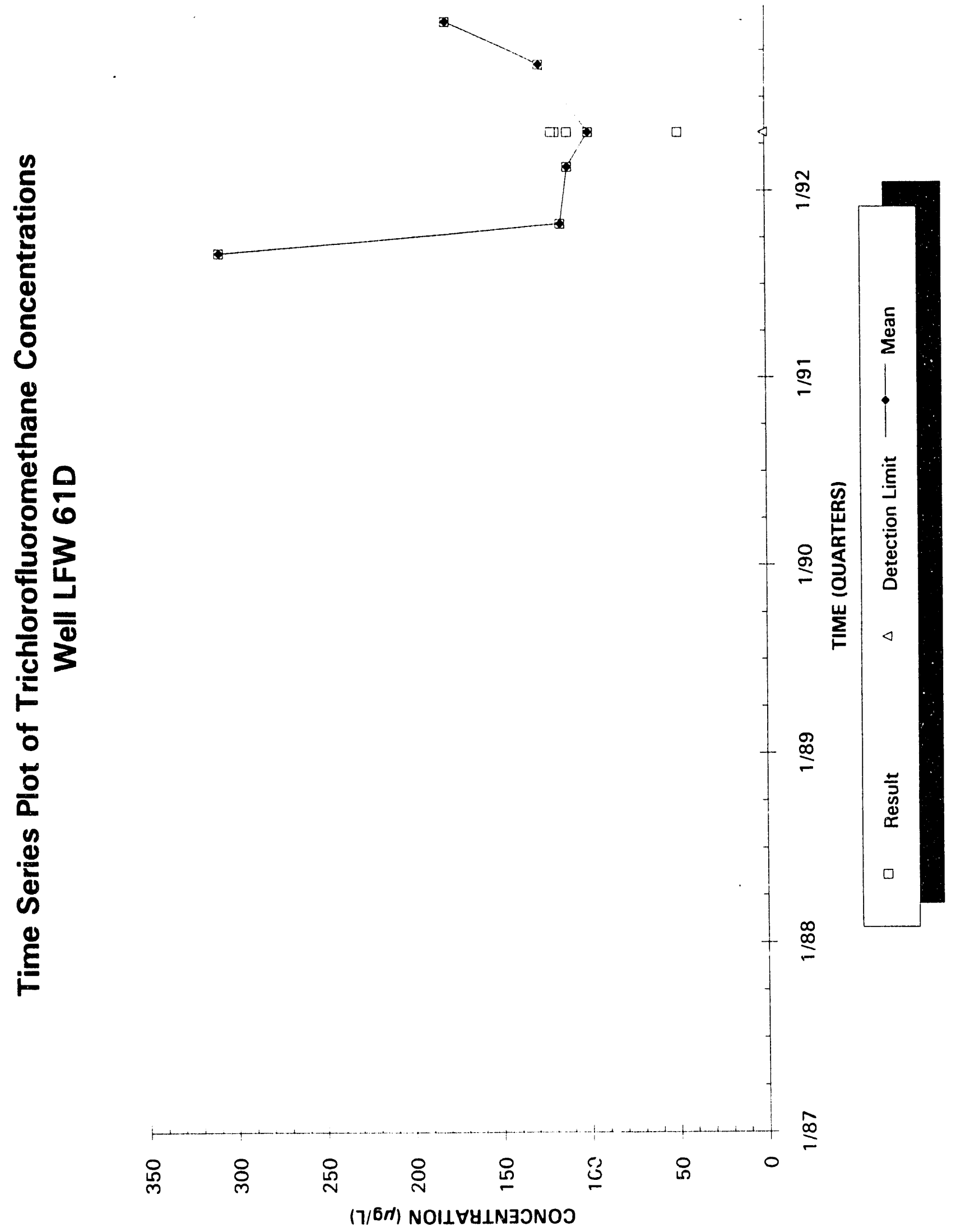
WSRC-TR-93-068

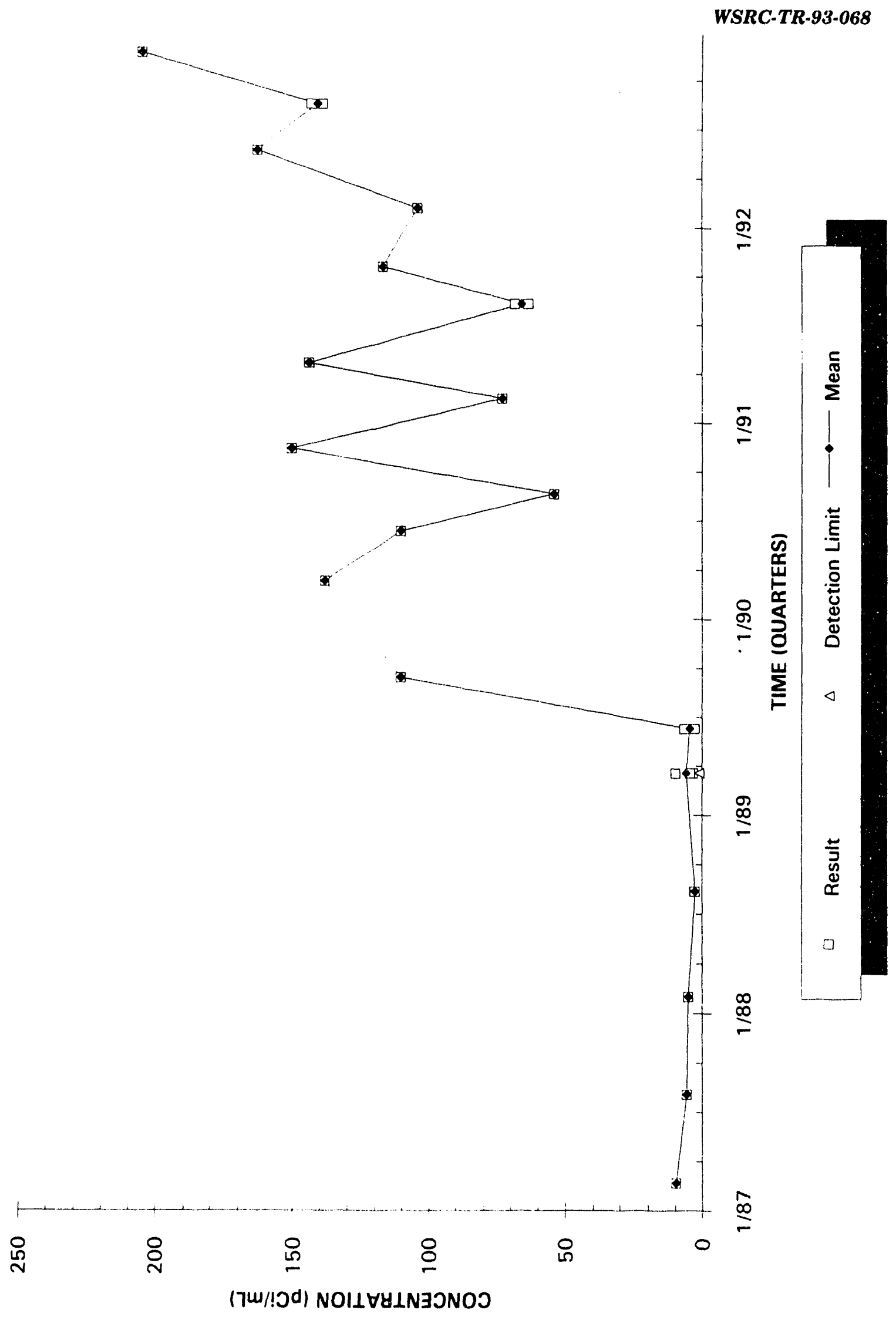




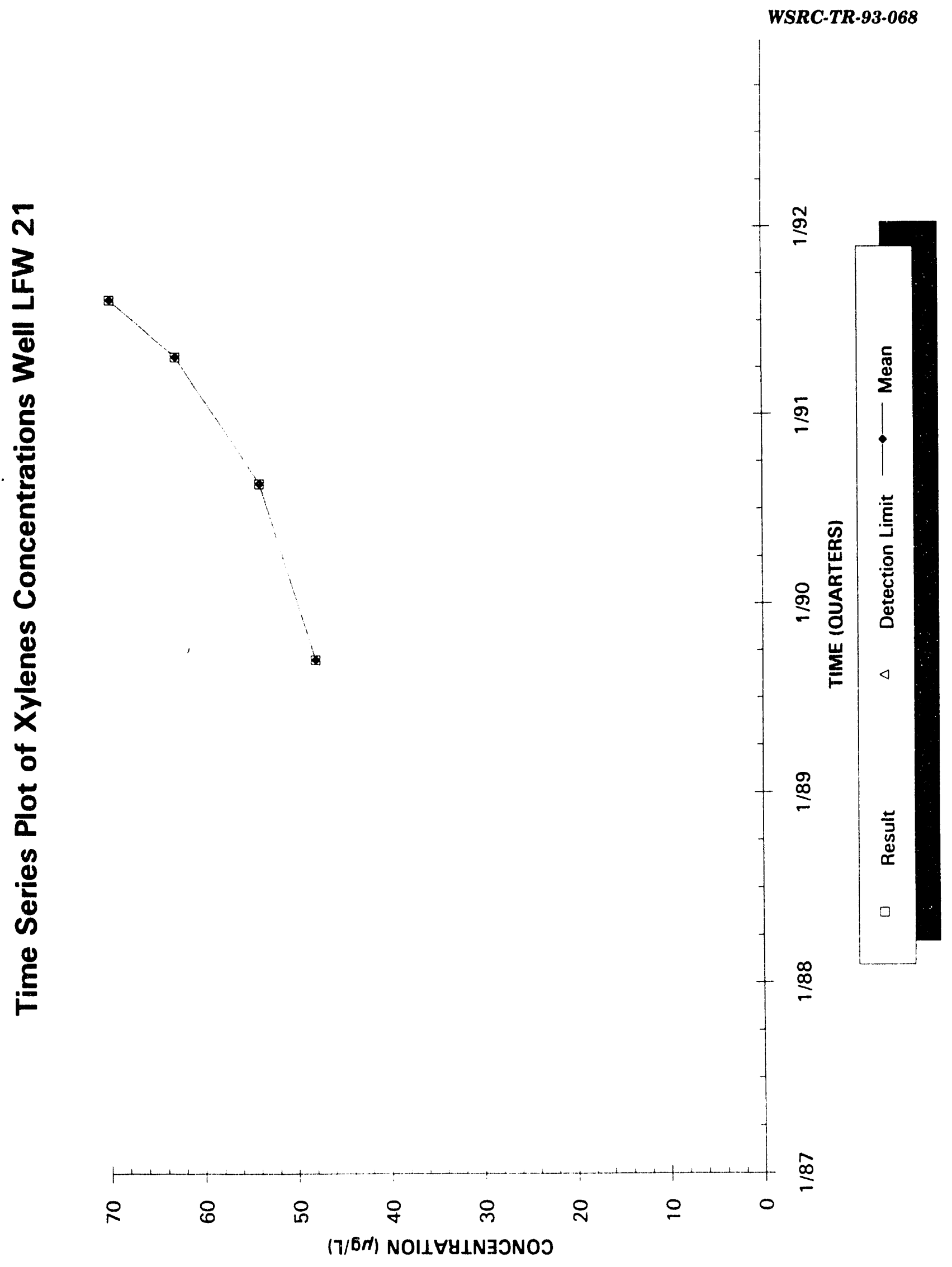



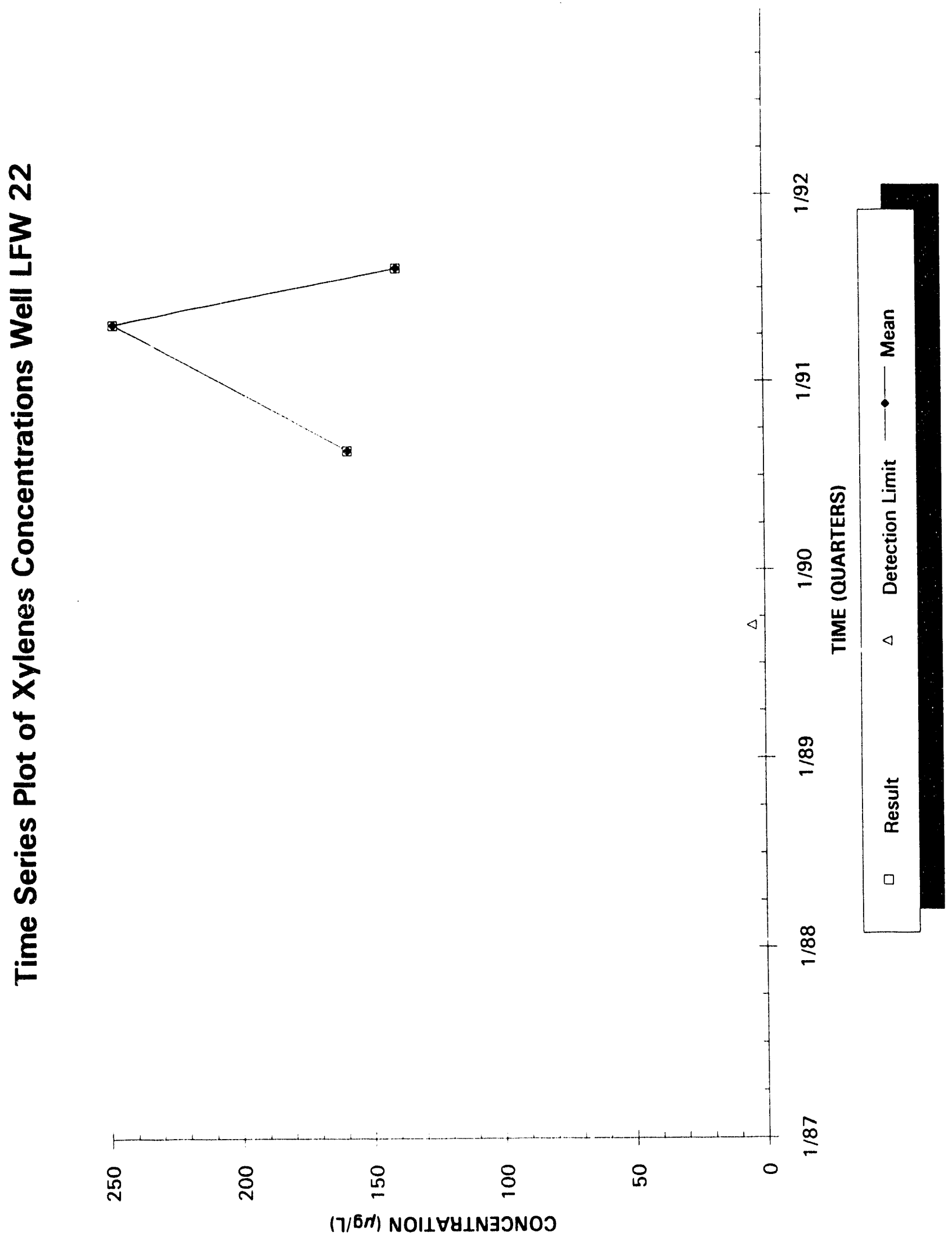
WSRC-TR-93-068

-

○

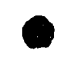

$\bullet$

$\bullet$

- 


\section{Appendix G - Hydrographs}




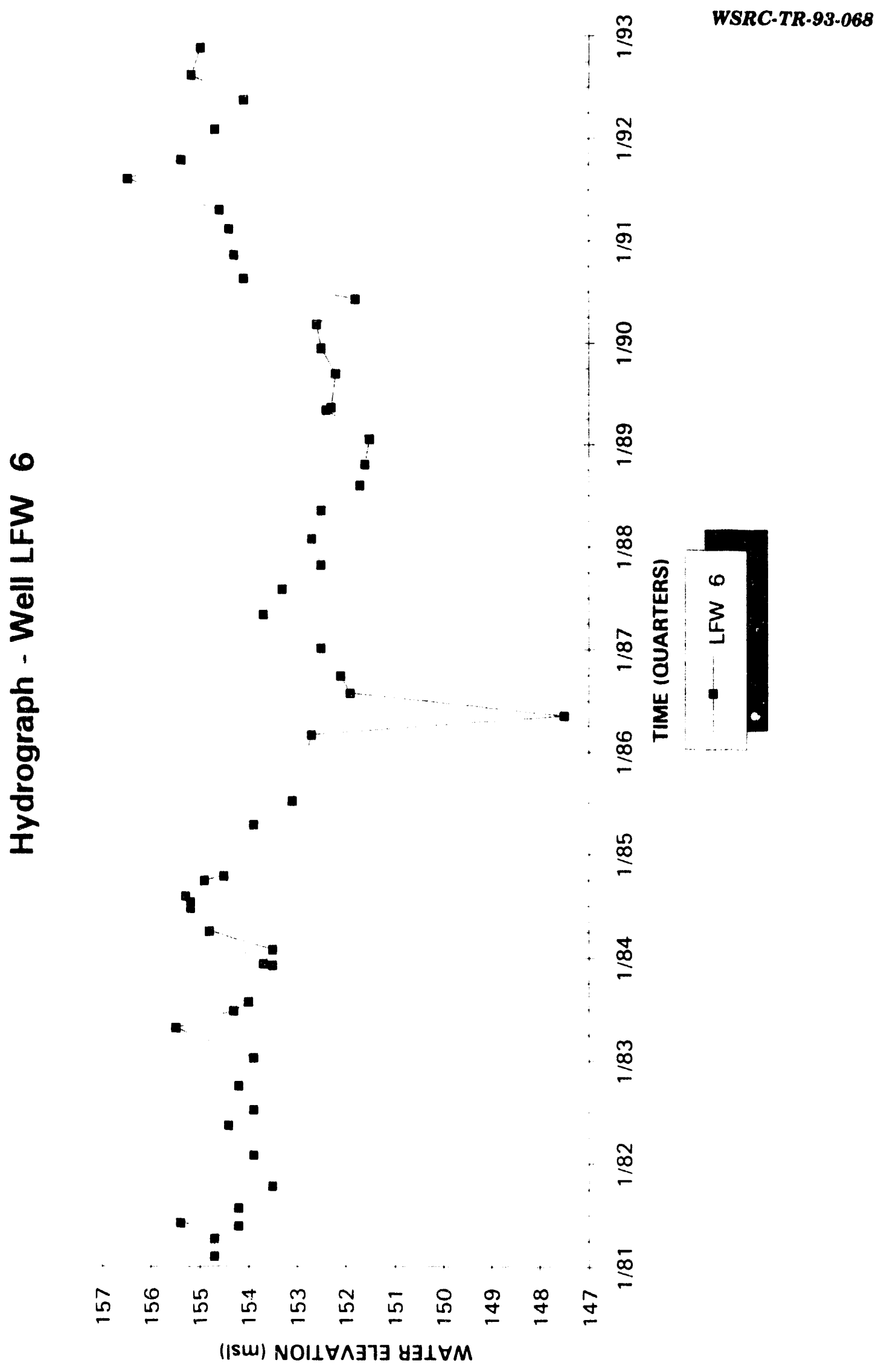




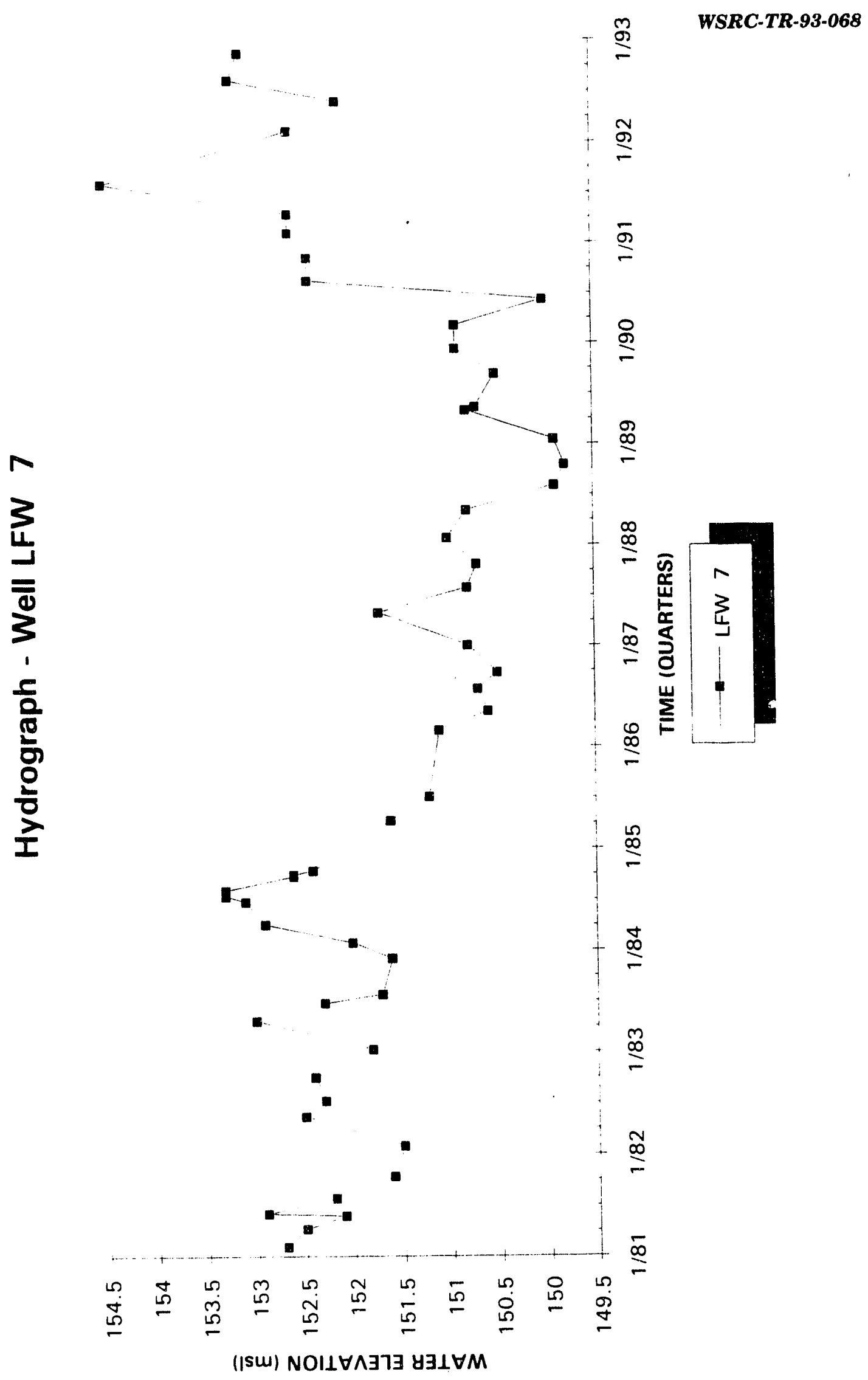




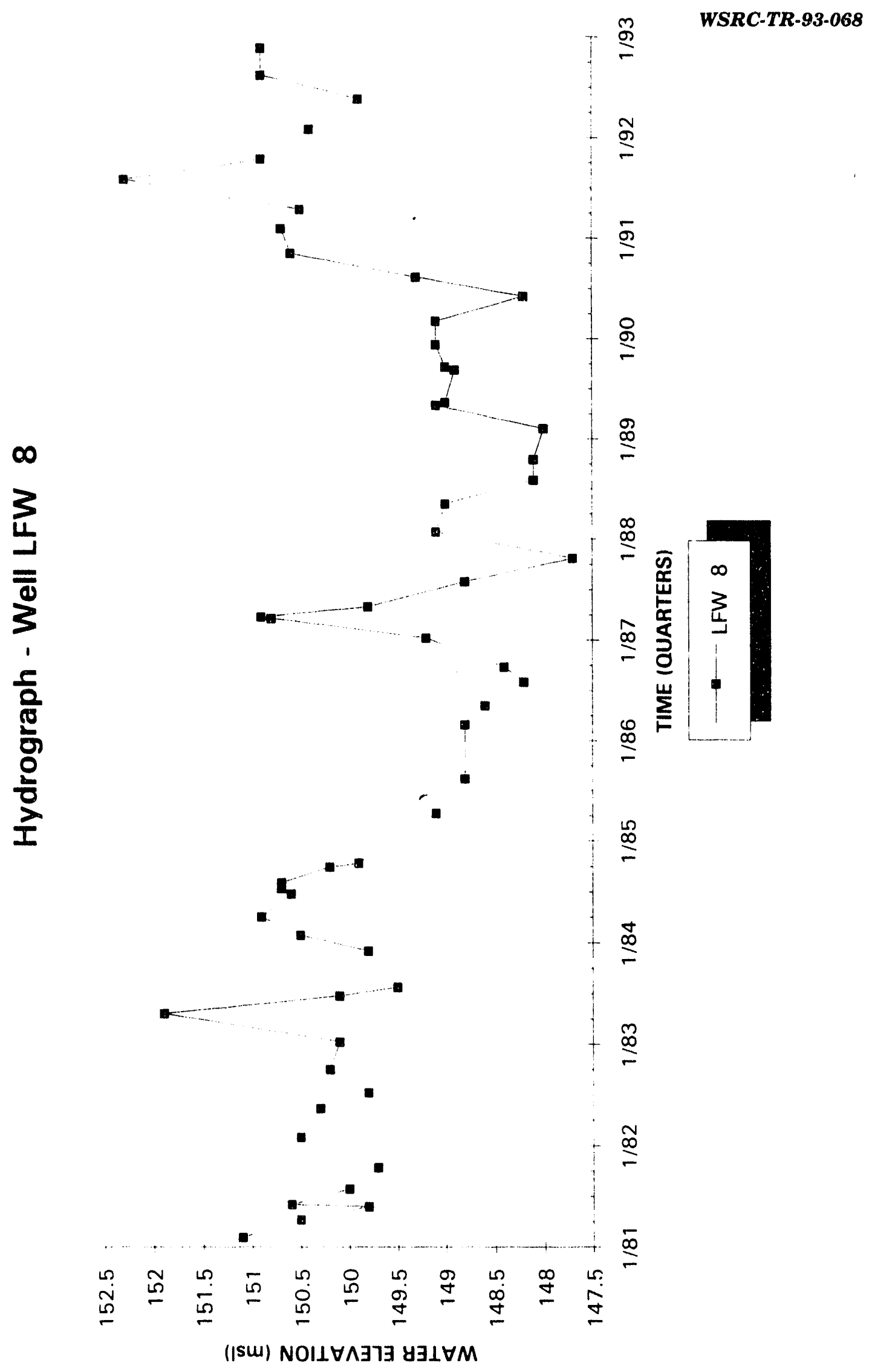

$\odot$ 


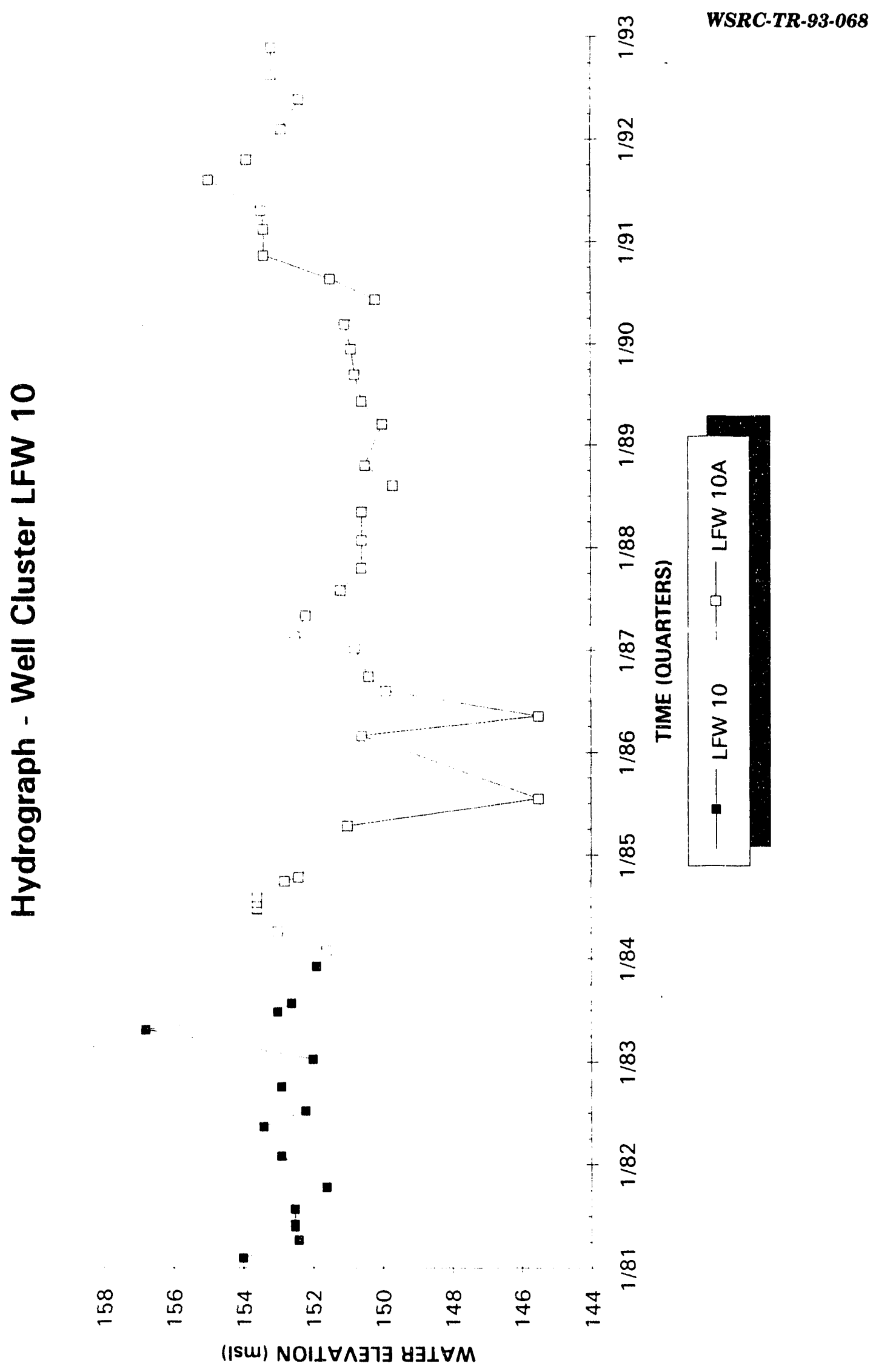




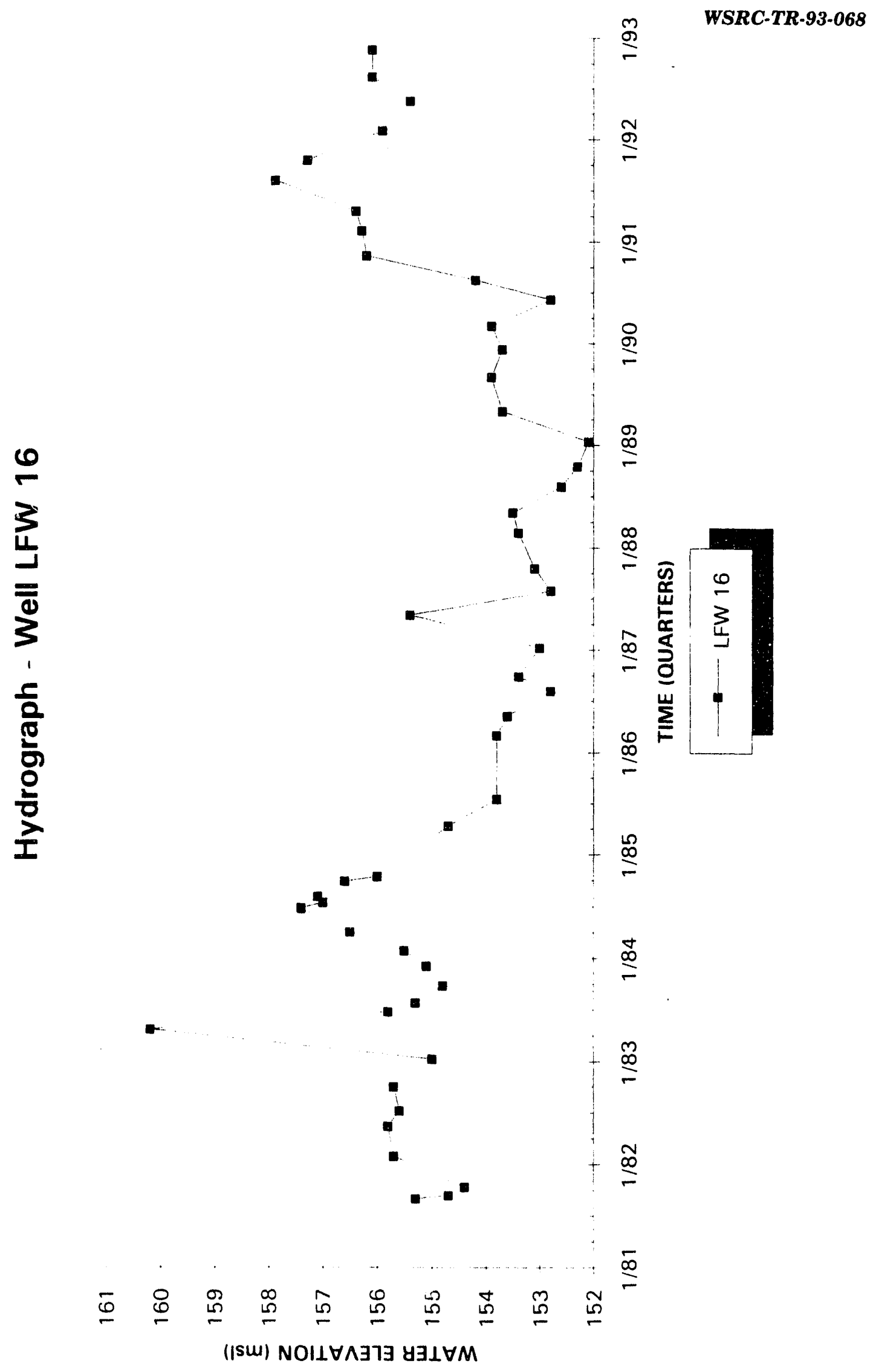




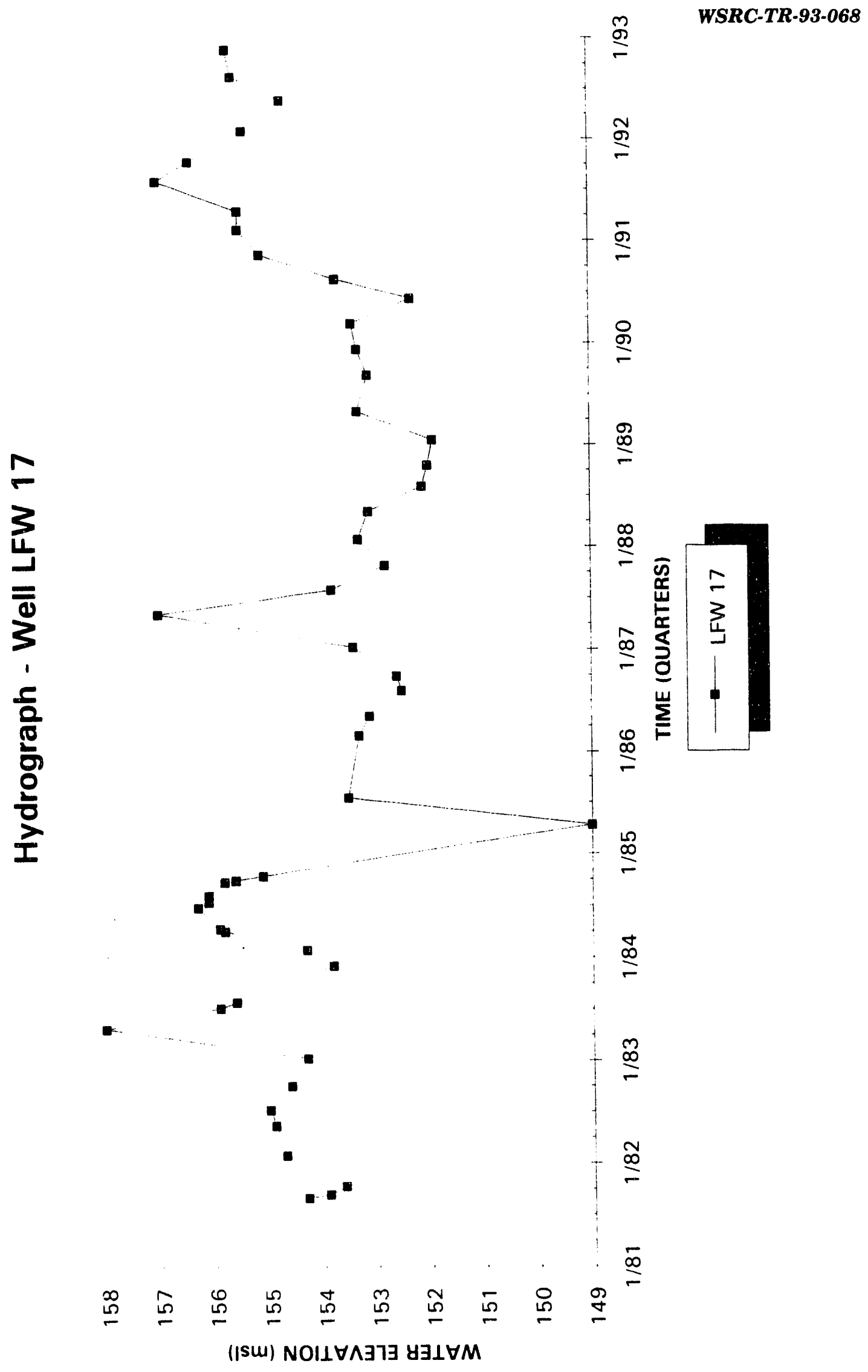




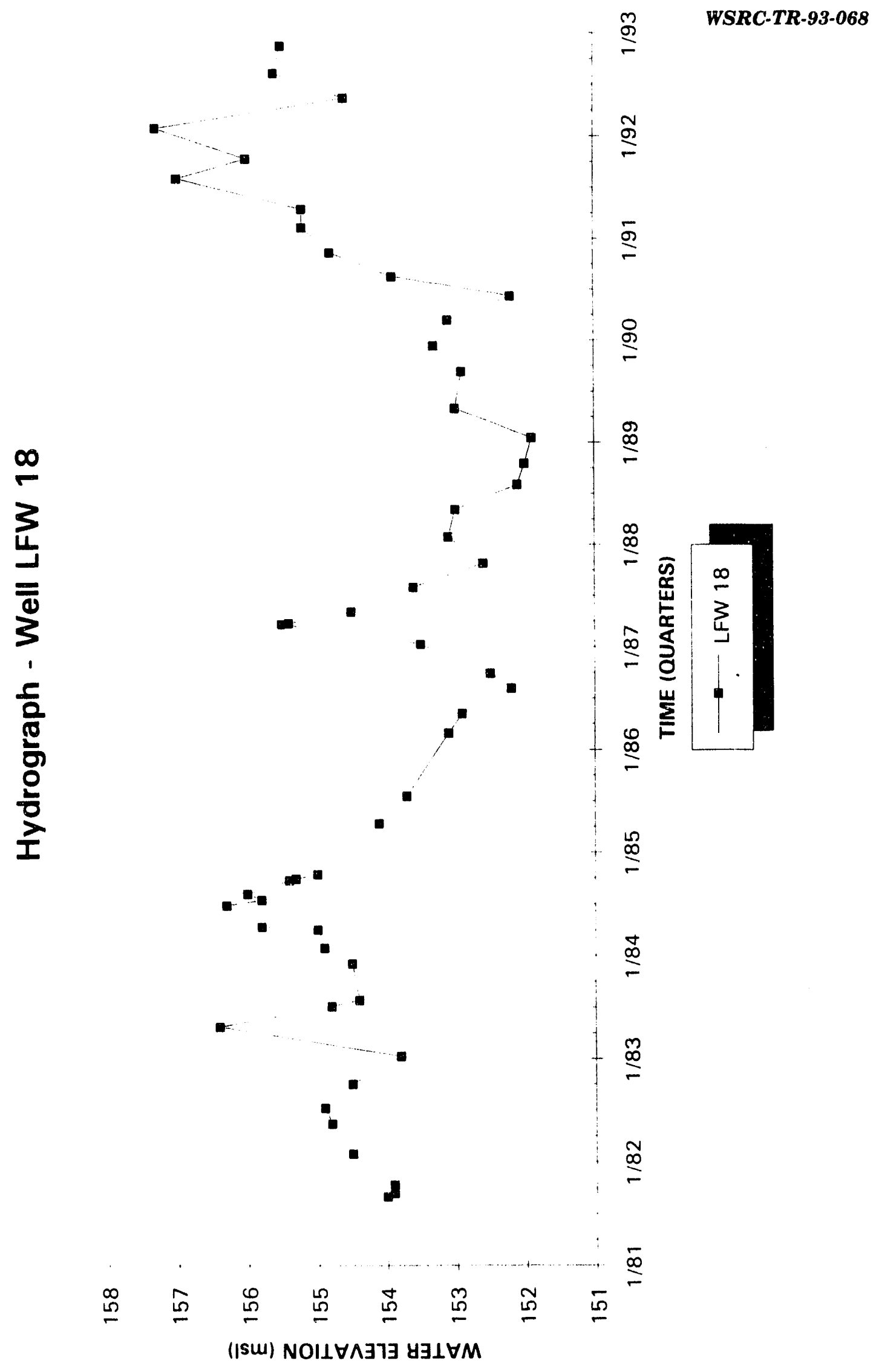




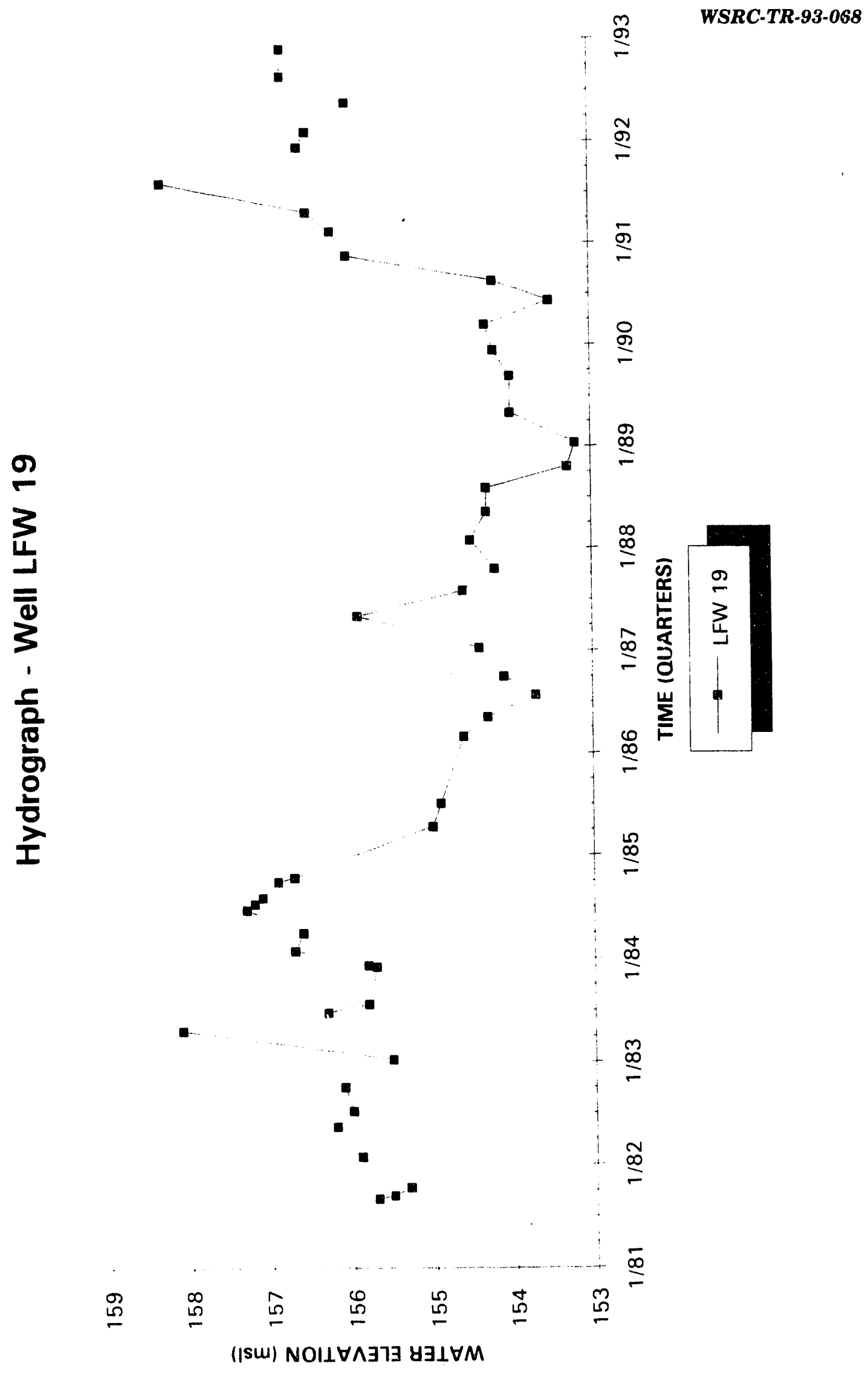




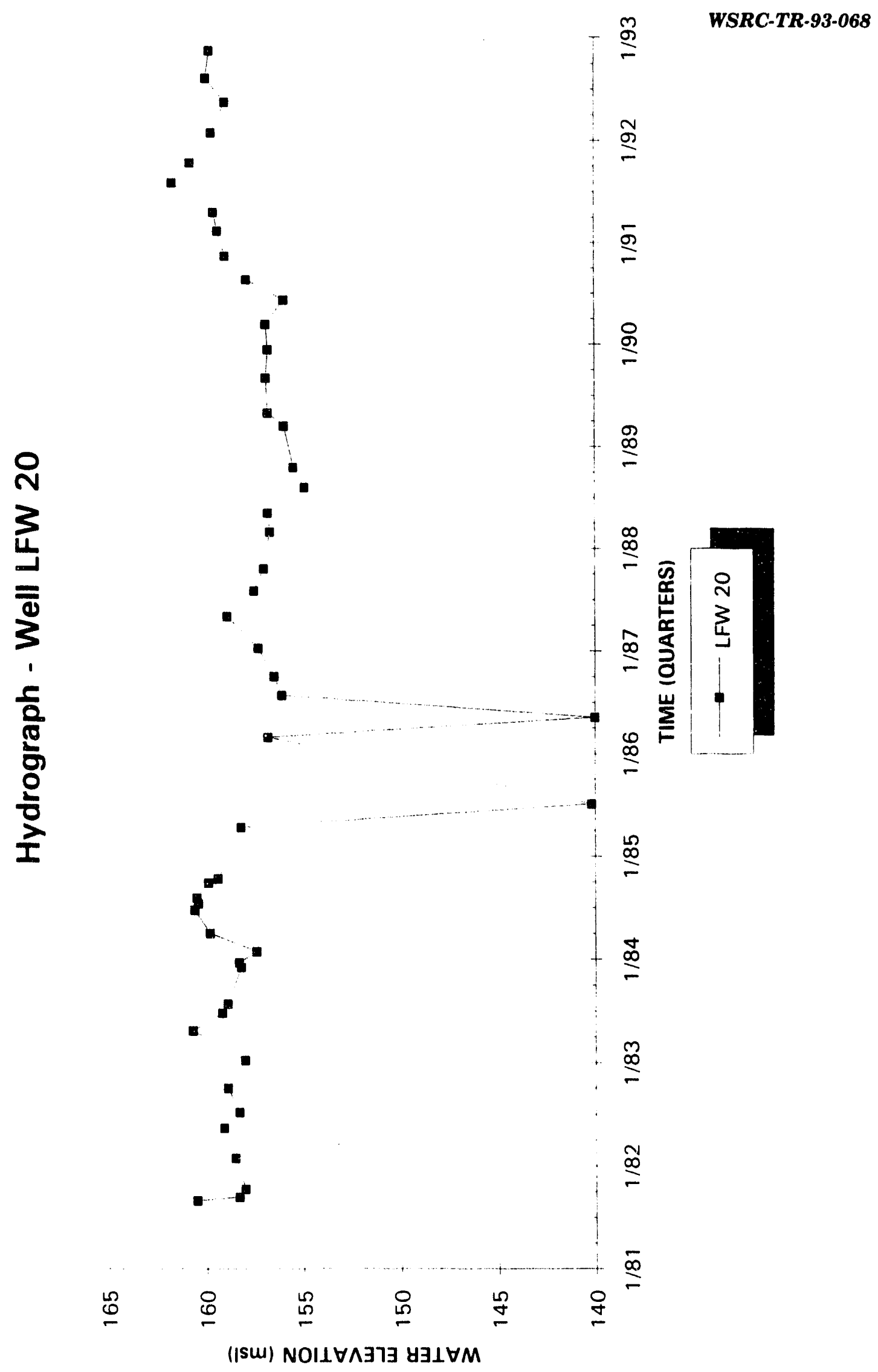




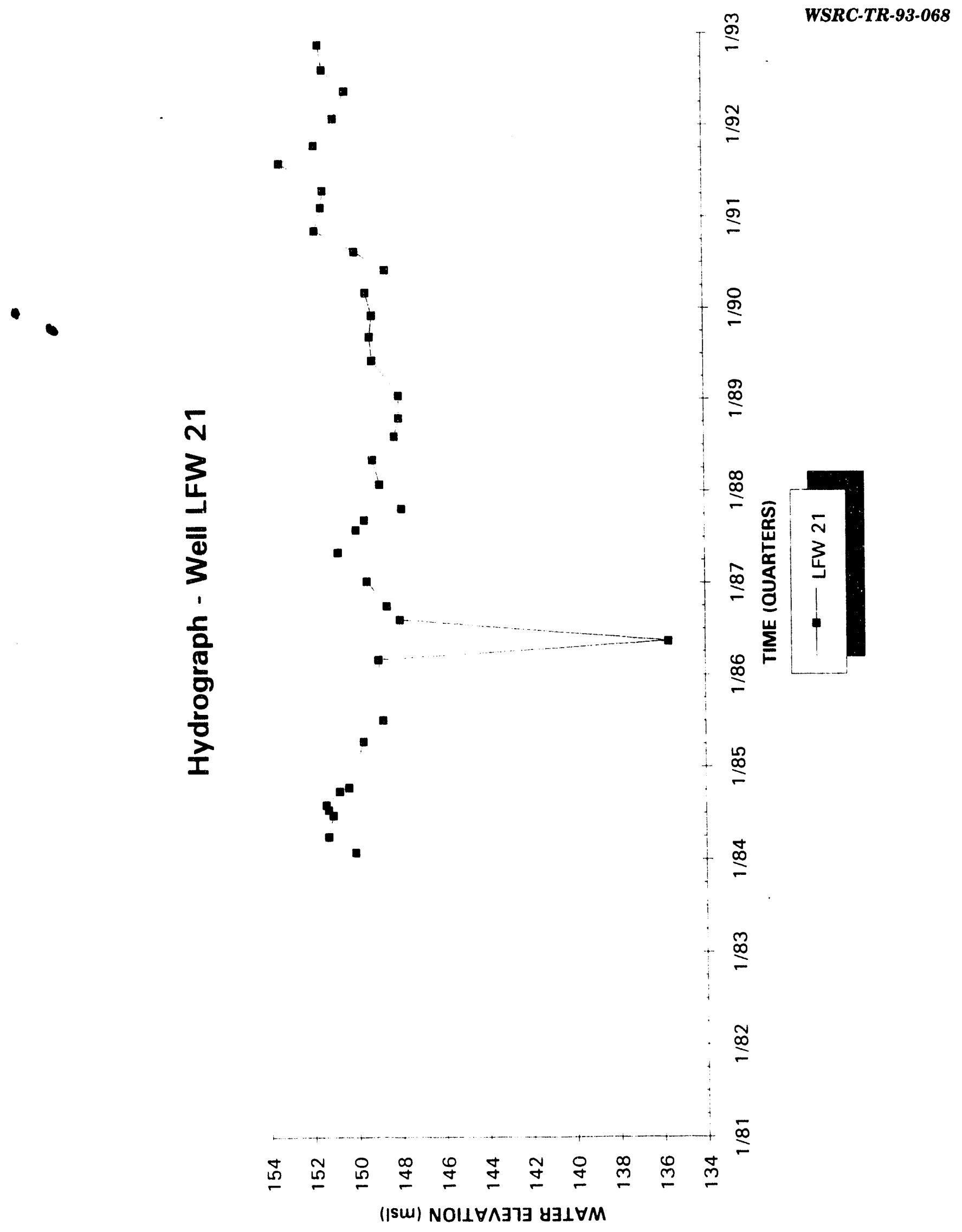




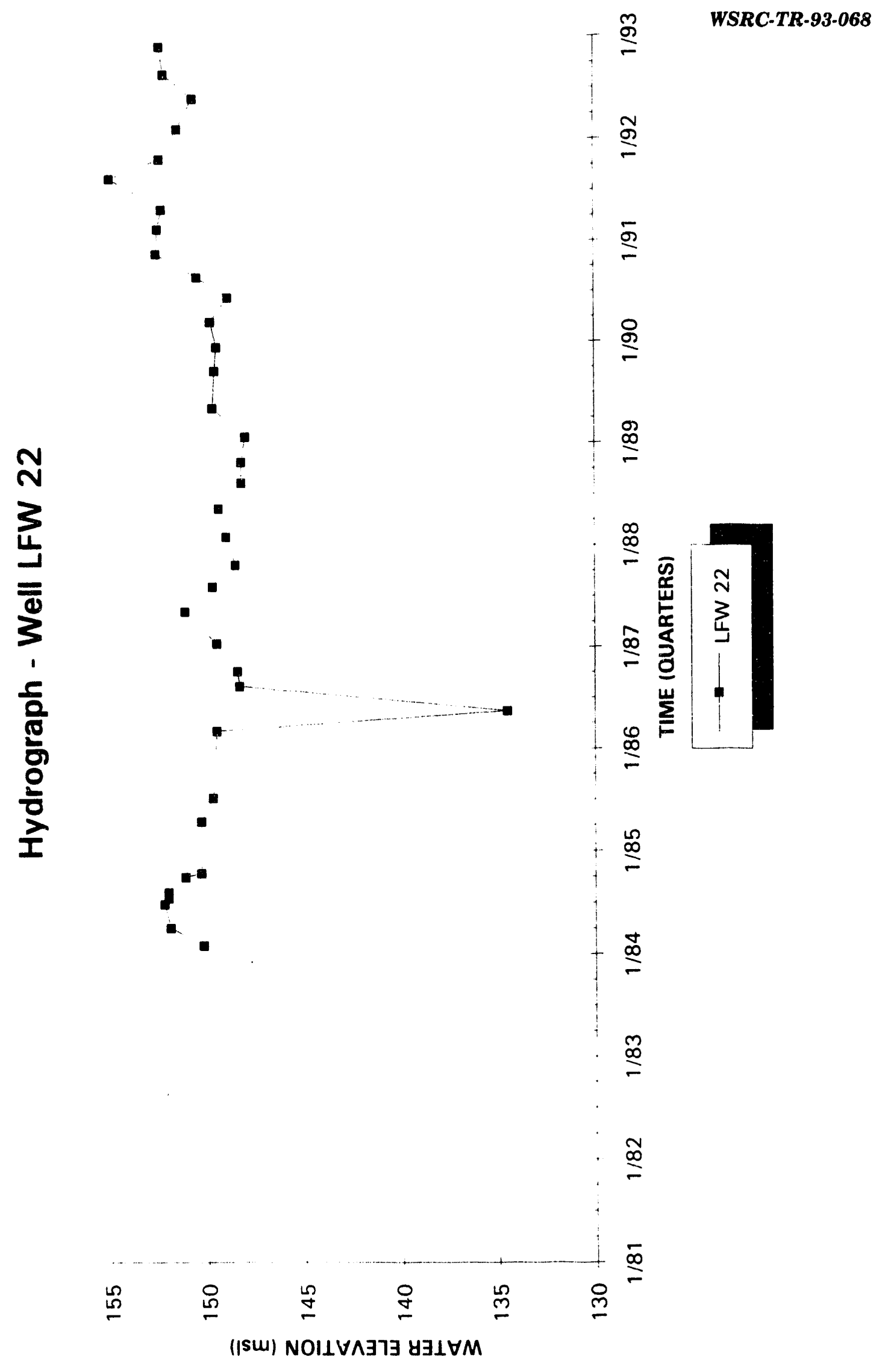




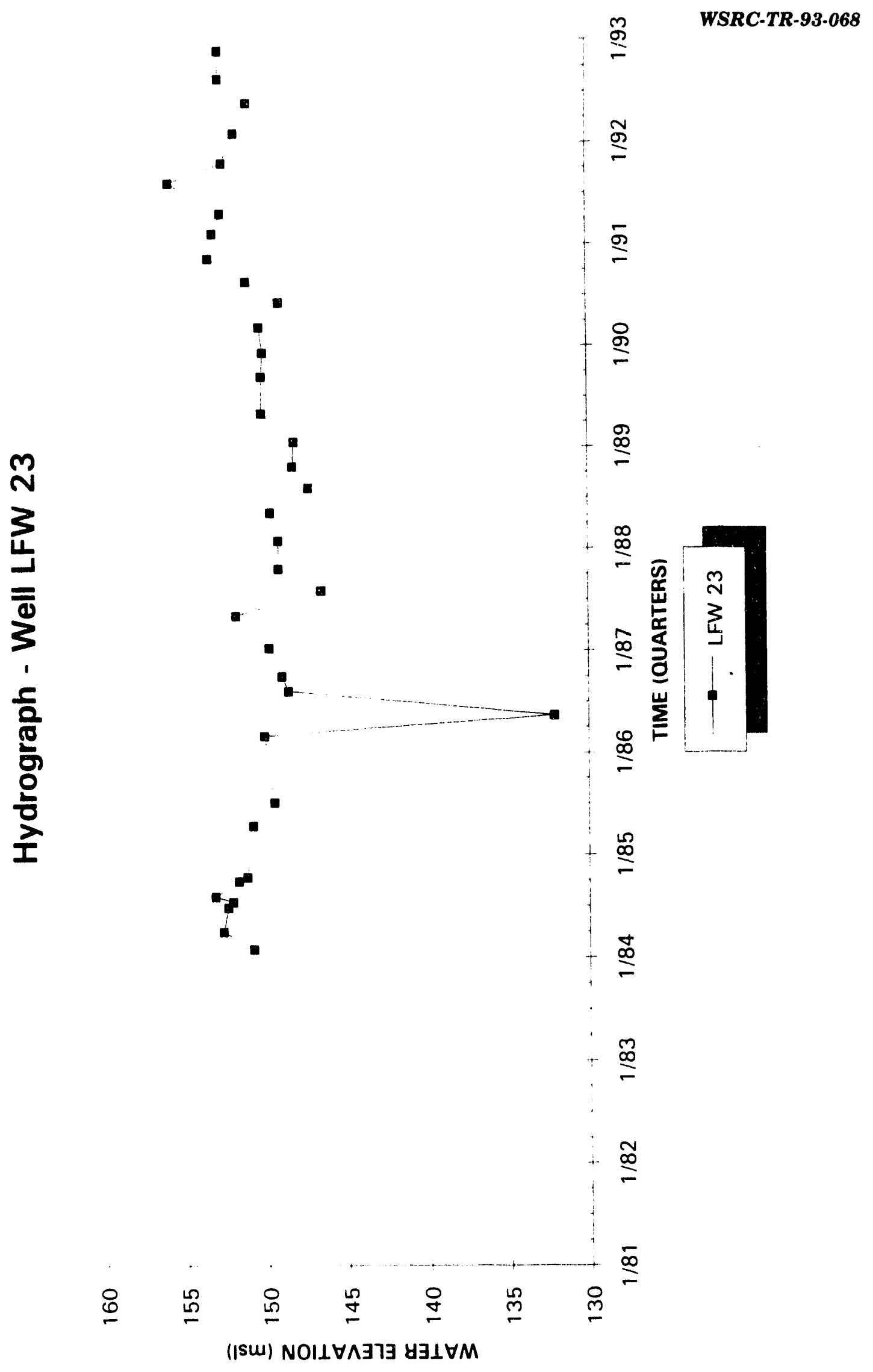




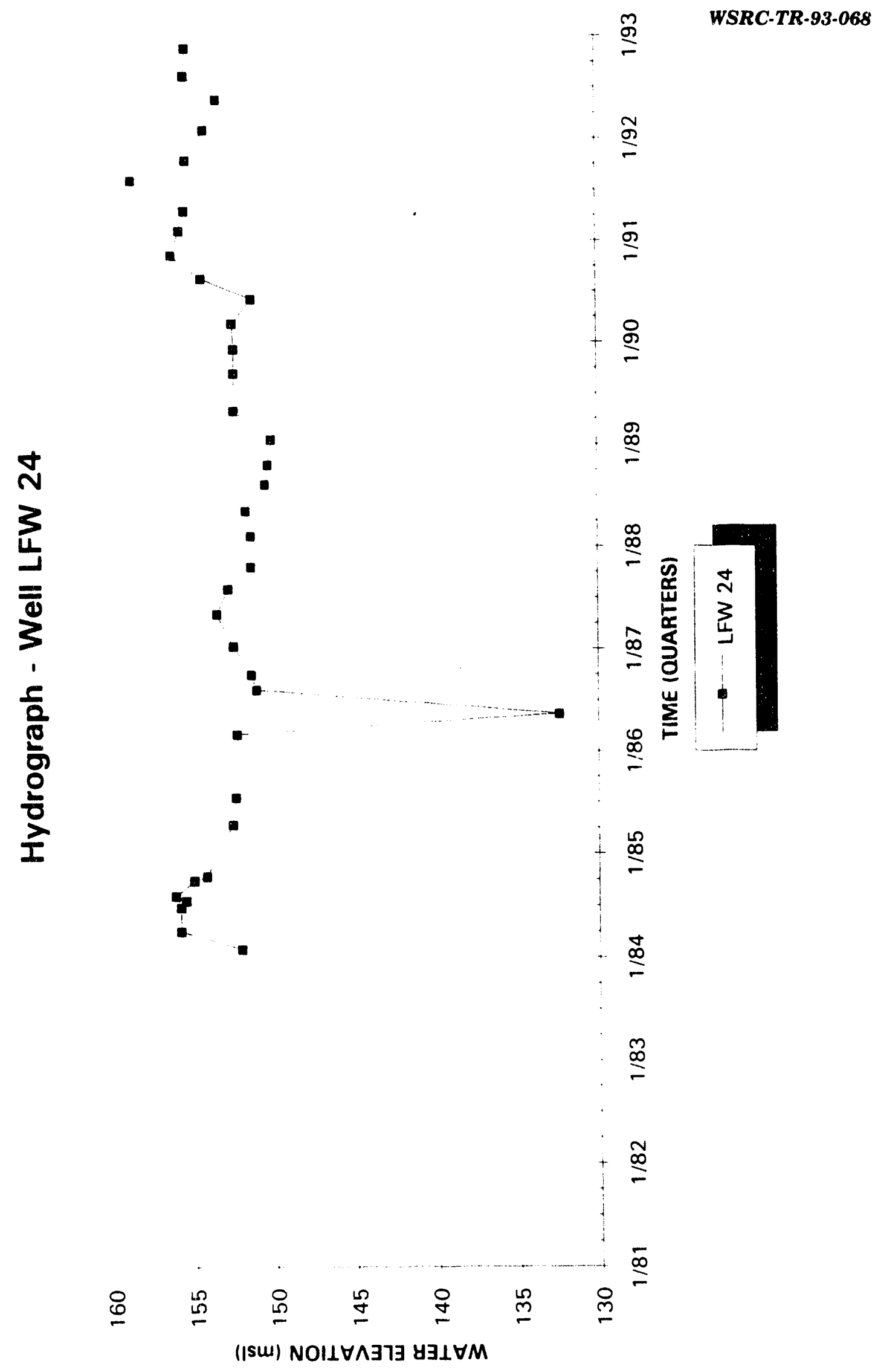




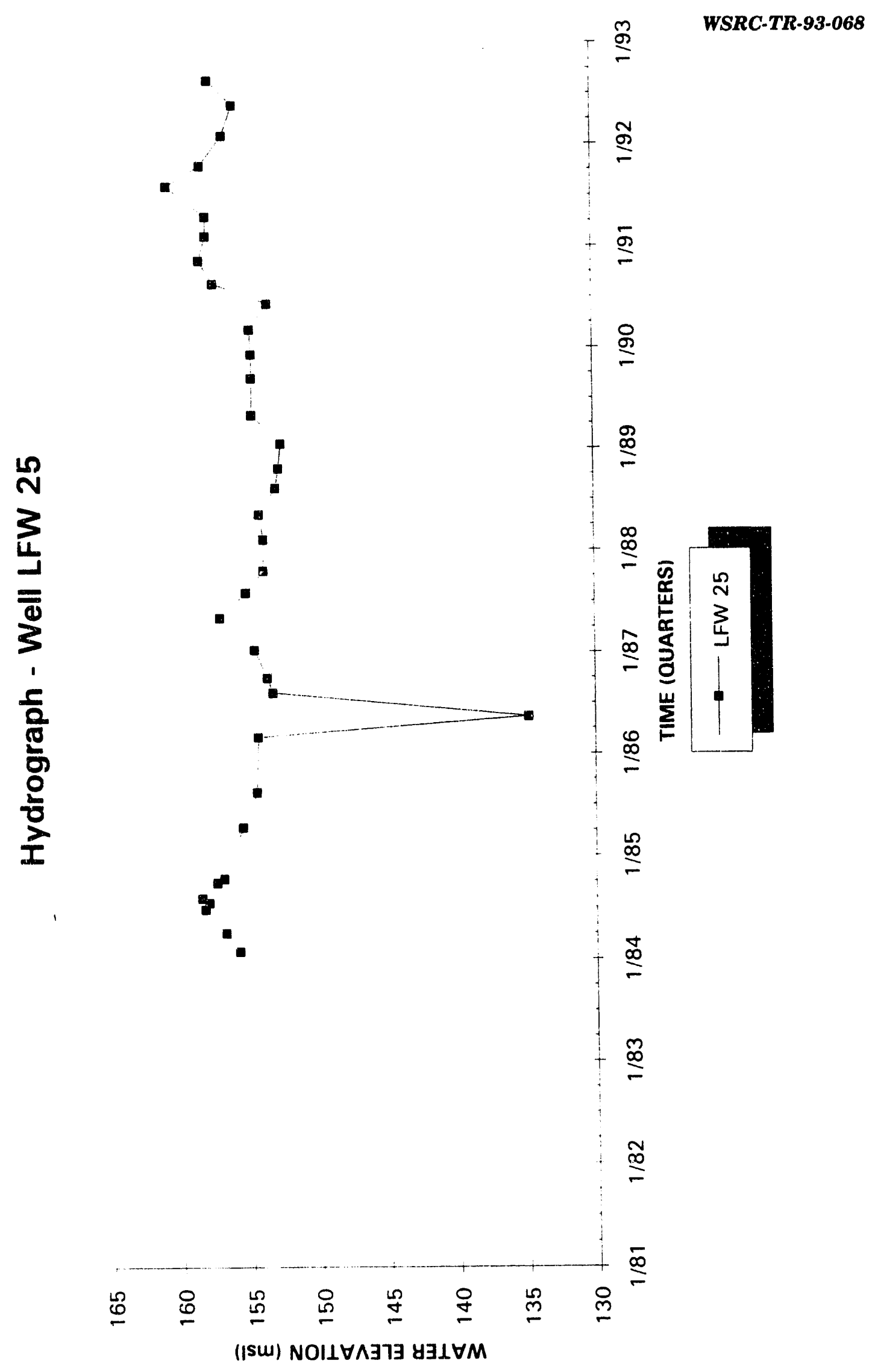




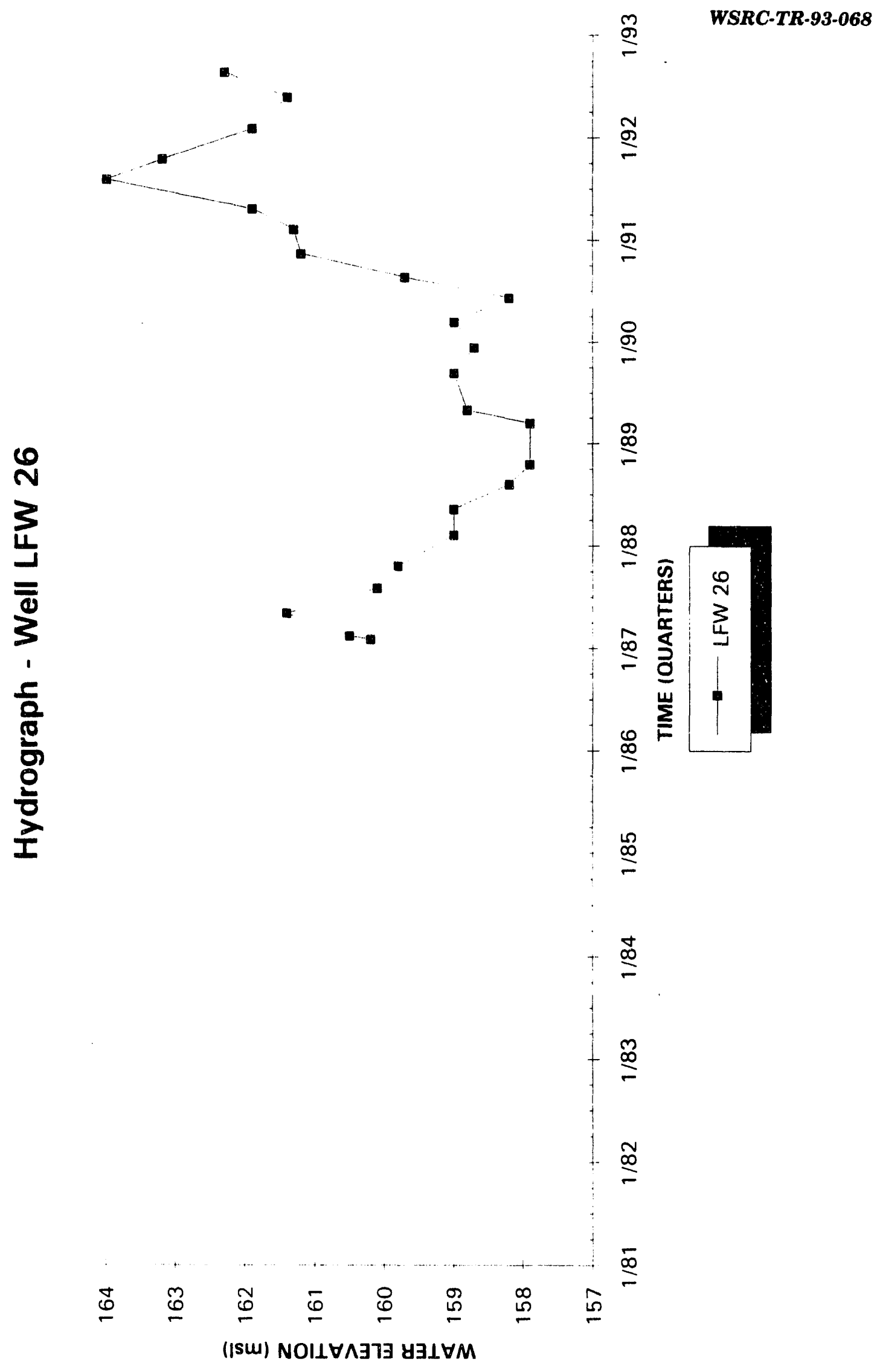




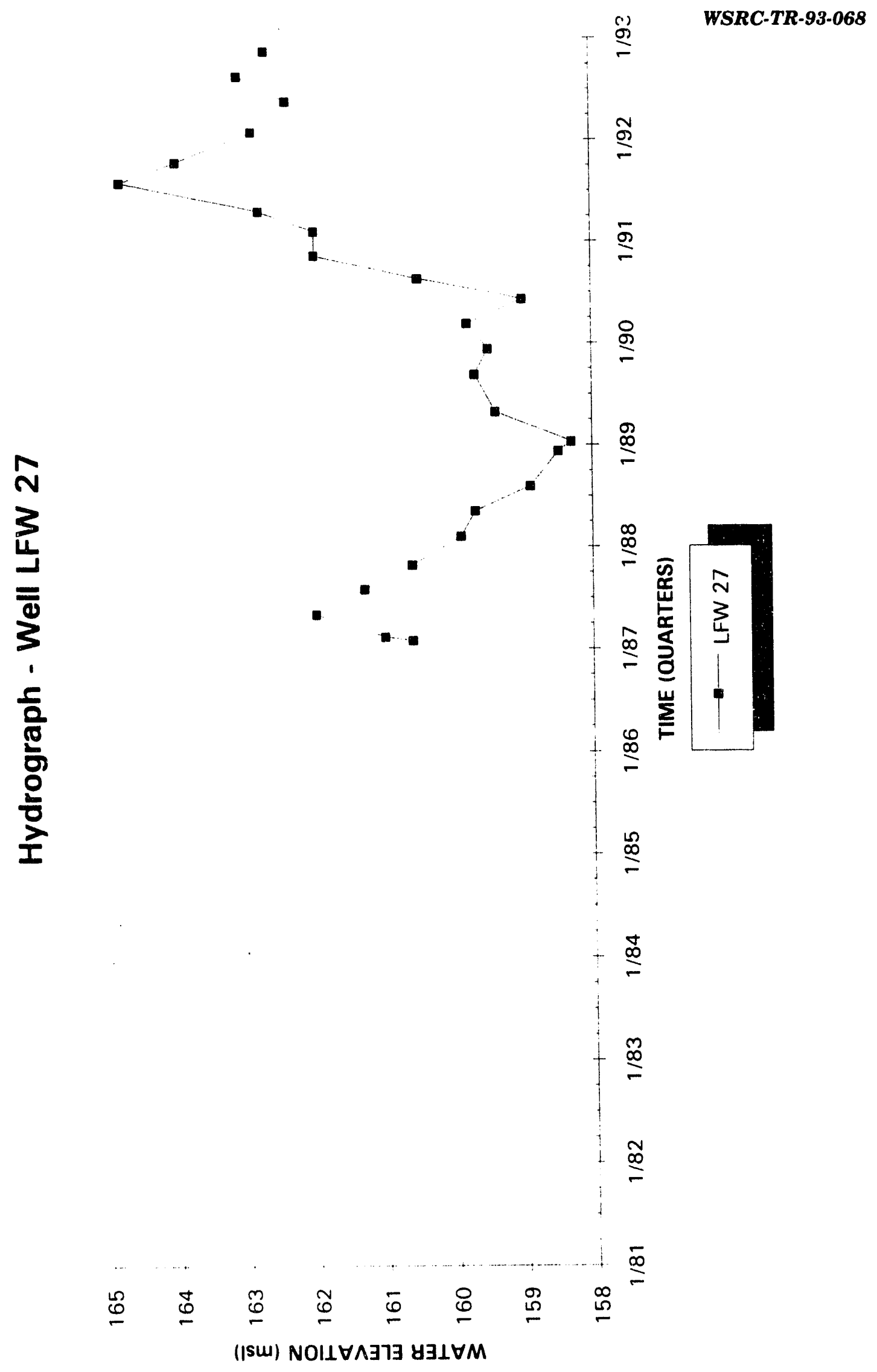




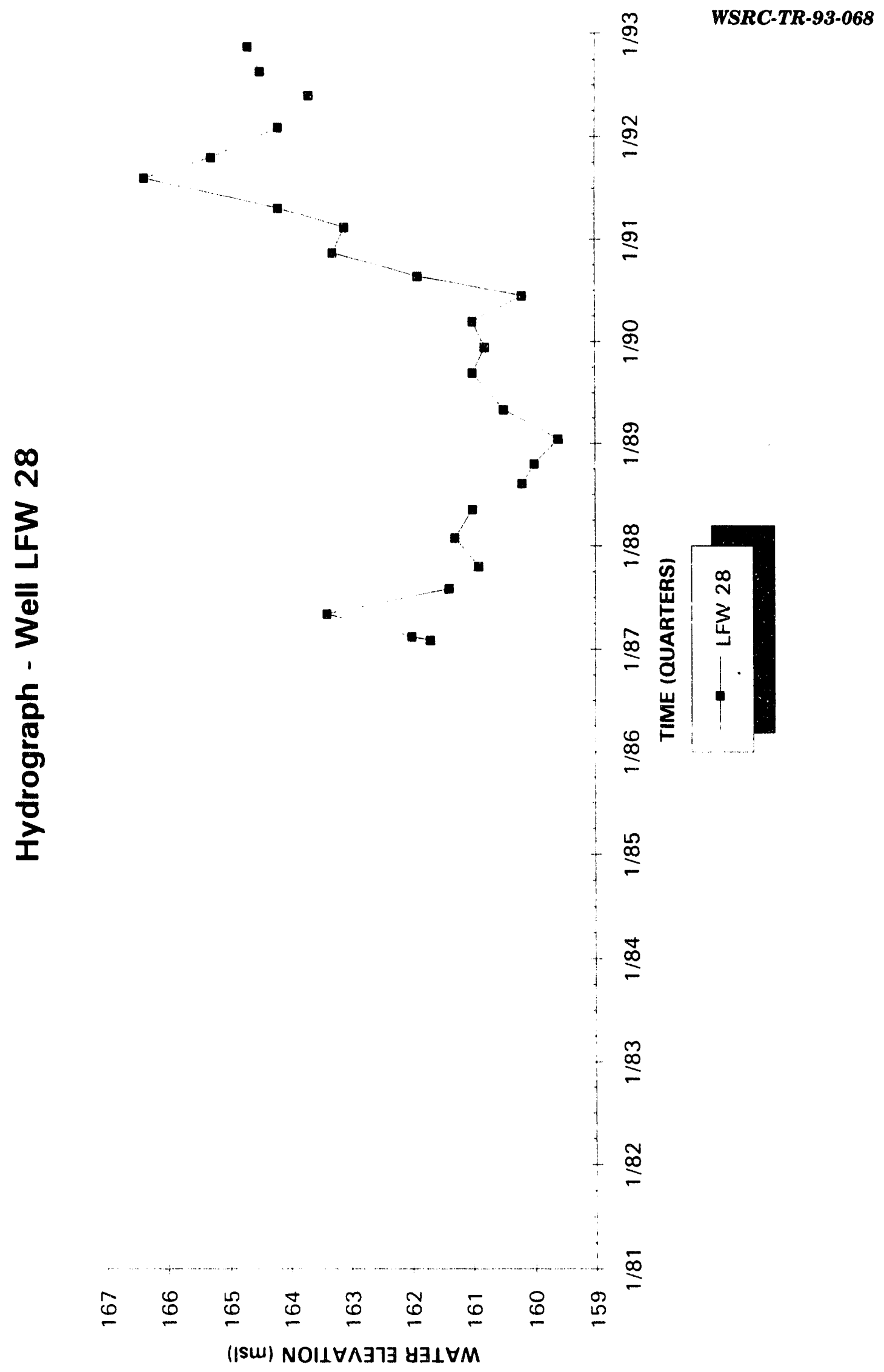




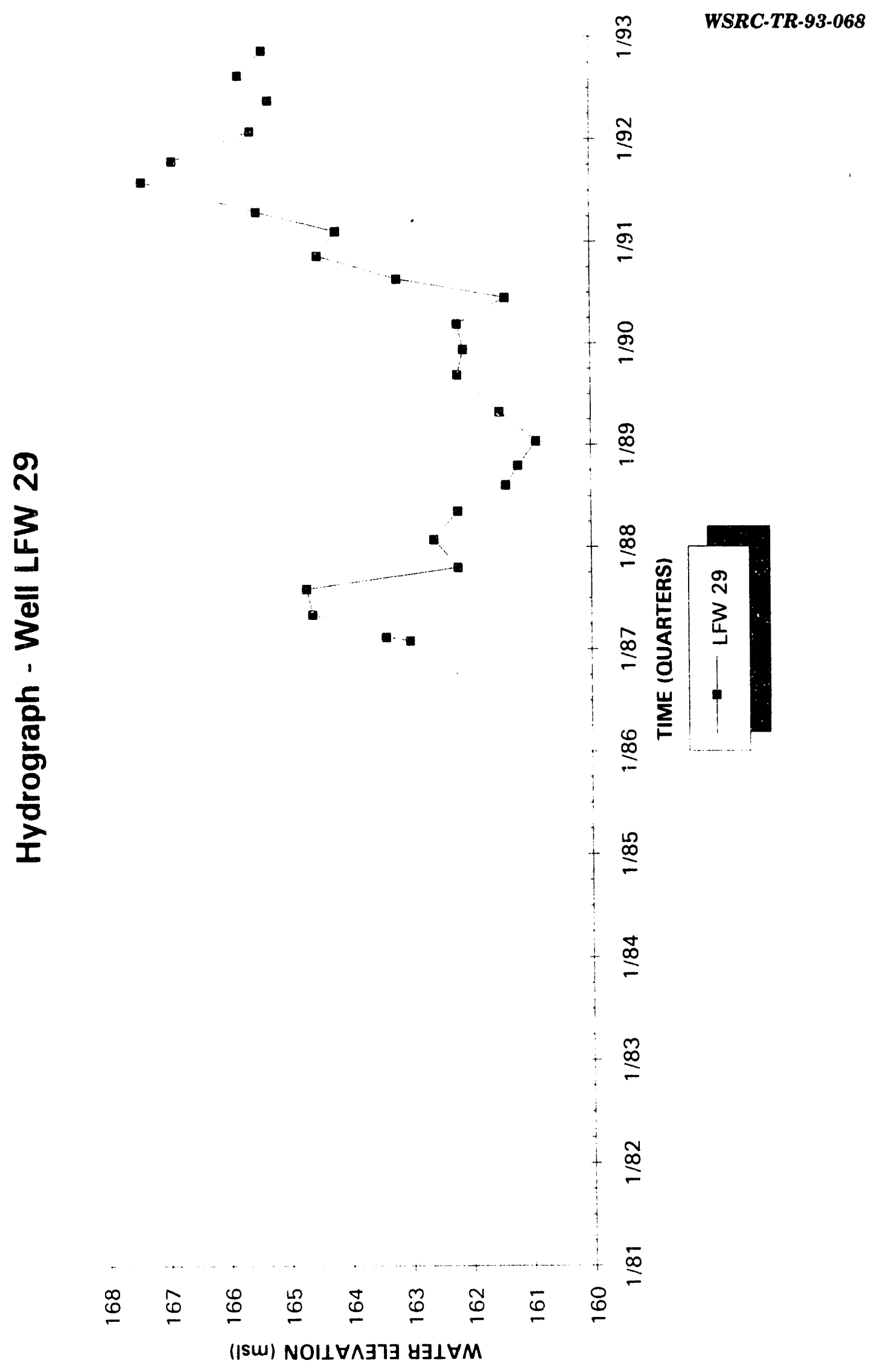




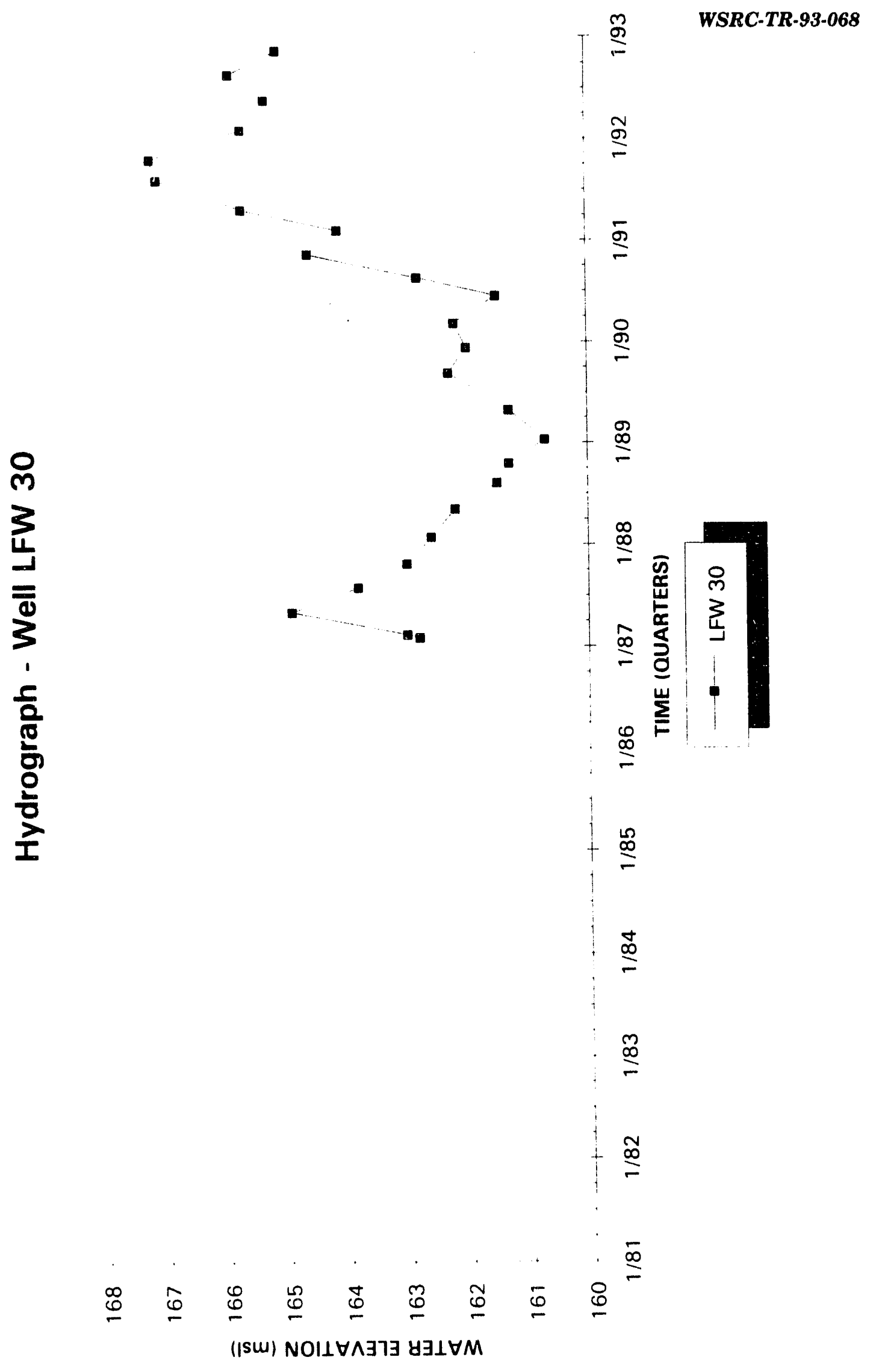




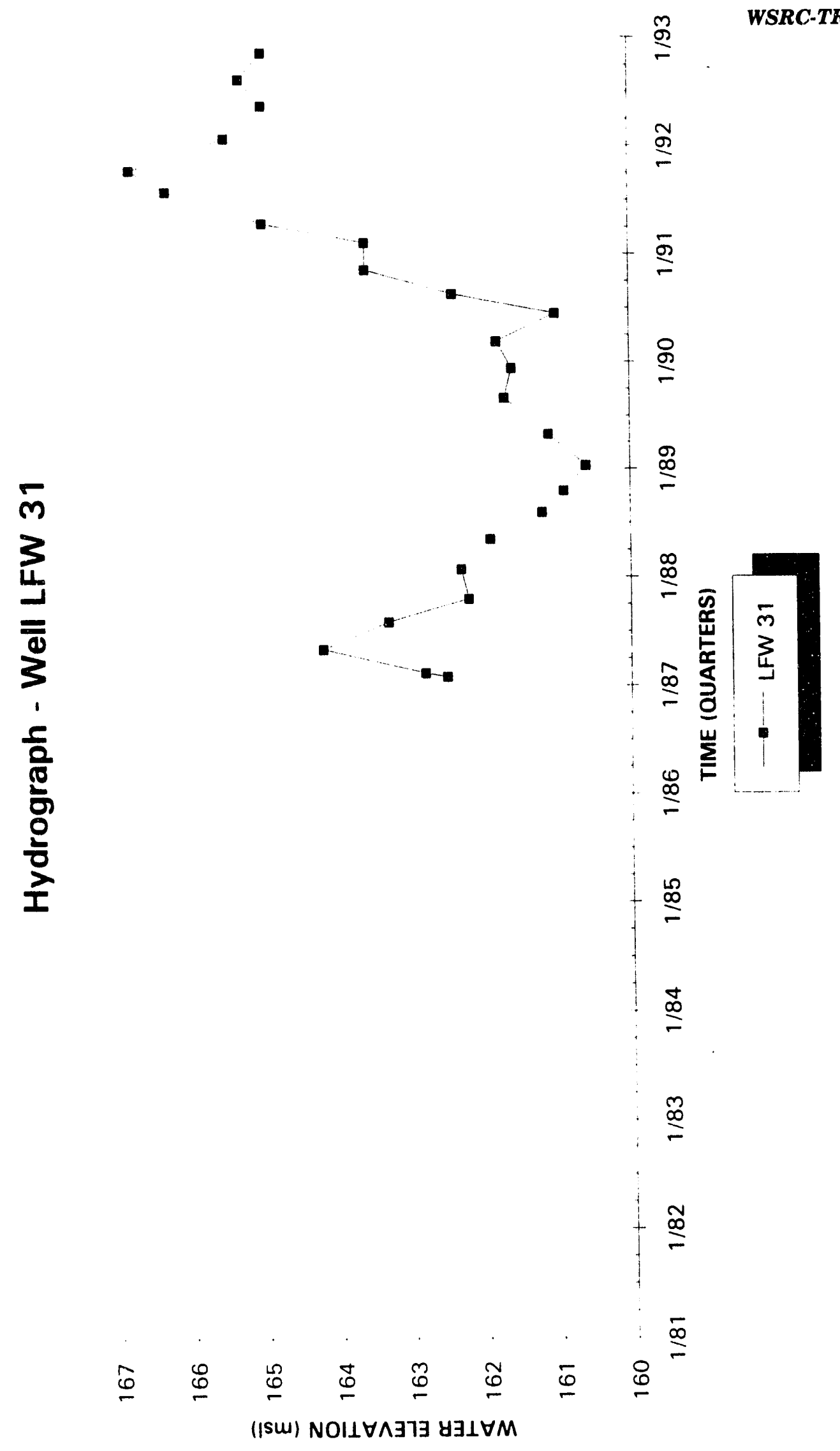




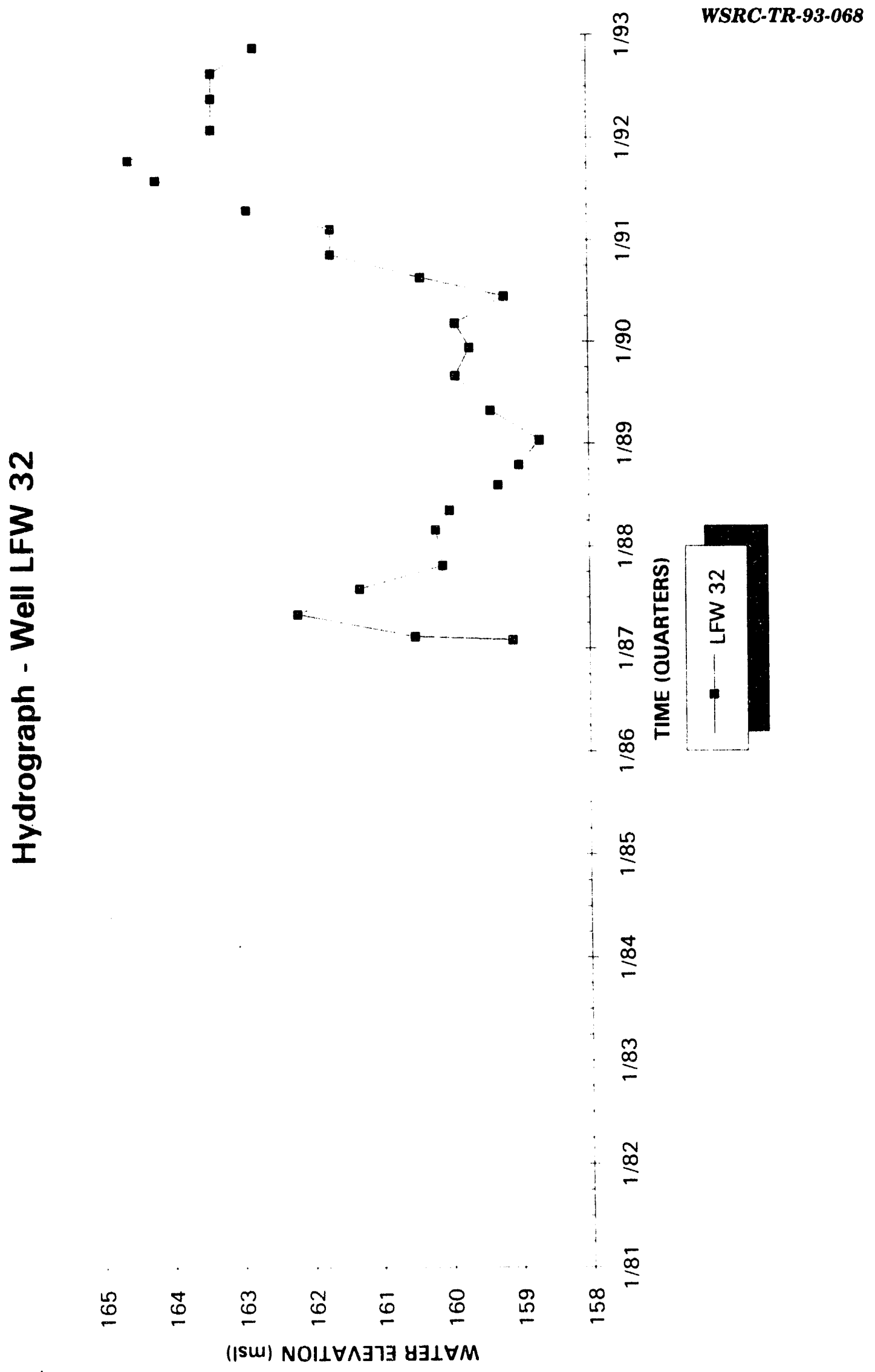




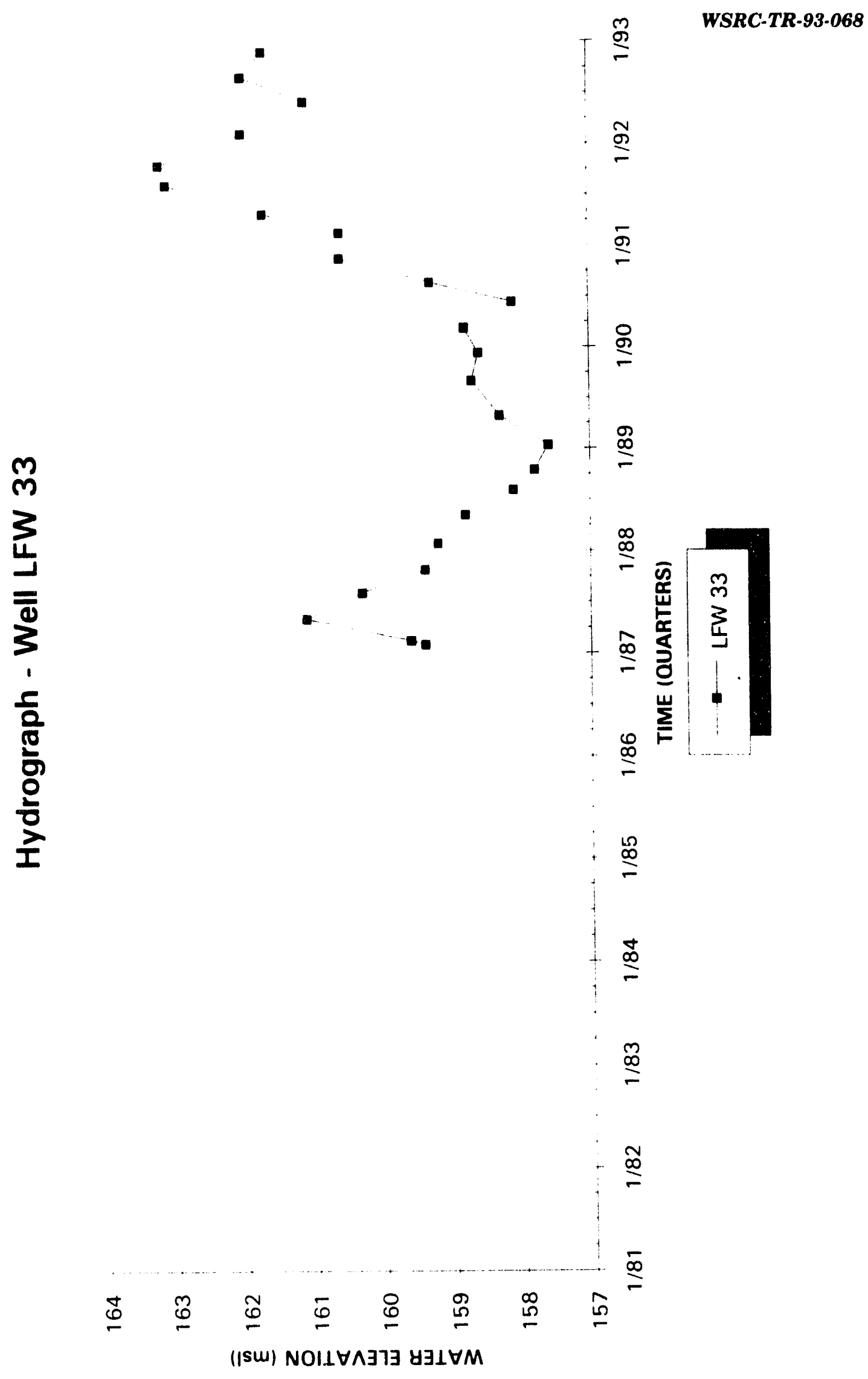




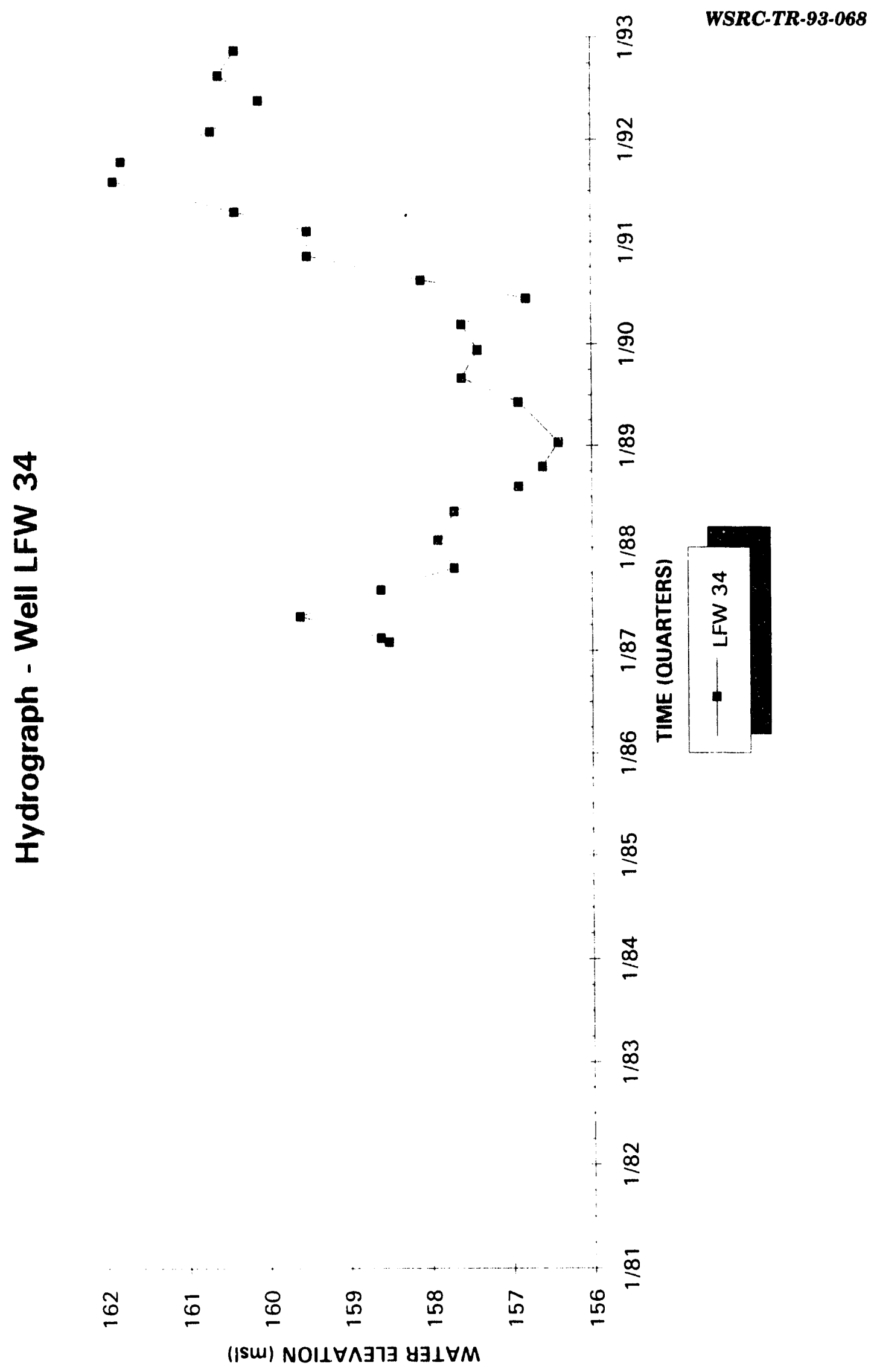




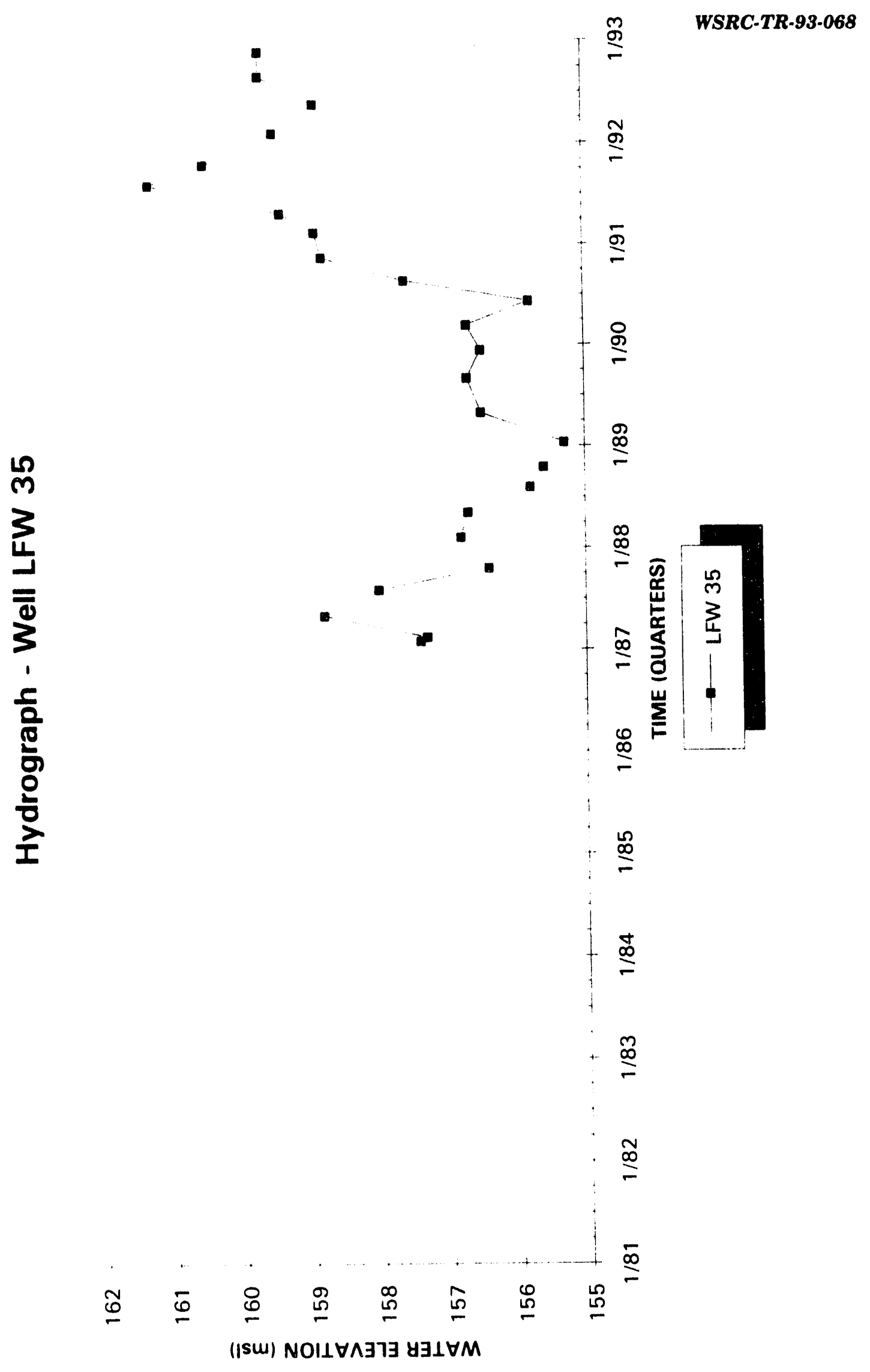




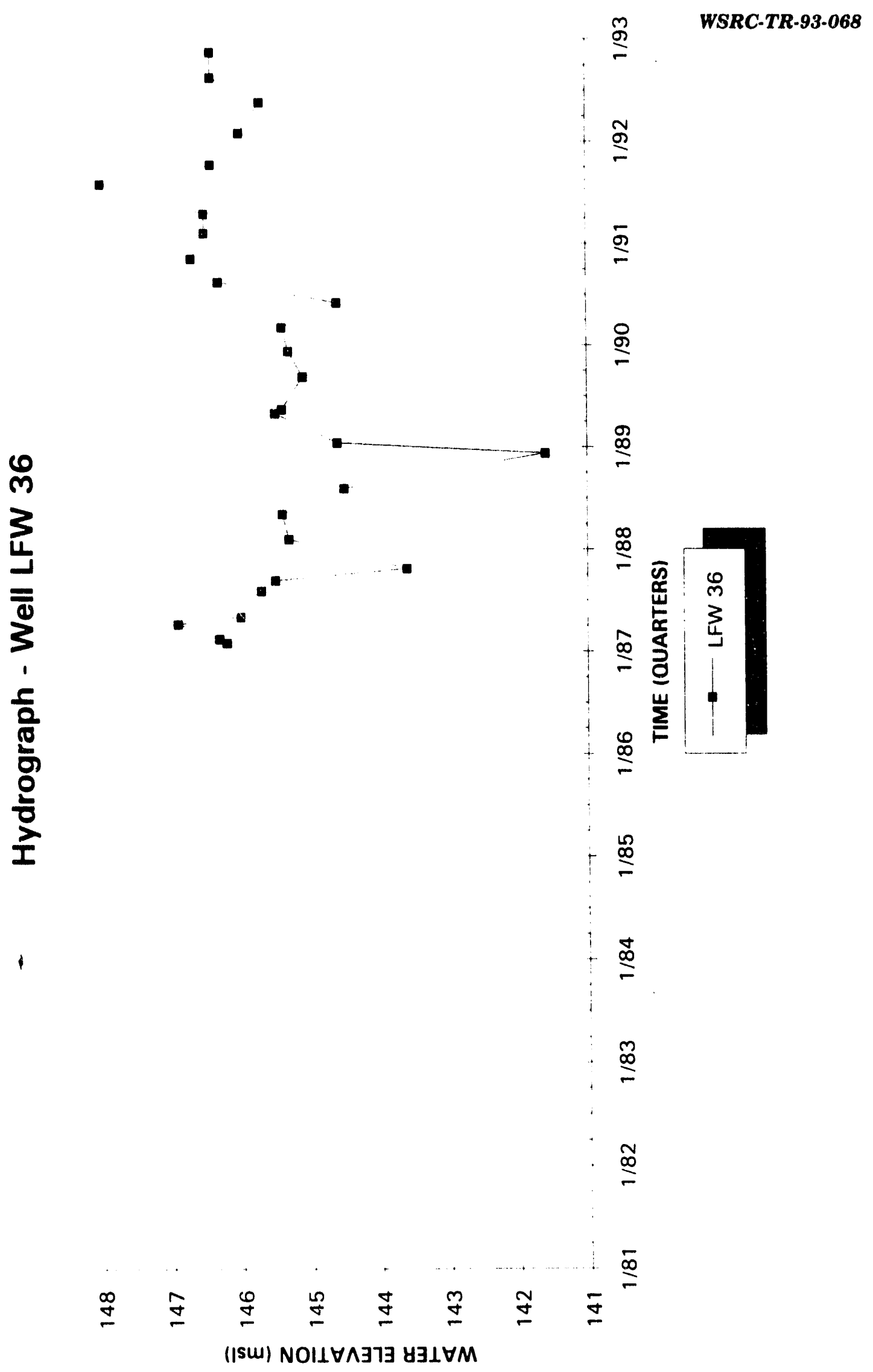




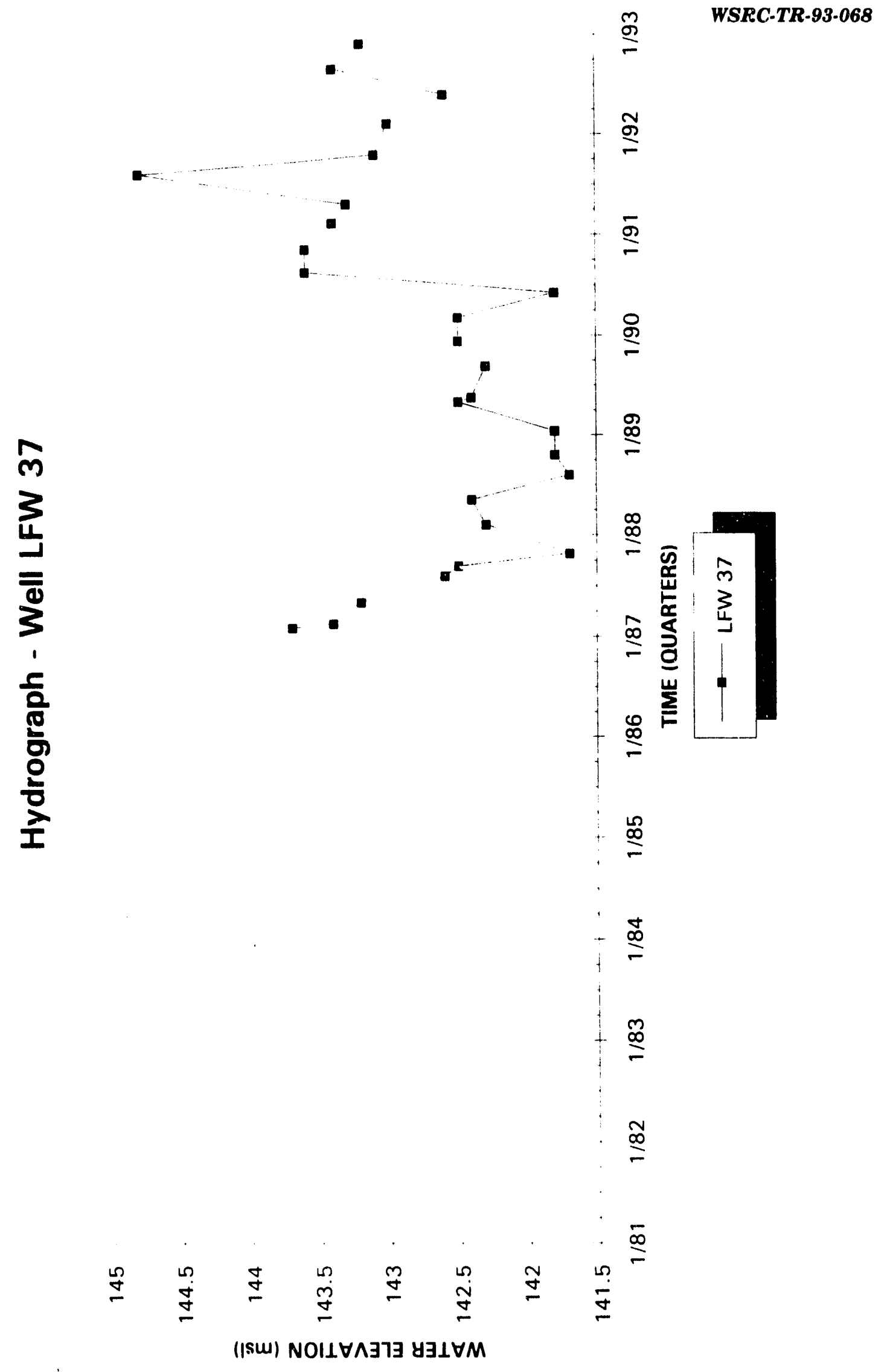




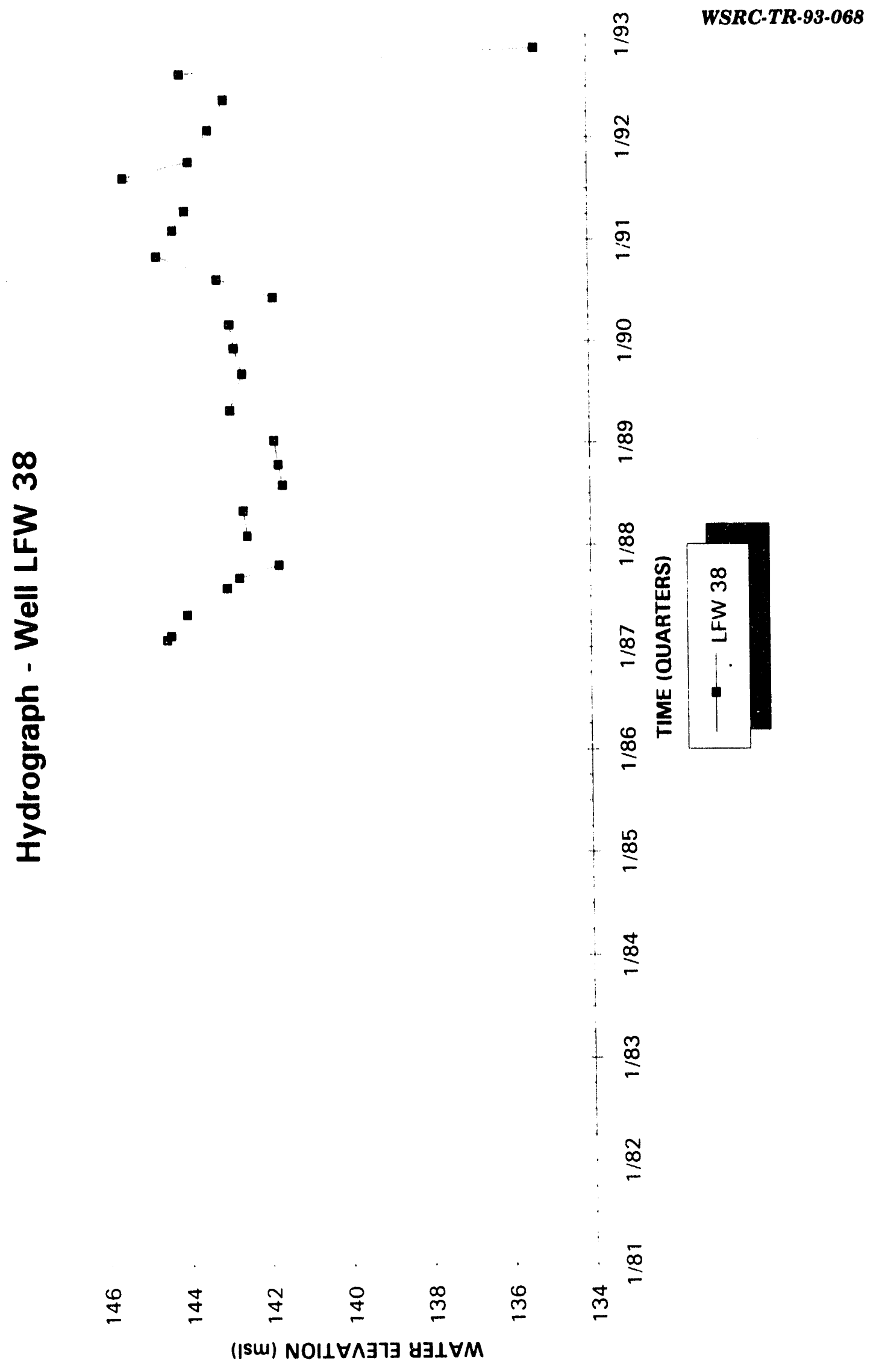

• 


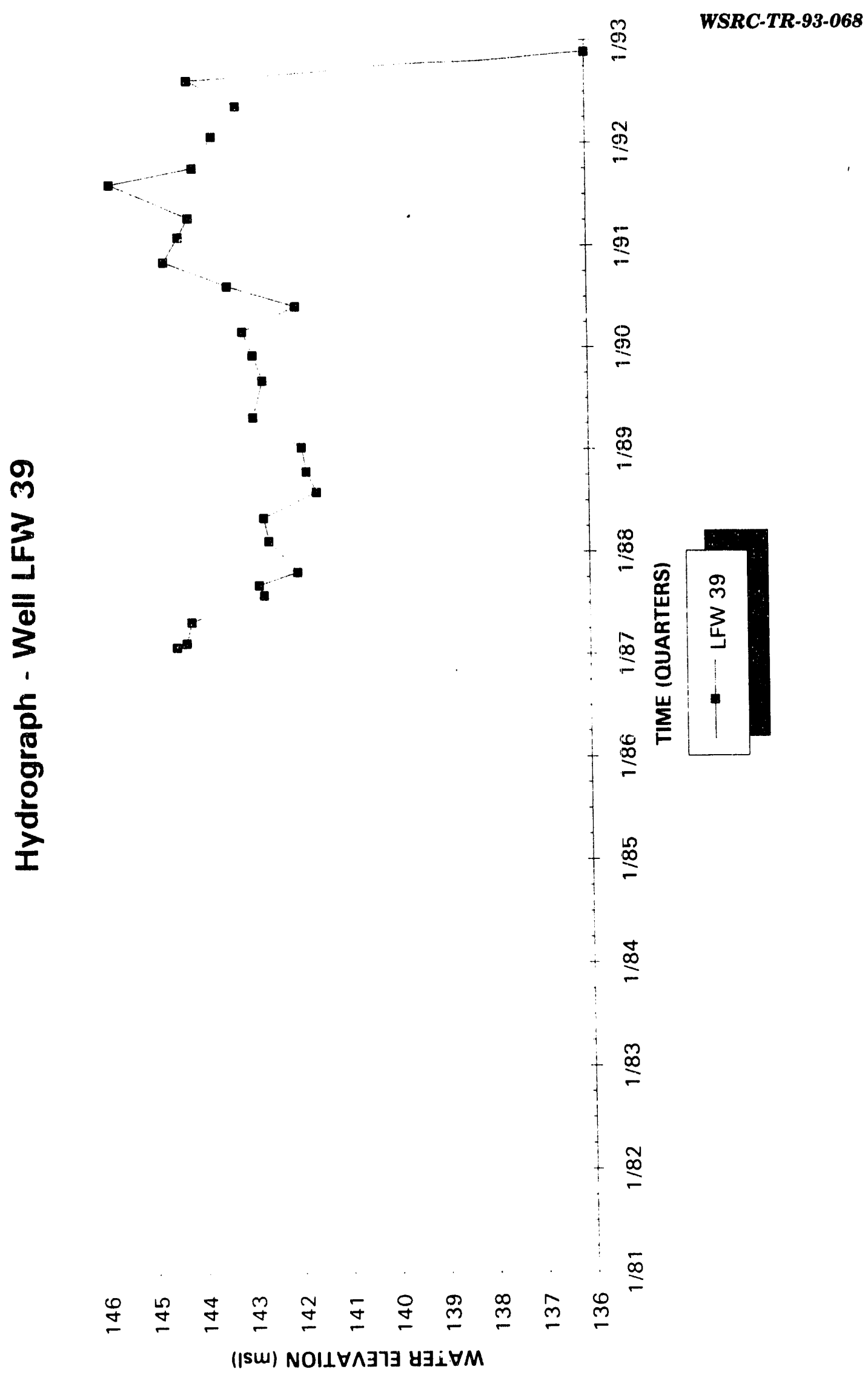




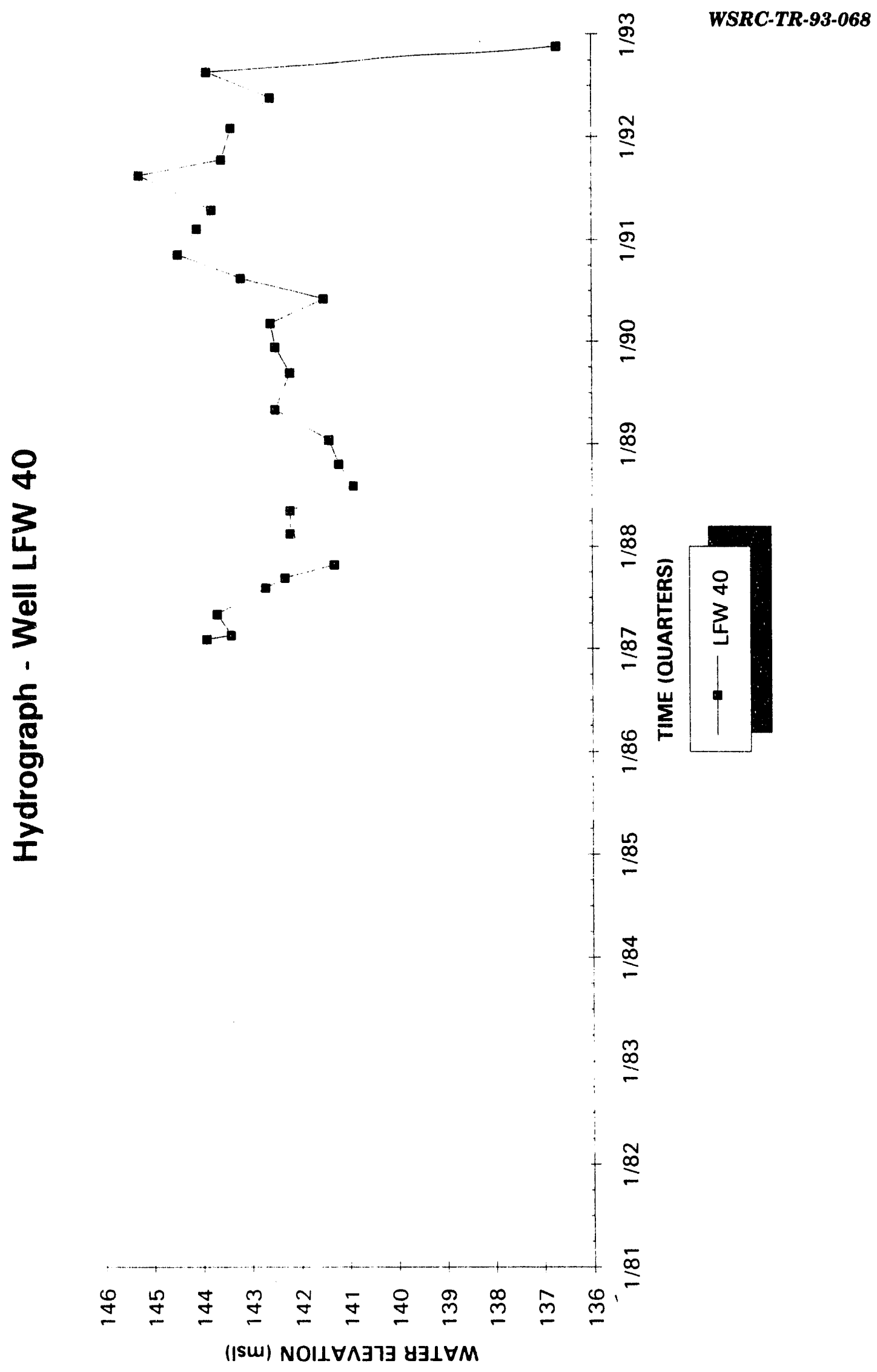




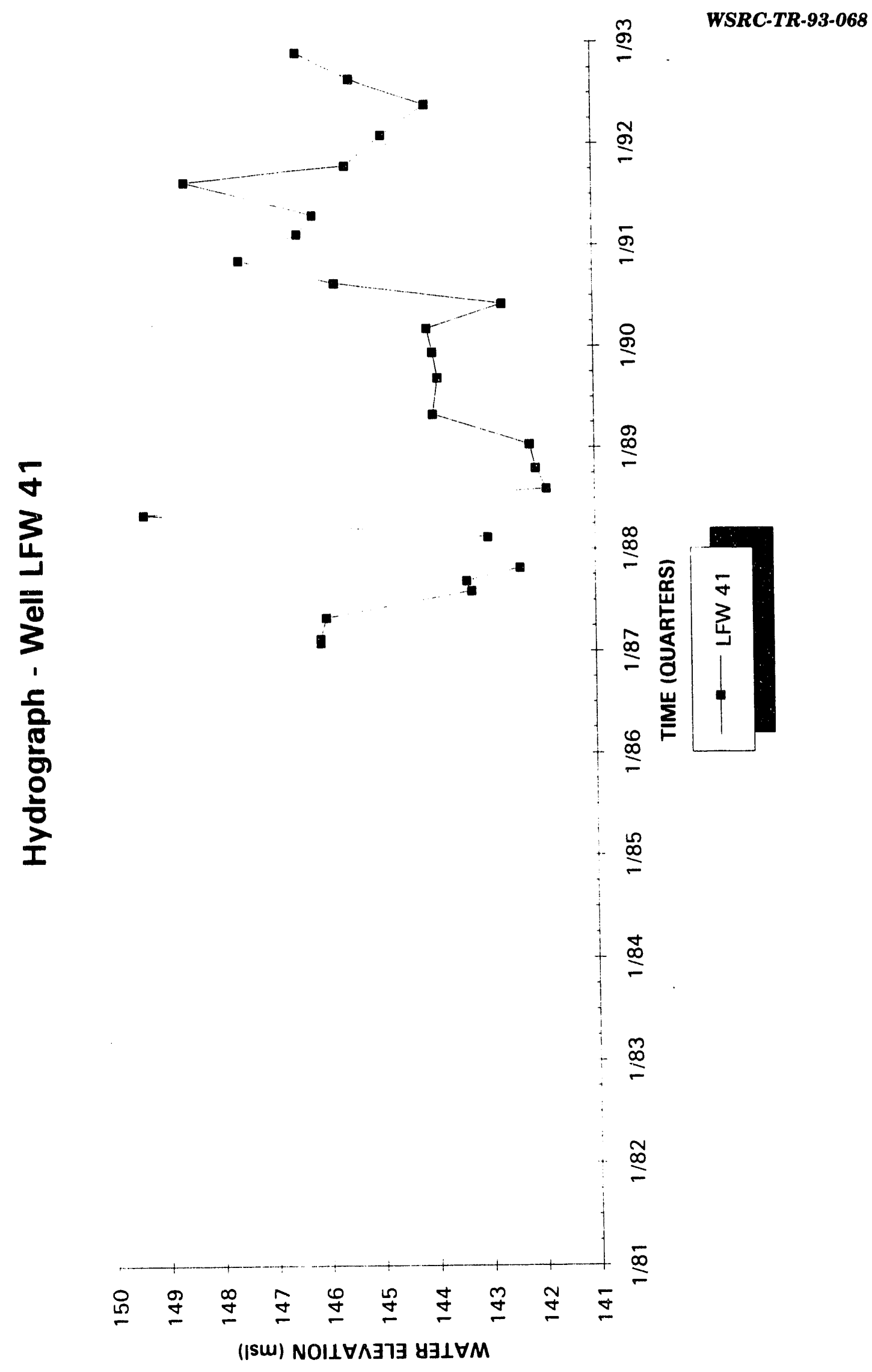




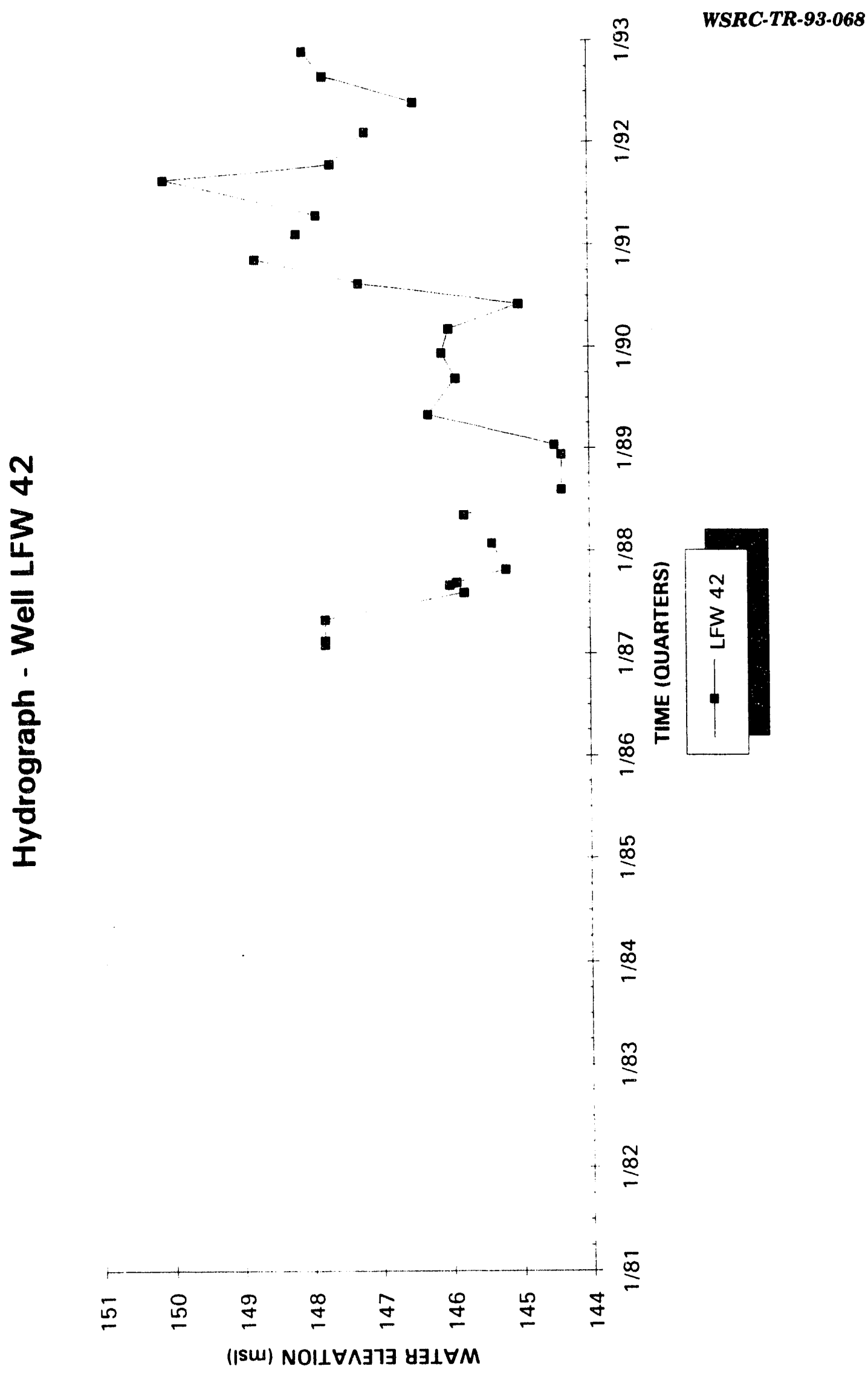




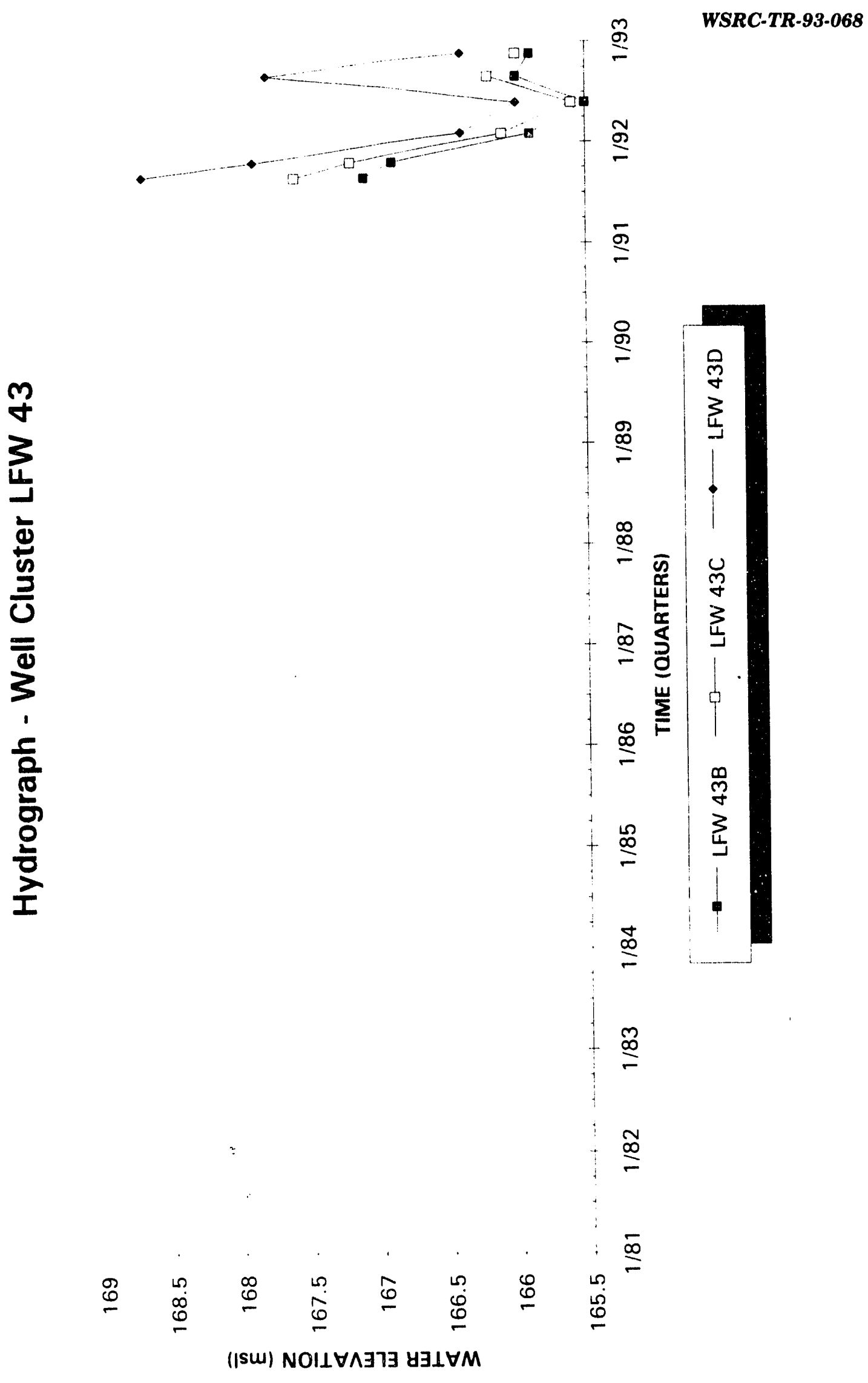




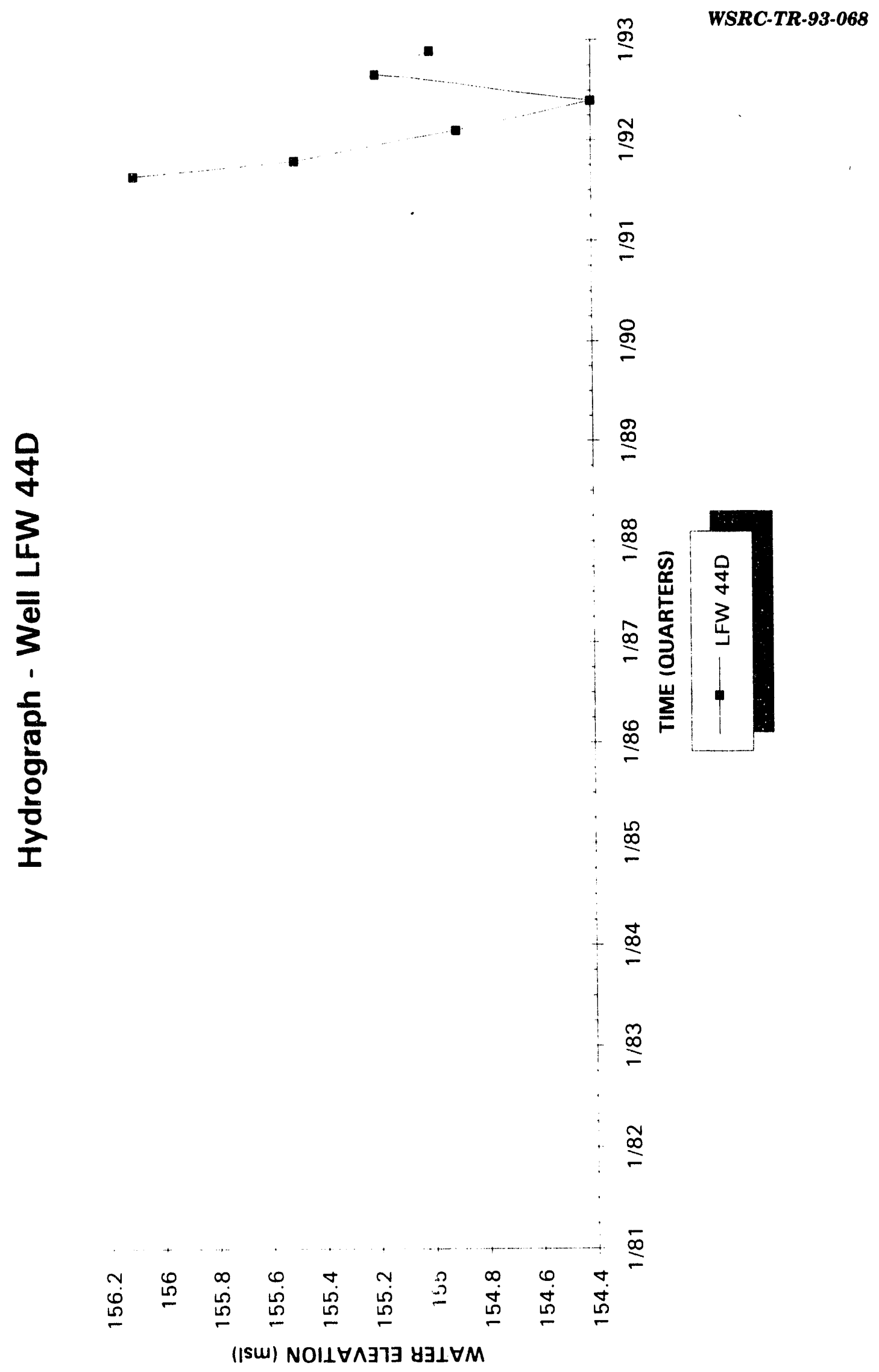




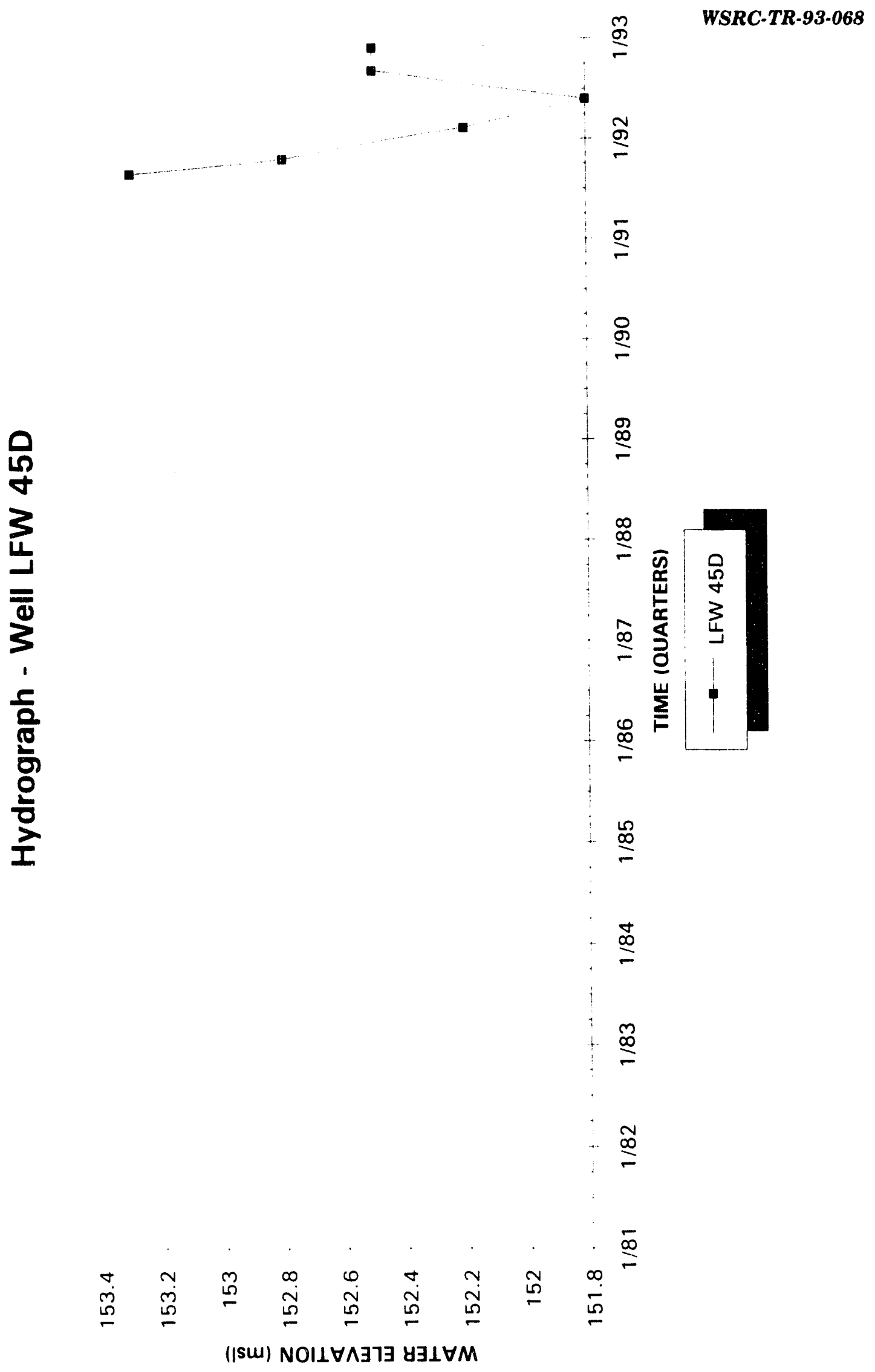




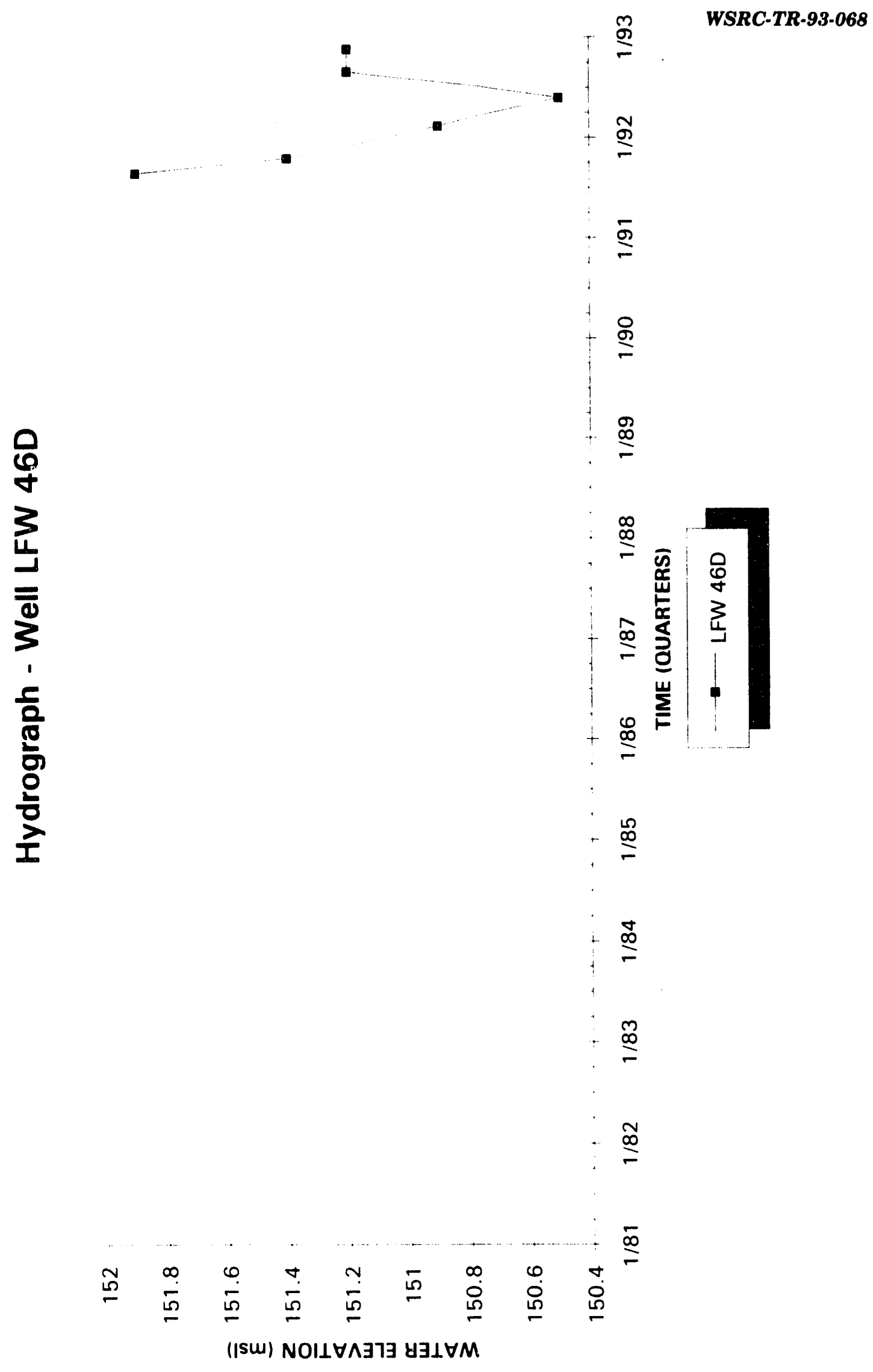




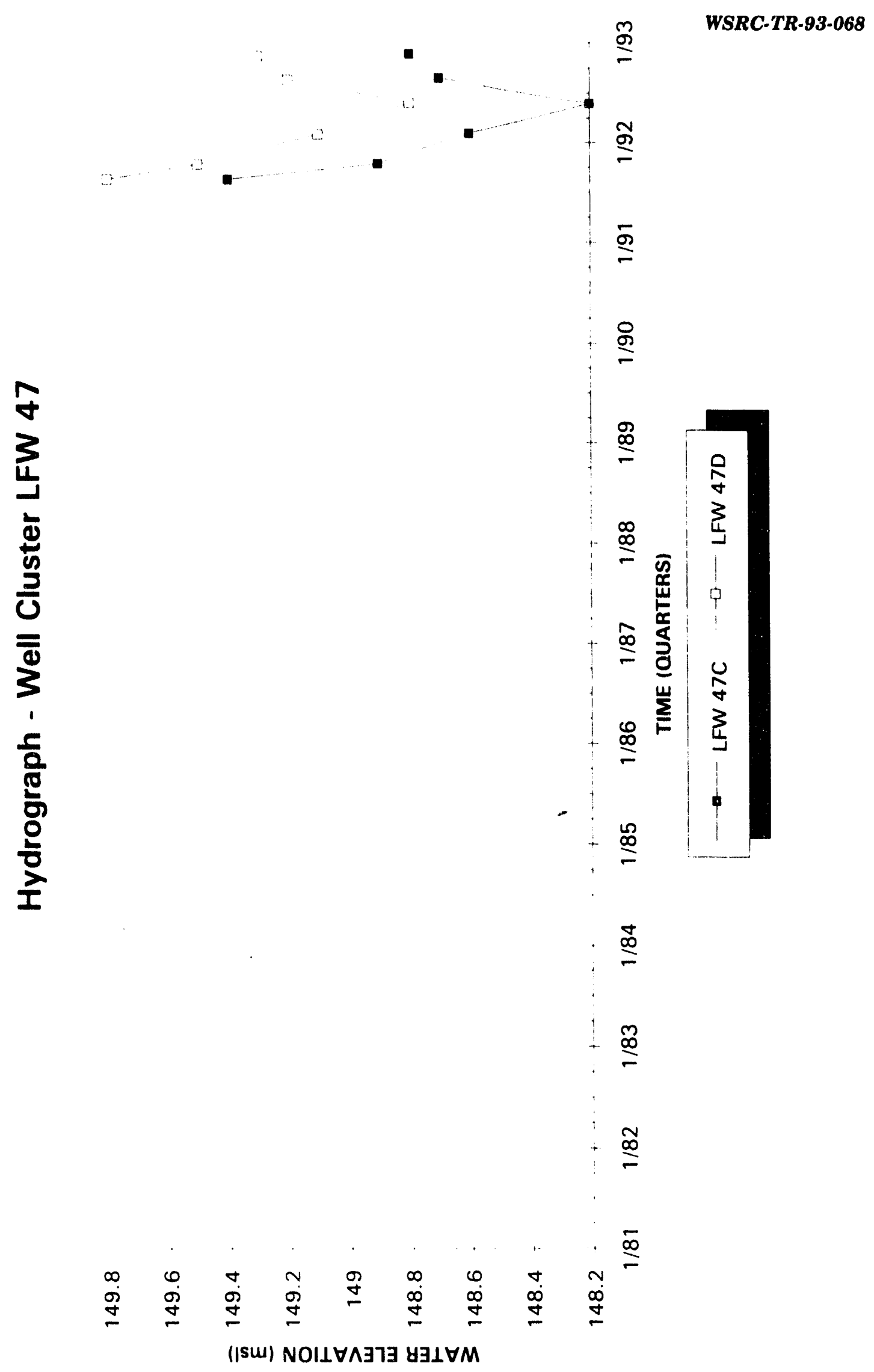




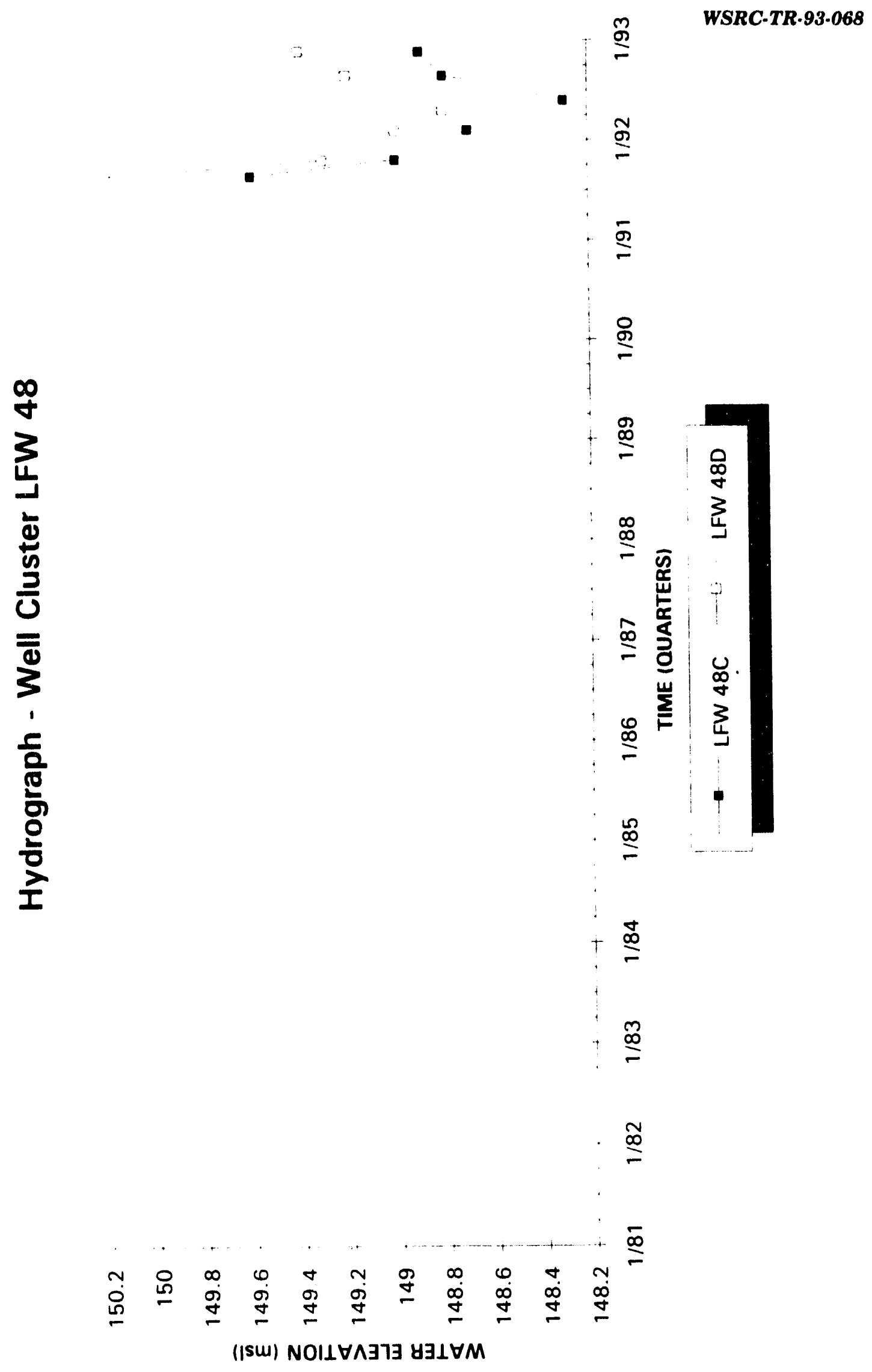




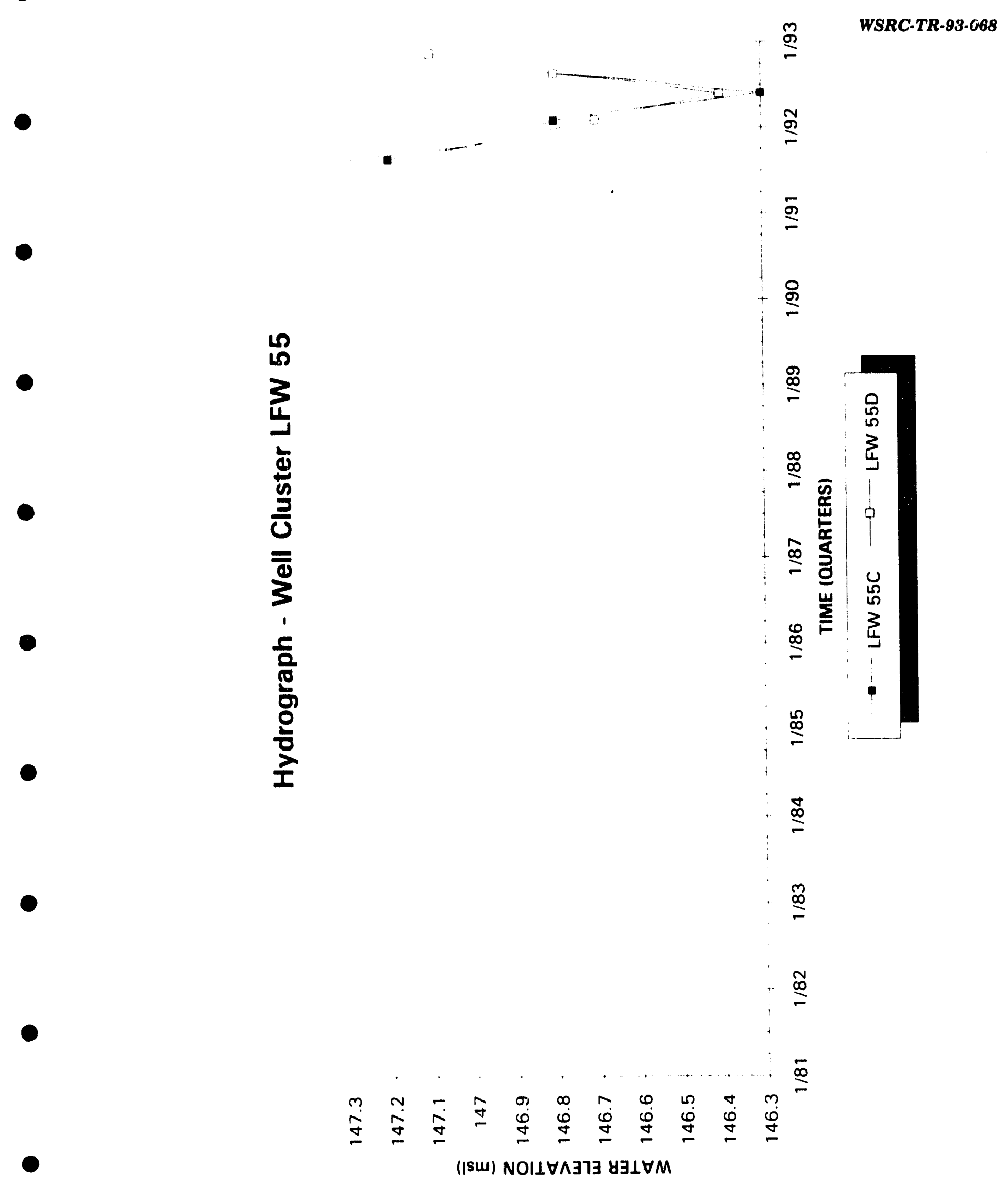




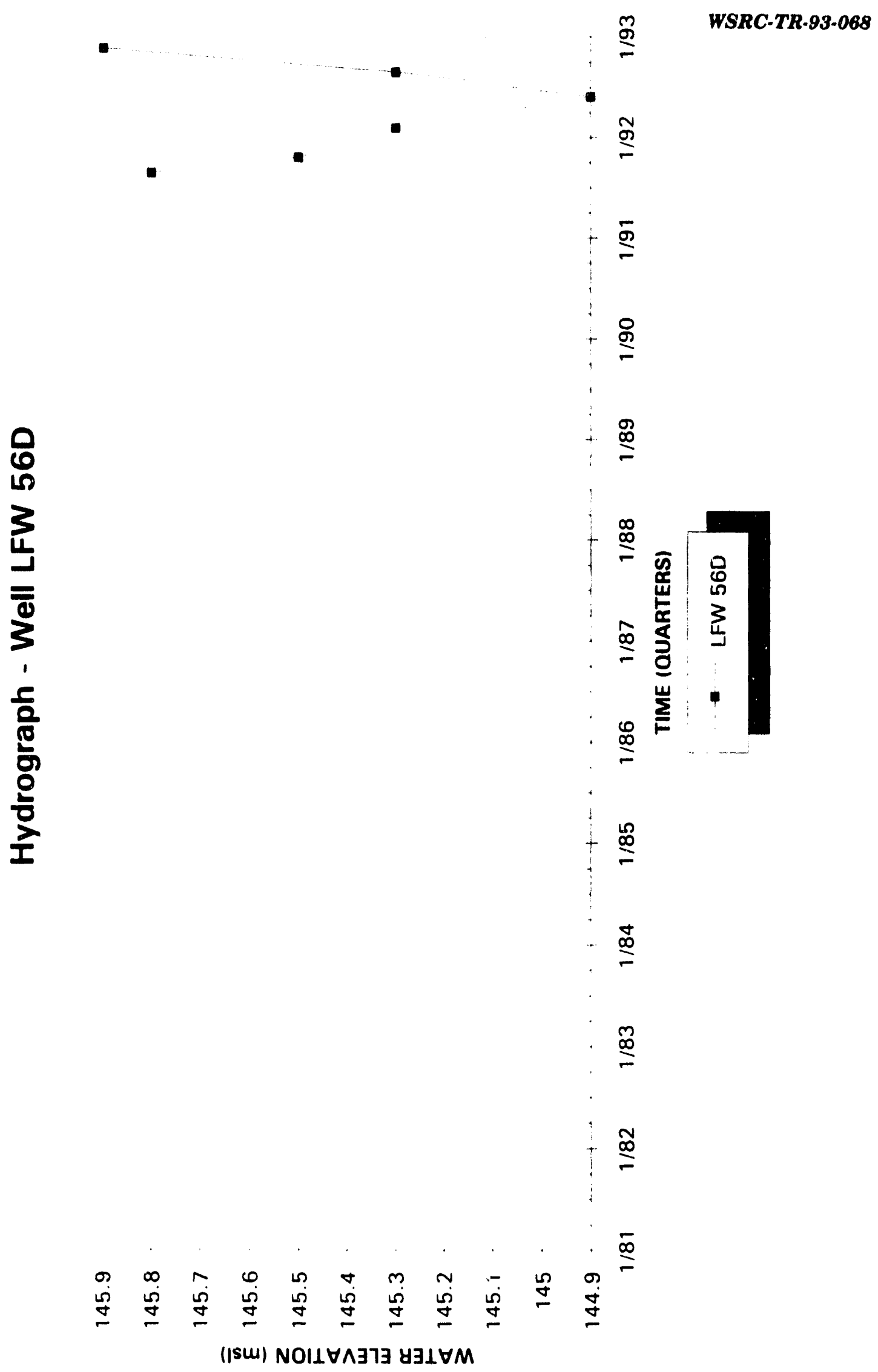




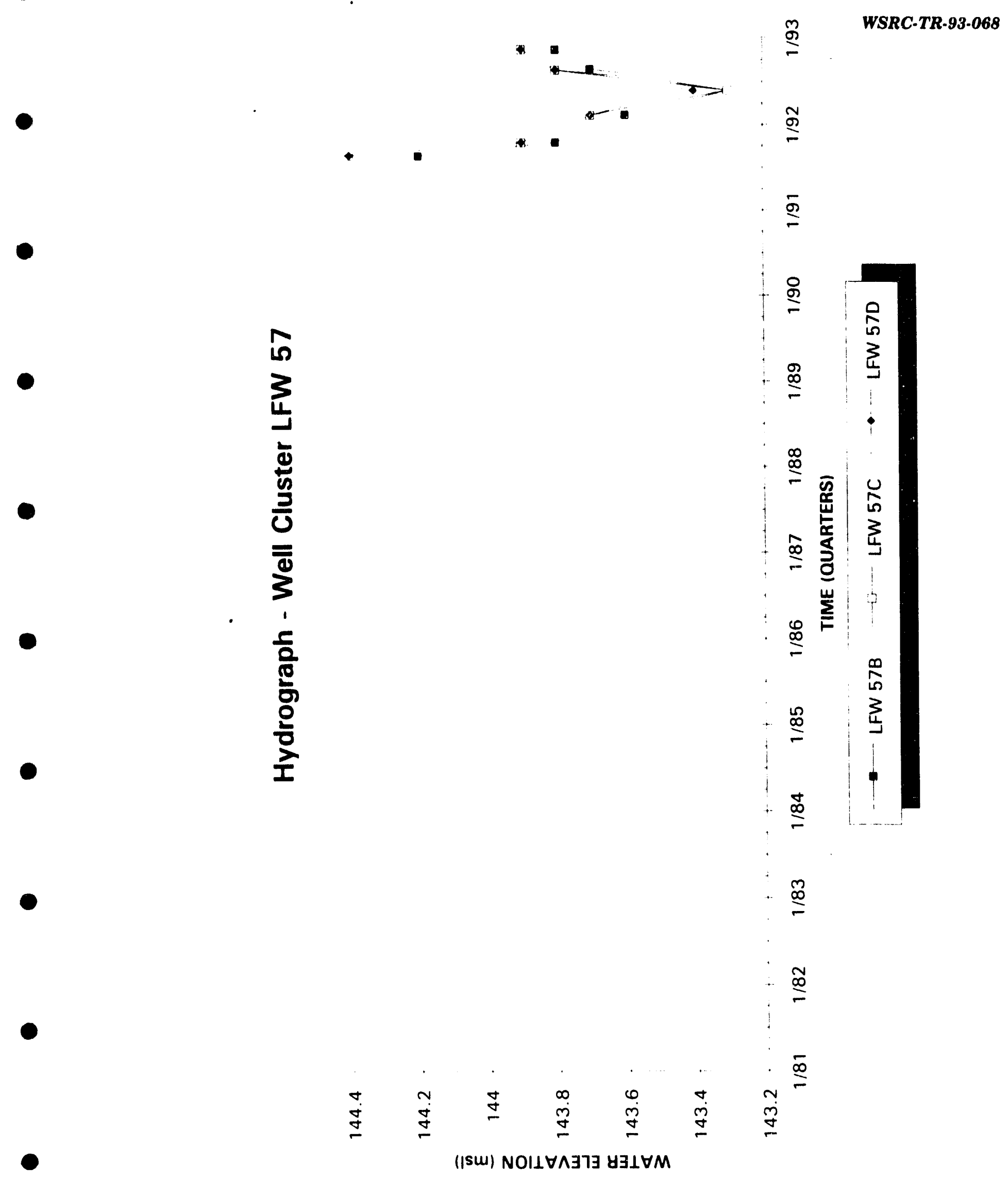




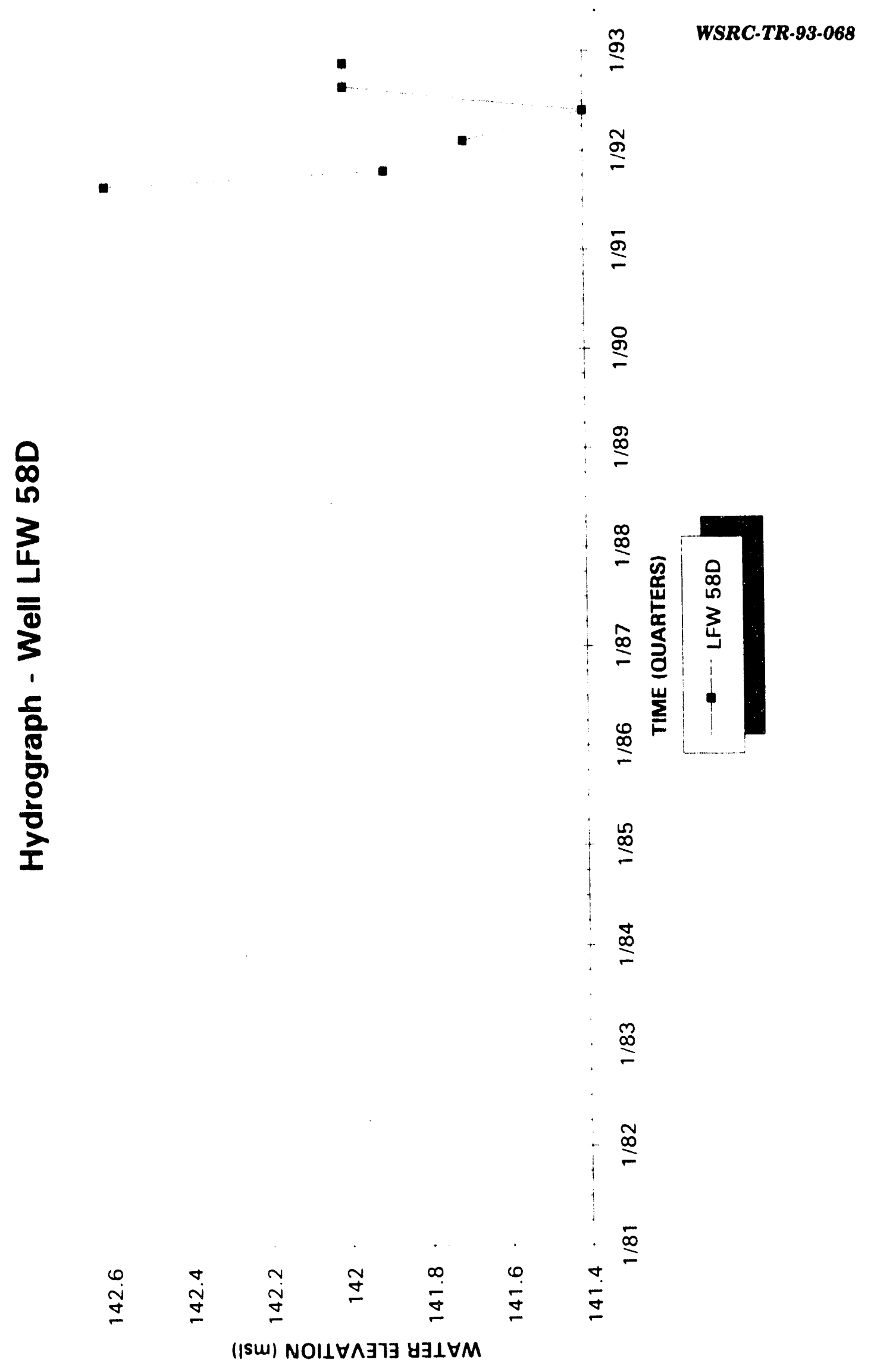




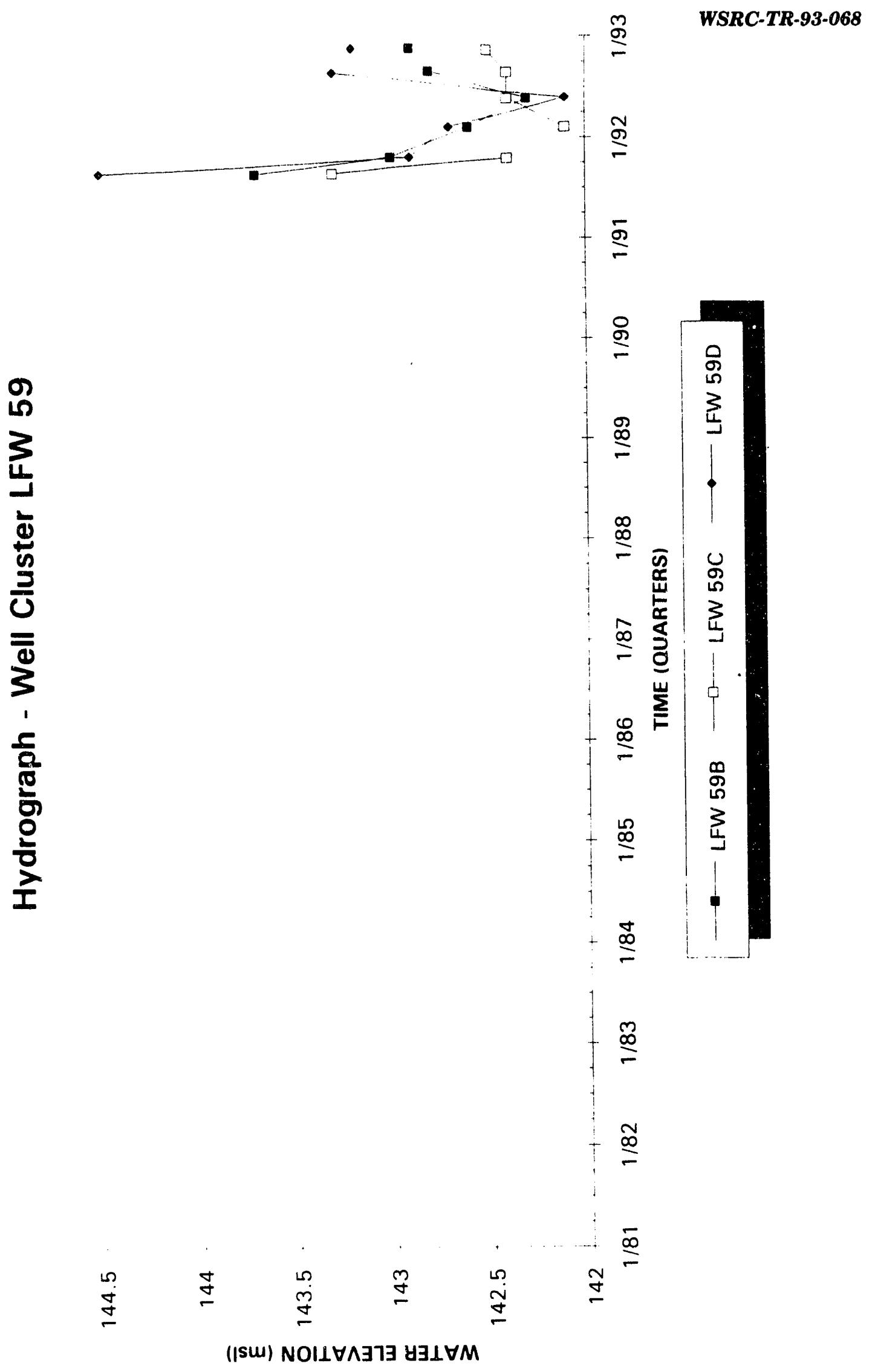




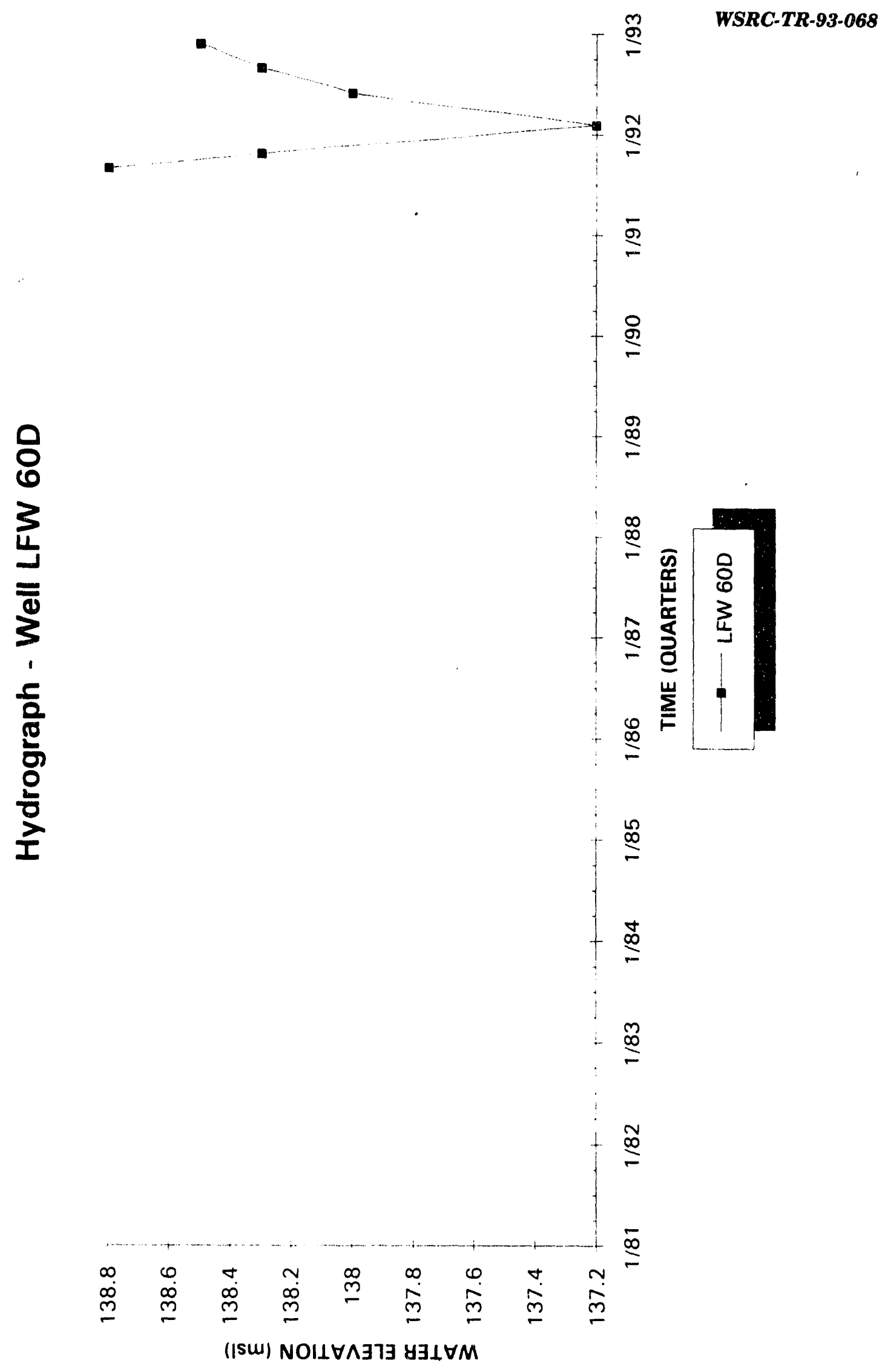

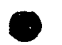

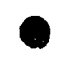

e

c

c 


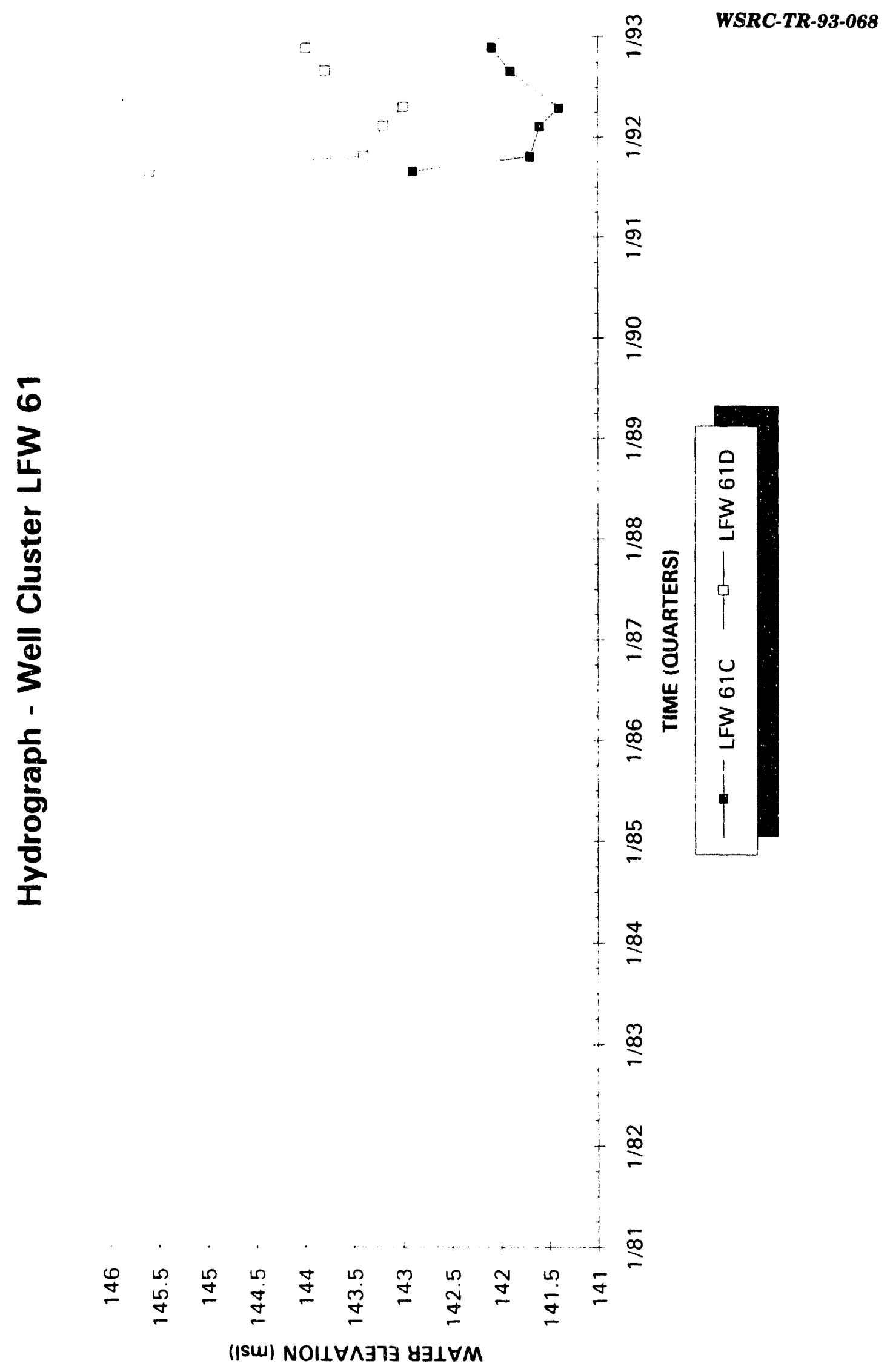




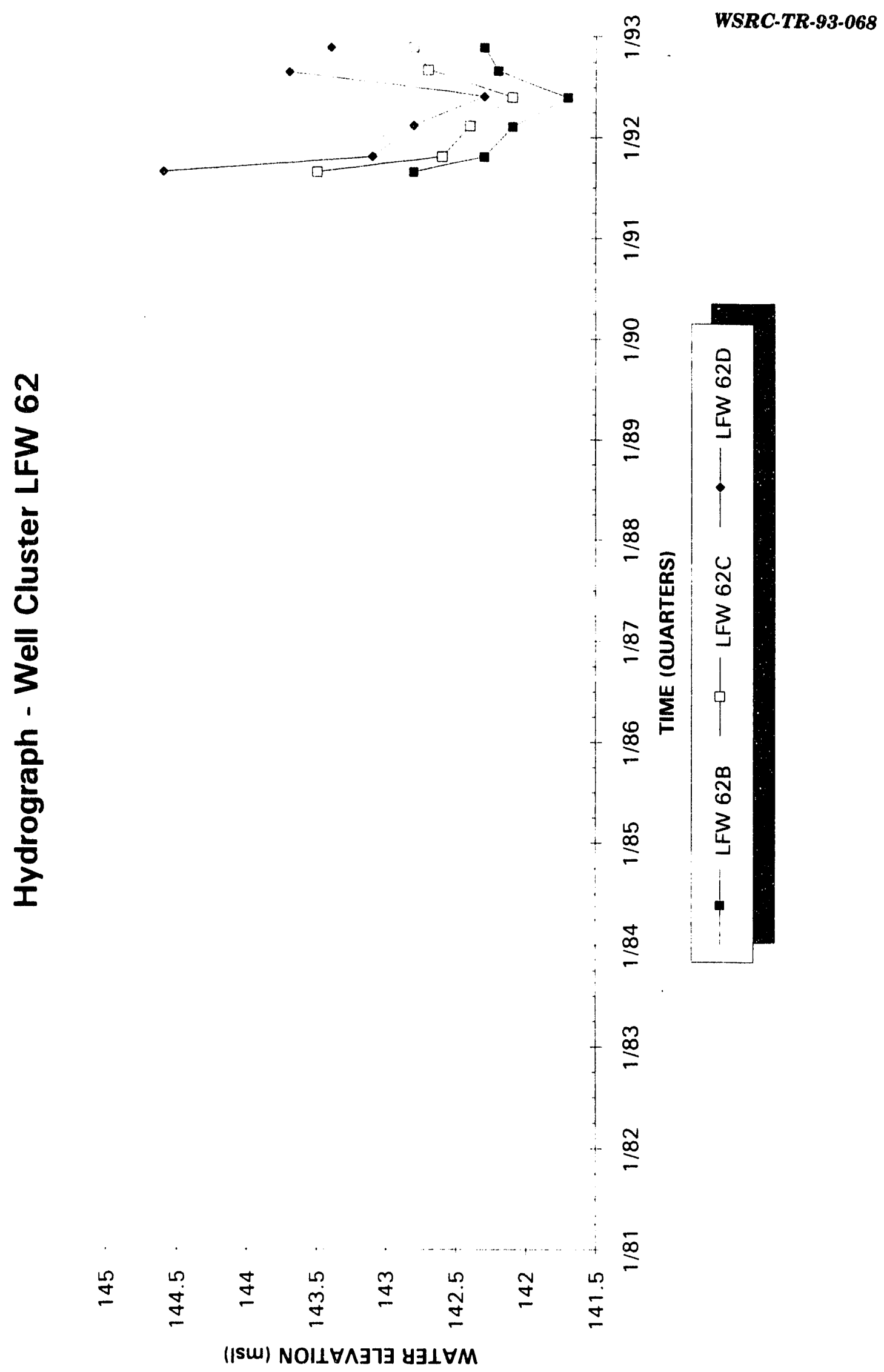



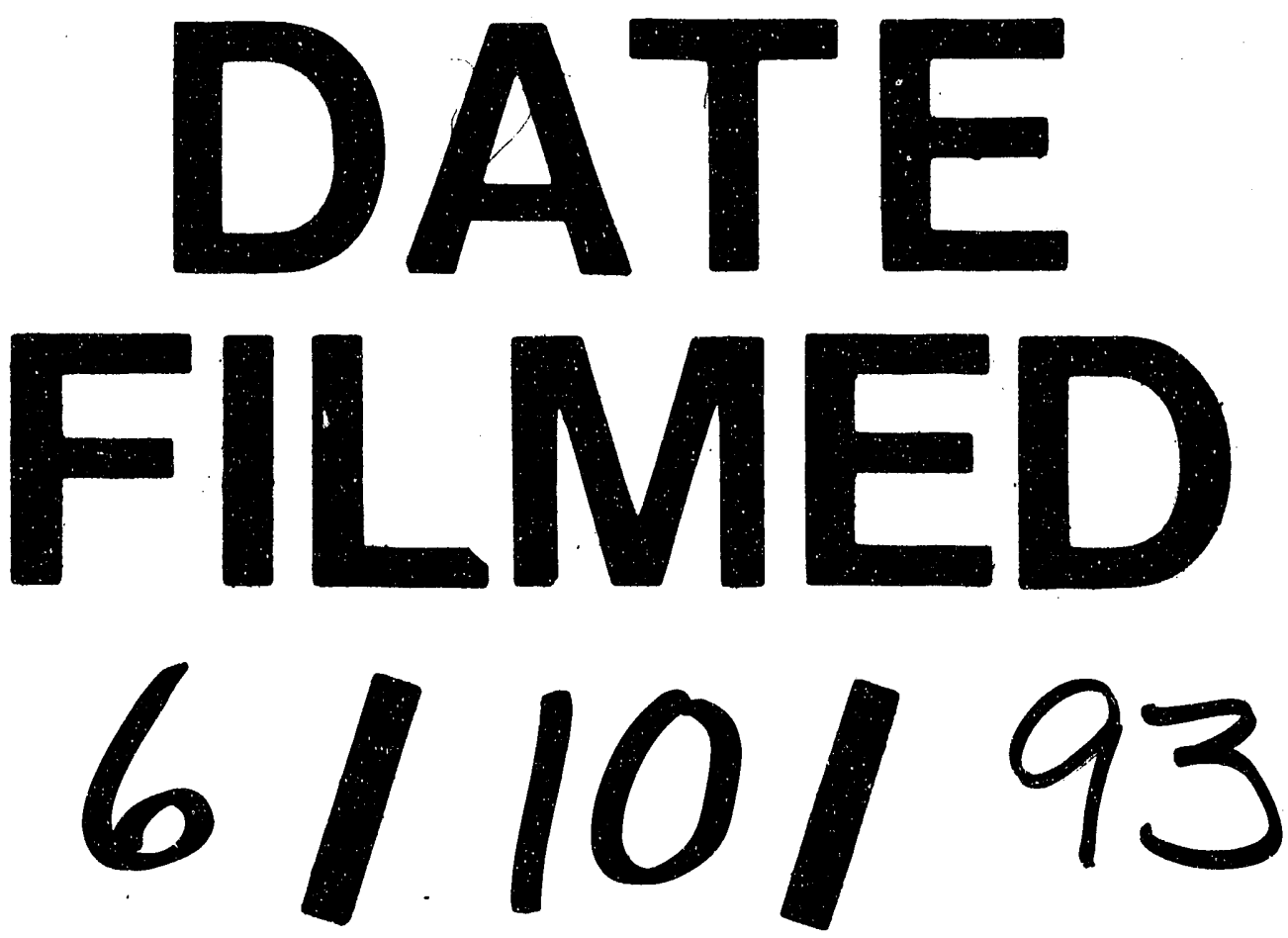
